\title{
Correlative Estimates of
}

Streamflow in the Upper Colorado River Basin

GEOLOGICAL SURVEY WATER-SUPPLY PAPER 1875

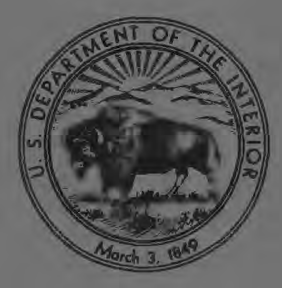




\title{
Correlative Estimates of Streamflow in the Upper Colorado River Basin
}

\author{
By L. E. CARROON
}

GEOLOGICAL SURVEY WATER-SUPPLY PAPER 1875
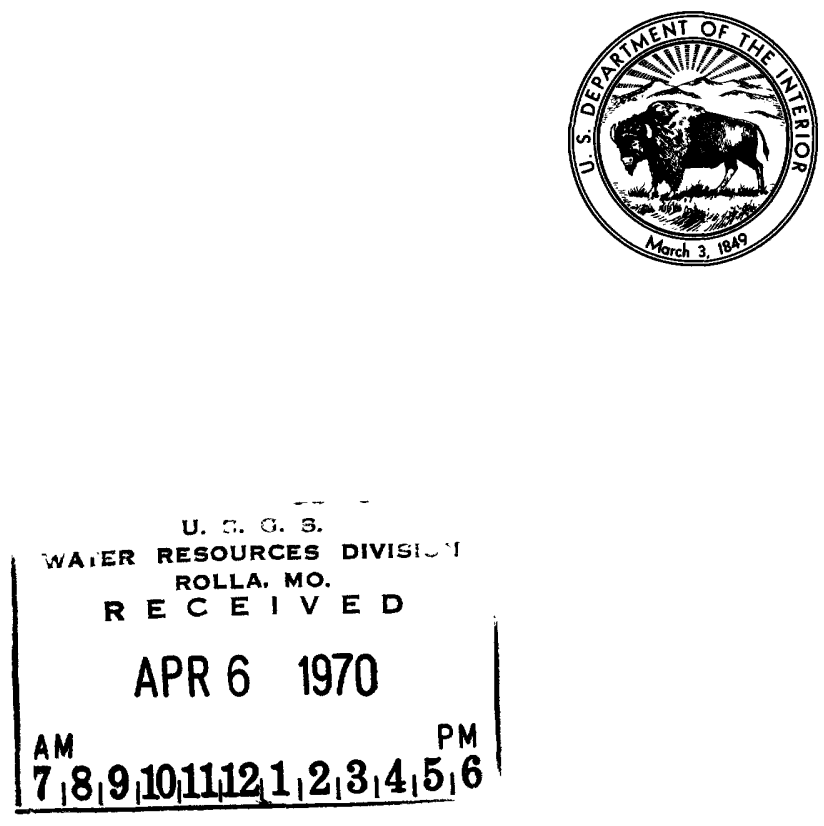


\title{
UNITED STATES DEPARTMENT OF THE INTERIOR
}

WALTER J. HICKEL, Secretary

\author{
GEOLOGICAL SURVEY
}

William T. Pecora, Director

Lilbrary of Congress catalog-card No. 78-603714 


\section{CONTENTS}

Page

Introduction

Purpose and scope. 1

Background of project. .

Hydrologic conditions..... 3

Acknowledgments.

Reports containing basic streamflow data_._. 5

Methods of analysis........ 5

Estimates of streamflow 8

Test of estimated streamflow. 9

Explanation of data.... 11

References.............. 15

Gaging-station records

Colorado River:

Grand Lake Outlet basin

North Inlet at Grand Lake, Colo... . . . . . . 18

Grand Lake:

East Inlet near Grand Lake, Colo... . . . 20

Willow Creek basin

Willow Creek near Granby, Colo...................... 22

Fraser River basin

Fraser River:

Ranch Creek near Tabernash, Colo

Meadow Creek near Tabernash, Colo .............. 26

Troublesome Creek basin

Troublesome Creek near Troublesome, Colo

Blue River basin

Blue River:

Rock Creek near Dillon, Colo

Cabin Creek basin

Cabin Creek:

Sunnyside Creek near Burns, Colo................. 32

Eagle River basin

Eagle River:

Turkey Creek at Red Cliff, Colo._. 34

Cross Creek near Minturn, Colo... .

Gore Creek near Minturn, Colo . . .

Roaring Fork River basin

Roaring Fork River at Aspen, Colo ...

Hunter Creek near Aspen, Colo. . .

Fryingpan River:

Lime Creek at Troutville, Colo............ 44

Lime Creek at Thomasville, Colo............. 46

Crystal River near Redstone, Colo._... 48 
Gaging-station records-Continued

Colorado River-Continued

Page

Canyon Creek basin

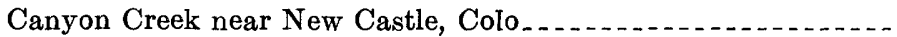

Elk Creek basin

Elk Creek at New Castle, Colo...............................

Battlement Creek basin

Battlement Creek near Grand Valley, Colo .................. 54

Plateau Creek basin

Plateau Creek at upper station near Collbran, Colo._........ 56

Big Creek at upper station near Collbran, Colo_..._._._. $\quad 58$

Cottonwood Creek at upper station near Molina, Colo _.... 60

Mesa Creek near Mesa, Colo......................... 62

Gunnison River basin

Gunnison River:

Cebolla Creek at Powderhorn, Colo

Smith Fork at Crawford, Colo _...

East Muddy Creek near Bardine, Colo.................. 68

West Muddy Creek near Ragged Mountain, Colo._._. $\quad 70$

Muddy Creek at Bardine, Colo...................... 72

Anthracite Creek near Floresta, Colo................ 74

Uncompahgre River:

Red Mountain Creek near Ironton, Colo. . . . . . . . . 76

Roubideau Creek at mouth, near Delta, Colo........... 78

Dolores River basin

Dolores River:

San Miguel River near Telluride, Colo. . . . . . . . . . 80

Fall Creek near Fall Creek, Colo................ 82

Leopard Creek at Noel, Colo................... 84

San Miguel River near Nucla, Colo............ 86

San Miguel River at Uravan, Colo.....

Dolores River at Gateway, Colo_..................... 90

Tributaries between Dolores River and Green River

Indian Creek near Monticello, Utah. .................. 92

Green River basin

Green River:

Horse Creek near Daniel, Wyo . . . . . .

Middle Piney Creek below South Fork, near Big Piney,

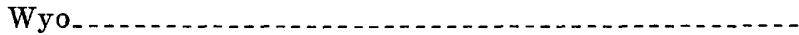

Blacks Fork:

Smith Fork at Mountainview, Wyo.............. 98

Henrys Fork:

Middle Fork Beaver Creek:

West Fork Beaver Creek near Lonetree, Wyo....

Yampa River:

Middle Fork Little Snake River (head of Snake River):

North Fork Little Snake River near Slater, Colo. -

Little Snake River:

Battle Creek near Encampment, Wyo.......... 104

Ashley Creek:

Dry Fork:

East Fork of Dry Fork near Dry Fork, Utah..... - 
Gaging-station records $\rightarrow$ Continued

Colorado River-Continued

Green River basin-Continued

Page

Ashley Creek at Sign of the Maine, near Vernal, Utah...... 108

Duchesne River near Hanna, Utah. ................. 110

Duchesne River at Hanna, Utah. . . . . . . . . . . . 112

White River below Trappers Lake, Colo _... . . . . . . 114

Price River near Wellington, Utah._._._. 116

Huntington Creek (head of San Rafael River):

Cottonwood Creek near Castle Dale, Utah............ 118

Ferron Creek near Castle Dale, Utah. ............ 120

San Rafael River near Castle Dale, Utah............... 122

Dirty Devil River basin

Fremont River (head of Dirty Devil River):

Muddy Creek:

Ivie Creek above diversions, near Emery, Utah........

San Juan River basin

East Fork San Juan River:

West Fork San Juan River above Borns Lake, near Pagosa

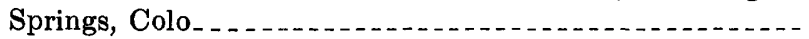

West Fork San Juan River near Pagosa Springs, Colo......

Rio Blanco:

Rito Blanco near Pagosa Springs, Colo...............

Navajo River:

Little Navajo River at Chromo, Colo . ............

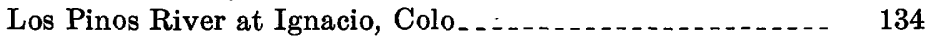

Animas River:

Falls Creek near Durango, Colo..............................

Junction Creek near Durango, Colo..................

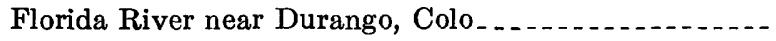

Florida River at Bondad, Colo. ...................

West Mancos River (head of Mancos River) near Mancos, Colo.

\section{ILLUSTRATIONS}

Plate 1. Map of Upper Colorado River Basin, showing location of streamflow gaging stations.......... In pocket

Figure 1. Index map, showing report area .

2. Graph showing seasonal distribution of streamflow at selected sites.

3. Histograms of annual streamflow at selected sites.......... 6

4. Graph showing relation of annual streamflow of Little Navajo River at Chromo, Colo., and Navajo River at Edith, Colo. 



\title{
CORRELATIVE ESTIMATES OF STREAMFLOW IN THE UPPER GOLORADO RIVER BASIN
}

\author{
By L. E. Carroon \\ INTRODUCTION \\ PURPOSE AND SCOPE
}

Most hydrologic analyses, whether for appraisal of the water resource, feasibility of a particular development, design of a system of operation, assessment of gains or losses from acts of man or natural changes, or almost any other use, require the extension in time of some streamflow records. In the Upper Colorado River Basin, streamflow records have been extended by various agencies from time to time to fulfill their individual needs, resulting in different estimates of flow for the same point and time. The purpose of this report is to provide correlative estimates of monthly mean discharge at discontinued streamflow gaging stations so that all users will have access to a single set of data.

Estimates of monthly mean discharge for periods other than the period of actual record are given for certain discontinued streamflow gaging stations. The stations selected were discontinued in recent years, but they have a sufficient length of record to permit development of a meaningful correlation with the records for a nearby long-term gaging station. Where possible, estimates of monthly mean discharge are made to provide a complete record, estimated or measured, from 1930 to 1965 . However, where the long-term gaging station record encompasses a shorter period, the estimates cover only that shorter period. Estimates for periods prior to 1930 were not made, although these may be computed for a few stations. Lack of a related long-term gagingstation record prevented the extension of records at many discontinued stations.

The area covered in this report (fig. 1) is that defined in the Colorado River Compact of 1922, as the Upper Basin-"those parts of the States of Arizona, Colorado, New Mexico, Utah, and Wyoming within and from which waters naturally drain into the Colorado River 
System above Lee Ferry," ${ }^{1}$ but excluding "all parts of said States located without the drainage area of the Colorado River System which are now and shall hereafter be beneficially served by waters diverted from the System above Lee Ferry" (Wilbur and Ely, 1948, p. A18).

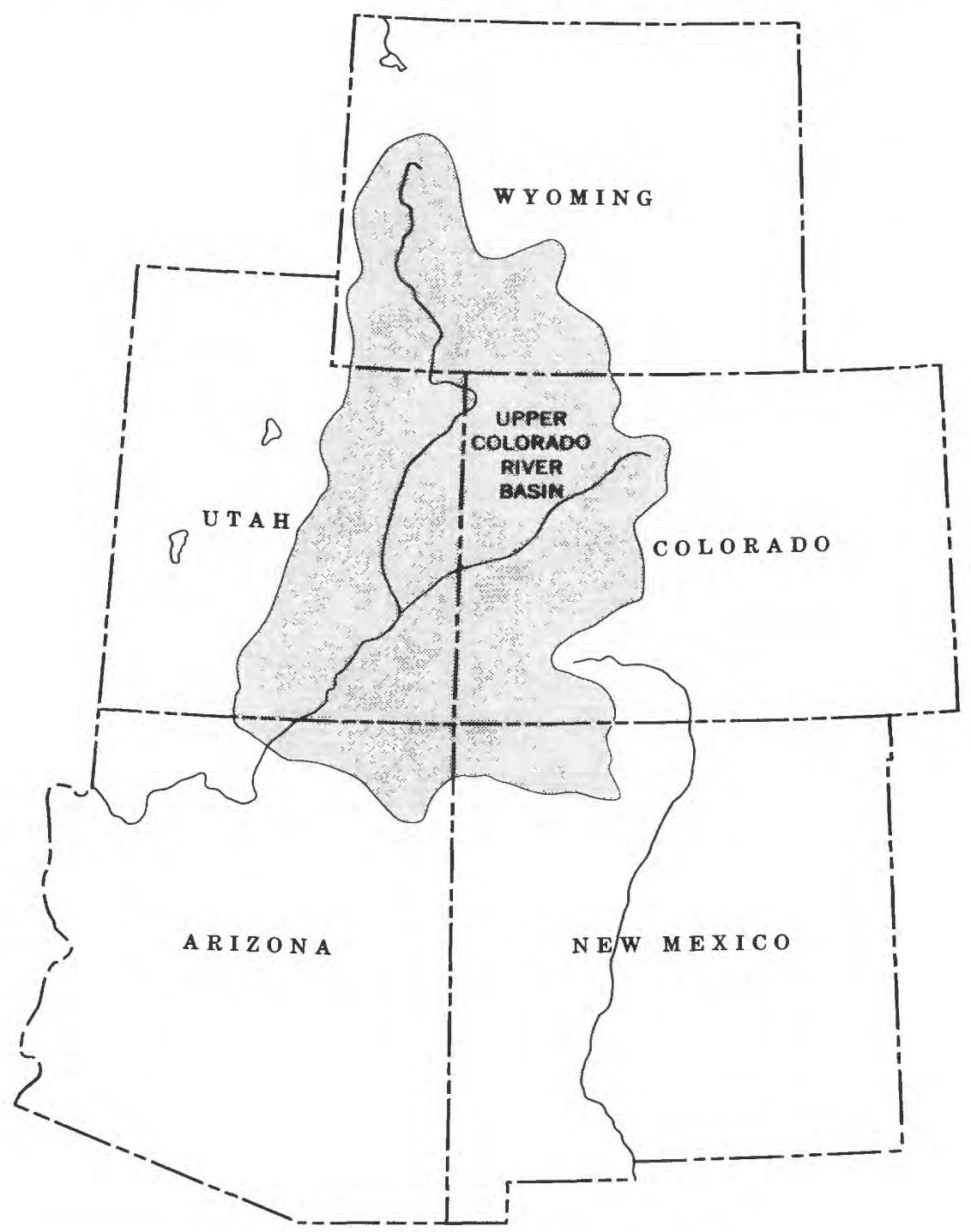

FIGURE 1.-Index map, showing report area.

1 The term "Lee Ferry" means a point in the main stream of the Colorado River 1 mile below the mouth of the Paria River. It is not the same location as the U.S. Geological Survey streamflow gaging station at Lees Ferry, Arizona, which is above the mouth of the Paria River. 
The U.S. Geological Survey, in the years 1954-57, made a nationwide appraisal of the adequacy of the stream-gaging network by means of correlation analysis. This appraisal indicated that records for certain streamflow gaging stations were sufficiently related to records for others that some stations could be discontinued with little loss of information. Consequently, a number of these stations were discontinued, but no systematic effort was made to extend records at the discontinued sites on the basis of information obtained at the continuing ones. Data users in the Upper Colorado River Basin considered using the relationships developed for the national appraisal as an economical means of extending records. However, further study of statistical theory indicated that although the relationships developed for the network appraisal adequately described the dependence between stations, they were unsuitable for extending streamflow records. Hence, a special study was initiated in 1964 to estimate monthly runoff for periods before or after the period of actual record at selected gaging stations. Results of the study are given in this report.

\section{HYDROLOGIC CONDITIONS}

The Upper Colorado River Basin contains approximately 109,300 square miles and has a great range in physical characteristics that affect streamflow characteristics and, hence, streamflow relationships. Most of the water in the Colorado River at Lees Ferry, Ariz., comes from the small part of the basin in the highest part of the Rocky Mountains, central and northern Colorado; the Wind River Range, Wyo.; the Uinta Mountains, Utah; and the San Juan Mountains, southwestern Colorado.

Most of the precipitation in these areas is snow, and the runoff is largely snowmelt. Because of this, $60-90$ percent of the annual streamflow usually occurs in the three months May-July (fig. 2). Because snowmelt is produced by heat, and the amount of heat varies inversely with latitude and altitude, streamflow characteristics are similar only where similar percentages of the drainage basins are in the same latitude and altitude zones. Latitude in the basin varies from approximately $35^{\circ} 30^{\prime}$ along the Continental Divide in New Mexico to about $43^{\circ} 30^{\prime}$ in western Wyoming, which is sufficient to cause considerable difference in snowmelt patterns from that source alone. The altitude of the basin ranges from about 3,000 feet near Lees Ferry, Ariz., to more than 14,000 feet in western Colorado.

Streams that derive any considerable part of their annual flow from 
rain are generally those which drain the arid or semiarid parts of the basin, where runoff is derived largely from thunderstorms. Flows in such streams generally have little relation to flows in nearby streams and frequently have little or no relation to flows in the more distant parts of the same stream.

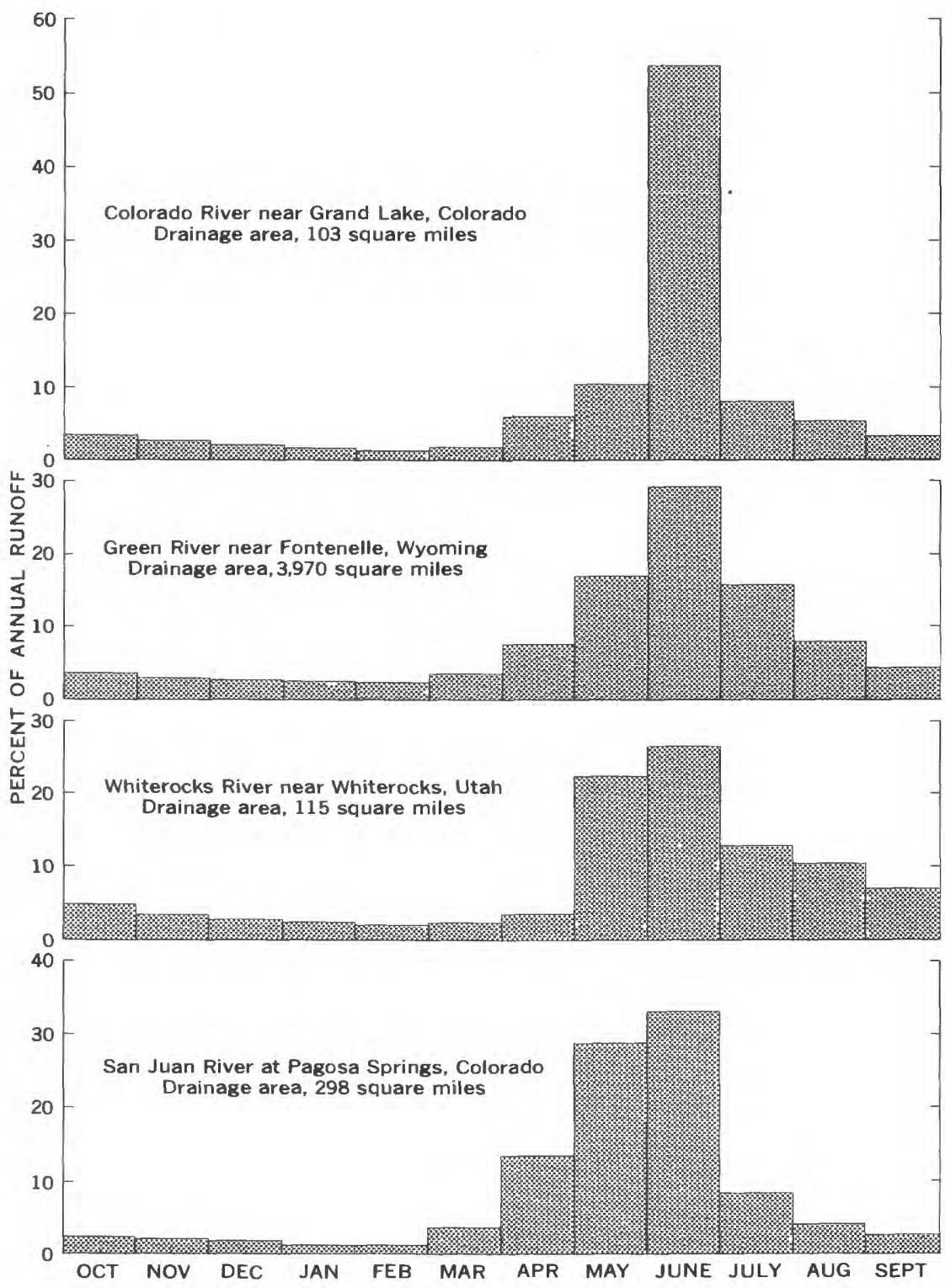

FIGURE 2.-Seasonal distribution of streamflow at selected sites. 
Figure 3 shows, by means of histograms of annual flow, the longterm trend at several key points in the basin. Thomas and others (1963) demonstrated by use of 5-year moving averages that flows since 1930 have averaged substantially less in the Upper Colorado River Basin than those that were observed earlier.

\section{ACKNOWLEDGMENTS}

The streamflow records which form the basis of the report have been compiled over many years by personnel of the various districts, Water Resources Division, U.S. Geological Survey, as a part of the nationwide program to investigate and appraise the Nation's water resources. The program has been carried out under the direction of the district chiefs and in cooperation with the various States, subdivisions thereof, municipalities, corporations, and Federal agencies, which are listed individually in the annual reports of streamflow for the area. This report has been prepared in the Rocky Mountain Region office, Water Resources Division, Denver, Colo., under the technical direction of M. R. Williams, Chief, Branch of Surface Water, Washington D.C.

\section{REPORTS CONTAINING BASIC STREAMFLOW DATA}

Streamflow data for the Upper Colorado River Basin have been published annually. Records prior to October 1, 1960, are contained in the annual series of Water-Supply Papers "Surface-Water Supply of the United States, Part 9, Colorado River Basin," and include figures of daily mean discharge. Annual and monthly mean discharges have been compiled and published in two volumes, Water-Supply Paper 1313 of the series "Compilation of Records of Surface Water of the United States through September 1950," and Water-Supply Paper 1733 of the series "Compilation of Records of Surface Waters of the United States, October 1950 to September 1960." Records of daily and monthly mean discharge since October 1960 have been published in the series of annual basic-data releases of surface-water records for each State. Data for the Upper Colorado River Basin are contained in the reports for Arizona, Colorado, New Mexico, Utah, and Wyoming. These data are to be compiled and published in Water-Supply Papers 1924 and 1925 of the series "Surface Water Supply of the United States, 1961-65."

\section{METHODS OF ANALYSIS}

In this report graphical methods of simple correlation of monthly mean discharge were used exclusively. That is, monthly discharge, in acre-feet, at a short-term streamflow gaging station was correlated with contemporaneous streamflow at a long-term gaging station and 

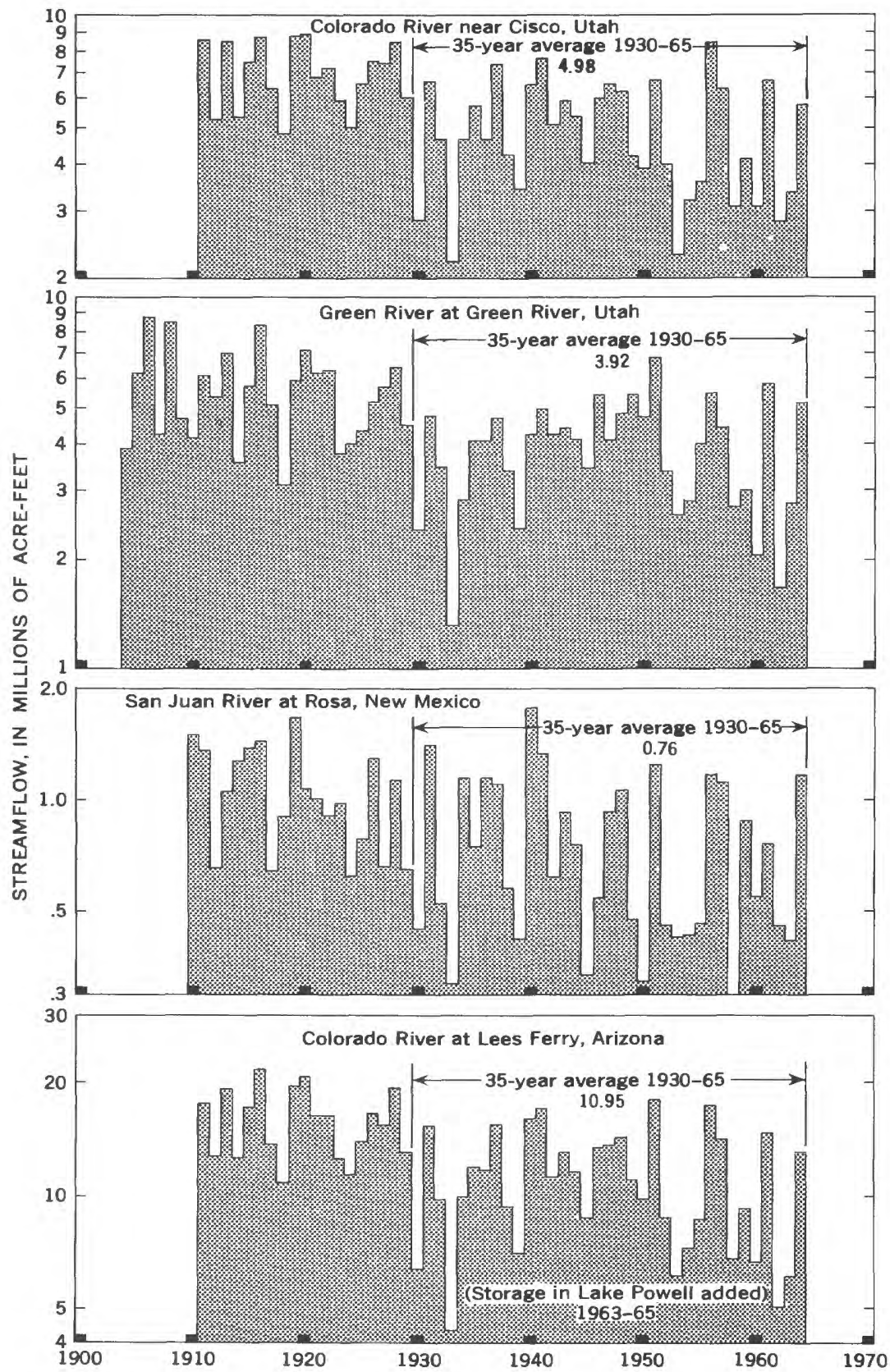

Figure 3-Histograms of annual streamflow at selected sites. 
a regression line was determined graphically for each month or group of months (season), as indicated by the data. Then, estimates of flow at the short-term station were made by entering the appropriate graph with the observed value at the long-term station and determining the short-term station value from the regression line.

For most streams, but not all, the graphical relationships between streamflow at one point and streamflow at a nearby point, or in an adjacent drainage, were found to form a straight line when logarithms of the discharge are used. Hence, logarithmic transformations of the data were used in all correlations by the simple expedient of using logarithmic coordinate paper.

The equation of a straight line on log paper is

$$
\log Y=b \log X-c,
$$

where $Y$ is the monthly mean discharge at the short-time gaging station, $X$ is the contemporaneous monthly mean discharge at the long-term gaging station, and $b$ and $c$ are constants that determine the position of the line. In a few calculations, further transformation by subtracting a constant from $Y$ or $X$ was used, resulting in the following formula :

$$
\begin{gathered}
\log (Y-a)=b \log X-c, \text { or } \\
\log Y=b \log (X-a)-c .
\end{gathered}
$$

In the few instances where curvilinear relations were found after transformation, the more complex equation was not determined.

Both correlation and regression analysis require that the data be statistically homogeneous - that is, of the same kind. Successive monthly streamflows are nonhomogeneous in the area of study because for most streams the seasonal streamflows result from different generative conditions and, hence, have different ranges of means and variances for each month. The nonhomogeneity of monthly streamflows for different calendar months was noted very early by Dennis (1921) and more recently by H. C. Riggs (written commun., 1966).

Because of the nonhomogeneity mentioned above, the methods of analysis given by Searcy (1960) and the method for seasonal adjustment developed by Somers (1954) were found not to apply in the basin under study. Both methods require that the individual monthly regressions be parallel, which has been found to be of infrequent occurrence.

The method of analysis used has been subjective. In the drawing of regression lines, the occasional outlying points, the regressions for adjacent months, and the relative position of the group of points in relation to the line of equal discharge per unit area (equal-yield line) 
have all been given weight based on judgment, and not on the mathematically determined line of best fit.

This method is believed to be justifiable because of the relatively short periods of concurrent record, most of which do not cover the extremes that have happened in the past and that may be exceeded in the future. Also, reasoning and the available evidence indicate that some cyclical (seasonal) relationship exists between regressions for the various months. For example, the May regression should have some general relation to the April and June regressions, although a readily workable mathematical expression has not come to the writer's attention. Only where plottings of adjacent months indicate that the relationships are the same in all probability are months grouped together.

In some correlations there are, as mentioned above, occasional outlying points that do not appear to be part of the general grouping for that month. These points may represent a single time when discharge was much greater or much less than the average for the month. Some are found to fit better in the correlation for an aajacent month; if so, the runoff conditions for that single month are assumed to be more like those generally prevailing in the adjacent month. Other outlying points may not fit in any group and can only be assumed to represent. unusual runoff in one basin but not in the adjacent basin.

\section{ESTIMATES OF STREAMFLOW}

The estimates of discharge for each streamflow station for each month have been obtained from the graphical relation for that month drawn to a large scale. The graph is entered with the monthly mean discharge, in acre-feet, for the independent (long-term) station, and an estimate of the concurrent discharge, in acre-feet, at the dependent station is read from the intercept on the regression line. The equation of the regression line is also determined for the user who may wish to duplicate the graphs to an enlarged scale. Use of the original graphs or application of the equations should give the same results, within the limits of accuracy being applied. The monthly estimates are rounded as follows:

1-99 acre-feet: one significant figure unless average annual total is less than 1,000 acre-feet; if less, all monthly figures greater than 10 acre-feet are rounded to two significant figures.

100-9,900 acre-feet: two significant figures.

10,000 acre-feet and above: three significant figures.

The annual figures shown are the sum of the 12 monthly estimates, rounded to one more significant figure than given for the individual months. 
Where the mean discharge for a month at the independent station was much greater or much less than that represented by the historical record, and where extrapolation would yield a value at the dependent station that seemed to be unreasonable when compared with concurrent streamflow at nearby points, it was necessary to assume that the discharge relation for that one month was more nearly represented by that for an adjacent month, or that, for extremely high discharge, the relationship approached equal discharge per unit area. Such individual values are noted in the tables wherever used.

Some streams decline to no flow during varying periods for some or most years. Such periods cannot be estimated by regression methods. Where several years contain months of no flow, flat estimates were used for those months based on the average flow for the month computed from the historic record. Such periods are noted in the tables.

In a few instances, flat estimates have also been made for periods of winter flow. These periods are also noted and the regression for that month is shown as a horizontal line.

Flow figures, previously estimated for the compilation reports (Water-Supply Papers 1313 and 1733) have not been used in defining regressions for this report. However, in those calculations where winter flow at the independent station was estimated for the 1950 compilation (Water-Supply Paper 1313) on the basis of hydrographic comparison to bridge short gaps in the record, the estimates have been reappraised by correlative procedures. If no inconsistency was found they were used as the basis for estimates at the dependent station. Such estimates are of poorer quality than the other estimates and should be used with caution. However, in most estimates these periods represent only 10-20 percent of the total annual flow, so that the effects on the annual streamflow figures are small.

\section{TEST OF ESTIMATED STREAMFLOW}

The estimated annual streamflow (sum of the 12 monthly figures) has been tested by plotting, to a large scale, a graphical correlation of annual flow, and determining the maximum departure of the estimated annual flow from the line of relation through the points representing the concurrent historical annual flow. (See fig. 4.)

Figure 4 shows that the spread of the estimated annual streamflows about the graphical line of relation is about the same as that for the historic record. All the estimates but one are within 20 percent of the line of relation. 


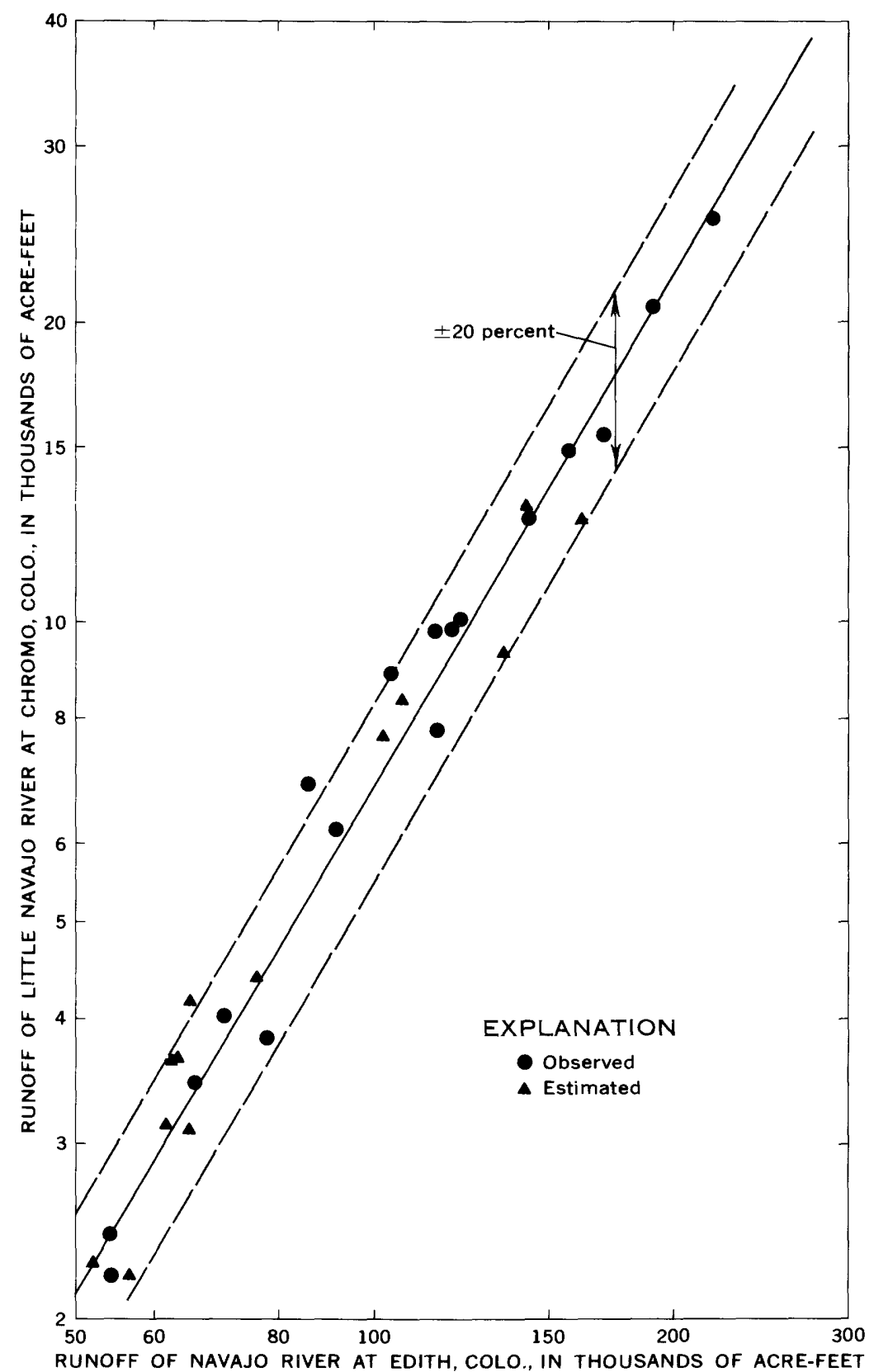

Frgure 4-Relation of annual streamflow of Little Navajo River at Chromo, Colo., and Navajo River at Edith, Colo. 


\section{EXPLANATION OF DATA}

The gaging-station records are arranged in downstream order. The order used is that adopted for use in the annual series of reports on surface-water supply beginning with the water-year 1951. Records are listed in a downstream direction along the main stem, and all stations on a tributary entering upstream from a main-stem station are listed before that station. A similar order is followed in listing stations on first rank, second rank, and other rank tributaries. As an added means of identification, each gaging station was assigned a number which is a part of the station name both in the heading of the description in text and as shown in plate 1 . The numbers were assigned in downstream order in each part (major basin). Gaps were left in the numbers to allow for new stations that may be established; hence, the numbers are not consecutive. The complete number of each station includes a part number and a six-digit station number. Because all stations in this report are in Part 9 (Colorado River Basin), the part number was omitted, and only the essential digits of the station numbers are shown. For example, the complete number $9-0795.00$ would appear as 795, just to the left of the station name.

The stations included in this report are those that were discontinued in recent years and that meet the following criteria:

1. If discontinued prior to October 1,1955 , have 15 or more years of record since October 1,1930 .

2. If discontinued after October 1,1955 , have 6 or more years of record.

3. Are free of extensive regulation or large-scale changes in storage or diversions.

4. Are not essentially equivalent to a currently active streamflow gaging station.

5. A correlative long-term streamflow station exists.

These criteria were selected after study of the trends in flow and the available records and by estimating the gain in information for various changes in the criteria. The investigation showed that 10-15 years of record frequently establish a reasonably good correlation. Some shorter term records, where the correlation is good (coefficients of correlation greater than 0.8 ) appear to give reasonably good figures of monthly streamflow. For purposes of this report a general guideline has been established that the annual flow (sum of 12 monthly estimates) should be within 25 percent of the annual regression line.

A number of stations meet all the criteria listed except the last and were omitted because no long-term station existed from which to make estimates. They are listed below with those stations which did not correlate owing to excessive diversion, regulation, or storage, or that are equivalent or nearly equivalent to an active station. 


\begin{tabular}{llcc}
\hline $\begin{array}{c}\text { Station } \\
\text { No. } \\
\text { (pl. i) }\end{array}$ & Name & $\begin{array}{c}\text { Period of } \\
\text { record }\end{array}$ & Remarks \\
\hline
\end{tabular}

150 Colorado River below Shadow Mountain Reservoir, Colo.

205 Willow Creek above Willow Creek Reservoir, Colo.

340 Fraser River at Granby, Colo

348 Little Muddy Creek near Parshall, Colo.

372 Skylark Creek near Parshall, Colo.

470 Blue River at Dillon, Colo... . . . 10-10 to 4-61

480

Snake River at Dillon, Colo_ _. _ 10-10 to 9-19

505 Tenmile Creek at Dillon, Colo....

608 Big Alkali Creek near Burns, Colo.

609 Catamount Creek near Burns, Colo.

673 Alkali Creek near Wolcott, Colo $10-58$ to 9-65

846 Fourmile Creek near Glenwood 10-57 to 9-65 Springs, Colo.

880 Baldy Creek near New Castle, $10-55$ to $9-61$ Colo.

907 East Divide Creek near Silt, Colo.

915 East Rifle Creek near Rifle, Colo.

958 Plateau Creek near Heiberger, Colo.

1825 Castle Creek near Moab, Utah...

1830 Courthouse Wash near Moab, Utah.

1870 Cottonwood Creek near Monticello, Utah.

1875 Indian Creek above Harts Draw, 10-49 to 9-57 near Monticello, Utah.

1890 Beaver Creek near Daniel, Wyo _ 10-38 to 9-54

1915 Cottonwood Creek near Daniel, $10-38$ to 9-54 Wyo.

1980 Pine Creek at Pinedale, Wyo_.... 10-03 to 10-04

$10-14$ to $9-54$ Wyo.

2110 Fontenelle Creek near Fontenelle, 5-14 to 9-19 Wyo.

2165 Green River at Green River, Wyo.

2190 Blacks Fork near Urie, W yo..... 10-13 to 9-24

2250 Blacks Fork near Green River, W yo.

2255 Green River near Linwood, Utah.
$10-29$ to $4-64$

10-10 to $9-19$

$10-29$ to $4-61$

$10-58$ to $9-65$

$10-55$ to $9-61$

$10-59$ to $9-65$

$10-36$ to $10-43$

$10-56$ to $9-64$

10-39 to $9-46$

$10-52$ to $9-64$

5-58 to $9-64$

$10-46$ to $3-65$

10-31 to $9-53$

$7-91$ to $8-91$

10-94 to 12-99

9-00 to $12-06$

9-14 to $10-45$

$10-37$ to $9-55$

Regulated.

Near equivalence to station 200.

Diversions to Moffat tunnel.

No correlation.

Do.

Near equivalence to station 466.

Site inundated by Dillon Reservoir.

Do.

No correlation.

Do.

Do.

Do.

Do.

Do.

Do.

Do.

Diversion to Buzzard Creek drainage.

No correlation.

Do.

Do.

Do.

Do.

Do.

Regulated.

Site inundated by Fontenelle Reservoir.

No correlation.

Near equivalence to station 2170.

No correlation, extensive diversions.

Near equivalence to station 2247.

$10-28$ to $3-63$ Site inundated by Flaming Gorge Reservoir. 


\begin{tabular}{|c|c|c|c|}
\hline $\begin{array}{l}\text { Station } \\
\text { No. } \\
\text { (pl. i) }\end{array}$ & Name & $\begin{array}{l}\text { Period of } \\
\text { record }\end{array}$ & Remarks \\
\hline
\end{tabular}

2270 East Fork Beaver Creek near $10-48$ to 9-62 No correlation.

Lonetree, Wyo.

2320 Sheep Creek near Manila, Utah_ _ 4-42

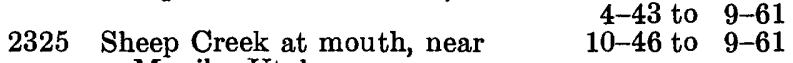
Manila. Utah.

2518 North Fork Little Snake River near Encampment, Wyo.

2605 Jones Hole Creek near Jensen, Utah.

2635

Brush Creek near Jensen, Utah....

2730

Duchesne River at Provo River Trail, near Hanna, Utah.

2865 Red Creek near Fruitland, Utah_-_ 10-17 to 9-22

3005 Uinta River at Fort Duchesne, Utah.

3010 Dry Gulch near Neola, Utah._._._10-50 to 9-58

3041 Big Beaver Creek near Buford, Colo.

3295 Fremont River near Fremont, Utah.

3300 Fremont River near Bicknell, Utah.

3325 Muddy Creek below Ivie Creek, near Emery, Utah.

3350 Colorado River at Hite, Utah... -

3505 San Juan River at Rosa, N. Mex.

3565 San Juan River near Blanco, N. Mex.

3570 San Juan River at Bloomfield, N. Mex.

3595 Animas River above Tacoma, Colo.

3620 Lightner Creek near Durango, Colo.

3629 Florida River near Hermosa, Colo.

3631 Salt Creek near Oxford, Colo

3660 Cherry Creek near Red Mesa, Colo.
10-56 to $9-65$

10-50 to $9-56$ 10-60 to $9-61$

4-39 to $9-65$

7-29 to $9-33$ 10-35 to $9-43$ 10-44 to $9-54$

9-99 to $\quad 9-20$ (fragmentary) 10-42 to 9-58

No correlation, extensive diversions.

No correlation, site inundated by Flaming Gorge Reservoir.

No correlation, transbasin diversion above station.

No correlation, spring fed.

No correlation, many diversions.

No correlation, most of flow diverted for use in Great Basin.

No correlation, regulation and diversion.

No correlation, many diversions.

No correlation, no flow much of time.

10-55 to 9-64 No correlation.

$7-49$ to $9-58$

Do.

5-09 to 12-12

10-37 to $9-58$

No correlation, regulated.

8-50 to $9-61$

$8-47$ to $9-58$

7-95 to 11-95

5-96 to $12-97$

4-98 to $12-98$

5-99 to 9-99

7-10 to 5-65

6-07 to $10-10$

(fragmentary)

10-27 to 1-55

1-10 to 9-11

8-27 to $12-31$

11-55 to 1-64

$10-45$ to $9-56$

No correlation, many diversions.

Site inundated by Lake Powell.

Site inundated by Navajo Reservoir.

Near equivalence to station 3555 .

No correlation, many diversions.

No correlation, part of flow bypasses gage.

7-27 to 9-49 No correlation.

10-55 to 9-63 Do.

10-56 to 9-63 Do.

5-28 to 9-50 Do. 
The data presented for each gaging station consist of a station description, a table of the previously published monthly and annual streamflow, in acre-feet, in the period 1930-65, a table of correlative estimates of monthly and annual streamflow, in acre-feet, and, on the facing page, a figure showing the correlations for each month or group of months.

The station description gives the name of the subbasin, the station name and number, location, altitude or datum, drainage area, records available, stations with which correlations were established, regression equation, average discharge, extremes of discharge, and remarks about diversions, storage, and quality of estimates.

The location and altitude of the gaging station is that for the lastused site, if more than one site was used in the period of record.

The "Records Available" paragraph lists all periods for which there are previously published records generally equivalent to those at the site given under "Location."

The "Estimates of Streamflow" paragraph gives the name or names of the station (s) with which correlations were established, the equation of the regression line used in making the estimates, and a table of constants used in the equation. Where more than one regression line has been used for a given month, the periods of use of each are given.

The average discharge for a station is the average of all complete water years of record available since 1930, both historical and estimated. For some stations, no suitable independent stations could be found to permit extensions to cover the complete period 1930-65. Hence, the average for a shorter period is shown.

Under "Extremes" are given the date and discharge of the highest and lowest momentary discharges during the period of historic record. In the instances where momentary data have not been determined, maximum or minimum daily figures are given. Where extremes are known outside the period of historic record, they are given in a second paragraph.

The "Remarks" paragraph contains information, where known, on diversion, utilization and storage, and changes during the period. A statement is given on the maximum deviation of the estimates of annual streamflow (sum of the 12 calendar monthly values) from the line of relation of the annual flows as a general guide to the reliability of the figures. In general, the annual figures are somewhat more reliable than those for individual months.

The table of monthly and annual streamflow summarizes previously published streamflow records. The figures represent flow past the station and are unadjusted for upstream storage or diversion unless otherwise stated under "Remarks" for the individual stations. Where 
the figures represent estimates prepared for previous reports they are footnoted, indicating that they were not used in the correlation analysis. Flows for partial months or partial years are not listed.

Values in the table of estimated monthly and annual streamflows have been picked graphically by entering the graph for the individual month with the long-term (primary or independent) value and estimating the short-term (secondary or dependent) value. The annual figures are the sums of the 12 monthly values.

The graphs for each station show the plotting position for the concurrent monthly flows for the long-term and short-term gaging stations and the regression line of best fit, determined graphically.

\section{REFERENCES}

Dennis, H. W., 1921, A method of adapting the records of streamflow at one point to another on the same stream: Am. Soc. Civil Engr. Trans., v. 84, p. 551-566.

Searcy, J. K., 1960, Graphical correlation of gaging-station records : U.S. Geol. Survey Water-Supply Paper 1541-C, p. 67-100.

Somers, W. P., 1964, Statistical analysis of streamflow relationships : $22 \mathrm{~d}$ Western Snow Conference, 1954, Salt Lake City, Proc., p. 26-32.

Thomas, H. E., and others, 1963, Effects of drought in the Colorado River basin : U.S. Geol. Survey Prof. Paper 372-F, 51 p.

Wilbur, R. L., and Ely, N., 1948, The Hoover Dam documents U.S. 80th Cong.,

2d sess., House Document 717, p. A17-A22. 

GAGING-STATION RECORDS 

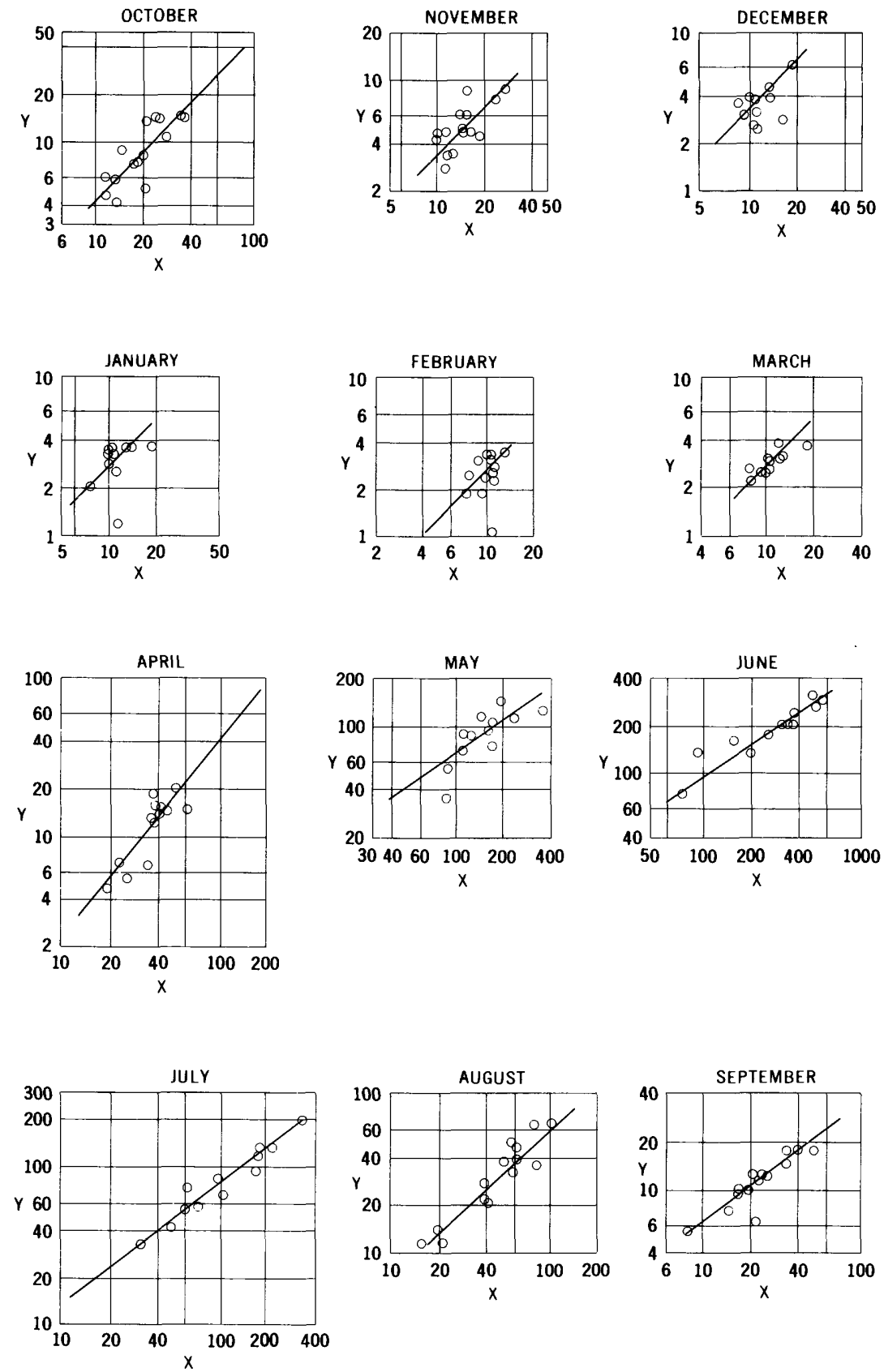

Relationships of monthly mean discharge of North Inlet at Grand Lake, Colo. $(Y)$, to sum of monthly mean discharges of Colorado River near Grand Lake, Colo., and Grand River ditch (X). Discharge in hundreds of acre-feet. 
125. North Inlet at Grand Lake, Colo.

Location.--Lat $40^{\circ} 15^{\prime} 10^{\prime \prime}$, long $105^{\circ} 48150^{\prime \prime}$, in $N E \frac{1}{4}$ sec.5, T.3 N., R.75 W., on right bank at north edge of town of Grand Lake, 300 't downstream from Tonahutu Creek and 1,600 ft upstream from high-water line of Grand Lake. Datum of gage 1s 8,434.48 ft above mean sea level (Bureau of Reclamation bench mark).

Drainage area. $--46.6 \mathrm{sq} \mathrm{mi}$.

Records available. --August 1905 to September 1909, october 1910 to September 1912, October 1947 to December 1955. Monthly discharge only for some periods. Prior to October 1947, published as North Inlet to Grand Lake at Grand Lake.

Estimates of streamflow.--June 1934 to September 1947, January 1956 to september 1965 , based on relationships of monthly mean discharge with Colorado River near Grand Lake, colo., adjusted for transmountain diversion through Grand River ditch. The regression equation used is:

$$
\log Y=b \log X-c
$$

(where $\mathrm{Y}$ is discharge of North Inlet at Grand Lake, Colo., and $X$ is the sum of discharges of Colorado River near Grand Lake, Colo. and Grand River ditch, all in acre-feet per month).

\begin{tabular}{r|r|r|r|r|r|r|r|r|r|r|r|r}
\hline & Mot. & Nov. & Dec. & Jan. & Feb. & Mar. & Apr. & May & June & July & Aug. & Sept. \\
\hline $\mathrm{b}$ & 1.02 & 1.00 & 1.02 & 1.02 & 1.02 & 1.03 & 1.26 & 0.67 & 0.66 & 0.75 & 0.90 & 0.74 \\
$\mathrm{c}$ & .43 & .45 & .54 & .61 & .64 & .55 & 1.44 & -1.15 & -1.35 & -.92 & -.15 & -.60 \\
\hline
\end{tabular}

Average discharge. --31 years $(1934-65), 45,120$ acre-feet per year (62.3 cfs).

Extremes, - - 1905-9, 1910-12, 1947-55: Maximum discharge, 1,110 cfs June 7, 1952; minimum dally, 1.5 cfs Feb. 5, 6, 1949.

Remarks. --Transmountain diversion above station by Eureka ditch. Flow of Eureka ditch rarely equals or exceeds 1 percent of flow in North Inlet. Estimates of annual flow are within about 20 percent of regression line.

\begin{tabular}{c|c|c|c|c|c|c|c|c|r|r|r|r|r}
\hline $\begin{array}{l}\text { Water } \\
\text { year }\end{array}$ & Oct. & Nov. & Dec. & Jan. & Feb. & Mar. & Apr. & May & June & July & Aug. & Sept. & Annual \\
\hline 1948 & 1,470 & 762 & 463 & 357 & 336 & 380 & 1,260 & 14,080 & 13,150 & 4,280 & 1,390 & 546 & 38,470 \\
1949 & 473 & 347 & 250 & 122 & 108 & 304 & 1,600 & 9,470 & 24,570 & 12,190 & 2,760 & 990 & 53,180 \\
1950 & 764 & 472 & 358 & 348 & 304 & 294 & 672 & 5,490 & 17,830 & 5,560 & 1,150 & 1,260 & 34,500 \\
1951 & 752 & 615 & 376 & 282 & 192 & 258 & 555 & 10,390 & 20,670 & 13,530 & 3,600 & 1,230 & 52,450 \\
1952 & 845 & 490 & 277 & 369 & 230 & 252 & 1,870 & 11,120 & 31,570 & 8,510 & 3,930 & 1,240 & 60,700 \\
1953 & 596 & 287 & 258 & 258 & 239 & 246 & 704 & 7,130 & 20,220 & 5,750 & 3,730 & 958 & 40,380 \\
1954 & 618 & 440 & 309 & 204 & 192 & 222 & 1,530 & 8,870 & 7,740 & 3,390 & 1,170 & 984 & 25,670 \\
1955 & 1,430 & 612 & 390 & 336 & 251 & 268 & 1,320 & 8,890 & 16,030 & 7,540 & 2,210 & 749 & 40,030 \\
1956 & 414 & 346 & 318 & - & - & - & - & - & - & - & - & - & - \\
\hline
\end{tabular}

Est1mated monthly and annual streamflow, in acre-feet

\begin{tabular}{|c|c|c|c|c|c|c|c|c|c|c|c|c|c|}
\hline $\begin{array}{l}\text { Water } \\
\text { year }\end{array}$ & oct. & Nov. & Dec. & Jan. & Feb. & Mar. & Apr. & May & June & July & Aug. & Sept. & Annual \\
\hline $\begin{array}{l}1934 \\
1935\end{array}$ & $\overline{440}$ & - 390 & $\overline{3} 30$ & $\overrightarrow{220}$ & 170 & 180 & $\overline{610}$ & 5,500 & $\begin{array}{r}7,000 \\
21,300\end{array}$ & $\begin{array}{r}1,600 \\
11,700\end{array}$ & $\begin{array}{l}1,100 \\
2,300\end{array}$ & $\begin{array}{l}820 \\
960\end{array}$ & $44, \overline{100}$ \\
\hline $\begin{array}{l}1936 \\
1937 \\
1938 \\
1939 \\
1940\end{array}$ & $\begin{array}{r}820 \\
780 \\
1,300 \\
860 \\
740\end{array}$ & $\begin{array}{l}330 \\
520 \\
780 \\
510 \\
390\end{array}$ & $\begin{array}{l}270 \\
450 \\
540 \\
370 \\
240\end{array}$ & $\begin{array}{l}310 \\
350 \\
430 \\
310 \\
200\end{array}$ & $\begin{array}{l}250 \\
320 \\
350 \\
240 \\
180\end{array}$ & $\begin{array}{l}260 \\
390 \\
330 \\
360 \\
270\end{array}$ & $\begin{array}{l}6,600 \\
1,000 \\
2,200 \\
1,900 \\
1,000\end{array}$ & $\begin{array}{l}14,700 \\
10,300 \\
11,700 \\
12,600 \\
9,100\end{array}$ & $\begin{array}{l}21,500 \\
14,700 \\
25,700 \\
16,800 \\
16,400\end{array}$ & $\begin{array}{r}9,400 \\
7,500 \\
11,800 \\
5,400 \\
5,400\end{array}$ & $\begin{array}{l}4,000 \\
2,000 \\
2,700 \\
1,400 \\
1,200\end{array}$ & $\begin{array}{r}1,100 \\
1,200 \\
2,000 \\
930 \\
1,200\end{array}$ & $\begin{array}{l}59,340 \\
39,510 \\
59,830 \\
41,680 \\
36,320\end{array}$ \\
\hline $\begin{array}{l}1941 \\
1942 \\
1943 \\
1944 \\
1945\end{array}$ & $\begin{array}{r}1,100 \\
900 \\
500 \\
760 \\
420\end{array}$ & $\begin{array}{l}560 \\
520 \\
570 \\
500 \\
340\end{array}$ & $\begin{array}{l}360 \\
490 \\
300 \\
280 \\
220\end{array}$ & $\begin{array}{l}260 \\
360 \\
240 \\
170 \\
190\end{array}$ & $\begin{array}{l}200 \\
230 \\
220 \\
160 \\
170\end{array}$ & $\begin{array}{l}250 \\
250 \\
270 \\
190 \\
220\end{array}$ & $\begin{array}{r}620 \\
1,300 \\
2,400 \\
540 \\
520\end{array}$ & $\begin{array}{l}12,200 \\
6,800 \\
8,000 \\
8,700 \\
8,400\end{array}$ & $\begin{array}{l}18,300 \\
19,700 \\
18,500 \\
18,600 \\
18,400\end{array}$ & $\begin{array}{r}7,200 \\
8,100 \\
8,000 \\
8,700 \\
13,200\end{array}$ & $\begin{array}{l}1,800 \\
1,400 \\
2,000 \\
1,200 \\
4,100\end{array}$ & $\begin{array}{r}1,200 \\
740 \\
710 \\
620 \\
1,000\end{array}$ & $\begin{array}{l}44,060 \\
40,790 \\
41,510 \\
40,420 \\
47,180\end{array}$ \\
\hline $\begin{array}{l}1946 \\
1947\end{array}$ & $\begin{array}{l}720 \\
780\end{array}$ & $\begin{array}{l}460 \\
560\end{array}$ & $\begin{array}{l}290 \\
320\end{array}$ & $\begin{array}{l}310 \\
230\end{array}$ & $\begin{array}{l}240 \\
200\end{array}$ & $\begin{array}{l}280 \\
280\end{array}$ & $\begin{array}{r}2,100 \\
590\end{array}$ & $\begin{array}{r}6,500 \\
11,300\end{array}$ & $\begin{array}{l}16,500 \\
21,000\end{array}$ & $\begin{array}{r}5,600 \\
14,400\end{array}$ & $\begin{array}{l}1,700 \\
3,200\end{array}$ & $\begin{array}{l}1,000 \\
1,300\end{array}$ & $\begin{array}{l}35,700 \\
54,160\end{array}$ \\
\hline $\begin{array}{l}1956 \\
1957 \\
1958 \\
1959 \\
1960\end{array}$ & $\begin{array}{r}\overline{510} \\
1,300 \\
440 \\
1,700\end{array}$ & $\begin{array}{l}- \\
310 \\
660 \\
360 \\
960\end{array}$ & $\begin{array}{l}- \\
240 \\
530 \\
290 \\
470\end{array}$ & $\begin{array}{l}250 \\
230 \\
340 \\
240 \\
280\end{array}$ & $\begin{array}{l}240 \\
190 \\
270 \\
200 \\
240\end{array}$ & $\begin{array}{l}260 \\
230 \\
300 \\
220 \\
540\end{array}$ & $\begin{array}{r}1,200 \\
400 \\
490 \\
510 \\
3,300\end{array}$ & $\begin{array}{r}12,500 \\
8,000 \\
13,000 \\
7,400 \\
9,400\end{array}$ & $\begin{array}{l}19,000 \\
23,300 \\
19,300 \\
19,900 \\
22,000\end{array}$ & $\begin{array}{r}4,000 \\
22,100 \\
4,000 \\
6,400 \\
7,200\end{array}$ & $\begin{array}{l}2,100 \\
5,600 \\
1,700 \\
2,800 \\
2,000\end{array}$ & $\begin{array}{r}740 \\
1,600 \\
760 \\
1,200 \\
1,000\end{array}$ & $\begin{array}{l}41,370 \\
62,710 \\
42,650 \\
39,960 \\
49,090\end{array}$ \\
\hline $\begin{array}{l}1961 \\
1962 \\
1963 \\
1964 \\
1965\end{array}$ & $\begin{array}{r}720 \\
3,700 \\
900 \\
680 \\
550\end{array}$ & $\begin{array}{r}450 \\
1,100 \\
340 \\
480 \\
360\end{array}$ & $\begin{array}{l}280 \\
700 \\
310 \\
230 \\
290\end{array}$ & $\begin{array}{l}240 \\
370 \\
180 \\
190 \\
270\end{array}$ & $\begin{array}{l}210 \\
320 \\
160 \\
160 \\
200\end{array}$ & $\begin{array}{l}220 \\
370 \\
260 \\
200 \\
220\end{array}$ & $\begin{array}{r}350 \\
3,200 \\
1,300 \\
320 \\
720\end{array}$ & $\begin{array}{r}8,100 \\
12,500 \\
8,800 \\
9,200 \\
8,700\end{array}$ & $\begin{array}{l}18,800 \\
22,700 \\
12,400 \\
16,000 \\
23,200\end{array}$ & $\begin{array}{r}6,500 \\
14,400 \\
3,400 \\
8,100 \\
14,300\end{array}$ & $\begin{array}{l}2,600 \\
3,900 \\
3,500 \\
2,800 \\
4,100\end{array}$ & $\begin{array}{l}2,900 \\
1,200 \\
1,500 \\
1,700 \\
1,700\end{array}$ & $\begin{array}{l}41,370 \\
64,460 \\
33,050 \\
39,460 \\
54,610\end{array}$ \\
\hline
\end{tabular}



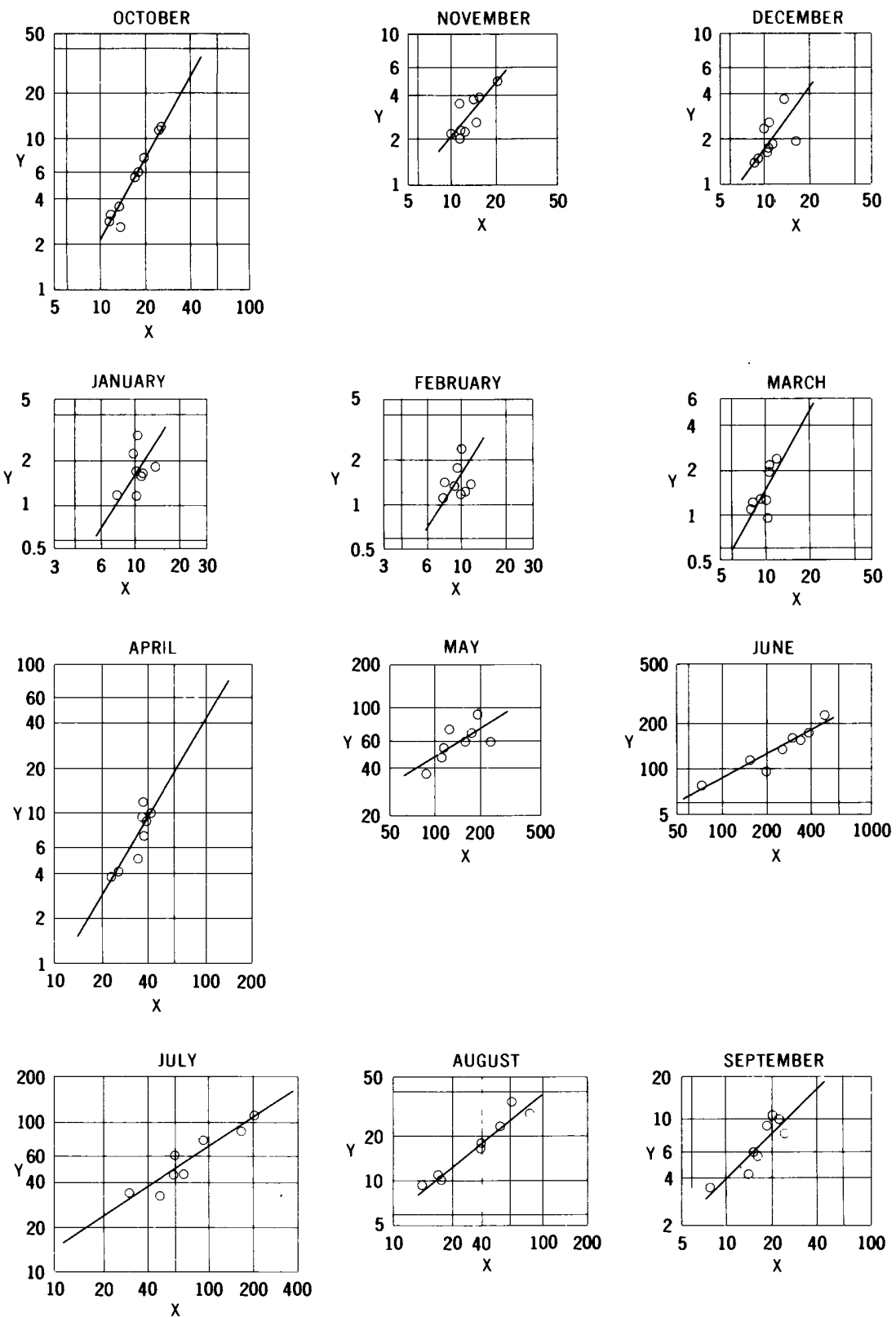

Relationships of monthly mean discharge of East Inlet near Grand Lake, Colo. (Y), to sum of monthly mean discharges of Colorado River near Grand Lake, Colo., and Grand River ditch (X). Discharge in hundreds of acre-feet. 


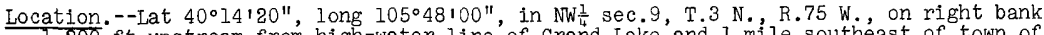
1,200 ft upstream from high-water line of Grand Lake and 1 mile southeast of town of Grand Lake. Datum of gage is $8,370.49 \mathrm{ft}$ above mean sea level (Bureau of Reclamation bench mark).

Drainage area. $--27.1 \mathrm{sq} \mathrm{mi}$.

Records available. --October 1947 to December 1955.

Estimates of streamflow. - June 1934 to September 1947, January 1956 to September 1965 , based on relationships of monthly mean discharge with Colorado River near Grand Lake adjusted for transmountain diversion through Grand River ditch. The regression equation used is:

$$
\log Y=b \log X-c
$$

(where $\mathrm{Y}$ is discharge of East Inlet near Grand Lake, Colo., and $\mathrm{X}$ is the sum of discharges of Colorado River near Grand Lake, Colo. and Grand River ditch, all in acrefeet per month).

Monthly values of constants in above equation

\begin{tabular}{l|l|l|l|l|l|l|l|l|l|l|l|l}
\hline & oct. & Nov. & Dec. & Jan. & Feb. & Mar. & Apr. & May & June & July & Aug. & Sept. \\
\hline b & 1.73 & 1.22 & 1.33 & 1.56 & 1.56 & 1.79 & 1.68 & 0.61 & 0.54 & 0.66 & 0.78 & 0.98 \\
c & 2.86 & 1.35 & 1.74 & 2.48 & 2.48 & 3.20 & 3.08 & -1.25 & -1.76 & -1.18 & -.46 & .34 \\
\hline
\end{tabular}

Average discharge. - -31 years (1934-65), 33,585 acre-feet per year (46.4 cfs).

Extremes.--1947-55: Maximum discharge, $710 \mathrm{cfs}$ June 17, 1949; minimum daily determined, $1.5 \mathrm{cf}$ 's Mar. 11 to Apr. 1, 1949.

Remarks. - No diversion above station. Estimated annual flow is within about 5 percent of regression line.

\begin{tabular}{c|c|c|c|c|r|r|r|r|r|r|r|r|r}
\hline $\begin{array}{c}\text { Water } \\
\text { year }\end{array}$ & oct. & Nov. & Dec. & Jan. & Feb. & Mar. & Apr. & May & June & July & Aug. & Sept. & Annual \\
\hline 1948 & 1,130 & 575 & 373 & 294 & 234 & 238 & 700 & 9,130 & 9,600 & 3,200 & 1,090 & 352 & 26,920 \\
1949 & 312 & 226 & 184 & 166 & 122 & 95 & 889 & 5,910 & 17,750 & 8,620 & 1,780 & 600 & 36,650 \\
1950 & 596 & 343 & 143 & 114 & 133 & 215 & 511 & 3,620 & 13,720 & 4,400 & 954 & 998 & 25,750 \\
1951 & 542 & 363 & 263 & 220 & 177 & 189 & 417 & 6,670 & 15,320 & 11,270 & 2,790 & 818 & 39,040 \\
1952 & 741 & 259 & 196 & 178 & 136 & 129 & 1,170 & 5,990 & 22,950 & 7,520 & 3,410 & 1,040 & 43,720 \\
1953 & 354 & 203 & 173 & 159 & 117 & 125 & 375 & 4,670 & 16,180 & 4,390 & 2,350 & 577 & 29,670 \\
1954 & 285 & 214 & 151 & 117 & 111 & 121 & 994 & 7,150 & 7,740 & 3,420 & 1,030 & 919 & 22,250 \\
1955 & 1,200 & 373 & 239 & 168 & 142 & 114 & 934 & 5,330 & 11,440 & 5,880 & 1,620 & 431 & 27,870 \\
1956 & 259 & 226 & 185 & - & - & - & - & - & - & - & - & - & - \\
\hline
\end{tabular}

\begin{tabular}{|c|c|c|c|c|c|c|c|c|c|c|c|c|c|}
\hline $\begin{array}{l}\text { Water } \\
\text { year }\end{array}$ & oct. & Nov. & Dec. & Jan. & Feb. & Mar. & Apr. & May & June & July & Aug. & Sept. & Annual \\
\hline $\begin{array}{l}1934 \\
1935\end{array}$ & $\overline{230}$ & $\overline{230}$ & $\overline{180}$ & $\overline{110}$ & - & - & - & $3, \overline{900}$ & $\begin{array}{r}6,500 \\
16,200\end{array}$ & & $\begin{array}{r}920 \\
1,800\end{array}$ & $\begin{array}{l}540 \\
680\end{array}$ & 32,830 \\
\hline $\begin{array}{l}1936 \\
1937 \\
1938 \\
1939 \\
1940\end{array}$ & $\begin{array}{r}410 \\
620 \\
1,400 \\
710 \\
560\end{array}$ & $\begin{array}{l}190 \\
320 \\
530 \\
320 \\
230\end{array}$ & $\begin{array}{l}140 \\
270 \\
340 \\
210 \\
120\end{array}$ & $\begin{array}{l}190 \\
230 \\
310 \\
180 \\
100\end{array}$ & $\begin{array}{r}150 \\
220 \\
250 \\
140 \\
90\end{array}$ & $\begin{array}{l}140 \\
270 \\
200 \\
240 \\
140\end{array}$ & $\begin{array}{r}a, 000 \\
670 \\
1,900 \\
1,600 \\
710\end{array}$ & $\begin{array}{l}9,500 \\
6,900 \\
7,800 \\
8,200 \\
6,100\end{array}$ & $\begin{array}{l}16,400 \\
11,900 \\
19,000 \\
13,400 \\
13,100\end{array}$ & $\begin{array}{l}7,500 \\
6,100 \\
9,100 \\
4,600 \\
4,600\end{array}$ & $\begin{array}{l}2,800 \\
1,500 \\
2,000 \\
1,200 \\
1,000\end{array}$ & $\begin{array}{r}810 \\
960 \\
1,800 \\
640 \\
870\end{array}$ & $\begin{array}{l}44,230 \\
29,960 \\
44,630 \\
31,440 \\
27,620\end{array}$ \\
\hline $\begin{array}{l}1941 \\
1942 \\
1943 \\
1944 \\
1945\end{array}$ & $\begin{array}{r}1,100 \\
790 \\
290 \\
590 \\
210\end{array}$ & $\begin{array}{l}350 \\
330 \\
210 \\
310 \\
200\end{array}$ & $\begin{array}{l}200 \\
300 \\
160 \\
150 \\
110\end{array}$ & $\begin{array}{r}140 \\
240 \\
130 \\
70 \\
90\end{array}$ & $\begin{array}{r}110 \\
130 \\
120 \\
80 \\
80\end{array}$ & $\begin{array}{r}140 \\
130 \\
140 \\
80 \\
100\end{array}$ & $\begin{array}{r}360 \\
960 \\
2,100 \\
290 \\
280\end{array}$ & $\begin{array}{l}8,000 \\
4,700 \\
5,500 \\
5,000 \\
5,800\end{array}$ & & $\begin{array}{r}5,900 \\
6,500 \\
6,500 \\
7,000 \\
10,000\end{array}$ & $\begin{array}{r}1,400 \\
1,100 \\
1,500 \\
970 \\
2,900\end{array}$ & $\begin{array}{l}890 \\
470 \\
440 \\
380 \\
720\end{array}$ & $\begin{array}{l}32,890 \\
30,850 \\
31,590 \\
30,420 \\
34,890\end{array}$ \\
\hline $\begin{array}{l}1946 \\
1947\end{array}$ & $\begin{array}{l}540 \\
620\end{array}$ & $\begin{array}{l}280 \\
350\end{array}$ & $\begin{array}{l}190 \\
210\end{array}$ & $\begin{array}{l}190 \\
120\end{array}$ & $\begin{array}{l}140 \\
100\end{array}$ & $\begin{array}{l}150 \\
150\end{array}$ & $\begin{array}{r}1,700 \\
330\end{array}$ & $\begin{array}{l}4,500 \\
7,500\end{array}$ & & $\begin{array}{r}4,700 \\
10,800\end{array}$ & & $\begin{array}{r}730 \\
1,000\end{array}$ & \\
\hline $\begin{array}{l}1956 \\
1957 \\
1958 \\
1959 \\
1960\end{array}$ & $\begin{array}{r}- \\
300 \\
1,400 \\
230 \\
2,300\end{array}$ & $\begin{array}{l}- \\
170 \\
440 \\
210 \\
690\end{array}$ & $\begin{array}{l}- \\
120 \\
330 \\
150 \\
290\end{array}$ & $\begin{array}{l}130 \\
110 \\
220 \\
130 \\
160\end{array}$ & $\begin{array}{l}140 \\
100 \\
170 \\
110 \\
140\end{array}$ & $\begin{array}{l}130 \\
110 \\
170 \\
100 \\
480\end{array}$ & $\begin{array}{r}830 \\
200 \\
260 \\
280 \\
3,200\end{array}$ & $\begin{array}{l}8,200 \\
5,500 \\
8,400 \\
5,100 \\
6,400\end{array}$ & $\begin{array}{l}14,800 \\
17,400 \\
15,000 \\
15,300 \\
16,600\end{array}$ & $\begin{array}{r}3,500 \\
15,800 \\
3,500 \\
5,300 \\
6,000\end{array}$ & $\begin{array}{l}1,600 \\
3,800 \\
1,300 \\
2,100 \\
1,600\end{array}$ & $\begin{array}{r}470 \\
1,300 \\
500 \\
870 \\
750\end{array}$ & $\begin{array}{l}30,470 \\
44,910 \\
31,690 \\
29,880 \\
38,610\end{array}$ \\
\hline $\begin{array}{l}1961 \\
1962 \\
1963 \\
1964 \\
1965\end{array}$ & $\begin{array}{r}530 \\
03,200 \\
780 \\
490 \\
340\end{array}$ & $\begin{array}{l}270 \\
840 \\
190 \\
290 \\
210\end{array}$ & $\begin{array}{l}150 \\
480 \\
160 \\
120 \\
150\end{array}$ & $\begin{array}{r}120 \\
250 \\
90 \\
90 \\
150\end{array}$ & $\begin{array}{r}110 \\
220 \\
80 \\
70 \\
110\end{array}$ & $\begin{array}{r}100 \\
250 \\
130 \\
80 \\
100\end{array}$ & $\begin{array}{r}160 \\
3,200 \\
900 \\
150 \\
440\end{array}$ & $\begin{array}{l}5,500 \\
8,100 \\
6,000 \\
6,200 \\
5,900\end{array}$ & $\begin{array}{l}14,700 \\
17,100 \\
10,400 \\
12,800 \\
17,400\end{array}$ & $\begin{array}{r}5,400 \\
10,800 \\
3,100 \\
6,500 \\
10,800\end{array}$ & $\begin{array}{l}2,000 \\
2,800 \\
2,500 \\
2,100 \\
2,900\end{array}$ & $\begin{array}{r}\varepsilon, 800 \\
960 \\
1,200 \\
800 \\
1,400\end{array}$ & $\begin{array}{l}31,840 \\
48,200 \\
25,530 \\
29,690 \\
39,900\end{array}$ \\
\hline
\end{tabular}

Estimated on basis of May regression.

Estimated on basis of September regression. 

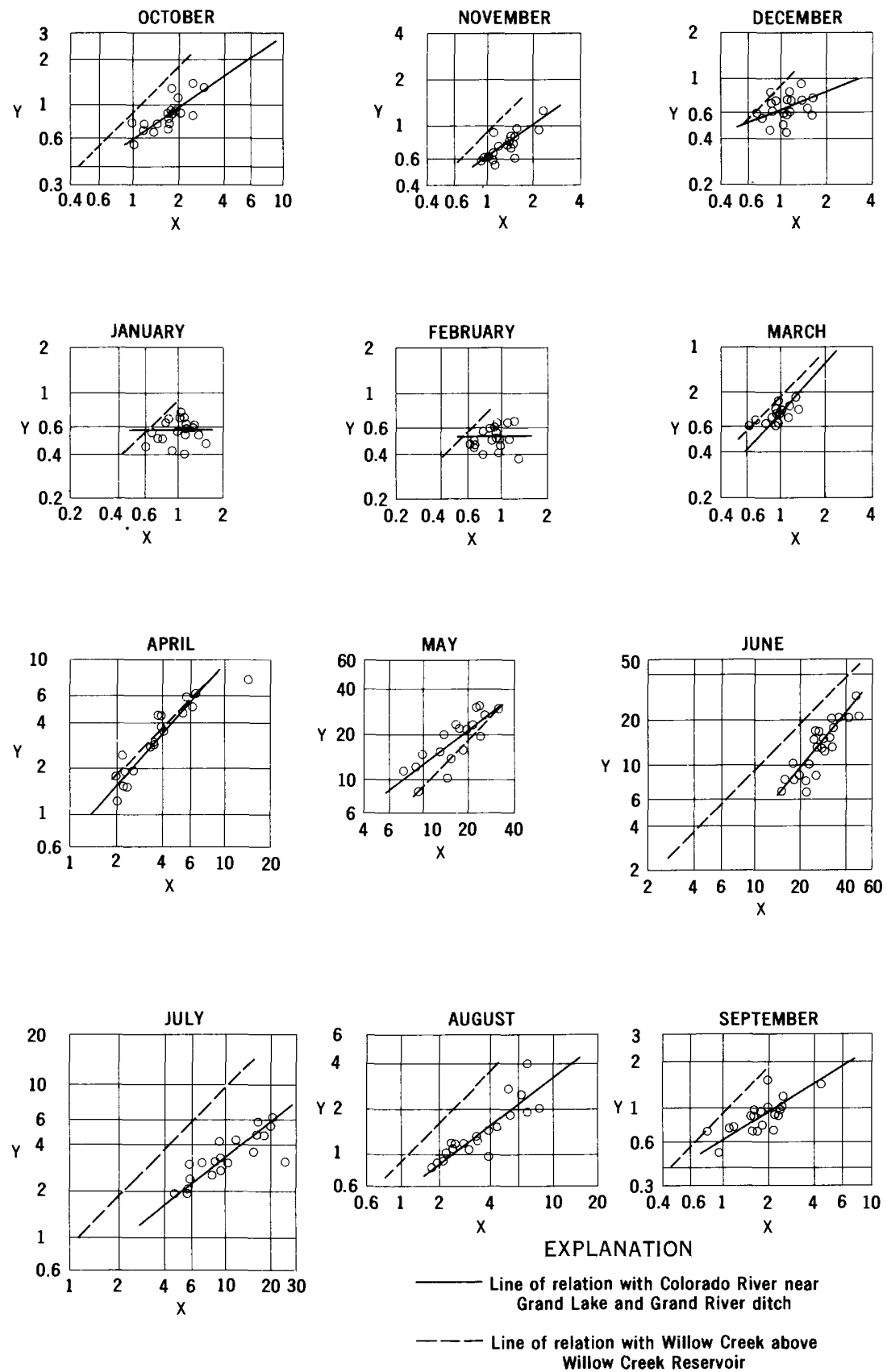

Relationships of monthly mean discharge of Willow Creek near Granby, Colo. (Y), to monthly mean discharge of Willow Creek above Willow Creek Reservoir, or sum of monthly mean discharges of Colorado River near Grand Lake, Colo., and Grand River ditch (X). Discharge in thousands of acre-feet. 
200. Willow Creek near Granby, Colo.

Location.--Lat $40^{\circ} 111$, long $106^{\circ} 001$, in NW $\frac{1}{4}$ sec.34, T.3 N., R.77 W., on right bank $10 \mathrm{ft}$ upstream from bridge on State Highway 125 , 100 ft downstream from Gold Run Creek, and 7 miles northwest of Granby. Datum of gage is $8,236.88 \mathrm{ft}$ above mean sea level, unadjusted.

Drainage area.--105 sq $\mathrm{mi}$.

Records available. --0ctober 1934 to September 1953. Prior to April 1935 monthly discharge only.

Estimates of streamflow.--October 1953 to September 1960 based on assumption that relationships of monthly mean discharge with Willow Creek above Willow Creek Reservoir, Colo, are equal to the square root of the drainage area ratio. October 1960 to September 1965; based on relationships of monthly mean discharge with Colorado River near Grand Lake, Colo. adjusted for transmountain diversion through Grand River ditch. The regression equation used is:

$$
\log \mathrm{Y}=\mathrm{b} \log \mathrm{X}-\mathrm{c}
$$

(where $\mathrm{Y}$ is discharge of Willow Creek near Granby, Colo., and $\mathrm{X}$ is discharge of Willow Creek above Willow Creek Reservoir, Colo., or sum of discharges of Colorado River near Grand Lake, Colo,, and Grand River ditch, all in acre-feet per month).

\begin{tabular}{c|r|r|r|r|r|r|r|r|r|r|r|r|r}
\hline & & Oct. & Nov. & Dec. & Jan. & Feb. & Mar. & Apr. & May & June & July & Aug. & Sept. \\
\hline oct. 1953 & b & 1.00 & 1.00 & 1.00 & 1.00 & 1.00 & 1.00 & 1.00 & 1.00 & 1.00 & 1.00 & 1.00 & 1.00 \\
Sept. 1960 & $\mathrm{c}$ & .04 & .04 & .04 & .04 & .04 & .04 & .04 & .04 & .04 & .04 & .04 & .04 \\
\hline oct. 1960 & $\mathrm{b}$ & .69 & .69 & .38 & - & - & 1.13 & 1.13 & .76 & 1.17 & .76 & .76 & .62 \\
Sept. 1965 & $\mathrm{c}$ & -.71 & -.72 & -1.66 & - & - & .53 & .54 & 1.06 & 1.05 & -.46 & -.48 & -.92 \\
\hline
\end{tabular}

Average discharge. --31 years $(1934-65), 46,404$ acre-feet per year (64.1 cf's).

Extremes.--1934-53: Maximum discharge, 956 cfs June 8, 1952; minimum daily, 6 cfs Feb. 1724, 1938, Dec. 15, 16, 27, 1941 .

Remarks. --Diversions above station for irrigation of hay meadows. Estimates of annual flow are within about 15 percent of regression line.

\begin{tabular}{|c|c|c|c|c|c|c|c|c|c|c|c|c|c|}
\hline $\begin{array}{l}\text { Water } \\
\text { year }\end{array}$ & Oct. & Nov. & Dec. & Jan. & Feb. & Mar. & Apr. & May & June & July & Aug. & Sept. & Annual \\
\hline 1935 & $\neq 553$ & $\neq 595$ & $\neq 492$ & $\neq 492$ & $\ddagger 444$ & $\neq 615$ & 1,480 & 11,360 & 20,440 & 3,490 & 986 & 696 & $\neq 41,640$ \\
\hline $\begin{array}{l}1936 \\
1937 \\
1938 \\
1939 \\
1940\end{array}$ & $\begin{array}{r}750 \\
1,280 \\
1,310 \\
944 \\
700\end{array}$ & $\begin{array}{l}595 \\
821 \\
948 \\
843 \\
661\end{array}$ & & $\begin{array}{l}400 \\
584 \\
460 \\
688 \\
498\end{array}$ & $\begin{array}{l}403 \\
666 \\
371 \\
629 \\
475\end{array}$ & $\begin{array}{l}799 \\
799 \\
686 \\
964 \\
732\end{array}$ & $\begin{array}{l}7,910 \\
2,710 \\
5,040 \\
4,570 \\
2,750\end{array}$ & $\begin{array}{l}29,700 \\
15,430 \\
29,140 \\
26,740 \\
13,640\end{array}$ & $\begin{array}{r}13,050 \\
7,930 \\
20,960 \\
10,280 \\
6,620\end{array}$ & $\begin{array}{l}4,240 \\
3,060 \\
4,560 \\
2,000 \\
1,880\end{array}$ & $\begin{array}{r}4,060 \\
1,360 \\
1,530 \\
1,120 \\
908\end{array}$ & $\begin{array}{r}1,510 \\
972 \\
1,410 \\
706 \\
706\end{array}$ & $\begin{array}{l}63,880 \\
36,320 \\
67,160 \\
50,190 \\
30,140\end{array}$ \\
\hline $\begin{array}{l}1941 \\
1942 \\
1943 \\
1944 \\
1945\end{array}$ & $\begin{array}{l}891 \\
709 \\
811 \\
762\end{array}$ & $\begin{array}{l}726 \\
625 \\
795 \\
639\end{array}$ & $\begin{array}{l}714 \\
617 \\
592\end{array}$ & $\begin{array}{l}420 \\
601 \\
676 \\
436 \\
553\end{array}$ & $\begin{array}{l}415 \\
495 \\
595 \\
463 \\
492\end{array}$ & $\begin{array}{l}610 \\
707 \\
748 \\
685 \\
638\end{array}$ & & $\begin{array}{r}19,360 \\
14,240 \\
15,100 \\
9,890 \\
19,320\end{array}$ & $\begin{array}{r}8,610 \\
14,520 \\
16,420 \\
13,100 \\
16,780\end{array}$ & & $\begin{array}{r}1,090 \\
1,210 \\
1,250 \\
895 \\
1,940\end{array}$ & $\begin{array}{l}873 \\
734 \\
732 \\
497 \\
902\end{array}$ & $\begin{array}{l}37,290 \\
40,920 \\
46,970 \\
32,350 \\
48,810\end{array}$ \\
\hline $\begin{array}{l}1946 \\
1947 \\
1948 \\
1949 \\
1950\end{array}$ & $\begin{array}{r}904 \\
938 \\
1,400 \\
764 \\
899\end{array}$ & $\begin{array}{r}748 \\
978 \\
1,240 \\
724 \\
902\end{array}$ & $\begin{array}{l}590 \\
809 \\
920 \\
590 \\
696\end{array}$ & $\begin{array}{l}615 \\
631 \\
748 \\
584 \\
680\end{array}$ & $\begin{array}{l}555 \\
565 \\
704 \\
633 \\
607\end{array}$ & $\begin{array}{l}900 \\
718 \\
831 \\
768 \\
752\end{array}$ & $\begin{array}{l}5,890 \\
2,440 \\
4,470 \\
3,790 \\
2,850\end{array}$ & $\begin{array}{r}8,360 \\
22,730 \\
21,700 \\
23,070 \\
12,010\end{array}$ & $\begin{array}{r}7,880 \\
15,110 \\
8,600 \\
20,790 \\
14,770\end{array}$ & $\begin{array}{l}2,340 \\
5,840 \\
1,910 \\
5,410 \\
2,960\end{array}$ & $\begin{array}{r}1,280 \\
1,860 \\
1,040 \\
1,430 \\
836\end{array}$ & $\begin{array}{r}764 \\
1,180 \\
696 \\
877 \\
877\end{array}$ & $\begin{array}{l}30,830 \\
53,800 \\
44,260 \\
59,610 \\
38,840\end{array}$ \\
\hline $\begin{array}{l}1951 \\
1952 \\
1953\end{array}$ & $\begin{array}{r}764 \\
1,110 \\
673\end{array}$ & $\begin{array}{l}748 \\
705 \\
546\end{array}$ & $\begin{array}{l}722 \\
566 \\
575\end{array}$ & $\begin{array}{l}561 \\
538 \\
535\end{array}$ & $\begin{array}{l}503 \\
496 \\
441\end{array}$ & $\begin{array}{l}681 \\
710 \\
641\end{array}$ & $\begin{array}{l}1,880 \\
4,430 \\
1,880\end{array}$ & $\begin{array}{l}21,880 \\
30,000 \\
11,290\end{array}$ & $\begin{array}{l}17,810 \\
28,910 \\
12,260\end{array}$ & $\begin{array}{l}5,150 \\
4,250 \\
3,030\end{array}$ & $\begin{array}{l}2,040 \\
2,520 \\
2,770\end{array}$ & $\begin{array}{l}982 \\
992 \\
960\end{array}$ & $\begin{array}{l}53,720 \\
75,230 \\
35,600\end{array}$ \\
\hline
\end{tabular}

* Estimated for 1950 Compilation.

\begin{tabular}{l|r|r|r|r|r|r|r|r|r|r|r|r|r|r}
\hline $\begin{array}{l}\text { Water } \\
\text { year }\end{array}$ & oct. & Nov. & Dec. & Jan. & Feb. & Mar. & Apr. & May & June & July & Aug. & Sept. & Annual \\
\hline 1954 & 730 & 670 & 570 & 470 & 460 & 500 & 3,900 & 9,200 & 2,700 & 950 & 720 & 510 & 21,380 \\
1955 & 680 & 650 & 520 & 530 & 400 & 590 & 3,700 & 11,600 & 7,900 & 2,300 & 1,300 & 570 & 30,740 \\
1956 & 580 & 560 & 580 & 580 & 500 & 610 & 3,800 & 28,200 & 12,200 & 2,000 & 1,100 & 390 & 51,100 \\
1957 & 390 & 680 & 530 & 510 & 520 & 550 & 1,700 & 16,800 & 42,400 & 14,000 & 3,500 & 1,600 & 83,180 \\
1958 & 1,300 & 930 & 750 & 710 & 730 & 810 & 1,900 & 23,900 & 9,900 & 1,500 & 920 & 640 & 43,990 \\
1959 & 610 & 660 & 510 & 460 & 440 & 650 & 1,900 & 12,500 & 9,000 & 2,100 & 1,400 & 970 & 31,200 \\
1960 & 2,100 & 1,500 & 910 & 760 & 700 & 1,600 & 8,700 & 19,000 & 15,100 & 3,000 & 1,200 & 860 & 55,430 \\
1961 & 850 & 720 & 600 & $e 570$ & $e 530$ & 560 & 1,100 & 15,400 & 13,800 & 2,500 & 1,700 & 2,000 & 45,330 \\
1962 & 2,600 & 1,400 & 830 & $e 570$ & $e 530$ & 1,000 & 7,800 & 25,000 & 19,000 & 5,500 & 2,400 & 1,000 & 67,630 \\
1963 & 990 & 600 & 610 & $e 570$ & $e 530$ & 670 & 3,300 & 17,200 & 6,500 & 1,300 & 2,200 & 1,200 & 35,670 \\
1964 & 820 & 770 & 560 & $e 570$ & 6530 & 500 & 1,000 & 17,800 & 10,200 & 3,100 & 1,800 & 930 & 38,580 \\
1965 & 710 & 640 & 610 & e570 & 6530 & 560 & 2,100 & 16,600 & 20,000 & 5,600 & 2,500 & 1,300 & 51,720 \\
\hline
\end{tabular}

e Flat estimate based on average streamflow for month. 

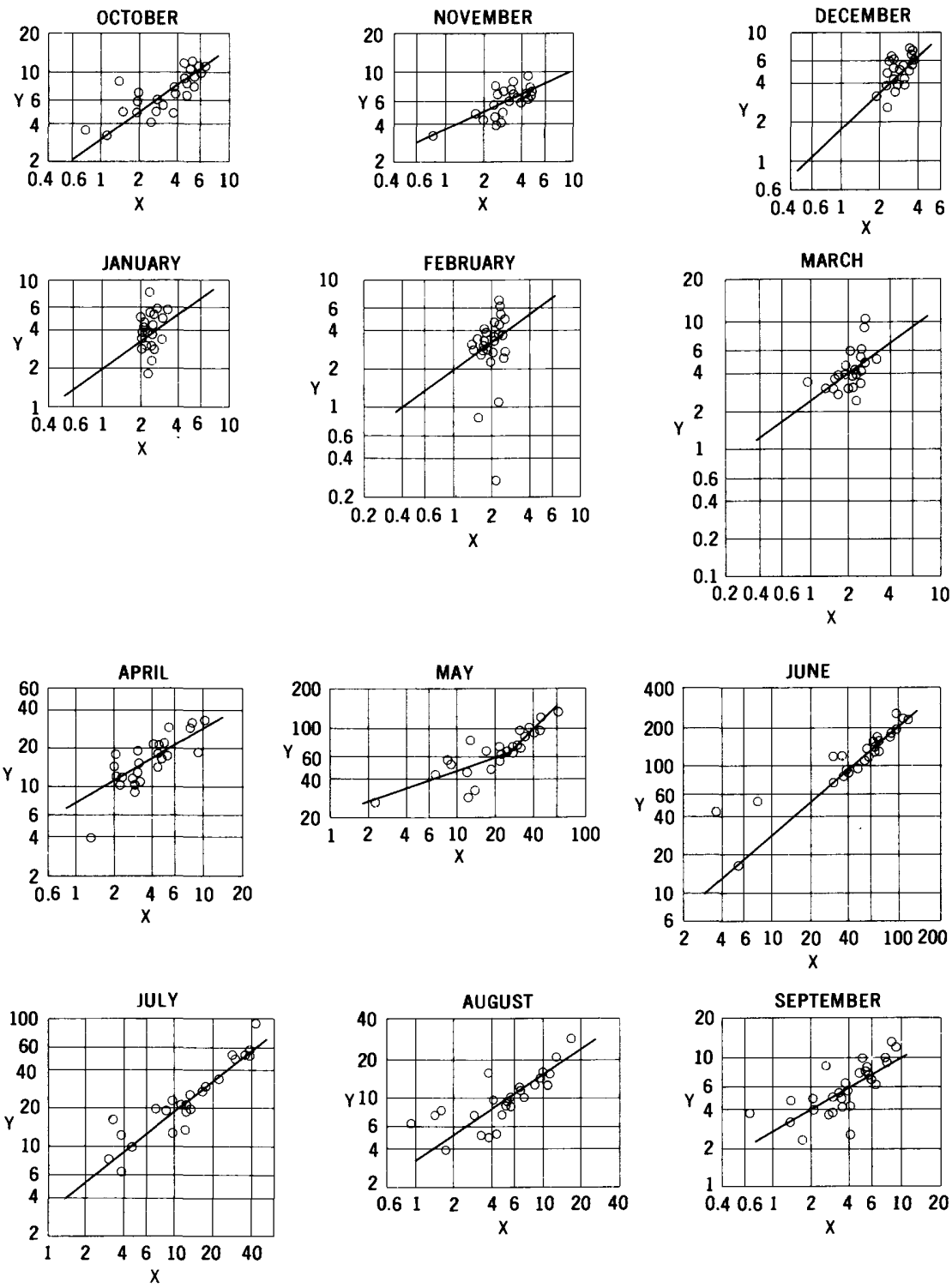

Relationships of monthly mean discharge of Ranch Creek near Tabernash, Colo. (Y), to monthly mean discharge of Ranch Creek near Fraser, Colo. (X). Discharge in hundreds of acre-feet.
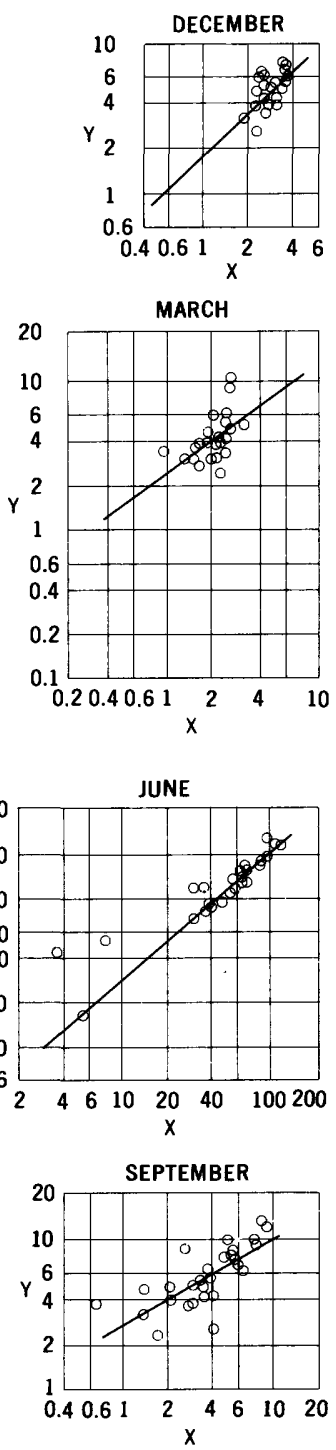
325. Ranch Creek near Tabernash, Colo.

Location. --Lat $39^{\circ} 59151^{\prime \prime}$, long $105^{\circ} 49122^{\prime \prime}$, in $\mathrm{NE} \frac{1}{4} \mathrm{NW} \frac{1}{4}$ sec.6, T.1 S., R.75 W., on right bank a quarter of a mile upstream from Meadow Creek and 1.2 miles east of Tabernash. Datum of gage is $8,339.79 \mathrm{ft}$ above mean sea level, datum of 1929 .

Drainage area. $--50.7 \mathrm{sq} \mathrm{mi}$.

Records available. --September 1934 to September 1960. Records since May 15, 1949, equivalent to earlier records if diversion to Moffat water tunnel is added to flow past station.

Estimates of streamflow. --August 1934, October 1960 to September 1965, based on relationships of monthly mean discharge with Ranch Creek near Fraser, Colo. The regression equation used is:

$\log Y=b \log X-c$

(where $Y$ is discharge of Ranch Creek near Tabernash, Colo., and X is discharge of Ranch Creek near Fraser, Colo., both in acre-feet per month).

\begin{tabular}{|c|c|c|c|c|c|c|c|c|c|c|c|c|}
\hline & oct. & Nov. & Dec. & Jan. & Feb. & Mar. & Apr. & *May & June & July & Aug. & Sept. \\
\hline b & 0.71 & 0.16 & 0.94 & 0.72 & 0.71 & 0.72 & 0.59 & 1.00 & 0.90 & 0.79 & 0.67 & 0.58 \\
\hline c & -1.05 & -1.64 & -.37 & -.86 & -.88 & -.96 & -1.69 & -2.58 & -.63 & -.90 & -1.18 & -1.31 \\
\hline
\end{tabular}

Average discharge. --31 years (1934-65), 25,054 acre-feet per year (34.6 cfs).

Extremes.--1934-60: Maximum discharge, 754 efs June 29, 1957; no flow Feb. 15 to Mar. 13, 1937, Feb. 13, 1947, and perhaps other days.

Remarks. - Transmountain diversion above station to Moffat water tunnel since May 1949. SmaIl diversion to Fraser River basin and several small diversions for irrigation of about 300 acres of hay meadows above station. Estimates of annual flow are within about 10 percent of regression line.

Monthly and annual streamflow, in acre-feet

\begin{tabular}{|c|c|c|c|c|c|c|c|c|c|c|c|c|c|}
\hline $\begin{array}{l}\text { Water } \\
\text { year }\end{array}$ & oct. & Nov. & Dec. & Jan. & Feb. & Mar. & Apr. & May & June & July & Aug. & Sept. & Annual \\
\hline & $\overline{401}$ & $\overline{625}$ & - & $\overline{365}$ & $\overline{314}$ & $\overrightarrow{370}$ & $\overline{994}$ & $\overline{2,950}$ & 14,040 & $5, \overrightarrow{590}$ & $1, \overline{450}$ & $\begin{array}{l}489 \\
693\end{array}$ & 28,230 \\
\hline 36 & $\begin{array}{r}656 \\
964 \\
1,150 \\
909 \\
580\end{array}$ & & $\begin{array}{l}553 \\
514 \\
740 \\
582\end{array}$ & & $\begin{array}{r}374 \\
27 \\
378 \\
471 \\
257\end{array}$ & $\begin{array}{r}338 \\
11 \\
430 \\
488 \\
401\end{array}$ & $\begin{array}{l}, 900 \\
, 860 \\
, 240 \\
, 790 \\
, 690\end{array}$ & $\begin{array}{r}13,670 \\
7,060 \\
10,330 \\
9,920 \\
6,680\end{array}$ & $\begin{array}{r}10,340 \\
5,990 \\
18,840 \\
7,880 \\
7,450\end{array}$ & $\begin{array}{l}2,930 \\
2,590 \\
4,940 \\
1,280 \\
2,020\end{array}$ & $\begin{array}{r}2,110 \\
1,220 \\
1,070 \\
505 \\
916\end{array}$ & $\begin{array}{r}1,030 \\
799 \\
1,360 \\
384 \\
1,010\end{array}$ & $\begin{array}{l}35,130 \\
22,040 \\
42,920 \\
25,390 \\
22,780\end{array}$ \\
\hline $\begin{array}{l}942 \\
43 \\
4\end{array}$ & $\begin{array}{r}1,030 \\
877 \\
544 \\
588 \\
486\end{array}$ & & & & & $\begin{array}{l}604 \\
417 \\
380 \\
465 \\
395\end{array}$ & & & $\begin{array}{r}9,370 \\
15,730 \\
11,850 \\
10,240 \\
10,710\end{array}$ & & $\begin{array}{r}1,040 \\
901 \\
1,170 \\
737 \\
3,030\end{array}$ & $\begin{array}{r}766 \\
540 \\
574 \\
376 \\
1,260\end{array}$ & \\
\hline $\begin{array}{l}46 \\
47 \\
48 \\
49\end{array}$ & $\begin{array}{r}1,190 \\
762 \\
1,090 \\
474 \\
762\end{array}$ & & & & $\begin{array}{r}611 \\
83 \\
452 \\
111 \\
250\end{array}$ & $\begin{array}{l}922 \\
277 \\
549 \\
246 \\
523\end{array}$ & & & $\begin{array}{r}7,620 \\
11,840 \\
7,780 \\
13,650 \\
6,650\end{array}$ & & $\begin{array}{r}934 \\
1,600 \\
874 \\
1,330 \\
532\end{array}$ & $\begin{array}{l}647 \\
952 \\
424 \\
647 \\
426\end{array}$ & $\begin{array}{l}31,810 \\
26,200 \\
31,830 \\
17,360\end{array}$ \\
\hline 800 & $\begin{array}{l}40 \\
82 \\
66 \\
48 \\
66\end{array}$ & & $\begin{array}{l}503 \\
403 \\
381 \\
322\end{array}$ & & & $\begin{array}{l}312 \\
389 \\
390 \\
312 \\
346\end{array}$ & $\begin{array}{l}2,950 \\
1,290 \\
1,170 \\
1,430\end{array}$ & & $\begin{array}{r}12,130 \\
19,720 \\
9,460 \\
1,300 \\
3,360\end{array}$ & & $\begin{array}{l}300 \\
630 \\
966 \\
415 \\
762\end{array}$ & $\begin{array}{l}857 \\
504 \\
490 \\
397\end{array}$ & $\begin{array}{r}30,240 \\
41,350 \\
21,600 \\
8,980 \\
13,920\end{array}$ \\
\hline $\begin{array}{l}557 \\
558\end{array}$ & $\begin{array}{r}325 \\
579 \\
1,090 \\
354 \\
871\end{array}$ & $\begin{array}{l}395 \\
652 \\
708 \\
432 \\
714\end{array}$ & $\begin{array}{l}393 \\
371 \\
720 \\
426 \\
615\end{array}$ & $\begin{array}{l}345 \\
304 \\
608 \\
397 \\
799\end{array}$ & $\begin{array}{l}285 \\
289 \\
540 \\
324 \\
690\end{array}$ & $\begin{array}{r}404 \\
309 \\
626 \\
423 \\
1,100\end{array}$ & $\begin{array}{r}1,800 \\
397 \\
918 \\
1,070 \\
2,930\end{array}$ & $\begin{array}{r}8,090 \\
5,600 \\
12,330 \\
5,270 \\
6,600\end{array}$ & $\begin{array}{r}4,140 \\
20,650 \\
8,950 \\
9,220 \\
13,380\end{array}$ & $\begin{array}{r}979 \\
9,460 \\
636 \\
1,640 \\
1,980\end{array}$ & $\begin{array}{r}770 \\
1,600 \\
537 \\
833 \\
640\end{array}$ & $\begin{array}{l}231 \\
862 \\
320 \\
476 \\
372\end{array}$ & $\begin{array}{l}18,160 \\
41,070 \\
27,980 \\
20,860 \\
30,690\end{array}$ \\
\hline
\end{tabular}

Est1mated monthly and annual streamflow, in acre-feet

\begin{tabular}{l|c|c|c|c|c|c|c|c|r|r|r|r|r}
\hline $\begin{array}{l}\text { Water } \\
\text { year }\end{array}$ & Oct. & Nov. & Dec. & Jan. & Feb. & Mar. & Apr. & May & June & July & Aug. & Sept. & Annual \\
\hline 1934 & - & - & - & - & - & - & - & - & - & - & 920 & - & - \\
& & & & & & - & & & - & & & \\
1961 & 300 & 450 & 150 & 150 & 150 & 200 & 720 & 3,900 & 2,000 & 620 & 480 & 560 & 9,680 \\
1962 & 1,700 & 1,000 & 800 & 490 & 310 & 440 & 2,900 & 6,300 & 8,700 & 3,900 & 390 & 230 & 27,160 \\
1963 & 210 & 350 & 150 & 170 & 150 & 220 & 1,100 & 3,000 & 730 & 410 & 650 & 490 & 7,630 \\
1964 & 290 & 310 & 150 & 130 & 110 & 130 & 800 & 4,000 & 860 & 470 & 350 & 250 & 7,850 \\
1965 & 340 & 300 & 100 & 140 & 130 & 190 & 1,000 & 4,400 & 7,200 & 2,500 & 1,800 & 1,470 & 18,570 \\
\hline
\end{tabular}



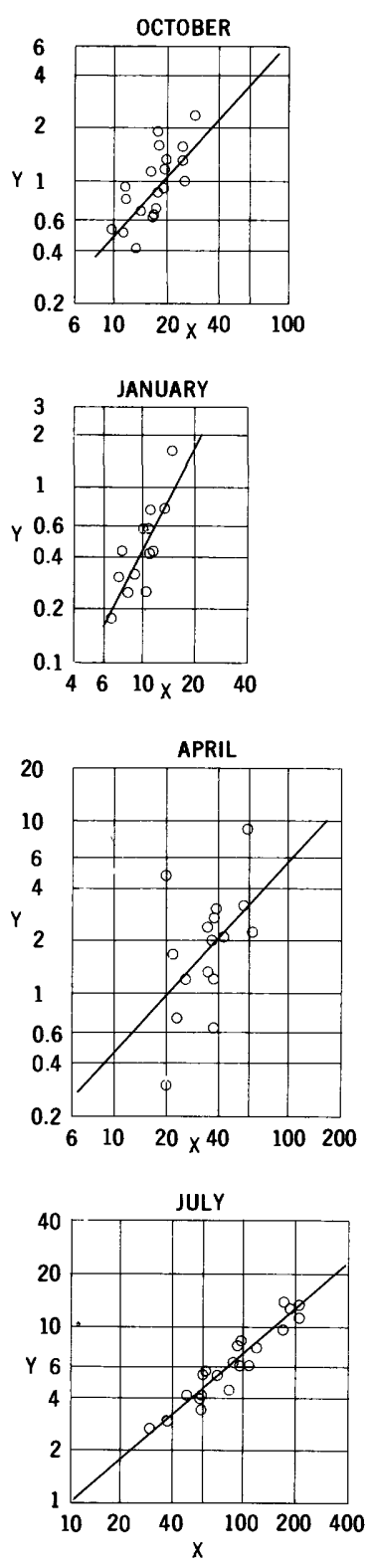
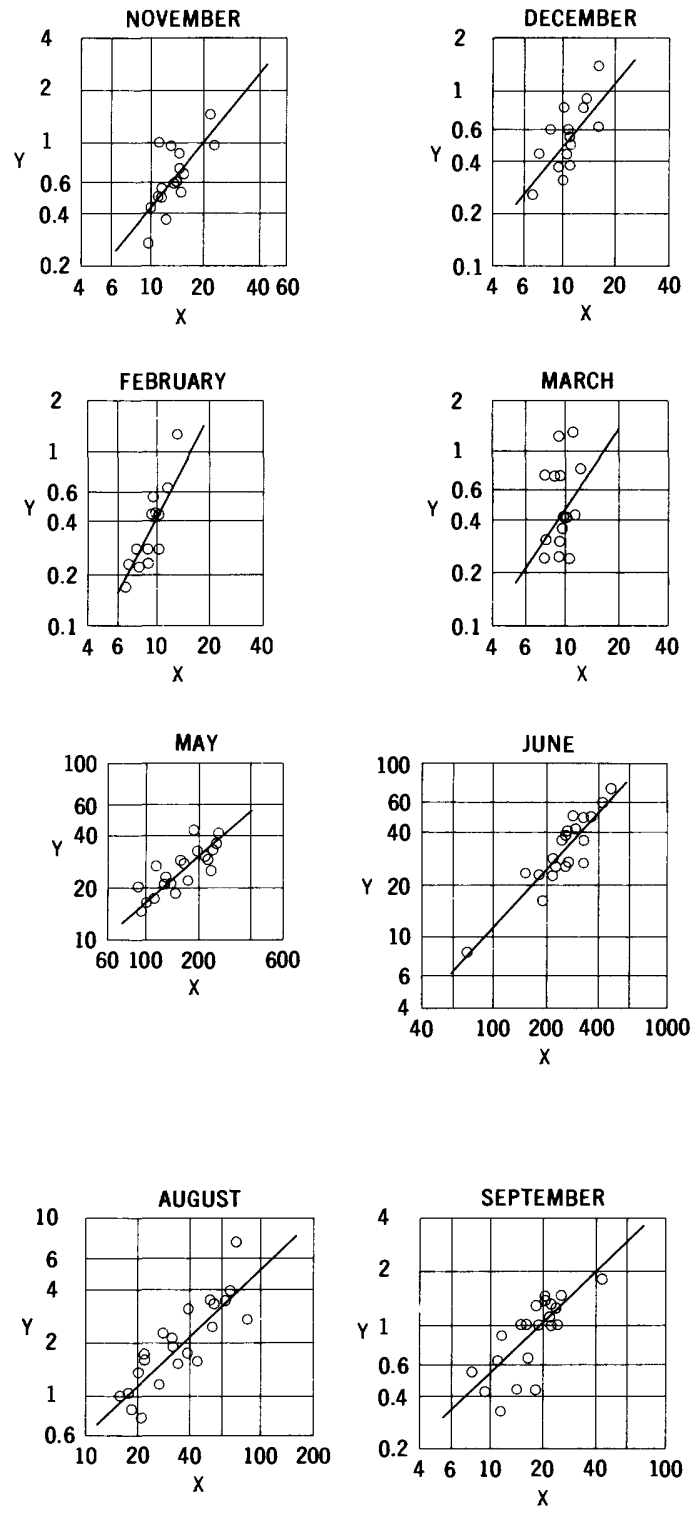

Relationships of monthly mean discharge of Meadow Creek near Tabernash, Colo. (Y), to sum of monthly mean discharges of Colorado River near Grand Lake, Colo., and Grand River ditch $(\mathrm{X})$. Discharge in hundreds of acre-feet. 
330. Meadow Creek near Tabernash, Colo.

Location. --Lat $40^{\circ} 02^{\prime} 55^{\prime \prime}$, long $105^{\circ} 46^{\prime} 30^{\prime \prime}$, in sec.15, T.1 N., R.75 W., on right bank $30 \mathrm{ft}$ upstream from bridge and 5 miles northeast of Tabernash. Altitude of gage is $9,780 \mathrm{ft}$ (from topographic map).

Drainage area. $--7.0 \mathrm{sq} \mathrm{mi}$, approximately.

Records available. --October 1935 to September 1956.

Estimates of streamflow.--June 1934 to September 1935 , October 1956 to September 1965 , based on relationships of monthly mean discharge with Colorado River near Grand Lake, Colo., adjusted for diversion through Grand River ditch. The regression equation used is:

$$
\log Y=b \log X-c
$$

(where $\mathrm{Y}$ is discharge of Meadow Creek near Tabemash, Colo., and X is the sum of discharges of Colorado River near Grand Lake, Colo. and'Grand River ditch, all in acrefeet per month).

\begin{tabular}{l|l|l|l|l|l|l|l|l|l|l|l|l}
\hline \multicolumn{10}{c}{ Monthly values of constants in above equation } \\
\hline b & Oct. & Nov. & Dec. & Jan. & Feb. & Mar. & Apr. & May & June & July & Aug. & Sept. \\
\hline c & 1.11 & 1.20 & 1.13 & 1.95 & 1.95 & 1.42 & 1.10 & 0.88 & 1.08 & 0.85 & 0.92 & 0.93 \\
\hline
\end{tabular}

Average discharge. --31 years $(1934-65), 7,919$ acre-f'eet per year $(10.9 \mathrm{cfs})$.

Extremes. --1935-56: Maximum discharge, 316 cf's June 10, 1952; probably no flow at times during ice periods.

Remarks. - No diversion above station. Estimates of annual flow are within about 15 percent of regression line.

Monthly and annual streamflow, in acre-feet

\begin{tabular}{|c|c|c|c|c|c|c|c|c|c|c|c|c|c|}
\hline $\begin{array}{l}\text { Water } \\
\text { year }\end{array}$ & oct. & Nov. & Dec. & Jan, & Feb. & Mar. & Apr. & May & June & July & Aug. & Sept. & Annual \\
\hline $\begin{array}{l}1936 \\
1937 \\
1938 \\
1939 \\
1940\end{array}$ & $\begin{array}{r}\$ 92 \\
190 \\
233 \\
89 \\
62\end{array}$ & $\begin{array}{r}\neq 60 \\
\neq 89 \\
142 \\
60 \\
49\end{array}$ & $\begin{array}{r}\neq 61 \\
\neq 92 \\
135 \\
55 \\
44\end{array}$ & $\begin{array}{r}\neq 61 \\
\neq 92 \\
166 \\
55 \\
31\end{array}$ & $\begin{array}{r}\neq 58 \\
\neq 83 \\
128 \\
44 \\
23\end{array}$ & $\begin{array}{r}\neq 92 \\
\$ 61 \\
129 \\
80 \\
31\end{array}$ & $\begin{array}{r}\$ 298 \\
\$ 179 \\
220 \\
319 \\
131\end{array}$ & $\begin{array}{r}\neq 4,920 \\
4,250 \\
2,840 \\
4,060 \\
2,870\end{array}$ & $\begin{array}{l}2,670 \\
2,270 \\
5,750 \\
2,500 \\
2,270\end{array}$ & $\begin{array}{l}762 \\
627 \\
972 \\
331 \\
406\end{array}$ & $\begin{array}{r}393 \\
211 \\
156 \\
162 \\
84\end{array}$ & $\begin{array}{l}140 \\
123 \\
182 \\
102 \\
110\end{array}$ & $\begin{array}{r}\neq 9,610 \\
\neq 8,270 \\
11,050 \\
7,860 \\
6,110\end{array}$ \\
\hline $\begin{array}{l}1941 \\
1942 \\
1943 \\
1944 \\
1945\end{array}$ & $\begin{array}{r}131 \\
133 \\
92 \\
69 \\
52\end{array}$ & $\begin{array}{r}\neq 70 \\
86 \\
\neq 65 \\
\neq 86 \\
27\end{array}$ & $\begin{array}{r}\neq 61 \\
\neq 92 \\
\neq 61 \\
\neq 61 \\
25\end{array}$ & $\begin{aligned} \neq 61 \\
\neq 92 \\
\neq 92 \\
\neq 61 \\
18\end{aligned}$ & $\begin{array}{l}\neq 56 \\
\neq 56 \\
\neq 56 \\
\neq 58 \\
17\end{array}$ & $\begin{array}{l}\neq 61 \\
\neq 61 \\
\ddagger 92 \\
\neq 61 \\
74\end{array}$ & $\begin{array}{r}\$ 60 \\
\neq 149 \\
\neq 298 \\
30 \\
476\end{array}$ & $\begin{array}{l}3,270 \\
1,620 \\
2,240 \\
1,840 \\
2,110\end{array}$ & $\begin{array}{l}2,500 \\
4,970 \\
3,820 \\
4,030 \\
3,440\end{array}$ & $\begin{array}{r}428 \\
839 \\
600 \\
589 \\
1,250\end{array}$ & $\begin{array}{l}224 \\
166 \\
188 \\
102 \\
670\end{array}$ & $\begin{array}{r}131 \\
87 \\
63 \\
42 \\
129\end{array}$ & $\begin{array}{r}\neq 7,050 \\
\neq 8,350 \\
\neq 7,670 \\
\neq 7,030 \\
8,290\end{array}$ \\
\hline $\begin{array}{l}1946 \\
1947 \\
1948 \\
1949 \\
1950\end{array}$ & $\begin{array}{r}110 \\
85 \\
155 \\
77 \\
157\end{array}$ & $\begin{array}{r}95 \\
65 \\
94 \\
55 \\
100\end{array}$ & $\begin{array}{l}80 \\
49 \\
80 \\
49 \\
61\end{array}$ & $\begin{array}{l}74 \\
25 \\
55 \\
43 \\
55\end{array}$ & $\begin{array}{l}56 \\
28 \\
46 \\
28 \\
28\end{array}$ & $\begin{array}{r}123 \\
74 \\
43 \\
43 \\
25\end{array}$ & $\begin{array}{l}893 \\
165 \\
119 \\
308 \\
235\end{array}$ & $\begin{array}{l}1,480 \\
2,980 \\
3,160 \\
2,730 \\
2,000\end{array}$ & $\begin{array}{l}2,870 \\
3,710 \\
1,620 \\
4,930 \\
3,560\end{array}$ & $\begin{array}{r}528 \\
1,130 \\
407 \\
1,350 \\
404\end{array}$ & $\begin{array}{r}117 \\
335 \\
133 \\
307 \\
99\end{array}$ & $\begin{array}{r}42 \\
147 \\
54 \\
107 \\
107\end{array}$ & $\begin{array}{r}6,470 \\
8,790 \\
5,970 \\
10,030 \\
6,830\end{array}$ \\
\hline $\begin{array}{l}1951 \\
1952 \\
1953 \\
1954 \\
1955\end{array}$ & $\begin{array}{r}63 \\
114 \\
55 \\
50 \\
99\end{array}$ & $\begin{array}{l}58 \\
71 \\
48 \\
43 \\
51\end{array}$ & $\begin{array}{l}61 \\
61 \\
43 \\
37 \\
31\end{array}$ & $\begin{array}{l}55 \\
74 \\
43 \\
43 \\
25\end{array}$ & $\begin{array}{l}44 \\
63 \\
44 \\
28 \\
22\end{array}$ & $\begin{array}{l}43 \\
74 \\
37 \\
31 \\
25\end{array}$ & $\begin{array}{r}119 \\
268 \\
71 \\
216 \\
201\end{array}$ & $\begin{array}{l}2,200 \\
2,480 \\
1,720 \\
2,030 \\
2,630\end{array}$ & $\begin{array}{r}4,800 \\
6,980 \\
4,190 \\
827 \\
2,350\end{array}$ & $\begin{array}{r}1,340 \\
779 \\
526 \\
261 \\
551\end{array}$ & $\begin{array}{r}265 \\
342 \\
241 \\
76 \\
173\end{array}$ & $\begin{array}{r}99 \\
145 \\
66 \\
101 \\
43\end{array}$ & $\begin{array}{r}9,150 \\
11,450 \\
7,090 \\
3,740 \\
6,200\end{array}$ \\
\hline 1956 & 42 & 36 & 37 & 31 & 23 & 25 & 63 & 3,570 & 2,700 & 294 & 150 & 33 & 7,000 \\
\hline
\end{tabular}

* Estimated for 1950 Compllation.

\begin{tabular}{l|r|r|r|r|r|r|r|r|r|r|r|r|r}
\hline $\begin{array}{l}\text { Water } \\
\text { year }\end{array}$ & Oct. & Nov. & Dec. & Jan. & Feb. & Mar. & Apr. & May & June & July & Aug. & Sept. & Annual \\
\hline 1934 & - & - & - & - & - & - & - & - & 650 & 110 & 90 & 70 & - \\
1935 & 50 & 50 & 50 & 30 & 20 & 20 & 110 & 1,300 & 4,200 & 1,100 & 200 & 80 & 7,210 \\
1957 & 60 & 40 & 30 & 30 & 20 & 40 & 80 & 2,000 & 4,800 & 2,200 & 500 & 160 & 9,960 \\
1958 & 150 & 90 & 80 & 60 & 50 & 50 & 90 & 3,800 & 3,600 & 310 & 150 & 60 & 8,490 \\
1959 & 50 & 40 & 40 & 30 & 30 & 30 & 100 & 1,900 & 3,700 & 520 & 260 & 110 & 6,810 \\
1960 & 210 & 150 & 70 & 40 & 40 & 120 & 480 & 2,600 & 4,400 & 610 & 180 & 90 & 8,990 \\
1961 & 80 & 60 & 40 & 30 & 30 & 30 & 70 & 2,100 & 3,500 & 540 & 230 & 330 & 7,040 \\
1962 & 490 & 180 & 110 & 80 & 60 & 70 & 470 & 3,600 & 4,600 & 1,300 & 350 & 120 & 11,430 \\
1963 & 110 & 40 & 40 & 20 & 20 & 40 & 210 & 2,400 & 1,200 & 260 & 310 & 140 & 5,290 \\
1964 & 80 & 60 & 30 & 20 & 20 & 30 & 60 & 2,500 & 2,600 & 700 & 250 & 100 & 6,450 \\
1965 & 60 & 40 & 40 & 40 & 30 & 30 & 130 & 2,300 & 4,800 & 1,800 & 370 & 170 & 9,810 \\
\hline
\end{tabular}



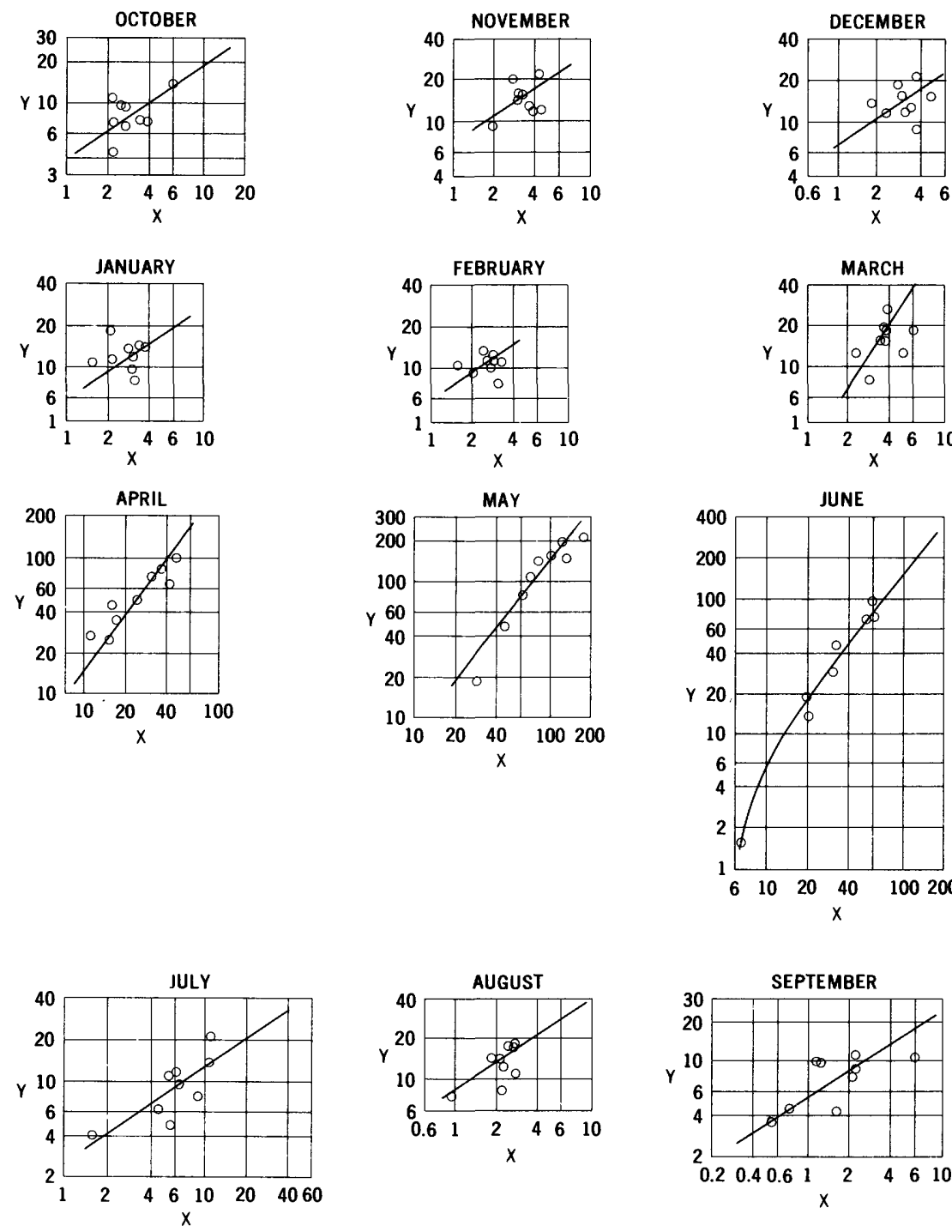

Relationships of monthly mean discharge of Troublesome Creek near Troublesome, Colo. (Y), to monthly mean discharge of East Fork Troublesome Creek near Troublesome, Colo. $(\mathrm{X})$. Discharge in hundreds of acre-feet. 
405. Troublesome Creek near Troublesome, Colo.

Location. - Lat $40^{\circ} 04^{1}$, long $106^{\circ} 19^{\prime}$, in sec.12, T.1 N., R.80 W., on left bank $50 \mathrm{ft}$ upstream from bridge on U. S. Highway 40 , half a mile upstream from mouth, and half a mile west of old Troublesome Post office. Datum of gage is 7,344.13 ft above mean sea level, datum of 1929 .

Drainage area. $--178 \mathrm{sq} \mathrm{mi}$.

Records available.--July 1904 to October 1905, October 1921 to September 1924, July 1937 to September 1956. Monthly discharge only for some periods. Published as "at Troublesome" $1904-5$.

Estimates of streamflow.--October 1956 to September 1965 , based on relationships of monthly mean discharge with East Fork Troublesome Creek near Troublesome, Colo. The regression equation used is:

$\log Y=b \log X-c$

(where $\mathrm{Y}$ is discharge of Troublesome Creek near Troublesome, Colo., and $\mathrm{X}$ is discharge of East Fork Troublesome Creek near Troublesome, Colo., both in acre-feet per month).

Monthiy values of constants in above equation

\begin{tabular}{c|c|c|c|r|r|r|r|r|r|r|r|l}
\hline & oct. & Nov. & Dec. & Jan. & Feb. & Mar. & Apr. & $*$ May & $*$ June & July & Aug. & Sept. \\
\hline $\mathrm{b}$ & 0.68 & 0.66 & 0.66 & 0.66 & 0.66 & 1.08 & 1.00 & 1.12 & 1.12 & 0.67 & 0.66 & 0.65 \\
$\mathrm{c}$ & -1.23 & -1.52 & -1.52 & -1.45 & -1.45 & -.46 & -.30 & .33 & .33 & -1.10 & -1.60 & -1.43 \\
\hline
\end{tabular}

Average discharge. --28 years $(1937-65), 36,770$ acre-feet per year (50.8 crs).

Extremes. - -1904-5, 1921-24, 1937-56: Maximum discharge, 1,230 cfs July 23, 1945, from rating curve extended above 370 cfs; minimum daily, i cfs July 19-21, 26, 27 , '1922.

Remarks.--Diversions for irrigation of about 4,000 acres above station. Estimates of annual flow are within about 10 percent of regression line.

Monthly and annual streamflow, in acre-feet

\begin{tabular}{|c|c|c|c|c|c|c|c|c|c|c|c|c|c|}
\hline $\begin{array}{l}\text { Water } \\
\text { year }\end{array}$ & oct. & Nov. & Dec. & Jan. & Feb. & Mar. & Apr. & May & June & July & Aug. & Sept. & Annual \\
\hline $\begin{array}{l}1937 \\
1938 \\
1939 \\
1940\end{array}$ & $\begin{array}{r}- \\
1,420 \\
746 \\
760\end{array}$ & $\begin{array}{c}- \\
2,210 \\
1,210 \\
1,590\end{array}$ & $\begin{array}{l}1,290 \\
1,540 \\
1,540\end{array}$ & $\begin{array}{r}- \\
978 \\
1,410 \\
1,350\end{array}$ & $\begin{array}{c}- \\
1,000 \\
1,110 \\
1,150\end{array}$ & $\begin{array}{c}- \\
2,570 \\
1,840 \\
1,840\end{array}$ & $\begin{array}{c}- \\
8,330 \\
6,460 \\
3,530\end{array}$ & $\begin{array}{r}- \\
20,640 \\
19,030 \\
8,000\end{array}$ & $\begin{array}{l}- \\
9,640 \\
3,000 \\
1,930\end{array}$ & $\begin{array}{r}2,100 \\
944 \\
480 \\
1,110\end{array}$ & $\begin{array}{r}1,700 \\
930 \\
1,390 \\
1,100\end{array}$ & $\begin{array}{r}659 \\
1,050 \\
865 \\
1,130\end{array}$ & $\begin{array}{c}- \\
51,000 \\
39,080 \\
25,030\end{array}$ \\
\hline $\begin{array}{l}1941 \\
1942 \\
1943 \\
1944 \\
1945\end{array}$ & $\begin{array}{r}\neq 1,730 \\
972 \\
1,090 \\
877 \\
687\end{array}$ & $\begin{array}{r}1,190 \\
2,040 \\
1,610 \\
1,890 \\
1,470\end{array}$ & $\begin{array}{r}\$ 861 \\
1,370 \\
2,070 \\
1,490 \\
1,350\end{array}$ & $\begin{array}{r} \pm 799 \\
1,100 \\
1,460 \\
1,100 \\
1,230\end{array}$ & $\begin{array}{r}\$ 778 \\
1,080 \\
1,100 \\
1,090 \\
1,330\end{array}$ & $\begin{array}{r}\neq 1,230 \\
1,280 \\
1,510 \\
1,290 \\
1,600\end{array}$ & $\begin{array}{l}+2,790 \\
7,330 \\
9,860 \\
2,160 \\
2,130\end{array}$ & $\begin{array}{r} \pm 15,210 \\
14,380 \\
10,650 \\
8,460 \\
13,100\end{array}$ & $\begin{array}{r}73,890 \\
7,130 \\
7,520 \\
6,240 \\
5,850\end{array}$ & $\begin{array}{r}\$ 349 \\
777 \\
1,370 \\
749 \\
2,570\end{array}$ & $\begin{array}{r}1,570 \\
1,460 \\
1,720 \\
711 \\
2,440\end{array}$ & $\begin{array}{r}\neq 1,210 \\
362 \\
948 \\
264 \\
1,130\end{array}$ & $\begin{array}{r}\$ 31,610 \\
39,280 \\
40,910 \\
26,320 \\
34,890\end{array}$ \\
\hline $\begin{array}{l}1946 \\
2947 \\
1948 \\
1949 \\
1950\end{array}$ & $\begin{array}{l}1,450 \\
1,110 \\
1,890 \\
1,330 \\
1,490\end{array}$ & $\begin{array}{l}1,440 \\
1,860 \\
2,530 \\
1,280 \\
1,520\end{array}$ & $\begin{array}{l}1,350 \\
1,760 \\
1,880 \\
1,340 \\
1,390\end{array}$ & $\begin{array}{r}1,350 \\
1,270 \\
1,250 \\
1,230 \\
962\end{array}$ & $\begin{array}{r}1,390 \\
1,080 \\
982 \\
1,150 \\
910\end{array}$ & $\left\{\begin{array}{l}1,840 \\
2,360 \\
1,200 \\
1,770 \\
1,680\end{array}\right.$ & $\begin{array}{l}7,670 \\
4,440 \\
6,570 \\
5,030 \\
4,610\end{array}$ & $\begin{array}{r}7,250 \\
16,160 \\
18,370 \\
14,530 \\
6,930\end{array}$ & $\begin{array}{r}2,330 \\
7,000 \\
2,290 \\
11,800 \\
4,890\end{array}$ & $\begin{array}{r}378 \\
2,400 \\
1,070 \\
3,200 \\
1,330\end{array}$ & $\begin{array}{l}1,170 \\
2,000 \\
2,050 \\
1,790 \\
1,230\end{array}$ & $\begin{array}{r}827 \\
1,400 \\
1,050 \\
1,780 \\
1,150\end{array}$ & $\begin{array}{l}28,440 \\
42,840 \\
41,130 \\
46,230 \\
28,090\end{array}$ \\
\hline $\begin{array}{l}1951 \\
1952 \\
1953 \\
1954 \\
1955\end{array}$ & $\begin{array}{r}984 \\
1,470 \\
1,260 \\
727 \\
692\end{array}$ & $\begin{array}{r}1,590 \\
1,890 \\
1,350 \\
1,290 \\
928\end{array}$ & $\begin{array}{l}1,700 \\
1,690 \\
1,580 \\
1,190 \\
1,170\end{array}$ & $\begin{array}{l}1,360 \\
1,250 \\
1,430 \\
1,190 \\
1,020\end{array}$ & $\begin{array}{r}1,240 \\
1,140 \\
1,180 \\
1,270 \\
930\end{array}$ & $\begin{array}{r}1,730 \\
1,240 \\
1,730 \\
1,550 \\
811\end{array}$ & $\begin{array}{l}3,760 \\
9,330 \\
3,160 \\
2,560 \\
4,550\end{array}$ & $\begin{array}{r}16,690 \\
26,810 \\
6,710 \\
1,920 \\
4,670\end{array}$ & $\begin{array}{r}9,450 \\
16,550 \\
5,310 \\
161 \\
1,410\end{array}$ & $\begin{array}{r}2,530 \\
2,590 \\
2,260 \\
418 \\
622\end{array}$ & $\begin{array}{r}2,460 \\
2,390 \\
2,040 \\
754 \\
1,240\end{array}$ & $\begin{array}{r}1,280 \\
1,600 \\
653 \\
428 \\
445\end{array}$ & $\begin{array}{l}44,770 \\
67,950 \\
28,660 \\
13,460 \\
18,490\end{array}$ \\
\hline 1956 & 460 & 1,460 & 1,890 & 1,370 & 1,350 & 1,930 & 4,890 & 14,610 & 4,600 & 1,170 & 1,790 & 1,000 & 36,520 \\
\hline
\end{tabular}

Estimated monthly and annual streamflow, in acre-feet

\begin{tabular}{l|r|l|l|l|l|l|l|l|r|r|r|r|r}
\hline $\begin{array}{l}\text { Water } \\
\text { year }\end{array}$ & Oct. & Nov. & Dec. & Jan. & Feb. & Mar. & Apr. & May & June & Ju1y & Aug. & Sept. & Annual \\
\hline 1957 & 730 & 1,200 & 1,200 & 1,200 & 1,200 & 1,700 & 2,000 & 12,800 & 30,000 & 3,000 & 3,600 & 1,600 & 60,230 \\
1958 & 1,200 & 2,100 & 2,000 & 1,500 & 1,400 & 2,200 & 3,000 & 18,800 & 4,200 & 890 & 1,700 & 870 & 39,860 \\
1959 & 800 & 1,400 & 1,500 & 1,300 & 1,200 & 1,600 & 1,800 & 5,800 & 3,000 & 980 & 2,300 & 1,200 & 22,880 \\
1960 & 1,700 & 2,300 & 1,500 & 1,400 & 1,200 & 3,500 & 10,000 & 10,600 & 5,700 & 1,200 & 1,600 & 1,200 & 41,900 \\
1961 & 1,000 & 1,600 & 1,400 & 1,100 & 1,200 & 1,900 & 1,800 & 6,800 & 3,300 & 790 & 1,300 & 2,100 & 24,290 \\
1962 & 2,600 & 2,000 & 1,600 & 1,300 & 1,400 & 2,000 & 16,700 & 25,800 & 8,900 & 1,800 & 2,300 & 1,100 & 67,500 \\
1963 & 1,100 & 1,700 & 1,300 & 1,100 & 1,400 & 2,300 & 2,500 & 4,300 & 940 & 590 & 2,000 & 1,100 & 20,330 \\
1964 & 700 & 1,200 & 720 & 780 & 850 & 940 & 1,300 & 7,900 & 3,500 & 950 & 2,200 & 820 & 21,860 \\
1965 & 640 & 1,200 & 1,100 & 940 & 1,000 & 1,200 & 4,100 & 16,900 & 12,700 & 1,700 & 3,000 & 1,600 & 46,080 \\
\hline
\end{tabular}



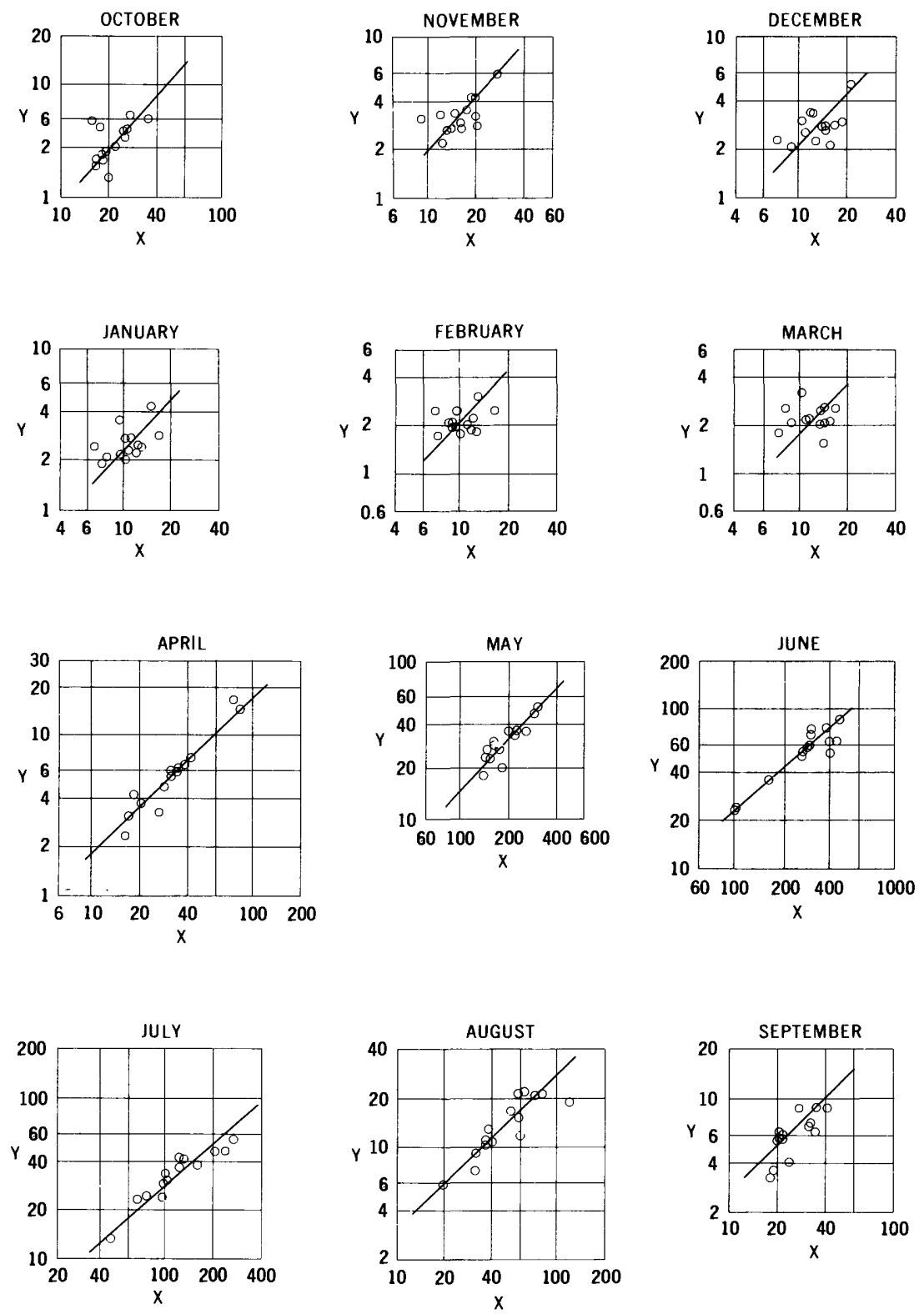

Relationships of monthly mean discharge of Rock Creek near Dillon, Colo. (Y), to monthly mean discharge of Tenmile Creek at Dillon, Colo. (X). Discharge in hundreds of acre-feet. 
520. Rock Creek near Dillon, Colo.

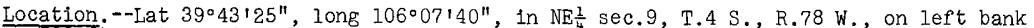
500 ft upstream from bridge on state Highway 9 , a quarter of a mile upstream from mouth, and 9 miles northwest of Dillon. Datum of gage is $8,502.52 \mathrm{ft}$ above mean sea level, unadjusted.

Drainage area. $--15.8 \mathrm{sq} \mathrm{mi}$.

Records avallable. --July 1942 to September 1956.

Estimates of streamflow.--October 1930 to July 1942 , October 1956 to April 1965 , based on relatjonships of monthly mean discharge with Tenmile Creek at Dilion, Colo. (Tenmile Creek at Dillon, Colo. discontinued April 1961; records for May 1961 to September 1965 were estimated for use in this relationship on the basis of discharge of Tenmile Creek below North Fork, at Frisco, Colo.). The regression equation used 18 :

$$
\log Y=b \log X-c
$$

(where $Y$ is discharge of Rock Creek near Dillon, Colo, and $X$ is discharge of Tenmile Creek at Dillon, Colo., estimated after April 1961, both in acre-feet per month).

Monthly values of constants in above equation

\begin{tabular}{r|r|r|r|r|r|r|r|r|r|r|r|r}
\hline & oct. & Nov. & Dec. & Jan. & Feb. & Mar. & Apr. & May & June & July & Aug. & Sept. \\
\hline b & 1.12 & 1.11 & 1.06 & 1.06 & 1.04 & 1.01 & 1.01 & 1.08 & 0.89 & 0.89 & 0.96 & 0.98 \\
$\mathrm{c}$ & 1.09 & 1.04 & .85 & .83 & .80 & .78 & .77 & 1.14 & .21 & .10 & .39 & .52 \\
\hline
\end{tabular}

Average discharge. --35 years $(1930-65), 16,570$ acre-feet per year (22.9 cfs).

Extremes.--1942-56: Maximum discharge, 260 cf's June 18, 1951, from rating curve extended above 190 cfs; minimum daily determined, 2.2 cfs Apr. 13, 17, 1945.

Remarks.--A few small diversions for irrigation of hay meadows above station. Estimates of annual flow are within about 10 percent of regression line.

Monthly and annual streamflow, in acre-feet

\begin{tabular}{c|c|c|c|c|c|c|c|c|c|c|c|c|c}
\hline $\begin{array}{l}\text { Water } \\
\text { year }\end{array}$ & oct. & Nov. & Dec. & Jan. & Feb. & Mar. & Apr. & May & June & July & Aug. & Sept. & Annual \\
\hline 1942 & - & - & - & - & - & - & - & - & & - & - & 1,090 & 557 \\
1943 & 314 & 270 & 265 & 234 & 206 & 220 & 1,690 & 3,670 & 6,690 & 4,300 & 2,120 & 899 & 20,880 \\
1944 & 500 & 343 & 345 & 192 & 249 & 326 & 312 & 3,020 & 5,770 & 3,360 & 923 & 355 & 15,700 \\
1945 & 259 & 224 & 234 & 246 & 194 & 154 & 238 & 2,760 & 4,990 & 3,880 & 1,920 & 628 & 15,730 \\
1946 & 516 & 424 & 338 & 277 & 211 & 258 & 1,490 & 2,140 & 5,380 & 2,440 & 1,300 & 544 & 15,320 \\
1947 & 374 & 357 & 277 & 221 & 189 & 209 & 372 & 3,550 & 5,190 & 4,870 & 2,090 & 877 & 18,580 \\
1948 & 610 & 585 & 516 & 435 & 306 & 258 & 658 & 5,030 & 7,640 & 3,010 & 1,120 & 627 & 20,800 \\
1949 & 533 & 335 & 307 & 357 & 211 & 258 & 561 & 3,080 & 6,330 & 4,750 & 1,530 & 711 & 18,960 \\
1950 & 644 & 417 & 215 & 277 & 250 & 246 & 595 & 2,770 & 6,340 & 3,080 & 718 & 579 & 16,130 \\
1951 & 341 & 295 & 268 & 242 & 184 & 224 & 427 & 3,560 & 6,180 & 5,660 & 2,150 & 691 & 20,220 \\
1952 & 458 & 288 & 283 & 250 & 222 & 205 & 725 & 3,490 & 8,520 & 4,300 & 2,210 & 878 & 21,830 \\
1953 & 403 & 274 & 283 & 221 & 178 & 209 & 330 & 1,920 & 6,360 & 3,630 & 1,670 & 542 & 16,020 \\
1954 & 364 & 277 & 229 & 207 & 198 & 219 & 612 & 2,480 & 2,310 & 1,340 & 584 & 580 & 9,400 \\
1955 & 585 & 318 & 212 & 213 & 173 & 182 & 622 & 2,460 & 3,560 & 2,370 & 1,180 & 404 & 12,280 \\
1956 & 336 & 322 & 299 & 285 & 260 & 254 & 482 & 4,750 & 5,820 & 2,460 & 1,030 & 326 & 16,620 \\
\hline
\end{tabular}

Estimated monthly and annual streamflow, in acre-feet

\begin{tabular}{|c|c|c|c|c|c|c|c|c|c|c|c|c|c|}
\hline $\begin{array}{l}\text { Water } \\
\text { year }\end{array}$ & oct. & Nov. & Dec. & Jan. & Feb. & Mar. & Apr. & May & June & July & Aug. & Sept. & Annual \\
\hline $\begin{array}{l}1931 \\
1932 \\
1933 \\
1934 \\
1935\end{array}$ & $\begin{array}{l}680 \\
460 \\
520 \\
410 \\
290\end{array}$ & $\begin{array}{l}450 \\
300 \\
340 \\
320 \\
260\end{array}$ & $\begin{array}{l}340 \\
210 \\
330 \\
290 \\
290\end{array}$ & $\begin{array}{l}280 \\
160 \\
280 \\
360 \\
290\end{array}$ & $\begin{array}{l}210 \\
170 \\
260 \\
250 \\
250\end{array}$ & $\begin{array}{l}270 \\
160 \\
270 \\
250 \\
200\end{array}$ & $\begin{array}{l}960 \\
560 \\
350 \\
840 \\
350\end{array}$ & $\begin{array}{l}2,800 \\
3,300 \\
2,300 \\
3,800 \\
1,200\end{array}$ & $\begin{array}{l}3,900 \\
5,800 \\
8,000 \\
2,300 \\
6,400\end{array}$ & $\begin{array}{l}1,600 \\
3,300 \\
3,200 \\
1,100 \\
3,000\end{array}$ & $\begin{array}{r}860 \\
1,500 \\
, 200 \\
860 \\
1,500\end{array}$ & $\begin{array}{l}540 \\
780 \\
760 \\
480 \\
840\end{array}$ & $\begin{array}{l}12,890 \\
16,700 \\
17,810 \\
11,260 \\
14,870\end{array}$ \\
\hline $\begin{array}{l}1936 \\
1937 \\
1938 \\
1939 \\
1940\end{array}$ & $\begin{array}{l}580 \\
340 \\
420 \\
540 \\
320\end{array}$ & $\begin{array}{l}440 \\
330 \\
450 \\
430 \\
210\end{array}$ & $\begin{array}{l}270 \\
310 \\
390 \\
340 \\
190\end{array}$ & $\begin{array}{l}290 \\
370 \\
270 \\
310 \\
170\end{array}$ & $\begin{array}{l}280 \\
330 \\
230 \\
210 \\
150\end{array}$ & $\begin{array}{l}280 \\
350 \\
260 \\
280 \\
200\end{array}$ & $\begin{array}{r}1,400 \\
500 \\
640 \\
720 \\
580\end{array}$ & $\begin{array}{l}7,000 \\
2,900 \\
3,400 \\
5,400 \\
2,500\end{array}$ & $\begin{array}{l}6,000 \\
3,400 \\
8,000 \\
4,500 \\
3,500\end{array}$ & $\begin{array}{l}3,500 \\
2,100 \\
3,400 \\
2,100 \\
1,900\end{array}$ & $\begin{array}{r}2,300 \\
960 \\
, 500 \\
960 \\
780\end{array}$ & $\begin{array}{r}800 \\
600 \\
, 000 \\
600 \\
600\end{array}$ & $\begin{array}{l}23,140 \\
12,590 \\
19,960 \\
16,390 \\
11,100\end{array}$ \\
\hline $\begin{array}{l}1941 \\
1942\end{array}$ & $\begin{array}{l}480 \\
400\end{array}$ & $\begin{array}{l}270 \\
310\end{array}$ & $\begin{array}{l}250 \\
240\end{array}$ & $\begin{array}{l}210 \\
220\end{array}$ & $\begin{array}{l}190 \\
240\end{array}$ & $\begin{array}{l}250 \\
220\end{array}$ & $\begin{array}{l}340 \\
580\end{array}$ & $\begin{array}{l}3,400 \\
2,300\end{array}$ & $\begin{array}{l}4,700 \\
6,000\end{array}$ & $\begin{array}{l}2,200 \\
3,700\end{array}$ & 1,000 & 600 & $\begin{array}{l}13,890 \\
15,860\end{array}$ \\
\hline $\begin{array}{l}1957 \\
1958 \\
1959 \\
1960\end{array}$ & $\begin{array}{l}270 \\
500 \\
360 \\
560\end{array}$ & $\begin{array}{l}270 \\
370 \\
240 \\
360\end{array}$ & $\begin{array}{l}290 \\
360 \\
250 \\
320\end{array}$ & $\begin{array}{l}270 \\
300 \\
280 \\
240\end{array}$ & $\begin{array}{l}180 \\
210 \\
230 \\
190\end{array}$ & $\begin{array}{l}160 \\
220 \\
200 \\
180\end{array}$ & $\begin{array}{l}270 \\
290 \\
520 \\
640\end{array}$ & $\begin{array}{l}1,300 \\
4,700 \\
2,000 \\
2,100\end{array}$ & $\begin{array}{l}\dot{8}, \\
6, \\
6, \\
6,\end{array}$ & $\begin{array}{l}9,100 \\
1,700 \\
2,600 \\
2,500\end{array}$ & $\begin{array}{r}8,800 \\
700 \\
100 \\
880\end{array}$ & $\begin{array}{l}920 \\
500 \\
620 \\
400\end{array}$ & $\begin{array}{l}24,630 \\
15,850 \\
15,000 \\
15,170\end{array}$ \\
\hline $\begin{array}{l}1961 \\
1962 \\
1963 \\
1964 \\
1965\end{array}$ & $\begin{array}{r}260 \\
1,300 \\
350 \\
350 \\
280\end{array}$ & $\begin{array}{l}240 \\
700 \\
260 \\
250 \\
230\end{array}$ & $\begin{array}{l}230 \\
500 \\
220 \\
220 \\
230\end{array}$ & $\begin{array}{l}180 \\
370 \\
180 \\
190 \\
250\end{array}$ & $\begin{array}{l}140 \\
280 \\
220 \\
150 \\
200\end{array}$ & $\begin{array}{l}160 \\
250 \\
200 \\
140 \\
160\end{array}$ & $\begin{array}{r}220 \\
1,200 \\
540 \\
200 \\
350\end{array}$ & $\begin{array}{l}2,100 \\
4,200 \\
2,200 \\
3,000 \\
2,600\end{array}$ & $\begin{array}{l}4,300 \\
7,200 \\
2,500 \\
5,000 \\
9,200\end{array}$ & $\begin{array}{l}1,800 \\
4,800 \\
1,400 \\
2,800 \\
6,800\end{array}$ & $\begin{array}{l}1,300 \\
1,500 \\
1,500 \\
1,400 \\
3,200\end{array}$ & $\begin{array}{r}, 400 \\
640 \\
840 \\
470 \\
970\end{array}$ & $\begin{array}{l}12,330 \\
22,940 \\
10,410 \\
14,170 \\
24,470\end{array}$ \\
\hline
\end{tabular}



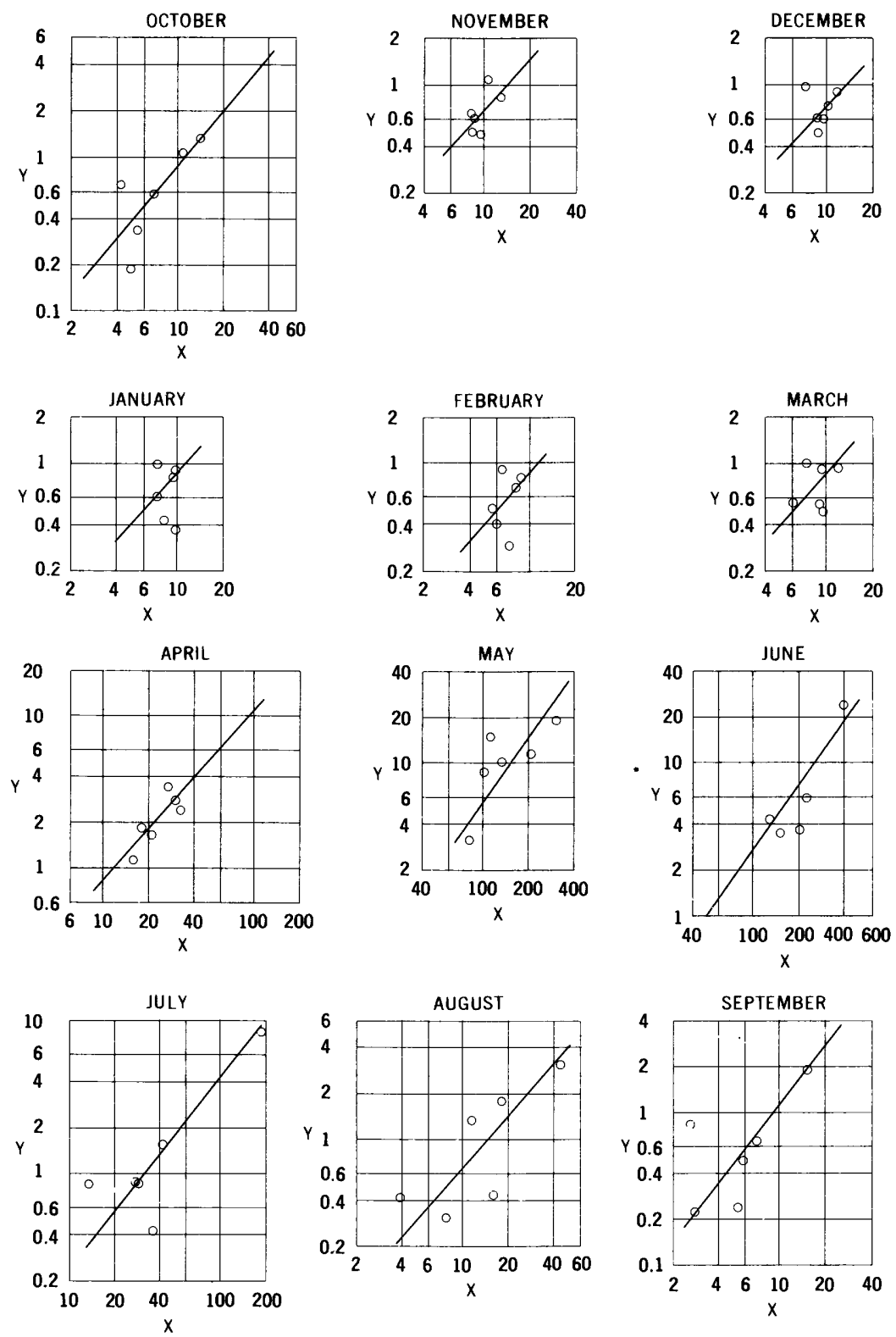

Relationships of monthly mean discharge of Sunnyside Creek near Burns, Colo. (Y), to monthly mean discharge of Piney River near State Bridge, Colo. (X). Discharge in hundreds of acre-feet. 
610. Sunnyside Creek near Burns, Colo.

Location. --Lat $39^{\circ} 58^{\prime}$, long $106^{\circ} 571$, in sec. 17 , T.1 S., R, 85 W., on left bank 6 ft downstream from bridge, 7 miles upstream from mouth, and $7 \frac{1}{2}$ miles northwest of Burns. Al titude of gage is $8,220 \mathrm{ft}^{\prime}$ (by barometer).

Drainage area.--10 sq mi, approximately.

Records available. --0ctober 1952 to September 1958.

Estimates of streamflow.--June 1944 to September 1952 , october 1958 to September 1965 ,

based on relationships of monthly mean discharge with Piney River near State Bridge,

colo. The regression equation used is:

$$
\log Y=b \log X-c
$$

(where $Y$ is discharge of Sunnyside Creek near Burns, Colo, and $X$ is discharge of Piney River near State Bridge, Colo., both in acre-feet per month).

\begin{tabular}{l|l|l|l|l|l|l|l|l|l|l|l|l}
\hline \multicolumn{10}{c}{ Monthly values of constants in above equation } \\
\hline & oct. & Nov. & Dec. & Jan. & Feb. & Mar. & Apr. & May & June & July & Aug. & Sept. \\
\hline $\mathrm{b}$ & 1.18 & 1.11 & 1.06 & 1.09 & 1.10 & 1.10 & 1.12 & 1.41 & 1.41 & 1.27 & 1.14 & 1.29 \\
$\mathrm{c}$ & 1.58 & 1.48 & 1.33 & 1.34 & 1.38 & 1.38 & 1.44 & 2.90 & 3.20 & 2.44 & 1.60 & 1.81 \\
\hline
\end{tabular}

Average discharge. - -21 years (1944-65), 3,128 acre-feet per year (4.32 cfs).

Extremes.--1952-58: Maximum discharge, $110 \mathrm{cfs}$ May 28, 1957; minimum daily, 0.1 cfs Aug. 31, 1955; Aug. 2-4, 1958.

Remarks. --Two small diversions above station for irrigation of about 50 acres above and below station. Estimates of annual flow are within about 20 percent of regression line.

\begin{tabular}{c|r|r|r|r|r|r|r|r|r|r|r|r|r}
\hline $\begin{array}{c}\text { Water } \\
\text { year }\end{array}$ & Oct. & Nov. & Dec. & Jan. & Feb. & Mar. & Apr. & May & June & July & Aug. & Sept. & Annual \\
\hline 1953 & 58 & 48 & 61 & 92 & 67 & 92 & 182 & 881 & 582 & 155 & 180 & 24 & 2,420 \\
1954 & 34 & 60 & 98 & 98 & 89 & 101 & 343 & 316 & 106 & 86 & 42 & 67 & 1,440 \\
1955 & 107 & 65 & 61 & 61 & 50 & 55 & 278 & 1,050 & 431 & 42 & 44 & 22 & 2,270 \\
1956 & 19 & 50 & 49 & 43 & 40 & 49 & 242 & 1,140 & 354 & 87 & 135 & 85 & 2,290 \\
1957 & 67 & 108 & 74 & 37 & 28 & 55 & 115 & 1,530 & 2,440 & 860 & 307 & 193 & 5,810 \\
1958 & 132 & 83 & 92 & 80 & 78 & 93 & 165 & 1,930 & 362 & 85 & 31 & 49 & 3,180 \\
\hline
\end{tabular}

Estimated monthly and annual streamflow, in acre-feet

\begin{tabular}{l|r|r|r|r|r|r|r|r|r|r|r|r|r}
\hline $\begin{array}{l}\text { Water } \\
\text { year }\end{array}$ & Oct. & Nov. & Dec. & Jan. & Feb. & Mar. & Apr. & May & June & July & Aug. & Sept. & Annual \\
\hline 1944 & - & - & - & - & - & - & - & - & 830 & 250 & 70 & 20 & - \\
1945 & 50 & 60 & 50 & 50 & 50 & 60 & 150 & 1,200 & 730 & 290 & 220 & 50 & 2,960 \\
1946 & 110 & 90 & 70 & 70 & 70 & 120 & 770 & 830 & 560 & 110 & 90 & 80 & 2,970 \\
1947 & 130 & 90 & 80 & 70 & 60 & 80 & 310 & 1,800 & 880 & 450 & 140 & 140 & 4,230 \\
1948 & 140 & 120 & 100 & 100 & 80 & 100 & 340 & 2,200 & 620 & 160 & 100 & 60 & 4,120 \\
1949 & 40 & 70 & 80 & 90 & 70 & 90 & 390 & 960 & 900 & 330 & 120 & 60 & 3,200 \\
1950 & 110 & 90 & 80 & 80 & 70 & 80 & 300 & 700 & 680 & 120 & 50 & 60 & 2,420 \\
1951 & 60 & 60 & 70 & 70 & 70 & 50 & 170 & 1,000 & 760 & 460 & 170 & 70 & 3,010 \\
1952 & 100 & 50 & 60 & 70 & 50 & 60 & 590 & 1,500 & 1,400 & 240 & 180 & 120 & 4,420 \\
1959 & 70 & 50 & 40 & 40 & 60 & 80 & 140 & 740 & 860 & 150 & 110 & 130 & 2,470 \\
1960 & 210 & 110 & 90 & 80 & 70 & 80 & 520 & 940 & 730 & 140 & 70 & 60 & 3,100 \\
1961 & 50 & 70 & 60 & 60 & 50 & 70 & 80 & 660 & 360 & 70 & 80 & 360 & 1,970 \\
1962 & 430 & 150 & 110 & 80 & 90 & 100 & 1,100 & 1,800 & 1,100 & 430 & 150 & 90 & 5,630 \\
1963 & 90 & 50 & 40 & 40 & 50 & 60 & 230 & 510 & 150 & 40 & 100 & 70 & 1,430 \\
1964 & 40 & 40 & 50 & 60 & 50 & 50 & 200 & 860 & 420 & 140 & 80 & 60 & 2,050 \\
1965 & 40 & 50 & 60 & 70 & 60 & 70 & 200 & 1,000 & 1,400 & 660 & 370 & 320 & 4,300 \\
\hline
\end{tabular}



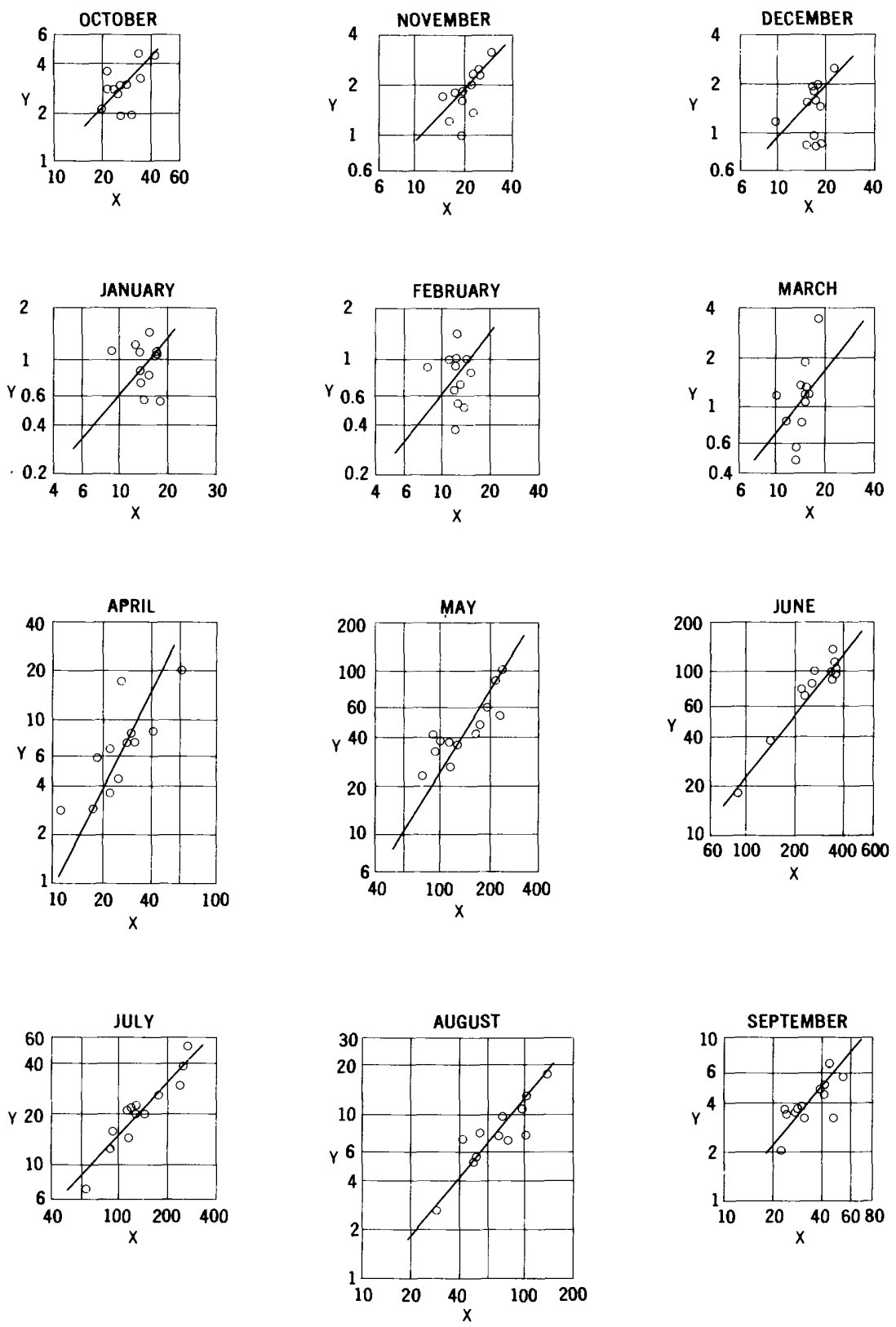

Relationships of monthly mean discharge of Turkey Creek at Red Cliff, Colo. $(Y)$, to the sum of monthly mean discharges of Blue River at Dillon, Colo., Boreas Pass ditch, and either East Hoosier ditch and West Hoosier ditch or Hoosier Pass tunnel (X). Discharge in hundreds of acre-feet. 
635. Turkey Creek at Red Cliff, Colo.

Location. --Lat $39^{\circ} 30150^{\prime \prime}$, long $106^{\circ} 22^{105^{\prime \prime}}$, in sec.19, T.6 S., R.80 W., on left bank at Red Cl1ff, $1,000 \mathrm{ft}$ upstream from mouth. Datum of gage is $8,641.79 \mathrm{ft}$ ' above mean sea level, datum of 1929.

Drainage area. $--28.6 \mathrm{sq} \mathrm{mi}$.

Records available.--July 1913 to September 1921, May 1944 to September 1956.

Estimates of streamflow. - October 1930 to May 1944, based on relationships of monthly mear discharge with BIue River at Dillon, Colo; adjusted for diversion through Boreas Pass ditch (from October 1932), East Hoosier ditch (June 1935 to September 1940), and West Hoosier ditch (June 1935 to September 1939), diversions prior to above dates were ungaged. October 1956 to Apri1 1961 , based on relationships of monthly mean discharge with Blue River at Dilion, Colo., adjusted for diversion through Boreas Pass ditch, and Hoosier Pass tunnel. The regression equation used is:

$$
\log Y=b \log X-c
$$

(where $Y$ is discharge of Turkey Creek at Red Cliff, Colo., and $X$ is the sum of discharges of Blue River at Dillon, Colo., Boreas Pass ditch, and either East Hoosier ditch and West Hoosier ditch, or Hoosier Pass tunnel, all in acre-feet per month).

Monthly values of constants in above equation

\begin{tabular}{l|l|l|l|l|l|l|l|l|l|l|l|l}
\hline & Oct. & Nov. & Dec. & Jan. & Feb. & Mar. & Apr. & May & June & July & Aug. & Sept. \\
\hline $\mathrm{b}$ & 1.02 & 1.06 & 1.07 & 1.16 & 1.26 & 1.25 & 2.03 & 1.63 & 1.24 & 1.05 & 1.21 & 1.20 \\
$\mathrm{c}$ & 1.04 & 1.23 & 1.23 & 1.70 & 2.01 & 1.90 & 4.13 & 3.13 & 1.60 & 1.03 & 1.75 & 1.61 \\
\hline
\end{tabular}

Average discharge. --30 years $(1930-60)$, 17,340 acre-feet per year $(24.0 \mathrm{cfs})$.

Extremes.--1913-21, 1944-56: Maximum discharge observed, 670 cfs June 13, 1918; min1mum daily, 0.4 cfs Feb. 21, 1955, Feb. 3, 1956

Remarks.--Diversion above station for municipal supply of Red Cliff. Small diversions for Irrigation of hay meadows above station. Estimates of annual flow are within about 25 percent of regression line.

\begin{tabular}{c|c|c|r|r|r|r|r|r|r|r|r|r|r}
\hline $\begin{array}{c}\text { Water } \\
\text { year }\end{array}$ & Oct. & Nov. & Dec. & Jan. & Feb. & Mar. & Apr. & May & June & July & Aug. & Sept. & Annual \\
\hline 1944 & - & - & - & - & - & - & - & - & 8,440 & 2,220 & 731 & 358 & - \\
1945 & 351 & 170 & 119 & 113 & 90 & 119 & 280 & 4,240 & 7,810 & 2,560 & 1,800 & 694 & 18,350 \\
1946 & 459 & 249 & 193 & 144 & 145 & 342 & 2,050 & 3,560 & 7,070 & 1,440 & 524 & 318 & 16,490 \\
1947 & 276 & 238 & 197 & 123 & 100 & 135 & 581 & 6,120 & 11,240 & 5,160 & 1,120 & 582 & 25,870 \\
1948 & 446 & 318 & 245 & 107 & 100 & 188 & 1,710 & 8,630 & 8,880 & 2,020 & 796 & 369 & 23,810 \\
1949 & 283 & 184 & 158 & 106 & 84 & 119 & 728 & 3,860 & 9,720 & 3,020 & 986 & 507 & 19,760 \\
1950 & 324 & 233 & 147 & 110 & 102 & 131 & 824 & 3,750 & 10,130 & 2,110 & 705 & 381 & 18,950 \\
1951 & 275 & 186 & 154 & 85 & 71 & 108 & 360 & 4,210 & 9,780 & 3,930 & 1,300 & 438 & 20,900 \\
1952 & 294 & 203 & 182 & 123 & 92 & 83 & 861 & 4,710 & 13,580 & 2,280 & 718 & 468 & 23,590 \\
1953 & 261 & 162 & 97 & 80 & 65 & 80 & 285 & 2,600 & 10,460 & 2,030 & 768 & 347 & 17,240 \\
1954 & 208 & 121 & 85 & 72 & 54 & 47 & 431 & 2,320 & 1,820 & 711 & 265 & 202 & 6,340 \\
1955 & 193 & 100 & 83 & 57 & 37 & 56 & 737 & 3,220 & 3,750 & 1,590 & 762 & 322 & 10,910 \\
1956 & 192 & 138 & 86 & 56 & 51 & 120 & 669 & 5,360 & 7,560 & 1,250 & 572 & 341 & 16,400 \\
\hline
\end{tabular}

Estimated monthly and annual streamflow, in acre-feet

\begin{tabular}{c|r|r|r|r|r|r|r|r|r|r|r|r|r}
\hline $\begin{array}{l}\text { Water } \\
\text { year }\end{array}$ & oct. & Nov. & Dec. & Jan. & Feb. & Mar. & Apr. & May & June & July & Aug. & Sept. & Annual \\
\hline 1931 & 430 & 220 & 180 & 80 & 60 & 90 & 780 & 2,900 & 6,000 & 1,500 & 580 & 350 & 13,270 \\
1932 & 280 & 150 & 130 & 70 & 80 & 110 & 620 & 4,800 & 6,200 & 2,300 & 900 & 470 & 16,110 \\
1933 & 290 & 160 & 150 & 80 & 70 & 110 & 280 & 1,900 & 9,700 & 1,900 & 540 & 390 & 14,470 \\
1934 & 270 & 160 & 150 & 80 & 80 & 100 & 1,100 & 5,600 & 2,700 & 780 & 500 & 350 & 11,870 \\
1935 & 210 & 160 & 120 & 70 & 60 & 80 & 280 & 940 & 7,000 & 2,000 & 900 & 580 & 12,400 \\
1936 & 310 & 170 & 120 & 90 & 90 & 120 & 2,800 & 14,000 & 9,600 & 2,200 & 1,800 & 680 & 31,970 \\
1937 & 370 & 180 & 100 & 30 & 50 & 110 & 860 & 2,500 & 4,200 & 1,400 & 500 & 430 & 10,730 \\
1938 & 260 & 170 & 130 & 60 & 60 & 90 & 540 & 4,300 & 12,000 & 2,300 & 880 & 920 & 21,710 \\
1939 & 410 & 220 & 170 & 90 & 70 & 140 & 1,300 & 10,000 & 5,800 & 1,300 & 440 & 330 & 20,270 \\
1940 & 230 & 140 & 100 & 50 & 50 & 80 & 360 & 2,500 & 3,900 & 1,100 & 340 & 430 & 9,280 \\
1941 & 340 & 160 & 130 & 70 & 60 & 80 & 180 & 4,400 & 6,600 & 1,800 & 580 & 400 & 14,900 \\
1942 & 350 & 210 & 170 & 100 & 80 & 100 & 800 & 3,700 & 8,800 & 1,800 & 660 & 350 & 17,120 \\
1943 & 230 & 180 & 140 & 50 & 10 & 60 & 2,400 & 4,000 & 7,600 & 2,000 & 840 & 400 & 17,940 \\
1944 & 250 & 160 & 110 & 40 & 30 & 60 & 160 & 2,500 & - & - & - & - & 15,060 \\
1957 & 180 & 180 & 180 & 110 & 110 & 100 & 380 & 2,400 & 12,000 & 5,000 & 1,900 & 640 & 23,180 \\
1958 & 360 & 240 & 220 & 130 & 100 & 130 & 330 & 9,800 & 18,200 & 1,200 & 460 & 330 & 21,500 \\
1959 & 230 & 160 & 160 & 110 & 100 & 110 & 290 & 2,600 & 8,600 & 1,800 & 680 & 320 & 15,160 \\
1960 & 340 & 220 & 190 & 100 & 90 & 120 & 1,300 & 2,200 & 7,600 & 1,800 & 540 & 300 & 14,790 \\
1961 & 240 & 170 & 140 & 70 & 50 & 70 & 160 & - & - & - & - & - & - \\
\hline
\end{tabular}



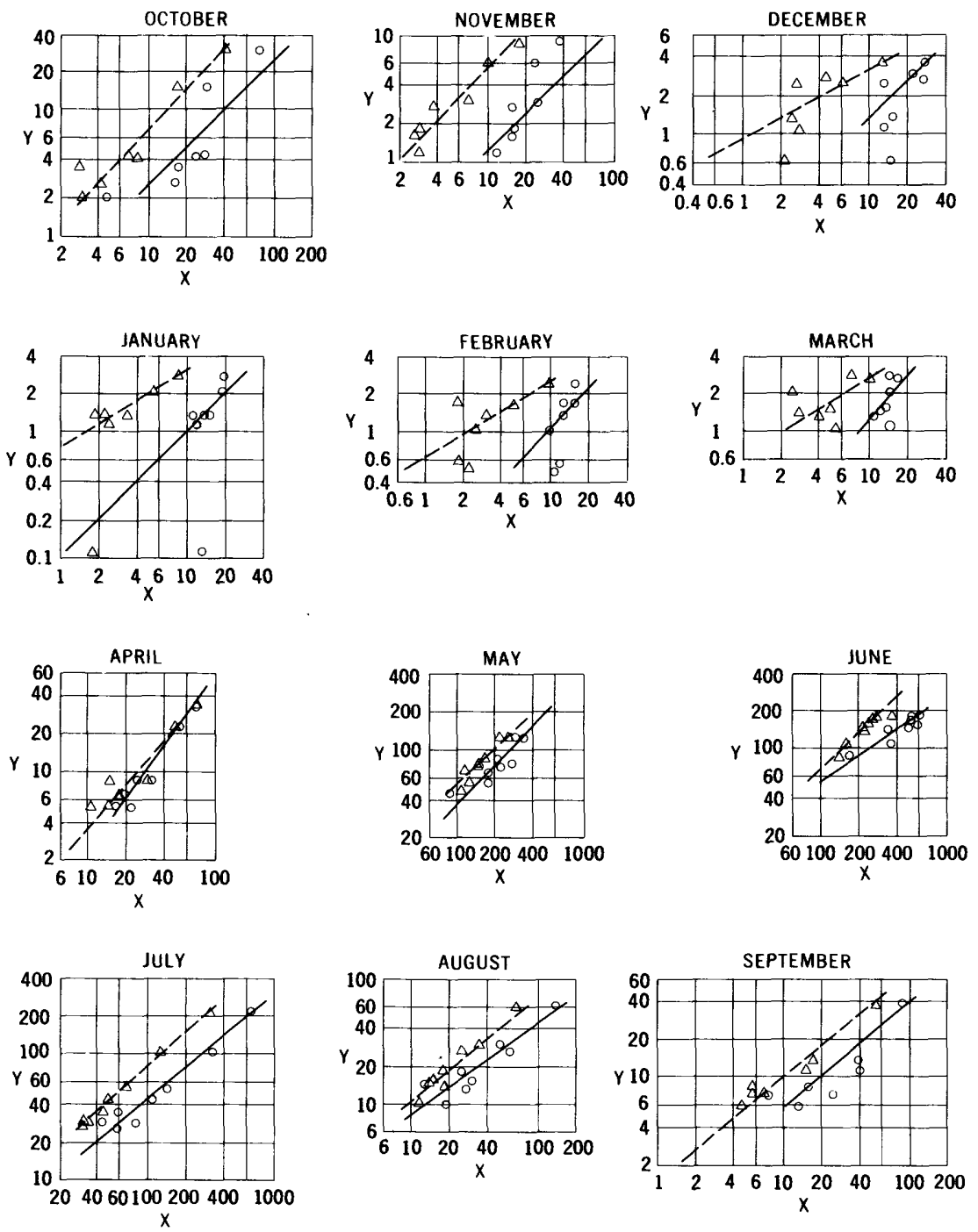

\section{EXPLANATION}

- Roaring Fork River at Aspen, Colo., adjusted for diversion

- $-\triangle$ Homestake Creek near Red Cliff, Colo.

Relationships of monthly mean discharge of Cross Creek near Minturn, Colo. (Y), to sum of monthly mean discharges of Roaring Fork River at Aspen, Colo., and Twin Lakes tunnel or monthly mean discharge of Homestake Creek near Red Cliff, Colo. (X). Discharge in hundreds of acre-feet. 
651. Cross Creek near Minturn, Colo.

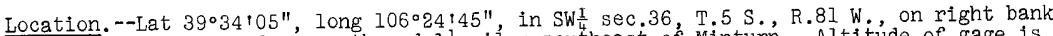
$0.4 \mathrm{mile}$ upstream from mouth and $1 \frac{1}{2}$ miles southeast of Minturn. Altitude of gage is $7,990 \mathrm{ft}$ (from topographic map).

Drainage area. $--33.5 \mathrm{sq} \mathrm{mi}$.

Records available. --May 1956 to September 1963.

Estimates of streanflow.--0ctober 1931 to June 1944, based on relationships of monthly mean discharge with Roaring Fork River at Aspen, Colo., adjusted for diversion through Twin Lakes tunnel; June 1944 to April 1956 and October 1963 to September 1965, based on relationships of monthly mean discharge with Homestake Creek near Red Cliff, Colo. The regression equation used is:

$\log Y=b \log X-c$

(where $Y$ is discharge of Cross Creek near Minturn, Colo, and $X$ is the sum of discharges of Roaring Fork River at Asper, Colo, and Twin Lakes tunnel, or discharge of Homestake creek near Red cliff, Colo., all in acre-feet per month).

\begin{tabular}{|c|c|c|c|c|c|c|c|c|c|c|c|c|c|}
\hline & & oct. & Nov. & Dec. & Jan. & Feb. & Mar. & Apr. & May & June & July & Aug. & Sept. \\
\hline $\begin{array}{l}\text { Oct } 1931 \\
\text { to } \\
\text { May } 1944\end{array}$ & c & $\begin{array}{r}1.00 \\
.60\end{array}$ & $\begin{array}{r}1.00 \\
.93\end{array}$ & $\begin{array}{r}1.00 \\
.89\end{array}$ & $\begin{array}{l}1.00 \\
1.00\end{array}$ & $\begin{array}{l}1.05 \\
1.11\end{array}$ & $\begin{array}{l}1.13 \\
1.31\end{array}$ & $\begin{array}{l}1.39 \\
1.79\end{array}$ & $\begin{array}{r}1.02 \\
.51\end{array}$ & $\begin{array}{r}0.67 \\
-1.06\end{array}$ & $\begin{array}{r}0.83 \\
-.32\end{array}$ & $\begin{array}{c}0.72 \\
-.76\end{array}$ & $\begin{array}{r}0.83 \\
-.26\end{array}$ \\
\hline $\begin{array}{l}\text { June } 1949 \text { to } \\
\text { Apr } 111956 \\
\text { Oct } 1963 \text { to } \\
\text { Sept } 1965\end{array}$ & c & $\begin{array}{r}1.06 \\
.35\end{array}$ & $\begin{array}{r}1.06 \\
-.45\end{array}$ & $\begin{array}{r}0.54 \\
-.88\end{array}$ & $\begin{array}{l}0.61 \\
-.64\end{array}$ & $\begin{array}{l}0.59 \\
-.62\end{array}$ & $\begin{array}{r}0.60 \\
-.60\end{array}$ & $\begin{array}{r}1.17 \\
.96\end{array}$ & $\begin{array}{l}0.93 \\
-.01\end{array}$ & $\begin{array}{r}0.93 \\
-.12\end{array}$ & $\begin{array}{r}0.87 \\
-.43\end{array}$ & $\begin{array}{r}0.85 \\
-.46\end{array}$ & $\begin{array}{r}0.81 \\
.54\end{array}$ \\
\hline
\end{tabular}

Average discharge. $-\mathbf{- 3 4}$ years $(1931-65), 36,570$ acre-feet per year $(50.5 \mathrm{cfs})$.

Extremes. --1956-63: Maximum discharge, 754 cfs June 30, 1957; minimum daily, 0.1 cfs Dec. 27-31, 1962; Jan. 6-8, 11-15, 1963.

Remarks.--Diversion above station for municipal supply of Minturn. Estimates of annual flow are within about 10 percent of regression line, prior to 1944 , and within about

5 percent thereafter.

\begin{tabular}{l|r|r|r|r|r|r|r|r|r|r|r|r|r|}
\hline $\begin{array}{l}\text { Water } \\
\text { year }\end{array}$ & Oct. & Nov. & Dec. & Jan. & Feb. & Mar. & Apr. & May & June & July & Aug. & Sept. & Annual \\
\hline 1956 & - & - & - & - & - & - & - & 12,740 & 13,800 & 3,520 & 1,470 & 214 & - \\
1957 & 209 & 118 & 111 & 115 & 105 & 131 & 655 & 4,720 & 18,400 & 21,830 & 5,880 & 1,110 & 53,370 \\
1958 & 442 & 295 & 253 & 209 & 167 & 153 & 529 & 12,700 & 14,500 & 2,930 & 1,010 & 590 & 33,780 \\
1959 & 356 & 160 & 131 & 138 & 170 & 202 & 854 & 5,470 & 18,520 & 4,410 & 1,540 & 841 & 32,790 \\
1960 & 1,570 & 612 & 287 & 138 & 138 & 274 & 2,280 & 6,690 & 17,170 & 5,550 & 1,360 & 740 & 36,810 \\
1961 & 262 & 266 & 250 & 136 & 58 & 141 & 533 & 7,580 & 10,790 & 2,710 & 1,820 & 3,870 & 28,420 \\
1962 & 3,050 & 926 & 370 & 275 & 240 & 261 & 3,430 & 7,750 & 15,530 & 10,690 & 2,670 & 737 & 45,930 \\
1963 & 425 & 182 & 61 & 11 & 50 & 107 & 855 & 8,420 & 8,750 & 2,980 & 2,940 & 1,350 & 26,130 \\
\hline
\end{tabular}

\begin{tabular}{|c|c|c|c|c|c|c|c|c|c|c|c|c|c|}
\hline \multirow{2}{*}{$\begin{array}{l}\text { Water } \\
\text { year }\end{array}$} & \multirow[b]{2}{*}{ oct. } & \multirow[b]{2}{*}{ Nov. } & & \multirow[b]{2}{*}{ Mar. } & \multirow[b]{2}{*}{ Apr. } & \multirow[b]{2}{*}{ May } & \multirow[b]{2}{*}{ June } & \multirow[b]{2}{*}{ July } & \multirow[b]{2}{*}{ Aug. } & \multirow[b]{2}{*}{ Sept. } & \multirow[b]{2}{*}{ Annual } \\
\hline & & & Dec. & Jan. & Feb. & & & & & & & & \\
\hline 1932 & $\neq 540$ & $\$ 170$ & $\neq 160$ & $\$ 120$ & $\$ 120$ & $\neq 200$ & $\neq 1,800$ & 9,000 & 15,300 & 8,300 & 3,000 & 1,100 & 39,820 \\
\hline 1933 & 670 & 290 & 260 & $\$ 180$ & $\neq 180$ & $\$ 240$ & $\$ 940$ & 7,000 & 17,000 & 4,500 & 2,100 & & 34,660 \\
\hline 1934 & $\neq 680$ & $\neq 210$ & $\$ 160$ & 100 & 130 & 210 & 3,300 & 10,900 &, 600 & & 1,000 & & 690 \\
\hline 1935 & 550 & 220 & 220 & 150 & 160 & 210 & 840 & 3,100 & 16,500 & 6,800 & 2,400 & 1,800 & 32,950 \\
\hline 1936 & 720 & 210 & 190 & 160 & 150 & 180 & 5,000 & 18,000 & 12,700 & 4,300 & 3,200 & 1,200 & 46,010 \\
\hline 1937 & 710 & & 220 & 150 & 140 & 180 & 820 & 11,600 & & & & 20 & \\
\hline 1938 & 510 & 210 & 190 & 150 & 130 & 160 & 1,500 & 7,200 & 18,800 & 8,600 & 2,700 & 2,300 & 42,450 \\
\hline 1939 & 880 & 320 & 320 & 190 & 200 & 240 & 2,100 & 14,300 & 11,700 & & 1,200 & 600 & 35,350 \\
\hline 1940 & 230 & 120 & 130 & 110 & 100 & 140 & 1,200 & 8,000 & 10,000 & 2,100 & 760 & 660 & 23,550 \\
\hline 1941 & 810 & 200 & 190 & 140 & 120 & 150 & 450 & 9,600 & 13,000 & $5, \infty 00$ & 1,700 & 940 & 32,300 \\
\hline 1942 & 830 & 250 & 220 & 140 & 130 & 140 & 1,000 & & 100 & & & 900 & 610 \\
\hline 1943 & 450 & 200 & 250 & 170 & 40 & 180 & 3,200 & 8,400 & 15,200 & 6,300 & 4,600 & 1,900 & 40,990 \\
\hline 1944 & 670 & 290 & 270 & 150 & 160 & 170 & 490 & 7,200 & 14,300 & 100 & 1,100 & 310 & 31,910 \\
\hline 1945 & 200 & 160 & 170 & 160 & 120 & 140 & 250 & 7,000 & 13,100 & 10,000 & 5,400 & 1,200 & 37,900 \\
\hline 1946 & 860 & 560 & 260 & 190 & 130 & 200 & 3,600 & 6,000 & 15,200 & 5,600 & 1,500 & 640 & 34,740 \\
\hline 1947 & 600 & 450 & 250 & 1 & 90 & 140 & 720 & & 000 & & & 2,000 & \\
\hline 1948 & 1,500 & 750 & 250 & 170 & 140 & 170 & 1,600 & 10,600 & 14,900 & 6,000 & 1,500 & 460 & 040 \\
\hline 1949 & 400 & 230 & 170 & 140 & 130 & 170 & 1,800 & 00 & 17,900 & 11,700 & 2,100 & 1,300 & 040 \\
\hline 19 & 1,200 & 620 & 310 & 250 & 180 & 190 & 1,600 & 7,000 & 17,000 & 5,400 & 970 & 740 & 35,460 \\
\hline 1951 & 320 & 200 & 220 & 150 & 120 & 160 & 900 & 8,600 & 18,200 & 13,300 & 3,400 & 740 & 310 \\
\hline 1952 & 600 & 270 & 200 & 180 & 130 & 150 & 1,400 & & 22,100 & & & 1,500 & \\
\hline 1953 & 390 & 240 & 200 & 190 & 150 & 150 & 740 & 5,600 & 18,500 & 7,600 & 2,700 & 580 & 37,240 \\
\hline 1954 & 260 & 240 & 190 & 160 & 120 & 130 & 1,200 & 6,600 & 5,600 & 2 , & 940 & 820 & 860 \\
\hline 1955 & 960 & 380 & 240 & 170 & 110 & 140 & 1,400 & 6,400 & 11,200 & 5,200 & 2,700 & 700 & 29,600 \\
\hline 1956 & 270 & 290 & 240 & 200 & 160 & 200 & 1,700 & - & - & - & - & - & 34,800 \\
\hline $\begin{array}{l}1964 \\
1965\end{array}$ & $\begin{array}{l}420 \\
350\end{array}$ & $\begin{array}{l}260 \\
240\end{array}$ & $\begin{array}{l}180 \\
220\end{array}$ & $\begin{array}{l}160 \\
200\end{array}$ & $\begin{array}{l}130 \\
140\end{array}$ & $\begin{array}{l}140 \\
150\end{array}$ & $\begin{array}{r}490 \\
1,100\end{array}$ & $\begin{array}{l}8,000 \\
7,400\end{array}$ & $\begin{array}{l}11,300 \\
19,100\end{array}$ & $\begin{array}{r}6,400 \\
16,800\end{array}$ & $\begin{array}{l}2,800 \\
5,600\end{array}$ & $\begin{array}{r}900 \\
2,400\end{array}$ & $\begin{array}{l}31,240 \\
53,700\end{array}$ \\
\hline
\end{tabular}

* Based on estimates for 1950 Compllation. 

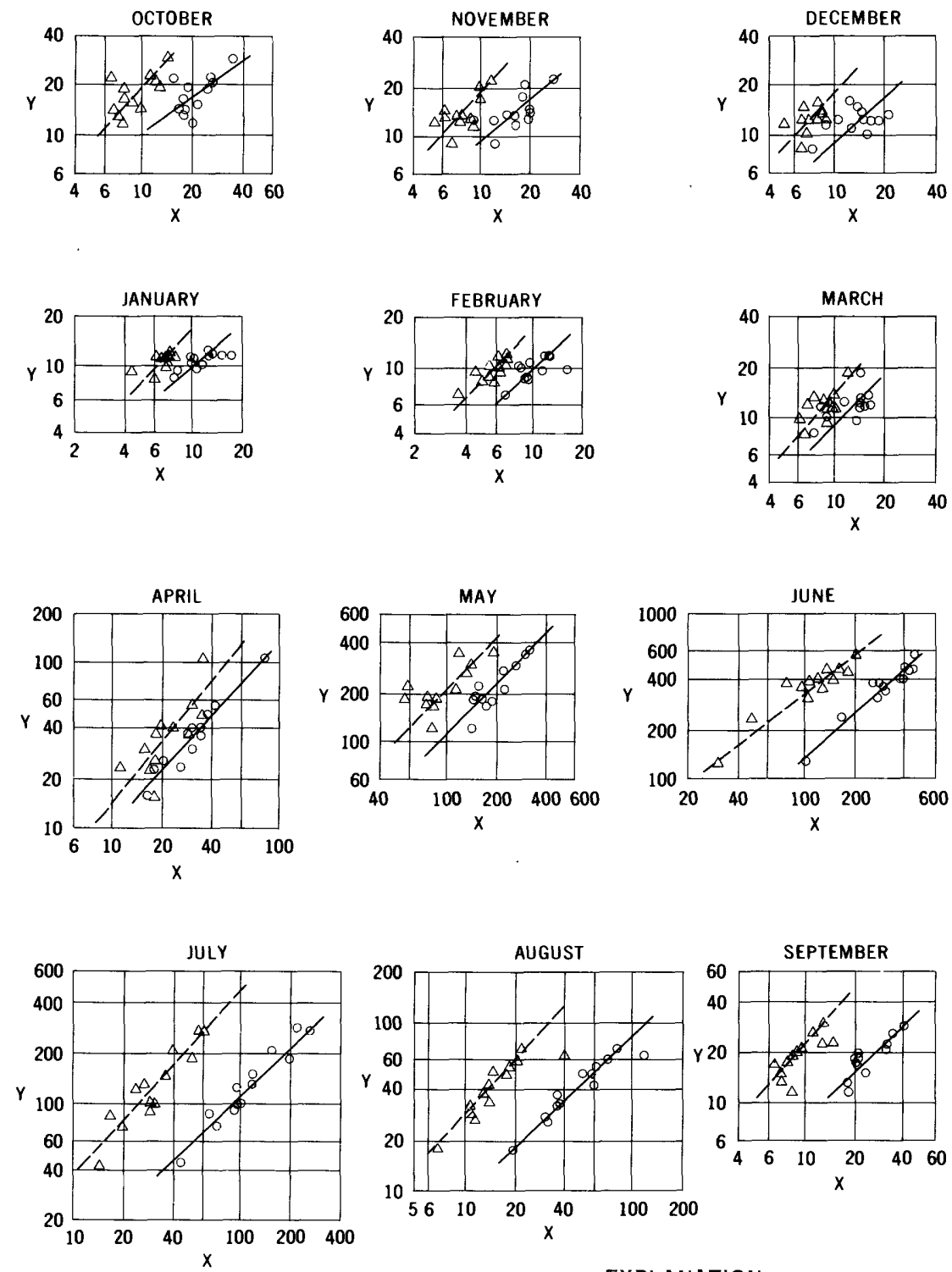

\section{EXPLANATION}

o Tenmile Creek at Dillon, Colo., adjusted for diversion

$--\triangle$ Eagle River at Red Cliff, Colo., adjusted for diversion

Relationships of monthly mean discharge of Gore Creek near Minturn, Colo. $(\mathrm{Y})$, to sum of monthly mean discharges of Tenmile Creek at Dillon, Colo., and Fremont Pass ditch, or sum of monthly mean discharges of Eagle River at Red Cliff, Colo., and Ewing, Wurtz, and Columbine ditches (X). Discharge in hundreds of acre-feet. 
665. Gore Creek near Minturn, Colo.

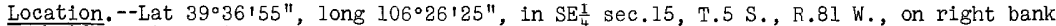
half a mile upstream from mouth and 2 miles north of Minturn.' Datum of gage is $7,756.24$ ft above mean sea level, unadjusted.

Drainage area. $--100 \mathrm{sq} \mathrm{mi}$.

Records available. --July 1911 to September 1914, May 1944 to September 1956.

Estimates of streamflow.--October 1930 to May 1944, based on relationships of monthly mean discharge with Tenmile Creek at Dillon, Colo., adjusted for diversion through Fremont Pass ditch; October 1956 to September 1965, based on relationships of monthly mean discharge with Eagle River at Red Cliff, Colo, , adjusted for diversion through Ewing, Wurtz, and Columbine ditches. The regression equation used is:

$\log Y=b \log X-c$

(where $\mathrm{Y}$ is discharge of Gore Creek near Minturn, Colo., and $\mathrm{X}$ is the sum of discharges of Tenmile Creek at Dilion, Colo, and Fremont Pass ditch, or the sum of discharges of Eagle River at Red Cliff, Colo, and Ewing, Wurtz, and Columbine ditches, all in acre-feet per month).

\begin{tabular}{|c|c|c|c|c|c|c|c|c|c|c|c|c|c|}
\hline & & oct. & Nov. & Dec. & Jan. & Feb. & Mar. & Apr. & May & June & July & Aug. & Sept. \\
\hline $\begin{array}{l}\text { Oct. } 1930 \\
\text { to } \\
\text { May } 1944\end{array}$ & b & $\begin{array}{l}0.72 \\
-.82\end{array}$ & $\begin{array}{l}0.84 \\
-.45\end{array}$ & & & $\begin{array}{l}0.95 \\
-.12\end{array}$ & $\begin{array}{c}0.97 \\
-.06\end{array}$ & $\begin{array}{r}1.09 \\
.24\end{array}$ & $\begin{array}{r}1.03 \\
.07\end{array}$ & $\begin{array}{r}0.90 \\
-.53\end{array} \mid$ & $\begin{array}{l}0.95 \\
-.24\end{array}$ & $\begin{array}{l}0.94 \\
-.07\end{array}$ & $\begin{array}{r}0.94 \\
-.16\end{array}$ \\
\hline $\begin{array}{l}\text { Oct. } 1956 \\
\text { to } \\
\text { Sept. } 1965\end{array}$ & b & $\begin{array}{l}1.10 \\
.01\end{array}$ & $\begin{array}{l}1.06 \\
-.07\end{array}$ & $\begin{array}{l}1.06 \\
-.06\end{array}$ & $\begin{array}{r}1.10 \\
.07\end{array}$ & & $\begin{array}{r}1.10 \\
.16\end{array}$ & $\begin{array}{r}1.22 \\
.50\end{array}$ & $\begin{array}{l}1.06 \\
-.08\end{array}$ & $\begin{array}{r}.76 \\
-1.47 \\
\end{array}$ & $\begin{array}{l}1.08 \\
-.34\end{array}$ & $\begin{array}{l}1.10 \\
-.14\end{array}$ & $\begin{array}{l}1.10 \\
-.04\end{array}$ \\
\hline
\end{tabular}

Average discharge. --35 years $(1930-65), 89,520$ acre-feet per year (124 cfs).

Extremes.--1944-56: Maximum discharge, 1,780 cfs June 7, 1952, from rating curve extended above 1,300 cfs; minimum daily, $10 \mathrm{cfs} F \mathrm{Feb}, 21$, 1955, At site I mile downstream a discharge of $1,540 \mathrm{cfs}$ was measured June 24 , 1912, and a stage $1.5 \mathrm{ft}$ higher than the meas urement was observed June 8, 1912.

Remarks.--Diversions for irrigation of about 600 acres of hay meadows above station. Estimates of annual flow are within about 10 percent of regression line.

\begin{tabular}{l|c|c|c|r|r|r|r|r|r|r|r|r|r}
\hline $\begin{array}{l}\text { Water } \\
\text { year }\end{array}$ & oct. & Nov. & Dec. & Jan. & Feb. & Mar. & Apr. & May & June & July & Aug. & Sept. & Annual \\
\hline 1944 & - & - & - & - & - & - & - & - & 38,460 & 12,570 & 2,640 & 1,170 & - \\
1945 & 1,200 & 932 & 845 & 833 & 847 & 1,140 & 1,560 & 18,500 & 39,150 & 20,070 & 6,530 & 2,250 & 93,860 \\
& & & & & & & & & & & & \\
1946 & 2,290 & 2,060 & 1,600 & 1,110 & 1,000 & 1,840 & 10,820 & 17,790 & 31,260 & 9,080 & 3,330 & 1,980 & 84,160 \\
1947 & 1,990 & 1,740 & 1,480 & 1,060 & 944 & 1,180 & 2,560 & 29,090 & 46,980 & 27,780 & 6,050 & 2,940 & 123,800 \\
1948 & 2,910 & 2,240 & 1,350 & 1,160 & 1,180 & 1,380 & 4,840 & 35,920 & 35,390 & 10,110 & 3,750 & 1,860 & 102,100 \\
1949 & 1,690 & 1,250 & 1,230 & 1,110 & 1,030 & 1,160 & 4,050 & 18,340 & 40,990 & 18,750 & 5,050 & 2,220 & 96,870 \\
1950 & 2,110 & 1,290 & 1,030 & 1,020 & 825 & 964 & 3,680 & 16,700 & 40,490 & 10,000 & 2,760 & 1,870 & 82,740 \\
1951 & 1,450 & 1,200 & 1,230 & 1,170 & 1,170 & 1,230 & 2,280 & 20,910 & 45,130 & 27,750 & 6,980 & 2,100 & 112,600 \\
1952 & 1,930 & 1,400 & 1,240 & 1,170 & 1,180 & 1,310 & 5,620 & 26,900 & 57,640 & 14,720 & 5,590 & 2,630 & 121,300 \\
1953 & 1,560 & 1,380 & 1,370 & 1,210 & 1,050 & 1,260 & 2,330 & 12,160 & 46,540 & 13,040 & 5,070 & 1,790 & 88,760 \\
1954 & 1,340 & 1,360 & 1,100 & 976 & 881 & 996 & 3,020 & 18,160 & 12,940 & 4,370 & 1,770 & 1,700 & 48,610 \\
1955 & 2,210 & 1,280 & 1,150 & 926 & 696 & 817 & 4,120 & 21,830 & 23,460 & 8,640 & 4,340 & 1,500 & 70,970 \\
1956 & 1,420 & 1,450 & 1,260 & 1,160 & 968 & 1,200 & 3,760 & 34,780 & 36,820 & 7,450 & 3,170 & 1,340 & 94,780 \\
\hline
\end{tabular}

Estimated monthly and annual streamflow, in acre-feet

\begin{tabular}{|c|c|c|c|c|c|c|c|c|c|c|c|c|c|}
\hline ater & t. & v. & e. & an. & eb. & ar. & pr. & lay & June & July & ug. & ept. & Annual \\
\hline $\begin{array}{l}1931 \\
1932 \\
1933 \\
1934 \\
1935\end{array}$ & $\begin{array}{l}0 \\
0 \\
0 \\
0 \\
0\end{array}$ & $\begin{array}{l}800 \\
300 \\
500 \\
400 \\
200\end{array}$ & $\begin{array}{l}, 300 \\
900 \\
, 300 \\
200 \\
200\end{array}$ & $\begin{array}{r}, 200 \\
730 \\
, 200 \\
, 400 \\
, 300\end{array}$ & $\begin{array}{r}940 \\
780 \\
1,100 \\
1,100 \\
1,100\end{array}$ & $\begin{array}{r}1,400 \\
860 \\
1,400 \\
1,300 \\
1,000\end{array}$ & & $\begin{array}{r}20,100 \\
24,100 \\
16,200 \\
27,000 \\
8,600\end{array}$ & & $\begin{array}{r}6,000 \\
13,500 \\
13,000 \\
4,400 \\
12,200\end{array}$ & & & \\
\hline $\begin{array}{l}19 \\
19 \\
19 \\
19 \\
19\end{array}$ & $\begin{array}{l}2,200 \\
1,600 \\
1,800 \\
2,100 \\
1,500\end{array}$ & & $\begin{array}{r}1,100 \\
1,200 \\
1,500 \\
1,400 \\
800\end{array}$ & & $\begin{array}{r}, 200 \\
, 400 \\
, 000 \\
920 \\
680\end{array}$ & & & & & & & & \\
\hline $\begin{array}{l}19 \\
19 \\
19 \\
19\end{array}$ & $\begin{array}{l}1,700 \\
1,600 \\
2,000\end{array}$ & $\begin{array}{l}100 \\
000 \\
100\end{array}$ & $\begin{array}{l}1,000 \\
1,000 \\
1,000 \\
1,100\end{array}$ & $\begin{array}{r}920 \\
950 \\
, 100 \\
760\end{array}$ & $\begin{array}{r}820 \\
1,000 \\
1,000 \\
680\end{array}$ & $\begin{array}{r}1,300 \\
1,100 \\
1,000 \\
970\end{array}$ & & & $\begin{array}{l}00 \\
000 \\
00\end{array}$ & & & & \\
\hline $\begin{array}{l}1957 \\
1958 \\
1959 \\
1960\end{array}$ & $\begin{array}{l}1,400 \\
2,000\end{array}$ & $\begin{array}{l}1,600 \\
1,200 \\
1,300\end{array}$ & $\begin{array}{r}1,400 \\
1,100 \\
980\end{array}$ & $\begin{array}{r}100 \\
920 \\
990\end{array}$ & $\begin{array}{l}760 \\
900 \\
650 \\
770\end{array}$ & $\begin{array}{l}760 \\
800 \\
660 \\
860\end{array}$ & & & & $\begin{array}{r}43,500 \\
9,500 \\
12,300 \\
14,800\end{array}$ & & & 99,20 \\
\hline $\begin{array}{l}1962 \\
1963 \\
1964 \\
1965\end{array}$ & & $\begin{array}{r}100 \\
, 500 \\
200 \\
100 \\
900\end{array}$ & $\begin{array}{r}920 \\
1,500 \\
1,100 \\
780 \\
870\end{array}$ & $\begin{array}{r}930 \\
, 000 \\
780 \\
840 \\
860\end{array}$ & $\begin{array}{r}800 \\
1,000 \\
660 \\
780 \\
720\end{array}$ & $\begin{array}{r}600 \\
840 \\
, 100 \\
820 \\
780\end{array}$ & $\begin{array}{l}1,300 \\
7,000 \\
2,600 \\
1,100 \\
2,400\end{array}$ & $\begin{array}{l}12,300 \\
28,700 \\
10,800 \\
18,200 \\
17,000\end{array}$ & $\begin{array}{l}24,100 \\
39,400 \\
15,100 \\
28,600 \\
49,400\end{array}$ & $\begin{array}{r}8,000 \\
17,300 \\
4,900 \\
10,000 \\
27,000\end{array}$ & $\begin{array}{l}4,400 \\
9,500\end{array}$ & $\begin{array}{l}4,500 \\
1,700 \\
3,000 \\
1,800 \\
4,800\end{array}$ & $\begin{array}{r}70,02 \\
115,53\end{array}$ \\
\hline
\end{tabular}



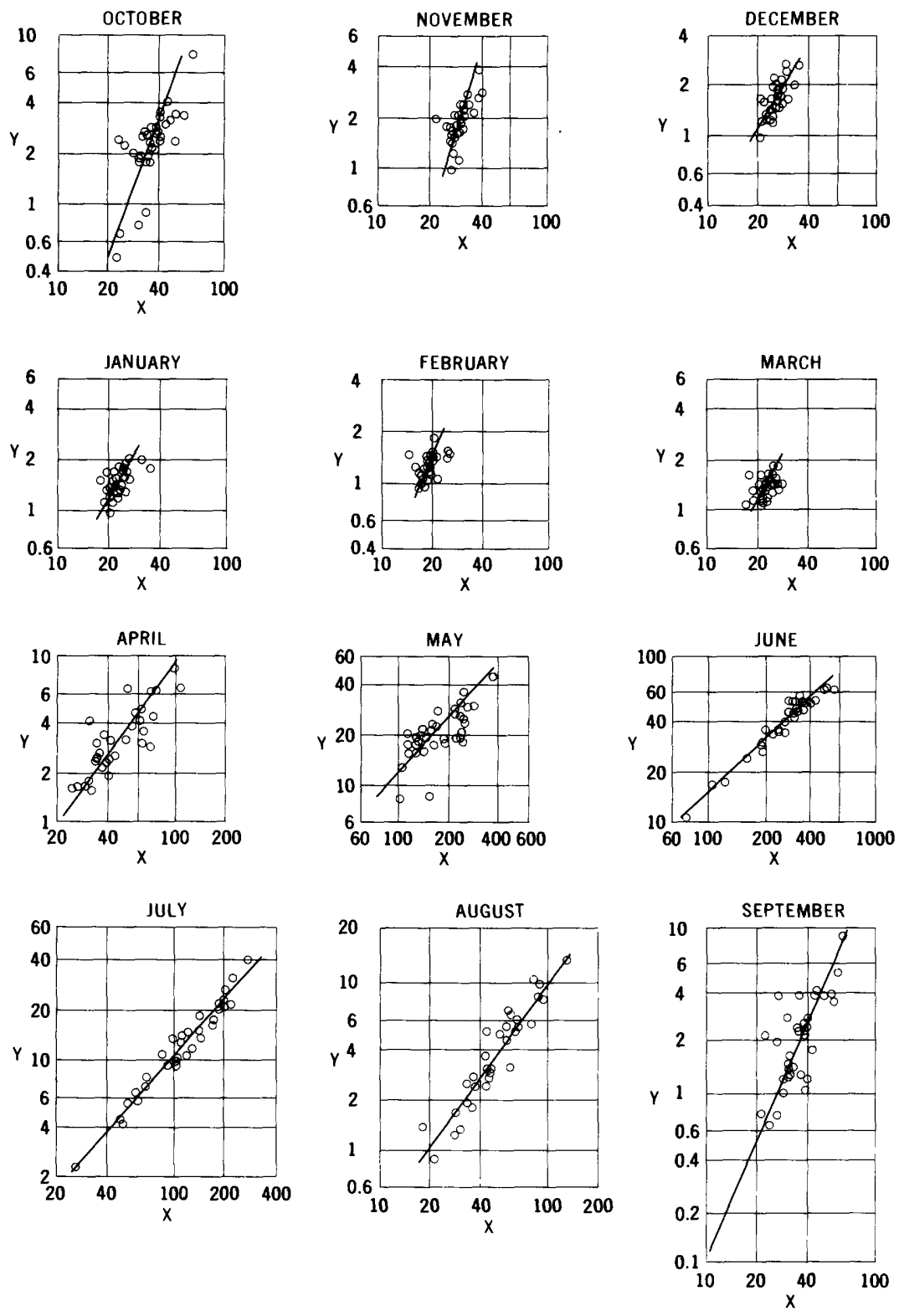

Relationships of sum of monthly mean discharges of Roaring Fork River at Aspen, Colo., and Twin Lakes tunnel $(Y)$, to sum of monthly mean discharges of Roaring Fork River at Glenwood Springs, Colo., Twin Lakes tunnel and Busk-Ivanhoe tunnel (X). Discharge in thousands of acre-feet. 
735. Roaring Fork River at Aspen, Colo.

Location. --Lat $39^{\circ} 11120^{\prime \prime}$, long $106^{\circ} 48^{\prime} 55^{\prime \prime}$, in sec.?, T.10 S., R.84 W. on right bank at As pen, three-quarters of a mile upstream' from Hunter creek.' Datum of gage is $7,884.58 \mathrm{ft}$ above mean sea level, datum of 1929 .

Drainage area. $--109 \mathrm{sq} \mathrm{mi}$.

Records available.--October 1910 to September 1921, October 1931 to September 1964. Records since May 24 , 1935, are equivalent to prior records if diversion to Twin Lanes tunnel is added to flow past station.

Estimates of streamflow.--0ctober 1930 to September 1931, based on relationships of monthly mean discharge with Roaring Fork River at Glenwood Springs, Colo., adjusted for flow through Twin Lakes tunnel and Busk-Ivanhoe tunnel. The regression equation used is:

$\log Y=b \log X-c$

(where $Y$ is the sum of discharges of Roaring Fork River at Aspen, Colo. and Twin Lakes tunnel, and $X$ is the sum of discharges of Roaring Fork River at Glenwood Springs, Colo.,

Twin Lakes tunnel, and Busk-Ivanhoe tunnel, all in acre-feet per month).
october 1964 to September 1965 based on records for Roaring Fork River near Aspen, Colo.

Monthly values of constants in above equation

\begin{tabular}{l|l|l|l|l|l|l|l|l|l|l|l|l}
\hline & oct. & Nov. & Dec. & Jan. & Feb. & Mar. & Apr. & May & June & July & Aug. & Sept. \\
\hline b & 2.76 & 3.22 & 1.63 & 1.84 & 2.41 & 1.92 & 1.39 & 1.12 & 0.95 & 1.13 & 1.39 & 2.35 \\
c & 9.23 & 1.13 & 3.95 & 4.83 & 7.22 & 5.17 & 2.98 & 1.50 & .56 & 1.65 & 2.95 & 7.39 \\
\hline
\end{tabular}

Average discharge. -35 years $(1930-65), 100,990$ acre-feet per year (139 cfs), including diversion by Twin Lakes tunnel.

Extremes.--1910-21, 1931-64: Maximum discharge, 3,170 cf's June 18, 1917 (from flood marks), from rating curve extended above 1,200 $\mathrm{cfs}$; minimum daily prior to construction of Twin Lakes tunnel, $15 \mathrm{cf}$ suly 15, 16, 1934; minimum daily since diversion through Twin Lakes tunnel, 0.4 cf's sept. 24, 27, 28, 1956.

Remarks.--Transmountain diversion through Twin Lakes tunnel to Arkansas River basin since May 24, 1935, not included in figures below. Salvation ditch diverts water above station for irrigation of about 1,000 acres below. Estimates of annual flow are within about 5 percent of regression line.

Monthly and annual streamflow, in acre-feet

\begin{tabular}{|c|c|c|c|c|c|c|c|c|c|c|c|c|c|}
\hline $\begin{array}{c}\text { Water } \\
\text { year }\end{array}$ & oct. & Nov, & Dec. & Jan. & Feb. & Mar. & Apr. & May & June & July & Aug. & Sept. & Annual \\
\hline $\begin{array}{l}1932 \\
1933 \\
1934 \\
1935\end{array}$ & $\begin{array}{r}\neq 2,150 \\
2,690 \\
\neq 2,710 \\
2,200\end{array}$ & $\begin{array}{r}\$ 1,490 \\
2,450 \\
\$ 1,790 \\
1,930\end{array}$ & $\begin{aligned} 1, & 230 \\
2, & 030 \\
\$ 1, & 230 \\
1, & 690\end{aligned}$ & $\begin{array}{r}\neq 1,230 \\
\neq 1,840 \\
984 \\
1,550\end{array}$ & $\begin{array}{l}\$ 1,150 \\
11,670 \\
1,170 \\
1,480\end{array}$ & $\begin{array}{l}1,540 \\
1,840 \\
1,650 \\
1,610\end{array}$ & $\begin{array}{l}4,170 \\
2,680 \\
6,430 \\
2,470\end{array}$ & $\begin{array}{r}23,990 \\
18,550 \\
28,630 \\
7,810\end{array}$ & $\begin{array}{l}46,270 \\
52,740 \\
10,320 \\
39,960\end{array}$ & $\begin{array}{r}21,570 \\
10,280 \\
2,340 \\
13,060\end{array}$ & & $\begin{array}{l}, 340 \\
, 620 \\
, 210 \\
, 160\end{array}$ & $\begin{array}{r}\neq 112,900 \\
\neq 103,000 \\
\neq 60,850 \\
80,210\end{array}$ \\
\hline $\begin{array}{l}1936 \\
1937 \\
1938 \\
1939 \\
1940\end{array}$ & $\begin{array}{l}2,360 \\
2,840 \\
1,810 \\
2,830 \\
625\end{array}$ & $\begin{array}{r}1,620 \\
2,060 \\
1,560 \\
2,340 \\
868\end{array}$ & $\begin{array}{l}1,300 \\
1,690 \\
1,300\end{array}$ & $\begin{array}{l}1,320 \\
1,480 \\
1,380 \\
1,560 \\
1,100\end{array}$ & $\begin{array}{r}1,280 \\
1,160 \\
1,050 \\
1,320 \\
908\end{array}$ & & $\begin{array}{l}7,770 \\
2,190 \\
3,280 \\
4,610 \\
2,660\end{array}$ & $\begin{array}{l}37,680 \\
18,470 \\
14,400 \\
22,560 \\
12,380\end{array}$ & $\begin{array}{l}26,740 \\
13,560 \\
37,790 \\
15,990 \\
10,660\end{array}$ & $\begin{array}{r}6,850 \\
4,700 \\
11,960 \\
3,290 \\
1,770\end{array}$ & & $\begin{array}{r}, 530 \\
714 \\
, 030 \\
815 \\
, 130\end{array}$ & $\begin{array}{l}95,150 \\
51,370 \\
81,330 \\
60,220 \\
34,910\end{array}$ \\
\hline $\begin{array}{l}1941 \\
1942 \\
1943 \\
1944 \\
1945\end{array}$ & $\begin{array}{r}2,230 \\
2,680 \\
1,520 \\
2,370 \\
675\end{array}$ & $\begin{array}{r}1,370 \\
1,770 \\
1,490 \\
1,960 \\
920\end{array}$ & & & $\begin{array}{r}980 \\
1,060 \\
1,160 \\
1,160 \\
944\end{array}$ & & & & & $\begin{array}{r}4,840 \\
12,690 \\
7,140 \\
7,740 \\
10,440\end{array}$ & & & \\
\hline $\begin{array}{l}1946 \\
1947 \\
1948 \\
1949 \\
1950\end{array}$ & $\begin{array}{l}1,990 \\
1,620 \\
2,390 \\
1,870 \\
2,380\end{array}$ & $\begin{array}{l}1,960 \\
1,440 \\
2,250 \\
1,630 \\
1,760\end{array}$ & & $\begin{array}{l}10 \\
90 \\
80 \\
90 \\
40\end{array}$ & $\begin{array}{l}1, \\
1, \\
1, \\
1, \\
1,\end{array}$ & & & & & $\begin{array}{r}5,110 \\
29,700 \\
6,340 \\
18,070 \\
4,990\end{array}$ & & & $\begin{array}{l}40 \\
000 \\
180 \\
50 \\
80\end{array}$ \\
\hline $\begin{array}{l}1951 \\
1952 \\
1953 \\
1954 \\
1955\end{array}$ & $\begin{array}{l}1,770 \\
1,720 \\
2,090 \\
2,000 \\
2,630\end{array}$ & & $\begin{array}{l}1,480 \\
1,550 \\
1,610 \\
1,470 \\
1,650\end{array}$ & & $\begin{array}{r}1,140 \\
1,270 \\
1,190 \\
1,120 \\
932\end{array}$ & & & & & & & & $\begin{array}{l}81,290 \\
59,390 \\
32,550 \\
43,460\end{array}$ \\
\hline $\begin{array}{l}1956 \\
1957 \\
1958 \\
1959 \\
1960\end{array}$ & $\begin{array}{r}679 \\
455 \\
2,290 \\
7,780 \\
2,890\end{array}$ & $\begin{array}{l}1,350 \\
1,060 \\
2,070 \\
1,540 \\
2,090\end{array}$ & $\begin{array}{l}1,260 \\
1,140 \\
2,400 \\
1,350 \\
2,070\end{array}$ & $\begin{array}{l}1,130 \\
1,120 \\
1,850 \\
1,280 \\
1,520\end{array}$ & $\begin{array}{r}1,010 \\
916 \\
1,360 \\
1,180 \\
1,190\end{array}$ & $\begin{array}{l}1,010 \\
1,260 \\
1,370 \\
1,380\end{array}$ & $\begin{array}{l}2,460 \\
1,870 \\
2,020 \\
2,390 \\
4,430\end{array}$ & & $\begin{array}{l}15,560 \\
49,700 \\
39,350 \\
28,830 \\
27,750\end{array}$ & $\mid \begin{array}{r}2,620 \\
55,190 \\
4,090 \\
4,270 \\
5,560\end{array}$ & $\left\{\begin{array}{l}1,030 \\
8,450 \\
1,410 \\
1,280 \\
1,220\end{array}\right.$ & $\begin{array}{r}111 \\
2,680 \\
1,010 \\
1,200 \\
742\end{array}$ & $\begin{array}{r}43,830 \\
131,000 \\
84,400 \\
57,910 \\
62,100\end{array}$ \\
\hline $\begin{array}{l}1961 \\
1962 \\
1963 \\
1964\end{array}$ & $\begin{array}{l}1,770 \\
4,640 \\
1,940 \\
1,640\end{array}$ & $\begin{array}{l}1,770 \\
1,700 \\
1,460\end{array}$ & $\begin{array}{l}1,220 \\
2,140 \\
1,210 \\
1,170\end{array}$ & $\begin{array}{l}1,800 \\
1,110 \\
1,230\end{array}$ & $\begin{array}{r}1,090 \\
1,340 \\
889 \\
1,040\end{array}$ & $\begin{array}{r}1,430 \\
1,250 \\
930\end{array}$ & $\begin{array}{l}1,570 \\
6,010 \\
2,400 \\
1,480\end{array}$ & $\begin{array}{r}12,600 \\
18,650 \\
9,800 \\
13,830\end{array}$ & $\begin{array}{r}18,370 \\
34,610 \\
5,920 \\
15,860\end{array}$ & $\begin{array}{r}2,680 \\
15,900 \\
1,120 \\
6,270\end{array}$ & $\begin{array}{l}2,740 \\
2,250 \\
3,350\end{array}$ & $\begin{array}{l}4,330 \\
1,580 \\
1,930 \\
2,450\end{array}$ & $\begin{array}{l}48,060 \\
93,610 \\
31,220 \\
50,710\end{array}$ \\
\hline
\end{tabular}

Estimated monthly and annual streamflow, in acre-feet

\begin{tabular}{l|l|l|l|l|l|l|l|l|l|l|l|l|l}
\hline $\begin{array}{l}\text { Water } \\
\text { year }\end{array}$ & Oct. & Nov. & Dec. & Jan. & Feb. & Mar. & Apr. & May & June & July & Aug. & Sept. & Annual \\
\hline 1931 & 2,600 & 1,600 & 1,700 & 1,500 & 1,000 & 1,100 & 1,600 & 10,200 & 24,500 & 5,600 & 1,500 & 1,600 & 54,500 \\
1965 & 1,700 & 1,400 & 1,500 & 1,400 & 1,200 & 1,200 & 2,800 & 10,900 & 30,700 & 32,500 & 5,400 & 4,100 & 94,800 \\
\hline
\end{tabular}



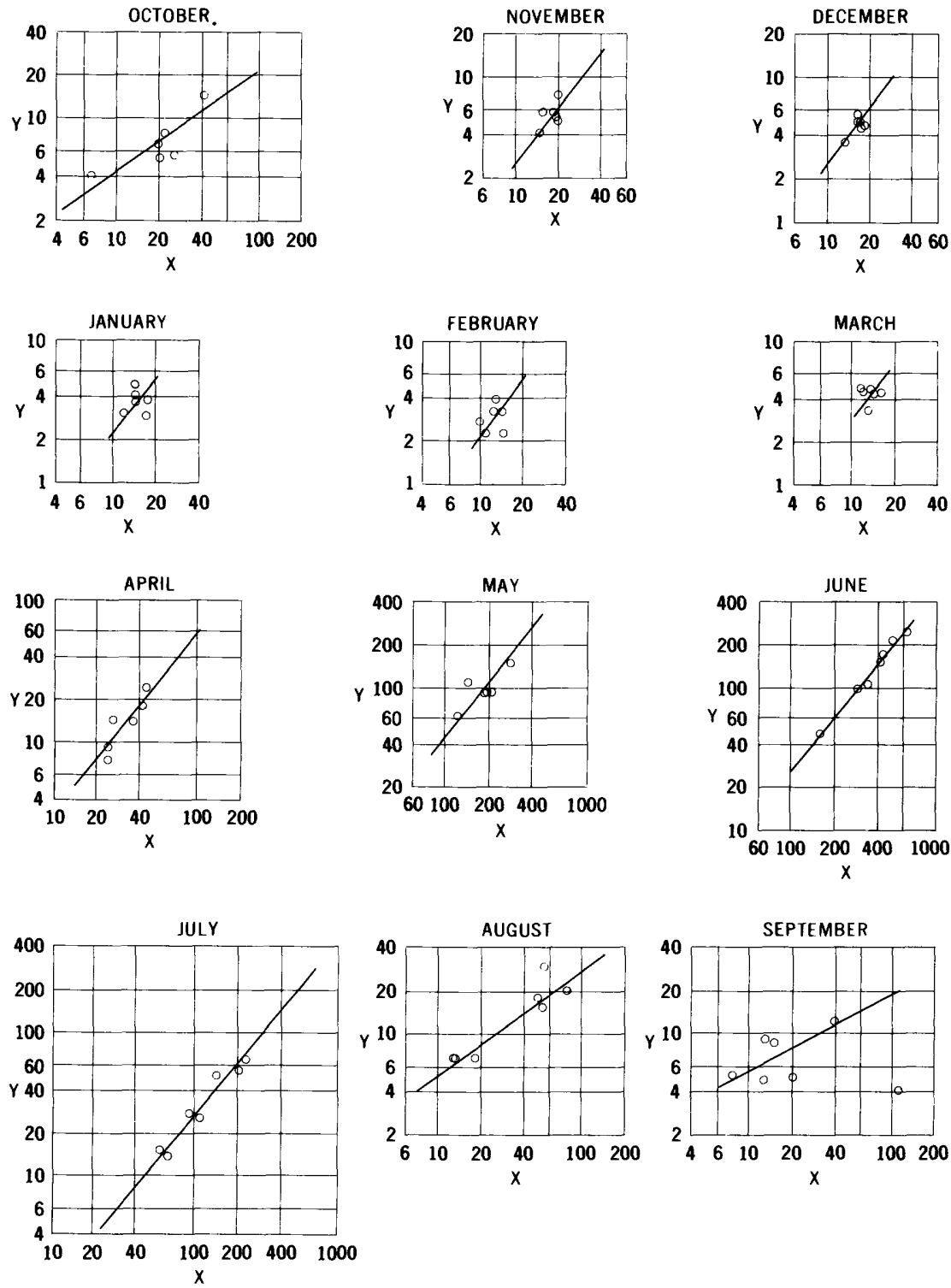

Relationships of monthly mean discharge of Hunter Creek near Aspen, Colo. (Y), to sum of monthly mean discharges of Roaring Fork River at Aspen, Colo., and Twin Lakes tunnel $(X)$. Discharge in hundreds of acre-feet. 
740. Hunter Creek near Aspen, Colo.

Location. - -Lat $39^{\circ} 12^{1} 20^{\prime \prime}$, long $106^{\circ} 48^{\prime} 00^{\prime \prime}$, in SW $\frac{1}{4}$ sec.5, T.10 S., R.84 W., on right bank $60 \mathrm{ft}$ upstream from headgate of Red Mountain ditch, $100 \mathrm{ft}$ upstream from road bridge, $1 \frac{1}{2}$ miles upstream from mouth, and $1 \frac{1}{2}$ miles northeast of Aspen. Altitude of gage 1 s $8,600 \mathrm{ft}$ (from topographic map).

Drainage area. $--40 \mathrm{sq} \mathrm{mi}$, approximately.

Records available. --June 1950 to September 1956.

Estimates of streamflow.--0ctober 1931 to May 1950, October 1956 to September 1965 , based on relationships of monthly mean discharge with Roaring Fork River at Aspen, Colo., adjusted for diversion through Twin Lakes tunnel since 1935. The regression equation used is:

$$
\log \mathrm{Y}=\mathrm{b} \log \mathrm{X}-\mathrm{c}
$$

(where $Y$ is discharge of Hunter Creek near Aspen Colo., and $X$ is the sum of discharges of Roaring Fork River at Aspen, Colo. and Twin Lakes tunnel, all in acre-feet per month).

\begin{tabular}{l|l|l|l|l|l|l|l|l|l|l|l|l}
\hline \multicolumn{10}{c}{ Monthly values of constants in above equation } \\
\hline Oct. & Nov. & Dec. & Jan. & Feb. & Mar. & Apr. & May & June & July & Aug. & Sept. \\
\hline b & 0.71 & 1.31 & 1.31 & 1.32 & 1.32 & 1.30 & 1.26 & 1.26 & 1.26 & 1.20 & 0.72 & 0.54 \\
c & -.50 & 1.55 & 1.55 & 1.62 & 1.62 & 1.43 & 1.27 & 1.38 & 1.64 & 1.37 & -.56 & -1.13 \\
\hline
\end{tabular}

Average discharge. -34 years $(1931-65), 40,422$ acre-feet per year (55.8 cfs).

Extremes.--1950-56: Maximum discharge, 1,010 cfs June 13, 1953, from rating curve extended above $580 \mathrm{cfs}$; minimum not determinéd.

Remarks. --Several small diversions above station for 1rrigation of hay meadows above and below station. Estimates of annual flow are within about 15 percent of regression line.

\begin{tabular}{|c|c|c|c|c|c|c|c|c|c|c|c|c|c|}
\hline $\begin{array}{l}\text { Water } \\
\text { year }\end{array}$ & Oct. & Nov. & Dec. & Jan. & Feb. & Mar. & Apr. & May & June & July & Aug. & Sept. & Annual \\
\hline 1950 & - & - & - & - & - & - & - & - & 15,020 & 2,510 & 689 & 876 & - \\
\hline $\begin{array}{l}1951 \\
1952 \\
1953 \\
1954 \\
1955\end{array}$ & $\begin{array}{r}676 \\
815 \\
572 \\
536 \\
1,460\end{array}$ & $\begin{array}{l}567 \\
576 \\
504 \\
531 \\
742\end{array}$ & $\begin{array}{l}555 \\
449 \\
462 \\
492 \\
492\end{array}$ & $\begin{array}{l}467 \\
301 \\
384 \\
430 \\
369\end{array}$ & $\begin{array}{l}400 \\
230 \\
331 \\
333 \\
278\end{array}$ & $\begin{array}{l}430 \\
333 \\
344 \\
364 \\
369\end{array}$ & $\begin{array}{r}935 \\
2,390 \\
752 \\
1,880 \\
1,440\end{array}$ & $\begin{array}{r}9,270 \\
11,130 \\
6,210 \\
9,390 \\
9,170\end{array}$ & $\begin{array}{r}17,190 \\
24,560 \\
21,170 \\
4,740 \\
10,180\end{array}$ & $\begin{array}{l}6,540 \\
5,550 \\
5,040 \\
1,400 \\
2,740\end{array}$ & $\begin{array}{r}1,570 \\
2,020 \\
2,970 \\
682 \\
1,810\end{array}$ & $\begin{array}{r}490 \\
1,250 \\
501 \\
922 \\
520\end{array}$ & $\begin{array}{l}39,090 \\
49,600 \\
39,240 \\
21,700 \\
29,570\end{array}$ \\
\hline 1956 & 402 & 405 & 355 & 307 & 230 & 458 & 1,450 & 14,890 & 10,530 & 1,470 & 678 & 429 & 31,600 \\
\hline
\end{tabular}

\begin{tabular}{|c|c|c|c|c|c|c|c|c|c|c|c|c|c|}
\hline $\begin{array}{l}\text { Water } \\
\text { year }\end{array}$ & oct. & Nov. & Dec. & Jan. & Feb. & Mar. & Apr. & May & June & July & Aug. & Sept. & Annual \\
\hline $\begin{array}{l}1932 \\
1933 \\
1934 \\
1935\end{array}$ & $\begin{array}{r}\neq 740 \\
860 \\
\neq 870 \\
750\end{array}$ & $\begin{array}{r}\neq 410 \\
790 \\
\neq 520 \\
580\end{array}$ & $\begin{array}{r}\$ 320 \\
630 \\
\neq 320 \\
490\end{array}$ & $\begin{array}{r}+290 \\
+500 \\
220 \\
400\end{array}$ & $\begin{array}{r}\$ 270 \\
\neq 440 \\
270 \\
370\end{array}$ & $\begin{array}{r}\$ 510 \\
\neq 640 \\
560 \\
540\end{array}$ & $\begin{array}{l}\$ 2,000 \\
\neq 1,100 \\
3,400 \\
1,000\end{array}$ & $\begin{array}{r}13,700 \\
9,900 \\
17,000 \\
3,700\end{array}$ & $\begin{array}{r}17,500 \\
20,400 \\
2,700 \\
19,800\end{array}$ & $\begin{array}{r}6,700 \\
2,700 \\
470 \\
5,100\end{array}$ & $\begin{array}{r}1,800 \\
1,300 \\
660 \\
1,500\end{array}$ & $\begin{array}{r}890 \\
950 \\
860 \\
1,200\end{array}$ & $\begin{array}{l}45,130 \\
40,210 \\
27,850 \\
35,430\end{array}$ \\
\hline $\begin{array}{l}1936 \\
1937 \\
1938 \\
1939 \\
1940\end{array}$ & $\begin{array}{r}900 \\
900 \\
710 \\
1,000 \\
400\end{array}$ & $\begin{array}{l}520 \\
620 \\
540 \\
900 \\
240\end{array}$ & $\begin{array}{l}400 \\
490 \\
420 \\
800 \\
240\end{array}$ & & $\begin{array}{l}360 \\
310 \\
270 \\
500 \\
200\end{array}$ & $\begin{array}{l}460 \\
470 \\
410 \\
640 \\
340\end{array}$ & $\begin{array}{r}5,000 \\
980 \\
1,700 \\
2,200 \\
1,400\end{array}$ & $\begin{array}{l}31,800 \\
18,400 \\
10,400 \\
24,000 \\
11,800\end{array}$ & $\begin{array}{r}12,000 \\
8,500 \\
24,800 \\
10,400 \\
7,700\end{array}$ & $\begin{array}{r}2,500 \\
2,600 \\
7,000 \\
1,700 \\
940\end{array}$ & $\begin{array}{r}2,000 \\
960 \\
1,700 \\
760 \\
480\end{array}$ & $\begin{array}{r}920 \\
660 \\
1,400 \\
580 \\
620\end{array}$ & $\begin{array}{l}57,270 \\
35,280 \\
49,740 \\
44,020 \\
24,620\end{array}$ \\
\hline $\begin{array}{l}1941 \\
1942 \\
1943 \\
1944 \\
1945\end{array}$ & $\begin{array}{r}990 \\
1,000 \\
650 \\
860 \\
350\end{array}$ & $\begin{array}{l}510 \\
660 \\
480 \\
800 \\
280\end{array}$ & & & $\begin{array}{l}250 \\
280 \\
300 \\
370 \\
230\end{array}$ & & $\begin{array}{r}580 \\
1,200 \\
3,300 \\
620 \\
680\end{array}$ & $\begin{array}{r}14,800 \\
9,600 \\
12,700 \\
10,300 \\
9,200\end{array}$ & $\begin{array}{l}12,600 \\
21,200 \\
17,200 \\
15,600 \\
14,200\end{array}$ & $\begin{array}{l}3,300 \\
5,400 \\
4,500 \\
4,700 \\
7,400\end{array}$ & & $\begin{array}{r}780 \\
760 \\
1,200 \\
440 \\
1,000\end{array}$ & $\begin{array}{l}36,020 \\
42,490 \\
44,630 \\
36,130 \\
37,000\end{array}$ \\
\hline $\begin{array}{l}1946 \\
1947 \\
1948 \\
1949 \\
1950\end{array}$ & $\begin{array}{r}860 \\
690 \\
1,000 \\
780 \\
830\end{array}$ & $\begin{array}{l}670 \\
470 \\
960 \\
480 \\
610\end{array}$ & $\begin{array}{l}660 \\
390 \\
600 \\
550 \\
520\end{array}$ & $\begin{array}{l}500 \\
450 \\
390 \\
510 \\
440\end{array}$ & $\begin{array}{l}370 \\
330 \\
360 \\
390 \\
340\end{array}$ & $\begin{array}{l}560 \\
460 \\
470 \\
620 \\
480\end{array}$ & $\begin{array}{l}3,300 \\
1,000 \\
1,300 \\
1,800 \\
1,400\end{array}$ & $\begin{array}{r}8,000 \\
15,100 \\
18,800 \\
8,200 \\
8,000\end{array}$ & $\begin{array}{l}16,600 \\
19,300 \\
17,200 \\
18,800\end{array}$ & $\begin{array}{r}3,300 \\
14,400 \\
3,900 \\
8,600 \\
-\end{array}$ & $\begin{array}{l}1,200 \\
2,400 \\
1,200 \\
1,600 \\
-\end{array}$ & $\begin{array}{r}680 \\
1,100 \\
580 \\
980 \\
-\end{array}$ & $\begin{array}{l}36,700 \\
56,090 \\
46,760 \\
43,310 \\
31,720\end{array}$ \\
\hline $\begin{array}{l}1957 \\
1958 \\
1959 \\
1960\end{array}$ & $\begin{array}{l}260 \\
920 \\
650 \\
960\end{array}$ & $\begin{array}{l}330 \\
860 \\
460 \\
790\end{array}$ & $\begin{array}{l}310 \\
900 \\
420 \\
700\end{array}$ & $\begin{array}{l}280 \\
560 \\
340 \\
410\end{array}$ & $\begin{array}{l}210 \\
390 \\
300 \\
300\end{array}$ & $\begin{array}{l}320 \\
430 \\
480 \\
470\end{array}$ & $\begin{array}{r}760 \\
860 \\
1,000 \\
2,400\end{array}$ & $\begin{array}{r}3,800 \\
19,500 \\
9,500 \\
9,200\end{array}$ & $\begin{array}{l}25,800 \\
18,600 \\
20,800 \\
20,300\end{array}$ & $\begin{array}{r}25,800 \\
2,000 \\
2,900 \\
3,900\end{array}$ & $\begin{array}{r}3,500 \\
840 \\
1,200 \\
1,100\end{array}$ & $\begin{array}{r}1,200 \\
660 \\
720 \\
490\end{array}$ & $\begin{array}{l}62,570 \\
46,520 \\
38,770 \\
41,010\end{array}$ \\
\hline $\begin{array}{l}1961 \\
1962 \\
1963 \\
1964 \\
1965\end{array}$ & $\begin{array}{r}640 \\
1,900 \\
810 \\
800 \\
720\end{array}$ & $\begin{array}{r}460 \\
, 500 \\
490 \\
510 \\
470\end{array}$ & $\begin{array}{l}360 \\
890 \\
410 \\
450 \\
480\end{array}$ & $\begin{array}{l}270 \\
570 \\
330 \\
340 \\
440\end{array}$ & $\begin{array}{l}270 \\
390 \\
240 \\
300 \\
350\end{array}$ & $\begin{array}{l}380 \\
570 \\
480 \\
330 \\
440\end{array}$ & $\begin{array}{r}620 \\
3,600 \\
1,300 \\
620 \\
1,300\end{array}$ & $\begin{array}{r}11,800 \\
15,900 \\
11,400 \\
13,100 \\
7,600\end{array}$ & $\begin{array}{r}12,400 \\
23,000 \\
4,600 \\
11,500 \\
19,800\end{array}$ & $\begin{array}{r}1,300 \\
10,800 \\
1,000 \\
4,300 \\
15,500\end{array}$ & $\begin{array}{l}1,000 \\
1,900 \\
1,700 \\
2,000 \\
3,000\end{array}$ & $\begin{array}{r}1,900 \\
910 \\
1,200 \\
1,000 \\
1,600\end{array}$ & $\begin{array}{l}31,400 \\
61,930 \\
23,960 \\
35,250 \\
51,700\end{array}$ \\
\hline
\end{tabular}

* Based on estimates for 1950 Compilation. 

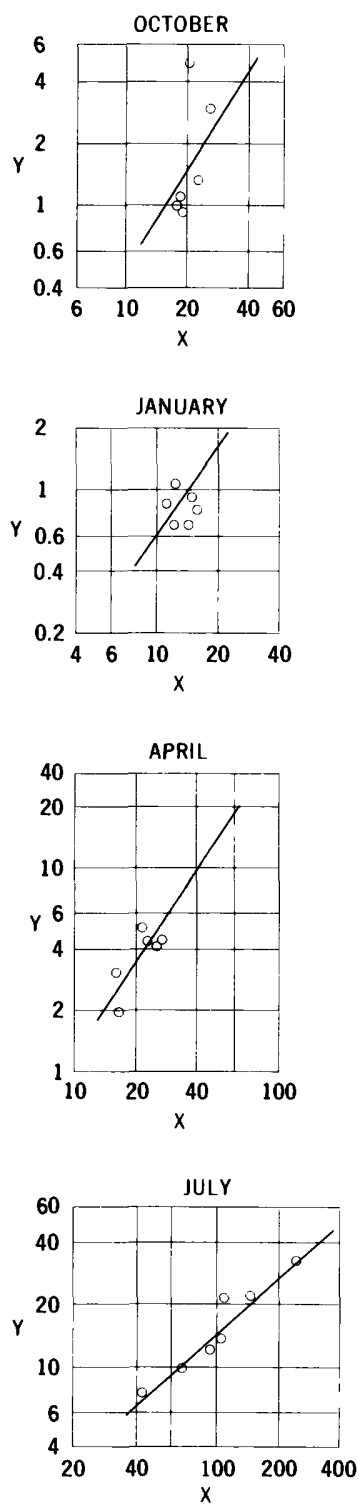
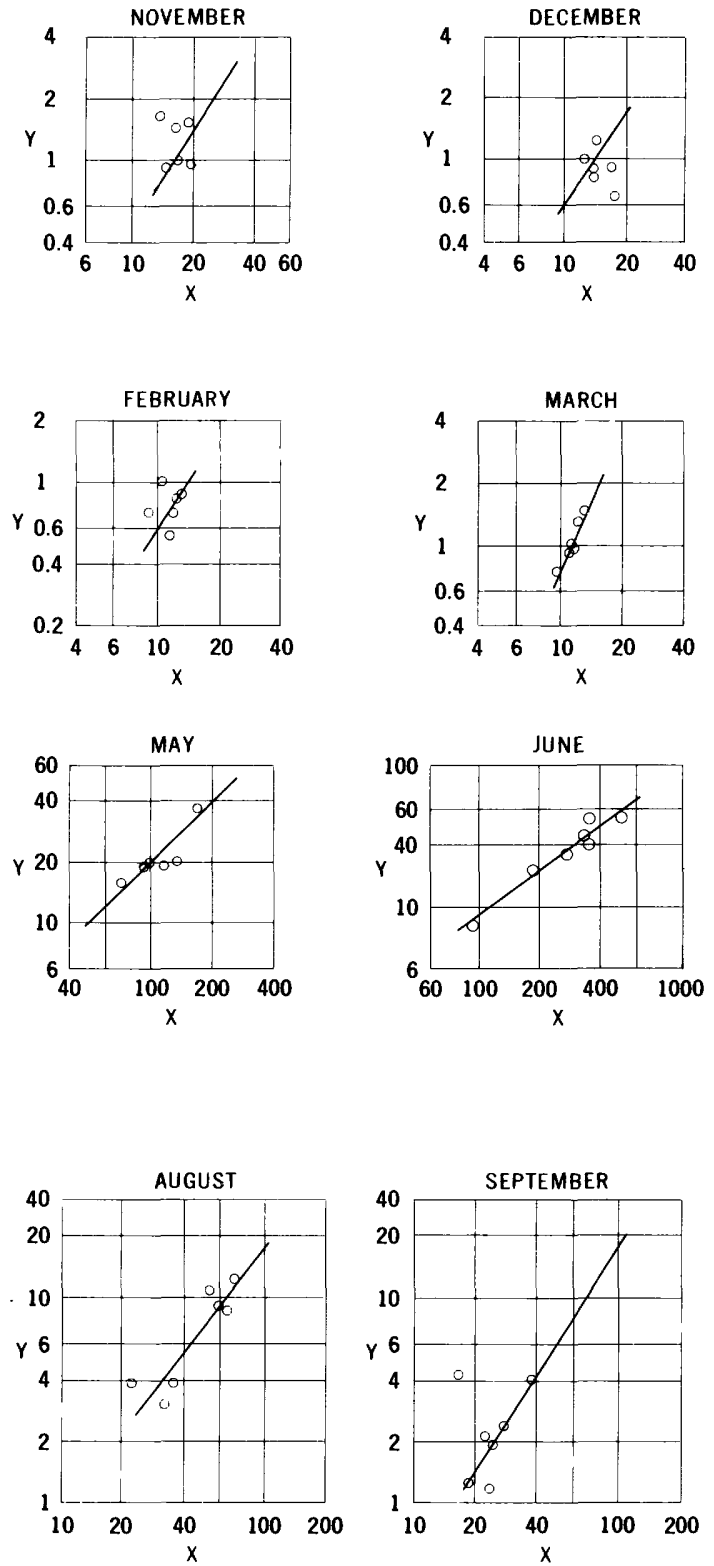

Relationships of monthly mean discharge of Lime Creek at Troutville, Colo. (Y), to sum of monthly mean discharges of Williams Fork near Leal, Colo., and August P. Gumlick tunnel (X). Discharge in hundreds of acre-feet. 
790. Lime Creek at Troutville, Colo.

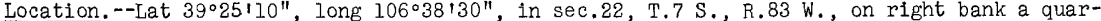
ter of a mile downstream from Woods Lake and three-quartérs of a mile west of Troutville. Altitude of gage is $9,390 \mathrm{ft}$ (from topographic map).

Drainage area. $--8.4 \mathrm{sq} \mathrm{mi}$, approximately.

Records available.--June 1950 to September 1956.

Estimates of streamflow.--July 1933 to May 1950, October 1956 to september 1965, based on relationships of monthly mean discharge with Williams Fork near Leal, Colo., adjusted for diversion through August P. Gumlick tunnel since 1940. The regression equation used is:

$\log Y=b \log X-c$

(where $Y$ is discharge of Lime creek at Troutvilie, Colo., and $X$ is the sum of discharges of Williams Fork near Leal, Colo. and August P. Gumlick tunnel, all in acre-feet per month).

Monthly values of constants in above equation

\begin{tabular}{l|l|l|l|l|l|l|l|l|l|l|l|l}
\hline & Oct. & Nov. & Dec. & Jan. & Feb. & Mar. & Apr. & May & June & July & Aug. & Sept. \\
\hline b & 1.60 & 1.60 & 1.50 & 1.50 & 1.50 & 2.18 & 1.52 & 1.01 & 0.73 & 0.88 & 1.32 & 1.62 \\
0 & 3.12 & 3.12 & 2.72 & 2.72 & 2.72 & 4.65 & 2.47 & .73 & -.33 & .36 & 2.01 & 3.18 \\
\hline
\end{tabular}

Average discharge. --32 years $(1933-65), 10,350$ acre-feet pe: year (14.3 cfs).

Extremes.--1950-56: Maximum discharge, 214 cfs June 13, 1953, from rating curve extended above $140 \mathrm{cfs}$; minimum daily, 0.3 cf's 0ct. 29-31, 1950 .

Remarks.--Flow regulated by several small lakes above station. No diversions above station. Estimates of annual flow are within 10 percent of regression line.

Monthly and annual streamflow, in acre-feet

\begin{tabular}{r|r|r|r|r|r|r|r|r|r|r|r|r|r}
\hline $\begin{array}{l}\text { Water } \\
\text { year }\end{array}$ & Oct. & Nov. & Dec. & Jan. & Feb. & Mar. & Apr. & May & June & July & Aug. & Sept. & Annual \\
\hline 1950 & - & - & - & - & - & - & - & - & 4,460 & 1,370 & 303 & 201 & - \\
& & & - & & - & & - & & & & & \\
1951 & 110 & 90 & 94 & 68 & 56 & 102 & 306 & 1,900 & 4,090 & 3,300 & 882 & 246 & 11,230 \\
1952 & 294 & 150 & 92 & 80 & 81 & 93 & 449 & 2,040 & 5,500 & 2,180 & 1,260 & 397 & 12,620 \\
1953 & 131 & 97 & 68 & 68 & 72 & 130 & 199 & 1,610 & 5,580 & 2,130 & 914 & 119 & 11,120 \\
1954 & 94 & 140 & 123 & 107 & 100 & 92 & 421 & 2,050 & 1,620 & 742 & 387 & 427 & 6,310 \\
1955 & 497 & 163 & 102 & 86 & 72 & 74 & 511 & 1,920 & 3,030 & 1,190 & 1,090 & 214 & 8,950 \\
1956 & 99 & 98 & 92 & 92 & 86 & 147 & 446 & 3,700 & 3,690 & 979 & 390 & 128 & 9,950 \\
\hline
\end{tabular}

\begin{tabular}{|c|c|c|c|c|c|c|c|c|c|c|c|c|c|}
\hline $\begin{array}{l}\text { Water } \\
\text { year }\end{array}$ & oct. & Nov. & Dec. & Jan. & Feb. & Mar. & Apr. & May & June & July & Aug. & Sept. & Annual \\
\hline $\begin{array}{l}1933 \\
1934 \\
1935\end{array}$ & $\begin{array}{l}- \\
160 \\
110\end{array}$ & $\begin{array}{l}- \\
90 \\
90\end{array}$ & $\begin{array}{r}100 \\
80\end{array}$ & $\begin{array}{r}- \\
90 \\
70\end{array}$ & $\begin{array}{l}- \\
70 \\
50\end{array}$ & $\begin{array}{r}160 \\
80\end{array}$ & $\begin{array}{l}\overline{820} \\
270\end{array}$ & $\begin{array}{l}3, \overline{800} \\
1,000\end{array}$ & $\begin{array}{c}\overline{2}, 100 \\
4,500\end{array}$ & $\begin{array}{r}1,900 \\
590 \\
2,200\end{array}$ & $\begin{array}{l}620 \\
370 \\
890\end{array}$ & $\begin{array}{l}250 \\
170 \\
310\end{array}$ & $\begin{array}{l}- \\
\overline{8}, 520 \\
9,650\end{array}$ \\
\hline $\begin{array}{l}1936 \\
1937 \\
1938 \\
1939 \\
1940\end{array}$ & $\begin{array}{l}200 \\
250 \\
250 \\
210 \\
150\end{array}$ & $\begin{array}{r}150 \\
130 \\
160 \\
140 \\
90\end{array}$ & $\begin{array}{r}100 \\
100 \\
130 \\
140 \\
60\end{array}$ & $\begin{array}{r}80 \\
80 \\
100 \\
110 \\
60\end{array}$ & $\begin{array}{l}70 \\
70 \\
80 \\
80 \\
50\end{array}$ & $\begin{array}{l}100 \\
110 \\
130 \\
130 \\
110\end{array}$ & $\begin{array}{r}1,200 \\
350 \\
450 \\
580 \\
380\end{array}$ & $\begin{array}{l}4,800 \\
2,600 \\
2,800 \\
4,100 \\
2,300\end{array}$ & $\begin{array}{l}4,100 \\
2,800 \\
6,500 \\
3,900 \\
3,200\end{array}$ & $\begin{array}{l}1,700 \\
1,300 \\
2,500 \\
1,200 \\
1,000\end{array}$ & $\begin{array}{r}1,100 \\
420 \\
750 \\
100 \\
290\end{array}$ & $\begin{array}{l}340 \\
240 \\
470 \\
160 \\
240\end{array}$ & $\begin{array}{r}13,940 \\
8,450 \\
14,320 \\
11,150 \\
7,930\end{array}$ \\
\hline $\begin{array}{l}1941 \\
1942 \\
1943 \\
1944 \\
1945\end{array}$ & $\begin{array}{l}220 \\
230 \\
150 \\
190 \\
120\end{array}$ & $\begin{array}{r}100 \\
100 \\
100 \\
130 \\
90\end{array}$ & $\begin{array}{r}100 \\
110 \\
100 \\
100 \\
80\end{array}$ & $\begin{array}{l}90 \\
80 \\
80 \\
80 \\
70\end{array}$ & $\begin{array}{l}60 \\
50 \\
60 \\
70 \\
60\end{array}$ & $\begin{array}{r}90 \\
90 \\
100 \\
90 \\
90\end{array}$ & $\begin{array}{l}230 \\
410 \\
910 \\
160 \\
210\end{array}$ & $\begin{array}{l}3,000 \\
2,100 \\
2,100 \\
1,700 \\
1,800\end{array}$ & $\begin{array}{l}3,800 \\
5,200 \\
4,300 \\
4,000 \\
3,600\end{array}$ & $\begin{array}{l}1,600 \\
1,900 \\
1,900 \\
1,900 \\
2,700\end{array}$ & $\begin{array}{r}530 \\
600 \\
750 \\
460 \\
1,700\end{array}$ & $\begin{array}{l}240 \\
180 \\
230 \\
120 \\
330\end{array}$ & $\begin{array}{r}10,060 \\
11,050 \\
10,780 \\
9,000 \\
10,850\end{array}$ \\
\hline $\begin{array}{l}1946 \\
1947 \\
1948 \\
1949 \\
1950\end{array}$ & $\begin{array}{l}280 \\
180 \\
330 \\
120 \\
180\end{array}$ & $\begin{array}{l}200 \\
130 \\
210 \\
100 \\
100\end{array}$ & $\begin{array}{r}160 \\
120 \\
160 \\
110 \\
90\end{array}$ & $\begin{array}{r}130 \\
80 \\
120 \\
80 \\
80\end{array}$ & $\begin{array}{l}90 \\
60 \\
80 \\
60 \\
60\end{array}$ & $\begin{array}{l}180 \\
130 \\
120 \\
110 \\
130\end{array}$ & $\begin{array}{r}1,600 \\
220 \\
420 \\
450 \\
370\end{array}$ & $\begin{array}{l}2,200 \\
2,600 \\
3,600 \\
1,900 \\
1,800\end{array}$ & $\begin{array}{c}3,900 \\
4,300 \\
3,800 \\
4,400 \\
-\end{array}$ & $\begin{array}{c}1,400 \\
3,800 \\
1,200 \\
2,600 \\
-\end{array}$ & $\begin{array}{r}500 \\
1,200 \\
450 \\
800 \\
-\end{array}$ & $\begin{array}{l}220 \\
470 \\
110 \\
220 \\
-\end{array}$ & $\begin{array}{r}10,860 \\
13,290 \\
10,600 \\
10,950 \\
9,144\end{array}$ \\
\hline $\begin{array}{l}1957 \\
1958 \\
1959 \\
1960\end{array}$ & $\begin{array}{l}120 \\
260 \\
130 \\
200\end{array}$ & $\begin{array}{r}100 \\
170 \\
90 \\
150\end{array}$ & $\begin{array}{r}90 \\
150 \\
100 \\
120\end{array}$ & $\begin{array}{r}90 \\
110 \\
80 \\
90\end{array}$ & $\begin{array}{l}60 \\
80 \\
60 \\
60\end{array}$ & $\begin{array}{r}100 \\
140 \\
80 \\
120\end{array}$ & $\begin{array}{l}220 \\
260 \\
260 \\
640\end{array}$ & $\begin{array}{l}1,100 \\
4,100 \\
1,700 \\
2,000\end{array}$ & $\begin{array}{l}4,400 \\
4,200 \\
4,000 \\
4,600\end{array}$ & $\begin{array}{r}4,300 \\
990 \\
1,300 \\
1,700\end{array}$ & $\begin{array}{r}1,500 \\
450 \\
530 \\
470\end{array}$ & $\begin{array}{l}440 \\
180 \\
150 \\
170\end{array}$ & $\begin{array}{r}12,520 \\
11,090 \\
8,480 \\
10,320\end{array}$ \\
\hline $\begin{array}{l}1961 \\
1962 \\
1963 \\
1964 \\
1965\end{array}$ & $\begin{array}{r}110 \\
890 \\
160 \\
80 \\
70\end{array}$ & $\begin{array}{r}100 \\
300 \\
100 \\
70 \\
70\end{array}$ & $\begin{array}{r}90 \\
180 \\
100 \\
60 \\
70\end{array}$ & $\begin{array}{r}50 \\
120 \\
80 \\
50 \\
80\end{array}$ & $\begin{array}{r}60 \\
100 \\
60 \\
50 \\
70\end{array}$ & $\begin{array}{r}90 \\
170 \\
100 \\
60 \\
80\end{array}$ & $\begin{array}{r}180 \\
1,200 \\
370 \\
180 \\
310\end{array}$ & $\begin{array}{l}1,900 \\
3,200 \\
1,700 \\
2,000 \\
1,600\end{array}$ & $\begin{array}{l}3,500 \\
4,600 \\
1,800 \\
3,100 \\
4,300\end{array}$ & $\begin{array}{r}1,100 \\
2,600 \\
600 \\
1,500 \\
3,200\end{array}$ & $\begin{array}{r}600 \\
710 \\
440 \\
500 \\
1,600\end{array}$ & $\begin{array}{l}830 \\
180 \\
170 \\
110 \\
430\end{array}$ & $\begin{array}{r}8,610 \\
14,250 \\
5,680 \\
7,760 \\
11,880\end{array}$ \\
\hline
\end{tabular}



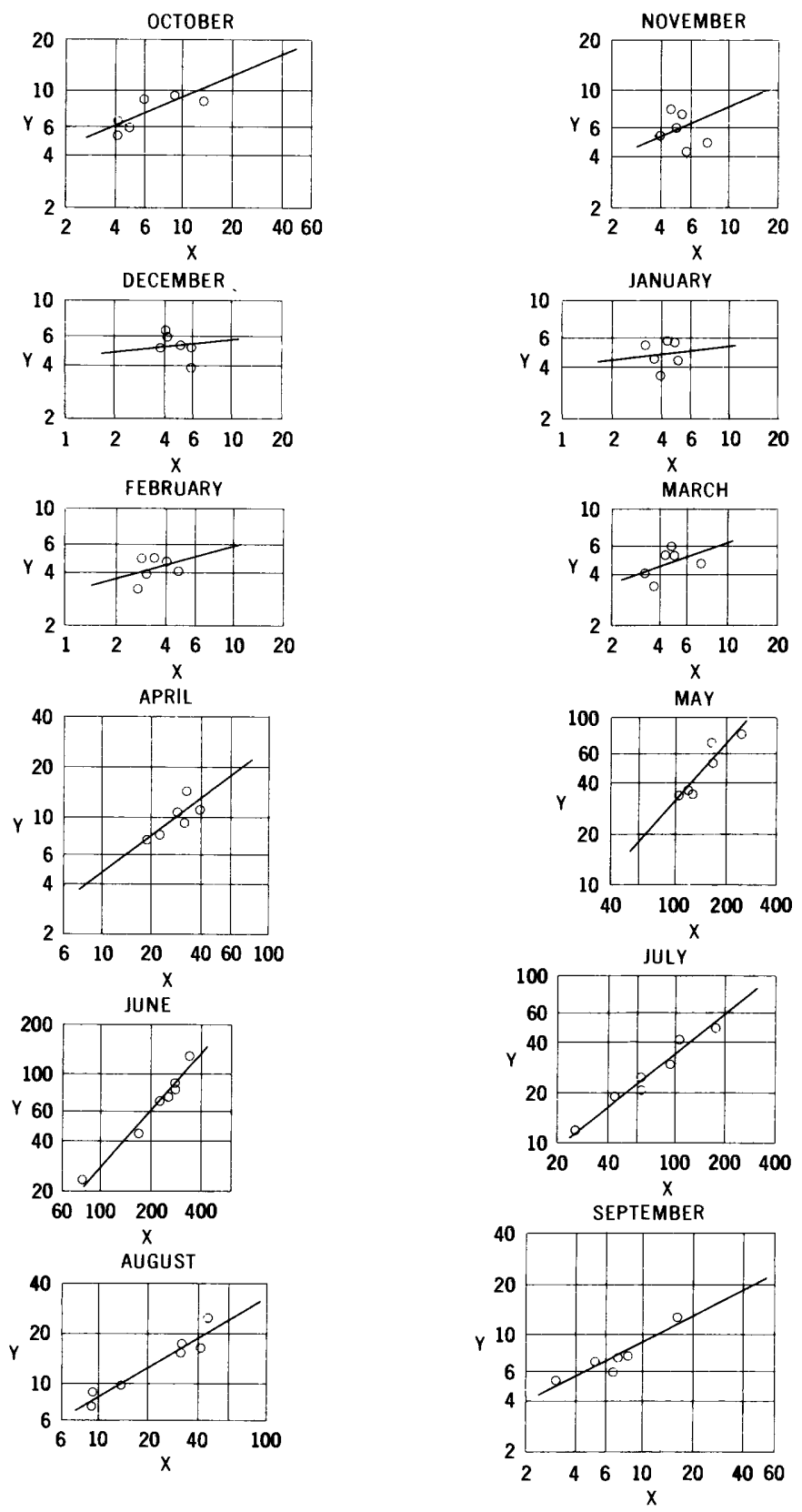

Relationships of monthly mean discharge of Lime Creek at Thomasville, Colo. (Y), to monthly mean discharge of Homestake Creek near Red Cliff, Colo. $(X)$. Discharge in hundreds of acre-feet. 
795. Lime creek at Thomasville, colo.

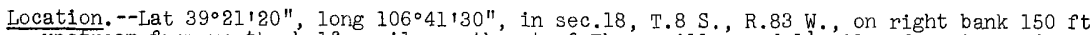
upstream from mouth, half a mile southeast of Thomasville', and $1 \frac{1}{2}$ miles downstream from Spring Creek. Altitude of gage is $8,050 \mathrm{ft}$ (from topogrephic map).

Dralnage area. $--32 \mathrm{sq} \mathrm{mi}$, approximately.

Records available. --June 1950 to September 1956.

Estimates of streamf'low.--June 1944 to May 1950, 0ctober 1956 to September 1965, based on relationships of monthly mean discharge with Homestake Creek near Red Cliff, Colo. The regression equation used is:

$$
\log Y=b \log X-c
$$

(where $Y$ is discharge of Lime Creek at Thomasville, Colo,, and X is discharge of Homestake Creek near Red Cliff, Colo., both in acre-feet per month).

Monthly values of constants in above equation

\begin{tabular}{c|c|c|c|c|c|c|c|c|c|c|c|c}
\hline & Oct. & Nov. & Dec. & Jan. & Feb. & Mar. & Apr. & May & June & July & Aug. & Sept. \\
\hline $\mathrm{b}$ & 0.43 & 0.43 & 0.11 & 0.11 & 0.28 & 0.36 & 0.75 & 1.13 & 1.13 & 0.79 & 0.60 & 0.52 \\
$\mathrm{c}$ & -1.68 & -1.61 & -2.43 & -2.40 & -1.93 & -1.72 & -.42 & 1.02 & 1.08 & -.37 & -1.12 & -1.40 \\
\hline
\end{tabular}

Average discharge. --21 years $(1944-65), 22,461$ acre-feet per year (31.0 cfs).

Extremes --1950-56: Maximum discharge, 344 cf's June 11, 1952: minimum daily, 4.5 cfs Feb. $21,1955$.

Remarks.--Small diversions for irrigation above station. Estimates of annual flow are within about 10 percent of regression line.

\begin{tabular}{c|c|c|c|c|c|c|c|c|c|c|c|c|c}
\hline $\begin{array}{l}\text { Water } \\
\text { year }\end{array}$ & oct. & Nov. & Dec. & Jan. & Feb. & Mar. & Apr. & May & Jur & July & Aug. & Sept. & Annual \\
\hline 1950 & - & - & - & - & - & - & - & - & 7,390 & 2,440 & 899 & 731 & - \\
& & & & & & & & & & & & & \\
1951 & 606 & 533 & 533 & 536 & 504 & 597 & 781 & 5,270 & 7,990 & 4,930 & 1,650 & 751 & 24,680 \\
1952 & 938 & 720 & 606 & 583 & 504 & 531 & 1,410 & 6,990 & 12,890 & 4,120 & 2,480 & 1,260 & 33,030 \\
1953 & 889 & 764 & 650 & 576 & 476 & 530 & 778 & 3,390 & 8,810 & 2,950 & 1,720 & 689 & 22,180 \\
1954 & 635 & 592 & 522 & 453 & 408 & 419 & 1,060 & 3,450 & 2,300 & 1,190 & 735 & 750 & 12,510 \\
1955 & 885 & 493 & 510 & 359 & 325 & 352 & 936 & 3,620 & 4,430 & 2,060 & 1,550 & 599 & 16,120 \\
1956 & 546 & 437 & 391 & 442 & 418 & 471 & 1,110 & 7,850 & 6,810 & 1,890 & 982 & 513 & 21,860 \\
\hline
\end{tabular}

\begin{tabular}{l|c|c|c|c|c|c|c|c|c|c|c|c|c|c}
\hline $\begin{array}{l}\text { Water } \\
\text { year }\end{array}$ & oct. & Nov. & Dec. & Jan. & Feb. & Mar. & Apr. & May & June & July & Aug. & Sept. & Annual \\
\hline 1944 & - & - & - & - & - & - & - & - & 6,600 & 2,900 & 890 & 450 & - \\
1945 & 560 & 480 & 510 & 480 & 410 & 440 & 380 & 4,400 & 6,000 & 4,200 & 2,700 & 1,000 & 21,560 \\
1946 & 1,000 & 810 & 550 & 490 & 440 & 550 & 2,000 & 3,700 & 7,100 & 2,500 & 1,100 & 700 & 20,940 \\
1947 & 880 & 740 & 540 & 470 & 360 & 450 & 740 & 6,000 & 8,000 & 5,200 & 1,900 & 1,500 & 26,780 \\
1948 & 1,300 & 910 & 540 & 480 & 450 & 490 & 1,200 & 7,200 & 6,800 & 2,600 & 1,100 & 570 & 23,640 \\
1949 & 740 & 560 & 510 & 470 & 440 & 490 & 1,300 & 4,300 & 8,600 & 4,700 & 1,400 & 1,100 & 24,610 \\
1950 & 1,200 & 840 & 570 & 520 & 500 & 540 & 1,200 & 4,400 & - & - & - & - & 21,230 \\
& & & & & & & & & & & & & \\
1957 & 550 & 470 & 500 & 460 & 400 & 460 & 700 & 3,200 & 11,800 & 8,200 & 2,600 & 1,100 & 30,440 \\
1958 & 820 & 690 & 540 & 500 & 480 & 490 & 600 & 7,600 & 6,600 & 1,400 & 920 & 620 & 21,260 \\
1959 & 550 & 460 & 490 & 460 & 360 & 390 & 610 & 4,000 & 8,500 & 1,900 & 1,100 & 680 & 19,500 \\
1960 & 1,200 & 810 & 530 & 480 & 420 & 570 & 1,500 & 3,700 & 8,000 & 2,500 & 1,200 & 680 & 21,590 \\
1961 & 640 & 530 & 500 & 460 & 370 & 400 & 470 & 4,700 & 4,600 & 1,300 & 1,200 & 2,200 & 17,370 \\
1962 & 1,800 & 1,000 & 590 & 530 & 590 & 640 & 2,100 & 5,000 & 7,400 & 4,000 & 1,400 & 760 & 25,810 \\
1963 & 860 & 470 & 490 & 450 & 390 & 510 & 1,000 & 5,600 & 4,000 & 1,400 & 1,700 & 1,200 & 18,070 \\
1964 & 750 & 590 & 500 & 480 & 430 & 440 & 580 & 5,200 & 4,900 & 2,800 & 1,700 & 880 & 19,250 \\
1965 & 700 & 570 & 530 & 490 & 450 & 470 & 950 & 4,700 & 9,400 & 6,600 & 2,800 & 1,600 & 29,260 \\
\hline
\end{tabular}



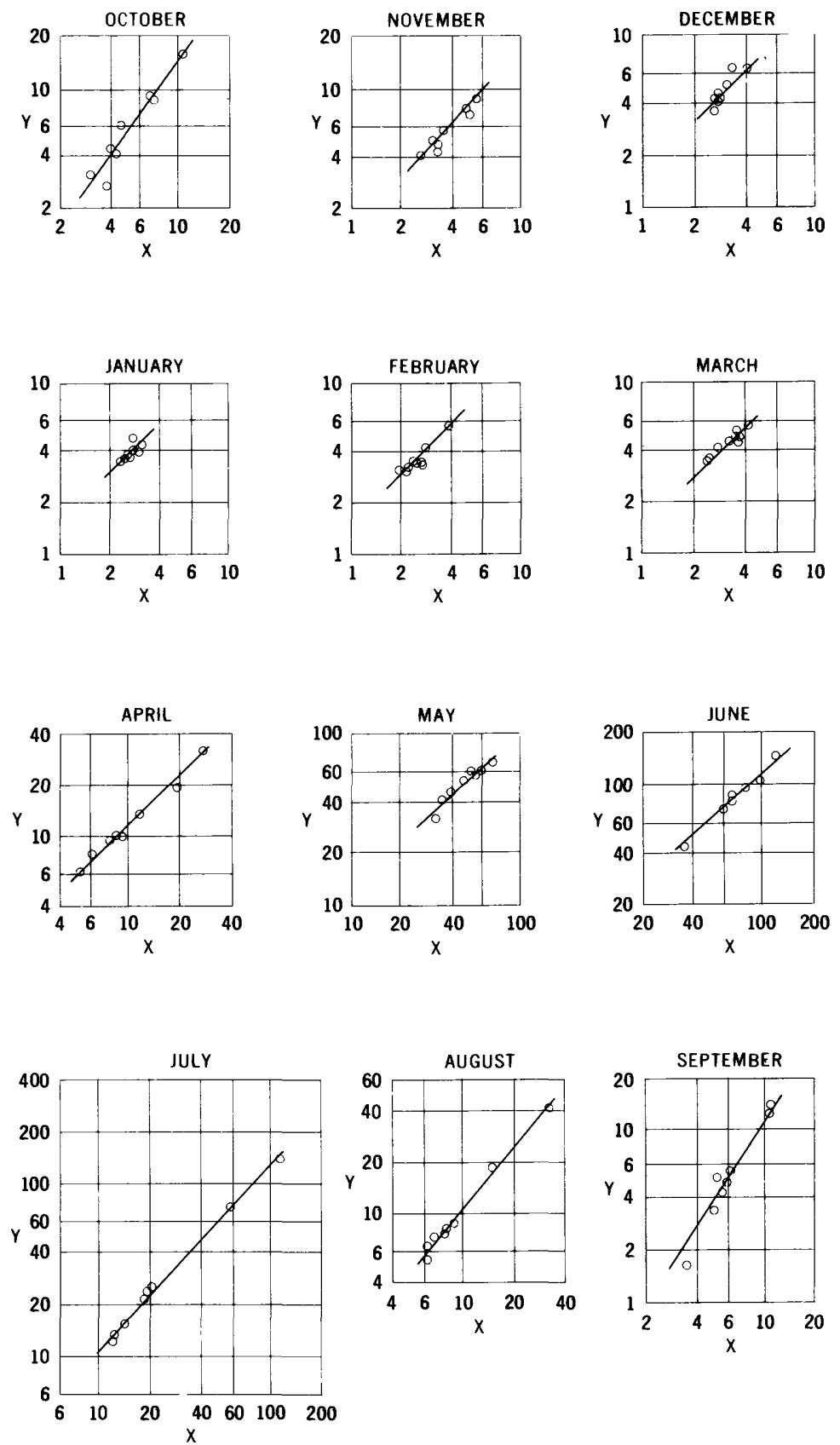

Relationships of monthly mean discharge of Crystal River near Redstone, Colo. (Y), to monthly mean discharge of Crystal River above Avalanche Creek near Redstone, Colo. (X). Discharge in thousands of acre-feet. 
825. Crystal River near Redstone, Colo.

Location. - Lat $39^{\circ} 18^{\prime}$, long $107^{\circ} 13^{\prime}$, in NE $\frac{1}{4}$ sec.9, T.9 S., R. 88 w., on right bank 20 ft downstream from private bridge, $75 \mathrm{ft}$ downstream from Nettle Creek, and 7 miles north of Redstone. Datum of gage is $6,483.77 \mathrm{ft}$ above mean sea level, datum of 1929 .

Drainage area. --220 sq $\mathrm{mi}$.

Records available. - May 1935 to September 1963.

Estimates of streamflow. -October 1963 to September 1965 based on relationships of monthly mean discharge with Crystal River above Avalanche Creek, near Redstone, Colo. The regression equation used is:

$\log Y=b \log X-c$

(where $\mathrm{Y}$ is discharge of Crystal River near Redstone, Colo., and X is discharge of Crystal River above Avalanche Creek, near Redstone, Colo., both' in acre-feet per month).

Monthly values of constants in above equation

\begin{tabular}{l|l|l|l|l|l|l|l|l|l|l|l|l}
\hline & Oct. & Nov. & Dec. & Jan. & Feb. & Mar. & Apr. & May & June & July & Aug. & Sept. \\
\hline $\mathrm{b}$ & 1.37 & 1.02 & 0.98 & 0.99 & 1.01 & 1.02 & 0.98 & -0.16 & 0.87 & 1.06 & 1.19 & 1.48 \\
$\mathrm{c}$ & 1.33 & -.12 & -.26 & -.21 & -.15 & -.07 & -.23 & -.55 & -.72 & .20 & .74 & 1.88 \\
\hline
\end{tabular}

Average discharge. -30 years $(1935-65)$, 256,830 acre-feet per year (355 cfs).

Extremes.--1935-63: Maximum discharge, 4,400 cfs June 21, 1938, from rating curve extended above $2,400 \mathrm{cfs}$; minimum daily, 16 cfs 0ct. 9, 1956.

Remarks. --Diversions above station for irrigation of about 115 acres above and about 2,040 acres below station. Estimates of annul flow are within about 5 percent of regression line.

\begin{tabular}{|c|c|c|c|c|c|c|c|c|c|c|c|c|c|}
\hline $\begin{array}{l}\text { Water } \\
\text { year }\end{array}$ & oct. & Nov. & Dec. & Jan. & Feb. & Mar. & Apr. & May & June & July & Aug. & Sept. & Annual \\
\hline 1935 & - & - & - & - & - & 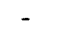 & - & $\neq 33,820$ & 104,400 & 56,540 & 15,660 & 10,160 & - \\
\hline $\begin{array}{l}1936 \\
1937 \\
1938 \\
1939 \\
1910\end{array}$ & $\begin{array}{l}7,220 \\
6,370 \\
6,640 \\
8,260 \\
6,180\end{array}$ & $\begin{array}{l}5,880 \\
5,030 \\
5,510 \\
6,430 \\
4,870\end{array}$ & $\begin{array}{l}5,010 \\
4,370 \\
5,040 \\
5,690 \\
3,900\end{array}$ & $\begin{array}{l}4,250 \\
4,080 \\
4,500 \\
5,040 \\
3,640\end{array}$ & $\begin{array}{l}3,810 \\
4,340 \\
3,630 \\
3,660 \\
3,320\end{array}$ & $\begin{array}{l}5,080 \\
4,910 \\
5,210 \\
5,500 \\
4,610\end{array}$ & $\begin{array}{l}20,380 \\
11,800 \\
17,220 \\
14,350 \\
13,380\end{array}$ & $\begin{array}{l}90,310 \\
78,270 \\
64,870 \\
68,570 \\
65,400\end{array}$ & $\begin{array}{r}93,160 \\
72,760 \\
132,000 \\
63,710 \\
58,850\end{array}$ & $\begin{array}{l}28,380 \\
31,540 \\
66,620 \\
17,840 \\
13,560\end{array}$ & $\begin{array}{r}15,040 \\
11,410 \\
17,600 \\
5,770 \\
6,170\end{array}$ & $\begin{array}{r}8,220 \\
9,210 \\
12,900 \\
9,690 \\
10,490\end{array}$ & $\begin{array}{l}286,700 \\
244,100 \\
341,700 \\
214,500 \\
194,400\end{array}$ \\
\hline $\begin{array}{l}1941 \\
1942 \\
1943 \\
1944 \\
1945\end{array}$ & $\begin{array}{r}10,310 \\
18,780 \\
5,240 \\
5,960 \\
7,190\end{array}$ & $\begin{array}{l}6,200 \\
9,740 \\
5,410 \\
5,120 \\
5,940\end{array}$ & & $\begin{array}{l}4,240 \\
4,820 \\
3,880 \\
4,410 \\
3,840\end{array}$ & $\begin{array}{l}4,020 \\
4,210 \\
3,660 \\
3,570 \\
3,600\end{array}$ & $\begin{array}{l}5,050 \\
4,800 \\
4,940 \\
3,950 \\
4,560\end{array}$ & $\begin{array}{r}10,210 \\
22,190 \\
22,800 \\
7,010 \\
7,130\end{array}$ & $\begin{array}{l}93,290 \\
58,930 \\
55,250 \\
56,960 \\
61,630\end{array}$ & $\begin{array}{r}99,830 \\
108,600 \\
104,400 \\
106,000 \\
82,090\end{array}$ & $\begin{array}{l}46,470 \\
45,930 \\
44,930 \\
69,050 \\
64,970\end{array}$ & $\begin{array}{l}13,030 \\
10,920 \\
17,270 \\
12,100 \\
15,520\end{array}$ & $\begin{array}{r}11,680 \\
4,970 \\
6,910 \\
4,400 \\
5,990\end{array}$ & $\begin{array}{l}309,200 \\
300,800 \\
278,900 \\
283,300 \\
266,400\end{array}$ \\
\hline $\begin{array}{l}1946 \\
1947 \\
1948 \\
1949 \\
1950\end{array}$ & $\begin{array}{r}6,330 \\
5,660 \\
11,470 \\
7,700 \\
6,400\end{array}$ & $\begin{array}{l}6,300 \\
6,040 \\
7,690 \\
5,250 \\
5,970\end{array}$ & $\begin{array}{l}5, \\
4, \\
5, \\
4, \\
5,\end{array}$ & $\begin{array}{l}1,350 \\
4,160 \\
4,720 \\
4,080 \\
4,380\end{array}$ & $\begin{array}{l}3,790 \\
3,630 \\
4,800 \\
4,040 \\
3,770\end{array}$ & $\begin{array}{l}4,660 \\
5,220 \\
5,100 \\
5,010 \\
4,050\end{array}$ & $\begin{array}{l}21,400 \\
11,030 \\
13,050 \\
14,000 \\
11,440\end{array}$ & $\begin{array}{l}53,070 \\
57,130 \\
68,730 \\
38,540 \\
34,360\end{array}$ & $\begin{array}{r}92,760 \\
79,680 \\
92,750 \\
103,900 \\
117,100\end{array}$ & $\begin{array}{l}23 \\
72 \\
40 \\
59 \\
40\end{array}$ & $\begin{array}{r}7,810 \\
18,480 \\
12,330 \\
9,970 \\
7,190\end{array}$ & $\left\{\begin{array}{r}6,130 \\
10,650 \\
5,100 \\
5,410 \\
4,980\end{array}\right.$ & $\begin{array}{l}236,000 \\
279,100 \\
271,700 \\
262,400 \\
215,600\end{array}$ \\
\hline $\begin{array}{l}1951 \\
1952 \\
1953 \\
1951 \\
1955\end{array}$ & $\begin{array}{r}4,380 \\
5,230 \\
4,430 \\
5,130 \\
10,390\end{array}$ & $\begin{array}{l}5,660 \\
5,080 \\
5,140 \\
5,800\end{array}$ & $\begin{array}{l}4,810 \\
4,910 \\
6,130 \\
4,190 \\
4,890\end{array}$ & $\begin{array}{l}4,300 \\
4,390 \\
5,150 \\
3,990 \\
4,150\end{array}$ & $\begin{array}{l}3,730 \\
3,670 \\
4,260 \\
3,380 \\
3,340\end{array}$ & $\begin{array}{l}4,250 \\
3,800 \\
5,750 \\
3,390 \\
3,930\end{array}$ & $\begin{array}{r}8,690 \\
21,340 \\
7,280 \\
10,510 \\
11,080\end{array}$ & $\begin{array}{l}48,100 \\
70,470 \\
23,860 \\
37,920 \\
43,210\end{array}$ & $\begin{array}{r}99,450 \\
140,700 \\
104,100 \\
37,890 \\
76,840\end{array}$ & $\begin{array}{l}55,780 \\
64,000 \\
30,590 \\
18,140 \\
36,720\end{array}$ & $\begin{array}{r}12,700 \\
23,370 \\
10,660 \\
5,440 \\
10,470\end{array}$ & $\begin{array}{l}4,790 \\
8,640 \\
4,130 \\
7,440 \\
2,390\end{array}$ & $\begin{array}{l}256,200 \\
356,200 \\
211,100 \\
142,500 \\
213,200\end{array}$ \\
\hline $\begin{array}{l}1956 \\
1957 \\
1958 \\
1959 \\
1960\end{array}$ & $\begin{array}{l}2,660 \\
3,140 \\
8,680 \\
4,080 \\
8,410\end{array}$ & $\begin{array}{l}4,240 \\
4,090 \\
7,680 \\
4,920 \\
6,900\end{array}$ & $\begin{array}{l}4,220 \\
3,590 \\
6,370 \\
4,430 \\
5,060\end{array}$ & $\begin{array}{l}3,780 \\
3,870 \\
4,380 \\
3,670 \\
4,020\end{array}$ & $\begin{array}{l}3,240 \\
3,470 \\
4,210 \\
3,160 \\
3,480\end{array}$ & $\begin{array}{l}4,770 \\
4,550 \\
4,960 \\
3,680 \\
5,280\end{array}$ & $\begin{array}{r}13,520 \\
10,170 \\
9,590 \\
7,910 \\
19,160\end{array}$ & $\begin{array}{l}62,140 \\
40,450 \\
68,920 \\
32,950 \\
44,790\end{array}$ & $\begin{array}{r}81,300 \\
140,700 \\
95,820 \\
95,400 \\
85,400\end{array}$ & $\begin{array}{r}15,570 \\
138,400 \\
23,250 \\
21,200 \\
24,990\end{array}$ & $\begin{array}{r}5,510 \\
41,520 \\
8,070 \\
8,780 \\
7,600\end{array}$ & $\begin{array}{r}1,650 \\
12,110 \\
4,940 \\
4,330 \\
3,370\end{array}$ & $\begin{array}{l}202,600 \\
406,000 \\
246,900 \\
194,500 \\
218,500\end{array}$ \\
\hline $\begin{array}{l}1961 \\
1962 \\
1963\end{array}$ & $\begin{array}{r}4,390 \\
14,760 \\
6,020\end{array}$ & $\begin{array}{l}4,820 \\
8,760 \\
5,780\end{array}$ & $\begin{array}{l}4,120 \\
5,380 \\
4,380\end{array}$ & $\begin{array}{l}3,560 \\
4,670 \\
3,790\end{array}$ & $\begin{array}{l}3,150 \\
5,610 \\
3,560\end{array}$ & $\begin{array}{l}4,240 \\
5,770 \\
4,500\end{array}$ & $\begin{array}{r}6,250 \\
31,040 \\
9,930\end{array}$ & $\begin{array}{l}52,430 \\
58,430 \\
61,130\end{array}$ & $\begin{array}{r}71,950 \\
106,800 \\
44,680\end{array}$ & $\begin{array}{l}13,240 \\
72,970 \\
12,170\end{array}$ & $\begin{array}{r}6,160 \\
18,130 \\
7,290\end{array}$ & $\begin{array}{r}13,440 \\
5,780 \\
5,150\end{array}$ & $\begin{array}{l}188,000 \\
339,100 \\
168,400\end{array}$ \\
\hline
\end{tabular}

* Estimated for 1950 Compilation.

Estimated monthly and annual streamflow, in acre-feet

\begin{tabular}{|c|c|c|c|c|c|c|c|c|c|c|c|c|c|}
\hline $\begin{array}{l}\text { Water } \\
\text { year }\end{array}$ & Oct. & Nov. & Dec. & Jan. & Feb. & Mar. & Apr. & May & June & July & Aug. & Sept. & Annual \\
\hline $\begin{array}{l}1964 \\
1965\end{array}$ & $\begin{array}{l}3,100 \\
3,900\end{array}$ & $\begin{array}{l}4,500 \\
5,000\end{array}$ & $\begin{array}{l}3,700 \\
4,300\end{array}$ & $\begin{array}{l}3,100 \\
4,200\end{array}$ & $\begin{array}{l}2,400 \\
3,700\end{array}$ & $\begin{array}{l}2,700 \\
3,900\end{array}$ & $\begin{array}{r}6,000 \\
12,800\end{array}$ & $\begin{array}{l}55,000 \\
50,200\end{array}$ & $\begin{array}{r}78,800 \\
112,000\end{array}$ & $\begin{array}{r}33,600 \\
100,000\end{array}$ & $\begin{array}{l}11,400 \\
22,700\end{array}$ & $\begin{array}{r}1,400 \\
15,200\end{array}$ & $\begin{array}{l}208,700 \\
337,900\end{array}$ \\
\hline
\end{tabular}



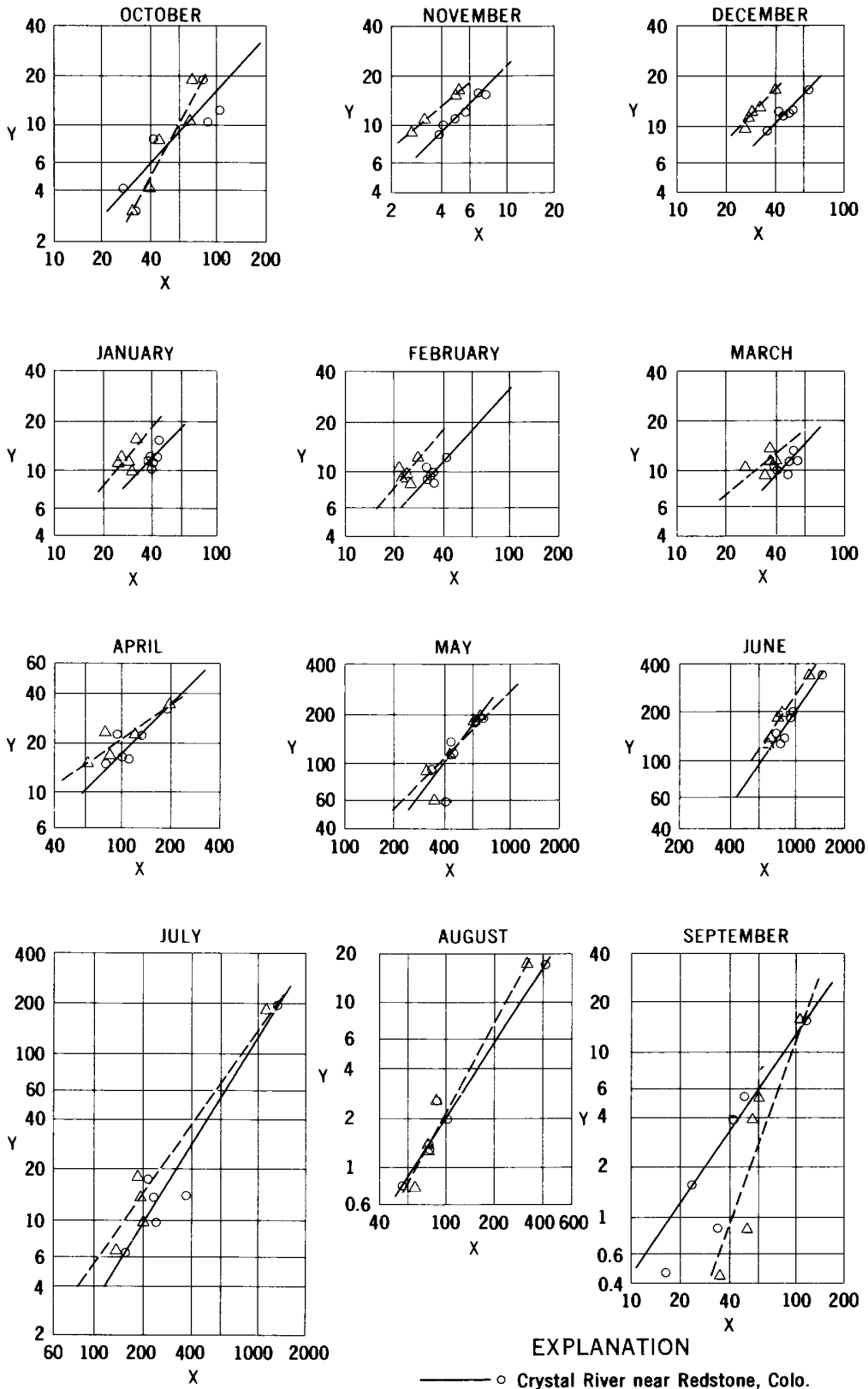

Crystal River near Redstone, Colo.

$-\ldots$ Crystal River above Avalanche Creek near Redstone, Colo.

Relationships of monthly mean discharge of Canyon Creek near New Castle, Colo. (Y), to monthly mean discharge of Crystal River near Redstone, Colo., or Crystal River above Avalanche Creek near Redstone, Colo. (X). Discharge in hundreds of acre-feet. 
855. Canyon Creek near New Castle, Colo.

Location. --Lat $39^{\circ} 34^{1} 30^{\prime \prime}$, long 107\%26'50", in NWli sec.36, T.5 S., R.90 W., on left bank $20 \mathrm{f}^{\prime} \mathrm{u}$ utream from the Denver \& Rio Grande Western Railroad Co. bridge $250 \mathrm{ft}$ upstream from mouth, and 5 miles east of New Castle. Altitude of gage is 5,620 ft (from riverprofile map).

Drainage area. $--54.3 \mathrm{sq} \mathrm{mi}$.

Records available. - - october 1954 to September 1960.

Estimates of streamflow.--May 1935 to September 1954, October 1960 to September 1963, based on relationships of monthly mean discharge with Crystal River near Redstone, Colo.; October 1963 to September 1965, based on relationships of monthly mean discharge with Crystal River above Avalanche Creek near Redstone, Colo. The regression equation used is:

$\log Y=b \log X-c$

(where $Y$ is discharge of Canyon Creek near New Castle, Colo, and $X$ is discharge of Crystal River near Redstone, Colo., or Crystal River ábove Ávalanche Creek near Redstone, colo., all in acre-feet per month).

\begin{tabular}{|c|c|c|c|c|c|c|c|c|c|c|c|c|c|}
\hline & & oct. & Nov. & Dec. & Jan. & Feb. & Mar. & $\mathrm{Apr}$ & May & June & July & Aug. & Sept. \\
\hline $\begin{array}{l}\text { May } 1935 \text { to } \\
\text { Sept } 1954 \\
\text { oct } 1960 \text { to } \\
\text { Sept } 1963\end{array}$ & c & $\begin{array}{l}1.10 \\
1.19\end{array}$ & $\begin{array}{r}0.99 \\
.58\end{array}$ & $\begin{array}{r}1.01 \\
.62\end{array}$ & & & $\begin{array}{r}1.06 \\
.83\end{array}$ & $\begin{array}{r}1.00 \\
.78\end{array}$ & $\begin{array}{r}1.00 \\
.56\end{array}$ & $\begin{array}{l}1.43 \\
2.85\end{array}$ & $\begin{array}{l}1.58 \\
3.82\end{array}$ & $\begin{array}{l}1.53 \\
3.81\end{array}$ & $\begin{array}{l}1.41 \\
2.56\end{array}$ \\
\hline $\begin{array}{l}\text { Oct } 1963 \\
\text { to } \\
\text { Sept } 1965\end{array}$ & b & $\begin{array}{l}1.91 \\
4.18\end{array}$ & $\begin{array}{r}0.85 \\
-.06\end{array}$ & $\begin{array}{r}1.04 \\
.52\end{array}$ & $\begin{array}{l}1.19 \\
1.02\end{array}$ & $\begin{array}{l}1.19 \\
1.02\end{array}$ & $\begin{array}{r}0.81 \\
-.19\end{array}$ & $\begin{array}{c}0.71 \\
-.47\end{array}$ & & $\begin{array}{l}1.47 \\
2.95\end{array}$ & $\begin{array}{l}1.43 \\
3.00\end{array}$ & $\begin{array}{l}1.80 \\
4.90\end{array}$ & $\begin{array}{l}2.86 \\
8.35\end{array}$ \\
\hline
\end{tabular}

Average discharge. --30 years $(1935-65), 47,510$ acre-feet per year $(65.6$ cf's).

Extremes.--1954-60: Maximum discharge, about 1,000 ef's June 29, 1957; minimum daily, 0.5 ef's oct. 8-10, 1956 .

Remarks.--Diversions above station for irrigation of about 1,100 acres, of which about 800 acres is along the Colorado River. Estimates of annual flow are within about 10 percent of regression line.

\begin{tabular}{c|r|r|l|r|r|r|r|r|r|r|r|r|r|r}
\hline $\begin{array}{l}\text { Water } \\
\text { year }\end{array}$ & Oct. & Nov. & Dec. & Jan. & Feb. & Mar. & Apr. & May & June & July & Aug. & Sept. & Annual \\
\hline 1955 & 1,230 & 1,210 & 1,220 & 1,160 & 930 & 974 & 1,550 & 13,250 & 14,650 & 1,370 & 195 & 155 & 37,890 \\
& & & & & & & & & & & & & \\
1956 & 413 & 1,040 & 1,240 & 1,190 & 918 & 1,130 & 2,200 & 17,870 & 12,470 & 629 & 78 & 47 & 39,220 \\
1957 & 307 & 916 & 946 & 978 & 835 & 932 & 1,610 & 5,850 & 33,220 & 19,090 & 1,730 & 1,550 & 67,960 \\
1958 & 1,060 & 1,580 & 1,670 & 1,500 & 1,200 & 1,330 & 2,230 & 18,690 & 19,600 & 1,360 & 125 & 529 & 50,870 \\
1959 & 823 & 1,120 & 1,140 & 1,090 & 1,040 & 1,060 & 1,450 & 9,030 & 18,420 & 1,730 & 257 & 388 & 37,550 \\
1960 & 1,920 & 1,610 & 1,290 & 1,130 & 960 & 1,150 & 3,230 & 10,140 & 13,450 & 957 & 139 & 85 & 36,060 \\
\hline
\end{tabular}

Estimated monthly and annual streamflow, in acre-feet

\begin{tabular}{|c|c|c|c|c|c|c|c|c|c|c|c|c|c|}
\hline $\begin{array}{l}\text { Vater } \\
\text { year }\end{array}$ & Oct. & Nov. & ec. & Jan. & $\mathrm{Feb}$. & Mar. & Apr. & May & June & July & Aug. & ept. & Annual \\
\hline 1935 & - & - & - & - & - & - & - & 0 & 200 & 0 & 380 & 0 & - \\
\hline $\begin{array}{l}1936 \\
1937 \\
1938 \\
1939 \\
1940\end{array}$ & $\begin{array}{r}1,100 \\
1,000 \\
1,000 \\
1,300 \\
960\end{array}$ & $\begin{array}{l}1,300 \\
1,200 \\
1,200 \\
1,500\end{array}$ & $\begin{array}{l}1,300 \\
1,100 \\
1,300 \\
1,500 \\
1,000\end{array}$ & $\begin{array}{l}1,200 \\
1,200 \\
1,300 \\
1,500 \\
1,100\end{array}$ & $\begin{array}{r}1,100 \\
1,200 \\
1,000 \\
1,000 \\
940\end{array}$ & $\begin{array}{l}1,200 \\
1,200 \\
1,300 \\
1,400 \\
1,100\end{array}$ & $\begin{array}{l}3,400 \\
2,000 \\
2,900 \\
2,400 \\
2,200\end{array}$ & & & & $\begin{array}{r}360 \\
230 \\
460 \\
80 \\
90\end{array}$ & & $\begin{array}{l}55,740 \\
45,830 \\
64,960 \\
41,440 \\
37,280\end{array}$ \\
\hline $\begin{array}{l}1941 \\
1942 \\
1943 \\
1944 \\
1945\end{array}$ & $\begin{array}{r}1,700 \\
3,300 \\
810 \\
930 \\
1,100\end{array}$ & $\begin{array}{l}1,400 \\
2,200 \\
1,200 \\
1,200 \\
1,400\end{array}$ & $\begin{array}{l}1,300 \\
1,800 \\
1,100 \\
1,200 \\
1,000\end{array}$ & $\begin{array}{l}1,200 \\
1,400 \\
1,200 \\
1,300 \\
1,100\end{array}$ & & $\begin{array}{r}1,300 \\
1,200 \\
1,200 \\
950 \\
1,100\end{array}$ & & & & & $\begin{array}{l}290 \\
220 \\
440 \\
260 \\
380\end{array}$ & $\begin{array}{r}1,400 \\
430 \\
690 \\
360 \\
560\end{array}$ & $\begin{array}{l}62,500 \\
57,050 \\
50,940 \\
51,900 \\
46,240\end{array}$ \\
\hline $\begin{array}{l}1946 \\
1947 \\
1948 \\
1949\end{array}$ & $\begin{array}{r}990 \\
880 \\
1,900 \\
1,200 \\
1,000\end{array}$ & $\begin{array}{l}1,400 \\
1,400 \\
1,800 \\
1,200 \\
1,400\end{array}$ & $\begin{array}{l}500 \\
200 \\
500 \\
200 \\
300\end{array}$ & $\begin{array}{l}1,300 \\
1,200 \\
1,400 \\
1,200 \\
1,300\end{array}$ & & $\begin{array}{r}1,100 \\
1,300 \\
1,200 \\
1,200 \\
980\end{array}$ & & $\begin{array}{r}14,300 \\
15,400 \\
18,700 \\
10,300 \\
9,300\end{array}$ & & & $\begin{array}{l}130 \\
490 \\
260 \\
190 \\
110\end{array}$ & $\begin{array}{r}580 \\
1,300 \\
450 \\
490 \\
430\end{array}$ & $\begin{array}{l}47,370 \\
51,410 \\
46,880 \\
46,720\end{array}$ \\
\hline $\begin{array}{l}1951 \\
1952 \\
1953 \\
1954\end{array}$ & $\begin{array}{l}660 \\
800 \\
670 \\
790\end{array}$ & $\begin{array}{l}1,200 \\
1,300 \\
1,200 \\
1,200\end{array}$ & $\begin{array}{l}1,300 \\
1,300 \\
1,600 \\
1,100\end{array}$ & $\begin{array}{l}1,300 \\
1,300 \\
1,500 \\
1,100\end{array}$ & $\begin{array}{r}1,000 \\
1,200 \\
950\end{array}$ & $\begin{array}{r}1,000 \\
920 \\
1,400 \\
800\end{array}$ & $\begin{array}{l}1,500 \\
3,600 \\
1,200 \\
1,800\end{array}$ & $\begin{array}{r}13,000 \\
19,200 \\
6,400 \\
10,200\end{array}$ & $\begin{array}{r}19,400 \\
32,200 \\
21,200 \\
4,900\end{array}$ & $\begin{array}{r}5,700 \\
1,800 \\
780\end{array}$ & $\begin{array}{r}280 \\
700 \\
210 \\
80\end{array}$ & $\begin{array}{l}960 \\
330 \\
770\end{array}$ & $\begin{array}{l}68,980 \\
38,710 \\
24,470\end{array}$ \\
\hline $\begin{array}{l}1961 \\
1962 \\
1963 \\
1964 \\
1965\end{array}$ & $\begin{array}{r}660 \\
2,500 \\
940 \\
350 \\
490\end{array}$ & $\begin{array}{l}1,100 \\
2,000 \\
1,300 \\
1,000 \\
1,100\end{array}$ & $\begin{array}{r}1,100 \\
1,700 \\
1,200 \\
970 \\
1,100\end{array}$ & $\begin{array}{r}1,000 \\
1,400 \\
1,100 \\
860 \\
1,200\end{array}$ & $\begin{array}{r}880 \\
1,600 \\
1,000 \\
630 \\
1,000\end{array}$ & $\begin{array}{r}1,000 \\
1,400 \\
1,100 \\
740 \\
970\end{array}$ & $\begin{array}{l}1,000 \\
5,200 \\
1,700 \\
1,200 \\
2,200\end{array}$ & $\begin{array}{l}21,800 \\
16,600 \\
13,600 \\
12,000\end{array}$ & $\begin{array}{r}12,300 \\
22,000 \\
6,200 \\
12,800 \\
23,200\end{array}$ & $\begin{array}{r}470 \\
7,000 \\
410 \\
2,400 \\
10,300\end{array}$ & $\begin{array}{l}100 \\
470 \\
120 \\
230 \\
660 \\
\end{array}$ & $\begin{array}{r}1,700 \\
540 \\
460 \\
230 \\
2,200\end{array}$ & $\begin{array}{l}67,610 \\
32,130 \\
34,810 \\
56,420\end{array}$ \\
\hline
\end{tabular}

* Based on estimates for 1950 compilation. 

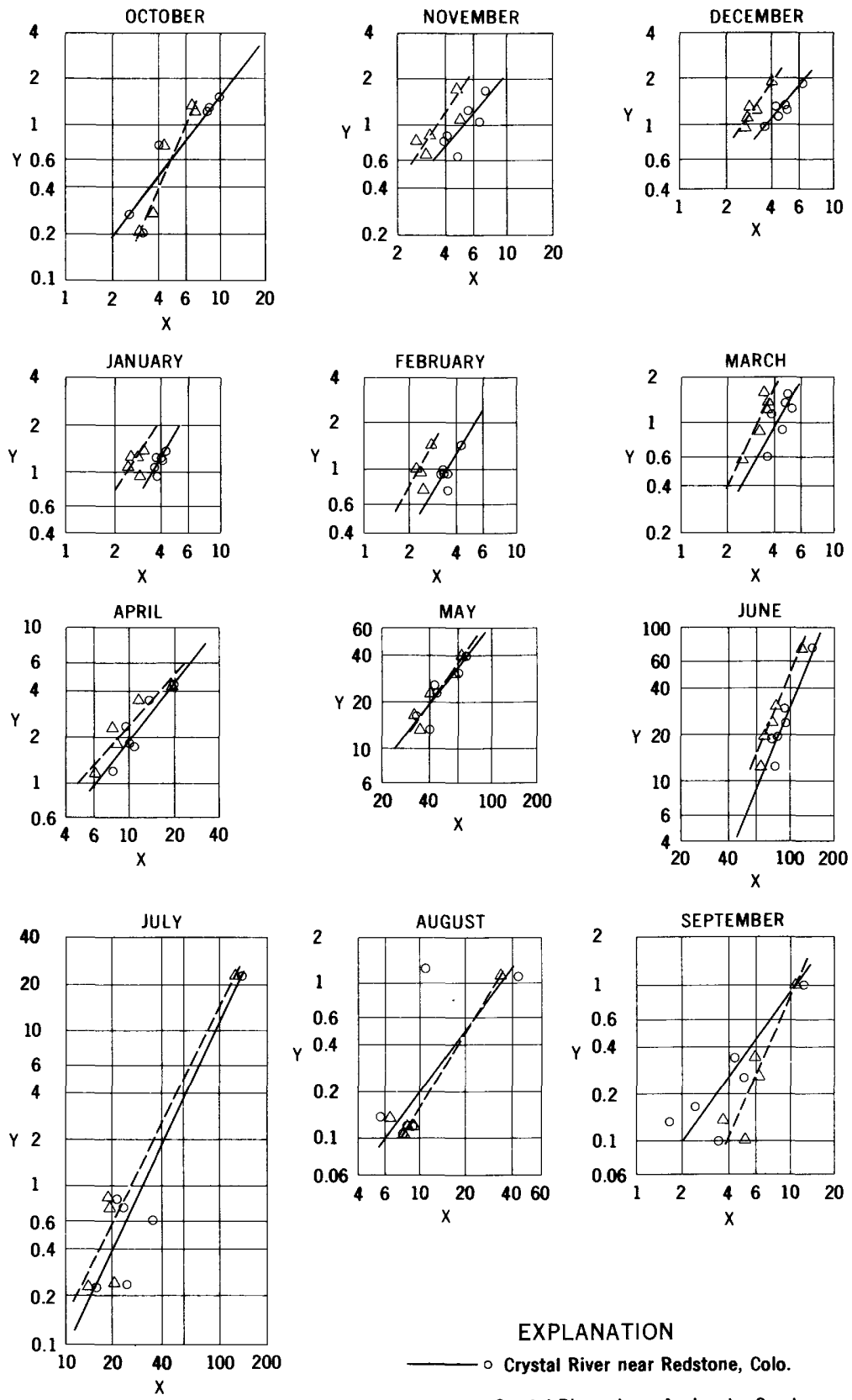

\section{EXPLANATION}

— C Crystal River near Redstone, Colo.

$--\triangle$ Crystal River above Avalanche Creek near Redstone, Colo.

Relationships of monthly mean discharge of Elk Creek at New Castle, Colo. (Y), to monthly mean discharge of Crystal River near Redstone, Colo., or Crystal River above Avalanche Creek near Redstone, Colo. (X). Discharge in thousands of acre-feet. 
875. Elk Creek at New Castle, Colo.

Location. --Lat 39.34'10", long 107.32140", in SWl sec.31, T.5 S., R.90 W., on left bank at upstream side of the Denver \& Rio Grande Western Rallroad Co. bridge in New Castle, $300 \mathrm{ft}$ upstream from mouth. Altitude of gage is $5,550 \mathrm{ft}$ (from river-profile map).

Drainage area. $--177 \mathrm{sq} \mathrm{mi}$.

Records available, --March 1922 to September 1924, October 1954 to September 1960.

Estimates of streamflow.--May 1935 to September 1954, October 1960 to September 1963, based on relationships of monthly mean discharge with Crystal River near Redstone, Colo; October 1963 to September 1965, based on relationships with Crystal RIver above Avalanche Creek, near Redstone, Colo. The regression equation used is:

$\log \mathrm{Y}=\mathrm{b} \log \mathrm{X}-\mathrm{c}$

(where $\mathrm{Y}$ is discharge of EIk Creek at New Castle, Colo, and $\mathrm{X}$ is discharge of Crystal River near Redstone, Colo., or Crystal River above Avalanche Creek, near Redstone, Colo., all in acre-feet per month).

Monthly values of constants in above equation

\begin{tabular}{|c|c|c|c|c|c|c|c|c|c|c|c|c|c|}
\hline & & Oct. & Nov. & Dec. & Jan. & Feb. & Mar. & Apr. & May & June & July & Aug. & Sept. \\
\hline $\begin{array}{l}\text { May } 1935 \\
\text { to } \\
\text { Sept } 1963\end{array}$ & c & $\begin{array}{l}1.31 \\
2.05\end{array}$ & $\begin{array}{l}1.15 \\
1.28\end{array}$ & $\begin{array}{l}1.15 \\
1.11\end{array}$ & $\begin{array}{l}1.62 \\
2.74\end{array}$ & $\begin{array}{l}1.62 \\
2.74\end{array}$ & $\begin{array}{l}1.70 \\
3.16\end{array}$ & $\begin{array}{l}1.27 \\
1.81\end{array}$ & $\begin{array}{l}1.27 \\
1.56\end{array}$ & $\begin{array}{r}2.43 \\
7.71\end{array}$ & $\begin{array}{l}2.10 \\
6.45\end{array}$ & $\begin{array}{l}1.36 \\
3.14\end{array}$ & $\begin{array}{l}1.36 \\
2.49\end{array}$ \\
\hline $\begin{array}{l}\text { Oct } 1963 \\
\text { to } \\
\text { Sept } 1965\end{array}$ & $b$ & $\begin{array}{l}2.26 \\
5.57\end{array}$ & $\begin{array}{l}1.47 \\
2.23\end{array}$ & $\begin{array}{l}1.41 \\
1.81\end{array}$ & $\begin{array}{l}1.52 \\
2.14\end{array}$ & $\begin{array}{l}1.78 \\
2.96\end{array}$ & $\begin{array}{l}1.12 \\
1.10\end{array}$ & $\begin{array}{l}1.34 \\
2.00\end{array}$ & $\begin{array}{l}1.40 \\
2.14\end{array}$ & $\begin{array}{l}2.02 \\
5.93\end{array}$ & $\begin{array}{l}1.64 \\
4.33\end{array}$ & $\begin{array}{l}1.64 \\
4.33\end{array}$ & $\begin{array}{l}2.22 \\
5.95\end{array}$ \\
\hline
\end{tabular}

Average discharge. - -30 years $(1935-65), 70,470$ acre-feet per year (97.3 cfs).

Extremes. - -1922-24, 1954-60: Maximum discharge, 1,770 cfs June 10, 1957; minimum daily. 1.I cfs Sept. 11, 1958, July 17, 18, 1960.

Remarks.--Diversions above station for irrigation of about 3,500 acres, of which about 1,500 acres is along the Colorado River. Estimates of annual flow are within about 25 percent of regression line.

\begin{tabular}{l|r|r|r|r|r|r|r|r|r|r|r|r|r}
\hline $\begin{array}{l}\text { Water } \\
\text { year }\end{array}$ & Oct. & Nov. & Dec. & Jan. & Feb. & Mar. & Apr. & May & June & July & Aug. & Sept. & Annual \\
\hline 1955 & 1,530 & 1,280 & 1,300 & 1,190 & 946 & 1,130 & 1,720 & 26,350 & 18,560 & 614 & 1,260 & 168 & 56,050 \\
& & & & & & & & & & & \\
1956 & 267 & 857 & 1,300 & 1,240 & 998 & 1,350 & 3,460 & 30,670 & 12,330 & 227 & 138 & 133 & 52,970 \\
1957 & 201 & 804 & 962 & 950 & 734 & 887 & 1,820 & 13,570 & 71,660 & 22,710 & 1,130 & 999 & 116,400 \\
1958 & 1,300 & 1,690 & 1,860 & 1,380 & 1,460 & 1,550 & 2,360 & 39,750 & 29,620 & 735 & 120 & 252 & 82,080 \\
1959 & 729 & 645 & 1,120 & 1,090 & 932 & 590 & 1,220 & 16,200 & 23,900 & 846 & 119 & 337 & 47,730 \\
1960 & 1,250 & 1,080 & 1,240 & 1,240 & 952 & 1,210 & 4,270 & 23,320 & 19,090 & 233 & 108 & 98 & 54,090 \\
\hline
\end{tabular}

Estimated monthly and annual streamilow, in acre-feet

\begin{tabular}{|c|c|c|c|c|c|c|c|c|c|c|c|c|c|}
\hline $\begin{array}{l}\text { Water } \\
\text { year }\end{array}$ & Oct. & Nov. & ec. & an. & eb. & Mar. & Apr. & May & June & July & Aug. & Sept. & Annual \\
\hline 1935 & - & - & - & - & - & - & - & $\pm 15,500$ & 31,400 &, 400 & 370 & 900 & - \\
\hline $\begin{array}{l}1936 \\
1937 \\
1938 \\
1939 \\
1940\end{array}$ & $\begin{array}{r}1,000 \\
850 \\
900 \\
1,200 \\
820\end{array}$ & $\begin{array}{r}1,100 \\
950 \\
1,000 \\
1,300 \\
910\end{array}$ & $\begin{array}{l}1,400 \\
1,200 \\
1,400 \\
1,600 \\
1,000\end{array}$ & $\begin{array}{l}1,400 \\
1,300 \\
1,500 \\
1,800 \\
1,100\end{array}$ & $\begin{array}{r}1,100 \\
1,400 \\
1,100 \\
1,100 \\
920\end{array}$ & $\begin{array}{l}1,400 \\
1,300 \\
1,400 \\
1,600 \\
1,200\end{array}$ & $\begin{array}{l}4,600 \\
2,300 \\
3,700 \\
2,900 \\
2,700\end{array}$ & $\begin{array}{l}53,700 \\
45,200 \\
36,600 \\
38,200 \\
36,000\end{array}$ & $\begin{array}{r}23,900 \\
13,000 \\
55,300 \\
9,400 \\
7,800\end{array}$ & $\begin{array}{r}780 \\
1,000 \\
4,900 \\
300 \\
1,700\end{array}$ & $\begin{array}{r}350 \\
240 \\
430 \\
90 \\
100\end{array}$ & $\begin{array}{r}680 \\
800 \\
1,100 \\
860 \\
950\end{array}$ & $\begin{array}{r}91,410 \\
69,540 \\
109,330 \\
60,350 \\
55,200\end{array}$ \\
\hline $\begin{array}{l}1941 \\
1942 \\
1943 \\
1944 \\
1945\end{array}$ & $\begin{array}{r}1,600 \\
3,500 \\
660 \\
790 \\
1,000\end{array}$ & $\begin{array}{r}1,200 \\
2,000 \\
1,000 \\
970 \\
1,100\end{array}$ & $\begin{array}{l}1,400 \\
2,000 \\
1,100 \\
1,300 \\
1,100\end{array}$ & $\begin{array}{l}1,400 \\
1,700 \\
1,200 \\
1,500 \\
1,100\end{array}$ & $\begin{array}{l}1,200 \\
1,400 \\
1,100 \\
1,000 \\
1,000\end{array}$ & $\begin{array}{r}1,300 \\
1,300 \\
1,300 \\
1,100 \\
1,100\end{array}$ & $\begin{array}{l}1,900 \\
5,100 \\
5,300 \\
1,200 \\
1,200\end{array}$ & $\begin{array}{l}56,500 \\
31,500 \\
29,000 \\
30,200 \\
33,300\end{array}$ & & $\begin{array}{l}2,200 \\
2,200 \\
2,100 \\
5,200 \\
4,600\end{array}$ & $\begin{array}{l}290 \\
230 \\
420 \\
260 \\
370\end{array}$ & $\begin{array}{r}1,000 \\
340 \\
530 \\
290 \\
430\end{array}$ & $\begin{array}{l}98,090 \\
85,670 \\
75,110 \\
76,110 \\
63,800\end{array}$ \\
\hline $\begin{array}{l}1946 \\
1947 \\
1948 \\
1949 \\
1950\end{array}$ & $\begin{array}{r}850 \\
730 \\
1,800 \\
1,100 \\
860\end{array}$ & $\begin{array}{l}1,200 \\
1,200 \\
1,500 \\
1,000 \\
1,100\end{array}$ & $\begin{array}{l}1,600 \\
1,300 \\
1,600 \\
1,200 \\
1,400\end{array}$ & $\begin{array}{l}1,400 \\
1,300 \\
1,600 \\
1,300 \\
1,400\end{array}$ & $\begin{array}{l}1,100 \\
1,000 \\
1,700 \\
1,300 \\
1,100\end{array}$ & $\begin{array}{r}1,200 \\
1,400 \\
1,400 \\
1,300 \\
940\end{array}$ & $\begin{array}{l}4,900 \\
2,100 \\
2,600 \\
2,900 \\
2,200\end{array}$ & $\begin{array}{l}27,600 \\
30,300 \\
38,300 \\
18,400 \\
15,900\end{array}$ & $\begin{array}{l}23,500 \\
16,200 \\
23,500 \\
31,000 \\
41,300\end{array}$ & $\begin{array}{r}530 \\
5,900 \\
1,700 \\
3,900 \\
1,600\end{array}$ & $\begin{array}{l}140 \\
460 \\
270 \\
200 \\
130\end{array}$ & $\begin{array}{l}450 \\
960 \\
350 \\
390 \\
340\end{array}$ & $\begin{array}{l}64,470 \\
62,850 \\
76,320 \\
63,990 \\
68,270\end{array}$ \\
\hline $\begin{array}{l}1951 \\
1952 \\
1953 \\
1954\end{array}$ & $\begin{array}{l}520 \\
660 \\
530 \\
650\end{array}$ & $\begin{array}{r}950 \\
1,100 \\
950 \\
970\end{array}$ & $\begin{array}{l}1,400 \\
1,400 \\
1,800 \\
1,100\end{array}$ & $\begin{array}{l}1,400 \\
1,400 \\
1,900 \\
1,200\end{array}$ & $\begin{array}{r}1,100 \\
1,100 \\
1,400 \\
950\end{array}$ & $\begin{array}{r}1,000 \\
840 \\
1,700 \\
690\end{array}$ & $\begin{array}{l}1,600 \\
4,900 \\
1,200 \\
2,000\end{array}$ & $\begin{array}{l}24,300 \\
39,500 \\
10,000 \\
18,000\end{array}$ & $\begin{array}{r}27,800 \\
64,700 \\
31,100 \\
2,700\end{array}$ & $\begin{array}{r}, 300 \\
4,500 \\
920 \\
310\end{array}$ & $\begin{array}{r}280 \\
660 \\
220 \\
90\end{array}$ & $\begin{array}{l}320 \\
730 \\
260 \\
590\end{array}$ & $\begin{array}{r}63,970 \\
121,490 \\
51,980 \\
29,250\end{array}$ \\
\hline $\begin{array}{l}1961 \\
1962 \\
1963 \\
1964 \\
1965\end{array}$ & $\begin{array}{r}520 \\
2,600 \\
800 \\
240 \\
350\end{array}$ & $\begin{array}{r}900 \\
1,800 \\
1,100 \\
720 \\
830\end{array}$ & $\begin{array}{r}1,100 \\
1,900 \\
1,200 \\
880 \\
1,100\end{array}$ & $\begin{array}{r}1,000 \\
1,600 \\
1,100 \\
820 \\
1,300\end{array}$ & $\begin{array}{r}840 \\
2,200 \\
1,000 \\
570 \\
1,200\end{array}$ & $\begin{array}{r}1,000 \\
1,700 \\
1,100 \\
380 \\
780\end{array}$ & $\begin{array}{l}1,000 \\
8,000 \\
1,800 \\
1,100 \\
2,600\end{array}$ & $\begin{array}{l}27,200 \\
31,200 \\
33,000 \\
26,800 \\
23,300\end{array}$ & $\begin{array}{r}12,700 \\
33,300 \\
4,000 \\
15,600 \\
41,000\end{array}$ & $\begin{array}{r}170 \\
5,900 \\
130 \\
1,200 \\
9,900\end{array}$ & $\begin{array}{l}110 \\
450 \\
130 \\
180 \\
480\end{array}$ & $\begin{array}{r}1,300 \\
410 \\
360 \\
220 \\
1,400\end{array}$ & $\begin{array}{l}47,840 \\
91,060 \\
45,720 \\
48,710 \\
84,240\end{array}$ \\
\hline
\end{tabular}

* Based on estimates made for 1950 Compilation. 

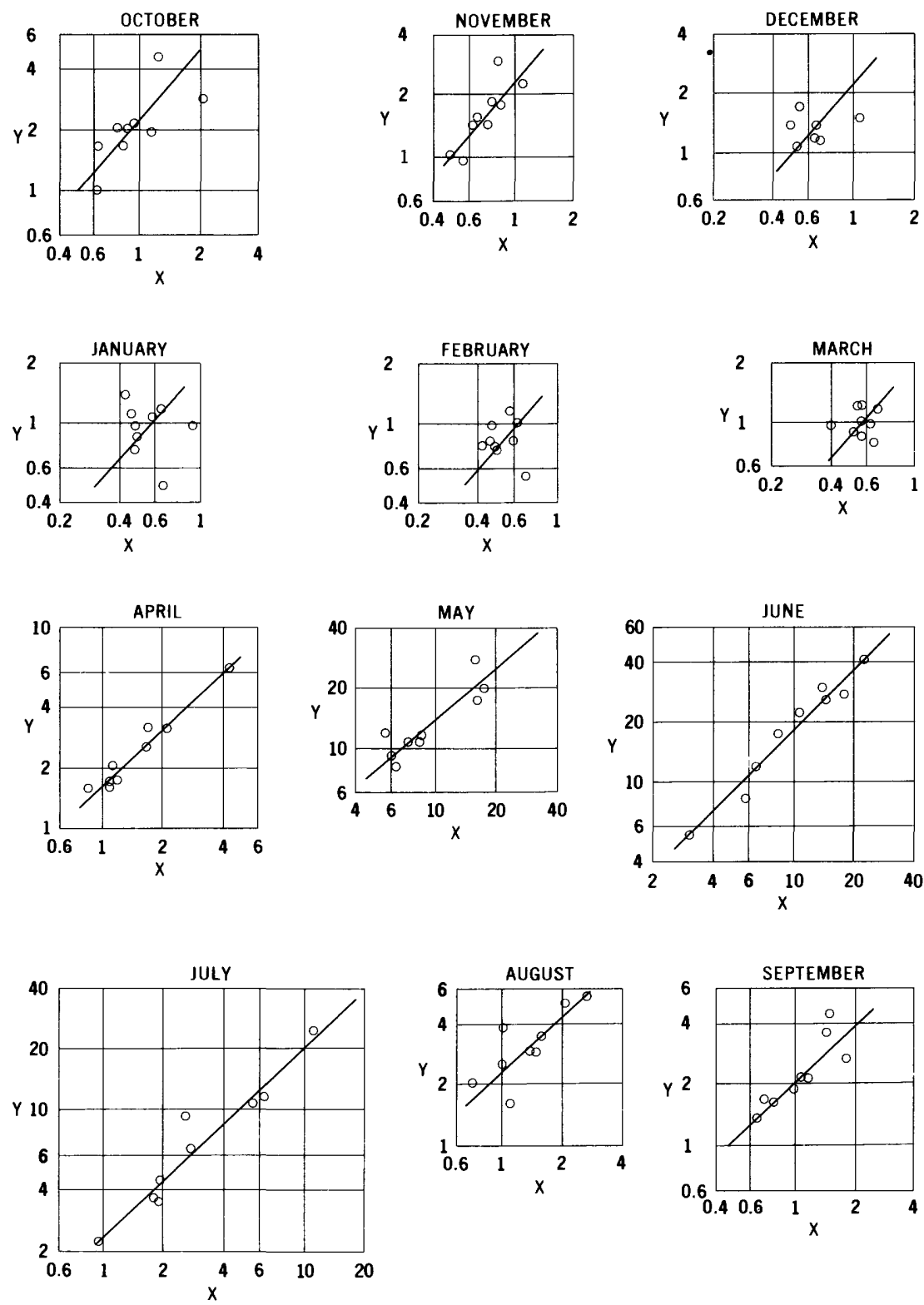

Relationships of monthly mean discharge of Battlement Creek near Grand Valley, Colo. $(\mathrm{Y})$, to monthly mean discharge of Beaver Creek near Rifle, Colo. $(\mathrm{X})$. Discharge in hundreds of acre-feet. 
926. Battlement Creek near Grand Valley, Colo.

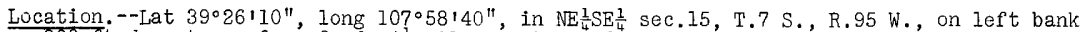
$300 \mathrm{f} t$ downstream from ford, $4 \frac{1}{2}$ miles upstream srom mouth, and 5 'miles southeast of Grand Valley. Altitude of gage is $6,630 \mathrm{ft}$ (from topographic map).

Drainage area. $--10.5 \mathrm{sq} \mathrm{mi}$.

Records avallable. --October 1956 to September 1965.

Estimates of streamilow.--October 1952 to September 1956, based on relationships of monthly mean discharge with Beaver Creek near Rifle, Colo. The regression equation used is:

$$
\log Y=b \log X-c
$$

(where $\mathrm{Y}$ is discharge of Battlement Creek near Grand Valley, Colo., and $\mathrm{X}$ is discharge of Beaver Creek near Rifle, Colo., both in acre-feet per month).

\begin{tabular}{l|l|l|l|l|l|l|l|l|l|l|l|l}
\hline \multicolumn{10}{c}{ Monthly values of constants in above equation } \\
\hline & Oct. & Nov. & Dec. & Jan. & Feb. & Mar. & Apr. & May & June & July & Aug. & Sept. \\
\hline b & 1.15 & 1.15 & 1.15 & 1.15 & 1.14 & 1.14 & 0.96 & 0.85 & 1.01 & 0.94 & 0.90 & 0.94 \\
c & -.04 & -.05 & -.05 & .01 & .04 & -.01 & -.28 & -.59 & -.24 & -.49 & -.57 & -.43 \\
\hline
\end{tabular}

Average discharge. -13 years $(1952-65), 5,469$ acre-feet per year $(7.55 \mathrm{cfs})$.

Extremes.--1956-65: Maximum discharge, $102 \mathrm{cfs}$ June 7, 1957; minimum not determined. Remarks. --Slight regulation by Battlement Reservoir. No diversion above station. Estimates of annual flow are within about 5 percent of regression line.

\begin{tabular}{|c|c|c|c|c|c|c|c|c|c|c|c|c|c|}
\hline $\begin{array}{l}\text { Water } \\
\text { year }\end{array}$ & Oct. & Nov. & Dec. & Jan. & Feb. & Mar. & Apr. & May & June & July & Aug. & Sept. & Annual \\
\hline $\begin{array}{l}1957 \\
1958 \\
1959 \\
1960\end{array}$ & $\begin{array}{l}100 \\
466 \\
218 \\
168\end{array}$ & $\begin{array}{r}95 \\
299 \\
182 \\
102\end{array}$ & $\begin{array}{r}86 \\
172 \\
120 \\
86\end{array}$ & $\begin{array}{r}86 \\
111 \\
98 \\
74\end{array}$ & $\begin{array}{l}83 \\
78 \\
99 \\
75\end{array}$ & $\begin{array}{r}90 \\
123 \\
97 \\
101\end{array}$ & $\begin{array}{l}206 \\
314 \\
159 \\
315\end{array}$ & $\begin{array}{r}1,180 \\
2,810 \\
831 \\
1,160\end{array}$ & $\begin{array}{r}4,090 \\
2,990 \\
833 \\
1,750\end{array}$ & $\begin{array}{r}2,510 \\
926 \\
364 \\
445\end{array}$ & $\begin{array}{l}556 \\
382 \\
251 \\
206\end{array}$ & $\begin{array}{l}446 \\
217 \\
168 \\
132\end{array}$ & $\begin{array}{l}9,530 \\
8,890 \\
3,420 \\
4,610\end{array}$ \\
\hline $\begin{array}{l}1961 \\
1962 \\
1963 \\
1964 \\
1965\end{array}$ & $\begin{array}{l}206 \\
286 \\
196 \\
209 \\
167\end{array}$ & $\begin{array}{l}158 \\
230 \\
145 \\
180 \\
143\end{array}$ & $\begin{array}{l}110 \\
151 \\
118 \\
142 \\
138\end{array}$ & $\begin{array}{r}49 \\
97 \\
109 \\
138 \\
119\end{array}$ & $\begin{array}{r}56 \\
103 \\
119 \\
83 \\
79\end{array}$ & $\begin{array}{r}86 \\
119 \\
122 \\
80 \\
99\end{array}$ & $\begin{array}{l}156 \\
634 \\
250 \\
169 \\
172\end{array}$ & $\begin{array}{r}1,080 \\
2,000 \\
917 \\
1,730 \\
1,070\end{array}$ & $\begin{array}{r}1,220 \\
2,560 \\
543 \\
2,270 \\
2,790\end{array}$ & $\begin{array}{r}346 \\
1,070 \\
219 \\
632 \\
1,160\end{array}$ & $\begin{array}{l}165 \\
350 \\
294 \\
289 \\
508\end{array}$ & $\begin{array}{l}273 \\
192 \\
219 \\
161 \\
369\end{array}$ & $\begin{array}{l}3,900 \\
7,790 \\
3,250 \\
6,080 \\
6,810\end{array}$ \\
\hline
\end{tabular}

\begin{tabular}{|c|c|c|c|c|c|c|c|c|c|c|c|c|c|}
\hline $\begin{array}{l}\text { Water } \\
\text { year }\end{array}$ & oct. & Nov. & Dec. & Jan. & Feb. & Mar. & Apr. & May & June & July & Aug. & Sept. & Annual \\
\hline $\begin{array}{l}1953 \\
1954 \\
1955\end{array}$ & $\begin{array}{l}160 \\
130 \\
240\end{array}$ & $\begin{array}{l}160 \\
110 \\
100\end{array}$ & $\begin{array}{r}160 \\
90 \\
90\end{array}$ & $\begin{array}{r}150 \\
60 \\
70\end{array}$ & $\begin{array}{l}90 \\
50 \\
50\end{array}$ & $\begin{array}{r}110 \\
70 \\
90\end{array}$ & $\begin{array}{l}170 \\
330 \\
150\end{array}$ & $\begin{array}{r}660 \\
1,300 \\
1,400\end{array}$ & $\begin{array}{r}1,800 \\
690 \\
1,900\end{array}$ & $\begin{array}{l}470 \\
360 \\
540\end{array}$ & $\begin{array}{l}250 \\
200 \\
260\end{array}$ & $\begin{array}{l}140 \\
160 \\
140\end{array}$ & $\begin{array}{l}4,320 \\
3,550 \\
5,030\end{array}$ \\
\hline 1956 & 120 & 100 & 100 & 70 & 60 & 90 & 190 & 1,200 & 1,200 & 400 & 290 & 100 & 3,920 \\
\hline
\end{tabular}



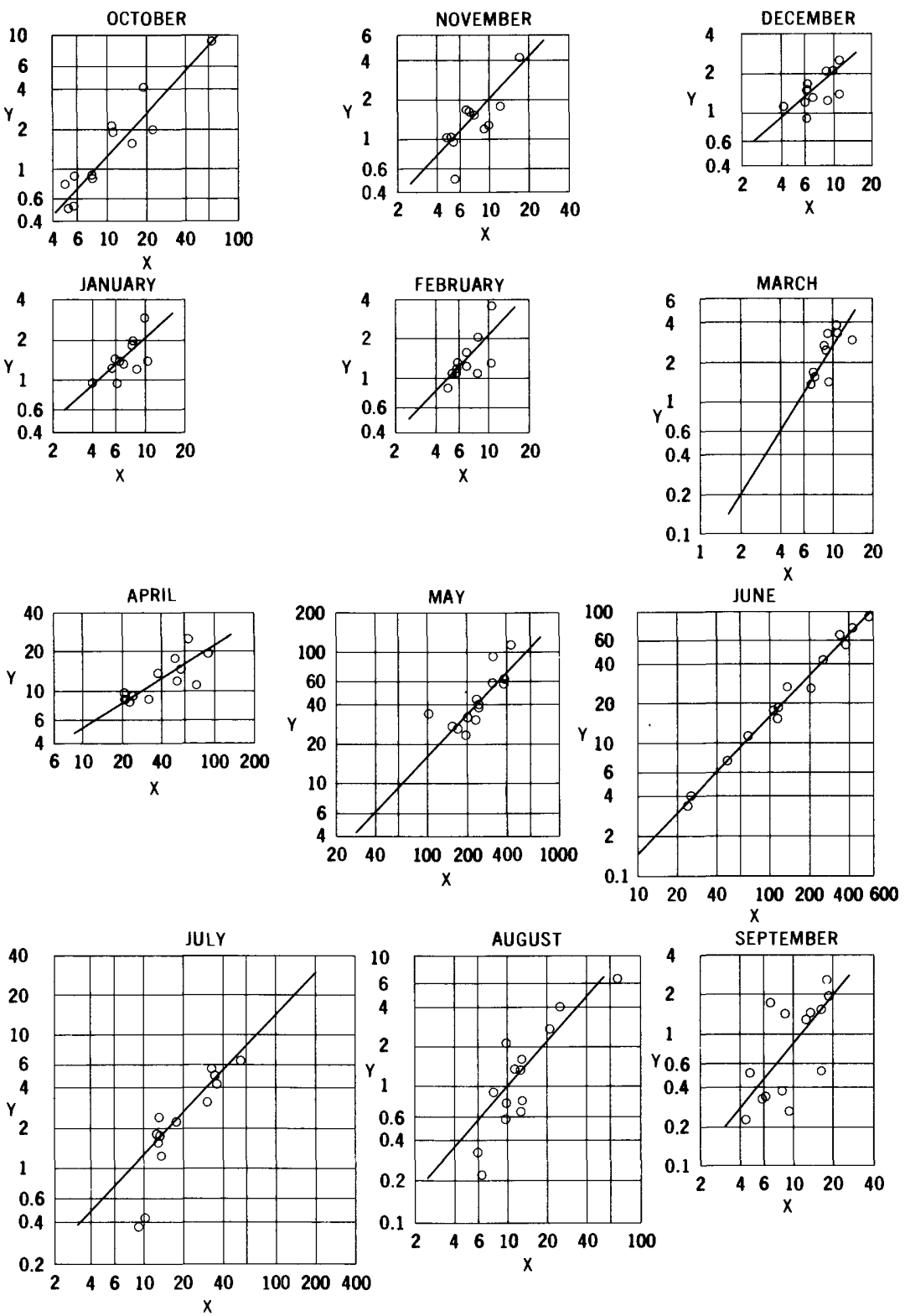

Relationships of monthly mean discharge of Plateau Creek at upper station, near Collbran, Colo. (Y), to monthly mean discharge of Plateau Creek near Collbran, Colo. (X). Discharge in hundreds of acre-feet. 
960. Plateau Creek at upper station, near Collbran, Colo.

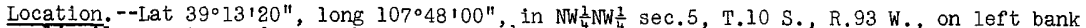
$1 \frac{1}{4}$ miles upstream from Park Creek and $8 \frac{1}{2}$ miles southeast of colibran. Aititude of gage is $7,885 \mathrm{ft}$ (from topographic map).

Drainage area. $--24 \mathrm{sq} \mathrm{mi}$, approximately.

Records ava1lable.--May 1937 to September 1943, May 1951 to April 1958. Monthly discharge only for some periods.

Estimates of streamflow.--october 1930 to April 1937, September 1943 to April 1951 , May 1958 to September 1965, based on relationships of monthly mean discharge with Plateau Creek near collbran, Colo. The regression equation used is:

$$
\log \mathrm{Y}=b \log \mathrm{X}-\mathrm{c}
$$

(where $\mathrm{Y}$ is discharge of Plateau Creek at upper station, near Collbran, Colo., and X Is discharge of Plateau Creek near Collbran, Colo., both in acre-feet per month).

\begin{tabular}{l|l|l|l|l|l|l|l|l|l|l|l|l}
\hline \multicolumn{10}{c}{ Monthly values of constants in above equation } \\
\hline b & oct. & Nov. & Dec. & Jan. & Feb. & Mar. & Apr. & May & June & July & Aug. & Sept. \\
\hline & 1.07 & 0.88 & 0.88 & 1.07 & 1.60 & 0.63 & 1.06 & 1.04 & 1.04 & 1.12 & 1.21 \\
$\mathrm{c}$ & 1.13 & .92 & .32 & .32 & .87 & 1.38 & -.83 & 1.05 & .96 & 1.02 & 1.36 & 1.70 \\
\hline
\end{tabular}

Average discharge. --35 years $(1931-65), 9,897$ acre-feet per year (13.7 cfs).

Extremes.--1937-43, 1951-58: Maximum discharge, 450 cfs May 27, 1942 from rating curve extended above 180 cfs; no flow at times.

Remarks.--One small reservoir (capacity, 200 acre-feet) and diversions for irrigation of about 730 acres above station. Erie Canal diverts water above station to Buzzard Creek drainage for irrigation of about 140 acres. Estimates of annual flow are within 15 percent of regression line.

\begin{tabular}{|c|c|c|c|c|c|c|c|c|c|c|c|c|c|}
\hline $\begin{array}{l}\text { Water } \\
\text { year }\end{array}$ & oct. & Nov. & Dec. & Jan. & Feb. & Mar. & Apr. & May & June & July & Aug. & sept. & Annual \\
\hline $\begin{array}{l}1937 \\
1938 \\
1939 \\
1940\end{array}$ & $\begin{array}{r}- \\
209 \\
152 \\
51\end{array}$ & $\begin{array}{l}- \\
129 \\
177 \\
103\end{array}$ & $\begin{array}{l}- \\
207 \\
138 \\
112\end{array}$ & $\begin{array}{l}- \\
183 \\
142 \\
96\end{array}$ & $\begin{array}{r}- \\
210 \\
136 \\
86\end{array}$ & $\begin{array}{l}- \\
325 \\
295 \\
327\end{array}$ & $\begin{array}{c}- \\
1,990 \\
1,200 \\
1,490\end{array}$ & $\begin{array}{l}6,260 \\
5,910 \\
4,410 \\
4,040\end{array}$ & $\begin{array}{r}1,880 \\
7,590 \\
751 \\
410\end{array}$ & $\begin{array}{r}424 \\
638 \\
37 \\
43\end{array}$ & $\begin{array}{r}138 \\
65 \\
32 \\
22\end{array}$ & $\begin{array}{r}174 \\
153 \\
38 \\
54\end{array}$ & $\begin{array}{r}- \\
17,610 \\
7,510 \\
6,830\end{array}$ \\
\hline $\begin{array}{l}1941 \\
1942 \\
1943\end{array}$ & $\begin{array}{r}194 \\
885 \\
\$ 203\end{array}$ & $\begin{array}{r}121 \\
\$ 407 \\
\$ 208\end{array}$ & $\begin{array}{r}123 \\
\neq 307 \\
\neq 154\end{array}$ & $\begin{array}{r}123 \\
\$ 277 \\
\$ 123\end{array}$ & $\begin{array}{r}111 \\
\$ 250 \\
\neq 139\end{array}$ & $\begin{array}{r}142 \\
\neq 293 \\
\neq 369\end{array}$ & $\begin{array}{r}979 \\
2,560 \\
\neq 1,710\end{array}$ & $\begin{array}{r}11,850 \\
9,490 \\
3,230\end{array}$ & $\begin{array}{l}4,320 \\
6,770 \\
2,720\end{array}$ & $\begin{array}{r}\$ 307 \\
561 \\
226\end{array}$ & $\begin{array}{r}80 \\
167 \\
676\end{array}$ & $\begin{array}{l}199 \\
145 \\
149\end{array}$ & $\begin{array}{r}\neq 18,550 \\
\neq 22,110 \\
\neq 9,910\end{array}$ \\
\hline $\begin{array}{l}1951 \\
1952 \\
1953 \\
1954 \\
1955\end{array}$ & $\begin{array}{r}- \\
87 \\
83 \\
87 \\
188\end{array}$ & $\begin{array}{l}- \\
104 \\
153 \\
165 \\
161\end{array}$ & $\begin{array}{l}- \\
124 \\
206 \\
142 \\
161\end{array}$ & $\begin{array}{l}-135 \\
193 \\
139 \\
149\end{array}$ & $\begin{array}{l}- \\
127 \\
161 \\
133 \\
125\end{array}$ & $\begin{array}{l}- \\
154 \\
240 \\
165 \\
155\end{array}$ & $\begin{array}{r}- \\
1,850 \\
824 \\
1,150 \\
928\end{array}$ & $\begin{array}{l}2,630 \\
5,970 \\
2,790 \\
2,350 \\
4,160\end{array}$ & $\begin{array}{r}1,740 \\
5,700 \\
2,630 \\
341 \\
1,580\end{array}$ & $\begin{array}{l}173 \\
492 \\
243 \\
181 \\
155\end{array}$ & $\begin{array}{r}58 \\
274 \\
214 \\
92 \\
136\end{array}$ & $\begin{array}{r}39 \\
130 \\
52 \\
268 \\
36\end{array}$ & $\begin{array}{r}- \\
15,150 \\
7,790 \\
5,210 \\
7,930\end{array}$ \\
\hline $\begin{array}{l}1956 \\
1957 \\
1958\end{array}$ & $\begin{array}{r}49 \\
75 \\
403\end{array}$ & $\begin{array}{r}50 \\
96 \\
417\end{array}$ & $\begin{array}{r}91 \\
131 \\
252\end{array}$ & $\begin{array}{r}96 \\
123 \\
301\end{array}$ & $\begin{array}{l}112 \\
112 \\
352\end{array}$ & $\begin{array}{l}260 \\
133 \\
384\end{array}$ & $\begin{array}{r}887 \\
873 \\
1,390\end{array}$ & $\begin{array}{c}3,080 \\
3,420 \\
-\end{array}$ & $\begin{array}{c}1,120 \\
9,390 \\
-\end{array}$ & $\begin{array}{r}126 \\
2,810\end{array}$ & $\begin{array}{r}79 \\
411 \\
-\end{array}$ & $\begin{array}{r}23 \\
254 \\
-\end{array}$ & $\begin{array}{r}5,970 \\
17,830 \\
-\end{array}$ \\
\hline
\end{tabular}

\# Estimated for 1950 Comp1lation.

Est1mated monthly and annual streamflow, in acre-feet

\begin{tabular}{|c|c|c|c|c|c|c|c|c|c|c|c|c|c|}
\hline $\begin{array}{l}\text { Water } \\
\text { year }\end{array}$ & oct. & Nov. & Dec. & Jan. & Feb. & Mar. & Apr. & May & June & July & Aug. & Sept. & Annual \\
\hline $\begin{array}{l}1931 \\
1932 \\
1933 \\
1934 \\
1935\end{array}$ & $\begin{array}{r}300 \\
150 \\
100 \\
70 \\
60\end{array}$ & $\begin{array}{l}300 \\
250 \\
140 \\
100 \\
100\end{array}$ & $\begin{array}{l}210 \\
260 \\
160 \\
140 \\
160\end{array}$ & $\begin{array}{l}130 \\
130 \\
150\end{array}$ & $\begin{array}{l}190 \\
260 \\
120 \\
170 \\
120\end{array}$ & $\begin{array}{l}320 \\
430 \\
370 \\
280 \\
150\end{array}$ & $\begin{array}{r}1,300 \\
1,900 \\
1,000 \\
1,700 \\
860\end{array}$ & $\begin{array}{l}3,500 \\
7,600 \\
2,900 \\
1,300 \\
1,900\end{array}$ & $\begin{array}{r}1,100 \\
5,500 \\
5,200 \\
150 \\
5,200\end{array}$ & $\begin{array}{r}200 \\
1,100 \\
370 \\
50 \\
530\end{array}$ & $\begin{array}{r}80 \\
270 \\
100 \\
50 \\
140\end{array}$ & $\begin{array}{r}180 \\
70 \\
70 \\
40 \\
90\end{array}$ & $\begin{array}{r}7,810 \\
18,020 \\
10,660 \\
4,180 \\
9,460\end{array}$ \\
\hline $\begin{array}{l}1936 \\
1937\end{array}$ & $\begin{array}{l}90 \\
60\end{array}$ & & $\begin{array}{l}170 \\
120\end{array}$ & & $\begin{array}{r}150 \\
90\end{array}$ & $\begin{array}{l}220 \\
120\end{array}$ & $\begin{array}{l}1,900 \\
1,100\end{array}$ & 4,500 & 1,000 & $\begin{array}{l}180 \\
-\end{array}$ & $\begin{array}{l}120 \\
-\end{array}$ & -60 & $\begin{array}{r}8,740 \\
10,616\end{array}$ \\
\hline $\begin{array}{l}1944 \\
1945\end{array}$ & $\begin{array}{l}150 \\
100\end{array}$ & $\begin{array}{l}290 \\
190\end{array}$ & $\begin{array}{l}180 \\
200\end{array}$ & & & $\begin{array}{l}210 \\
230\end{array}$ & $\begin{array}{l}760 \\
860\end{array}$ & & $\begin{array}{l}6,700 \\
5,200\end{array}$ & $\begin{array}{r}1,000 \\
740\end{array}$ & $\begin{array}{l}160 \\
390\end{array}$ & $\begin{array}{l}50 \\
80\end{array}$ & \\
\hline $\begin{array}{l}1946 \\
1947 \\
1948 \\
1949 \\
1950\end{array}$ & $\begin{array}{r}120 \\
260 \\
190 \\
80 \\
120\end{array}$ & $\begin{array}{l}200 \\
290 \\
220 \\
160 \\
130\end{array}$ & $\begin{array}{l}210 \\
240 \\
200 \\
170 \\
140\end{array}$ & $\begin{array}{l}200 \\
180 \\
190 \\
170\end{array}$ & $\begin{array}{l}180 \\
170 \\
190 \\
180 \\
150\end{array}$ & $\begin{array}{l}290 \\
320 \\
230 \\
200 \\
190\end{array}$ & $\begin{array}{l}2,000 \\
1,300 \\
1,500 \\
1,400 \\
1,100\end{array}$ & & & $\begin{array}{l}190 \\
580 \\
270 \\
420 \\
370\end{array}$ & $\begin{array}{l}250 \\
200 \\
130 \\
110 \\
100\end{array}$ & $\begin{array}{l}90 \\
90 \\
50 \\
70 \\
70\end{array}$ & $\begin{array}{r}9,120 \\
13,150 \\
11,260 \\
10,980 \\
9,470\end{array}$ \\
\hline 1951 & 70 & 110 & 140 & 130 & 100 & 110 & 740 & - & - & - & - & - & 6,040 \\
\hline $\begin{array}{l}1958 \\
1959 \\
1960\end{array}$ & $\begin{array}{r}- \\
100 \\
100\end{array}$ & $\begin{array}{l}110 \\
120\end{array}$ & $\begin{array}{l}- \\
120 \\
130\end{array}$ & $\begin{array}{l}120 \\
110\end{array}$ & $\begin{array}{l}- \\
100 \\
120\end{array}$ & $\begin{array}{l}- \\
190 \\
200\end{array}$ & $\begin{array}{r}-\overline{860} \\
1,200\end{array}$ & & $\begin{array}{r}2,600 \\
880 \\
1,500\end{array}$ & $\begin{array}{l}170 \\
130 \\
160\end{array}$ & $\begin{array}{l}100 \\
100 \\
130\end{array}$ & $\begin{array}{l}80 \\
40 \\
50\end{array}$ & $\begin{array}{r}12,550 \\
5,320 \\
7,220\end{array}$ \\
\hline $\begin{array}{l}1961 \\
1962 \\
1963 \\
1964 \\
1965\end{array}$ & $\begin{array}{r}80 \\
360 \\
130 \\
70 \\
60\end{array}$ & $\begin{array}{r}130 \\
210 \\
140 \\
50 \\
50\end{array}$ & $\begin{array}{r}130 \\
130 \\
110 \\
80 \\
70\end{array}$ & $\begin{array}{r}120 \\
130 \\
90 \\
70 \\
60\end{array}$ & $\begin{array}{r}50 \\
110 \\
70 \\
60 \\
50\end{array}$ & $\begin{array}{r}20 \\
260 \\
60 \\
40 \\
30\end{array}$ & $\begin{array}{r}230 \\
1,600 \\
270 \\
200 \\
50\end{array}$ & $\begin{array}{r}1,200 \\
1,500 \\
500 \\
1,300 \\
460\end{array}$ & $\begin{array}{r}540 \\
4,100 \\
250 \\
420 \\
2,000\end{array}$ & $\begin{array}{l}170 \\
700 \\
150 \\
180 \\
370\end{array}$ & $\begin{array}{l}140 \\
180 \\
140 \\
170 \\
170\end{array}$ & $\begin{array}{r}230 \\
120 \\
70 \\
90 \\
250\end{array}$ & $\begin{array}{l}3,040 \\
9,400 \\
1,980 \\
2,730 \\
3,620\end{array}$ \\
\hline
\end{tabular}



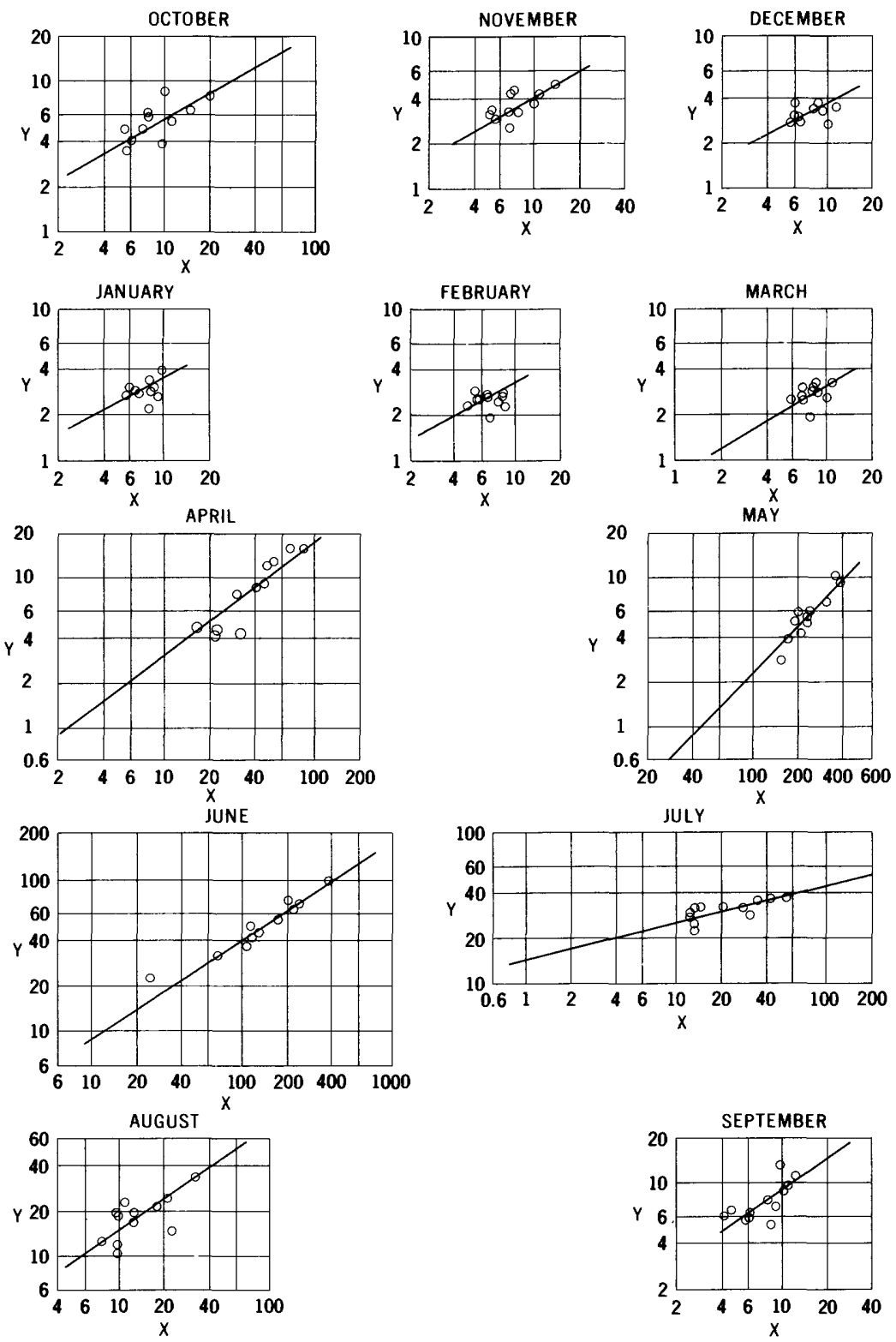

Relationships of monthly mean discharge of Big Creek at upper station, near Collbran, Colo. (Y), to monthly mean discharge of Plateau Creek near Collbran, Colo. $(X)$. Discharge in hundreds of acre-feet. 
995. Big Creek at upper station, near Collbran, Colo.

Location --Lat $39^{\circ} 08^{\prime}$, long $107^{\circ} 55^{\prime}$, in NE $\frac{1}{4}$ sec.5 T. 11 S., R. 94 W., on right bank at downstream side of bridge, half a mile downstream from Barter Creek " 8 miles south of Collbran, and 9 miles upstream from mouth. Altitude of gage is $8,590 \mathrm{ft}$ (from topographic map).

Drainage area. --17 sq mi, approximately.

Records available. - -June 1945 to September 1956.

Estimates of streamflow.--October 1930 to June 1945, October 1956 to September 1965, based on relationships of monthly mear discharge with Plateau Creek near collbran, Colo. The regression equation used is:

$\log \mathrm{Y}=\mathrm{b} \log \mathrm{X}-\mathrm{c}$

(where $Y$ is discharge of Big Creek at upper station near Collbran, Colo., and $X$ is discharge of Plateau Creek near Collbran, Colo., both in acre-feet per month).

Monthly values of constants in above equation

\begin{tabular}{l|l|l|l|l|l|l|l|l|l|l|l|l}
\hline & Oct. & Nov. & Dec. & Jan. & Feb. & Mar. & Apr. & May & June & July & Aug. & Sept. \\
\hline b & 0.57 & 0.55 & 0.51 & 0.53 & 0.53 & 0.60 & 0.74 & 1.05 & 0.65 & 0.24 & 0.68 & 0.68 \\
c & $\mathbf{1 . 0 3}$ & -.94 & -1.03 & -.95 & -.92 & -.68 & -.25 & -.80 & -.99 & -2.69 & -1.13 & -.89 \\
\hline
\end{tabular}

Average discharge. -35 years $(1930-65), 19,572$ acre-feet per year (27.0 ofs).

Extremes.--1945-56: Maximum discharge, 474 cfs May 21, 1948, from rating curve extended above $130 \mathrm{cfs}$; minimum not determined.

Remarks. -Flow regulated by several small storage reservoirs above station. No diversion above station. Estimates of annual flow are within about 15 percent of regression line.

Monthly and annual streamflow, in acre-feet

\begin{tabular}{l|c|c|c|c|c|c|c|r|r|r|r|r|r}
\hline $\begin{array}{c}\text { Water } \\
\text { year }\end{array}$ & oct. & Nov. & Dec. & Jan. & Feb. & Mar. & Apr. & May & June & July & Aug. & Sept. & Annual \\
\hline 1945 & - & - & - & - & - & - & - & - & - & 3,770 & 3,320 & 1,300 & - \\
1946 & 239 & 359 & 264 & 264 & 262 & 257 & 1,560 & 5,820 & 4,420 & 3,140 & 1,450 & 883 & 19,520 \\
1947 & 782 & 471 & 348 & 400 & 246 & 320 & 862 & 9,180 & 5,410 & 3,550 & 2,150 & 946 & 24,660 \\
1948 & 630 & 419 & 330 & 281 & 225 & 330 & 1,260 & 10,040 & 4,180 & 3,160 & 1,660 & 635 & 23,150 \\
1949 & 479 & 315 & 340 & 307 & 278 & 285 & 928 & 5,530 & 6,830 & 2,810 & 2,250 & 776 & 21,130 \\
1950 & 370 & 241 & 278 & 221 & 189 & 191 & 427 & 4,290 & 6,300 & 3,170 & 1,930 & 521 & 18,130 \\
1951 & 398 & 286 & 290 & 264 & 234 & 249 & 470 & 3,930 & 3,580 & 2,200 & 1,190 & 579 & 13,670 \\
1952 & 617 & 311 & 275 & 277 & 265 & 295 & 1,210 & 6,820 & 9,770 & 3,530 & 2,400 & 1,130 & 26,900 \\
1953 & 569 & 436 & 370 & 338 & 278 & 337 & 412 & 2,810 & 7,250 & 3,160 & 1,870 & 656 & 18,490 \\
1954 & 336 & 316 & 307 & 293 & 254 & 263 & 1,580 & 5,210 & 2,250 & 2,910 & 1,220 & 696 & 15,640 \\
1955 & 533 & 428 & 369 & 307 & 250 & 246 & 451 & 6,040 & 4,840 & 2,730 & 1,920 & 566 & 18,680 \\
1956 & 474 & 327 & 307 & 307 & 288 & 311 & 770 & 5,050 & 3,130 & 2,430 & 1,040 & 612 & 15,050 \\
\hline
\end{tabular}

Estimated monthly and annual streamflow, in acre-feet

\begin{tabular}{|c|c|c|c|c|c|c|c|c|c|c|c|c|c|}
\hline $\begin{array}{l}\text { Water } \\
\text { year }\end{array}$ & oct. & Nov. & Dec. & Jan. & Feb. & Mar. & Apr. & May & June & July & Aug. & Sept. & Annual \\
\hline $\begin{array}{l}1931 \\
1932 \\
1933 \\
1934 \\
1935\end{array}$ & $\begin{array}{r}880 \\
620 \\
490 \\
+390 \\
380\end{array}$ & $\begin{array}{r}480 \\
430 \\
320 \\
+280 \\
280\end{array}$ & $\begin{array}{r}360 \\
410 \\
310 \\
+280 \\
310\end{array}$ & $\begin{array}{r}270 \\
370 \\
270 \\
+270 \\
290\end{array}$ & $\begin{array}{l}310 \\
350 \\
240 \\
290 \\
240\end{array}$ & $\begin{array}{l}320 \\
380 \\
350 \\
310 \\
250\end{array}$ & $\begin{array}{r}920 \\
1,400 \\
640 \\
1,300 \\
540\end{array}$ & $\begin{array}{r}5,000 \\
10,600 \\
4,100 \\
1,900 \\
2,700\end{array}$ & $\begin{array}{r}3,000 \\
8,600 \\
8,200 \\
910 \\
8,200\end{array}$ & $\begin{array}{l}2,800 \\
4,200 \\
3,300 \\
2,100 \\
3,500\end{array}$ & $\begin{array}{r}1,300 \\
2,700 \\
1,500 \\
940 \\
1,800\end{array}$ & $\begin{array}{r}1,400 \\
780 \\
780 \\
600 \\
960\end{array}$ & $\begin{array}{r}17,040 \\
30,840 \\
20,500 \\
9,570 \\
19,450\end{array}$ \\
\hline $\begin{array}{l}1936 \\
1937 \\
1938 \\
1939 \\
1940\end{array}$ & $\begin{array}{l}470 \\
390 \\
580 \\
700 \\
390\end{array}$ & $\begin{array}{l}360 \\
330 \\
390 \\
430 \\
260\end{array}$ & $\begin{array}{l}320 \\
270 \\
350 \\
380 \\
230\end{array}$ & $\begin{array}{l}330 \\
240 \\
310 \\
360 \\
210\end{array}$ & $\begin{array}{l}270 \\
200 \\
290 \\
350 \\
230\end{array}$ & $\begin{array}{l}280 \\
230 \\
320 \\
370 \\
290\end{array}$ & $\begin{array}{r}1,400 \\
720 \\
1,600 \\
1,000 \\
1,100\end{array}$ & $\begin{array}{l}6,400 \\
9,400 \\
9,200 \\
5,400 \\
5,800\end{array}$ & $\begin{array}{r}2,900 \\
4,400 \\
10,200 \\
2,400 \\
1,600\end{array}$ & $\begin{array}{l}2,800 \\
3,500 \\
3,800 \\
2,500 \\
2,600\end{array}$ & $\begin{array}{l}1,600 \\
1,600 \\
1,700 \\
1,000 \\
1,100\end{array}$ & $\begin{array}{r}700 \\
680 \\
1,300 \\
780 \\
1,300\end{array}$ & $\begin{array}{l}17,830 \\
21,960 \\
30,040 \\
15,650 \\
15,110\end{array}$ \\
\hline $\begin{array}{l}1941 \\
1942 \\
1943 \\
1944 \\
1945\end{array}$ & $\begin{array}{r}860 \\
1,600 \\
470 \\
620 \\
500\end{array}$ & $\begin{array}{l}370 \\
570 \\
350 \\
470 \\
380\end{array}$ & $\begin{array}{l}340 \\
450 \\
280 \\
340 \\
360\end{array}$ & $\begin{array}{l}330 \\
410 \\
260 \\
300 \\
310\end{array}$ & $\begin{array}{l}290 \\
340 \\
260 \\
280 \\
260\end{array}$ & $\begin{array}{l}290 \\
340 \\
380 \\
280 \\
290\end{array}$ & $\begin{array}{r}520 \\
1,200 \\
1,400 \\
470 \\
540\end{array}$ & $\begin{array}{r}10,300 \\
7,400 \\
4,700 \\
7,600 \\
7,400\end{array}$ & $\begin{array}{l}7,300 \\
8,800 \\
4,800 \\
9,700 \\
8,300\end{array}$ & $\begin{array}{c}3,400 \\
3,400 \\
2,900 \\
4,100 \\
-\end{array}$ & $\begin{array}{c}1,800 \\
1,800 \\
5,400 \\
1,900 \\
-\end{array}$ & $\begin{array}{r}1,400 \\
820 \\
1,100 \\
680 \\
-\end{array}$ & $\begin{array}{l}27,500 \\
27,130 \\
22,300 \\
26,740 \\
26,730\end{array}$ \\
\hline $\begin{array}{l}1957 \\
1958 \\
1959 \\
1960\end{array}$ & $\begin{array}{l}370 \\
800 \\
420 \\
490\end{array}$ & $\begin{array}{l}270 \\
530 \\
280 \\
300\end{array}$ & $\begin{array}{l}290 \\
370 \\
270 \\
270\end{array}$ & $\begin{array}{l}260 \\
350 \\
250 \\
240\end{array}$ & $\begin{array}{l}230 \\
330 \\
230 \\
240\end{array}$ & $\begin{array}{l}240 \\
320 \\
270 \\
270\end{array}$ & $\begin{array}{l}520 \\
800 \\
540 \\
820\end{array}$ & $\begin{array}{l}2,300 \\
8,800 \\
3,700 \\
4,900\end{array}$ & $\begin{array}{r}12,300 \\
5,400 \\
2,700 \\
3,700\end{array}$ & $\begin{array}{l}5,200 \\
2,700 \\
2,600 \\
2,700\end{array}$ & $\begin{array}{l}2,800 \\
1,500 \\
1,500 \\
1,700\end{array}$ & $\begin{array}{r}1,400 \\
880 \\
620 \\
680\end{array}$ & $\begin{array}{l}26,180 \\
22,780 \\
13,380 \\
16,310\end{array}$ \\
\hline $\begin{array}{l}1961 \\
1962 \\
1963 \\
1964 \\
1965\end{array}$ & $\begin{array}{l}450 \\
980 \\
560 \\
420 \\
380\end{array}$ & $\begin{array}{l}320 \\
410 \\
320 \\
200 \\
200\end{array}$ & $\begin{array}{l}270 \\
270 \\
250 \\
210 \\
200\end{array}$ & $\begin{array}{l}250 \\
260 \\
210 \\
180 \\
170\end{array}$ & $\begin{array}{l}150 \\
230 \\
180 \\
170 \\
160\end{array}$ & $\begin{array}{l}110 \\
300 \\
170 \\
140 \\
140\end{array}$ & $\begin{array}{r}110 \\
1,200 \\
140 \\
100 \\
280\end{array}$ & $\begin{array}{r}1,900 \\
2,200 \\
780 \\
2,000 \\
660\end{array}$ & $\begin{array}{l}2,000 \\
7,100 \\
1,200 \\
1,700 \\
4,500\end{array}$ & $\begin{array}{l}2,700 \\
3,800 \\
2,700 \\
2,800 \\
1,400\end{array}$ & $\begin{array}{l}1,700 \\
2,100 \\
1,800 \\
2,000 \\
2,000\end{array}$ & $\begin{array}{r}1,600 \\
1,100 \\
820 \\
900 \\
1,700\end{array}$ & $\begin{array}{r}11,560 \\
19,950 \\
9,130 \\
10,820 \\
11,790\end{array}$ \\
\hline
\end{tabular}

* Based on estimates for 1950 Compliation. 

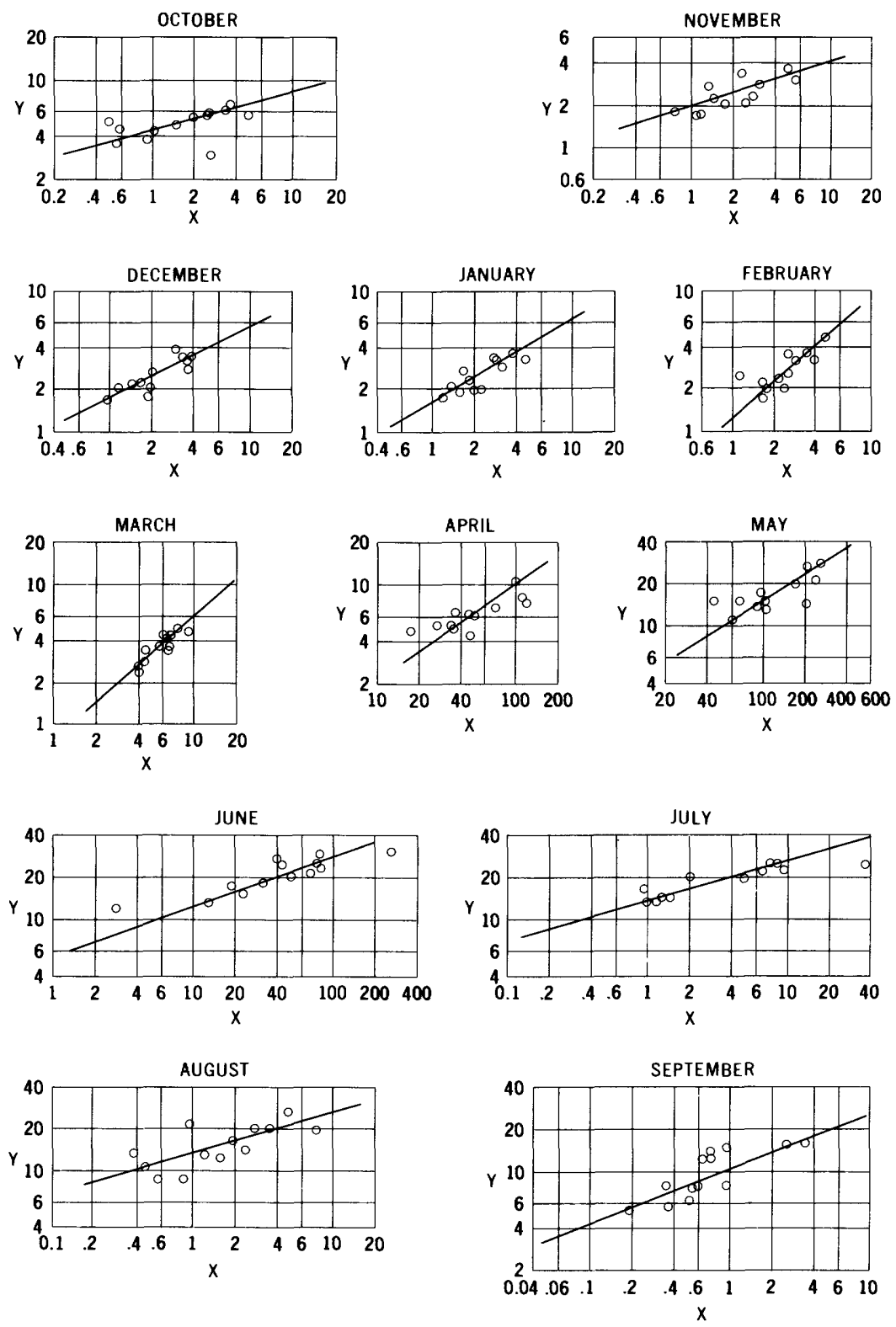

Relationships of monthly mean discharge of Cottonwood Creek at upper station, near Molina, Colo. ( $\mathrm{Y}$ ), to monthly mean discharge of Buzzard Creek near Collbran, Colo. (X). Discharge in hundreds of acre-feet. 
1005. Cottonwood Creek at upper station, near Molina, Colo.

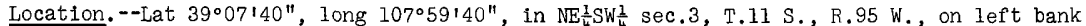
6 miles southeast of Molina and 7 miles upstream from mouth. Altitude of gage is $7,685 \mathrm{ft}$ (from topographic map).

Drainage area. - - $-16 \mathrm{sq} \mathrm{mi}$, approximately.

Records ava1lab1e. --May 1945 to September 1957.

Estimates of streamflow.--October 1930 to May 1945, October 1957 to September 1965, based on relationships of monthly mean discharge with Buzzard Creek near Collbran, Colo. The regression equation used is:

$\log Y=b \log X-c$

(where $Y$ is discharge of Cottonwood Creek at upper station near Molina, Colo., and $X$ is discharge of Buzzard Creek near Collbran, Colo., both in acre-feet per monthi).

\begin{tabular}{c|c|c|c|c|c|c|c|c|c|c|c|c}
\hline \multicolumn{10}{c}{ Monthly values of constants in above equation } \\
\hline b & oct. & Nov. & Dec. & Jan. & Feb. & Mar. & Apr. & May & June & July & Aug. & Sept. \\
\hline & 0.29 & 0.31 & 0.52 & 0.60 & 0.88 & 0.88 & 0.70 & 0.52 & 0.35 & 0.28 & 0.28 & 0.39 \\
\hline & -2.06 & -1.67 & -1.20 & -1.02 & -.36 & -.17 & -.20 & -1.12 & -2.03 & -2.56 & -2.56 & -2.25 \\
\hline
\end{tabular}

Average discharge. --35 years $(1930-65), 11,400$ acre-feet per year $(15.7 \mathrm{cfs})$

Extremes.--1945-57: Maximum discharge, 101 cf's May 18, 1948, from rating curve extended above 45 cfs; minimum not determined.

Remarks. -Flow regulated by several small reservoirs (combined capacity, about 3,200 acre$\left.f^{\prime} t\right)$. No diversion above station. Estimates of annual flow are within about 20 percent of regression line.

Monthly and annual streamflow, in acre-feet

\begin{tabular}{r|c|c|c|r|r|r|r|r|r|r|r|r|r}
\hline $\begin{array}{c}\text { Water } \\
\text { year }\end{array}$ & oct. & Nov. & Dec. & Jan. & Feb. & Mar. & Apr. & May & June & July & Aug. & Sept. & Annual \\
\hline 1945 & - & - & - & - & - & - & - & - & 2,560 & 2,280 & 1,930 & 1,380 & - \\
1946 & 701 & 301 & 316 & 299 & 337 & 482 & 1,070 & 1,730 & 1,890 & 1,410 & 1,260 & 799 & 10,600 \\
1947 & 630 & 280 & 330 & 277 & 265 & 378 & 618 & 2,700 & 2,190 & 2,450 & 2,010 & 1,560 & 13,690 \\
1948 & 594 & 366 & 341 & 379 & 483 & 504 & 814 & 2,880 & 2,740 & 2,420 & 1,640 & 1,230 & 14,390 \\
1949 & 563 & 238 & 277 & 338 & 333 & 440 & 682 & 2,030 & 2,920 & 2,500 & 2,150 & 1,250 & 13,720 \\
1950 & 603 & 339 & 387 & 332 & 378 & 455 & 614 & 1,530 & 2,500 & 1,960 & 1,280 & 799 & 11,180 \\
1951 & 454 & 273 & 219 & 209 & 245 & 361 & 470 & 1,190 & 1,540 & 1,370 & 891 & 621 & 7,840 \\
1952 & 387 & 171 & 173 & 234 & 256 & 382 & 738 & 2,150 & 2,330 & 2,220 & 2,000 & 1,490 & 12,530 \\
1953 & 440 & 226 & 265 & 344 & 364 & 450 & 506 & 1,310 & 2,020 & 2,040 & 1,400 & 771 & 10,140 \\
1954 & 496 & 209 & 206 & 198 & 201 & 298 & 635 & 1,590 & 1,250 & 1,420 & 1,080 & 569 & 8,150 \\
1955 & 307 & 208 & 215 & 197 & 178 & 246 & 508 & 1,380 & 1,740 & 1,650 & 1,350 & 801 & 8,780 \\
1956 & 508 & 182 & 201 & 213 & 223 & 350 & 483 & 1,580 & 1,340 & 1,310 & 885 & 528 \\
1957 & 369 & 172 & 167 & 179 & 205 & 280 & 435 & 1,460 & 3,090 & 2,480 & 2,680 & 1,570 & 13,090 \\
\hline
\end{tabular}

Estimated monthly and annual streamflow, in acre-feet

\begin{tabular}{|c|c|c|c|c|c|c|c|c|c|c|c|c|c|}
\hline $\begin{array}{l}\text { Water } \\
\text { year }\end{array}$ & oct. & Nov. & Dec. & Jan. & Feb. & Mar. & Apr. & May & June & July & Aug. & Sept. & Annual \\
\hline $\begin{array}{l}1931 \\
1932 \\
1933 \\
1934 \\
1935\end{array}$ & & 10 & $\begin{array}{l}480 \\
480 \\
580 \\
240 \\
130\end{array}$ & & $\begin{array}{l}580 \\
700 \\
780 \\
330 \\
110\end{array}$ & $\begin{array}{r}750 \\
, 000 \\
, 100 \\
510 \\
320\end{array}$ & $\begin{array}{l}670 \\
960 \\
380 \\
480 \\
440\end{array}$ & $\begin{array}{r}1,700 \\
2,700 \\
2,000 \\
830 \\
1,700\end{array}$ & & & $\begin{array}{r}1,200 \\
2,100 \\
1,200 \\
870 \\
1,000\end{array}$ & $\begin{array}{r}1,800 \\
1,200 \\
1,300 \\
620 \\
1,200\end{array}$ & $\begin{array}{r}12,030 \\
16,130 \\
13,890 \\
6,400 \\
10,390\end{array}$ \\
\hline $\begin{array}{l}1936 \\
1937 \\
1938 \\
1939 \\
1940\end{array}$ & $\begin{array}{l}500 \\
520 \\
700 \\
680 \\
450\end{array}$ & & & & & $\begin{array}{l}430 \\
250 \\
760 \\
780 \\
680\end{array}$ & $\begin{array}{r}920 \\
520 \\
1,300 \\
800 \\
720\end{array}$ & & & & $\begin{array}{r}1,500 \\
1,300 \\
1,100 \\
1,200 \\
940\end{array}$ & $\begin{array}{r}860 \\
850 \\
1,900 \\
850 \\
870\end{array}$ & $\begin{array}{r}10,160 \\
10,510 \\
15,000 \\
9,810 \\
7,780\end{array}$ \\
\hline $\begin{array}{l}1941 \\
1942 \\
1943 \\
1944 \\
1945\end{array}$ & $\begin{array}{r}690 \\
.000 \\
580 \\
650 \\
550\end{array}$ & $\begin{array}{l}290 \\
270 \\
310\end{array}$ & $\begin{array}{l}210 \\
480 \\
300 \\
290 \\
210\end{array}$ & $\begin{array}{l}280 \\
260 \\
260\end{array}$ & $\begin{array}{l}200 \\
450 \\
330 \\
330 \\
270\end{array}$ & $\begin{array}{l}320 \\
460 \\
590 \\
270 \\
330\end{array}$ & $\begin{array}{r}650 \\
1,500 \\
780 \\
420 \\
470\end{array}$ & & $\begin{array}{l}2,500 \\
3,100 \\
2,000 \\
2,900 \\
-\end{array}$ & $\begin{array}{l}2,200 \\
2,600 \\
1,600 \\
2,700 \\
-\end{array}$ & $\begin{array}{l}1,600 \\
1,500 \\
2,700 \\
1,000 \\
-\end{array}$ & $\begin{array}{r}1,600 \\
940 \\
1,700 \\
650 \\
-\end{array}$ & $\begin{array}{l}13,260 \\
15,970 \\
12,450 \\
12,540 \\
12,750\end{array}$ \\
\hline $\begin{array}{l}1958 \\
1959 \\
1960\end{array}$ & $\begin{array}{l}760 \\
430 \\
560\end{array}$ & & $\begin{array}{l}360 \\
240 \\
120\end{array}$ & & & $\begin{array}{l}760 \\
240 \\
580\end{array}$ & $\begin{array}{l}960 \\
430 \\
890\end{array}$ & & & & & $\begin{array}{l}840 \\
520 \\
330\end{array}$ & $\begin{array}{r}12,800 \\
8,010 \\
9,110\end{array}$ \\
\hline $\begin{array}{l}1961 \\
1962 \\
1963 \\
1964 \\
1965\end{array}$ & $\begin{array}{l}330 \\
800 \\
580 \\
470 \\
490\end{array}$ & $\begin{array}{l}250 \\
240\end{array}$ & $\begin{array}{l}160 \\
290 \\
240 \\
150 \\
220\end{array}$ & $\begin{array}{l}120 \\
300 \\
240 \\
150 \\
260\end{array}$ & $\begin{array}{l}140 \\
610 \\
450 \\
120 \\
320\end{array}$ & $\begin{array}{r}360 \\
560 \\
1,000 \\
130 \\
200\end{array}$ & $\begin{array}{r}380 \\
1,300 \\
390 \\
630 \\
800\end{array}$ & $\begin{array}{r}1,500 \\
2,300 \\
760 \\
2,000 \\
2,000\end{array}$ & $\begin{array}{l}1,400 \\
2,600 \\
1,000 \\
2,000 \\
2,800\end{array}$ & & $\begin{array}{l}1,300 \\
1,400 \\
1,100 \\
2,000 \\
2,100\end{array}$ & $\begin{array}{r}2,000 \\
1,100 \\
920 \\
1,300 \\
2,400\end{array}$ & $\begin{array}{r}9,370 \\
14,350 \\
8,360 \\
11,100 \\
14,930\end{array}$ \\
\hline
\end{tabular}



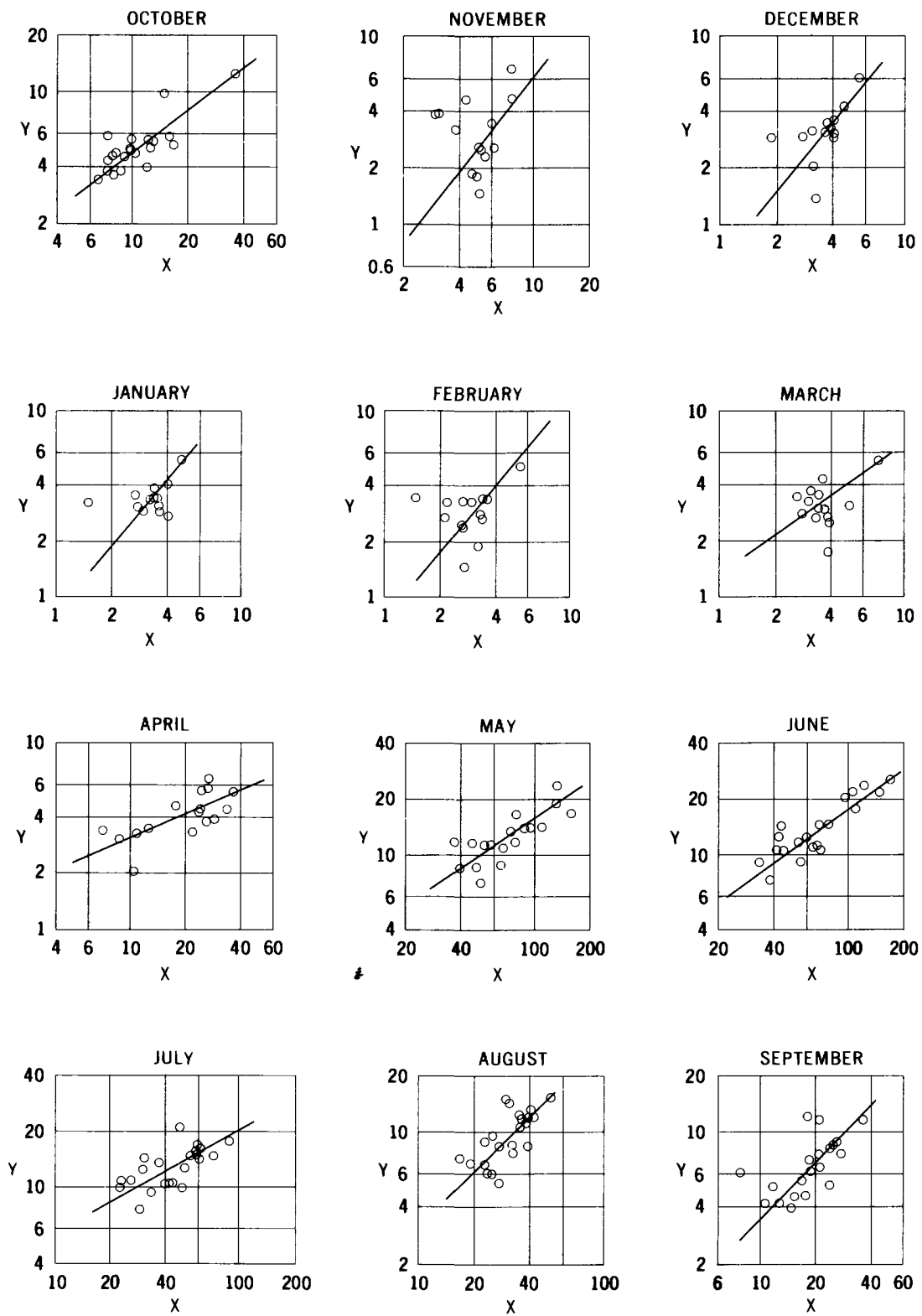

Relationships of monthly mean discharge of Mesa Creek near Mesa, Colo. (Y), to monthly mean discharge of Surface Creek near Cedaredge, Colo. (X). Discharge in hundreds of acre-feet. 
1045. Mesa Creek near Mesa, Colo.

Location. --Lat $39^{\circ} 05^{\prime}$, long $108^{\circ} 07^{\prime}$, in SW $\frac{1}{4}$ sec.16, T.11 S., R.96 W., on right bank an elghth of a mile upstream from unnamed stream, $5 \frac{1}{2}$ miles southeast' of Mesa, and $7 \frac{1}{2}$ miles upstream from mouth. Altitude of gage is 7,400 ft (by barometer).

Drainage area. - -About $7 \mathrm{sq} \mathrm{mi}$.

Records available. --May 1937 to September 1960. Prior to April 1941 monthly discharge only.

Estimates of streamflow.--october 1960 to September 1965, based on relationships of monthly mean discharge with Surface Creek near Cedaredge, Colo. The regression equation used is:

$$
\log Y=b \log X-c
$$

(where $\mathrm{Y}$ is discharge of Mesa Creek near Mesa, Colo., and $\mathrm{X}$ is discharge of Surface Creek near cedaredge, Colo., both in acre-feet per month).

\begin{tabular}{|c|c|c|c|c|c|c|c|c|c|c|c|c|}
\hline \multicolumn{13}{|c|}{ Monthly values } \\
\hline & oct. & Nov. & Dec. & Jan. & Feb. & Mar. & Apr. & May & June & July & Aug. & Sept. \\
\hline $\mathrm{b}$ & 0.75 & 1.28 & 1.20 & 1.17 & 1.19 & 0.71 & 0.43 & 0.67 & 0.72 & 0.56 & 0.99 & 1.03 \\
\hline c & -.43 & 1.05 & .58 & .42 & .51 & -.68 & -2.20 & -.52 & -.37 & -1.08 & .48 & .56 \\
\hline
\end{tabular}

Average discharge. - -28 years $(1937-65), 8,259$ acre-feet per year (11.4 cfs).

Extremes,--1937-60: Maximum discharge observed, 140 efs May 12, 1941, from rating curve extended above $50 \mathrm{cfs}$; minimum daily, $0.6 \mathrm{cf}$ ' Mar. $9,11-14,1946$, Mar. 18-20, 1948.

Remarks.--Some regulation by small reservoirs above station. No diversion above station. Estimates of annul flow are within about 20 percent of regression line.

Monthly and annual streamflow, in acre-feet

\begin{tabular}{|c|c|c|c|c|c|c|c|c|c|c|c|c|c|}
\hline \begin{tabular}{c|} 
water \\
year \\
\end{tabular} & oct. & Nov. & Dec. & Jan. & Feb. & Mar. & Apr. & May & June & July & Aug. & Sept. & Annual \\
\hline $\begin{array}{l}1937 \\
1938 \\
1939 \\
1940\end{array}$ & $\begin{array}{l}- \\
490 \\
786 \\
430\end{array}$ & $\begin{array}{l}- \\
263 \\
513 \\
304\end{array}$ & $\begin{array}{l}- \\
316 \\
538 \\
212\end{array}$ & $\begin{array}{l}- \\
136 \\
588 \\
215\end{array}$ & $\begin{array}{l}- \\
246 \\
368 \\
230\end{array}$ & $\begin{array}{l}- \\
291 \\
352 \\
277\end{array}$ & $\begin{array}{l}- \\
474 \\
686 \\
568\end{array}$ & $\begin{array}{l}1,280 \\
1,320 \\
1,550 \\
1,640\end{array}$ & $\begin{array}{r}980 \\
1,940 \\
1,230 \\
1,420\end{array}$ & $\begin{array}{l}1,050 \\
1,760 \\
1,240 \\
1,450\end{array}$ & $\begin{array}{r}934 \\
1,450 \\
739 \\
682\end{array}$ & $\begin{array}{l}598 \\
742 \\
609 \\
451\end{array}$ & $\begin{array}{l}- \\
9,430 \\
9,200 \\
7,880\end{array}$ \\
\hline $\begin{array}{l}1941 \\
1942 \\
1943 \\
1944 \\
1945\end{array}$ & $\begin{array}{r}516 \\
1,230 \\
581 \\
452 \\
554\end{array}$ & $\begin{array}{r}\$ 391 \\
600 \\
435 \\
154 \\
420\end{array}$ & $\begin{array}{r}+369 \\
590 \\
529 \\
228 \\
480\end{array}$ & $\begin{array}{r}\$ 369 \\
615 \\
492 \\
289 \\
394\end{array}$ & $\begin{array}{r}\$ 305 \\
555 \\
489 \\
286 \\
355\end{array}$ & $\begin{array}{r}+314 \\
664 \\
492 \\
322 \\
418\end{array}$ & $\begin{array}{l}313 \\
815 \\
787 \\
329 \\
418\end{array}$ & $\begin{array}{l}2,370 \\
2,140 \\
1,160 \\
1,470 \\
1,380\end{array}$ & $\begin{array}{l}2,380 \\
2,200 \\
1,260 \\
1,760 \\
1,470\end{array}$ & $\begin{array}{l}2,120 \\
1,590 \\
1,050 \\
1,490 \\
1,490\end{array}$ & $\begin{array}{r}1,420 \\
1,530 \\
841 \\
1,310 \\
1,200\end{array}$ & $\begin{array}{r}1,180 \\
1,230 \\
556 \\
856 \\
860\end{array}$ & $\begin{array}{r}\$ 12,050 \\
13,760 \\
8,670 \\
8,930 \\
9,440\end{array}$ \\
\hline $\begin{array}{l}1946 \\
1947 \\
1948 \\
1949 \\
1950\end{array}$ & $\begin{array}{l}553 \\
506 \\
570 \\
448 \\
481\end{array}$ & $\begin{array}{l}36.4 \\
146 \\
470 \\
320 \\
230\end{array}$ & $\begin{array}{l}392 \\
351 \\
422 \\
324 \\
393\end{array}$ & $\begin{array}{l}359 \\
337 \\
404 \\
350 \\
356\end{array}$ & $\begin{array}{l}288 \\
327 \\
338 \\
337 \\
328\end{array}$ & $\begin{array}{l}182 \\
372 \\
174 \\
309 \\
439\end{array}$ & $\begin{array}{l}649 \\
441 \\
385 \\
377 \\
424\end{array}$ & $\begin{array}{r}1,170 \\
1,400 \\
1,410 \\
1,340 \\
885\end{array}$ & $\begin{array}{l}1,060 \\
1,430 \\
1,240 \\
1,440 \\
1,060\end{array}$ & $\begin{array}{l}1,100 \\
1,450 \\
1,290 \\
1,710 \\
1,050\end{array}$ & $\begin{array}{r}899 \\
1,240 \\
1,040 \\
1,190 \\
836\end{array}$ & $\begin{array}{l}617 \\
792 \\
652 \\
770 \\
526\end{array}$ & $\begin{array}{l}7,630 \\
8,800 \\
8,400 \\
8,910 \\
6,910\end{array}$ \\
\hline $\begin{array}{l}1951 \\
1952 \\
1953 \\
1954 \\
1955\end{array}$ & $\begin{array}{l}502 \\
396 \\
466 \\
359 \\
378\end{array}$ & $\begin{array}{l}253 \\
346 \\
256 \\
188 \\
258\end{array}$ & $\begin{array}{l}308 \\
342 \\
290 \\
206 \\
316\end{array}$ & $\begin{array}{l}285 \\
345 \\
313 \\
311 \\
276\end{array}$ & $\begin{array}{l}262 \\
275 \\
144 \\
268 \\
187\end{array}$ & $\begin{array}{l}271 \\
299 \\
296 \\
348 \\
247\end{array}$ & $\begin{array}{l}304 \\
440 \\
329 \\
556 \\
346\end{array}$ & $\begin{array}{r}714 \\
1,690 \\
849 \\
1,140 \\
1,180\end{array}$ & $\begin{array}{r}906 \\
2,130 \\
1,130 \\
912 \\
1,090\end{array}$ & $\begin{array}{r}954 \\
I, 620 \\
I, 350 \\
990 \\
990\end{array}$ & $\begin{array}{r}543 \\
1,110 \\
964 \\
605 \\
865\end{array}$ & $\begin{array}{l}470 \\
900 \\
450 \\
418 \\
458\end{array}$ & $\begin{array}{l}5,770 \\
9,890 \\
6,840 \\
6,300 \\
6,590\end{array}$ \\
\hline $\begin{array}{l}1956 \\
1957 \\
1958 \\
1959 \\
1960\end{array}$ & $\begin{array}{l}479 \\
342 \\
976 \\
546 \\
380\end{array}$ & $\begin{array}{l}465 \\
180 \\
675 \\
387 \\
387\end{array}$ & $\begin{array}{l}312 \\
136 \\
599 \\
308 \\
288\end{array}$ & $\begin{array}{l}288 \\
293 \\
556 \\
386 \\
334\end{array}$ & $\begin{array}{l}233 \\
248 \\
503 \\
324 \\
342\end{array}$ & $\begin{array}{l}266 \\
282 \\
531 \\
355 \\
324\end{array}$ & $\begin{array}{l}463 \\
338 \\
566 \\
205 \\
333\end{array}$ & $\begin{array}{r}1,100 \\
971 \\
1,910 \\
861 \\
1,130\end{array}$ & $\begin{array}{r}1,070 \\
2,560 \\
2,030 \\
732 \\
1,150\end{array}$ & $\begin{array}{r}1,080 \\
1,800 \\
1,500 \\
761 \\
1,060\end{array}$ & $\begin{array}{r}675 \\
1,530 \\
1,210 \\
604 \\
772\end{array}$ & $\begin{array}{r}402 \\
1,190 \\
718 \\
423 \\
515\end{array}$ & $\begin{array}{r}6,830 \\
9,870 \\
11,770 \\
5,890 \\
7,010\end{array}$ \\
\hline
\end{tabular}

* Estimated for 1950 compilation.

\begin{tabular}{l|c|c|c|c|c|c|c|c|r|r|r|r|r}
\hline $\begin{array}{l}\text { Water } \\
\text { year }\end{array}$ & oct. & Nov. & Dec. & Jan. & Feb. & Mar. & Apr. & May & June & July & Aug. & Sept. & Annual \\
\hline 1961 & 380 & 90 & 140 & 170 & 170 & 230 & 280 & 1,000 & 1,100 & 1,100 & 870 & 510 & 6,040 \\
1962 & 420 & 170 & 240 & 260 & 240 & 280 & 550 & 1,300 & 1,800 & 1,400 & 1,200 & 690 & 8,550 \\
1963 & 360 & 100 & 160 & 170 & 160 & 330 & 390 & 1,000 & 660 & 960 & 640 & 510 & 5,440 \\
1964 & 330 & 90 & 130 & 150 & 140 & 200 & 240 & 1,100 & 980 & 1,200 & 920 & 710 & 6,190 \\
1965 & 490 & 110 & 130 & 160 & 130 & 240 & 320 & 1,300 & 1,700 & 1,500 & 1,300 & 870 & 8,250 \\
\hline
\end{tabular}



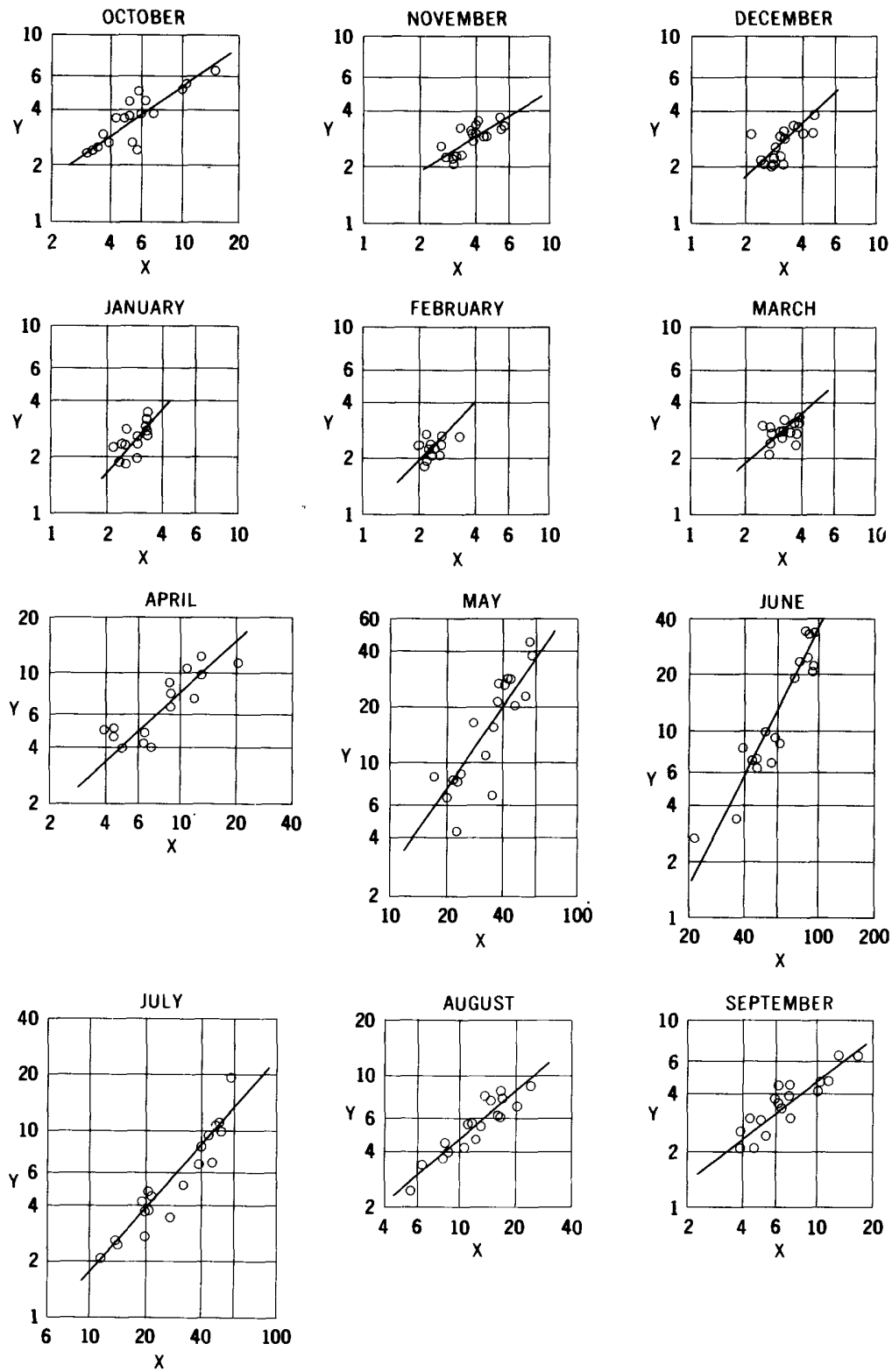

Relationships of sum of monthly mean discharges of Cebolla Creek at Powderhorn, Colo., and Tabor ditch (Y), to monthly mean discharge of Lake Fork at Gateview, Colo. (X). Discharge in thousands of acre-feet. 
1220. Cebolla Creek at Powderhorn, Colo.

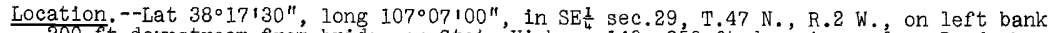
$200 \mathrm{ft}$ downstream from bridge on State Highway $149,250 \mathrm{ft}$ downstream from Powderhorn creek, and half a mile north of Powderhorn. Altitude of gage is $8,000 \mathrm{ft}$ (from topographic map).

Drainage area. $--334 \mathrm{sq} \mathrm{mi}$.

Records ava1labIe. --October 1937 to December 1955.

Estimates of streamflow.--January 1956 to September 1965, based on relationships of monthly mean discharge, adjusted for transmountain diversion through Tabor ditch, with Lake Fork at Gateview, Colo. The regression equation used is:

$$
\log (Y+d)=b \log X-c
$$

(where $\mathrm{Y}$ is discharge of Cebolla creek near Powderhorn, Colo., $d$ is diversion through Tabor ditch at Spring Creek Pass, Colo., and $X$ is discharge of Lake Fork at Gateview, colo., all in acre-feet per month).

\begin{tabular}{l|c|c|r|r|r|r|r|r|r|r|r|r}
\hline \multicolumn{10}{c}{ Monthly values of constants in above equation } \\
\hline & Oct. & Nov. & Dec. & Jan. & Feb. & Mar. & Apr. & May & June & July & Aug. & Sept. \\
\hline b & 0.68 & 0.62 & 0.90 & 1.15 & 1.02 & 0.85 & 0.91 & 1.47 & 1.99 & 1.13 & 0.82 & 0.77 \\
c & -1.01 & -1.24 & -.29 & .58 & .07 & -.49 & -.26 & 2.46 & 5.40 & 1.28 & -.38 & -.59 \\
\hline
\end{tabular}

Average discharge. --28 years $(1937-65), 72,073$ acre-feet per year (99.6 cfs).

Extremes.--1937-55: Maximum discharge, 2,150 cfs May 16, 1944, from rating curve extended above $710 \mathrm{cfs}$; minimum dally, 20 cf's Nov. 11, 1950.

Remarks.--Diversions for irrigation of about 2,800 acres above station. Tabor ditch diverts water above station to Rio Grande Basin. Estimates of annual flow are within about 25 percent of regression line.

Monthly and annual streamflow, in acre-feet

\begin{tabular}{|c|c|c|c|c|c|c|c|c|c|c|c|c|c|}
\hline $\begin{array}{l}\text { Water } \\
\text { year }\end{array}$ & oct. & Nov. & Dec. & Jan. & Feb. & Mar. & Apr. & May & June & JuIy & Aug. & Sept. & Annual \\
\hline $\begin{array}{l}1938 \\
1939 \\
1940\end{array}$ & $\begin{array}{r}\mp 3,690 \\
5,480 \\
2,680\end{array}$ & $\begin{array}{r}\neq 2,680 \\
\neq 3,680 \\
2,520\end{array}$ & $\begin{array}{r}\neq 2,150 \\
\neq 2,150 \\
2,950\end{array}$ & $\begin{aligned} \neq 1, & 840 \\
\neq 1, & 840 \\
2, & 270\end{aligned}$ & $\begin{array}{r}\neq 1,940 \\
\neq 1,670 \\
1,950\end{array}$ & $\begin{array}{r}\$ 2,770 \\
* 3,050 \\
2,100\end{array}$ & $\begin{array}{r}10,860 \\
9,000 \\
4,020\end{array}$ & $\begin{array}{r}21,950 \\
15,500 \\
6,680\end{array}$ & $\begin{array}{r}20,930 \\
7,950 \\
3,260\end{array}$ & & $\begin{array}{l}4,580 \\
3,630 \\
2,470\end{array}$ & $\begin{array}{l}4,700 \\
3,020 \\
2,400\end{array}$ & $\begin{array}{r}\neq 84,870 \\
\neq 59,390 \\
35,360\end{array}$ \\
\hline $\begin{array}{l}1941 \\
1942 \\
1943 \\
1944 \\
1945\end{array}$ & $\begin{array}{l}2,450 \\
6,520 \\
3,630 \\
4,470 \\
3,700\end{array}$ & $\begin{array}{l}2,300 \\
4,630 \\
2,860 \\
3,090 \\
2,740\end{array}$ & $\begin{array}{r}2,050 \\
3,770 \\
2,550 \\
\$ 2,150 \\
2,020\end{array}$ & $\begin{array}{r}1,970 \\
2,540 \\
2,340 \\
\neq 1,840 \\
1,890\end{array}$ & $\begin{array}{r}2,090 \\
2,340 \\
1,670 \\
\neq 1,730 \\
2,340\end{array}$ & $\begin{array}{r}3,050 \\
2,870 \\
2,340 \\
\$ 2,150 \\
2,980\end{array}$ & $\begin{array}{r}4,960 \\
10,730 \\
8,570 \\
75,880 \\
5,080\end{array}$ & $\begin{array}{l}28,480 \\
27,210 \\
11,070 \\
44,760 \\
16,690\end{array}$ & $\begin{array}{r}23,660 \\
32,870 \\
6,630 \\
33,970 \\
9,780\end{array}$ & $\begin{array}{r}10,930 \\
9,350 \\
3,500 \\
9,900 \\
5,180\end{array}$ & $\begin{array}{l}7,410 \\
6,010 \\
8,840 \\
6,030 \\
8,400\end{array}$ & $\begin{array}{l}4,300 \\
3,940 \\
6,500 \\
3,790 \\
4,510\end{array}$ & $\begin{array}{r}93,650 \\
112,800 \\
60,500 \\
\neq 119,800 \\
65,310\end{array}$ \\
\hline $\begin{array}{l}1946 \\
1947 \\
1948 \\
1949 \\
1950\end{array}$ & $\begin{array}{l}5,100 \\
3,680 \\
5,370 \\
3,800 \\
4,480\end{array}$ & $\begin{array}{l}3,330 \\
3,050 \\
3,300 \\
2,940 \\
3,530\end{array}$ & $\begin{array}{l}3,020 \\
2,880 \\
2,900 \\
3,000 \\
3,240\end{array}$ & $\begin{array}{l}2,830 \\
2,360 \\
2,680 \\
2,770 \\
3,430\end{array}$ & $\begin{array}{l}2,380 \\
2,210 \\
2,620 \\
2,670 \\
2,620\end{array}$ & $\begin{array}{l}2,860 \\
3,160 \\
3,350 \\
3,280 \\
2,980\end{array}$ & $\begin{array}{r}7,380 \\
6,610 \\
12,060 \\
9,960 \\
7,750\end{array}$ & $\begin{array}{r}8,830 \\
23,230 \\
38,480 \\
26,680 \\
8,170\end{array}$ & $\begin{array}{r}8,440 \\
18,880 \\
24,750 \\
34,670 \\
6,970\end{array}$ & $\begin{array}{r}4,580 \\
10,580 \\
8,110 \\
19,560 \\
3,700\end{array}$ & $\begin{array}{l}5,430 \\
7,620 \\
5,320 \\
7,810 \\
3,380\end{array}$ & $\begin{array}{l}3,440 \\
6,560 \\
3,590 \\
4,460 \\
2,530\end{array}$ & $\begin{array}{r}57,620 \\
90,820 \\
112,500 \\
121,600 \\
52,780\end{array}$ \\
\hline $\begin{array}{l}1951 \\
1952 \\
1953 \\
1954 \\
1955\end{array}$ & $\begin{array}{l}2,500 \\
2,430 \\
3,830 \\
2,940 \\
2,670\end{array}$ & $\begin{array}{l}2,170 \\
2,260 \\
2,960 \\
2,950 \\
2,020\end{array}$ & $\begin{array}{l}2,290 \\
3,240 \\
2,830 \\
2,550 \\
2,110\end{array}$ & $\begin{array}{l}2,410 \\
3,230 \\
2,970 \\
2,350 \\
1,870\end{array}$ & $\begin{array}{l}2,350 \\
2,060 \\
2,480 \\
2,270 \\
1,810\end{array}$ & $\begin{array}{l}2,800 \\
2,570 \\
3,130 \\
2,720 \\
2,440\end{array}$ & $\begin{array}{r}3,990 \\
11,560 \\
4,180 \\
4,740 \\
4,610\end{array}$ & $\begin{array}{r}7,900 \\
20,650 \\
8,480 \\
4,250 \\
6,630\end{array}$ & $\begin{array}{r}6,180 \\
22,250 \\
9,120 \\
2,660 \\
7,010\end{array}$ & $\begin{array}{l}2,650 \\
6,720 \\
4,520 \\
4,260 \\
2,590\end{array}$ & $\begin{array}{l}3,900 \\
6,870 \\
5,540 \\
4,430 \\
4,220\end{array}$ & $\begin{array}{l}2,050 \\
4,720 \\
2,970 \\
2,960 \\
2,070\end{array}$ & $\begin{array}{l}88,560 \\
53,010 \\
39,080 \\
40,050\end{array}$ \\
\hline 1956 & 2,350 & 2,260 & 2,150 & - & - & - & - & - & - & - & - & - & - \\
\hline
\end{tabular}

* Estimated for 1950 Compllation.

\begin{tabular}{c|c|c|r|r|r|r|r|r|r|r|r|r|r}
\hline $\begin{array}{l}\text { Water } \\
\text { year }\end{array}$ & oct. & Nov. & Dec. & Jan. & Feb. & Mar. & Apr. & May & June & July & Aug. & Sept. & Annual \\
\hline 1956 & - & - & - & 1,800 & 1,800 & 2,700 & 5,400 & 13,700 & 5,500 & 1,700 & 2,600 & 1,700 & 43,660 \\
1957 & 2,000 & 2,300 & 2,100 & 1,900 & 2,100 & 2,500 & 6,000 & 7,600 & 29,800 & 17,600 & 10,500 & 5,300 & 89,700 \\
1958 & 3,800 & 3,500 & 3,700 & 3,100 & 2,700 & 2,900 & 7,200 & 40,100 & 22,200 & 4,500 & 4,600 & 3,400 & 101,700 \\
1959 & 3,400 & 2,900 & 2,900 & 2,400 & 2,400 & 2,500 & 4,500 & 8,500 & 9,000 & 2,400 & 4,100 & 2,800 & 47,800 \\
1960 & 3,900 & 3,000 & 2,200 & 2,000 & 2,000 & 4,400 & 11,500 & 13,600 & 14,700 & 4,300 & 3,600 & 2,600 & 67,800 \\
1961 & 3,100 & 2,600 & 2,100 & 1,900 & 1,900 & 2,500 & 4,200 & 18,200 & 9,100 & 2,400 & 4,800 & 4,400 & 57,200 \\
1962 & 4,800 & 3,700 & 3,400 & 3,100 & 3,200 & 2,900 & 13,200 & 20,200 & 13,900 & 8,300 & 4,700 & 3,100 & 84,500 \\
1963 & 3,500 & 3,000 & 2,400 & 2,100 & 2,700 & 3,700 & 6,600 & 18,200 & 1,700 & 2,100 & 4,400 & 4,000 & 54,400 \\
1964 & 2,900 & 2,700 & 2,300 & 2,100 & 2,000 & 2,200 & 3,400 & 15,500 & 6,900 & 3,500 & 5,400 & 3,200 & 52,100 \\
1965 & 3,100 & 2,700 & 2,800 & 2,700 & 2,600 & 2,700 & 9,300 & 18,400 & 18,600 & 13,000 & 8,800 & 5,600 & 90,300 \\
\hline
\end{tabular}



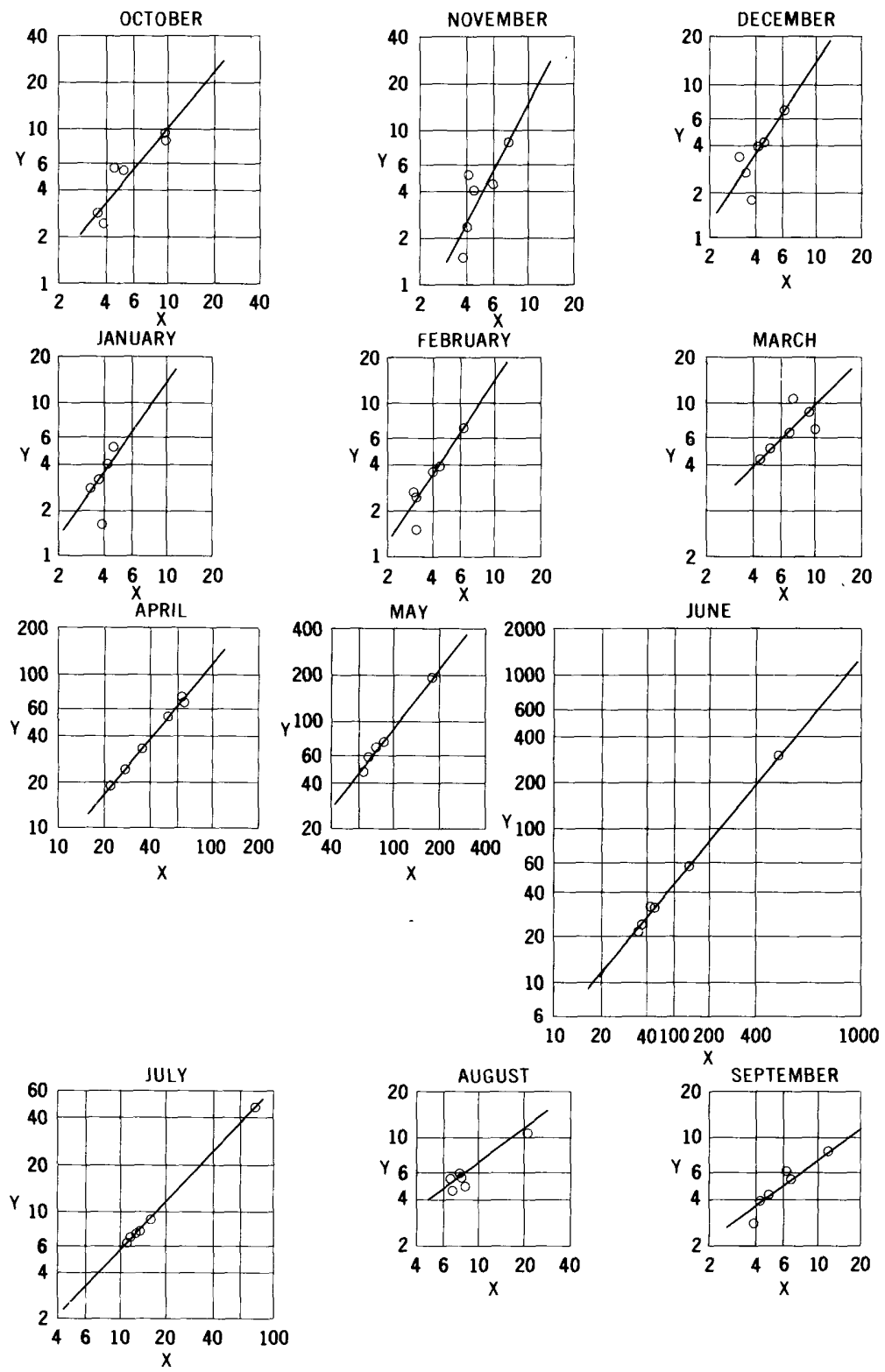

Relationships of monthly mean discharge of Smith Fork at Crawford, Colo. (Y), to monthly mean discharge of Smith Fork near Crawford, Colo. (X). Discharge in hundreds of acre-feet. 
1290. Smith Fork at Crawford, Colo.

Location.--Lat $38^{\circ} 43^{\prime}$, long $107^{\circ} 35^{\prime}$, in SE $\frac{1}{4}$ sec.29, T.15 S., R.91 W., on right bank $100 \mathrm{ft}$ upstream from former bridge site, $i \frac{1}{2}$ miles northeast of Crawford, and 2 miles upstream from Iron Creek.

Drainage area. - -63 sq mi, approximately.

Records available. - - October 1954 to September 1960.

Estimates of streamflow. -October 1935 to september 1954, October 1960 to September 1965, based on relationships of monthly mean discharge with smith Fork near Crawford, Colo. The regression equation used is:

$$
\log Y=\mathrm{b} \log \mathrm{X}-\mathrm{c}
$$

(where $Y$ is discharge of Smith Fork at Crawford, Colo., and $X$ is discharge of Smith Fork near Crawford, Colo., both in acre-feet per monthi.

Monthly values of constants in above equation

\begin{tabular}{l|r|r|r|r|r|r|r|r|r|r|r|l}
\hline & Oct. & Nov. & Dec. & Jan. & Feb. & Mar. & Apr. & May & June & July & Aug. & Sept. \\
\hline $\mathrm{b}$ & 1.18 & 1.97 & 1.48 & 1.48 & 1.48 & 0.99 & 1.21 & 1.29 & 1.20 & 1.05 & 0.72 & 0.72 \\
$\mathrm{c}$ & .54 & 2.48 & 1.30 & 1.30 & 1.30 & -.02 & .77 & 1.23 & .90 & .40 & -.68 & -.68 \\
\hline
\end{tabular}

Average discharge. --30 years $(1935-65), 27,327$ acre-feet per year (37.7 cfs).

Extremes.--1954-60: Maximum discharge, about 1,200 cfs June 5 or 6, 1957; minimum daily, 1.9 cfs Dec. 5, 1955.

Remarks. -Natural flow affected by diversions above station for irrigation above and below station, one diversion to Cottonwood Creek drainage, one small diversion to Iron Creek drainage, and water imported from Curecanti Creek drainage. Estimates of annual flow

are within about 10 percent of regression line.

\begin{tabular}{c|c|c|c|r|r|r|r|r|r|r|r|r|r}
\hline $\begin{array}{c}\text { Water } \\
\text { year }\end{array}$ & Oct. & Nov. & Dec. & Jan. & Feb. & Mar. & Apr. & May & June & July & Aug. & Sept. & Annual \\
\hline 1955 & 560 & 514 & 395 & 324 & 254 & 432 & 2,380 & 5,870 & 2,410 & 750 & 494 & 387 & 14,770 \\
& & & & & & & & & & & \\
1956 & 289 & 156 & 176 & 166 & 150 & 685 & 5,280 & 7,230 & 2,190 & 621 & 462 & 283 & 17,690 \\
1957 & 247 & 247 & 261 & 288 & 395 & 645 & 3,290 & 19,130 & 29,590 & 4,780 & 1,060 & 809 & 60,740 \\
1958 & 826 & 838 & 670 & 522 & 695 & 100 & 7,050 & 22,900 & 5,570 & 889 & 573 & 603 & 42,340 \\
1959 & 540 & 412 & 414 & 410 & 357 & 517 & 1,890 & 4,760 & 3,100 & 684 & 590 & 534 & 14,210 \\
1960 & 926 & 458 & 337 & 284 & 266 & 883 & 6,620 & 5,900 & 3,100 & 731 & 541 & 383 & 21,430 \\
\hline
\end{tabular}

\begin{tabular}{|c|c|c|c|c|c|c|c|c|c|c|c|c|c|}
\hline $\begin{array}{l}\text { Water } \\
\text { year }\end{array}$ & oct. & Nov. & Dec. & Jan. & Feb. & Mar. & Apr. & May & June & July & Aug. & Sept. & Annual \\
\hline $\begin{array}{l}1936 \\
1937 \\
1938 \\
1939 \\
1940\end{array}$ & $\begin{array}{r}430 \\
1,200 \\
680 \\
820 \\
370\end{array}$ & $\begin{array}{r}310 \\
2,000 \\
440 \\
720 \\
270\end{array}$ & $\begin{array}{r}230 \\
1,800 \\
430 \\
680 \\
390\end{array}$ & $\begin{array}{r}270 \\
1,500 \\
390 \\
580 \\
350\end{array}$ & $\begin{array}{r}280 \\
1,200 \\
330 \\
550 \\
310\end{array}$ & $\begin{array}{r}660 \\
1,600 \\
840 \\
1,600 \\
1,200\end{array}$ & & $\begin{array}{l}12,000 \\
11,100 \\
15,600 \\
10,200 \\
16,100\end{array}$ & $\begin{array}{l}2,900 \\
2,300 \\
7,000 \\
2,300 \\
2,300\end{array}$ & $\begin{array}{l}, 300 \\
, 100 \\
, 100 \\
580 \\
620\end{array}$ & $\begin{array}{r}1,000 \\
560 \\
720 \\
520 \\
460\end{array}$ & $\begin{array}{l}840 \\
500 \\
840 \\
600 \\
510\end{array}$ & $\begin{array}{l}28,720 \\
29,960 \\
36,170 \\
26,650 \\
30,080\end{array}$ \\
\hline $\begin{array}{l}1941 \\
1942 \\
1943 \\
1944 \\
1945\end{array}$ & $\begin{array}{r}\neq 520 \\
2,500 \\
370 \\
700 \\
\$ 460\end{array}$ & $\begin{array}{r}\neq 350 \\
2,500 \\
310 \\
520 \\
\$ 390\end{array}$ & $\begin{array}{r}\neq 390 \\
1,300 \\
390 \\
450 \\
\neq 520\end{array}$ & $\begin{array}{r}\neq 430 \\
980 \\
320 \\
410 \\
\neq 560\end{array}$ & $\begin{array}{r}\$ 4.50 \\
880 \\
560 \\
360 \\
\neq 570\end{array}$ & $\begin{array}{r}880 \\
940 \\
940 \\
540 \\
\neq 900\end{array}$ & $\begin{array}{r}3,600 \\
1,000 \\
8,800 \\
1,100 \\
3,200\end{array}$ & $\begin{array}{r}14,000 \\
18,200 \\
8,700 \\
16,100 \\
17,400\end{array}$ & $\begin{array}{r}4,800 \\
10,100 \\
4,800 \\
10,000 \\
6,400\end{array}$ & $\begin{array}{r}1,400 \\
1,300 \\
1,300 \\
\neq 1,600 \\
1,700\end{array}$ & $\begin{array}{r}680 \\
590 \\
780 \\
+580 \\
810\end{array}$ & $\begin{array}{r}780 \\
420 \\
610 \\
¥ 400 \\
540\end{array}$ & $\begin{array}{l}28,280 \\
50,710 \\
27,880 \\
32,760 \\
33,370\end{array}$ \\
\hline $\begin{array}{l}1947 \\
1948 \\
1949 \\
1950\end{array}$ & $\begin{array}{r}840 \\
560 \\
1,600 \\
440 \\
390\end{array}$ & $\begin{array}{r}760 \\
380 \\
, 300 \\
260 \\
350\end{array}$ & $\begin{array}{l}500 \\
230 \\
620 \\
320 \\
310\end{array}$ & $\begin{array}{l}410 \\
220 \\
560 \\
350 \\
330\end{array}$ & $\begin{array}{l}350 \\
290 \\
480 \\
230 \\
410\end{array}$ & $\begin{array}{l}820 \\
760 \\
760 \\
560 \\
480\end{array}$ & & $\begin{array}{r}5,900 \\
8,900 \\
20,600 \\
9,400 \\
7,400\end{array}$ & $\begin{array}{l}3,000 \\
5,000 \\
3,300 \\
6,200 \\
4,200\end{array}$ & $\begin{array}{r}840 \\
1,400 \\
960 \\
1,400 \\
1,100\end{array}$ & $\begin{array}{l}630 \\
680 \\
580 \\
620 \\
500\end{array}$ & $\begin{array}{l}440 \\
660 \\
440 \\
390 \\
400\end{array}$ & $\begin{array}{l}21,890 \\
21,580 \\
38,700 \\
24,770 \\
22,270\end{array}$ \\
\hline $\begin{array}{l}1951 \\
1952 \\
1953 \\
1954\end{array}$ & $\begin{array}{l}430 \\
270\end{array}$ & $\begin{array}{l}230 \\
280\end{array}$ & $\begin{array}{l}310 \\
260 \\
310 \\
260\end{array}$ & $\begin{array}{l}210 \\
240 \\
250 \\
240\end{array}$ & $\begin{array}{l}190 \\
200 \\
320 \\
250\end{array}$ & $\begin{array}{l}540 \\
420 \\
700 \\
360\end{array}$ & & & & $\begin{array}{r}840 \\
1,300 \\
900 \\
430\end{array}$ & $\begin{array}{l}500 \\
680 \\
720 \\
400\end{array}$ & $\begin{array}{l}380 \\
510 \\
380 \\
540\end{array}$ & $\begin{array}{r}13,930 \\
37,430 \\
17,930 \\
8,810\end{array}$ \\
\hline $\begin{array}{l}1961 \\
1962 \\
1963 \\
1964 \\
1965\end{array}$ & $\begin{array}{r}380 \\
1,100 \\
400 \\
380 \\
230\end{array}$ & $\begin{array}{l}250 \\
680 \\
280 \\
310 \\
280\end{array}$ & $\begin{array}{l}280 \\
460 \\
270 \\
350 \\
340\end{array}$ & $\begin{array}{l}280 \\
440 \\
210 \\
280 \\
280\end{array}$ & $\begin{array}{l}220 \\
680 \\
300 \\
230 \\
420\end{array}$ & $\begin{array}{l}510 \\
680 \\
730 \\
400 \\
860\end{array}$ & $\begin{array}{r}1,500 \\
13,800 \\
3,000 \\
1,500 \\
4,300\end{array}$ & $\begin{array}{r}7,000 \\
10,700 \\
5,200 \\
11,500 \\
12,300\end{array}$ & $\begin{array}{r}2,300 \\
4,300 \\
1,000 \\
3,400 \\
11,500\end{array}$ & $\begin{array}{r}560 \\
1,400 \\
240 \\
720 \\
2,300\end{array}$ & $\begin{array}{l}4.70 \\
600 \\
480 \\
530 \\
530\end{array}$ & $\begin{array}{l}810 \\
400 \\
480 \\
430 \\
880\end{array}$ & $\begin{array}{l}14,560 \\
35,240 \\
12,590 \\
20,030 \\
34,320\end{array}$ \\
\hline
\end{tabular}

* Based on estimates for 1950 Compilation. 

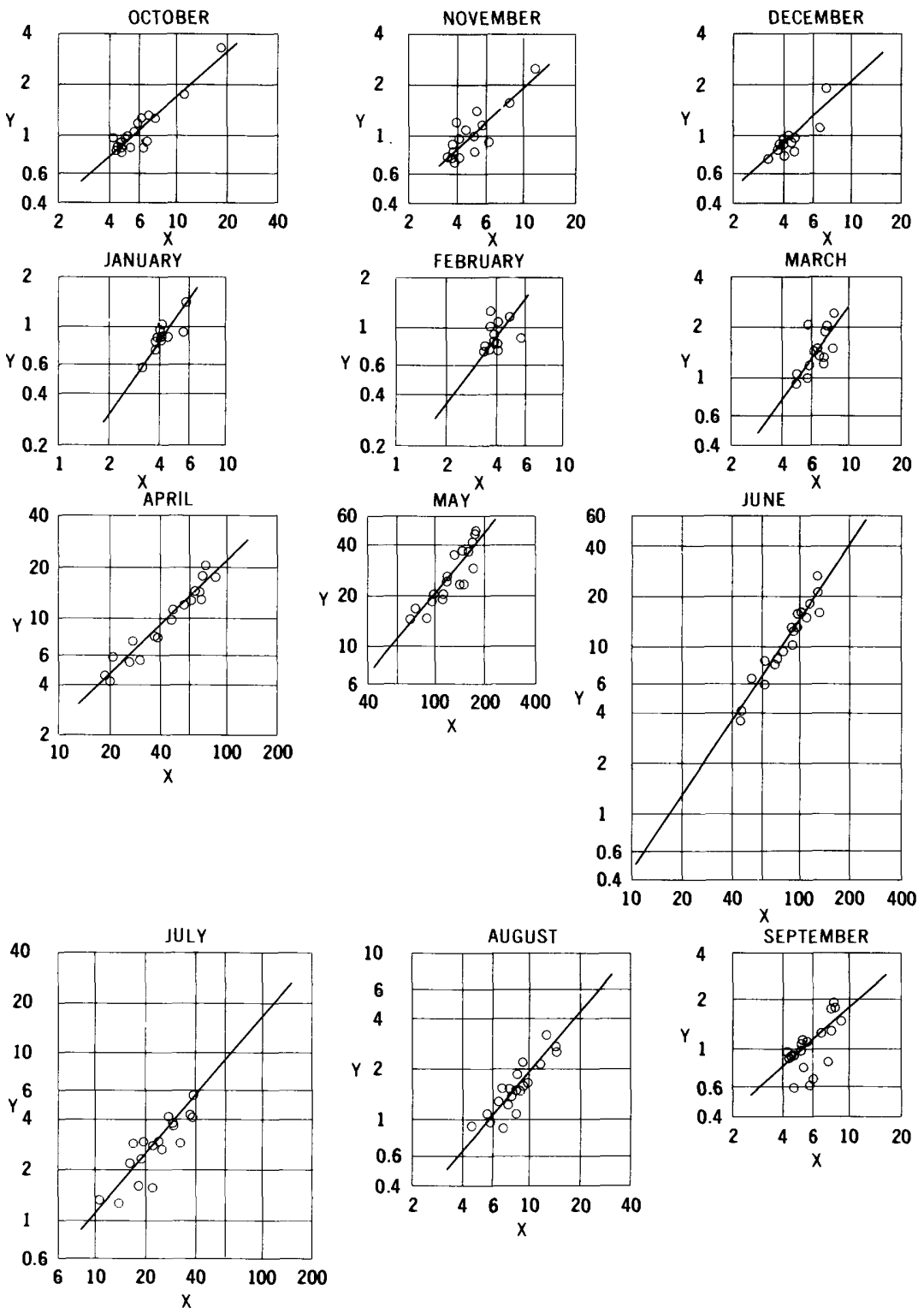

Relationships of monthly mean discharge of East Muddy Creek near Bardine, Colo. (Y), to monthly mean discharge of North Fork Gunnison River near Somerset, Colo., adjusted for change in contents of Paonia Reservoir (X). Discharge in thousands of acre-feet. 
1305. East Muddy Creek near Bardine, Colo.

Location.--Lat $39^{\circ} 01^{\prime}$, long $107^{\circ} 22^{\prime}$, in sec.17, T.12 S., R.89 W., on left bank 5 f't from State Highway 133, a quarter of a mile downstream from Spring Creek, $1 \frac{1}{2}$ miles upstream from West Muddy Creek, and $6 \frac{1}{2}$ miles upstream from Bardine. Datum of gage is 6,654.78 $\mathrm{ft}$ above mean sea level, datum of 1929 .

Drainage area. $--136 \mathrm{sq} \mathrm{mi}$, approximately.

Records available.--October 1934 to September 1953.

Estimates of streamflow.--October 1933 to September 1934, October 1953 to September 1965, based on relationships of monthly mean discharge with North Fork Gunnison River near Somerset, Colo., adjusted for storage in Paonia Reservoir since February 1962. The regression equation used is:

$\log Y=b \log X-c$

(where $Y$ is discharge of East Muddy Creek near Bardine, Colo, and $X$ is discharge of North Fork Gunnison River near Somerset, Colo., adjusted for change in contents of Paonia Reservoir, all in acre-feet per month).

Monthly values of constants in above equation

\begin{tabular}{l|r|r|r|r|r|r|r|l|l|l|l|l}
\hline & oct. & Nov. & Dec. & Jan. & Feb. & Mar. & Apr. & May & June & July & Aug. & Sept. \\
\hline $\mathrm{b}$ & 0.88 & 0.88 & 0.89 & 1.42 & 1.35 & 1.35 & 0.96 & 1.21 & 1.50 & 1.17 & 1.17 & 0.89 \\
$\mathrm{c}$ & .29 & .22 & .24 & 2.19 & 1.92 & 1.99 & .47 & 1.74 & 3.35 & 1.64 & 1.40 & .30 \\
\hline
\end{tabular}

Average discharge. --32 years $(1933-65), 59,831$ acre-feet per year (82.6 cf's).

Extremes.--1934-53: Maximum discharge, 2,190 cf's May 13, 1941, from rating curve extended above $800 \mathrm{cf} s$; minimum not determined.

Remarks.--Diversions for irrigation of about 2,000 acres of hay meadows above station. Estimates of annual flow are within about 20 percent of regression line.

\begin{tabular}{|c|c|c|c|c|c|c|c|c|c|c|c|c|c|}
\hline $\begin{array}{l}\text { Nater } \\
\text { year }\end{array}$ & oct. & Nov. & Dec. & Jan. & Feb. & Mar. & Apr. & May & June & July & Aug. & Sept. & Annual \\
\hline 1935 & $\neq 615$ & $\neq 655$ & $\$ 676$ & $\neq 615$ & ¥555 &, 050 & 5,120 & 20,040 & 18,100 & , 650 &, 520 & 1,290 & $\neq 52,890$ \\
\hline $\begin{array}{l}1936 \\
1937 \\
1938 \\
1939 \\
1940\end{array}$ & $\begin{array}{r}845 \\
802 \\
1,330 \\
1,280 \\
799\end{array}$ & $\begin{array}{r}841 \\
1,250 \\
1,210 \\
\neq 1,41 \\
83\end{array}$ & & & & & & & $\begin{array}{r}6,030 \\
6,610 \\
21,950 \\
4,290 \\
3,620\end{array}$ & & $\begin{array}{l}, 100 \\
, 540 \\
, 380 \\
972 \\
933\end{array}$ & $\begin{array}{r}613 \\
1,110 \\
1,830 \\
853 \\
794\end{array}$ & $\begin{array}{l}\neq 53,270 \\
\neq 49,650 \\
\neq 97,730 \\
\neq 43,230 \\
\neq 39,000\end{array}$ \\
\hline $\begin{array}{l}1941 \\
1942 \\
1943 \\
1944 \\
1945\end{array}$ & $\begin{array}{r}1,290 \\
3,340 \\
966 \\
1,190 \\
968\end{array}$ & $\begin{array}{r}\mp 827 \\
2,530 \\
897 \\
1,450 \\
1,050\end{array}$ & $\begin{array}{r}\neq 615 \\
1,910 \\
853 \\
922 \\
986\end{array}$ & $\begin{array}{r}\neq 676 \\
1,410 \\
585 \\
861 \\
994\end{array}$ & $\begin{array}{r}7722 \\
1,170 \\
730 \\
748 \\
1,260\end{array}$ & & $\begin{array}{r}5,600 \\
17,320 \\
12,920 \\
4,460 \\
5,790\end{array}$ & & & & & $\begin{array}{r}1,920 \\
1,000 \\
1,780 \\
920 \\
678\end{array}$ & $\begin{array}{r}\$ 82,900 \\
86,870 \\
49,720 \\
94,660 \\
71,480\end{array}$ \\
\hline $\begin{array}{l}1946 \\
1947 \\
1948 \\
1949 \\
1950\end{array}$ & $\begin{array}{r}926 \\
854 \\
1,780 \\
926 \\
1,050\end{array}$ & $\begin{array}{r}976 \\
843 \\
1,630 \\
762 \\
1,110\end{array}$ & $\begin{array}{r}972 \\
811 \\
1,140 \\
910 \\
891\end{array}$ & $\begin{array}{r}1,050 \\
738 \\
958 \\
877 \\
863\end{array}$ & $\begin{array}{r}1,060 \\
893 \\
877 \\
778 \\
1,010\end{array}$ & & & $\begin{array}{l}14,880 \\
35,360 \\
41,680 \\
26,320 \\
24,200\end{array}$ & $\begin{array}{r}8,450 \\
13,440 \\
9,550 \\
16,020 \\
10,500\end{array}$ & $\begin{array}{l}1,890 \\
4,360 \\
2,970 \\
3,650 \\
2,840\end{array}$ & $\begin{array}{r}883 \\
2,180 \\
1,890 \\
1,270 \\
1,300\end{array}$ & $\begin{array}{r}586 \\
1,500 \\
1,000 \\
1,150 \\
1,130\end{array}$ & $\begin{array}{l}70,750 \\
79,000 \\
65,140 \\
58,510\end{array}$ \\
\hline $\begin{array}{l}1951 \\
1952 \\
1953\end{array}$ & $\begin{array}{l}837 \\
839 \\
982\end{array}$ & $\begin{array}{l}992 \\
722 \\
770\end{array}$ & $\begin{array}{l}954 \\
904 \\
765\end{array}$ & & $\begin{array}{l}815 \\
805 \\
740\end{array}$ & $\begin{array}{r}1,170 \\
908 \\
1,370\end{array}$ & $\begin{array}{r}5,510 \\
20,370 \\
4,170\end{array}$ & $\begin{array}{l}18,860 \\
42,860 \\
16,710\end{array}$ & $\begin{array}{r}7,990 \\
16,390 \\
12,780\end{array}$ & $\begin{array}{l}1,560 \\
2,920 \\
1,630\end{array}$ & $\begin{array}{l}1,240 \\
2,760 \\
1,650\end{array}$ & $\begin{array}{r}924 \\
1,330 \\
902\end{array}$ & $\begin{array}{l}41,680 \\
91,700 \\
43,290\end{array}$ \\
\hline
\end{tabular}

* Estimated for 1950 Compilation.

Estimated monthly and annual streamflow, in acre-feet

\begin{tabular}{l|r|r|r|r|r|r|r|r|r|r|r|r|r}
\hline $\begin{array}{l}\text { Water } \\
\text { year }\end{array}$ & Oct. & Nov. & Dec. & Jan. & Feb. & Mar. & Apr. & May & June & July & Aug. & Sept. & Annual \\
\hline 1934 & $\neq 1,100$ & $\neq 1,100$ & 4980 & $\$ 740$ & $\$ 680$ & $\neq 2,400$ & 7,300 & 8,800 & 500 & 380 & 600 & 590 & 25,170 \\
1954 & 720 & 960 & 720 & 620 & 740 & 910 & 6,500 & 8,500 & 1,300 & 990 & 920 & 1,200 & 24,080 \\
1955 & 1,300 & 980 & 860 & 740 & 650 & 1,200 & 5,800 & 19,200 & 6,400 & 1,900 & 1,700 & 750 & 41,480 \\
1956 & 630 & 800 & 860 & 840 & 740 & 2,200 & 9,100 & 22,000 & 5,300 & 1,200 & 940 & 590 & 45,200 \\
1957 & 580 & 720 & 720 & 660 & 920 & 1,700 & 6,500 & 33,000 & 53,500 & 18,500 & 6,000 & 1,800 & 124,600 \\
1958 & 1,600 & 1,600 & 1,400 & 1,000 & 1,500 & 1,800 & 8,500 & 39,100 & 12,200 & 1,700 & 1,200 & 990 & 72,590 \\
1959 & 760 & 840 & 820 & 590 & 590 & 820 & 4,100 & 14,000 & 6,300 & 1,100 & 1,200 & 1,000 & 32,120 \\
1960 & 1,500 & 1,200 & 800 & 610 & 600 & 1,800 & 11,600 & 17,600 & 6,600 & 1,300 & 1,100 & 810 & 15,520 \\
1961 & 700 & 780 & 780 & 440 & 460 & 1,000 & 4,400 & 16,800 & 4,300 & 870 & 920 & 2,100 & 33,550 \\
1962 & 2,300 & 1,500 & 1,100 & 730 & 1,600 & 2,500 & 22,500 & 34,800 & 16,000 & 5,100 & 1,400 & 850 & 90,380 \\
1963 & 930 & 720 & 610 & 600 & 1,200 & 1,600 & 7,500 & 13,500 & 1,600 & 750 & 1,000 & 660 & 30,670 \\
1964 & 700 & 780 & 800 & 450 & 430 & 480 & 4,300 & 28,200 & 8,000 & 2,800 & 2,000 & 1,000 & 49,940 \\
1965 & 550 & 920 & 830 & 850 & 640 & 730 & 9,300 & 32,900 & 20,900 & 9,200 & 3,600 & 2,500 & 82,920 \\
\hline
\end{tabular}

F Based on estimates for 1950 Compllation. 

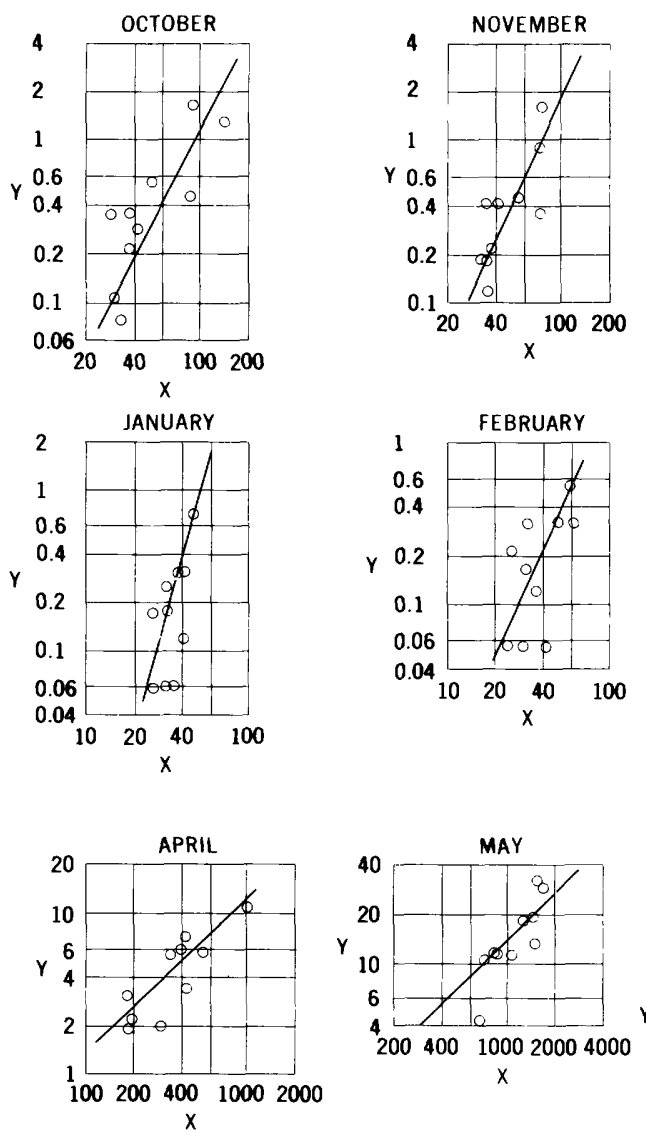
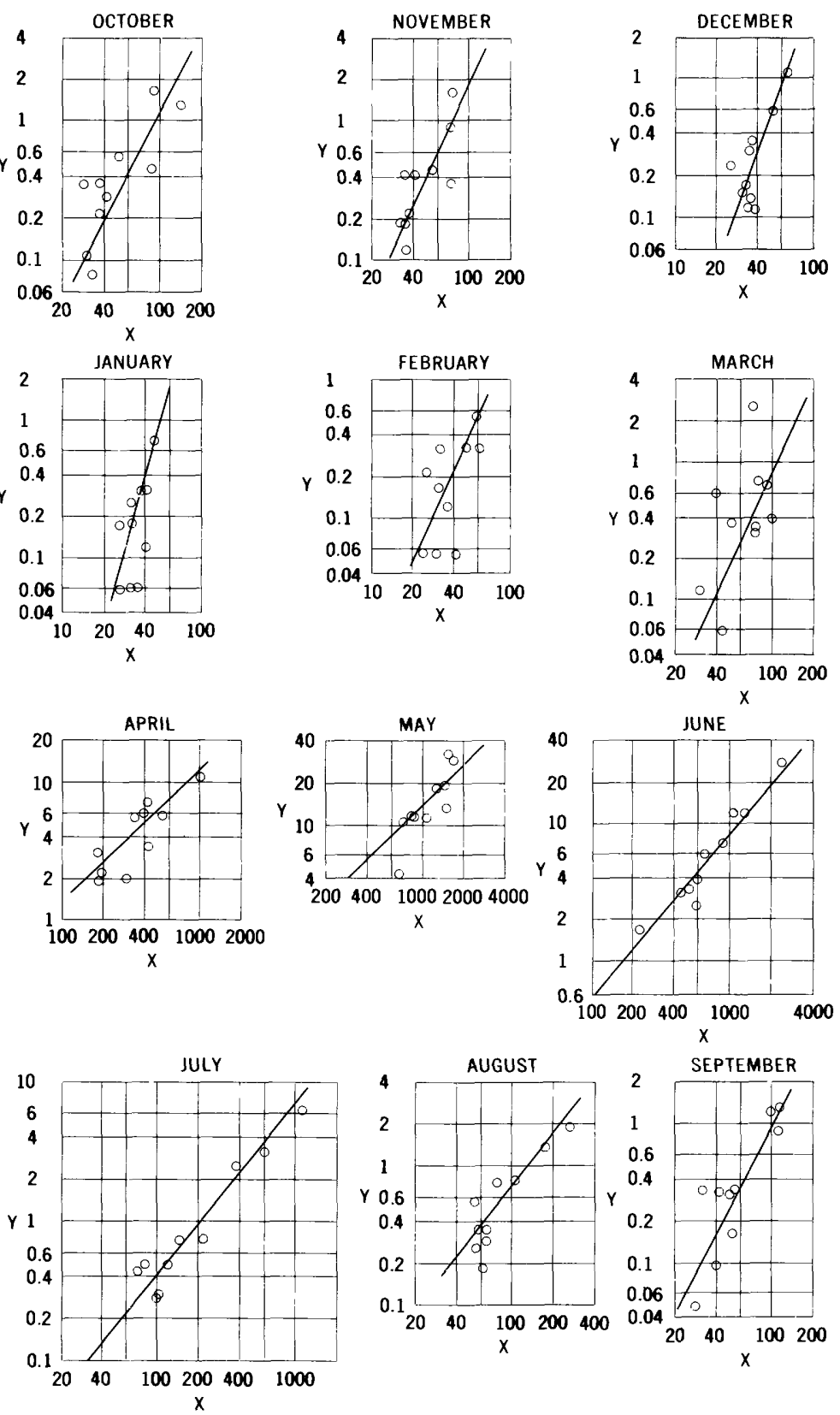

Relationships of monthly mean discharge of West Muddy Creek near Ragged Mountain, Colo. (Y), to monthly mean discharge of North Fork Gunnison River near Somerset, Colo. (X). Discharge in hundreds of acre-feet. 
1306. West Muddy Creek near Ragged Mountain, Coio.

Location.--Lat $39^{\circ} 07^{\prime} 50^{\prime \prime}$, long $107^{\circ} 34130^{\prime \prime}$, in NW $\frac{1}{4}$ sec.5, T.11 S., R.91 W., on left bank $100 \mathrm{f}^{\prime} \mathrm{t}$ upstream from Gold Creek and $9 \frac{1}{2}$ 'miles northwest of Ragged Mountain. Altitude of gage is 8,658 $\mathrm{ft}$ (from topographic map).

Drainage area. $--7.52 \mathrm{sq} \mathrm{mi}$.

Records available. --October 1955 to September 1965.

Estimates of streamf low.--October 1933 to September 1955, based on relationships of monthly mean discharge with North Fork Gunn1son River near Somerset, Colo, adjusted for storage in Paonia Reservoir since February 1962. The regression equation used is:

$$
\log \mathrm{Y}=\mathrm{b} \log \mathrm{X}-\mathrm{c}
$$

(where $Y$ is discharge of West Muddy Creek near Ragged Mountain, Colo., and X Is discharge of North Fork Gunnison River near Somerset, Colo., adjusted for change in contents of Paonia Reservoir, all in acre-feet per month).

Monthly values of constants in above equation

\begin{tabular}{l|l|l|l|l|l|l|l|l|l|l|l|l}
\hline & oct. & Nov. & Dec. & Jan. & Feb. & Mar. & Apr. & May & June & July & Aug. & Sept. \\
\hline b & 1.91 & 2.12 & 2.86 & 3.62 & 2.18 & 2.21 & 0.94 & 0.96 & 1.17 & 1.23 & 1.25 & 1.92 \\
c & 5.57 & 6.25 & 8.80 & 10.42 & 6.50 & 6.89 & 1.61 & 1.66 & 2.93 & 3.30 & 3.15 & 5.72 \\
\hline
\end{tabular}

Average discharge. --32 years $(1933-65), 3,408$ acre-feet per year (4.71 cfs).

Extremes.--1955-65: Maximum discharge, about 260 cfs May 10, 1962 (from flood mark); no flow at times in 1956, 1959-60.

Remarks. --No diversion above station. Estimates of annual flow are within about 5 percent of 'regression line.

Monthly and annual streamflow, in acre-feet

\begin{tabular}{|c|c|c|c|c|c|c|c|c|c|c|c|c|c|}
\hline $\begin{array}{l}\text { Water } \\
\text { year }\end{array}$ & oct. & Nov. & Dec. & Jan. & $\mathrm{Feb}$. & Mar. & Apr. & May & June & July & Aug. & Sept. & Annual \\
\hline $\begin{array}{l}1956 \\
1957 \\
1958 \\
1959 \\
1960\end{array}$ & $\begin{array}{c}8.1 \\
11 \\
174 \\
29 \\
46\end{array}$ & $\begin{array}{r}12 \\
19 \\
165 \\
22 \\
45\end{array}$ & $\begin{array}{r}12 \\
15 \\
112 \\
14 \\
31\end{array}$ & $\begin{array}{c}12 \\
6.1 \\
74 \\
6.1 \\
25\end{array}$ & $\begin{array}{l}12 \\
5.6 \\
56 \\
5.6 \\
17\end{array}$ & $\begin{array}{c}69 \\
31 \\
74 \\
6.1 \\
33\end{array}$ & $\begin{array}{l}710 \\
198 \\
603 \\
306 \\
585\end{array}$ & $\begin{array}{l}1,100 \\
1,350 \\
2,920 \\
1,090 \\
1,140\end{array}$ & $\begin{array}{r}343 \\
2,820 \\
740 \\
259 \\
406\end{array}$ & $\begin{array}{r}30 \\
634 \\
73 \\
29 \\
52\end{array}$ & $\begin{array}{r}26 \\
191 \\
35 \\
29 \\
19\end{array}$ & $\begin{array}{l}4.8 \\
85 \\
31 \\
16 \\
9.5\end{array}$ & $\begin{array}{l}2,340 \\
5,370 \\
5,060 \\
1,510 \\
2,410\end{array}$ \\
\hline $\begin{array}{l}1961 \\
1962 \\
1963 \\
1964 \\
1965\end{array}$ & $\begin{array}{r}22 \\
138 \\
57 \\
37 \\
35\end{array}$ & $\begin{array}{l}18 \\
93 \\
37 \\
42 \\
41\end{array}$ & $\begin{array}{l}18 \\
61 \\
25 \\
12 \\
37\end{array}$ & $\begin{array}{c}18 \\
31 \\
18 \\
6.1 \\
31\end{array}$ & $\begin{array}{c}22 \\
33 \\
33 \\
5.8 \\
33\end{array}$ & $\begin{array}{r}37 \\
39 \\
250 \\
12 \\
61\end{array}$ & $\begin{array}{r}218 \\
1,130 \\
571 \\
198 \\
343\end{array}$ & $\begin{array}{r}1,180 \\
5,270 \\
457 \\
1,850 \\
1,960\end{array}$ & $\begin{array}{r}324 \\
1,220 \\
170 \\
611 \\
1,200\end{array}$ & $\begin{array}{r}49 \\
252 \\
44 \\
76 \\
323\end{array}$ & $\begin{array}{r}55 \\
75 \\
35 \\
79 \\
142\end{array}$ & $\begin{array}{r}128 \\
32 \\
33 \\
34 \\
119\end{array}$ & $\begin{array}{l}2,090 \\
6,370 \\
1,730 \\
2,960 \\
4,320\end{array}$ \\
\hline
\end{tabular}

Estimated monthly and annual streamflow, in acre-feet

\begin{tabular}{|c|c|c|c|c|c|c|c|c|c|c|c|c|c|}
\hline $\begin{array}{l}\text { Water } \\
\text { year }\end{array}$ & oct. & Nov. & Dec. & Jan. & Feb. & Mar. & Apr. & May & June & July & Aug. & Sept. & Annual \\
\hline $\begin{array}{l}1934 \\
1935\end{array}$ & $\begin{array}{r}\neq 50 \\
10\end{array}$ & $\begin{array}{r}\$ 50 \\
20\end{array}$ & $\neq 40$ & $\begin{array}{l}\neq 30 \\
\neq 20\end{array}$ & $\begin{array}{l}\neq 20 \\
\neq 10\end{array}$ & $\begin{array}{l}\$ 70 \\
\neq 20\end{array}$ & $\begin{array}{l}430 \\
330\end{array}$ & $\begin{array}{r}700 \\
1,400\end{array}$ & $\begin{array}{r}60 \\
1,000\end{array}$ & $\begin{array}{r}10 \\
130\end{array}$ & $\begin{array}{l}20 \\
50\end{array}$ & $\begin{array}{r}8 \\
40\end{array}$ & $\begin{array}{l}7,490 \\
3,050\end{array}$ \\
\hline 1936 & 30 & 20 & 10 & 20 & 10 & 40 & 900 & 1,900 & 480 & 80 & 60 & 30 & 3,580 \\
\hline 1937 & 20 & 20 & 30 & 20 & 10 & 30 & 490 & 2,000 & 390 & 80 & 50 & 20 & 160 \\
\hline 1938 & 60 & 50 & 50 & 50 & 20 & 80 & 920 & 2,300 & 1,200 & 150 & 50 & 60 & 4,990 \\
\hline 1939 & 50 & 50 & 50 & 40 & 10 & 90 & 620 & 1,500 & 330 & 40 & 40 & 50 & 2,870 \\
\hline 1940 & 30 & 20 & 20 & 10 & 10 & 50 & 520 & 1,500 & 320 & 40 & 30 & 30 & 2,580 \\
\hline 1941 & 70 & 20 & 20 & 30 & 20 & 40 & 400 & 2,500 & 840 & 160 & 60 & 60 & 4,220 \\
\hline 1942 & 390 & 240 & 160 & 160 & 40 & 30 & $I, 100$ & 2,200 & 940 & 120 & 60 & 20 & 160 \\
\hline 1943 & 20 & 20 & 20 & 20 & 20 & 50 & 920 & 1,300 & 600 & 90 & 100 & 60 & 3,220 \\
\hline 1944 & 40 & 40 & 40 & 40 & 20 & 20 & 250 & 2,400 & 1,200 & 220 & 60 & 20 & 4,350 \\
\hline 1945 & 30 & 40 & 40 & 40 & 20 & 30 & 280 & 2,000 & 800 & 210 & 110 & 40 & 3,640 \\
\hline 1946 & 50 & 60 & 50 & 40 & 20 & 40 & 720 & 1,000 & 480 & 60 & 40 & 20 & 2,580 \\
\hline 1947 & 50 & 40 & 50 & 30 & 20 & 60 & 370 & 1,800 & 760 & 210 & 90 & 70 & 3,550 \\
\hline 1948 & 140 & 110 & 130 & 130 & 50 & 40 & 820 & 2,300 & 660 & 100 & 60 & 20 & 4,560 \\
\hline 1949 & 30 & 20 & 30 & 50 & 20 & 30 & 620 & 1,600 & 880 & 160 & 50 & 30 & 3,520 \\
\hline 1950 & 40 & 30 & 30 & 40 & 20 & 20 & 800 & 1,600 & 760 & 110 & 40 & 30 & 3,520 \\
\hline 1951 & 20 & 20 & 30 & 40 & 20 & 30 & 350 & 1,400 & 560 & 110 & 50 & 20 & 2,650 \\
\hline 1952 & 30 & 20 & 40 & 60 & 20 & 20 & 980 & 2,500 & 1,200 & 180 & 110 & 60 & 5,220 \\
\hline 1953 & 30 & 30 & 30 & 30 & 20 & 30 & 270 & 1,100 & 760 & 90 & 70 & 20 & 2,480 \\
\hline 1954 & 20 & 30 & 20 & 20 & 20 & 20 & 400 & 680 & 120 & 40 & 40 & 40 & 1,450 \\
\hline 1955 & 70 & 30 & 30 & 30 & 10 & 30 & 380 & 1,300 & 450 & 70 & 60 & 10 & 2,470 \\
\hline
\end{tabular}

* Based on estimates for 1950 compllation. 

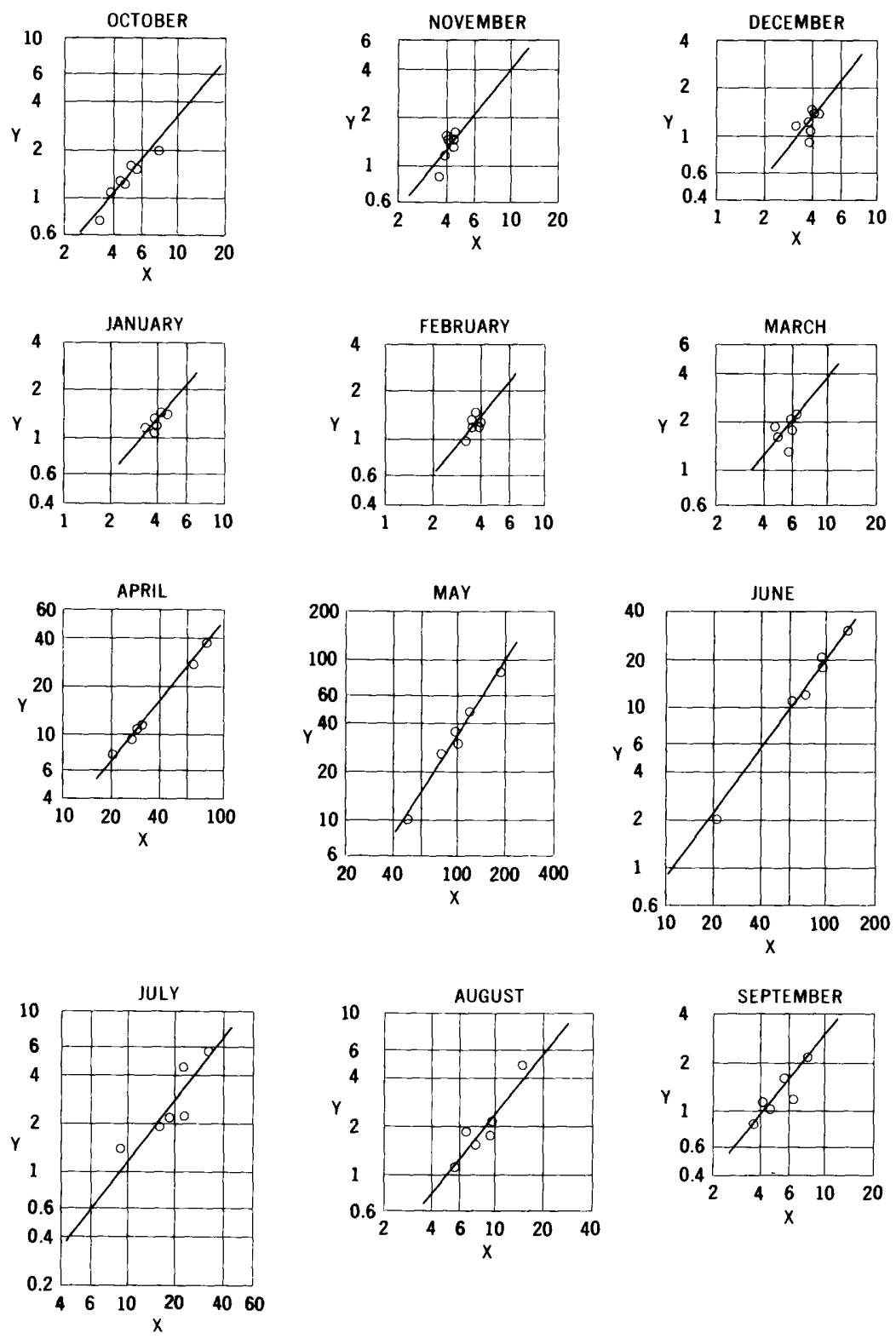

Relationships of monthly mean discharge of Muddy Creek at Bardine, Colo. (Y), to monthly mean discharge of North Fork Gunnison River near Somerset, Colo. (X). Discharge in thousands of acre-feet:- 
1315. Muddy Creek at Bardine, Colo.

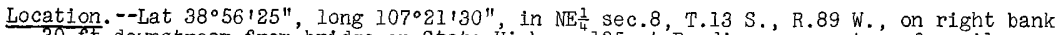
$30 \mathrm{ft}$ downstream from 'bridge on State Highway 135 at Bardine, a quarter of a mile upstream from confluence with Anthracite Creek, li miles downstream from Deep creek, and $5 \frac{1}{2}$ miles east of Somerset. Datum of gage is $6,266.07 \mathrm{ft}$ above mean sea level, datum of 1929 .

Drainage area. $--246 \mathrm{sq} \mathrm{mi}$.

Records avallable. - - October 1949 to December 1955.

Estimates of streamflow. --October 1933 to September 1949, January 1956 to January 1962 , based on relationships of monthly mean discharge with North Fork Gunnison R1ver near somerset, colo. The regression equation used is:

$$
\log Y=b \log X-c
$$

(where $Y$ is discharge of Muddy Creek at Bardine, Colo., and $X$ is discharge of North Fork Gunnison River near Somerset, Colo., both in acre-feet'per month).

\begin{tabular}{l|l|l|l|l|l|l|l|l|l|l|l|l}
\hline \multicolumn{10}{c}{ Monthly values of constants in above equation } \\
\hline b & Oct. & Nov. & Dec. & Jan. & Feb. & Mar. & Apr. & May & June & July & Aug. & Sept. \\
\hline $\mathrm{c}$ & 1.19 & 1.23 & 1.26 & 1.18 & 1.20 & 1.20 & 1.23 & 1.57 & 1.35 & 1.29 & 1.23 & 1.20 \\
\hline
\end{tabular}

Average discharge. --28 years $(1933-61), 102,440$ acre-feet per year (14l cfs).

Extremes. $-1949-55$ : Maximum discharge, 3,400 cfs May 4, 1952; minimum daily, $8.4 \mathrm{cfs}$ Sept. $1-3,1954$.

Remarks. - Natural flow of stream affected by small diversions to nearby drainage areas for Irrigation, irrigation of about 2,500 acres above station, and storage in Overland Reservoir (capacity 2,660 acre-feet). Completely regulated by Paonia Reservoir after January 1962. Estimates of annual flow are within about 20 percent of regression line.

Monthly and annual streamflow, in acre-feet

\begin{tabular}{r|c|c|c|c|c|c|c|c|c|c|c|c|c}
\hline $\begin{array}{l}\text { Water } \\
\text { year }\end{array}$ & Oct. & Nov. & Dec. & Jan. & Peb. & Mar. & Apr. & May & June & July & Aug. & Sept. & Annual \\
\hline 1950 & 1,540 & 1,610 & 1,230 & 1,480 & 1,500 & 1,350 & 28,650 & 48,090 & 21,000 & 4,580 & 1,880 & 1,610 & 114,500 \\
1951 & 1,310 & 1,580 & 1,400 & 1,210 & 1,240 & 1,800 & 9,350 & 30,590 & 12,260 & 2,240 & 1,560 & 1,030 & 65,570 \\
1952 & 1,270 & 1,180 & 1,400 & 1,410 & 1,340 & 1,650 & 39,640 & 85,130 & 30,750 & 5,640 & 4,800 & 2,160 & 176,400 \\
1953 & 1,620 & 1,450 & 1,430 & 1,360 & 1,230 & 2,240 & 7,600 & 26,640 & 18,290 & 2,180 & 2,180 & 1,150 & 67,370 \\
1954 & 1,120 & 1,430 & 1,170 & 1,180 & 1,200 & 1,870 & 11,490 & 10,170 & 2,040 & 1,420 & 1,110 & 1,200 & 35,400 \\
1955 & 2,000 & 1,330 & 1,120 & 1,100 & 994 & 2,120 & 11,190 & 36,230 & 11,580 & 1,910 & 1,750 & 849 & 72,170 \\
1956 & 746 & 900 & 922 & - & - & - & - & - & - & - & - & - & - \\
\hline
\end{tabular}

Estimated monthly and annual streamflow, in acre-feet

\begin{tabular}{|c|c|c|c|c|c|c|c|c|c|c|c|c|c|}
\hline $\begin{array}{l}\text { Water } \\
\text { year }\end{array}$ & oct. & Nov. & Dec. & Jan. & Feb. & Mar. & Apr. & May & June & July & Aug. & Sept. & Annual \\
\hline $\begin{array}{l}1934 \\
1935\end{array}$ & $\begin{array}{r}\mp 1,800 \\
800\end{array}$ & $\begin{array}{l}800 \\
, 000\end{array}$ & $\begin{array}{l}500 \\
100\end{array}$ & 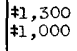 & $\begin{array}{r}1,100 \\
\$ 920\end{array}$ & & $\begin{array}{r}15,000 \\
9,700\end{array}$ & & r, & $\begin{array}{r}390 \\
3,800\end{array}$ & $\begin{array}{r}700 \\
1,700\end{array}$ & $\begin{array}{r}640 \\
1,800\end{array}$ & $\begin{array}{l}38,430 \\
84,920\end{array}$ \\
\hline $\begin{array}{l}1936 \\
1937 \\
1938 \\
1939 \\
1940\end{array}$ & $\begin{array}{l}1,500 \\
1,200 \\
2,100 \\
1,900 \\
1,300\end{array}$ & $\begin{array}{l}1,300 \\
1,300 \\
1,900 \\
2,000 \\
1,200\end{array}$ & $\begin{array}{r}840 \\
1,300 \\
1,600 \\
1,700 \\
1,000\end{array}$ & $\begin{array}{r}1,100 \\
1,100 \\
1,500 \\
1,300 \\
920\end{array}$ & $\begin{array}{l}1,100 \\
1,100 \\
1,500 \\
1,000 \\
1,100\end{array}$ & $\begin{array}{l}2,400 \\
2,300 \\
3,700 \\
3,800 \\
2,900\end{array}$ & $\begin{array}{l}34,000 \\
15,400 \\
35,600 \\
21,200 \\
16,500\end{array}$ & $\begin{array}{l}61,000 \\
64,000 \\
76,000 \\
41,500 \\
40,800\end{array}$ & $\begin{array}{r}11,000 \\
8,400 \\
30,800 \\
7,200 \\
7,000\end{array}$ & & $\begin{array}{l}800 \\
500 \\
700 \\
200 \\
860\end{array}$ & & $\begin{array}{r}118,740 \\
101,200 \\
163,100 \\
86,000 \\
75,880\end{array}$ \\
\hline $\begin{array}{l}1941 \\
1942 \\
1943 \\
1944 \\
1945\end{array}$ & $\begin{array}{l}2,300 \\
6,800 \\
1,200 \\
1,700 \\
1,400\end{array}$ & $\begin{array}{l}1,300 \\
4,700 \\
1,100 \\
1,700 \\
1,700\end{array}$ & $\begin{array}{l}00 \\
00 \\
00 \\
00 \\
00\end{array}$ & $\begin{array}{l}1,200 \\
2,200 \\
1,100 \\
1,300 \\
1,400\end{array}$ & $\begin{array}{l}1,300 \\
1,800 \\
1,500 \\
1,300 \\
1,300\end{array}$ & $\begin{array}{l}2,500 \\
2,300 \\
2,700 \\
1,600 \\
2,000\end{array}$ & $\begin{array}{r}12,000 \\
44,500 \\
35,000 \\
6,400 \\
7,600\end{array}$ & $\begin{array}{l}88,000 \\
73,500 \\
30,000 \\
82,000 \\
64,000\end{array}$ & $\begin{array}{l}00 \\
00 \\
00 \\
00 \\
00\end{array}$ & $\begin{array}{l}00 \\
00 \\
00 \\
00 \\
00\end{array}$ & & & $\begin{array}{r}139,300 \\
170,200 \\
95,800 \\
138,400 \\
112,700\end{array}$ \\
\hline $\begin{array}{l}1946 \\
1947 \\
1948 \\
1949\end{array}$ & $\begin{array}{l}2,000 \\
1,900 \\
3,700 \\
1,300\end{array}$ & $\begin{array}{l}1,700 \\
3,100 \\
1,100\end{array}$ & $\begin{array}{l}1,600 \\
2,500 \\
1,300\end{array}$ & $\begin{array}{l}1,000 \\
1,300 \\
2,000 \\
1,500\end{array}$ & $\begin{array}{l}1,400 \\
2,100 \\
1,200\end{array}$ & $\begin{array}{l}2,600 \\
3,000 \\
2,500 \\
2,200\end{array}$ & $\begin{array}{l}26,000 \\
10,900 \\
30,800 \\
21,000\end{array}$ & $\begin{array}{l}21,100 \\
54,200 \\
82,000 \\
45,500\end{array}$ & $\begin{array}{l}11,200 \\
18,300 \\
15,600 \\
22,200\end{array}$ & & & $\begin{array}{l}1,100 \\
2,500 \\
1,000 \\
1,300\end{array}$ & $\begin{array}{r}74,900 \\
105,900 \\
150,000 \\
104,900\end{array}$ \\
\hline $\begin{array}{l}1956 \\
1957 \\
1958 \\
1959 \\
1960\end{array}$ & $\begin{array}{r}- \\
760 \\
2,900 \\
1,100 \\
2,800\end{array}$ & $\begin{array}{r}- \\
780 \\
2,800 \\
1,200 \\
1,800\end{array}$ & $\begin{array}{r}- \\
960 \\
2,500 \\
1,100 \\
1,100\end{array}$ & $\begin{array}{l}1,400 \\
1,100 \\
1,700 \\
1,000 \\
1,100\end{array}$ & $\begin{array}{l}1,200 \\
1,500 \\
2,500 \\
1,000 \\
1,000\end{array}$ & $\begin{array}{l}3,400 \\
2,600 \\
2,800 \\
1,400 \\
2,700\end{array}$ & $\begin{array}{r}17,300 \\
11,400 \\
16,000 \\
6,200 \\
24,100\end{array}$ & $\begin{array}{l}38,500 \\
63,000 \\
81,500 \\
21,200 \\
28,800\end{array}$ & $\begin{array}{r}8,600 \\
70,500 \\
18,200 \\
10,000 \\
10,400\end{array}$ & $\mid \begin{array}{r}1,200 \\
24,300 \\
1,900 \\
1,200 \\
1,400\end{array}$ & $\begin{array}{l}1,100 \\
8,000 \\
1,400 \\
1,400 \\
1,300\end{array}$ & $\begin{array}{r}630 \\
3,000 \\
1,300 \\
1,300 \\
960\end{array}$ & $\begin{array}{r}75,900 \\
187,900 \\
135,300 \\
48,100 \\
77,460\end{array}$ \\
\hline $\begin{array}{l}1961 \\
1962\end{array}$ & $\begin{array}{r}980 \\
5,000\end{array}$ & $\begin{array}{l}1,100 \\
2,700\end{array}$ & & $\begin{array}{r}820 \\
1,200\end{array}$ & $\begin{array}{l}800 \\
-\end{array}$ & 1,700 & 6,600 & 27,100 & 7,000 & 900 & 1,100 & $\begin{array}{c}3,600 \\
-\end{array}$ & 52,800 \\
\hline
\end{tabular}

* Based on estimates for 1950 Compilation. 

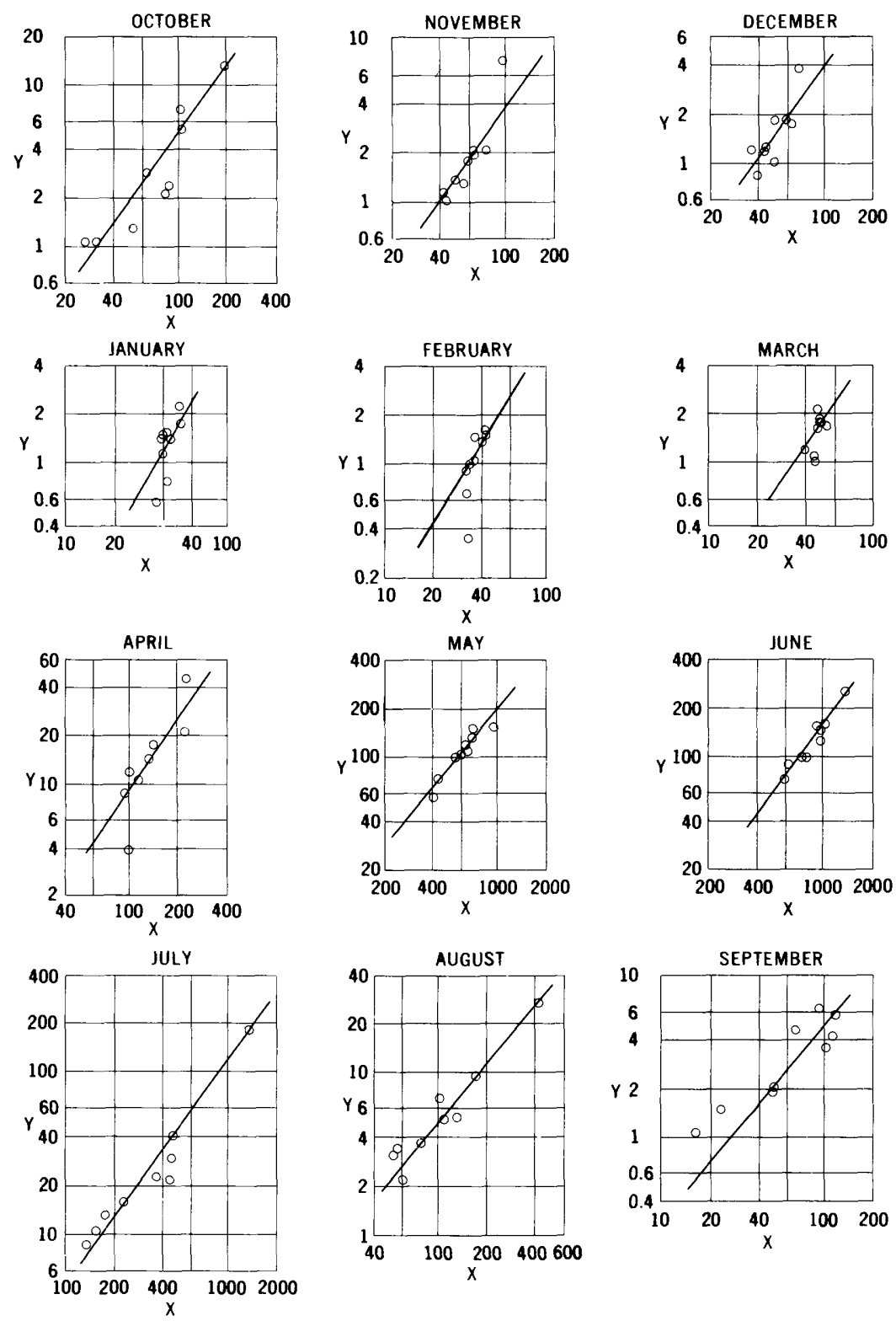

Relationships of monthly mean discharge of Anthracite Creek near Floresta, Colo. (Y), to monthly mean discharge of Crystal River near Redstone, Colo. $(X)$. Discharge in hundreds of acre-feet. 
1320. Anthracite Creek near Floresta, Colo.

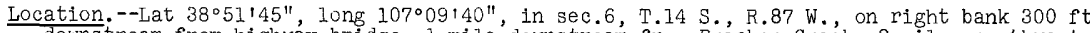
downstream from highway bridge, 1 mile downstream from Bracken Creek, 2 miles northwest of old Floresta, and 9 miles west of Crested Butte. Altitude of gage is 8,830 ft (from topographic mapl.

Drainage area. $--17.5 \mathrm{sq} \mathrm{mi}$.

Records available.--october 1938 to September 1943, October 1954 to September 1958. Monthly discharge only for some periods.

Estimates of streamflow.--May 1935 to September 1938, October 1943 to September 1954, October 1958 to september 1965, based on relationships of monthly mean discharge with Crystal River near Redstone, Colo. (Crystal River near Redstore, Colo. discontinued Sept. 30 , 1963; records for October 1963 to September 1965, were estimated for use in this relationship on the basis of discharge of Crystal River above Avalanche Creek, near Redstone, colo.). The regression equation used is:

$$
\log Y=b \log X-c
$$

(where $\mathrm{Y}$ is discharge of Anthracite Creek near Floresta, Colo., and $\mathrm{X}$ is discharge of Crystal River near Redstone, Colo., both in acre-feet per month).

Monthly values of constants in above equation

\begin{tabular}{l|l|l|l|l|l|l|l|l|l|l|l|l}
\hline & oct. & Nov. & Dec. & Jan. & Feb. & Mar. & Apr. & May & June & July & Aug. & Sept. \\
\hline b & 1.44 & 1.40 & 1.39 & 1.73 & 1.62 & 1.51 & 1.47 & 1.19 & 1.29 & 1.38 & 1.22 & 1.20 \\
c & 3.03 & 3.02 & 2.96 & 4.16 & 2.72 & 3.33 & 2.90 & 1.65 & 2.30 & 2.83 & 2.19 & 2.09 \\
\hline
\end{tabular}

Average discharge. --30 years $(1936-65), 31,230$ acre-feet per year (43.1 cfs).

Extremes.--1938-43, 1954-58: Maximum discharge, 709 cfs June 28, 1957; minimum discharge not determined.

Remarks.--One sme11 diversion above station to Coal Creek in East River basin. Estimates of annual flow are within about 5 percent of regression line.

Monthly and annual streamflow, in acre-feet

\begin{tabular}{|c|c|c|c|c|c|c|c|c|c|c|c|c|c|}
\hline $\begin{array}{l}\text { Water } \\
\text { year }\end{array}$ & Oct. & Nov. & Dec. & Jan. & Feb. & Mar. & Apr. & May & June & July & Aug. & Sept. & Annual \\
\hline $\begin{array}{l}1939 \\
1940\end{array}$ & $\begin{array}{l}214 \\
282\end{array}$ & $\begin{array}{l}196 \\
137\end{array}$ & $\begin{array}{r}184 \\
85\end{array}$ & $\begin{array}{r}173 \\
58\end{array}$ & $\begin{array}{r}150 \\
35\end{array}$ & $\begin{array}{l}170 \\
105\end{array}$ & $\begin{array}{l}1,720 \\
1,400\end{array}$ & $\begin{array}{l}13,100 \\
10,910\end{array}$ & & $\begin{array}{r}1,300 \\
848\end{array}$ & $\begin{array}{l}333 \\
218\end{array}$ & $\begin{array}{l}636 \\
358\end{array}$ & $\begin{array}{l}26,880 \\
21,780\end{array}$ \\
\hline $\begin{array}{l}1941 \\
1942 \\
1943\end{array}$ & $\begin{array}{r}\$ 530 \\
1,340 \\
130\end{array}$ & $\begin{array}{r}\neq 208 \\
\neq 734 \\
128\end{array}$ & $\begin{array}{r}\$ 184 \\
\$ 369 \\
123\end{array}$ & $\begin{array}{r}¥ 154 \\
¥ 215 \\
110\end{array}$ & $\begin{array}{r}\neq 139 \\
\neq 167 \\
108\end{array}$ & $\begin{array}{r}\neq 184 \\
\neq 215 \\
185\end{array}$ & $\begin{array}{r}\neq 1,190 \\
\neq 2,060 \\
4,540\end{array}$ & $\begin{array}{r}\neq 15,060 \\
10,400 \\
10,130\end{array}$ & $\begin{array}{l}14,030 \\
15,880 \\
12,090\end{array}$ & $\begin{array}{l}4,040 \\
2,920 \\
2,160\end{array}$ & $\begin{array}{l}526 \\
515 \\
978\end{array}$ & $\begin{array}{l}422 \\
191 \\
464\end{array}$ & $\begin{array}{r} \pm 36,670 \\
\pm 35,000 \\
31,150\end{array}$ \\
\hline 1955 & 710 & 178 & 100 & 77 & 67 & 123 & 1,070 & 7,420 & 9,990 & 2,250 & 698 & 153 & 22,840 \\
\hline $\begin{array}{l}1956 \\
1957 \\
1958\end{array}$ & $\begin{array}{l}108 \\
108 \\
242\end{array}$ & $\begin{array}{l}101 \\
112 \\
202\end{array}$ & $\begin{array}{l}119 \\
121 \\
172\end{array}$ & $\begin{array}{l}137 \\
145 \\
139\end{array}$ & $\begin{array}{r}91 \\
103 \\
153\end{array}$ & $\begin{array}{l}164 \\
112 \\
188\end{array}$ & $\begin{array}{r}1,410 \\
395 \\
867\end{array}$ & $\begin{array}{r}11,810 \\
5,620 \\
14,380\end{array}$ & $\begin{array}{r}9,630 \\
24,670 \\
15,410\end{array}$ & $\begin{array}{r}1,040 \\
18,410 \\
1,580\end{array}$ & $\begin{array}{r}308 \\
2,690 \\
364\end{array}$ & $\begin{array}{l}106 \\
569 \\
201\end{array}$ & $\begin{array}{l}25,020 \\
53,060 \\
33,900\end{array}$ \\
\hline
\end{tabular}

* Estimated for 1950 Compilation.

\begin{tabular}{|c|c|c|c|c|c|c|c|c|c|c|c|c|c|}
\hline $\begin{array}{l}\text { Water } \\
\text { year }\end{array}$ & Oct. & Nov. & Dec. & Jan. & Feb. & Mar. & Apr. & May & June & July & Aug. & Sept. & Annual \\
\hline 1935 & - & - & - & - & - & - & - & $\neq 5,400$ & 15,200 & 5,400 & 850 & 500 & - \\
\hline $\begin{array}{l}1936 \\
1937 \\
1938\end{array}$ & $\begin{array}{l}350 \\
290 \\
310\end{array}$ & $\begin{array}{l}180 \\
140 \\
160\end{array}$ & $\begin{array}{l}150 \\
130 \\
150\end{array}$ & $\begin{array}{l}130 \\
120 \\
140\end{array}$ & $\begin{array}{l}120 \\
150 \\
110\end{array}$ & $\begin{array}{l}190 \\
180 \\
190\end{array}$ & $\begin{array}{l}2,600 \\
1,200 \\
2,100\end{array}$ & $\begin{array}{l}17,800 \\
14,900 \\
12,000\end{array}$ & $\begin{array}{r}13,200 \\
9,600 \\
20,400\end{array}$ & $\begin{array}{l}2,100 \\
2,400 \\
6,800\end{array}$ & $\begin{array}{l}810 \\
580 \\
970\end{array}$ & $\begin{array}{l}390 \\
450 \\
870\end{array}$ & $\begin{array}{l}38,020 \\
30,140 \\
44,000\end{array}$ \\
\hline $\begin{array}{l}1944 \\
1945\end{array}$ & $\begin{array}{l}260 \\
340\end{array}$ & $\begin{array}{l}150 \\
180\end{array}$ & $\begin{array}{l}140 \\
110\end{array}$ & $\begin{array}{l}140 \\
110\end{array}$ & $\begin{array}{l}100 \\
110\end{array}$ & $\begin{array}{l}130 \\
160\end{array}$ & $\begin{array}{l}550 \\
560\end{array}$ & $\begin{array}{l}10,200 \\
11,100\end{array}$ & $\begin{array}{l}15,600 \\
11,300\end{array}$ & $\begin{array}{l}7,100 \\
6,500\end{array}$ & $\begin{array}{l}620 \\
840\end{array}$ & $\begin{array}{l}180 \\
270\end{array}$ & $\begin{array}{l}35,170 \\
31,580\end{array}$ \\
\hline $\begin{array}{l}1946 \\
1947 \\
1948 \\
1949 \\
1950\end{array}$ & $\begin{array}{l}280 \\
240 \\
660 \\
380 \\
290\end{array}$ & $\begin{array}{l}200 \\
190 \\
260 \\
150 \\
180\end{array}$ & $\begin{array}{l}180 \\
140 \\
170 \\
130 \\
150\end{array}$ & $\begin{array}{l}140 \\
130 \\
160 \\
120 \\
140\end{array}$ & $\begin{array}{l}120 \\
110 \\
170 \\
130 \\
120\end{array}$ & $\begin{array}{l}160 \\
200 \\
190 \\
180 \\
130\end{array}$ & $\begin{array}{l}2,800 \\
1,100 \\
1,400 \\
1,500 \\
1,100\end{array}$ & $\begin{array}{r}9,400 \\
10,200 \\
12,800 \\
6,400 \\
5,600\end{array}$ & $\begin{array}{l}13,100 \\
10,700 \\
13,100 \\
15,400 \\
17,700\end{array}$ & $\begin{array}{l}1,600 \\
7,600 \\
3,400 \\
5,800 \\
3,400\end{array}$ & $\begin{array}{r}370 \\
1,000 \\
630 \\
490 \\
330\end{array}$ & $\begin{array}{l}280 \\
530 \\
220 \\
240 \\
210\end{array}$ & $\begin{array}{l}28,630 \\
32,140 \\
33,160 \\
30,920 \\
29,350\end{array}$ \\
\hline $\begin{array}{l}1951 \\
1952 \\
1953 \\
1954\end{array}$ & $\begin{array}{l}170 \\
220 \\
170 \\
210\end{array}$ & $\begin{array}{l}150 \\
170 \\
150 \\
150\end{array}$ & $\begin{array}{l}140 \\
140 \\
200 \\
120\end{array}$ & $\begin{array}{l}130 \\
140 \\
180 \\
110\end{array}$ & $\begin{array}{l}110 \\
110 \\
140 \\
100\end{array}$ & $\begin{array}{l}140 \\
120 \\
220 \\
100\end{array}$ & $\begin{array}{r}760 \\
2,800 \\
580 \\
1,000\end{array}$ & $\begin{array}{r}8,400 \\
13,300 \\
3,600 \\
6,400\end{array}$ & $\begin{array}{r}14,500 \\
22,100 \\
15,200 \\
4,100\end{array}$ & $\begin{array}{l}5,200 \\
6,400 \\
2,300 \\
1,100\end{array}$ & $\begin{array}{r}660 \\
1,400 \\
530 \\
230\end{array}$ & $\begin{array}{l}200 \\
420 \\
170 \\
350\end{array}$ & $\begin{array}{l}30,560 \\
47,320 \\
23,440 \\
13,970\end{array}$ \\
\hline $\begin{array}{l}1959 \\
1960\end{array}$ & $\begin{array}{l}150 \\
430\end{array}$ & $\begin{array}{l}140 \\
220\end{array}$ & $\begin{array}{l}130 \\
150\end{array}$ & $\begin{array}{l}100 \\
120\end{array}$ & $\begin{array}{r}90 \\
100\end{array}$ & $\begin{array}{l}110 \\
200\end{array}$ & $\begin{array}{r}660 \\
=, 400\end{array}$ & $\begin{array}{l}5,300 \\
7,800\end{array}$ & $\begin{array}{l}15,800 \\
11,800\end{array}$ & $\begin{array}{l}1,400 \\
1,700\end{array}$ & $\begin{array}{l}420 \\
350\end{array}$ & $\begin{array}{l}180 \\
130\end{array}$ & $\begin{array}{l}22,480 \\
25,400\end{array}$ \\
\hline $\begin{array}{l}1961 \\
1962 \\
1963 \\
1964 \\
1965\end{array}$ & $\begin{array}{l}170 \\
950 \\
260 \\
110 \\
140\end{array}$ & $\begin{array}{l}140 \\
310 \\
170 \\
120 \\
140\end{array}$ & $\begin{array}{l}110 \\
210 \\
120 \\
100 \\
120\end{array}$ & $\begin{array}{r}100 \\
150 \\
110 \\
80 \\
130\end{array}$ & $\begin{array}{r}90 \\
230 \\
110 \\
60 \\
110\end{array}$ & $\begin{array}{r}140 \\
220 \\
150 \\
70 \\
120\end{array}$ & $\begin{array}{r}470 \\
4,900 \\
910 \\
440 \\
1,300\end{array}$ & $\begin{array}{r}9,300 \\
10,400 \\
11,000 \\
9,800 \\
8,800\end{array}$ & $\begin{array}{r}9,400 \\
15,600 \\
5,200 \\
10,100 \\
17,000\end{array}$ & $\begin{array}{r}790 \\
7,600 \\
780 \\
3,000 \\
16,000\end{array}$ & $\begin{array}{r}290 \\
1,000 \\
340 \\
580 \\
1,300\end{array}$ & $\begin{array}{l}700 \\
260 \\
220 \\
180 \\
820\end{array}$ & $\begin{array}{l}21,700 \\
41,830 \\
19,370 \\
24,640 \\
45,980\end{array}$ \\
\hline
\end{tabular}

* Based on estimates for 1950 Compilation. 

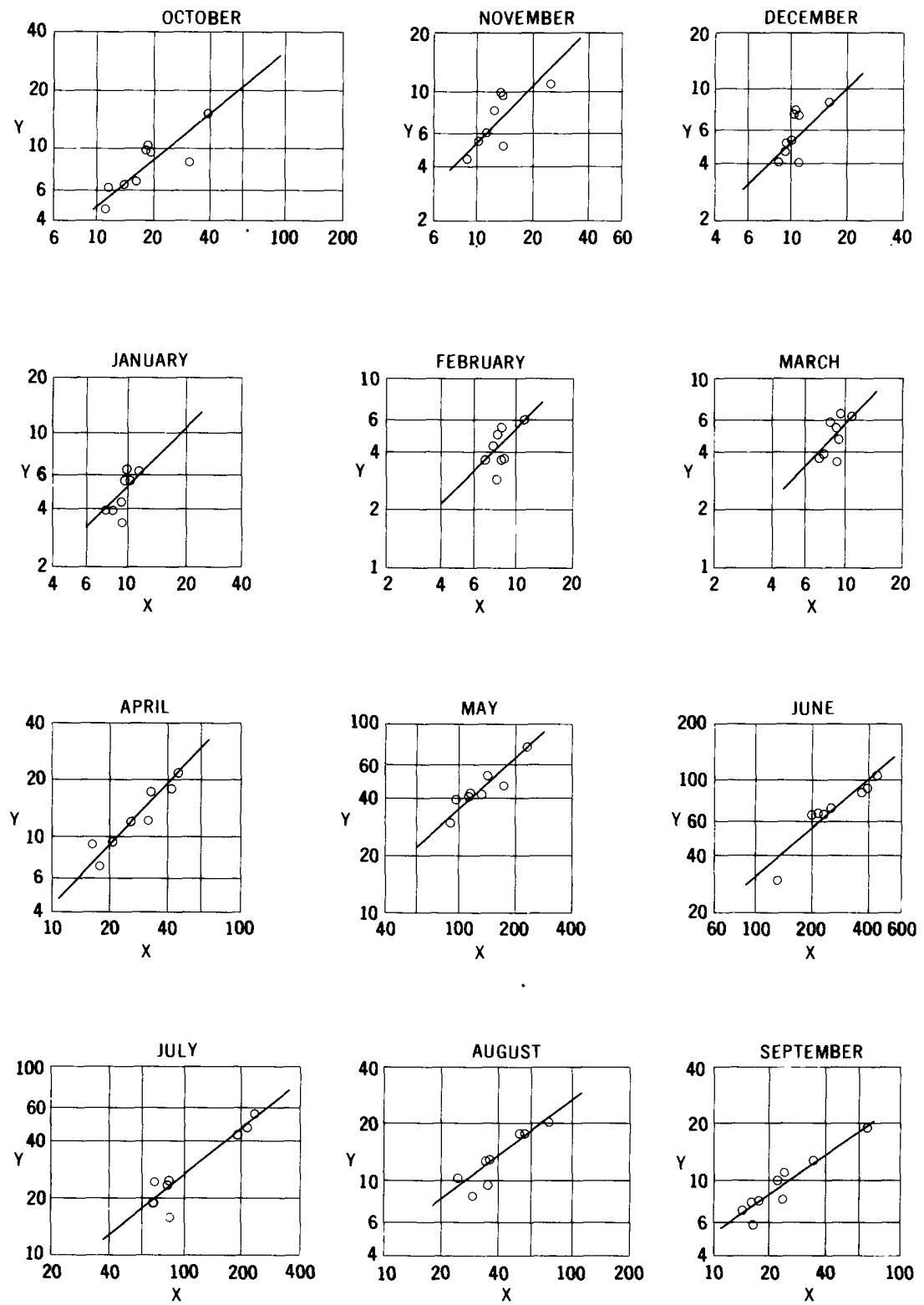

Relationships of monthly mean discharge of Red Mountain Creek near Ironton, Colo. (Y), to monthly mean discharge of Animas River at Howardsville, Colo. $(X)$. Discharge in hundreds of acre-feet. 
1445. Red Mountain Creek near Ironton, Colo.

Location. --Lat $37^{\circ} 57^{14} 5^{\prime \prime}$, long $107^{\circ} 39140^{\prime \prime}$, in $N \frac{1}{2}$ sec. 29 , T. 43 N., R.7 W., on right bank

$100 \mathrm{f}$ t from U. S. Highway 550, 2 miles northeast of Ironton, 2 miles upstream from mouth, and 4 miles south of Ouray. Datum of gage is $9,585.58 \mathrm{ft}$ above mean sea level (Bureau of Reclamation bench mark).

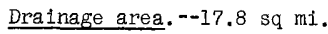

Records available. --August 1947 to December 1955.

Estimates of streamflow.--october 1935 to August 1947, January 1956 to September 1965 , based on relationships of monthly mean discharge with Animas River at Howardsville, Colo. The regression equation used is:

$$
\log Y=b \log X-c
$$

(where $Y$ is discharge of Red Mountain Creek near Ironton, Colo,, and X Is discharge of Animas River at Howardsville, Colo., both in acre-feet per month).

\begin{tabular}{r|r|r|r|r|r|r|r|r|r|r|r|r}
\hline \multicolumn{10}{c}{ Monthly values of constants in above equation } \\
\hline b & Oct. & Nov. & Dec. & Jan. & Feb. & Mar. & Apr. & May & June & July & Aug. & Sept. \\
\hline & 0.82 & 0.98 & 0.98 & 0.98 & 0.98 & 1.06 & 1.06 & 0.89 & 0.85 & 0.80 & 0.75 & 0.70 \\
$\mathrm{c}$ & -.23 & .22 & .22 & .20 & .21 & .12 & .53 & .00 & -.20 & -.22 & -.42 & -.61 \\
\hline
\end{tabular}

Average discharge. -30 years $(1935-65), 24,128$ acre-feet per year $(33.3 \mathrm{cfs})$.

Extremes:-1947-55: Maximum discharge, 331 cfs June 18, 1949; minimum daily, 3.0 cfs Feb. 1,1951 .

Remarks. - Water is imported above station by Red Mountain ditch from Mineral Creek in San Juan River basin. No diversion above station. Estimates of annual flow are within about 10 percent of regression line.

Monthly and annual streamflow, in acre-feet

\begin{tabular}{r|r|r|r|r|r|r|r|r|r|r|r|r|r}
\hline $\begin{array}{l}\text { Water } \\
\text { year }\end{array}$ & Oct. & Nov. & Dec. & Jan. & Feb. & Mar. & Apr. & May & June & July & Aug. & Sept. & Annual \\
\hline 1947 & - & - & - & - & - & - & - & - & - & & - & - & 1,910 \\
1948 & 1,540 & 1,040 & 827 & 652 & 603 & 639 & 1,210 & 7,520 & 8,510 & 4,240 & 1,740 & 1,110 & 29,630 \\
1949 & 1,040 & 932 & 712 & 588 & 559 & 657 & 1,730 & 5,330 & 10,140 & 5,530 & 1,770 & 1,030 & 30,020 \\
1950 & 942 & 944 & 719 & 593 & 385 & 488 & 2,170 & 3,970 & 6,480 & 2,310 & 1,020 & 768 & 20,790 \\
1951 & 647 & 514 & 460 & 400 & 447 & 596 & 905 & 4,100 & 6,480 & 2,460 & 1,270 & 773 & 19,050 \\
1952 & 623 & 520 & 506 & 440 & 374 & 394 & 1,190 & 4,720 & 10,690 & 4,730 & 2,020 & 1,300 & 27,510 \\
1953 & 946 & 746 & 754 & 641 & 511 & 560 & 954 & 3,090 & 7,190 & 2,400 & 1,280 & 692 & 19,760 \\
1954 & 615 & 586 & 518 & 395 & 380 & 383 & 1,780 & 4,200 & 3,040 & 1,560 & 827 & 809 & 15,090 \\
1955 & 824 & 492 & 397 & 345 & 298 & 368 & 689 & 3,940 & 6,670 & 1,890 & 958 & 567 & 17,440 \\
1956 & 479 & 434 & 404 & - & - & - & - & - & - & - & - & - & - \\
\hline
\end{tabular}

Estimated monthly and annual streamflow, in acre-feet

\begin{tabular}{|c|c|c|c|c|c|c|c|c|c|c|c|c|c|}
\hline $\begin{array}{l}\text { Water } \\
\text { year }\end{array}$ & oct. & Nov. & Dec. & Jan. & Feb. & Mar. & Apr. & May & June & JuIy & Aug. & Sept. & Annual \\
\hline $\begin{array}{l}1936 \\
1937 \\
1938 \\
1939 \\
1940\end{array}$ & $\begin{array}{r}\$ 860 \\
800 \\
850 \\
1,700 \\
760\end{array}$ & $\begin{array}{r}\$ 840 \\
990 \\
840 \\
1,200 \\
640\end{array}$ & $\begin{array}{r}\neq 700 \\
\neq 660 \\
\neq 660 \\
\neq 700 \\
520\end{array}$ & $\begin{array}{r}\neq 730 \\
\neq 640 \\
\neq 670 \\
\neq 600 \\
510\end{array}$ & $\begin{array}{r}\neq 650 \\
\neq 540 \\
\neq 540 \\
\$ 490 \\
450\end{array}$ & $\begin{array}{r}\neq 800 \\
\neq 620 \\
\neq 760 \\
650 \\
540\end{array}$ & $\begin{array}{r}\neq 2,600 \\
\neq 2,200 \\
1,800 \\
1,800 \\
2,000\end{array}$ & $\begin{array}{l}9,100 \\
9,000 \\
4,900 \\
6,800 \\
7,300\end{array}$ & $\begin{array}{r}5,400 \\
5,300 \\
10,400 \\
5,800 \\
5,100\end{array}$ & $\begin{array}{l}2,100 \\
1,900 \\
4,700 \\
1,800 \\
1,200\end{array}$ & $\begin{array}{r}2,100 \\
980 \\
1,600 \\
980 \\
760\end{array}$ & $\begin{array}{r}1,100 \\
980 \\
1,600 \\
1,400 \\
1,100\end{array}$ & $\begin{array}{l}26,980 \\
24,610 \\
29,320 \\
23,920 \\
20,080\end{array}$ \\
\hline $\begin{array}{l}1941 \\
1942 \\
1943 \\
1944 \\
1945\end{array}$ & $\begin{array}{r}1,400 \\
3,100 \\
620 \\
930 \\
780\end{array}$ & $\begin{array}{r}800 \\
1,800 \\
530 \\
1,100 \\
690\end{array}$ & $\begin{array}{l}570 \\
800 \\
510 \\
670 \\
490\end{array}$ & $\begin{array}{l}500 \\
820 \\
530 \\
560 \\
440\end{array}$ & $\begin{array}{l}420 \\
640 \\
470 \\
500 \\
430\end{array}$ & $\begin{array}{l}520 \\
710 \\
520 \\
660 \\
440\end{array}$ & $\begin{array}{r}600 \\
1,400 \\
3,100 \\
610 \\
600\end{array}$ & $\begin{array}{l}6,700 \\
4,900 \\
4,600 \\
5,600 \\
4,900\end{array}$ & $\begin{array}{l}8,300 \\
9,400 \\
6,600 \\
9,200 \\
7,200\end{array}$ & $\begin{array}{l}6,100 \\
4,700 \\
2,900 \\
5,000 \\
3,600\end{array}$ & $\begin{array}{l}2,200 \\
1,700 \\
2,100 \\
1,600 \\
1,700\end{array}$ & $\begin{array}{r}1,900 \\
1,000 \\
1,500 \\
830 \\
830\end{array}$ & $\begin{array}{l}30,010 \\
30,970 \\
23,980 \\
27,260 \\
22,100\end{array}$ \\
\hline $\begin{array}{l}1946 \\
1947\end{array}$ & $\begin{array}{l}960 \\
800\end{array}$ & $\begin{array}{l}950 \\
780\end{array}$ & $\begin{array}{l}680 \\
690\end{array}$ & $\begin{array}{l}540 \\
560\end{array}$ & $\begin{array}{l}450 \\
490\end{array}$ & $\begin{array}{l}600 \\
510\end{array}$ & $\begin{array}{r}2,800 \\
800\end{array}$ & $\begin{array}{l}3,600 \\
6,300\end{array}$ & $\begin{array}{l}7,500 \\
7,800\end{array}$ & $\begin{array}{l}2,400 \\
4,800\end{array}$ & $\begin{array}{l}1,100 \\
2,100\end{array}$ & $\begin{array}{l}860 \\
-\end{array}$ & $\begin{array}{l}22,440 \\
27,540\end{array}$ \\
\hline $\begin{array}{l}1956 \\
1957 \\
1958 \\
1959 \\
1960\end{array}$ & $\begin{array}{r}- \\
470 \\
960 \\
930 \\
1,300\end{array}$ & $\begin{array}{r}- \\
380 \\
1,100 \\
740 \\
1,200\end{array}$ & $\begin{array}{l}- \\
370 \\
850 \\
660 \\
730\end{array}$ & $\begin{array}{l}500 \\
350 \\
680 \\
640 \\
700\end{array}$ & $\begin{array}{l}440 \\
300 \\
460 \\
560 \\
570\end{array}$ & $\begin{array}{l}620 \\
380 \\
560 \\
680 \\
640\end{array}$ & $\begin{array}{r}1,500 \\
970 \\
930 \\
840 \\
2,300\end{array}$ & $\begin{array}{l}6,200 \\
2,900 \\
8,200 \\
4,200 \\
4,600\end{array}$ & $\begin{array}{r}6,300 \\
10,800 \\
8,500 \\
6,700 \\
8,400\end{array}$ & $\begin{array}{l}1,600 \\
7,300 \\
2,500 \\
1,500 \\
2,800\end{array}$ & $\begin{array}{r}850 \\
2,900 \\
1,400 \\
1,200 \\
1,000\end{array}$ & $\begin{array}{r}600 \\
1,600 \\
1,200 \\
790 \\
780\end{array}$ & $\begin{array}{l}19,930 \\
28,720 \\
27,340 \\
19,440 \\
25,020\end{array}$ \\
\hline $\begin{array}{l}1961 \\
1962 \\
1963 \\
1964 \\
1965\end{array}$ & $\begin{array}{r}730 \\
1,600 \\
900 \\
660 \\
690\end{array}$ & $\begin{array}{r}660 \\
1,100 \\
750 \\
520 \\
490\end{array}$ & $\begin{array}{l}560 \\
720 \\
600 \\
430 \\
430\end{array}$ & $\begin{array}{l}530 \\
630 \\
580 \\
410 \\
450\end{array}$ & $\begin{array}{l}470 \\
590 \\
570 \\
340 \\
360\end{array}$ & $\begin{array}{l}540 \\
630 \\
740 \\
370 \\
360\end{array}$ & $\begin{array}{r}800 \\
3,200 \\
1,500 \\
490 \\
1,800\end{array}$ & $\begin{array}{l}6,200 \\
5,300 \\
6,400 \\
5,600 \\
4,900\end{array}$ & $\begin{array}{l}6,800 \\
7,500 \\
3,800 \\
5,400 \\
8,500\end{array}$ & $\begin{array}{l}1,600 \\
3,900 \\
1,600 \\
1,800 \\
6,100\end{array}$ & $\begin{array}{l}1,100 \\
1,400 \\
1,100 \\
1,100 \\
2,500\end{array}$ & $\begin{array}{r}1,400 \\
1,000 \\
1,000 \\
800 \\
1,900\end{array}$ & $\begin{array}{l}21,390 \\
27,570 \\
19,540 \\
17,920 \\
28,480\end{array}$ \\
\hline
\end{tabular}

F Based on estimates for 1950 Compilation. 

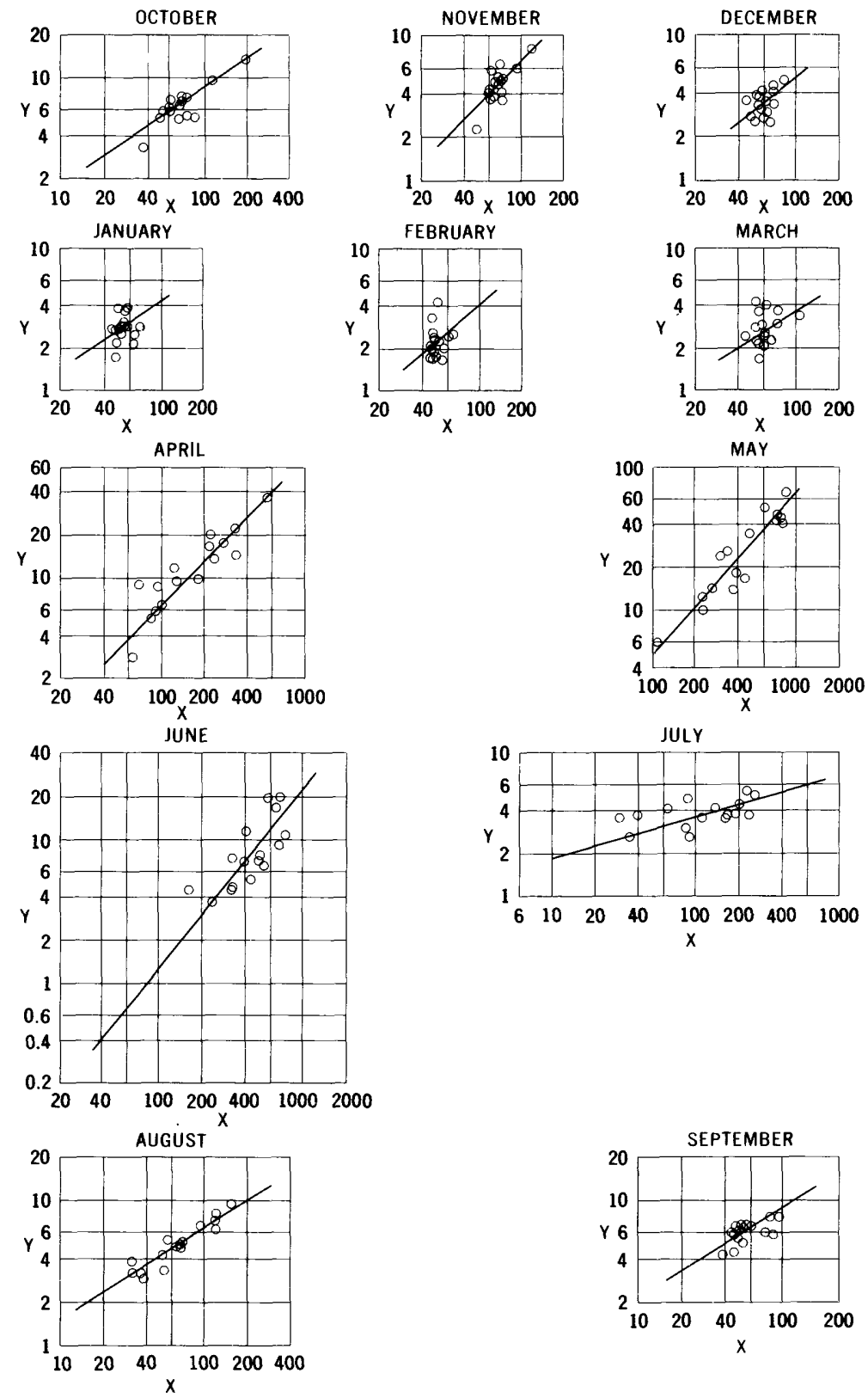

Relationships of monthly mean discharge of Roubideau Creek at mouth, near Delta, Colo. (Y), to monthly mean discharge of Gunnison River near Grand Junction, Colo. $(X)$. Discharge in thousands of acre-feet. 
1505. Roubideau Creek at mouth, near Delta, Colo.

Location. --Lat $38^{\circ} 44^{\prime}$, long $108^{\circ} 09 \mathrm{I}$, in sec.19, T.15 S., R.96 W., on left bank $90 \mathrm{ft}$ upstream from railroad bridge, a quarter of a mile upstream from mouth, and 5 miles west of Delta. Datum of gage is $4,864.34 \mathrm{ft}$ above mean sea level, datum of 1929 .

Drainage area. $--245 \mathrm{sq} \mathrm{mi}$.

Records available. --October 1938 to September 1954.

Estimates of streamflow.--October 1930 to September 1938, October 1954 to september 1965 based on relationships of monthly mean discharge with Gunnison River near Grand Junction, colo. The regression equation used is:

$$
\log Y=b \log X-c
$$

(where $Y$ is discharge of Roubideau Creek at mouth, near Delta, Colo., and $X$ is discharge of Gunnison River near Grand Junction, Colo., both in acre-feet per'month).

Monthly values of constants in above equation

\begin{tabular}{l|l|l|r|r|r|r|r|r|r|r|r|c}
\hline \multicolumn{10}{c}{ Monthly values of constants in above equation } \\
\hline & Oct. & Nov. & Dec. & Jan. & Feb. & Mar. & Apr. & May & June & July & Aug. & Sept. \\
\hline $\mathrm{b}$ & 0.67 & 1.00 & 0.75 & 0.67 & 0.85 & 0.64 & 1.02 & 1.13 & 1.25 & 0.29 & 0.63 & 0.63 \\
$\mathrm{c}$ & -.59 & 1.18 & .05 & -.28 & .64 & -.36 & 1.28 & 1.96 & 3.25 & -2.11 & -.66 & -.83 \\
\hline
\end{tabular}

Average discharge. --35 years $(1930-65), 84,757$ acre-feet per year (117 cfs).

Extremes.--1938-54: Maximum discharge, 2,950 cf's Aug. 5, 1945, from rating curve extended above 1,300 cf's; minimum observed, il cf's Feb. 1, igsi (discharge measurement).

Remarks. --Part of discharge is return flow from irrigated lands under lower end of Ironstone Canal from Uncompahgre River. Diversions for irrigation of a few hundred acres above station. Estimates of annual flow are within about 25 percent of regression line.

\begin{tabular}{|c|c|c|c|c|c|c|c|c|c|c|c|c|c|}
\hline $\begin{array}{l}\text { Water } \\
\text { year }\end{array}$ & oct. & Nov. & Dec. & Jan. & Feb. & Mar. & Apr. & May & June & July & Aug. & Sept. & Annual \\
\hline $\begin{array}{l}1939 \\
1940\end{array}$ & $\begin{array}{r}75,530 \\
5,880\end{array}$ & $\begin{array}{r}\begin{array}{r}73,570 \\
3,940\end{array} \\
\end{array}$ & $\begin{array}{r}\mp 2,460 \\
2,710\end{array}$ & $\begin{array}{r}\neq 2,150 \\
1,760\end{array}$ & $\begin{array}{r}\neq 1,670 \\
1,750\end{array}$ & $\begin{array}{r} \pm 3,310 \\
2,630\end{array}$ & $\begin{array}{r}16,730 \\
9,500 \\
,\end{array}$ & $\begin{array}{l}13,810 \\
25,700\end{array}$ & $\begin{array}{l}3,700 \\
4,470\end{array}$ & $\begin{array}{l}2,700 \\
3,610\end{array}$ & $\begin{array}{l}2,910 \\
3,700\end{array}$ & $\begin{array}{l}6,810 \\
6,450\end{array}$ & $\begin{array}{r}\neq 65,350 \\
72,100\end{array}$ \\
\hline $\begin{array}{l}1941 \\
1942 \\
1943 \\
1944\end{array}$ & $\begin{array}{r}5,350 \\
13,730 \\
5,990 \\
7,280 \\
6,890\end{array}$ & $\begin{array}{l}3,600 \\
8,220 \\
3,720 \\
4,900 \\
5,080\end{array}$ & $\begin{array}{l}3,210 \\
4,730 \\
2,990 \\
3,480 \\
2,920\end{array}$ & $\begin{array}{l}3,890 \\
2,780 \\
3,730 \\
2,590 \\
2,860\end{array}$ & $\begin{array}{l}4,350 \\
2,430 \\
3,280 \\
1,910 \\
2,690\end{array}$ & $\begin{array}{l}4,000 \\
3,690 \\
3,580 \\
2,750 \\
4,280\end{array}$ & $\begin{array}{r}11,770 \\
36,690 \\
17,360 \\
6,470 \\
5,860\end{array}$ & $\begin{array}{l}67,610 \\
41,740 \\
18,550 \\
47,620 \\
52,210\end{array}$ & $\begin{array}{r}19,170 \\
9,170 \\
7,000 \\
19,120 \\
11,520\end{array}$ & $\begin{array}{l}3,770 \\
3,660 \\
3,580 \\
5,530 \\
3,570\end{array}$ & $\begin{array}{l}6,820 \\
4,720 \\
9,590 \\
4,310 \\
8,100\end{array}$ & $\begin{array}{l}8,050 \\
7,020 \\
6,060 \\
6,030 \\
6,810\end{array}$ & $\begin{array}{r}141,600 \\
138,600 \\
85,700 \\
112,000 \\
112,800\end{array}$ \\
\hline $\begin{array}{l}.948 \\
.949 \\
950\end{array}$ & $\begin{array}{l}7,270 \\
6,860 \\
9,490 \\
6,020 \\
7,100\end{array}$ & $\begin{array}{l}6,030 \\
4,570 \\
4,020\end{array}$ & $\begin{array}{l}4,100 \\
3,780 \\
4,130 \\
4,360 \\
3,750\end{array}$ & $\begin{array}{l}2,860 \\
2,720 \\
3,880 \\
2,670 \\
3,020\end{array}$ & $\begin{array}{l}2,600 \\
2,350 \\
2,520 \\
2,250 \\
2,010\end{array}$ & $\begin{array}{l}2,920 \\
2,170 \\
3,040 \\
2,270 \\
2,100\end{array}$ & $\begin{array}{r}9,960 \\
8,600 \\
22,580 \\
13,650 \\
20,380\end{array}$ & $\begin{array}{l}12,120 \\
16,940 \\
41,020 \\
34,530 \\
23,960\end{array}$ & $\begin{array}{r}4,840 \\
8,000 \\
6,690 \\
16,860 \\
7,430\end{array}$ & & & & $\begin{array}{r}74,060 \\
114,500 \\
102,400 \\
86,630\end{array}$ \\
\hline $\begin{array}{l}1951 \\
1952 \\
1953 \\
1954\end{array}$ & $\begin{array}{l}3,310 \\
5,270 \\
5,260 \\
6,170\end{array}$ & $\begin{array}{l}2,700 \\
4,150 \\
5,760 \\
4,750\end{array}$ & $\begin{array}{l}2,640 \\
3,530 \\
3,330 \\
2,510\end{array}$ & $\begin{array}{l}2,670 \\
2,830 \\
2,550 \\
2,190\end{array}$ & $\begin{array}{l}1,710 \\
2,200 \\
1,810 \\
2,100\end{array}$ & $\begin{array}{l}1,730 \\
2,280 \\
2,560 \\
2,490\end{array}$ & $\begin{array}{r}2,840 \\
14,640 \\
5,200 \\
8,970\end{array}$ & $\begin{array}{l}14,700 \\
43,970 \\
10,230 \\
6,040\end{array}$ & $\begin{array}{r}4,590 \\
10,670 \\
5,240 \\
2,760\end{array}$ & $\begin{array}{l}2,640 \\
4,440 \\
3,060 \\
3,790\end{array}$ & $\begin{array}{l}3,240 \\
7,020 \\
4,780 \\
3,170\end{array}$ & $\begin{array}{l}4,420 \\
6,300 \\
6,030 \\
7,140\end{array}$ & $\begin{array}{r}47,190 \\
107,400 \\
55,810 \\
52,080\end{array}$ \\
\hline
\end{tabular}

f Est1mated for 1950 compilation.

\begin{tabular}{l|c|c|c|c|c|c|c|c|r|r|r|r|r}
\hline $\begin{array}{l}\text { Water } \\
\text { year }\end{array}$ & oct. & Nov. & Dec. & Jan. & Feb. & Mar. & Apr. & May & June & July & Aug. & Sept. & Annual \\
\hline 1931 & 7,800 & 4,700 & 3,800 & 3,300 & 2,600 & 2,400 & 4,000 & 6,400 & 1,700 & 2,700 & 2,500 & 3,800 & 45,700 \\
1932 & 7,900 & 5,100 & 3,700 & 2,600 & 3,000 & 3,100 & 23,000 & 44,200 & 9,200 & 4,400 & 3,700 & 5,400 & 115,300 \\
1933 & 6,000 & 4,500 & 3,100 & 2,300 & 1,900 & 2,800 & 4,900 & 20,700 & 10,700 & 3,500 & 3,800 & 5,600 & 69,800 \\
1934 & 5,800 & 4,100 & 3,300 & 2,600 & 2,100 & 2,200 & 4,800 & 8,000 & 330 & 1,900 & 2,000 & 3,000 & 40,130 \\
1935 & 2,600 & 2,000 & 3,000 & 2,500 & 1,800 & 2,200 & 3,800 & 14,600 & 11,400 & 4,100 & 4,300 & 6,300 & 58,600 \\
1936 & 6,400 & 4,100 & 3,000 & 2,700 & 2,100 & 2,500 & 19,600 & 39,000 & 5,000 & 3,400 & 4,400 & 6,400 & 98,600 \\
1937 & 5,600 & 4,200 & 3,100 & 2,500 & 2,000 & 2,800 & 10,800 & 38,800 & 4,200 & 3,500 & 3,200 & 4,700 & 85,400 \\
1938 & 6,000 & 4,200 & 3,500 & 2,900 & 2,100 & 3,100 & 24,000 & 40,000 & 15,800 & 4,400 & 7,100 & 10,100 & 123,200 \\
1955 & 6,500 & 3,400 & 3,000 & 2,600 & 1,900 & 2,600 & 7,000 & 14,400 & 3,400 & 3,000 & 4,300 & 4,900 & 57,000 \\
1956 & 4,500 & 3,600 & 3,300 & 2,700 & 2,100 & 2,600 & 9,400 & 18,200 & 4,200 & 2,800 & 2,900 & 3,400 & 59,700 \\
1957 & 4,300 & 3,700 & 2,900 & 2,800 & 2,500 & 2,500 & 8,800 & 33,400 & 27,400 & 6,400 & 10,600 & 9,800 & 115,100 \\
1958 & 9,100 & 7,300 & 4,700 & 3,200 & 3,100 & 3,200 & 17,000 & 56,000 & 11,200 & 3,200 & 3,700 & 6,200 & 127,900 \\
1959 & 5,600 & 4,700 & 3,600 & 3,000 & 2,300 & 2,400 & 3,600 & 8,600 & 4,100 & 2,700 & 4,200 & 5,400 & 50,200 \\
1960 & 8,600 & 4,800 & 3,000 & 2,700 & 1,900 & 3,400 & 18,000 & 14,200 & 5,800 & 3,100 & 3,200 & 5,100 & 73,800 \\
1961 & 5,500 & 3,900 & 3,000 & 2,400 & 1,900 & 2,500 & 4,400 & 14,800 & 3,200 & 2,700 & 3,800 & 9,400 & 57,500 \\
1962 & 9,200 & 5,700 & 3,300 & 2,800 & 2,600 & 2,500 & 26,800 & 35,100 & 8,900 & 4,600 & 4,200 & 7,000 & 112,700 \\
1963 & 6,900 & 4,500 & 3,200 & 2,600 & 3,100 & 3,200 & 6,600 & 10,000 & 1,200 & 2,800 & 4,200 & 6,100 & 54,400 \\
1964 & 5,800 & 4,300 & 3,000 & 2,500 & 2,100 & 2,100 & 5,000 & 24,200 & 5,400 & 3,500 & 6,200 & 6,800 & 70,900 \\
1965 & 5,700 & 4,300 & 3,400 & 2,900 & 2,100 & 2,400 & 15,200 & 35,800 & 14,000 & 5,750 & 8,600 & 12,800 & 112,950 \\
\hline
\end{tabular}



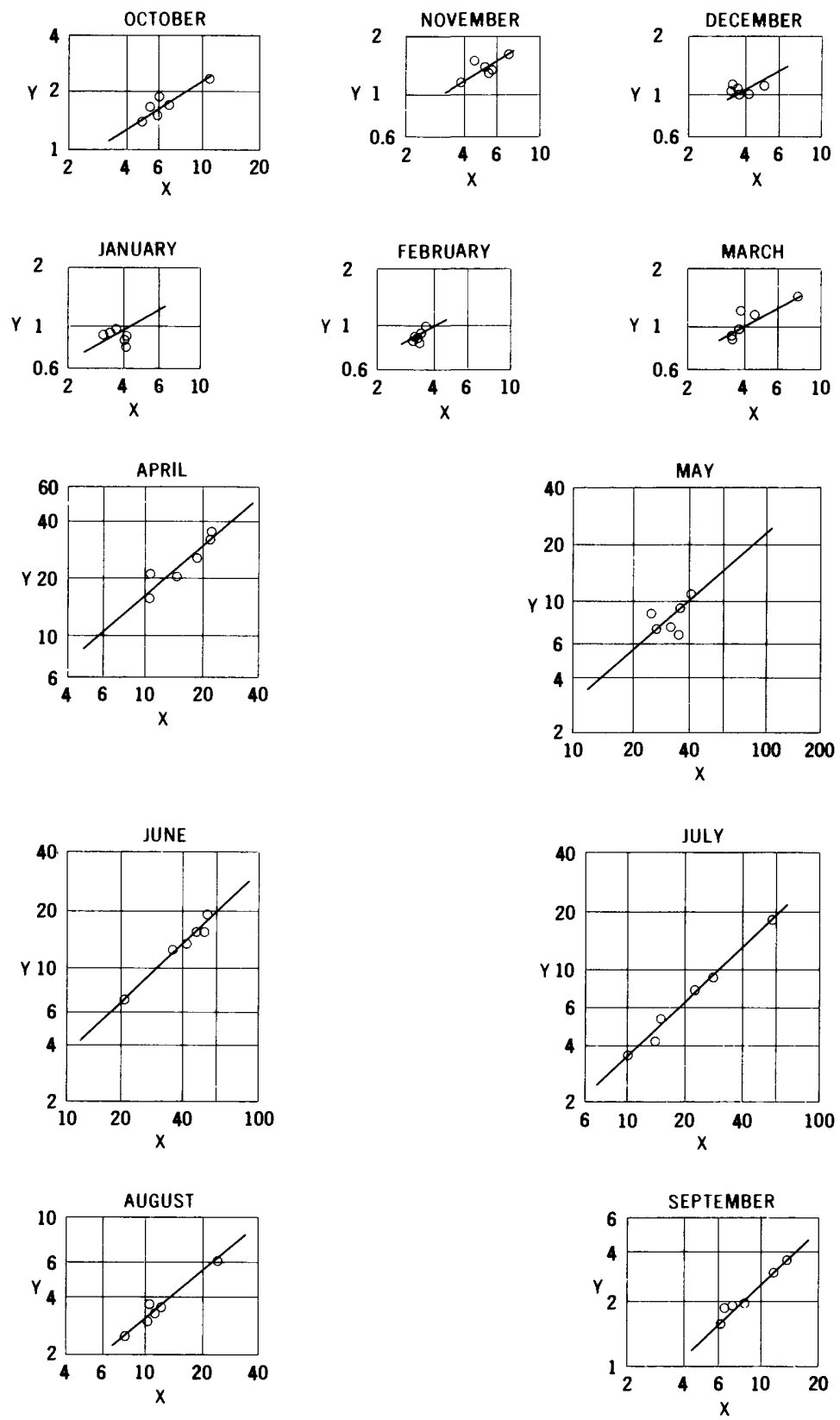

Relationships of monthly mean discharge of San Miguel River near Telluride, Colo. (Y), to monthly mean discharge of San Miguel River near Placerville, Colo. (X). Discharge in thousands of acre-feet. 
1712. San Miguel River near Telluride, Colo.

Location.--Lat $37^{\circ} 56^{\prime} 55^{\prime \prime}$, long 107052135", in NWI sec.33, T.43 N., R. 9 W., on left bank $0.1 \mathrm{mile}$ upstream from Remine Creek, 0.3 mile downstream from bridge on state Highway $145,0.5$ mile downstream from Prospect Creek, 1.6 miles upstream from South Fork and 3.5 miles west of Telluride. Datum of gage is $8,622.81 \mathrm{ft}$ above mean sea level (levels by Bureau of Reclamation).

Drainage area. $-42.8 \mathrm{sq} \mathrm{mi}$.

Records available. --October 1959 to September 1965.

Estimates of streamflow.--October 1930 to September 1934, April 1942 to September 1959 based on relationships of monthly mean discharge with San Miguel River near Placerville, Colo. The regression equation used is:

$$
\log Y=b \log X-c
$$

(where $Y$ is discharge of San Miguel River near Telluride, Colo., and $X$ is discharge of San Miguel River near Placerville, Colo,, both in acre-feet per month).

Monthly values of constants in above equation

\begin{tabular}{l|l|l|l|l|l|l|l|l|l|l|l|l}
\hline & oct. & Nov. & Dec. & Jan. & Feb. & Mar. & Apr. & May & June & July & Aug. & Sept. \\
\hline $\mathrm{b}$ & 0.63 & 0.60 & 0.53 & 0.54 & 0.54 & 0.53 & 0.84 & 0.85 & 0.92 & 0.93 & 0.80 & 0.92 \\
$\mathrm{c}$ & -.83 & -.90 & -1.11 & -1.05 & -1.06 & -1.10 & .14 & -.09 & .10 & .17 & -.28 & .26 \\
\hline
\end{tabular}

Average discharge. --27 years (1930-34, 1942-65), 46,310 acre-feet per year (64.0 cfs).

Extremes. --1959-65: Maximum discharge, 616 of s July 12, 1965; minimum daily determined, 12 crs Mar. 8, 1961, Mar. 9, 11, 1964.

Remarks.--Several small diversions for Irrigation of hay meadows above station. Estimates of annual flow are within about 15 percent of regression line.

\begin{tabular}{|c|c|c|c|c|c|c|c|c|c|c|c|c|c|}
\hline $\begin{array}{l}\text { Water } \\
\text { year }\end{array}$ & oct. & Nov. & Dec. & Jan. & Feb. & Mar. & Apr. & May & June & July & Aug. & Sept. & Annual \\
\hline 1960 & 1,890 & 1,490 &, 110 & 958 & 869 & 1,160 & 3,480 & 7,090 & 19,030 & 7,680 & 2,530 & 1,700 & 48,990 \\
\hline $\begin{array}{l}1961 \\
1962 \\
1963 \\
1964 \\
1965\end{array}$ & $\begin{array}{l}1,480 \\
2,330 \\
1,720 \\
1,420 \\
1,680\end{array}$ & $\begin{array}{l}1,310 \\
1,610 \\
1,290 \\
1,370 \\
1,140\end{array}$ & $\begin{array}{r}1,090 \\
1,100 \\
996 \\
1,050 \\
984\end{array}$ & $\begin{array}{l}984 \\
922 \\
799 \\
922 \\
922\end{array}$ & $\begin{array}{l}912 \\
994 \\
948 \\
805 \\
829\end{array}$ & $\begin{array}{r}1,200 \\
984 \\
1,470 \\
906 \\
895\end{array}$ & $\begin{array}{l}2,020 \\
3,210 \\
2,120 \\
1,570 \\
2,540\end{array}$ & $\begin{array}{r}10,830 \\
7,370 \\
8,560 \\
9,360 \\
6,610\end{array}$ & $\begin{array}{r}15,890 \\
13,440 \\
6,990 \\
12,670 \\
15,130\end{array}$ & $\begin{array}{r}4,200 \\
9,140 \\
3,440 \\
5,470 \\
17,950\end{array}$ & $\begin{array}{l}3,330 \\
2,920 \\
3,540 \\
3,500 \\
6,120\end{array}$ & $\begin{array}{l}3,100 \\
2,100 \\
2,130 \\
2,070 \\
3,660\end{array}$ & $\begin{array}{l}46,350 \\
46,120 \\
34,000 \\
41,110 \\
58,660\end{array}$ \\
\hline
\end{tabular}

Estimated monthly and annual streamflow, in acre-feet

\begin{tabular}{|c|c|c|c|c|c|c|c|c|c|c|c|c|c|}
\hline $\begin{array}{l}\text { Nater } \\
\text { year }\end{array}$ & t. & ov. & c. & Jan. & eb. & Mar & Apr. & May & June & July & Aug. & Sept. & Annual \\
\hline & 0 & 0 & $\begin{array}{l}000 \\
100\end{array}$ & $\begin{array}{r}960 \\
, 000\end{array}$ & $\begin{array}{l}.000 \\
980 \\
960 \\
, 000\end{array}$ & & & $\begin{array}{r}3 \\
10 \\
6 \\
6\end{array}$ & & & & & \\
\hline $\begin{array}{l}194 \\
194 \\
194\end{array}$ & $1, \overline{600}$ & $\overrightarrow{400}$ & $\begin{array}{l}- \\
, 300 \\
, 200 \\
200\end{array}$ & $\begin{array}{l}1, \overline{300} \\
1,100 \\
1,100\end{array}$ & $\begin{array}{l}- \\
, 000 \\
, 000\end{array}$ & $\begin{array}{l}- \\
1,300 \\
1,100 \\
1,100\end{array}$ & & $\begin{array}{r}15 \\
7 \\
14 \\
10\end{array}$ & & & & & $\begin{array}{c}- \\
46,000 \\
66,400 \\
50,800\end{array}$ \\
\hline $\begin{array}{l}194 \\
194 \\
194\end{array}$ & 0 & $\begin{array}{l}700 \\
300 \\
300\end{array}$ & $\begin{array}{l}300 \\
100 \\
100\end{array}$ & $\begin{array}{r}960 \\
980 \\
100 \\
980 \\
980\end{array}$ & $\begin{array}{r}980 \\
900 \\
100 \\
000 \\
920\end{array}$ & & & & & & & & $\begin{array}{l}54,280 \\
60,900 \\
58,280 \\
35,800\end{array}$ \\
\hline $\begin{array}{l}195 \\
195 \\
195 \\
19\end{array}$ & $\begin{array}{l}1, \\
1, \\
2,\end{array}$ & $\begin{array}{l}.000 \\
200 \\
200 \\
200\end{array}$ & $\begin{array}{r}980 \\
960 \\
, 100 \\
, 000 \\
980\end{array}$ & $\begin{array}{r}920 \\
920 \\
, 100 \\
, 000 \\
940\end{array}$ & $\begin{array}{l}880 \\
900 \\
940 \\
940 \\
860\end{array}$ & $\begin{array}{r}940 \\
960 \\
1,100 \\
1,000 \\
1,000\end{array}$ & $\begin{array}{r}890 \\
2,500 \\
1,600 \\
1,500 \\
1,700\end{array}$ & $\begin{array}{r}4, \\
31, \\
5, \\
5, \\
6,\end{array}$ & & & & & \\
\hline $\begin{array}{l}19 \\
19\end{array}$ & 900 & $\begin{array}{l}000 \\
000 \\
700 \\
300\end{array}$ & .000 & $\begin{array}{l}900 \\
920 \\
, 000 \\
, 000\end{array}$ & $\begin{array}{r}860 \\
860 \\
980 \\
, 000\end{array}$ & $\begin{array}{r}1,000 \\
900 \\
1,100 \\
1,000\end{array}$ & $\begin{array}{l}1,600 \\
1,600 \\
4,000 \\
1,300\end{array}$ & $\begin{array}{r}7,400 \\
8,000 \\
21,000 \\
4,800\end{array}$ & & $\begin{array}{l}00 \\
00 \\
00 \\
00\end{array}$ & & & $\begin{array}{l}72,120 \\
68,880 \\
32,700 \\
\end{array}$ \\
\hline
\end{tabular}



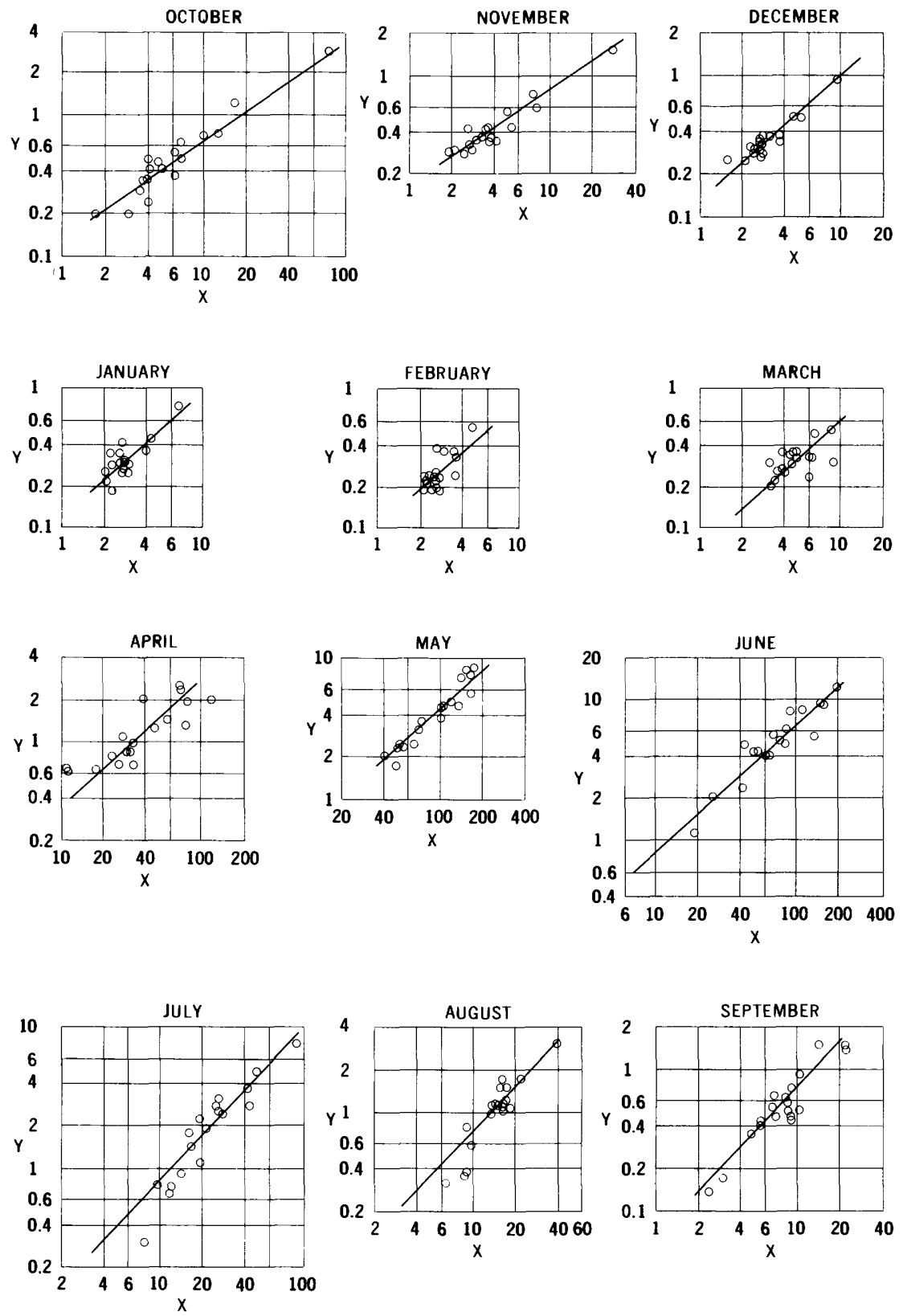

Relationships of monthly mean discharge of Fall Creek near Fall Creek, Colo. (Y), to monthly mean discharge of Dolores River at Dolores, Colo. (X). Discharge in thousands of acre-feet. 
1720. Fall Creek near Fall Creek, Colo.

Location.--Lat $37^{\circ} 581$, long $108^{\circ} 01^{\prime}$, in sec.24, T.43 N., R.11 W., on left bank 2.7 miles upstream from mouth and 2.8 miles south of town of Fáll creek. Datum of gage is 7,928.79 ft above mean sea level (Bureau of Reclamation bench mark).

Drainage area. $--33.5 \mathrm{sq} \mathrm{mi}$.

Records available. --August 1941 to September 1959.

Estimates of streamf 10w. --October 1930 to July 1941, October 1959 to September 1965, based on relationships of monthly mean discharge with Dolores River at Dolores, Colo. 'The regression equation used is:

$$
\log \mathrm{Y}=\mathrm{b} \log \mathrm{X}-\mathrm{c}
$$

(where $Y$ is discharge of Fall Creek near Fall Creek, Colo., and $X$ is discharge of Dolores River at Dolores, Colo., both in acre-feet per month).

Monthly values of constants in above equation

\begin{tabular}{l|r|r|r|r|r|r|r|r|r|r|r|l}
\hline & oct. & Nov. & Dec. & Jan. & Feb. & Mar. & Apr. & May & June & July & Aug. & Sept. \\
\hline $\mathrm{b}$ & 0.69 & 0.69 & 0.88 & 0.88 & 0.88 & 0.91 & 0.91 & 0.90 & 0.90 & 1.06 & 1.06 & 1.06 \\
$\mathrm{c}$ & -.08 & -.14 & .52 & .56 & .61 & .87 & 1.09 & .85 & .70 & 1.31 & 1.37 & 1.35 \\
\hline
\end{tabular}

Average discharge. --35 years $(1930-65), 16,930$ acre-feet per year (23.4 cfs).

Extremes.--1941-59: Maximum discharge, 1,390 cfs May 4, 1957, from rating curve extended above $240 \mathrm{cfs}$ on basis of slope-area measurement of peak flow; minimum daily determined, 1.6 cfs sept. 26-28, 1951.

Remarks.--Slight regulation by Sylvan Lake Reservoir (capacity, 230 acre-feet). One diversion above station to Beaver and Saltado Creek basins for irrigation of about 2,000 acres. Diversions for irrigation of 200 acres above station. Estimates of annual flow are within about 15 percent of regression line.

\begin{tabular}{|c|c|c|c|c|c|c|c|c|c|c|c|c|c|}
\hline \multicolumn{14}{|c|}{ Monthly and annual streamflow, in acre-fee } \\
\hline $\begin{array}{l}\text { Water } \\
\text { year }\end{array}$ & oct. & Nov. & Dec. & Jan. & Feb. & Mar. & Apr. & May & June & July & Aug. & Sept. & Annual \\
\hline $\begin{array}{l}1941 \\
1942 \\
1943 \\
1944 \\
1945\end{array}$ & $\begin{array}{r}- \\
2,370 \\
492 \\
518 \\
430\end{array}$ & $\begin{array}{r}1,530 \\
417 \\
541 \\
347\end{array}$ & $\begin{array}{l}- \\
922 \\
369 \\
369 \\
363\end{array}$ & $\begin{array}{l}- \\
738 \\
338 \\
277 \\
400\end{array}$ & $\begin{array}{l}- \\
555 \\
389 \\
230 \\
372\end{array}$ & $\begin{array}{l}- \\
523 \\
492 \\
369 \\
375\end{array}$ & $\begin{array}{r}- \\
2,080 \\
1,970 \\
696 \\
689\end{array}$ & $\begin{array}{l}3, \overline{370} \\
4,690 \\
?, 800 \\
4,520\end{array}$ & $\begin{array}{l}8, \overline{370} \\
5,780 \\
9,380 \\
4,910\end{array}$ & $\begin{array}{l}3,120 \\
2,280 \\
4,940 \\
2,810\end{array}$ & $\begin{array}{l}1,790 \\
1,190 \\
1,550 \\
1,160 \\
1,590\end{array}$ & $\begin{array}{r}1,370 \\
666 \\
740 \\
516 \\
450\end{array}$ & $\begin{array}{c}- \\
31,010 \\
19,510 \\
26,800 \\
17,260\end{array}$ \\
\hline $\begin{array}{l}1946 \\
1947 \\
1948 \\
1949 \\
1950\end{array}$ & $\begin{array}{r}575 \\
679 \\
1,280 \\
368 \\
391\end{array}$ & $\begin{array}{l}363 \\
426 \\
728 \\
302 \\
342\end{array}$ & $\begin{array}{l}246 \\
338 \\
492 \\
320 \\
277\end{array}$ & $\begin{array}{l}215 \\
246 \\
357 \\
283 \\
258\end{array}$ & $\begin{array}{l}194 \\
194 \\
374 \\
261 \\
250\end{array}$ & $\begin{array}{l}369 \\
246 \\
338 \\
556 \\
335\end{array}$ & $\begin{array}{l}2,070 \\
1,100 \\
2,570 \\
1,290 \\
1,460\end{array}$ & $\begin{array}{l}2,450 \\
4,970 \\
?, 210 \\
4,530 \\
2,430\end{array}$ & $\begin{array}{l}4,060 \\
5,040 \\
6,210 \\
5,450 \\
4,250\end{array}$ & $\begin{array}{l}1,450 \\
2,610 \\
2,440 \\
2,780 \\
1,810\end{array}$ & $\begin{array}{r}992 \\
1,740 \\
1,170 \\
1,090 \\
595\end{array}$ & $\begin{array}{r}644 \\
1,470 \\
476 \\
456 \\
443\end{array}$ & $\begin{array}{l}13,630 \\
19,060 \\
23,640 \\
17,490 \\
12,840\end{array}$ \\
\hline $\begin{array}{l}1951 \\
1952 \\
1953 \\
1954 \\
1955\end{array}$ & $\begin{array}{l}359 \\
204 \\
501 \\
247 \\
760\end{array}$ & $\begin{array}{l}425 \\
274 \\
327 \\
357 \\
416\end{array}$ & $\begin{array}{l}338 \\
287 \\
290 \\
369 \\
300\end{array}$ & $\begin{array}{l}184 \\
273 \\
283 \\
338 \\
285\end{array}$ & $\begin{array}{l}194 \\
245 \\
243 \\
250 \\
227\end{array}$ & $\begin{array}{l}260 \\
213 \\
305 \\
307 \\
271\end{array}$ & $\begin{array}{r}651 \\
1,320 \\
794 \\
992 \\
646\end{array}$ & $\begin{array}{l}1,700 \\
5,660 \\
2,320 \\
2,320 \\
3,140\end{array}$ & $\begin{array}{l}2,390 \\
9,150 \\
4,040 \\
1,150 \\
4,220\end{array}$ & $\begin{array}{r}774 \\
3,720 \\
1,130 \\
672 \\
926\end{array}$ & $\begin{array}{r}323 \\
1,260 \\
1,150 \\
368 \\
1,110\end{array}$ & $\begin{array}{l}138 \\
926 \\
402 \\
579 \\
542\end{array}$ & $\begin{array}{r}7,740 \\
23,530 \\
11,780 \\
7,950 \\
12,840\end{array}$ \\
\hline $\begin{array}{l}1956 \\
1957 \\
1958 \\
1959\end{array}$ & $\begin{array}{l}300 \\
204 \\
774 \\
427\end{array}$ & $\begin{array}{l}288 \\
283 \\
586 \\
349\end{array}$ & $\begin{array}{l}277 \\
246 \\
492 \\
307\end{array}$ & $\begin{array}{l}246 \\
258 \\
430 \\
246\end{array}$ & $\begin{array}{l}2.01 \\
239 \\
333 \\
222\end{array}$ & $\begin{array}{l}310 \\
282 \\
338 \\
235\end{array}$ & $\begin{array}{r}863 \\
859 \\
2,350 \\
636\end{array}$ & $\begin{array}{l}3,590 \\
3,280 \\
8,720 \\
2,070\end{array}$ & $\begin{array}{r}4,740 \\
11,910 \\
8,010 \\
2,010\end{array}$ & $\begin{array}{r}740 \\
7,800 \\
1,970 \\
313\end{array}$ & $\begin{array}{r}385 \\
3,080 \\
1,170 \\
809\end{array}$ & $\begin{array}{r}171 \\
1,480 \\
513 \\
352\end{array}$ & $\begin{array}{r}22,110 \\
29,920 \\
25,690 \\
7,980\end{array}$ \\
\hline
\end{tabular}

Estimated monthly and annual streamflow, in acre-feet

\begin{tabular}{c|r|r|r|r|r|r|r|r|r|r|r|r|r}
\hline $\begin{array}{l}\text { Water } \\
\text { year }\end{array}$ & Oct. & Nov. & Dec. & Jan. & Feb. & Mar. & Apr. & May & June & July & Aug. & Sept. & Annual \\
\hline 1931 & 430 & 350 & 290 & 270 & 260 & 290 & 470 & 2,200 & 2,300 & 760 & 350 & 360 & 8,330 \\
1932 & 560 & 420 & 440 & 360 & 500 & 520 & 2,100 & 7,200 & 7,200 & 2,900 & 1,100 & 500 & 23,800 \\
1933 & 400 & 310 & 330 & 330 & 190 & 260 & 470 & 2,800 & 5,600 & 1,700 & 450 & 620 & 13,460 \\
1934 & 520 & 430 & 350 & 270 & 270 & 290 & 900 & 1,700 & 550 & 270 & 220 & 180 & 5,950 \\
1935 & 240 & 250 & 240 & 200 & 180 & 290 & 1,000 & 3,100 & 8,200 & 3,000 & 1,200 & 830 & 18,730 \\
1936 & 520 & 370 & 270 & 230 & 220 & 520 & 2,000 & 4,600 & 3,400 & 1,100 & 1,500 & 640 & 15,370 \\
1937 & 410 & 500 & 410 & 360 & 220 & 340 & 2,200 & 7,000 & 4,200 & 1,900 & 560 & 380 & 18,480 \\
1938 & 470 & 370 & 290 & 270 & 240 & 580 & 2,500 & 5,200 & 8,200 & 2,900 & 650 & 1,000 & 22,670 \\
1939 & 630 & 510 & 400 & 300 & 250 & 600 & 1,300 & 3,200 & 2,000 & 440 & 280 & 620 & 10,530 \\
1940 & 340 & 340 & 200 & 190 & 220 & 390 & 1,100 & 4,000 & 2,900 & 930 & 630 & 690 & 11,930 \\
1941 & 810 & 500 & 360 & 310 & 320 & 520 & 960 & 8,400 & 9,000 & 5,600 & - & - & 29,940 \\
1960 & 450 & 470 & 250 & 220 & 180 & 520 & 1,900 & 3,800 & 5,400 & 1,300 & 600 & 300 & 15,390 \\
1961 & 410 & 370 & 290 & 230 & 180 & 270 & 910 & 4,100 & 3,600 & 1,000 & 920 & 1,000 & 13,280 \\
1962 & 710 & 600 & 380 & 350 & 450 & 310 & 1,900 & 4,200 & 4,700 & 2,000 & 1,100 & 520 & 17,220 \\
1963 & 470 & 400 & 350 & 250 & 310 & 660 & 1,100 & 2,900 & 1,300 & 920 & 760 & 560 & 9,980 \\
1964 & 370 & 350 & 260 & 220 & 180 & 130 & 410 & 3,700 & 3,000 & 1,200 & 1,500 & 680 & 12,000 \\
1965 & 320 & 330 & 330 & 310 & 250 & 220 & 1,500 & 4,700 & 7,300 & 5,800 & 2,300 & 1,400 & 24,760 \\
\hline
\end{tabular}



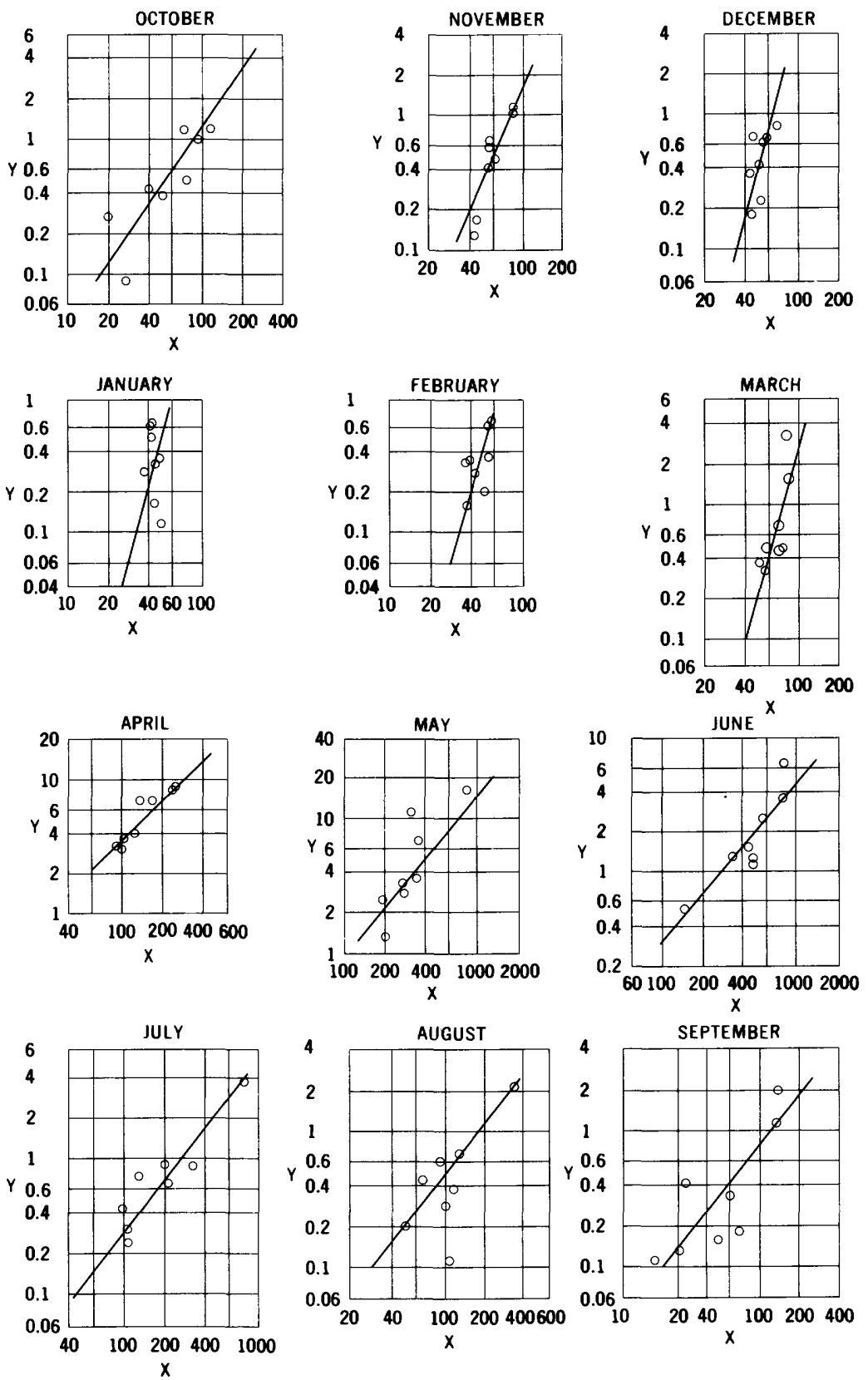

Relationships of monthly mean discharge of Leopard Creek at Noel, Colo. (Y), to monthły mean discharge of Uncompahgre River at Colona, Colo. $(\mathrm{X})$. Discharge in hundreds of acre-feet. 
1721. Leopard Creek at Noel, Colo.

Location. --Lat $38^{\circ} 06^{\prime 1} 10^{\prime \prime}$, long $107^{\circ} 55^{\prime} 10^{\prime \prime}$, in SE $\frac{1}{4}$ SW $\frac{1}{4}$ sec.36, T.45 N., R.10 W., on right bank $10 \mathrm{ft}$ downstream from abandoned railroad, $0.6 \mathrm{miles}$ west of Noel, and $2 \mathrm{miles}$ up stream from Dead Horse Canyon. Altitude of gage is 8,700 ft (from topographic map).

Drainage area. $--9.11 \mathrm{sq} \mathrm{mi}$.

Records ava1lable. --October 1955 to September 1963.

Estimates of streamf low.--0ctober 1930 to September 1955, October 1963 to September 1965, based on relationships of monthly mean discharge with Uncompahgre River at Colona, colo. The regression equation used is:

$$
\log Y=b \log X-c
$$

(where $\mathrm{Y}$ is discharge of Leopard Creek at Noel, Colo., and $\mathrm{X}$ is discharge of Uncompahgre River at Colona, Colo., both in acre-feet per month).

Monthly values of constants in above equation

\begin{tabular}{c|c|c|c|c|c|c|c|c|c|c|c|c}
\hline & oct. & Nov. & Dec. & Jan. & Feb. & Mar. & Apr. & May & June & July & Aug. & Sept. \\
\hline b & 1.44 & 2.20 & 3.70 & 3.70 & 3.46 & 3.55 & 0.98 & 1.22 & 1.16 & 1.27 & 1.25 & 1.23 \\
& 3.65 & 6.62 & 12.12 & 11.95 & 11.15 & 11.71 & 1.36 & 2.90 & 3.14 & 3.64 & 3.32 & 3.04 \\
\hline
\end{tabular}

Average discharge. --35 years $(1930-65), 1,828$ acre-feet per year (2.52 cfs).

Extremes:- $-1955-63$ : Maximum discharge, 100 cfs Apr. 30, 1961; minimum daily, 0.1 cfs for many days in $1956-57,1959$.

Remarks.--One diversion above station to Dallas Creek drainage. Small diversions for irrigation of hay meadows above station. Estimates of annual flow are within about 25 percent of regression line.

\begin{tabular}{|c|c|c|c|c|c|c|c|c|c|c|c|c|c|}
\hline $\begin{array}{c}\text { Water } \\
\text { year }\end{array}$ & Oct. & Nov. & Dec. & Jan. & Feb. & Mar. & Apr. & May & June & July & Aug. & Sept. & Annual \\
\hline $\begin{array}{l}1956 \\
1957 \\
1958 \\
1959 \\
1960\end{array}$ & $\begin{array}{c}28 \\
9.3 \\
125 \\
40 \\
107\end{array}$ & $\begin{array}{r}17 \\
13 \\
114 \\
47 \\
65\end{array}$ & $\begin{array}{l}23 \\
18 \\
82 \\
62 \\
36\end{array}$ & $\begin{array}{l}17 \\
12 \\
66 \\
36 \\
29\end{array}$ & $\begin{array}{l}16 \\
20 \\
63 \\
28 \\
33\end{array}$ & $\begin{array}{r}46 \\
37 \\
68 \\
32 \\
150\end{array}$ & $\begin{array}{l}412 \\
364 \\
885 \\
314 \\
840\end{array}$ & $\begin{array}{r}287 \\
1,170 \\
1,720 \\
262 \\
343\end{array}$ & $\begin{array}{l}130 \\
659 \\
344 \\
115 \\
252\end{array}$ & $\begin{array}{r}43 \\
373 \\
64 \\
30 \\
87\end{array}$ & $\begin{array}{r}20 \\
219 \\
37 \\
11 \\
44\end{array}$ & $\begin{array}{r}11 \\
201 \\
18 \\
13 \\
40\end{array}$ & $\begin{array}{r}1,050 \\
3,100 \\
3,590 \\
990 \\
2,030\end{array}$ \\
\hline $\begin{array}{l}1961 \\
1962 \\
1963\end{array}$ & $\begin{array}{r}44 \\
127 \\
51\end{array}$ & $\begin{array}{r}41 \\
105 \\
57\end{array}$ & $\begin{array}{l}42 \\
66 \\
68\end{array}$ & $\begin{array}{l}34 \\
52 \\
65\end{array}$ & $\begin{array}{l}35 \\
37 \\
48\end{array}$ & $\begin{array}{r}45 \\
47 \\
327\end{array}$ & $\begin{array}{l}725 \\
727 \\
322\end{array}$ & $\begin{array}{l}730 \\
381 \\
137\end{array}$ & $\begin{array}{r}155 \\
125 \\
54\end{array}$ & $\begin{array}{l}73 \\
85 \\
24\end{array}$ & $\begin{array}{l}70 \\
88 \\
60\end{array}$ & $\begin{array}{r}115 \\
16 \\
33\end{array}$ & $\begin{array}{l}2,110 \\
1,800 \\
1,250\end{array}$ \\
\hline
\end{tabular}

Estimated monthly and annual streamflow, in acre-feet

\begin{tabular}{|c|c|c|c|c|c|c|c|c|c|c|c|c|c|}
\hline $\begin{array}{l}\text { Water } \\
\text { year }\end{array}$ & Oct. & Nov. & Dec. & Jan. & Feb. & Mar. & Apr. & May & June & July & Aug. & sept. & Annual \\
\hline $\begin{array}{l}1931 \\
1932 \\
1933 \\
1934 \\
1935\end{array}$ & $\begin{array}{r}90 \\
120 \\
40 \\
120 \\
30\end{array}$ & $\begin{array}{l}\neq 40 \\
\neq 50 \\
\neq 20 \\
50 \\
20\end{array}$ & $\begin{array}{r}\neq 6 \\
\neq 30 \\
\neq 6 \\
\neq 40 \\
\neq 6\end{array}$ & $\begin{array}{r}\neq 4 \\
\neq 30 \\
\neq 7 \\
\neq 50 \\
\neq 4\end{array}$ & $\begin{array}{r}\neq 8 \\
\neq 30 \\
\neq 6 \\
\neq 30 \\
\neq 6\end{array}$ & $\begin{array}{r}\neq 70 \\
\neq 100 \\
\neq 30 \\
\neq 40 \\
\neq 20\end{array}$ & $\begin{array}{l}340 \\
670 \\
330 \\
580 \\
230\end{array}$ & $\begin{array}{l}190 \\
670 \\
370 \\
330 \\
170\end{array}$ & $\begin{array}{r}110 \\
250 \\
230 \\
30 \\
250\end{array}$ & $\begin{array}{r}30 \\
140 \\
50 \\
9 \\
120\end{array}$ & $\begin{array}{l}30 \\
60 \\
30 \\
20 \\
80\end{array}$ & $\begin{array}{l}40 \\
40 \\
80 \\
20 \\
70\end{array}$ & $\begin{array}{r}958 \\
2,190 \\
1,200 \\
1,320 \\
1,010\end{array}$ \\
\hline $\begin{array}{l}1936 \\
1937 \\
1938 \\
1939 \\
1940\end{array}$ & $\begin{array}{r}80 \\
50 \\
90 \\
140 \\
40\end{array}$ & $\begin{array}{l}40 \\
30 \\
40 \\
90 \\
30\end{array}$ & $\begin{array}{l}20 \\
30 \\
30 \\
60 \\
20\end{array}$ & $\begin{array}{l}20 \\
30 \\
20 \\
50 \\
30\end{array}$ & $\begin{array}{l}20 \\
20 \\
20 \\
20 \\
20\end{array}$ & $\begin{array}{r}40 \\
70 \\
110 \\
400 \\
170\end{array}$ & $\begin{array}{r}630 \\
680 \\
1,100 \\
700 \\
640\end{array}$ & $\begin{array}{l}450 \\
640 \\
630 \\
450 \\
570\end{array}$ & $\begin{array}{l}110 \\
110 \\
450 \\
140 \\
190\end{array}$ & $\begin{array}{r}30 \\
50 \\
180 \\
30 \\
30\end{array}$ & $\begin{array}{r}70 \\
40 \\
70 \\
30 \\
.20\end{array}$ & $\begin{array}{r}30 \\
60 \\
130 \\
50 \\
50\end{array}$ & $\begin{array}{l}1,540 \\
1,810 \\
2,870 \\
2,160 \\
1,810\end{array}$ \\
\hline $\begin{array}{l}1941 \\
1942 \\
1943 \\
1944 \\
1945\end{array}$ & $\begin{array}{r}150 \\
490 \\
70 \\
80 \\
60\end{array}$ & $\begin{array}{r}50 \\
220 \\
40 \\
70 \\
40\end{array}$ & $\begin{array}{r}40 \\
220 \\
50 \\
60 \\
20\end{array}$ & $\begin{array}{l}40 \\
80 \\
60 \\
30 \\
40\end{array}$ & $\begin{array}{l}30 \\
40 \\
50 \\
20 \\
30\end{array}$ & $\begin{array}{r}100 \\
70 \\
50 \\
30 \\
30\end{array}$ & $\begin{array}{r}380 \\
1,500 \\
570 \\
410 \\
380\end{array}$ & $\begin{array}{l}970 \\
960 \\
260 \\
900 \\
660\end{array}$ & $\begin{array}{l}310 \\
400 \\
170 \\
350 \\
210\end{array}$ & $\begin{array}{r}230 \\
180 \\
70 \\
250 \\
120\end{array}$ & $\begin{array}{r}120 \\
70 \\
150 \\
50 \\
150\end{array}$ & $\begin{array}{r}100 \\
50 \\
80 \\
30 \\
30\end{array}$ & $\begin{array}{l}2,520 \\
4,280 \\
1,620 \\
2,280 \\
1,770\end{array}$ \\
\hline $\begin{array}{l}1946 \\
1947 \\
1948 \\
1949 \\
1950\end{array}$ & $\begin{array}{r}90 \\
80 \\
160 \\
60 \\
70\end{array}$ & $\begin{array}{r}60 \\
60 \\
110 \\
50 \\
60\end{array}$ & $\begin{array}{l}30 \\
40 \\
80 \\
50 \\
40\end{array}$ & $\begin{array}{l}40 \\
50 \\
50 \\
60 \\
40\end{array}$ & $\begin{array}{l}40 \\
10 \\
60 \\
50 \\
50\end{array}$ & $\begin{array}{r}100 \\
50 \\
70 \\
120 \\
40\end{array}$ & $\begin{array}{r}730 \\
390 \\
1,000 \\
860 \\
540\end{array}$ & $\begin{array}{l}200 \\
440 \\
800 \\
460 \\
130\end{array}$ & $\begin{array}{l}160 \\
240 \\
220 \\
330 \\
150\end{array}$ & $\begin{array}{r}60 \\
150 \\
100 \\
190 \\
70\end{array}$ & $\begin{array}{r}60 \\
120 \\
60 \\
40 \\
10\end{array}$ & $\begin{array}{r}50 \\
130 \\
30 \\
30 \\
10\end{array}$ & $\begin{array}{l}1,620 \\
1,760 \\
2,740 \\
2,300 \\
1,210\end{array}$ \\
\hline $\begin{array}{l}1951 \\
1952 \\
1953 \\
1954 \\
1955\end{array}$ & $\begin{array}{r}20 \\
30 \\
50 \\
30 \\
100\end{array}$ & $\begin{array}{l}20 \\
30 \\
30 \\
70 \\
30\end{array}$ & $\begin{array}{l}20 \\
40 \\
40 \\
40 \\
20\end{array}$ & $\begin{array}{l}40 \\
30 \\
60 \\
30 \\
30\end{array}$ & $\begin{array}{l}20 \\
10 \\
30 \\
30 \\
10\end{array}$ & $\begin{array}{l}20 \\
20 \\
40 \\
10 \\
30\end{array}$ & $\begin{array}{l}170 \\
670 \\
330 \\
270 \\
330\end{array}$ & $\begin{array}{l}150 \\
480 \\
220 \\
150 \\
220\end{array}$ & $\begin{array}{r}110 \\
330 \\
230 \\
40 \\
140\end{array}$ & $\begin{array}{r}40 \\
130 \\
60 \\
40 \\
30\end{array}$ & $\begin{array}{r}30 \\
110 \\
50 \\
20 \\
60\end{array}$ & $\begin{array}{l}10 \\
60 \\
10 \\
60 \\
10\end{array}$ & $\begin{array}{r}650 \\
1,940 \\
1,150 \\
790 \\
1,010\end{array}$ \\
\hline $\begin{array}{l}1964 \\
1965\end{array}$ & $\begin{array}{l}40 \\
60\end{array}$ & $\begin{array}{l}60 \\
40\end{array}$ & $\begin{array}{l}20 \\
50\end{array}$ & $\begin{array}{l}20 \\
50\end{array}$ & $\begin{array}{l}10 \\
20\end{array}$ & $\begin{array}{l}30 \\
30\end{array}$ & $\begin{array}{l}480 \\
640\end{array}$ & $\begin{array}{l}500 \\
370\end{array}$ & $\begin{array}{l}180 \\
220\end{array}$ & $\begin{array}{r}80 \\
250\end{array}$ & $\begin{array}{l}100 \\
130\end{array}$ & $\begin{array}{r}50 \\
120\end{array}$ & $\begin{array}{l}1,570 \\
1,980\end{array}$ \\
\hline
\end{tabular}

\# Based on estimates for 1950 compllation. 

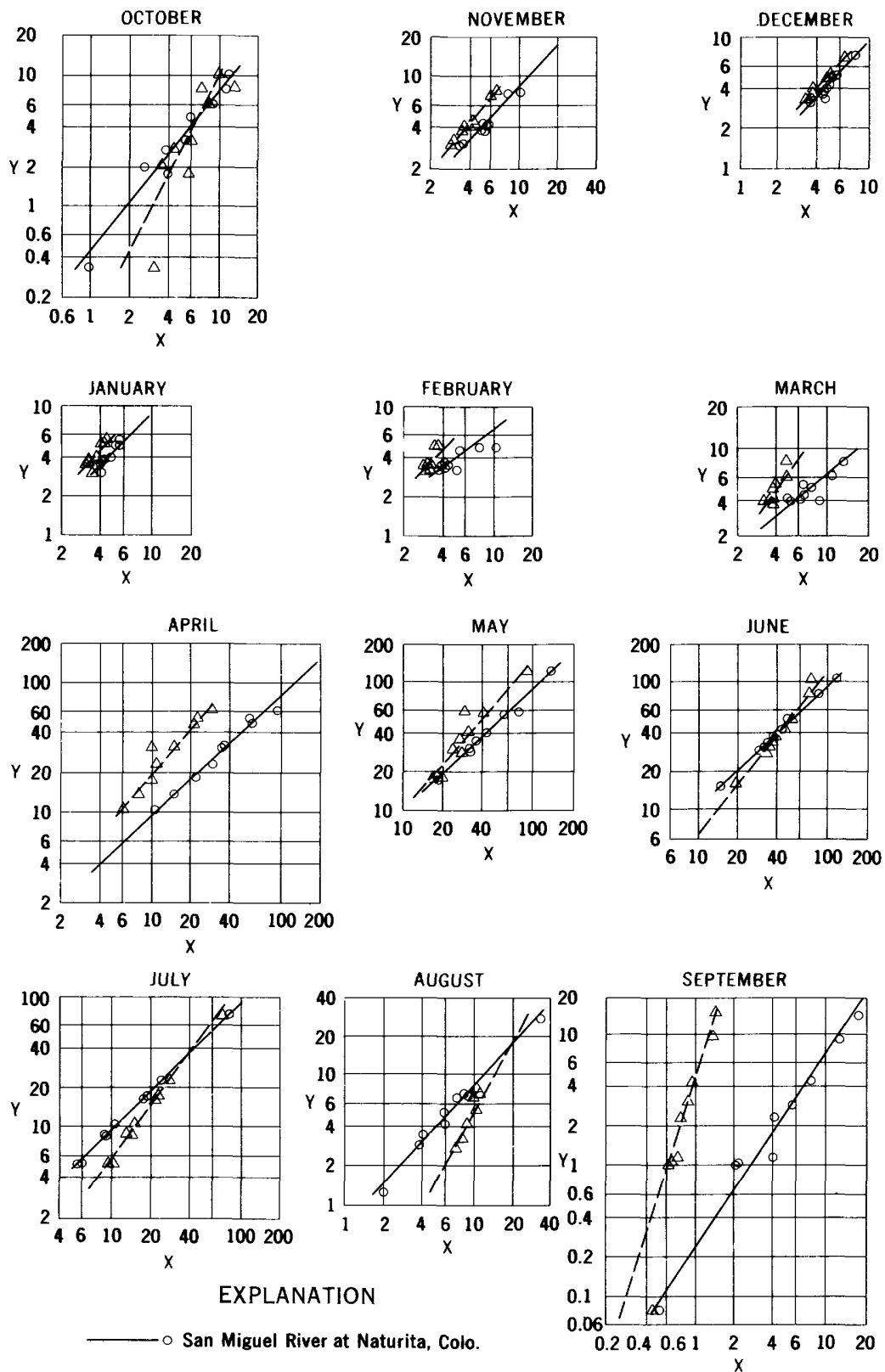

$--\triangle$ San Miguel River near Placerville, Colo.

Relationships of monthly mean discharge of San Miguel River near Nucla, Colo. $(Y)$, to monthly mean discharge of San Miguel River near Placerville, Colo., or San Miguel River at Naturita, Colo. (X). Discharge in thousands of acrefeet. 
1740. San Miguel River near Nucla, Colo.

Location. - Lat $38^{\circ} 15^{\prime}$, Iong $108^{\circ} 24^{\prime}$, in $\mathrm{NE} \frac{1}{4} \mathrm{sec} .10$, T.46 N., R.14 W., on right bank 0.4 miles upstream from highway bridge, three-quarters of a mile upstrearn from Cottonwood Creek, and $8 \frac{1}{2}$ miles east of Nucla. Altitude of gage is $5,800 \mathrm{ft}$ (from topographic map).

Drainage area. $--660 \mathrm{sq} \mathrm{mi}$, approximately.

Records available. - -october 1953 to september 1962.

Estimates of streamflow.--October 1930 to September 1934 , based on relationships of monthly mean discharge with San Miguel River near Placerville, Colo.; May 1940 to September 1953, October 1962 to September 1965 , based on relationships of monthly mean discharge with

San Miguel River at Naturita, Colo. The regression equation used is:

$$
\log Y=b \log X-c
$$

(where $Y$ is discharge of San Miguel River near Nucla, Colo., and X is discharge of San Miguel River near Placerville, Colo., or San Miguel River at Naturita, Colo., all in acre-feet per month).

Monthly values of constants in above equation

\begin{tabular}{l|l|r|r|r|r|r|r|r|r|r|r|r|r}
\hline & & Oct. & Nov. & Dec. & Jan. & Feb. & Mar. & Apr. & May & June & July & Aug. & Sept. \\
\hline $\begin{array}{l}\text { Oet 1930 } \\
\text { to }\end{array}$ & b & 1.97 & 1.20 & 1.08 & 1.08 & 1.08 & 1.39 & 1.16 & 1.16 & 1.25 & 1.36 & 1.79 & 2.92 \\
Sept 1934 & c & 3.86 & .73 & .26 & .23 & .22 & 1.32 & .35 & .64 & 1.19 & 1.69 & 3.46 & 7.86 \\
\hline $\begin{array}{l}\text { May 1940 } \\
\text { to }\end{array}$ & b & 1.24 & 1.06 & 1.09 & 1.00 & 0.78 & 0.84 & 0.93 & 0.94 & 0.92 & 0.97 & 1.07 & 1.45 \\
Sept 1965 & c & 1.05 & .56 & .38 & .06 & -.72 & -.46 & -.25 & -.24 & -.35 & -.10 & .37 & 1.87 \\
\hline
\end{tabular}

Average discharge. --29 years (1931-34, 1940-65), 197,900 acre-feet per year (273 cfs). Extremes.-1953-62: Maximum discharge, 3,810 cf's Apr. 22, 1958; minimum daily 0.5 cf's oct. $11-22,1956$.

Remarks.--Slight regulation by Lake Hope and Trout Lake of western Colorado Power Co. (combined capacity 5,040 acre-feet). Natural flow of stream also affected by water exported from Beaver Creek to Naturita Creek drainage for irrigation of about 12,000 acres, diversions for irrigation of about 5,000 acres above station, and diversion by Colorado Cooperative Canal 4 miles upstream for irrigation below. Éstimates of annual flow are within about 5 percent of regression line.

\begin{tabular}{l|l|l|l|r|r|r|r|r|r|r|r|r|r|r}
\hline $\begin{array}{l}\text { Water } \\
\text { year }\end{array}$ & oct. & Nov. & Dec. & Jan. & Feb. & Mar. & Apr. & May & June & July & Aug. & Sept. & Annual \\
\hline 1954 & 2,770 & 4,080 & 4,460 & 4,890 & 3,720 & 4,190 & 10,270 & 18,120 & 15,460 & 8,800 & 3,460 & 4,400 & 84,620 \\
1955 & 6,090 & 4,460 & 3,430 & 3,970 & 3,520 & 4,010 & 23,240 & 30,960 & 33,820 & 10,250 & 7,210 & 1,010 & 132,000 \\
& & & & & & & & & & & & & & \\
1956 & 2,050 & 3,190 & 4,050 & 3,710 & 3,400 & 5,530 & 18,040 & 28,690 & 30,480 & 5,310 & 1,250 & 78 & 105,800 \\
1957 & 337 & 5,080 & 3,190 & 3,060 & 3,230 & 4,080 & 31,740 & 59,140 & 102,900 & 71,500 & 26,400 & 14,150 & 322,800 \\
1958 & 8,020 & 8,010 & 7,250 & 4,920 & 4,910 & 6,360 & 61,430 & 118,900 & 78,740 & 16,150 & 4,260 & 2,940 & 321,900 \\
1959 & 3,220 & 4,460 & 4,950 & 5,560 & 4,620 & 4,190 & 13,660 & 17,430 & 29,020 & 5,210 & 6,660 & 1,040 & 100,000 \\
1960 & 4,890 & 4,660 & 4,210 & 3,750 & 3,510 & 8,220 & 54,020 & 35,180 & 50,660 & 16,980 & 2,900 & 1,140 & 190,100 \\
1961 & 1,760 & 3,870 & 3,710 & 3,570 & 3,220 & 4,490 & 32,200 & 55,840 & 41,280 & 8,570 & 7,010 & 9,360 & 174,900 \\
1962 & 10,560 & 7,540 & 5,180 & 3,830 & 4,990 & 5,010 & 48,880 & 40,010 & 36,810 & 22,820 & 5,100 & 2,340 & 193,100 \\
\hline
\end{tabular}

\begin{tabular}{|c|c|c|c|c|c|c|c|c|c|c|c|c|c|}
\hline $\begin{array}{l}\text { Vate } \\
\text { year }\end{array}$ & oct. & Nov. & Dec. & Jan. & $\mathrm{F} \in \mathrm{b}$. & Mar. & Apr. & May & June & July & Aug. & Sept. & Annual \\
\hline $\begin{array}{l}1 \\
1 \\
1\end{array}$ & & $\begin{array}{l}00 \\
00\end{array}$ & $\begin{array}{l}200 \\
900 \\
900 \\
200\end{array}$ & $\begin{array}{l}4,600 \\
4,200 \\
4,200 \\
4,600\end{array}$ & $\begin{array}{l}4,500 \\
4,300 \\
4,200 \\
4,500\end{array}$ & $\begin{array}{l}5,300 \\
5,300 \\
7,000 \\
6,400\end{array}$ & $\begin{array}{l}11,100 \\
45,200 \\
12,100 \\
22,000\end{array}$ & & & $\begin{array}{r}7,600 \\
28,500 \\
13,300 \\
3,500\end{array}$ & $\begin{array}{r}3,500 \\
11,300 \\
3,100 \\
2,800\end{array}$ & $\begin{array}{l}300 \\
600 \\
000 \\
000\end{array}$ & $\begin{array}{r}86,400 \\
218,300 \\
133,900 \\
94,500\end{array}$ \\
\hline 1940 & - & - & - & - & - & - & - & 50,900 & 40,400 & 9,100 & 4,500 & 510 & - \\
\hline $\begin{array}{l}1941 \\
1942 \\
1943 \\
1944 \\
1945\end{array}$ & $\begin{array}{r}9,300 \\
\times 37,400 \\
5,300 \\
4,000 \\
4,600\end{array}$ & $\begin{array}{r}5,500 \\
17,600 \\
6,500 \\
5,000 \\
5,400\end{array}$ & $\begin{array}{r}5,000 \\
10,000 \\
7,400 \\
4,800 \\
5,200\end{array}$ & $\begin{array}{l}5,000 \\
8,100 \\
7,000 \\
4,200 \\
4,300\end{array}$ & $\begin{array}{l}4,400 \\
5,200 \\
4,900 \\
4,100 \\
4,200\end{array}$ & $\begin{array}{l}6,700 \\
7,800 \\
5,200 \\
4,800 \\
4,400\end{array}$ & $\mid \begin{array}{r}50,500 \\
133,000 \\
40,800 \\
21,400 \\
23,800\end{array}$ & $\begin{array}{l}137, \\
128, \\
38, \\
718 \\
78\end{array}$ & $\begin{array}{l}89,500 \\
82,300 \\
38,300 \\
80,000 \\
46,200\end{array}$ & $\begin{array}{l}57,000 \\
34,100 \\
17,800 \\
37,200 \\
25,300\end{array}$ & $\begin{array}{r}22,500 \\
11,000 \\
20,000 \\
9,600 \\
14,600\end{array}$ & $\begin{array}{r}18,500 \\
4,000 \\
7,000 \\
1,900 \\
2,400\end{array}$ & $\begin{array}{l}410,900 \\
478,500 \\
199,100 \\
295,000 \\
218,900\end{array}$ \\
\hline $\begin{array}{l}1946 \\
1947 \\
1948 \\
1949 \\
1950\end{array}$ & $\begin{array}{r}5,400 \\
5,200 \\
11,500 \\
4,700 \\
4,200\end{array}$ & $\begin{array}{l}5,300 \\
5,200 \\
7,800 \\
4,200 \\
4,500\end{array}$ & $\begin{array}{l}4,400 \\
4,800 \\
6,700 \\
4,500 \\
4,600\end{array}$ & $\begin{array}{l}4,000 \\
4,500 \\
6,700 \\
4,900 \\
5,700\end{array}$ & og & $\begin{array}{l}5,800 \\
5,000 \\
6,300 \\
4,900 \\
5,000\end{array}$ & $\begin{array}{l}30,300 \\
17,200 \\
70,500 \\
33,800 \\
26,000\end{array}$ & $\begin{array}{l}25,300 \\
41,600 \\
80,500 \\
47,000 \\
21,600\end{array}$ & & $\begin{array}{l}600 \\
100 \\
400 \\
600 \\
400\end{array}$ & $\begin{array}{r}9,100 \\
16,200 \\
9,800 \\
9,200 \\
3,000\end{array}$ & $\begin{array}{r}4,600 \\
18,400 \\
2,000 \\
1,800 \\
1,000\end{array}$ & $\begin{array}{l}149,400 \\
196,700 \\
287,100 \\
225,900 \\
127,800\end{array}$ \\
\hline $\begin{array}{l}195 \\
195 \\
195\end{array}$ & $\begin{array}{l}2,900 \\
2,300 \\
5,000\end{array}$ & $\begin{array}{l}3,200 \\
3,100 \\
4,400\end{array}$ & $\begin{array}{l}4,000 \\
3,400 \\
5,600\end{array}$ & $\begin{array}{l}3,800 \\
4,100 \\
5,500\end{array}$ & $\begin{array}{l}3,100 \\
3,600 \\
3,900\end{array}$ & $\begin{array}{l}3,200 \\
3,700 \\
4,400\end{array}$ & $\begin{array}{r}3,500 \\
52,300 \\
15,200\end{array}$ & $\begin{array}{l}13,800 \\
66,200 \\
24,800\end{array}$ & & $\begin{array}{r}9,200 \\
31,700 \\
14,700\end{array}$ & $\begin{array}{r}5,900 \\
12,900 \\
6,900\end{array}$ & $\begin{array}{r}I, 800 \\
5,400 \\
980\end{array}$ & $\begin{array}{r}80,800 \\
260,200 \\
137,080\end{array}$ \\
\hline $\begin{array}{l}1963 \\
1964 \\
1965\end{array}$ & $\begin{array}{l}5,900 \\
1,800\end{array}$ & $\begin{array}{l}4,800 \\
4,600 \\
3,500\end{array}$ & $\begin{array}{l}4,800 \\
3,200 \\
4,300\end{array}$ & $\begin{array}{l}00 \\
00 \\
00\end{array}$ & $\begin{array}{l}5,100 \\
2,900\end{array}$ & $\begin{array}{r}10,600 \\
2,900 \\
3,700\end{array}$ & & & & & $\begin{array}{r}5,900 \\
8,800 \\
19,900\end{array}$ & $\begin{array}{r}3,600 \\
1,900 \\
12,700\end{array}$ & $\begin{array}{l}200 \\
900 \\
600\end{array}$ \\
\hline
\end{tabular}

* Estimated on basis of August regression. 

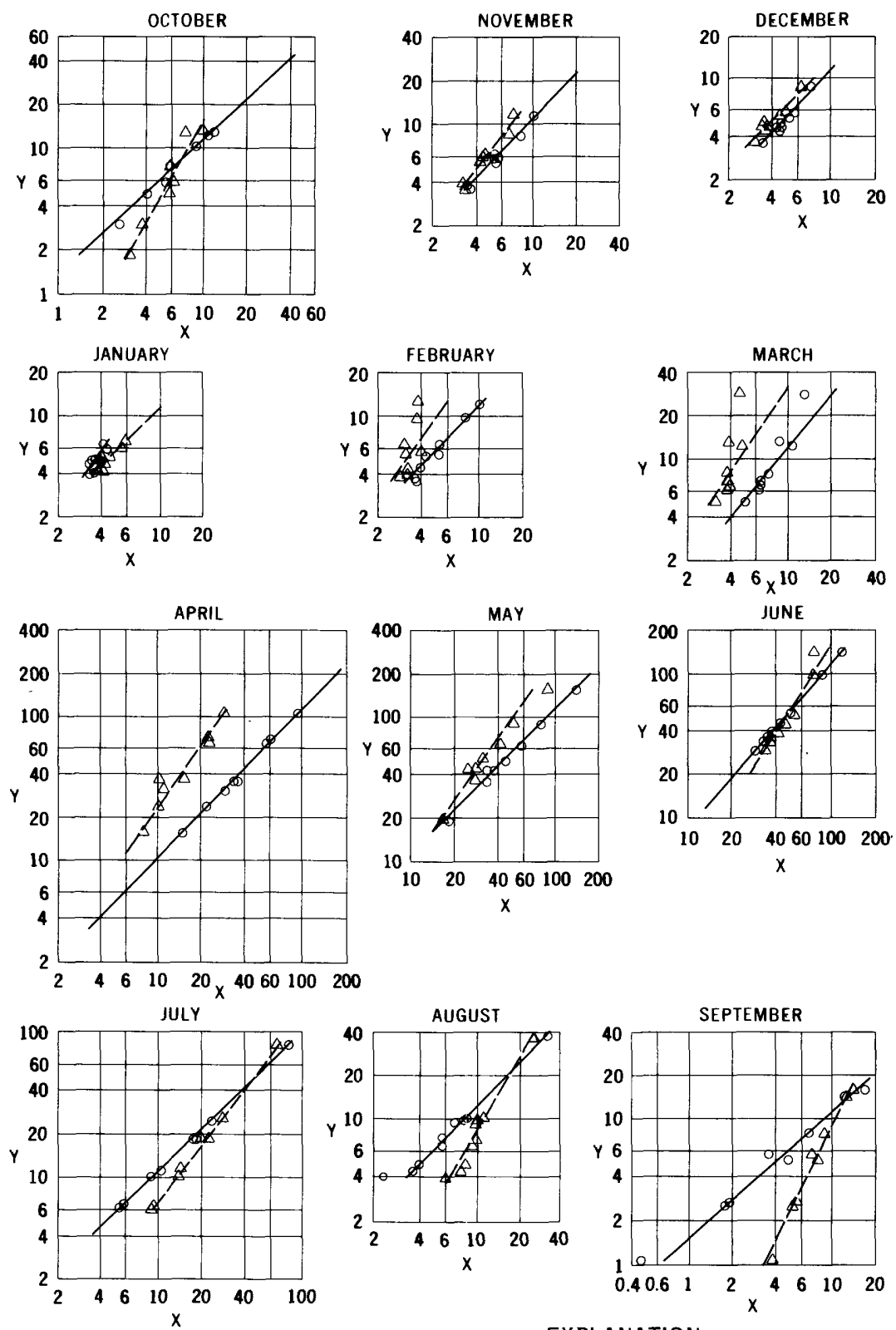

EXPLANATION

$-\longrightarrow \triangle$ San Miguel River near Placerville, Colo.

San Miguel River at Naturita, Colo.

Relationships of monthly mean discharge of San Miguel River at Uravan, Colo. $(\mathrm{Y})$, to monthly mean discharge of San Miguel River near Placerville, Colo., or San Miguel River at Naturita, Colo. (X). Discharge in thousands of acrefeet. 
1770. San Miguel River at Uravan, Colo.

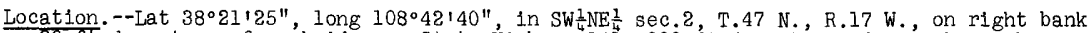
$20 \mathrm{ft}$ downs tream from bridge on State Highway 141 , $300 \mathrm{ft}$ downstream from Tabeguache Creek, and $1 \frac{1}{4}$ miles southeast of Uravan. Altitude of gage is 5,000 ft (from topographic map).

Drainage area. $--1,550 \mathrm{sq} \mathrm{mi}$, approximately.

Records available. - -August 1954 to September 1962.

Estimates of streamflow.--October 1930 to September 1934, based on relationships of monthly mean discharge with San Miguel River near Placerville, Colo.; May 1940 to July 1954 , 0ctober 1962 to September 1965, based on relationships of monthly mean discharge with San Miguel River at Naturita, Colo. The regression equation used is:

$\log Y=b \log X-c$

(where $Y$ is discharge of San Miguel River at Uravan, Colo, and X is discharge of San Miguel River near Placerville, Colo., or San Miguel River at Naturita, Colo., all in acre-feet per month).

Monthly values of constants in above equation

\begin{tabular}{|c|c|c|c|c|c|c|c|c|c|c|c|c|c|}
\hline & & & ov. & & ran. & &. & pr. & \{a & & $\mathrm{y}$ & ag. & pt. \\
\hline $\begin{array}{l}\text { et } 1 \\
\text { to } \\
\text { ept }\end{array}$ & & 2.77 & 30 & -.04 & 1.57 & 1.40 & 1.35 & $\begin{array}{l}1.45 \\
1.44 \\
\end{array}$ & $\begin{array}{l}1.44 \\
1.77 \\
\end{array}$ & $\begin{array}{l}1.54 \\
2.50 \\
\end{array}$ & $\begin{array}{l}1.30 \\
1.38 \\
\end{array}$ & $\begin{array}{l}1.62 \\
2.58 \\
\end{array}$ & .04 \\
\hline $\begin{array}{l}\text { ay } 1940 \\
\text { to } \\
\text { ept } 1965\end{array}$ & & $\begin{array}{r}0.93 \\
-.35\end{array}$ & $\begin{array}{l}1.00 \\
-.03\end{array}$ & $\begin{array}{r}1.06 \\
.19\end{array}$ & $\begin{array}{l}1.03 \\
.07\end{array}$ & $\begin{array}{r}1.03 \\
.05\end{array}$ & $\begin{array}{r}1.21 \\
.76\end{array}$ & $\begin{array}{r}1.04 \\
.14\end{array}$ & $\begin{array}{r}1.01 \\
-.01\end{array}$ & $\begin{array}{r}1.12 \\
.54 \\
\end{array}$ & $\begin{array}{l}0.96 \\
-.20\end{array}$ & $\begin{array}{r}0.96 \\
-.22 \\
\end{array}$ & $\begin{array}{r}0.89 \\
-.52\end{array}$ \\
\hline
\end{tabular}

Average discharge. --29 years (1930-34, 1940-65), 256,500 acre-feet per year (354 cfs).

Extremes.--1954-62: Maximum discharge, 6,690 cfs Apr. 19, 1958; minimum daily, 14 cfs Sept. $16,18-20,1956$.

Remarks. -Natural flow of stream affected by storage reservoirs, diversions for irrigation of about 28,000 acres above station, and return flow from irrigated areas. Estimates of annual flow October 1930 to September 1934 are within about 10 percent of regression line. Estimates of annual flow after May 1940 are within about 5 percent of regression line.

\begin{tabular}{l|r|r|r|r|r|r|r|r|r|r|r|r|r|r}
\hline $\begin{array}{l}\text { Water } \\
\text { year }\end{array}$ & oct. & Nov. & Dec. & Jan. & Feb. & Mar. & Apr. & May & June & July & Aug. & Sept. & Annual \\
\hline 1954 & $-\vec{r}$ & - & $-\vec{r}$ & - & - & - & - & - & - & - & 4,760 & 8,100 & - \\
1955 & 10,500 & 5,440 & 4,610 & 4,940 & 5,220 & 13,020 & 30,890 & 43,120 & 36,790 & 11,320 & 9,710 & 2,600 & 178,200 \\
1956 & 3,020 & 3,620 & 4,520 & 3,930 & 3,690 & 6,570 & 23,850 & 36,400 & 33,240 & 6,610 & 4,060 & 1,000 & 130,500 \\
1957 & 1,880 & 3,820 & 3,560 & 4,940 & 6,350 & 5,020 & 36,460 & 90,880 & 140,500 & 80,330 & 36,380 & 16,040 & 426,200 \\
1958 & 12,730 & 11,330 & 8,880 & 6,430 & 12,520 & 12,350 & 106,700 & 156,600 & 95,900 & 18,430 & 6,400 & 5,390 & 452,700 \\
1959 & 5,800 & 6,040 & 5,440 & 5,850 & 5,510 & 6,220 & 15,770 & 19,730 & 29,380 & 6,330 & 9,100 & 2,710 & 117,900 \\
1960 & 7,790 & 5,970 & 4,860 & 4,640 & 4,410 & 28,220 & 65,250 & 43,830 & 52,610 & 18,440 & 4,310 & 3,710 & 244,000 \\
1961 & 4,920 & 6,270 & 4,600 & 4,000 & 3,790 & 7,010 & 35,870 & 64,310 & 44,950 & 10,130 & 9,890 & 14,960 & 210,700 \\
1962 & 13,180 & 8,360 & 5,840 & 4,810 & 9,820 & 7,960 & 69,950 & 51,160 & 38,920 & 24,990 & 7,380 & 5,840 & 248,200 \\
\hline
\end{tabular}

Estimated monthly and annual streamflow, in acre-feet

\begin{tabular}{|c|c|c|c|c|c|c|c|c|c|c|c|c|c|}
\hline $\begin{array}{l}\text { ater } \\
\text { ear }\end{array}$ & Oct. & Nov. & Dec. & Jan. & eb. & Mer. & Apr. & lay & June & Ju1y & Aug. & Sept. & Annue] \\
\hline $\begin{array}{l}19 \\
19 \\
19\end{array}$ & $\begin{array}{l}8 \\
5 \\
9\end{array}$ & & & $\begin{array}{l}0 \\
0 \\
0 \\
0\end{array}$ & $\begin{array}{l}300 \\
100 \\
300\end{array}$ & $\begin{array}{r}9,000 \\
9,000 \\
11,800 \\
10,800\end{array}$ & $\begin{array}{l}11,500 \\
64,000 \\
12,800 \\
26,700\end{array}$ & $\begin{array}{l}14 \\
78 \\
31 \\
34\end{array}$ & $\begin{array}{l}00 \\
00 \\
00 \\
00\end{array}$ & $\begin{array}{r}9 \\
31 \\
15 \\
4\end{array}$ & & $\begin{array}{l}100 \\
500 \\
600 \\
800\end{array}$ & $\begin{array}{l}291,000 \\
165,100 \\
121,000\end{array}$ \\
\hline 94 & - & - & - & - & - & - & - & 65,500 & 44,000 & 11,400 &, 700 &, 100 & - \\
\hline $\begin{array}{l}1941 \\
1942 \\
1943 \\
1944 \\
1945\end{array}$ & $\begin{array}{r}13,200 \\
43,400 \\
8,700 \\
7,000 \\
7,700\end{array}$ & $\begin{array}{r}7,000 \\
21,100 \\
8,300 \\
6,400 \\
6,900\end{array}$ & $\begin{array}{r}5,900 \\
11,500 \\
8,600 \\
5,700 \\
6,100\end{array}$ & $\begin{array}{r}6,300 \\
10,200 \\
8,800 \\
5,200 \\
5,400\end{array}$ & $\begin{array}{l}6,700 \\
8,200 \\
7,600 \\
6,000 \\
6,100\end{array}$ & $\begin{array}{r}12,300 \\
15,000 \\
8,600 \\
7,600 \\
6,600\end{array}$ & $\begin{array}{l}68,500 \\
201,000 \\
53,400 \\
26,200 \\
29,500\end{array}$ & $\begin{array}{l}190,000 \\
176,000 \\
49,200 \\
160,000 \\
104,000\end{array}$ & & $\begin{array}{l}70,300 \\
42,400 \\
22,400 \\
46,100 \\
31,400\end{array}$ & & $\begin{array}{r}19,600 \\
7,600 \\
10,700 \\
4,800 \\
5,500\end{array}$ & $\begin{array}{l}544,100 \\
656,200 \\
252,900 \\
389,100 \\
279,500\end{array}$ \\
\hline $\begin{array}{l}1946 \\
1947 \\
1948 \\
1949 \\
1950\end{array}$ & $\begin{array}{r}8, \\
8, \\
15, \\
7 \\
7,\end{array}$ & $\begin{array}{l}6,800 \\
6,700 \\
9,800 \\
5,500 \\
5,800\end{array}$ & $\begin{array}{l}5,200 \\
5,600 \\
7,800 \\
5,300 \\
5,400\end{array}$ & $\begin{array}{l}5,100 \\
5,700 \\
7,600 \\
6,100 \\
7,100\end{array}$ & $\begin{array}{r}5,400 \\
5,800 \\
11,000 \\
7,400 \\
6,800\end{array}$ & $\begin{array}{r}9,800 \\
8,100 \\
11,300 \\
7,800 \\
8,100\end{array}$ & $\begin{array}{l}38,400 \\
20,500 \\
99,000 \\
43,400 \\
32,300\end{array}$ & & & & $\begin{array}{r}12,400 \\
21,200 \\
13,400 \\
12,700 \\
4,600\end{array}$ & & $\begin{array}{l}188,000 \\
240,900 \\
381,600 \\
291,700 \\
158,900\end{array}$ \\
\hline $\begin{array}{l}1951 \\
1952 \\
1953 \\
1954\end{array}$ & $\begin{array}{l}4,600 \\
8,200 \\
4,700\end{array}$ & $\begin{array}{l}4,200 \\
4,100 \\
5,800 \\
5,400\end{array}$ & $\begin{array}{l}4,700 \\
4,000 \\
6,600 \\
5,300\end{array}$ & $\begin{array}{l}4,800 \\
5,100 \\
7,000 \\
5,700\end{array}$ & $\begin{array}{l}4,200 \\
5,100 \\
5,500 \\
4,700\end{array}$ & $\begin{array}{l}4,200 \\
5,300 \\
6,800 \\
5,200\end{array}$ & $\begin{array}{r}3,400 \\
70,200 \\
17,800 \\
10,700\end{array}$ & $\begin{array}{l}16,200 \\
87,000 \\
30,300 \\
20,700\end{array}$ & $\begin{array}{l}26,200 \\
88,000 \\
51,600 \\
13,700\end{array}$ & $\begin{array}{l}11,500 \\
39,200 \\
18,300 \\
10,400\end{array}$ & $\begin{array}{r}8 \\
17 \\
9\end{array}$ & $\begin{array}{l}500 \\
100 \\
200\end{array}$ & $\begin{array}{r}98,500 \\
339,000 \\
170,700 \\
99,360\end{array}$ \\
\hline $\begin{array}{l}1963 \\
1964 \\
1965\end{array}$ & $\begin{array}{l}9,400 \\
3,800 \\
7,000\end{array}$ & $\begin{array}{l}6,200 \\
6,000 \\
4,700\end{array}$ & $\begin{array}{l}5,600 \\
3,800 \\
5,000\end{array}$ & $\begin{array}{l}5,700 \\
3,700 \\
5,700\end{array}$ & $\begin{array}{l}7,900 \\
3,800 \\
5,100\end{array}$ & $\begin{array}{r}23 \\
3 \\
5\end{array}$ & & $\begin{array}{l}27,300 \\
64,000 \\
74,500\end{array}$ & $\begin{array}{l}12,900 \\
33,300 \\
62,500\end{array}$ & $\left\{\begin{array}{r}7,300 \\
12,100 \\
60,000\end{array}\right.$ & $\begin{array}{r}8,400 \\
12,000 \\
25,400\end{array}$ & $\begin{array}{l}7,100 \\
4,800 \\
5,400\end{array}$ & $\begin{array}{l}138,300 \\
178,700 \\
341,000\end{array}$ \\
\hline
\end{tabular}



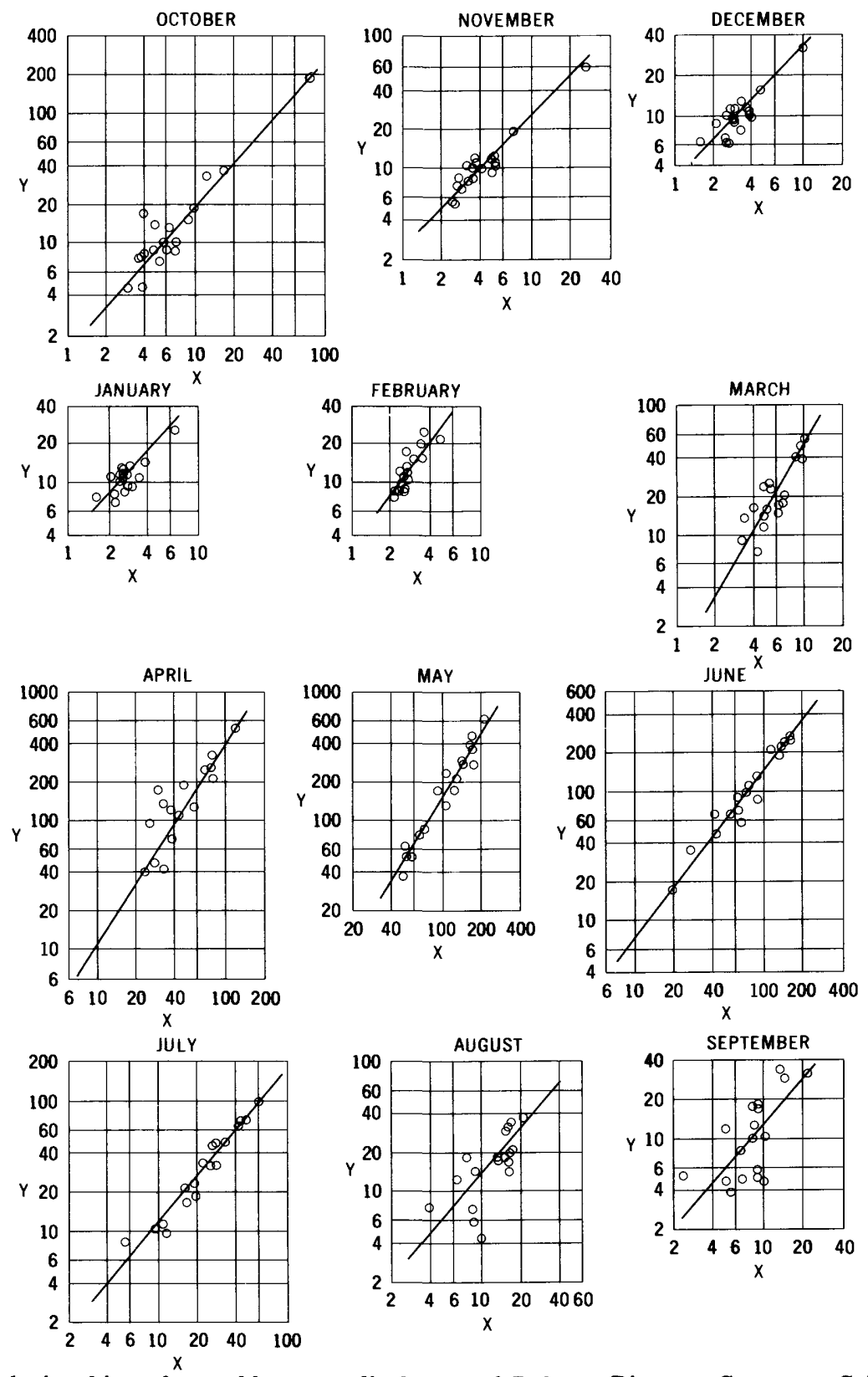

Relationships of monthly mean discharge of Dolores River at Gateway, Colo. $(Y)$, to monthly mean discharge of Dolores River at Dolores, Colo. (X). Discharge in thousands of acre-feet. 
1795. Dolores River at Gateway, Colo.

Location.--Lat $38^{\circ} 40155^{\prime \prime}$, Iong $108^{\circ} 58150^{\prime \prime}$, in SW $\frac{1}{4}$ sec.15, T.51 N., R.19 W., on right bank $500 \mathrm{ft}$ downstream from bridge on State Highway $141,0.3$ mile northwest of Gateway, 0.3 mile downstream from West Creek, and 8 miles upstream from Colorado-Utah State

line. Datum of gage is 4,547.76 ft above mean sea level, datum of 1929 .

Drainage area. $--4,350 \mathrm{sq} \mathrm{mi}$, approximately.

Records available. --October 1936 to December 1954.

Estimates of streamflow.--October 1930 to September 1936, January 1955 to September 1965, based on relationships of monthly mean discharge with Dolores River at Dolores, Colo. The regression equation used is:

$$
\log Y=b \log X-c
$$

(where $\mathrm{Y}$ is discharge of Dolores River at Gateway, Colo., and $\mathrm{X}$ is discharge of Dolores River at Dolores, Colo., both in acre-feet per month).

Monthly values of constants in above equation

\begin{tabular}{r|r|r|r|r|r|r|r|r|r|r|r|r}
\hline & oct. & Nov. & Dec. & Jan. & Feb. & Mar. & Apr. & May & June & July & Aug. & Sept. \\
\hline $\mathrm{b}$ & 1.12 & 1.03 & 1.04 & 1.11 & 1.34 & 1.74 & 1.55 & 1.64 & 1.30 & 1.19 & 1.15 & 1.17 \\
$\mathrm{c}$ & .19 & -.28 & -.39 & -.26 & .49 & 2.23 & 2.15 & 3.00 & 1.33 & .69 & .46 & .56 \\
\hline
\end{tabular}

Average discharge. --35 years $(1930-65), 586,310$ acre-feet per year (810 cfs).

Extremes. - -1936-54: Maximum discharge, 15,400 cfs May 14, 194l; minimum daily, 23 cfs Sept. 6, 1950.

Remarks.--Diversions for irrigation of about 35,000 acres above station and about 37,000 acres in Montezuma Valley in the San Juan River basin. Estimates of annual flow are within about 20 percent of regression line.

\begin{tabular}{|c|c|c|c|c|c|c|c|c|c|c|c|c|c|}
\hline \\
\hline & ct. & Nov. & Dec. & Jan. & Feb. & Mar. & Apr. & May & June & July & Aug. & Sept. & Annual \\
\hline 37 & $\begin{array}{r}714,450 \\
10,590 \\
15,590 \\
7,800\end{array}$ & \begin{tabular}{|r|}
$\mid 11,900$ \\
8,090 \\
11,040 \\
6,870
\end{tabular} & 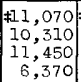 & $\begin{array}{r}+11,070 \\
10,160 \\
9,660 \\
7,890\end{array}$ & \begin{tabular}{|r|}
12,220 \\
11,260 \\
9,260 \\
12,380
\end{tabular} & $\begin{array}{l}22,73 \\
39,80 \\
54,80 \\
17,38\end{array}$ & $121,300=$ & $\begin{array}{r}277,000 \\
215,800 \\
87,590 \\
271,700\end{array}$ & \begin{tabular}{|r|}
72,080 \\
192,900 \\
35,460 \\
66,680
\end{tabular} & $\begin{array}{r}33,970 \\
48,310 \\
8,390 \\
11,690\end{array}$ & $\begin{array}{r}18,930 \\
14,780 \\
7,580 \\
7,220\end{array}$ & $\begin{array}{l}12,120 \\
34,330 \\
17,910 \\
17,020\end{array}$ & $\begin{array}{r}\$ 760,400 \\
888,700 \\
379,200 \\
454,300\end{array}$ \\
\hline & $\begin{array}{r}34,320 \\
197,100 \\
9,250 \\
10,700 \\
7,490\end{array}$ & $\mid \begin{array}{r}12,060 \\
61,070 \\
9,930 \\
9,190 \\
11,940\end{array}$ & $\begin{array}{l}13,090 \\
33,800 \\
11,550 \\
10,030 \\
11,480\end{array}$ & $\left|\begin{array}{r}13,590 \\
25,830 \\
11,540 \\
9,440 \\
11,690\end{array}\right|$ & $\begin{array}{l}20,050 \\
22,090 \\
13,900 \\
12,150 \\
15,680\end{array}$ & $\begin{array}{l}20,470 \\
16,760 \\
14,050\end{array}$ & $\begin{array}{l}212,200 d \\
96,91 \mathrm{dA} \\
134,10 \mathrm{~d}\end{array}$ & $\begin{array}{l}614,800 \\
387,100 \\
133,300 \\
463,200 \\
296,300\end{array}$ & & & & $\begin{array}{r}32,190 \\
8,320 \\
18,790 \\
4,780 \\
5,850\end{array}$ & \\
\hline & & & & & $\begin{array}{l}10,010 \\
10,810 \\
25,580 \\
17,540 \\
15,960\end{array}$ & & & $\begin{array}{r}53,470 \\
172,600 \\
270,900 \\
233,400 \\
77,680\end{array}$ & $\mid \begin{array}{r}110,600 \\
132,600 \\
221,500 \\
66,990\end{array}$ & & $\begin{array}{r}17,810 \\
36,740 \\
18,420 \\
19,850 \\
4,410\end{array}$ & & \\
\hline 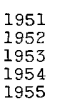 & $\begin{array}{l}4, \\
4, \\
8,\end{array}$ & $\begin{array}{r}5,360 \\
5,460 \\
7,250 \\
11,140 \\
8,360\end{array}$ & $\begin{array}{l}6, \\
9, \\
7,\end{array}$ & $\left|\begin{array}{r}7,150 \\
12,640 \\
10,950 \\
8,290\end{array}\right|$ & $\begin{array}{l}8,700 \\
8,870\end{array}$ & $\begin{array}{r}7,460 \\
13,920 \\
11,740 \\
9,310 \\
\end{array}$ & $\begin{array}{l}40,450 \\
41,470\end{array}$ & $\begin{array}{r}37,330 \\
364,500 \\
63,820 \\
52,590\end{array}$ & $\begin{array}{l}55,800 \\
90,810 \\
17,710\end{array}$ & $\begin{array}{r}10,710 \\
64,430 \\
18,680 \\
9,650\end{array}$ & $\begin{array}{r}12,530 \\
20,700 \\
18,590 \\
5,880\end{array}$ & $\left|\begin{array}{r}5,200 \\
10,800 \\
3,910 \\
12,790\end{array}\right|$ & $\begin{array}{l}293,400 \\
203,200\end{array}$ \\
\hline
\end{tabular}

₹ Estimated for 1950 Compllation.

Estimated monthly and annual streamflow, in acre-feet

\begin{tabular}{|c|c|c|c|c|c|c|c|c|c|c|c|c|c|}
\hline $\begin{array}{l}\text { Water } \\
\text { year }\end{array}$ & oct. & Nov. & Dec. & Jan. & Feb. & Mar. & Apr. & May & June & July & Aug. & Sept. & Annual \\
\hline $\begin{array}{l}1931 \\
1932 \\
1933 \\
1934 \\
1935\end{array}$ & $\begin{array}{r}9,100 \\
13,700 \\
8,000 \\
12,200 \\
3,500\end{array}$ & $\begin{array}{l}7,100 \\
9,600 \\
6,100 \\
9,700 \\
4,400\end{array}$ & $\begin{array}{r}8,400 \\
13,600 \\
10,000 \\
10,300 \\
6,600\end{array}$ & $\begin{array}{r}10,600 \\
15,300 \\
13,900 \\
10,600 \\
7,300\end{array}$ & $\begin{array}{r}12,500 \\
35,000 \\
7,900 \\
13,200 \\
7,000\end{array}$ & $\begin{array}{l}13,800 \\
41,400 \\
11,200 \\
13,800 \\
13,900\end{array}$ & $\begin{array}{r}17,500 \\
258,000 \\
17,100 \\
55,500 \\
66,500\end{array}$ & $\begin{array}{r}43,000 \\
397,000 \\
69,500 \\
27,400 \\
81,000\end{array}$ & $\begin{array}{r}33,400 \\
177,000 \\
122,000 \\
4,200 \\
212,000\end{array}$ & $\begin{array}{r}10,700 \\
47,100 \\
26,000 \\
3,300 \\
48,300\end{array}$ & $\begin{array}{r}6,100 \\
22,000 \\
7,900 \\
3,700 \\
23,100\end{array}$ & $\begin{array}{r}5,700 \\
8,200 \\
10,300 \\
2,600 \\
14,000\end{array}$ & $\begin{array}{r}177,900 \\
1,037,900 \\
309,900 \\
166,500 \\
487,600\end{array}$ \\
\hline 1936 & 12,000 & 7,900 & 7,400 & 8,800 & 10,200 & 42,000 & 226,000 & 172,000 & 60,000 & 16,100 & 30,200 & 10,600 & 603,200 \\
\hline 1955 & - & - & - & 9,400 & b. & 10,000 & 26,600 & 500 & 62000 & 18 & 27,200 & 8,100 & 295,100 \\
\hline $\begin{array}{l}1956 \\
1957 \\
1958 \\
1959 \\
1960\end{array}$ & $\begin{array}{r}6,000 \\
2,600 \\
25,100 \\
7,300 \\
9,500\end{array}$ & $\begin{array}{r}5,100 \\
4,600 \\
20, \operatorname{oog} \\
7,200 \\
11,200\end{array}$ & $\begin{array}{r}9,500 \\
5,200 \\
18,200 \\
7,900 \\
7,000\end{array}$ & $\begin{array}{r}12,700 \\
11,800 \\
19,100 \\
8,500 \\
8,300\end{array}$ & $\begin{array}{r}11,900 \\
13,100 \\
19,000 \\
9,800 \\
7,100\end{array}$ & $\begin{array}{r}50,200 \\
11,500 \\
24,700 \\
9,500 \\
42,800\end{array}$ & $\begin{array}{r}65,500 \\
60,500 \\
231,000 \\
12,400 \\
97,000\end{array}$ & $\begin{array}{r}96,000 \\
164,000 \\
400,000 \\
35,600 \\
120,000\end{array}$ & $\begin{array}{r}51,500 \\
373,000 \\
136,000 \\
25,600 \\
117,000\end{array}$ & $\begin{array}{r}14,700 \\
164,000 \\
27, \text {,od } \\
8,700 \\
19,200\end{array}$ & $\begin{array}{l}12,000 \\
66,500 \\
20,200 \\
11,900 \\
11,000\end{array}$ & $\begin{array}{r}3,200 \\
32,800 \\
10,400 \\
5,300 \\
4,700\end{array}$ & $\begin{array}{l}338,300 \\
909,600 \\
951,500 \\
149,700 \\
554,800\end{array}$ \\
\hline $\begin{array}{l}1961 \\
1962 \\
1963 \\
1964 \\
1965\end{array}$ & $\begin{array}{r}8,200 \\
20,400 \\
10,400 \\
7,000 \\
5,600\end{array}$ & $\begin{array}{r}7,900 \\
16,200 \\
9,200 \\
7,200 \\
6,700\end{array}$ & $\begin{array}{r}8,200 \\
11,600 \\
10,200 \\
7,200 \\
9,600\end{array}$ & $\begin{array}{r}9, \text { ood } \\
15, \text { ood } \\
9,700 \\
8,300 \\
12,600\end{array}$ & $\begin{array}{r}7,500 \\
29,000 \\
17,200 \\
7,000 \\
12,500\end{array}$ & $\begin{array}{r}12,300 \\
15,500 \\
66,000 \\
2,800 \\
8,500\end{array}$ & $\begin{array}{r}57 \\
210 \\
80 \\
13 \\
131\end{array}$ & $\begin{array}{r}139,000 \\
145,000 \\
73,000 \\
117,000 \\
176,000\end{array}$ & $\begin{array}{r}64,200 \\
96,000 \\
14,800 \\
49,900 \\
182,000\end{array}$ & $\begin{array}{r}14,700 \\
32,000 \\
13,000 \\
17,400 \\
102,000\end{array}$ & $\begin{array}{l}17,000 \\
20,300 \\
14,100 \\
28,600 \\
45,500\end{array}$ & $\begin{array}{r}18,000 \\
8,500 \\
9,300 \\
11,300 \\
24,000\end{array}$ & $\begin{array}{l}363,200 \\
619,500 \\
326,900 \\
277,600 \\
716,000\end{array}$ \\
\hline
\end{tabular}



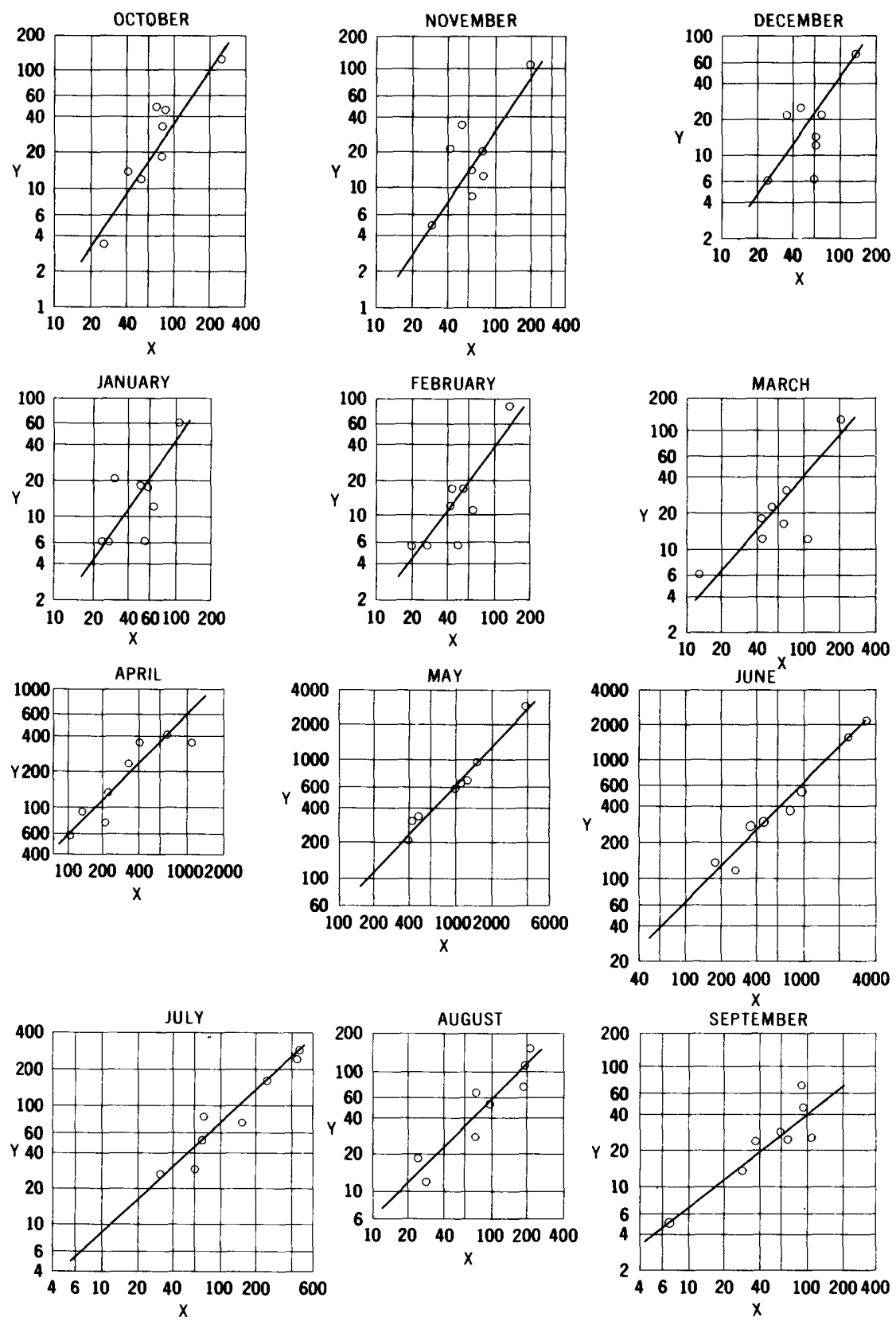

Relationships of monthly mean discharge of Indian Creek near Monticello, Utah $(Y)$, to monthly mean discharge of Indian Creek above Cottonwood Creek, near Monticello, Utah $(X)$. Discharge in acre-feet. 
1860. Indian Creek near Monticello, Utah

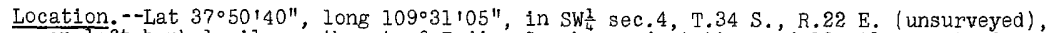
on left bank 1 mile northwest of Indian Creek guard station and 10 miles west of Monticello. Altitude of gage is $8,700 \mathrm{ft}$ (from topographic map).

Drainage area. $--4.70 \mathrm{sq} \mathrm{mi}$.

Records available. - - October 1949 to september 1957.

Estimates of streamflow.--0ctober 1957 to September 1965, based on relationships of monthly mean discharge with Indian Creek above Cottonwood Creek, near Monticello, Utah. The regression equation used is:

$$
\log Y=b \log X-c
$$

(where $\mathrm{Y}$ is discharge of Indian Creek near Monticello, Utah, and $\mathrm{X}$ is discharge of Indian Creek above Cottonwood Creek, near Monticello, Utah, both in acre-feet per month).

\begin{tabular}{l|l|l|l|l|l|r|r|r|r|r|r|r}
\hline \multicolumn{10}{c}{ Monthly values of constants 1n above equat1on } \\
\hline b & Oct. & Nov. & Dec. & Jan. & Feb. & Mar. & Apr. & May & June & July & Aug. & Sept. \\
\hline c & 1.49 & 1.46 & 1.44 & 1.44 & 1.35 & 1.16 & 1.02 & 1.06 & 1.02 & 0.91 & 0.99 & 0.80 \\
\hline
\end{tabular}

Average discharge. - -16 years (1949-65), 1,913 acre-feet per year (2.64 cfs).

Extremes.--1949-57: Maximum discharge, 122 cfs Aug. 6, 1955, from rating curve extended above $50 \mathrm{cfs}$ on basis of logarithmic plotting; no fiow for several days in september. october, and November 1956.

Remarks. - A tunnel diverts water about a mile above station (diversion began June 1952) to San Juan River basin for domestic use and irrigation in the vicinity of Blanding. Estimates of annual flow are within about 10 percent of regression line.

\begin{tabular}{l|c|c|c|l|l|l|r|r|r|r|r|r|r|r}
\hline $\begin{array}{c}\text { Water } \\
\text { year }\end{array}$ & Oct. & Nov. & Dec. & Jan. & Feb. & Mar. & Apr. & May & June & July & Aug. & Sept. & Annual \\
\hline 2950 & 123 & 104 & 71 & 61 & 83 & 123 & 410 & 638 & 557 & 158 & 67 & 46 & 2,440 \\
& & & & & & & & & & & & & & \\
2951 & 34 & 20 & 22 & 18 & 17 & 22 & 58 & 294 & 278 & 80 & 56 & 71 & 970 \\
2952 & 50 & 34 & 25 & 18 & 17 & 31 & 354 & 2,770 & 1,580 & 243 & 74 & 25 & 5,220 \\
1953 & 19 & 12 & 12 & 12 & 11 & 16 & 133 & 328 & 298 & 51 & 29 & 14 & 935 \\
1954 & 12 & 8.5 & 6.1 & 6.1 & 5.6 & 12 & 234 & 663 & 117 & 29 & 12 & 26 & 1,130 \\
1955 & 47 & 14 & 14 & 6.1 & 5.6 & 12 & 356 & 957 & 379 & 71 & 156 & 24 & 2,040 \\
1956 & 14 & 21 & 22 & 21 & 12 & 18 & 91 & 212 & 134 & 26 & 19 & 5.0 & 595 \\
1957 & 3.4 & 4.8 & 6.1 & 6.1 & 5.6 & 6.1 & 74 & 663 & 2,180 & 285 & 112 & 29 & 3,380 \\
\hline
\end{tabular}

\begin{tabular}{c|c|c|c|c|c|c|c|c|r|r|r|r|r}
\hline $\begin{array}{c}\text { Water } \\
\text { year }\end{array}$ & Oct. & Nov. & Dec. & Jan. & Feb. & Mar. & Apr. & May & June & July & Aug. & Sept. & Annual \\
\hline 1958 & 80 & 90 & 20 & 30 & 20 & 40 & 460 & 2,400 & 890 & 100 & 40 & 40 & 4,210 \\
1959 & 20 & 30 & 10 & 10 & 7 & 10 & 70 & 90 & 30 & 6 & 7 & 4 & 294 \\
1960 & 3 & 2 & 4 & 5 & 3 & 20 & 280 & 590 & 430 & 40 & 40 & 20 & 1,440 \\
1961 & 50 & 20 & 30 & 10 & 6 & 20 & 200 & 580 & 300 & 40 & 60 & 60 & 1,380 \\
1962 & 80 & 40 & 30 & 10 & 30 & 50 & 880 & 920 & 440 & 100 & 10 & 20 & 2,610 \\
1963 & 30 & 20 & 20 & 20 & 20 & 50 & 140 & 360 & 80 & 6 & 70 & 70 & 886 \\
1964 & 20 & 20 & 8 & 4 & 4 & 6 & 110 & 360 & 160 & 40 & 40 & 10 & 782 \\
1965 & 7 & 9 & 10 & 10 & 10 & 20 & 110 & 570 & 1,000 & 310 & 130 & 100 & 2,290 \\
\hline
\end{tabular}



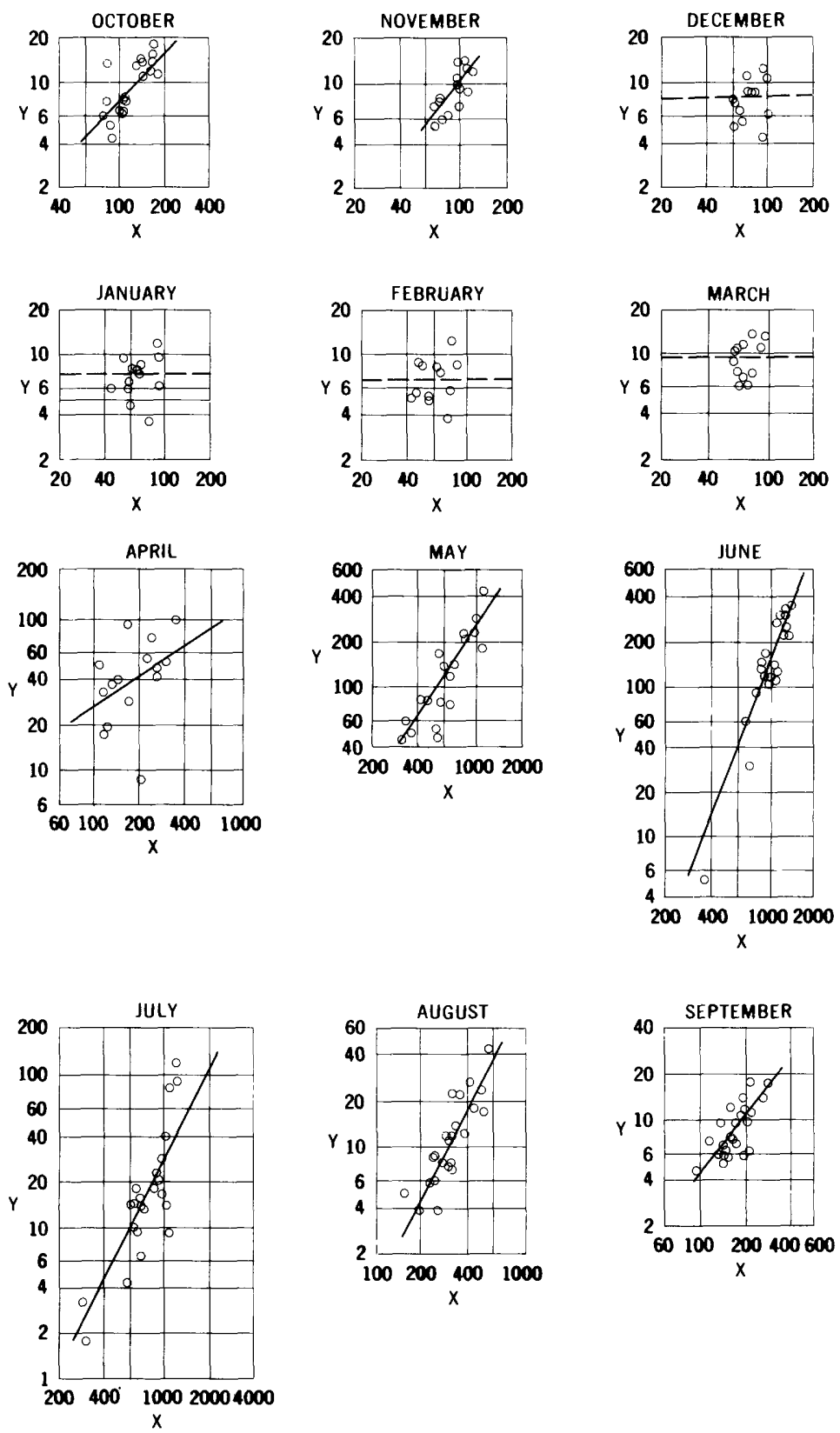

Relationships of monthly mean discharge of Horse Creek near Daniel, Wyo. ( $Y$ ), to monthly mean discharge of Green River at Warren Bridge near Daniel, Wyo. (X). Discharge in hundreds of acre-feet. 
1900. Horse Creek near Daniel, Wyo.

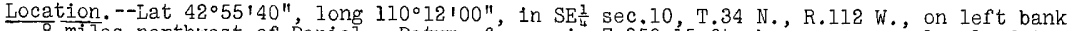
8 miles northwest of Daniel. Datum of gage is 7,350 .15 it above mean sea level, datum of 1929 .

Drainage area. --124 sq $\mathrm{mi}$.

Records available. --October 1931 to September 1954.

Estimates of streamflow.--October 1954 to September 1965, based on relationships of monthly mean discharge with Green River at Warren Bridge, near Daniel, Wyo. The regression equation used is:

$$
\log Y=b \log X-c
$$

(where $Y$ is discharge of Horse Creek near Daniel, Wyo., and $X$ is discharge of Green River at Warren Bridge, near Daniel, Wyo., both in acre-f'eet per month).

Monthly values of constants in above equation

\begin{tabular}{c|c|c|c|c|c|c|c|c|c|c|c|c}
\hline & oct. & Nov. & Dec. & Jan. & Feb. & Mar. & Apr. & May & June & July & Aug. & Sept. \\
\hline b & 1.06 & 1.26 & - & - & - & - & 0.67 & 1.50 & 2.60 & 1.96 & 1.88 & 1.26 \\
c & 1.35 & 1.98 & - & - & - & - & -.72 & 3.08 & 8.87 & 6.34 & 5.40 & 2.40 \\
\hline
\end{tabular}

Average discharge. --34 years $(1931-65), 47,060$ acre-feet per year (65.0 cfs).

Extremes.--1931-54: Maximum discharge, 1,670 cfs May 31, 1936, from rating curve extended above 800 cf's; minimum daily, 1 cf's July 22,23 , 1940.

Remarks. - Water rights totaling about 232 cf's (priorities 1889-1937) for irrigation of about 16,300 acres (part of which is above and part below station), adjudicated by Wyoming for diversion above station. Estimates of annual flow are within about 25 percent of regression line.

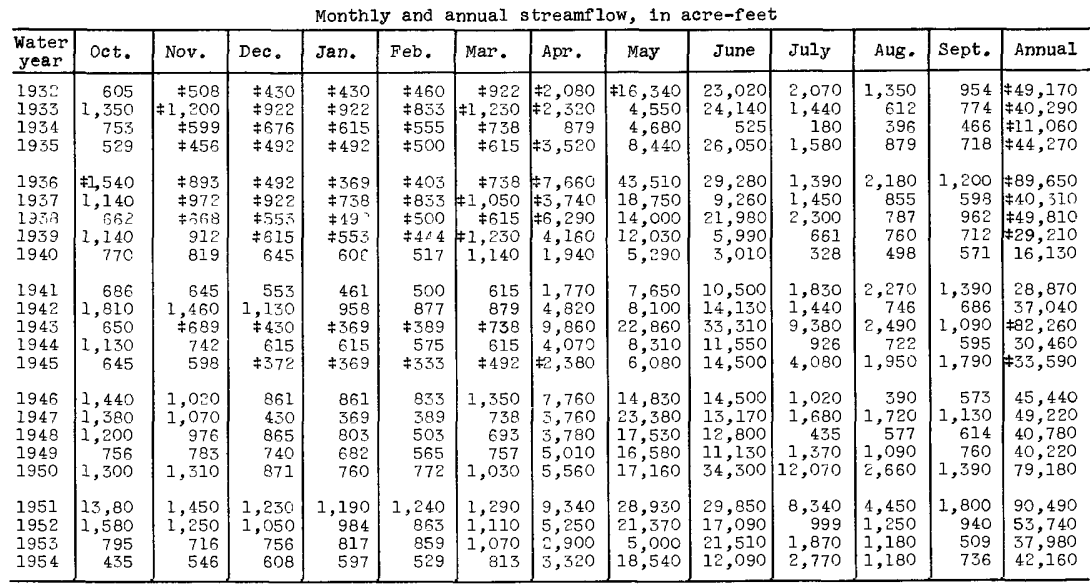

* Estimated for 1950 Compilation.

Estimated monthly and annual streamflow, in acre-feet

\begin{tabular}{l|r|r|r|r|r|r|r|r|r|r|r|r|r}
\hline $\begin{array}{l}\text { Water } \\
\text { year }\end{array}$ & Oct. & Nov. & Dec. & Jan. & Feb. & Mar. & Apr. & May & June & July & Aug. & Sept. & Annual \\
\hline \hline 1955 & 620 & 760 & $e 800$ & $e 750$ & $e 690$ & $e 930$ & 3,100 & 9,200 & 11,000 & 950 & 890 & 610 & 30,300 \\
1956 & 650 & 580 & $e 800$ & $e 750$ & $e 690$ & $e 930$ & 5,100 & 30,000 & 55,000 & 1,900 & 1,400 & 690 & 98,490 \\
1957 & 780 & 900 & $e 800$ & $e 750$ & $e 690$ & $e 930$ & 2,700 & 14,000 & 28,000 & 3,800 & 2,500 & 1,200 & 57,050 \\
1958 & 1,200 & 1,100 & $e 800$ & $e 750$ & $e 690$ & $e 930$ & 3,300 & 20,000 & 8,800 & 380 & 770 & 790 & 39,510 \\
1959 & 680 & 800 & $e 800$ & $e 750$ & $e 690$ & $e 930$ & 3,100 & 6,900 & 35,000 & 1,200 & 1,200 & 730 & 52,780 \\
1960 & 960 & 880 & $e 800$ & $e 750$ & $e 690$ & $e 930$ & 3,400 & 5,700 & 7,000 & 490 & 630 & 750 & 22,980 \\
1961 & 940 & 940 & $e 800$ & $e 750$ & $e 690$ & $e 930$ & 2,600 & 8,200 & 9,700 & 360 & 440 & 1,300 & 27,650 \\
1962 & 900 & 1,000 & $e 800$ & $e 750$ & $e 690$ & $e 930$ & 4,200 & 20,000 & 24,000 & 2,500 & 1,700 & 600 & 58,070 \\
1965 & 900 & 970 & $e 800$ & $e 750$ & $e 690$ & $e 930$ & 2,600 & 11,000 & 16,000 & 1,300 & 880 & 2,100 & 38,920 \\
1964 & 1,000 & 960 & $e 800$ & $e 750$ & $e 690$ & $e 930$ & 4,300 & 13,000 & 15,000 & 4,000 & 1,500 & 520 & 43,450 \\
1965 & 630 & 700 & $e 800$ & $e 750$ & $e 690$ & $e 930$ & 3,200 & 12,500 & 40,000 & 4,900 & 3,300 & 1,100 & 69,500 \\
\hline
\end{tabular}

e Flat estimate based on average discharge for month. 

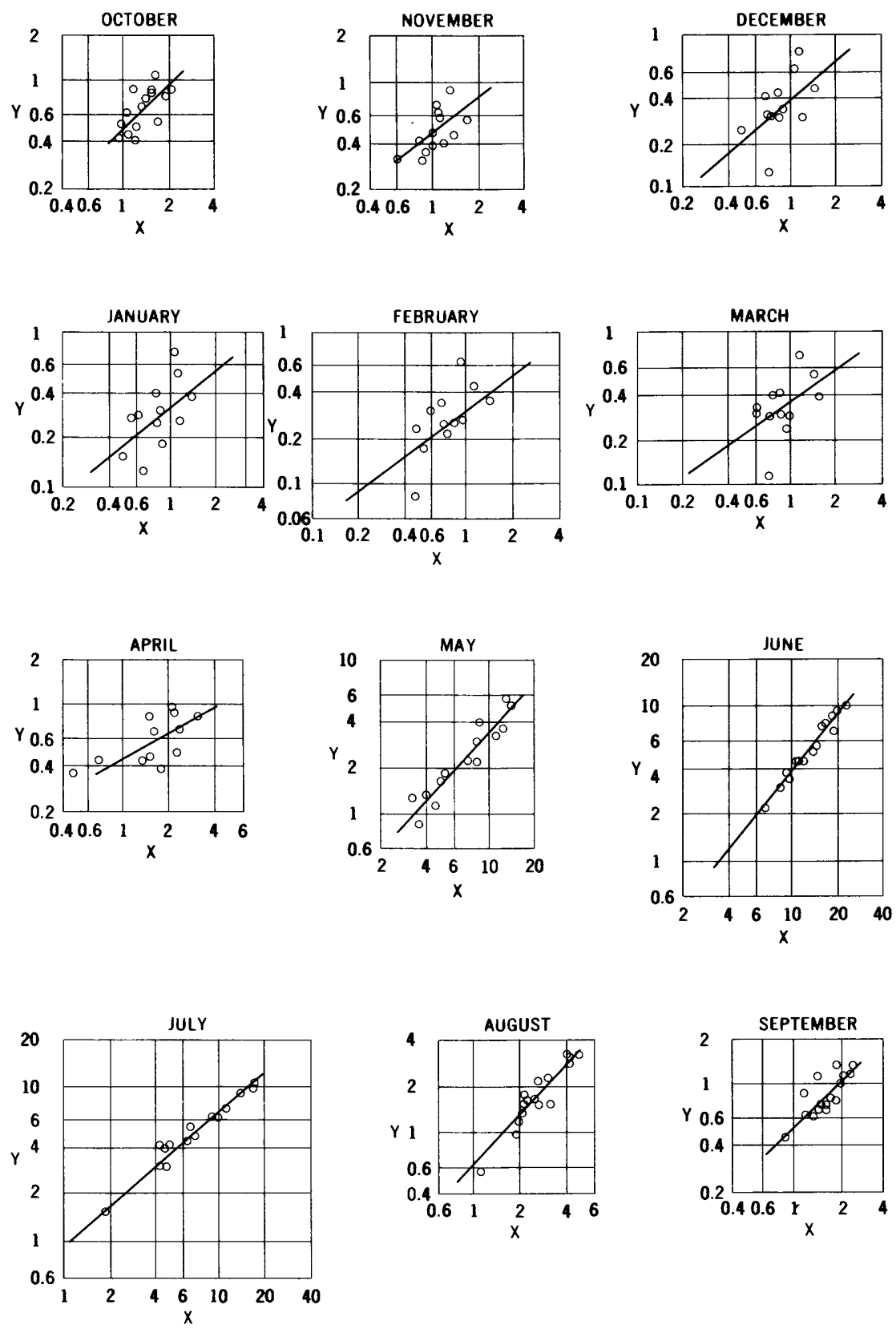

Relationships of monthly mean discharge of Middle Piney Creek below South Fork, near Big Piney, Wyo. (Y), to monthly mean discharge of North Piney Creek near Mason, Wyo. (X). Discharge in thousands of acre-feet. 
2060. Middle Piney Creek below South Fork, near Big Piney, Wyo.

Location. --Lat $42^{\circ} 36^{\prime} 10^{\prime \prime}$, long $110^{\circ} 27120^{\prime \prime}$, in sec.7, T.30 N., R.114 W., on left bank 1 mile downstream from South Fork and 18 miles northwest of Big Piney. Altitude of gage is $7,980 \mathrm{ft}$ (from topographic map).

Drainage area. $--34.3 \mathrm{sq} \mathrm{mi}$.

Records available. --July 1939 to September 1954.

Estimates of streamf'low.--October 1931 to July 1939, October 1954 to September 1965, based on relationships of monthly mean discharge with North Piney Creek near Mason, Wyo. The regression equation used is:

$$
\log Y=b \log X-c
$$

(where $Y$ is discharge of Middle Piney Creek below South Fork near Big Piney, Wyo., and $X$ is discharge of North Piney Creek near Mason, Wyo., both in acre-feet per'month).

Monthly values of constants in above equation

\begin{tabular}{l|c|c|c|c|c|c|c|c|c|c|c|c}
\hline & Oct. & Nov. & Dec. & Jan. & Feb. & Mar. & Apr. & May & June & July & Aug. & Sept. \\
\hline $\mathrm{b}$ & 0.96 & 0.77 & 0.84 & 0.82 & 0.75 & 0.71 & 0.55 & 1.08 & 1.25 & 0.87 & 1.06 & 0.96 \\
$\mathrm{c}$ & .19 & -.36 & -.06 & -.06 & -.24 & -.43 & -.99 & .80 & 1.44 & -.34 & .39 & .16 \\
\hline
\end{tabular}

Average discharge. --34 years $(1931-65), 19,060$ acre-feet per year (26.3 cfs).

Extremes.--1939-54: Maximum discharge, 254 cfs June 29, 1943, from rating curve extended above $190 \mathrm{cfs}$; minimum not determined, occurred during period of ice effect.

Remarks.--Flow regulated by Middle Piney Lake (usable capacity, 4,200 acre-ft). Adjud1cated diversions for irrigation of about 400 acres of hay meadows above station. Estimates of annual flow are within about 10 percent of regression line.

\begin{tabular}{|c|c|c|c|c|c|c|c|c|c|c|c|c|c|}
\hline \multicolumn{14}{|c|}{ Monthly and annual } \\
\hline $\begin{array}{l}\text { Water } \\
\text { year } \\
\end{array}$ & oct. & Nov. & Dec. & Jan. & Feb. & Mar. & Apr. & May & June & July & Aug. & Sept. & Annual \\
\hline $\begin{array}{l}1939 \\
1940\end{array}$ & $\overline{627}$ & -468 & $\overline{307}$ & $\overline{-}_{123}$ & $\overline{173}$ & $\overline{307}$ & $\overline{437}$ & $1, \overline{1} 40$ & $2, \overline{210}$ & 1,560 & $\begin{array}{r}1,600 \\
578\end{array}$ & $\begin{array}{l}871 \\
453\end{array}$ & 8,380 \\
\hline $\begin{array}{l}1941 \\
1942 \\
1943 \\
1944 \\
1945\end{array}$ & $\begin{array}{r}420 \\
676 \\
523 \\
1,100 \\
861\end{array}$ & $\begin{array}{r}319 \\
382 \\
\neq 441 \\
873 \\
708\end{array}$ & $\begin{array}{r}246 \\
92 \\
+369 \\
799 \\
615\end{array}$ & $\begin{array}{r}154 \\
135 \\
+369 \\
738 \\
553\end{array}$ & $\begin{array}{r}83 \\
183 \\
+278 \\
633 \\
444\end{array}$ & $\begin{array}{r}123 \\
289 \\
+369 \\
738 \\
553\end{array}$ & $\begin{array}{r}357 \\
863 \\
\neq 2,080 \\
952 \\
655\end{array}$ & $\begin{array}{l}1,680 \\
1,280 \\
4,960 \\
1,860 \\
1,350\end{array}$ & $\begin{array}{l}3,720 \\
3,430 \\
8,630 \\
4,330 \\
2,980\end{array}$ & $\begin{array}{l}3,050 \\
3,040 \\
9,310 \\
4,410 \\
6,250\end{array}$ & $\begin{array}{l}1,510 \\
1,170 \\
2,860 \\
1,750 \\
1,500\end{array}$ & $\begin{array}{r}736 \\
634 \\
1,360 \\
1,120 \\
1,020\end{array}$ & $\begin{array}{r}12,400 \\
12,170 \\
\neq 1,550 \\
19,300 \\
17,490\end{array}$ \\
\hline $\begin{array}{l}1946 \\
1947 \\
1948 \\
1949 \\
1950\end{array}$ & $\begin{array}{l}851 \\
550 \\
861 \\
447 \\
764\end{array}$ & 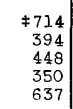 & $\begin{array}{r}+553 \\
307 \\
295 \\
338 \\
405\end{array}$ & $\begin{array}{r}\mathbf{\$ 4 9 2} \\
277 \\
264 \\
307 \\
284\end{array}$ & $\begin{array}{r}+555 \\
233 \\
213 \\
250 \\
304\end{array}$ & $\begin{array}{r}\$ 738 \\
338 \\
246 \\
307 \\
402\end{array}$ & $\begin{array}{r}\neq 2,080 \\
833 \\
381 \\
477 \\
891\end{array}$ & $\begin{array}{l}3,990 \\
5,740 \\
2,180 \\
2,260 \\
2,910\end{array}$ & $\begin{array}{l}7,470 \\
7,700 \\
5,560 \\
4,470 \\
9,490\end{array}$ & $\begin{array}{r}5,410 \\
7,330 \\
4,120 \\
4,040 \\
10,560\end{array}$ & $\begin{array}{r}2,180 \\
3,080 \\
978 \\
1,330 \\
3,160\end{array}$ & $\begin{array}{r}791 \\
1,130 \\
614 \\
695 \\
1,320\end{array}$ & $\begin{array}{r}\$ 25,820 \\
27,910 \\
16,160 \\
15,270 \\
31,130\end{array}$ \\
\hline $\begin{array}{l}1951 \\
1952 \\
1953 \\
1954\end{array}$ & $\begin{array}{l}807 \\
871 \\
512 \\
417\end{array}$ & $\begin{array}{l}565 \\
587 \\
410 \\
304\end{array}$ & $\begin{array}{l}461 \\
430 \\
296 \\
131\end{array}$ & $\begin{array}{l}381 \\
400 \\
259 \\
186\end{array}$ & $\begin{array}{l}355 \\
345 \\
255 \\
262\end{array}$ & $\begin{array}{l}400 \\
418 \\
296 \\
293\end{array}$ & $\begin{array}{l}842 \\
684 \\
426 \\
454\end{array}$ & $\begin{array}{r}5,130 \\
3,580 \\
862 \\
3,260\end{array}$ & $\begin{array}{r}10,140 \\
6,980 \\
5,090 \\
4,400\end{array}$ & $\begin{array}{l}9,770 \\
4,300 \\
6,340 \\
4,810\end{array}$ & $\begin{array}{l}3,160 \\
1,660 \\
2,260 \\
1,530\end{array}$ & $\begin{array}{r}1,170 \\
811 \\
738 \\
668\end{array}$ & $\begin{array}{l}33,180 \\
21,070 \\
17,740 \\
16,720\end{array}$ \\
\hline
\end{tabular}

* Estimated for 1950 Compilation.

\begin{tabular}{|c|c|c|c|c|c|c|c|c|c|c|c|c|c|}
\hline $\begin{array}{l}\text { Water } \\
\text { year }\end{array}$ & oct. & Nov. & Dec. & Jan. & Peb. & Mar. & Apr. & May & June & July & Aug. & Sept. & Annual \\
\hline $\begin{array}{l}1932 \\
1933 \\
1934 \\
1935\end{array}$ & $\begin{array}{l}540 \\
650 \\
400 \\
410\end{array}$ & $\begin{array}{r}\$ 400 \\
\$ 400 \\
\$ 360 \\
350\end{array}$ & $\begin{array}{l}\neq 290 \\
\neq 290 \\
\neq 250 \\
\neq 250\end{array}$ & $\begin{array}{l}\neq 220 \\
\neq 220 \\
\neq 200 \\
\neq 200\end{array}$ & $\begin{array}{l}\neq 200 \\
\neq 200 \\
\neq 170 \\
\neq 170\end{array}$ & $\begin{array}{l}\$ 290 \\
\$ 290 \\
\neq 260 \\
\neq 260\end{array}$ & $\begin{array}{r}\neq 400 \\
\neq 410 \\
\neq 560 \\
580\end{array}$ & $\begin{array}{r}2,100 \\
880 \\
2,000 \\
1,300\end{array}$ & $\begin{array}{r}6,900 \\
5,300 \\
930 \\
5,700\end{array}$ & $\begin{array}{l}6,500 \\
3,200 \\
1,000 \\
4,200\end{array}$ & $\begin{array}{l}2,100 \\
1,000 \\
500 \\
1,500\end{array}$ & $\begin{array}{l}870 \\
520 \\
360 \\
740\end{array}$ & $\begin{array}{r}20,810 \\
13,360 \\
6,990 \\
15,660\end{array}$ \\
\hline $\begin{array}{l}1936 \\
1937 \\
1938 \\
1939\end{array}$ & $\begin{array}{l}540 \\
660 \\
580 \\
860\end{array}$ & $\begin{array}{l}\neq 430 \\
\neq 530 \\
\neq 440 \\
\neq 430\end{array}$ & $\begin{array}{l}\neq 290 \\
\neq 350 \\
\neq 290 \\
\neq 290\end{array}$ & $\begin{array}{l}\neq 220 \\
\neq 260 \\
\neq 220 \\
\neq 220\end{array}$ & $\begin{array}{l}\neq 190 \\
\neq 200 \\
\neq 180 \\
\neq 180\end{array}$ & $\begin{array}{l}\neq 260 \\
\neq 290 \\
\neq 290 \\
\neq 350\end{array}$ & $\begin{array}{l}\neq 430 \\
\neq 410 \\
\neq 650 \\
\neq 740\end{array}$ & $\begin{array}{r}4,400 \\
+1,800 \\
2,200 \\
3,700 \\
\end{array}$ & $\begin{array}{l}9,300 \\
3,500 \\
7,000 \\
4,100\end{array}$ & $\begin{array}{l}4,200 \\
3,600 \\
4,400 \\
3,700\end{array}$ & $\begin{array}{c}2,000 \\
1,600 \\
2,000 \\
-\end{array}$ & $\begin{array}{l}880 \\
710 \\
960 \\
-\end{array}$ & $\begin{array}{l}23,140 \\
13,910 \\
19,210 \\
17,040\end{array}$ \\
\hline 1955 & 650 & 440 & 300 & 230 & 180 & 240 & 380 &, 300 & 3,800 & 3,600 & 1,300 & 740 & 13,160 \\
\hline $\begin{array}{l}1956 \\
1957 \\
1958 \\
1959 \\
1960\end{array}$ & $\begin{array}{l}540 \\
620 \\
700 \\
460 \\
800\end{array}$ & $\begin{array}{l}330 \\
480 \\
480 \\
480 \\
530\end{array}$ & $\begin{array}{l}230 \\
350 \\
350 \\
350 \\
370\end{array}$ & $\begin{array}{l}180 \\
270 \\
270 \\
260 \\
310\end{array}$ & $\begin{array}{l}180 \\
270 \\
240 \\
240 \\
260\end{array}$ & $\begin{array}{l}290 \\
380 \\
350 \\
350 \\
460\end{array}$ & $\begin{array}{l}720 \\
510 \\
480 \\
500 \\
770\end{array}$ & $\begin{array}{l}5,200 \\
2,300 \\
3,400 \\
1,200 \\
1,400\end{array}$ & $\begin{array}{r}11,200 \\
8,900 \\
6,500 \\
6,600 \\
4,000\end{array}$ & $\begin{array}{l}4,600 \\
8,600 \\
3,000 \\
4,200 \\
2,800\end{array}$ & $\begin{array}{l}1,700 \\
2,100 \\
1,300 \\
2,000 \\
1,100\end{array}$ & $\begin{array}{l}800 \\
950 \\
710 \\
940 \\
570\end{array}$ & $\begin{array}{l}25,970 \\
25,730 \\
17,780 \\
17,580 \\
13,370\end{array}$ \\
\hline $\begin{array}{l}1961 \\
1962 \\
1963 \\
1964 \\
1965\end{array}$ & $\begin{array}{l}640 \\
660 \\
720 \\
790 \\
670\end{array}$ & $\begin{array}{l}480 \\
510 \\
550 \\
590 \\
510\end{array}$ & $\begin{array}{l}230 \\
280 \\
420 \\
280 \\
340\end{array}$ & $\begin{array}{l}200 \\
210 \\
270 \\
220 \\
330\end{array}$ & $\begin{array}{l}200 \\
230 \\
300 \\
290 \\
330\end{array}$ & $\begin{array}{l}350 \\
320 \\
370 \\
390 \\
480\end{array}$ & $\begin{array}{l}530 \\
510 \\
500 \\
480 \\
740\end{array}$ & $\begin{array}{l}1,400 \\
2,600 \\
1,700 \\
2,000 \\
2,300\end{array}$ & $\begin{array}{l}3,100 \\
6,500 \\
5,300 \\
5,900 \\
9,300\end{array}$ & & $\begin{array}{l}1,200 \\
1,800 \\
1,700 \\
2,000 \\
2,800\end{array}$ & $\begin{array}{r}750 \\
930 \\
1,300 \\
800 \\
1,300\end{array}$ & $\begin{array}{l}11,080 \\
20,150 \\
17,430 \\
20,940 \\
28,500\end{array}$ \\
\hline
\end{tabular}

* Based on estimates for 1950 compilation. 

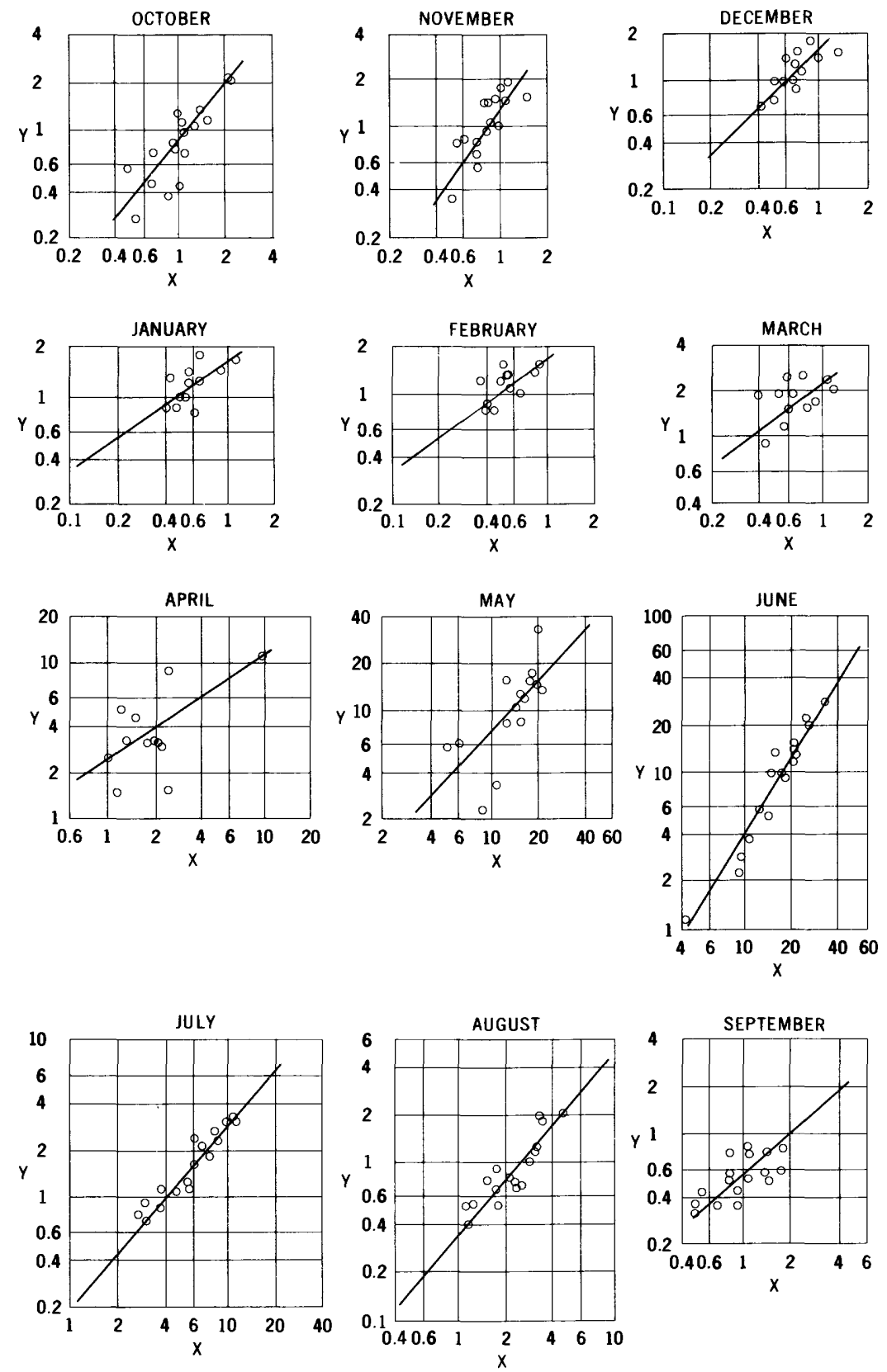

Relationships of monthly mean discharge of Smith Fork at Mountainview, Wyo. (Y), to sum of monthly mean discharges of East Fork and West Fork of Smith Fork near Robertson, Wyo. (X). Discharge in thousands of acre-feet. 
2215. Smith Fork at Mountainview, Wyo.

Location.--Lat $41^{\circ} 16^{\prime}$, long $110^{\circ} 20^{\prime}$, in sec.23, T.15 N., R.115 W., on right bank just downstream from highway bridge in southwestern edge of Mountainview. Altitude of gage is $6,830 \mathrm{ft}$ (by barometer).

Drainage area. - -192 sq mi.

Records available. --May 1941 to December 1957.

Estimates of streamflow.--July 1939 to April 1941, January 1958 to September 1965, based on relationships of monthly mean discharge with the sum of East Fork of Smith Fork near Robertson, wyo., and West Fork of Smith Fork near Robertson, Wyo. The regression equation used is:

$\log Y=b \log X-c$

(where $Y$ is discharge of Smith Fork at Mountainview, Wyo, and $X$ is the sum of discharges of East Fork of Smith Fork near Robertson, Wyo., and West Fork of Smith Fork near Robertson, Wyo., all in acre-feet per month).

\begin{tabular}{c|r|r|r|r|r|r|r|r|r|r|r|r}
\hline \multicolumn{10}{c}{ Monthly values of constants in above equation } \\
\hline oct. & Nov. & Dec. & Jan. & Feb. & Mar. & Apr. & May & June & July & Aug. & Sept. \\
\hline b & 1.23 & 1.43 & 0.96 & 0.69 & 0.71 & 0.73 & 0.67 & 1.07 & 1.68 & 1.17 & 1.17 & 0.87 \\
\hline & .75 & 1.18 & -.31 & -1.15 & -1.09 & -1.13 & -1.37 & .43 & 3.11 & 1.22 & .98 & -.15 \\
\hline
\end{tabular}

Average discharge. --26 years $(1939-65), 37,860$ acre-feet per year (52.3 cfs).

Extremes.--1941-57: Maximum discharge, 1,100 cfs June 13, 1953; minimum daily, 2.5 cf's Aug. 30, 1948.

Remarks.--D1versions above station for irrigation of about 17,800 acres, part of which is above and part below station. One small reservoir (capacity, about 300 acre-ft) for irrigation above station. Estimates of annual flow are within about 25 percent of regression line.

Monthly and annual streamflow, in acre-feet

\begin{tabular}{|c|c|c|c|c|c|c|c|c|c|c|c|c|c|}
\hline $\begin{array}{l}\text { Water } \\
\text { year }\end{array}$ & oct. & Nov. & Dec. & Jan. & Feb. & Mar. & Apr. & May & June & July & Aug. & Sept. & Annua] \\
\hline $\begin{array}{l}1941 \\
1942 \\
1943 \\
1944 \\
1945\end{array}$ & $\begin{array}{r}2, \overline{120} \\
381 \\
742 \\
944\end{array}$ & $\begin{array}{r}- \\
1,970 \\
579 \\
1,080 \\
926\end{array}$ & $\begin{array}{r}1, \overrightarrow{530} \\
964 \\
720 \\
627\end{array}$ & $\begin{array}{r}1, \overline{170} \\
1,010 \\
678 \\
764\end{array}$ & $\begin{array}{r}- \\
1,170 \\
829 \\
680 \\
899\end{array}$ & $\begin{array}{l}1,390 \\
1,370 \\
1,010 \\
1,400\end{array}$ & $\begin{array}{l}5,160 \\
5,550 \\
3,740 \\
2,630\end{array}$ & $\begin{array}{r}13,360 \\
16,420 \\
10,290 \\
17,020 \\
8,310\end{array}$ & $\begin{array}{r}12,070 \\
10,570 \\
5,880 \\
14,310 \\
9,290\end{array}$ & $\begin{array}{l}2,420 \\
1,260 \\
1,100 \\
3,000 \\
3,120\end{array}$ & $\begin{array}{r}1,160 \\
536 \\
710 \\
815 \\
2,050\end{array}$ & $\begin{array}{l}587 \\
359 \\
437 \\
783 \\
579\end{array}$ & $\begin{array}{c}- \\
43,660 \\
29,100 \\
44,580 \\
31,540\end{array}$ \\
\hline $\begin{array}{l}1946 \\
1947 \\
1948 \\
1949 \\
1950\end{array}$ & $\begin{array}{r}1,020 \\
1,120 \\
1,310 \\
271 \\
1,150\end{array}$ & $\begin{array}{r}1,490 \\
1,750 \\
1,510 \\
830 \\
1,010\end{array}$ & $\begin{array}{r}1,410 \\
1,770 \\
1,530 \\
988 \\
990\end{array}$ & $\begin{array}{r}1,480 \\
799 \\
1,850 \\
986 \\
1,350\end{array}$ & $\begin{array}{r}1,390 \\
1,000 \\
1,270 \\
871 \\
1,210\end{array}$ & $\begin{array}{l}1,970 \\
2,240 \\
1,880 \\
1,840 \\
1,820\end{array}$ & $\begin{array}{l}11,060 \\
3,180 \\
5,030 \\
2,960 \\
4,540\end{array}$ & $\begin{array}{r}8,310 \\
16,860 \\
15,050 \\
11,760 \\
15,210\end{array}$ & $\begin{array}{r}2,320 \\
13,720 \\
3,760 \\
13,470 \\
15,430\end{array}$ & $\begin{array}{r}1,140 \\
2,660 \\
775 \\
1,850 \\
1,610\end{array}$ & $\begin{array}{r}530 \\
1,210 \\
540 \\
696 \\
643\end{array}$ & $\begin{array}{l}428 \\
781 \\
311 \\
502 \\
826\end{array}$ & $\begin{array}{l}32,550 \\
47,090 \\
34,820 \\
37,020 \\
45,790\end{array}$ \\
\hline $\begin{array}{l}1951 \\
1952 \\
1953 \\
1954 \\
1955\end{array}$ & $\begin{array}{r}813 \\
2,140 \\
711 \\
456 \\
441\end{array}$ & $\begin{array}{r}1,050 \\
1,560 \\
687 \\
787 \\
548\end{array}$ & $\begin{array}{r}1,280 \\
1,490 \\
889 \\
394 \\
683\end{array}$ & $\begin{array}{r}994 \\
1,730 \\
1,270 \\
1,240 \\
843\end{array}$ & $\begin{array}{r}1,090 \\
1,520 \\
1,330 \\
1,520 \\
783\end{array}$ & $\begin{array}{l}1,540 \\
1,610 \\
2,350 \\
1,490 \\
1,160\end{array}$ & $\begin{array}{l}3,100 \\
9,140 \\
3,180 \\
1,530 \\
1,500\end{array}$ & $\begin{array}{r}12,590 \\
32,880 \\
5,690 \\
2,230 \\
3,280\end{array}$ & $\begin{array}{r}9,960 \\
20,460 \\
22,390 \\
1,140 \\
2,860\end{array}$ & $\begin{array}{r}2,120 \\
2,300 \\
1,150 \\
715 \\
924\end{array}$ & $\begin{array}{r}1,920 \\
1,980 \\
756 \\
408 \\
928\end{array}$ & $\begin{array}{l}511 \\
831 \\
353 \\
528 \\
561\end{array}$ & $\begin{array}{l}36,970 \\
77,540 \\
40,760 \\
13,040 \\
14,510\end{array}$ \\
\hline $\begin{array}{l}1956 \\
1957 \\
1958\end{array}$ & $\begin{array}{r}714 \\
577 \\
1,290\end{array}$ & $\begin{array}{r}780 \\
348 \\
1,420\end{array}$ & $\begin{array}{r}1,370 \\
731 \\
1,110\end{array}$ & $\begin{array}{c}1,440 \\
851 \\
-\end{array}$ & $\begin{array}{c}1,200 \\
793 \\
-\end{array}$ & $\begin{array}{c}2,490 \\
902 \\
-\end{array}$ & $\begin{array}{c}3,040 \\
2,410 \\
-\end{array}$ & $\begin{array}{r}14,240 \\
6,110 \\
-\end{array}$ & $\begin{array}{r}5,340 \\
29,750 \\
-\end{array}$ & $\begin{array}{r}860 \\
3,000 \\
-\end{array}$ & $\begin{array}{l}780 \\
984 \\
-\end{array}$ & $\begin{array}{l}352 \\
754 \\
-\end{array}$ & $\begin{array}{c}32,610 \\
47,210 \\
\end{array}$ \\
\hline
\end{tabular}

\begin{tabular}{l|c|c|c|c|c|c|c|c|c|c|c|c|c}
\hline $\begin{array}{l}\text { Water } \\
\text { year }\end{array}$ & Oct. & Nov. & Dec. & Jan. & Feb. & Mar. & Apr. & May & June & July & Aug. & Sept. & Annual \\
\hline 1939 & - & - & - & - & - & - & - & - & - & 570 & 320 & 710 & - \\
1940 & 970 & 420 & $\$ 550$ & $\neq 750$ & $\neq 720$ & $\$ 1,200$ & 3,800 & 12,300 & 1,000 & 220 & 130 & 640 & 22,700 \\
1941 & 1,600 & 1,400 & 1,200 & 1,000 & 810 & 1,300 & 2,300 & - & - & - & - & - & 39,210 \\
1958 & - & - & - & 1,400 & 1,400 & 2,000 & 3,300 & 13,300 & 6,600 & 800 & 530 & 660 & 33,810 \\
1959 & 500 & 540 & 810 & 870 & 800 & 1,100 & 1,800 & 7,200 & 12,800 & 1,300 & 740 & 710 & 29,170 \\
1960 & 1,500 & 1,000 & 420 & 590 & 730 & 2,000 & 5,100 & 8,900 & 5,800 & 690 & 260 & 460 & 27,450 \\
1961 & 700 & 860 & 1,000 & 1,100 & 1,000 & 2,100 & 4,500 & 7,200 & 2,500 & 370 & 460 & 1,500 & 23,290 \\
1962 & 2,700 & 2,000 & 1,300 & 1,300 & 1,600 & 2,300 & 7,600 & 10,800 & 10,600 & 2,200 & 760 & 460 & 43,620 \\
1963 & 630 & 440 & 320 & 350 & 350 & 730 & 2,600 & 8,000 & 5,500 & 1,400 & 790 & 1,000 & 22,110 \\
1964 & 1,000 & 910 & 950 & 1,200 & 1,000 & 1,400 & 1,900 & 14,600 & 13,300 & 2,400 & 960 & 570 & 40,190 \\
1965 & 660 & 920 & 1,300 & 1,500 & 1,300 & 1,600 & 3,100 & 10,000 & 60,500 & 6,800 & 4,300 & 2,100 & 94,080 \\
\hline
\end{tabular}

* Based on estimates made for 1950 Compilation. 

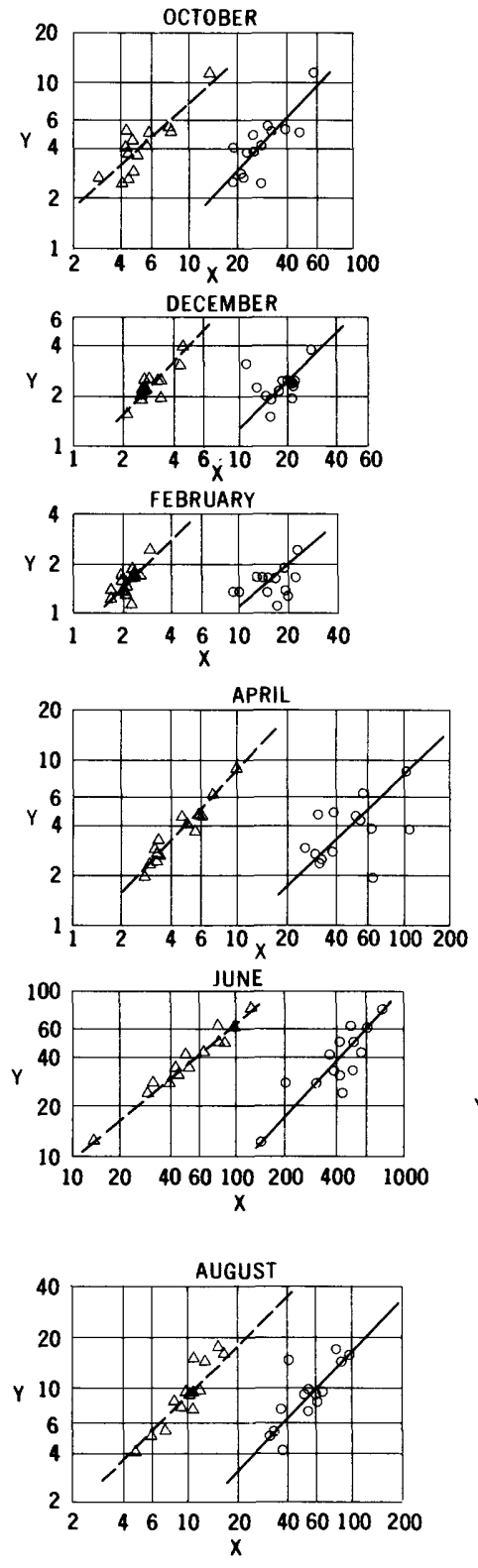
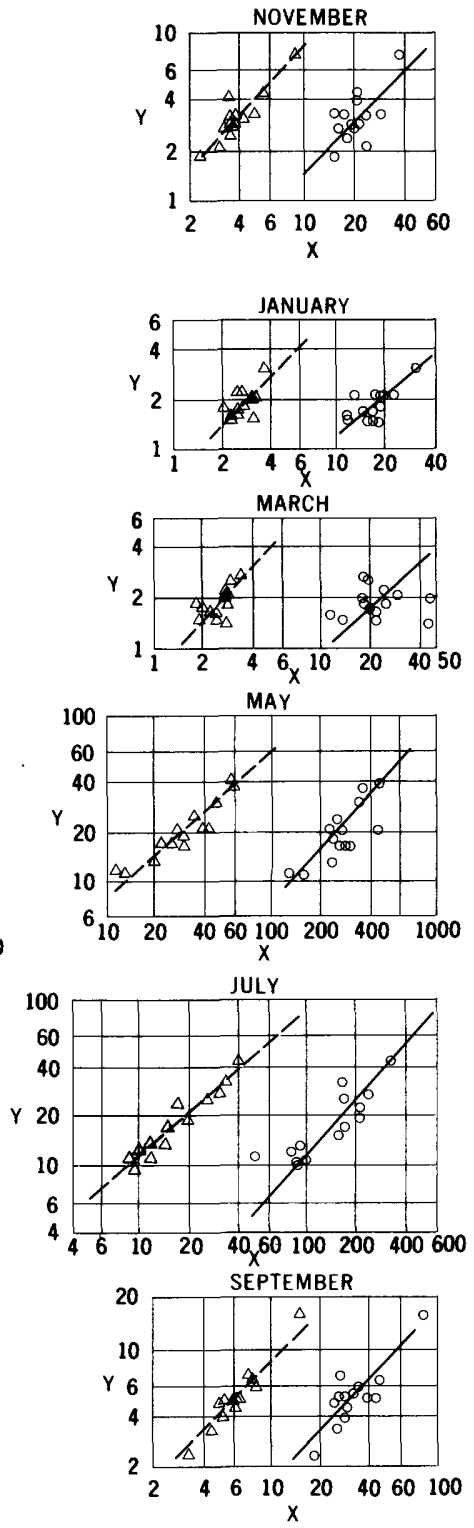

\section{EXPLANATION}

o Blacks Fork near Millburne, Wyo.

- $-\triangle$ Middle Fork Beaver Creek near Lonetree, Wyo.

Relationships of monthly mean discharge of West Fork Beaver Creek near Lonetree, Wyo. (Y), to monthly mean discharge of Blacks Fork near Millburne, Wyo., or Middle Fork Beaver Creek near Lonetree, Wyo. (X). Discharge in hundreds of acre-feet. 
2275. West Fork Beaver Creek near Lonetree, Wyo.

Location. --Lat $40^{\circ} 56150^{\prime \prime}$, long $110^{\circ} 13^{100 "}$, in SW $\frac{1}{4}$ sec.35, T.3 N., R.15 E., Salt Lake meridian, on right bank at forest boundary, an eighth of a mile upstream from Fellow

Creek, $3 \frac{1}{2}$ miles south of Utah-Wyoming State line, and $7 \frac{1}{2}$ miles southwest of Lonetree.

Altitude of gage is $8,700 \mathrm{ft}$ (from topographic map).

Drainage area. $--23 \mathrm{sq} \mathrm{ml}$, approximately.

Records ava1lable. --october 1948 to September 1962.

Estimates of streamflow.--July 1939 to September 1948, based on relationships of monthly mean discharge with Blacks Fork near Millburne, Wyo.; October 1962 to September 1965 , based on relationships with Middle Fork Beaver Creek near Lonetree, Wyo. The regres sion equation used is:

$\log \mathrm{Y}=\mathrm{b} \log \mathrm{X}-\mathrm{c}$

(where $Y$ is discharge of West Fork Beaver Creek near Lonetree, Wyo., and $X$ is discharge of Blacks Fork near Millburne, Wyo., or Middle Fork Beaver Creek near Lonetree, Wyo., all in acre-feet per month).

\begin{tabular}{|c|c|c|c|c|c|c|c|c|c|c|c|c|c|}
\hline & & Oct. & Nov. & Dec. & Jan. & Feb, & Mar. & Apr. & May & June & July & Aug. & Sept. \\
\hline $\begin{array}{l}\text { July } 1939 \\
\text { to } \\
\text { Sept. } 1948\end{array}$ & $\begin{array}{l}b \\
c\end{array}$ & $\begin{array}{r}1.03 \\
.93\end{array}$ & $\begin{array}{r}1.03 \\
.93\end{array}$ & $\begin{array}{r}0.98 \\
.83\end{array}$ & $\begin{array}{r}0.84 \\
.44\end{array}$ & $\begin{array}{r}0.87 \\
.57\end{array}$ & $\begin{array}{r}0.90 \\
.72\end{array}$ & $\begin{array}{r}0.97 \\
.97\end{array}$ & $\begin{array}{l}1.10 \\
1.53\end{array}$ & $\begin{array}{l}1.10 \\
1.47\end{array}$ & $\begin{array}{l}1.14 \\
1.50\end{array}$ & $\begin{array}{r}1.04 \\
.93\end{array}$ & $\begin{array}{r}1.02 \\
.89\end{array}$ \\
\hline $\begin{array}{l}\text { oct. } 1962 \\
\text { to } \\
\text { Sept. } 1965\end{array}$ & $\begin{array}{l}b \\
c\end{array}$ & $\begin{array}{r}0.89 \\
-.19\end{array}$ & $\begin{array}{r}1.04 \\
.20\end{array}$ & $\begin{array}{r}1.02 \\
.15\end{array}$ & $\begin{array}{r}1.00 \\
.14\end{array}$ & $\begin{array}{r}1.00 \\
.14\end{array}$ & $\begin{array}{r}1.07 \\
.28\end{array}$ & $\begin{array}{r}1.07 \\
.27\end{array}$ & $\begin{array}{l}0.89 \\
-.22\end{array}$ & $\begin{array}{l}0.84 \\
-.16\end{array}$ & $\begin{array}{l}0.86 \\
-.48\end{array}$ & $\begin{array}{l}0.97 \\
-.04\end{array}$ & $\begin{array}{r}0.99 \\
.05\end{array}$ \\
\hline
\end{tabular}

Average discharge. --26 years $(1939-65), 12,380$ acre-feet per year (17.1 cfs).

Extremes.--1948-62: Maximum discharge, 417 cfs June 13, 1953, from rating curve extended above $250 \mathrm{cfs}$ on basis of logarithmic plotting; minimum observed, 2.0 cfs Mar. 1, 1954 (discharge measurement).

Remarks.--No diversion above station. Estimates of annual flow are within about 5 percent of regression line.

Monthly and annual streamflow, in acre-feet

\begin{tabular}{c|c|c|c|c|c|c|c|c|c|c|c|c|c|c}
\hline $\begin{array}{l}\text { Water } \\
\text { year }\end{array}$ & oct. & Nov. & Dec. & Jan. & Feb. & Mar. & Apr. & May & June & July & Aug. & Sept. & Annual \\
\hline 1949 & 373 & 323 & 222 & 160 & 139 & 160 & 442 & 2,090 & 5,190 & 2,490 & 893 & 399 & 12,880 \\
1950 & 559 & 439 & 305 & 215 & 167 & 200 & 456 & 1,900 & 3,460 & 1,900 & 781 & 511 & 10,890 \\
1951 & 247 & 206 & 201 & 169 & 139 & 154 & 241 & 1,670 & 3,480 & 2,330 & 1,330 & 506 & 10,670 \\
1952 & 499 & 323 & 246 & 188 & 167 & 263 & 614 & 3,680 & 6,200 & 3,010 & 1,550 & 655 & 17,400 \\
1953 & 509 & 400 & 246 & 215 & 167 & 246 & 447 & 1,110 & 6,240 & 1,770 & 921 & 337 & 12,610 \\
1954 & 277 & 316 & 241 & 154 & 111 & 149 & 423 & 1,690 & 1,210 & 1,210 & 529 & 507 & 6,820 \\
1955 & 297 & 234 & 192 & 154 & 139 & 166 & 231 & 1,690 & 2,740 & 992 & 739 & 451 & 8,020 \\
1956 & 374 & 292 & 227 & 215 & 184 & 206 & 374 & 2,060 & 2,400 & 1,070 & 420 & 236 & 8,060 \\
1957 & 265 & 184 & 154 & 154 & 128 & 144 & 198 & 1,100 & 8,030 & 4,350 & 1,690 & 584 & 16,980 \\
1958 & 429 & 285 & 246 & 215 & 167 & 184 & 270 & 3,780 & 3,270 & 1,030 & 757 & 665 & 11,300 \\
1959 & 399 & 269 & 219 & 175 & 139 & 170 & 281 & 1,390 & 4,440 & 1,570 & 909 & 546 & 10,510 \\
1960 & 534 & 320 & 194 & 174 & 144 & 205 & 377 & 2,430 & 4,300 & 1,290 & 488 & 484 & 10,940 \\
1961 & 459 & 280 & 246 & 215 & 167 & 184 & 299 & 2,110 & 2,750 & 1,160 & 1,440 & 1,630 & 10,940 \\
1962 & 1,140 & 764 & 389 & 307 & 232 & 221 & 879 & 3,080 & 5,110 & 2,790 & 964 & 524 & 16,400 \\
\hline
\end{tabular}

Estimated monthly and annual streamflow, in acre-feet

\begin{tabular}{|c|c|c|c|c|c|c|c|c|c|c|c|c|c|}
\hline $\begin{array}{l}\text { Water } \\
\text { year }\end{array}$ & Oct. & Nov. & Dec. & Jan. & Feb. & Mar. & Apr. & May & June & July & Aug. & Sept. & Annual \\
\hline $\begin{array}{l}1939 \\
1940\end{array}$ & $43 \overline{0}$ & $25 \overline{0}$ & $17 \overline{0}$ & $15 \overline{0}$ & 130 & $160^{\circ}$ & $37 \overline{0}$ & $3, \overline{200}$ & $1, \overline{500}$ & $\begin{array}{r}\neq 900 \\
420\end{array}$ & $\begin{array}{l}570 \\
330\end{array}$ & $\begin{array}{l}560 \\
660\end{array}$ & $\overline{7,770}$ \\
\hline $\begin{array}{l}1941 \\
1942 \\
1943 \\
1944 \\
1945\end{array}$ & $\begin{array}{l}800 \\
600 \\
360 \\
370 \\
440\end{array}$ & $\begin{array}{r}370 \\
460 \\
\neq 320 \\
\neq 310 \\
\neq 270\end{array}$ & $\begin{array}{r}260 \\
\neq 350 \\
\neq 270 \\
\neq 240 \\
\neq 160\end{array}$ & $\begin{array}{r}210 \\
\neq 230 \\
\neq 210 \\
\neq 170 \\
\neq 150\end{array}$ & $\begin{array}{r}130 \\
\$ 170 \\
\$ 160 \\
\$ 180 \\
\$ 140\end{array}$ & $\begin{array}{r}160 \\
\$ 190 \\
\$ 260 \\
\$ 210 \\
\$ 170\end{array}$ & $\begin{array}{r}280 \\
\neq 250 \\
\neq 1,200 \\
\neq 300 \\
\neq 200\end{array}$ & $\begin{array}{r}3,400 \\
2,300 \\
2,600 \\
+2,500 \\
1,800\end{array}$ & $\begin{array}{l}4,700 \\
4,600 \\
3,100 \\
4,600 \\
3,700\end{array}$ & $\begin{array}{l}2,500 \\
2,000 \\
2,000 \\
2,800 \\
3,500\end{array}$ & $\begin{array}{r}1,300 \\
660 \\
1,000 \\
800 \\
1,900\end{array}$ & $\begin{array}{l}680 \\
440 \\
420 \\
380 \\
710\end{array}$ & $\begin{array}{l}14,790 \\
12,250 \\
11,900 \\
12,860 \\
13,140\end{array}$ \\
\hline $\begin{array}{l}1946 \\
1947 \\
1948\end{array}$ & $\begin{array}{l}440 \\
320 \\
680\end{array}$ & $\begin{array}{l}400 \\
280 \\
470\end{array}$ & $\begin{array}{l}290 \\
230 \\
300\end{array}$ & $\begin{array}{l}220 \\
110 \\
230\end{array}$ & $\begin{array}{l}170 \\
110 \\
180\end{array}$ & $\begin{array}{l}210 \\
170 \\
180\end{array}$ & $\begin{array}{r}1,400 \\
260 \\
350\end{array}$ & $\begin{array}{l}2,200 \\
3,700 \\
3,800\end{array}$ & $\begin{array}{l}2,800 \\
3,900 \\
2,800\end{array}$ & $\begin{array}{r}1,100 \\
3,000 \\
630\end{array}$ & $\begin{array}{r}620 \\
1,400 \\
600\end{array}$ & $\begin{array}{l}300 \\
770 \\
370\end{array}$ & $\begin{array}{l}10,150 \\
14,250 \\
10,590\end{array}$ \\
\hline $\begin{array}{l}1963 \\
1964 \\
1965\end{array}$ & $\begin{array}{l}430 \\
420 \\
360\end{array}$ & $\begin{array}{l}310 \\
340 \\
330\end{array}$ & $\begin{array}{l}190 \\
230 \\
310\end{array}$ & $\begin{array}{l}150 \\
150 \\
270\end{array}$ & $\begin{array}{l}130 \\
140 \\
200\end{array}$ & $\begin{array}{l}140 \\
140 \\
190\end{array}$ & $\begin{array}{l}190 \\
190 \\
350\end{array}$ & $\begin{array}{l}2,100 \\
3,300 \\
1,300\end{array}$ & $\begin{array}{l}2,100 \\
5,800 \\
9,400\end{array}$ & $\begin{array}{l}1,400 \\
3,300 \\
8,100\end{array}$ & $\begin{array}{r}700 \\
1,100 \\
3,500\end{array}$ & $\begin{array}{r}580 \\
500 \\
1,500\end{array}$ & $\begin{array}{r}8,420 \\
15,610 \\
25,810\end{array}$ \\
\hline
\end{tabular}

* Based on estimates for 1950 Compilation. 

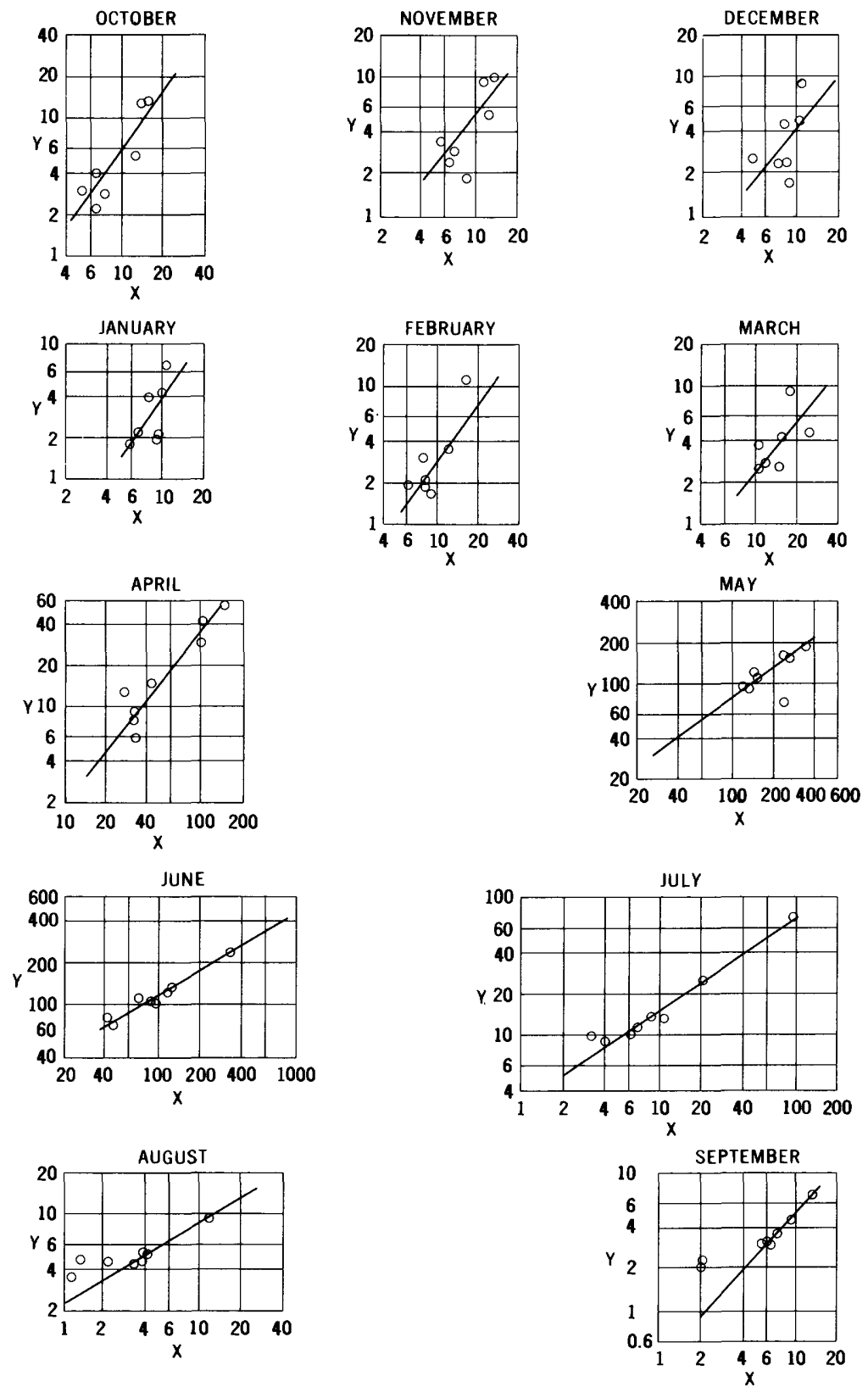

Relationships of monthly mean discharge of North Fork Little Snake River near Slater, Colo. (Y), to monthly mean discharge of Slater Fork near Slater, Colo. $(X)$. Discharge in hundreds of acre-feet. 
2519. North Fork Little Snake River near Slater, Colo.

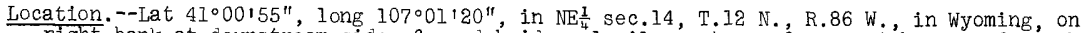
right bank at downstream side of road bridge, 1 mile upstream from west branch of North Fork, 3 miles upstream from mouth, and 19 miles east of Slater. Altitude of gage is

$7,350 \mathrm{ft}$ (from topographic map).

Drainage area. $--29.3 \mathrm{sq} \mathrm{mi}$.

Records available. -April 1956 to September 1963.

Estimates of streamflow. --August 1931 to March 1956, october 1963 to September 1965, based on relationships of inonthly mean discharge with Ślater Fork near Slater, Colo. The regression equation used is:

$\log Y=b \log X-c$

(where $\mathrm{Y}$ is discharge of North Fork Little Snake River near Slater, Colo., and X is discharge of Slater Fork near Slater, Colo., both in acre-feet per month).

\begin{tabular}{c|c|c|c|c|c|c|c|c|c|c|c|c}
\hline \multicolumn{10}{c}{ Monthly values of constants in above equation } \\
\hline b & oct. & Nov. & Dec. & Jan. & Feb. & Mar. & Apr. & May & June & July & Aug. & Sept. \\
\hline $\mathrm{c}$ & 1.42 & 1.25 & 1.26 & 1.45 & 1.40 & 1.23 & 1.28 & 0.73 & 0.58 & 0.67 & 0.59 & 1.09 \\
\hline
\end{tabular}

Average discharge. -34 years $(1932-65), 34,883$ acre-feet per year (48.2 cfs).

Extremes ${ }^{--1956-63: ~ M a x i m u m ~ d i s c h a r g e, ~} 628$ cf's June 7, 1957; minimum daily, 2.4 cfs Jan. 13, $14,19,1963$.

Remarks.--No diversion above station. Estimates of annual flow are within about 10 percent of regression line.

\begin{tabular}{r|r|r|r|r|r|r|r|r|r|r|r|r|r}
\hline $\begin{array}{l}\text { Water } \\
\text { year }\end{array}$ & Oct. & Nov. & Dec. & Jan. & Feb. & Mar. & Apr. & May & June & July & Aug. & Sept. & Annual \\
\hline 1956 & - & - & - & - & - & - & 2,990 & 16,300 & 10,370 & 1,020 & 438 & 240 & - \\
1957 & 226 & 190 & 172 & 197 & 211 & 277 & 918 & 7,390 & 23,620 & 6,990 & 932 & 462 & 41,580 \\
1958 & 552 & 557 & 492 & 430 & 361 & 430 & 1,500 & 18,440 & 12,040 & 1,150 & 466 & 320 & 36,740 \\
1959 & 281 & 297 & 246 & 215 & 194 & 246 & 798 & 9,360 & 11,300 & 1,340 & 454 & 355 & 25,090 \\
1960 & 1,330 & 950 & 461 & 400 & 316 & 461 & 4,230 & 12,180 & 10,160 & 1,380 & 357 & 212 & 32,440 \\
1961 & 306 & 250 & 240 & 221 & 200 & 373 & 1,280 & 9,770 & 7,940 & 988 & 507 & 702 & 22,780 \\
1962 & 1,300 & 1,070 & 900 & 674 & 1,100 & 922 & 5,440 & 15,710 & 13,090 & 2,510 & 458 & 308 & 43,480 \\
1963 & 418 & 349 & 254 & 182 & 176 & 256 & 593 & 11,130 & 6,960 & 907 & 528 & 301 & 22,050 \\
\hline
\end{tabular}

Estimated monthly and annual streamflow, in acre-feet

\begin{tabular}{|c|c|c|c|c|c|c|c|c|c|c|c|c|c|}
\hline $\begin{array}{l}\text { Water } \\
\text { year }\end{array}$ & oct. & Nov. & Dec. & Jan. & Feb. & Mar. & Apr. & May & June & July & Aug. & Sept. & Annual \\
\hline $\begin{array}{l}1931 \\
1932 \\
1933 \\
1934 \\
1935\end{array}$ & $\begin{array}{l}- \\
390 \\
350 \\
190 \\
520\end{array}$ & $\begin{array}{l}- \\
220 \\
340 \\
210 \\
410\end{array}$ & $\begin{array}{r}- \\
160 \\
270 \\
\neq 160 \\
\neq 270\end{array}$ & $\begin{array}{r}150 \\
210 \\
\neq 150 \\
\neq 210\end{array}$ & $\begin{array}{r}160 \\
160 \\
\neq 160 \\
\neq 160\end{array}$ & $\begin{array}{r}- \\
320 \\
220 \\
350 \\
\neq 310\end{array}$ & $\begin{array}{r}- \\
4,300 \\
330 \\
1,800 \\
1,000\end{array}$ & $\begin{array}{r}- \\
21,000 \\
10,300 \\
3,200 \\
14,000\end{array}$ & $\begin{array}{r}18,500 \\
14,000 \\
4,000 \\
18,500\end{array}$ & $\begin{array}{r}- \\
4,100 \\
690 \\
520 \\
2,200\end{array}$ & $\begin{array}{l}340 \\
800 \\
370 \\
330 \\
480\end{array}$ & $\begin{array}{l}180 \\
270 \\
120 \\
220 \\
160\end{array}$ & $\begin{array}{l}\overline{50}, 370 \\
27,360 \\
11,290 \\
38,220\end{array}$ \\
\hline $\begin{array}{l}1936 \\
1937 \\
1938 \\
1939 \\
1940\end{array}$ & $\begin{array}{l}250 \\
480 \\
700 \\
700 \\
500\end{array}$ & $\begin{array}{l}330 \\
630 \\
700 \\
620 \\
320\end{array}$ & $\begin{array}{r}270 \\
\$ 390 \\
650 \\
540 \\
270\end{array}$ & $\begin{array}{r}\$ 210 \\
\neq 260 \\
490 \\
540 \\
250\end{array}$ & $\begin{array}{r}\neq 160 \\
\neq 160 \\
440 \\
400 \\
260\end{array}$ & $\begin{array}{r}\$ 350 \\
\$ 300 \\
550 \\
6 \ni 0 \\
350\end{array}$ & & $\begin{array}{l}13,000 \\
16,000 \\
17,000 \\
11,000 \\
12,000\end{array}$ & $\begin{array}{r}8,000 \\
15,300 \\
16,800 \\
8,000 \\
8,800\end{array}$ & $\begin{array}{r}1,000 \\
3,700 \\
2,400 \\
710 \\
880\end{array}$ & $\begin{array}{l}250 \\
700 \\
600 \\
410 \\
270\end{array}$ & $\begin{array}{l}170 \\
240 \\
490 \\
400 \\
280\end{array}$ & $\begin{array}{l}26,590 \\
39,360 \\
44,820 \\
26,410 \\
26,480\end{array}$ \\
\hline $\begin{array}{l}1941 \\
1942 \\
1943 \\
1944 \\
1945\end{array}$ & $\begin{array}{r}690 \\
2,100 \\
480 \\
600 \\
540\end{array}$ & $\begin{array}{r}560 \\
1,000 \\
570 \\
420 \\
450\end{array}$ & $\begin{array}{l}340 \\
560 \\
450 \\
340 \\
330\end{array}$ & & $\begin{array}{l}250 \\
350 \\
300 \\
290 \\
250\end{array}$ & $\begin{array}{l}440 \\
390 \\
390 \\
290 \\
340\end{array}$ & $\begin{array}{r}1,100 \\
4,100 \\
3,400 \\
700 \\
780\end{array}$ & $\begin{array}{r}15,800 \\
14,900 \\
9,100 \\
14,000 \\
18,200\end{array}$ & $\begin{array}{l}12,900 \\
15,100 \\
14,900 \\
16,100 \\
21,000\end{array}$ & & $\begin{array}{r}800 \\
390 \\
520 \\
400 \\
1,400\end{array}$ & $\begin{array}{l}420 \\
210 \\
180 \\
280 \\
500\end{array}$ & $\begin{array}{l}35,170 \\
41,630 \\
32,290 \\
36,110 \\
50,330\end{array}$ \\
\hline $\begin{array}{l}1946 \\
1947 \\
1948 \\
1949 \\
1950\end{array}$ & $\begin{array}{r}800 \\
910 \\
870 \\
690 \\
1,200\end{array}$ & & $\begin{array}{l}590 \\
600 \\
710 \\
490 \\
500\end{array}$ & $\begin{array}{l}450 \\
450 \\
590\end{array}$ & $\begin{array}{l}350 \\
380 \\
340 \\
290 \\
370\end{array}$ & $\begin{array}{l}560 \\
780 \\
410 \\
380 \\
360\end{array}$ & $\begin{array}{l}5,700 \\
1,800 \\
2,400 \\
3,200 \\
3,200\end{array}$ & $\begin{array}{r}9,700 \\
18,000 \\
15,000 \\
19,000 \\
16,000\end{array}$ & $\begin{array}{r}10,000 \\
16,000 \\
9,800 \\
19,000 \\
18,000\end{array}$ & & $\begin{array}{l}610 \\
790 \\
430 \\
560 \\
420\end{array}$ & $\begin{array}{l}350 \\
400 \\
150 \\
330 \\
410\end{array}$ & $\begin{array}{l}220 \\
520 \\
700 \\
320 \\
750\end{array}$ \\
\hline $\begin{array}{l}1951 \\
1952 \\
1953 \\
1954 \\
1955\end{array}$ & $\begin{array}{l}340 \\
720\end{array}$ & $\begin{array}{l}370 \\
440 \\
440 \\
600\end{array}$ & $\begin{array}{l}420 \\
430 \\
410 \\
280 \\
430\end{array}$ & $\begin{array}{l}350 \\
500 \\
400 \\
270 \\
370\end{array}$ & $\begin{array}{l}280 \\
350 \\
310 \\
180 \\
240\end{array}$ & $\begin{array}{l}440 \\
340 \\
410 \\
250 \\
240\end{array}$ & $\begin{array}{r}1,300 \\
3,900 \\
800 \\
2,600 \\
1,600\end{array}$ & $\begin{array}{r}11,400 \\
19,800 \\
10,300 \\
9,400 \\
12,000\end{array}$ & $\begin{array}{r}11,000 \\
19,500 \\
15,500 \\
6,300 \\
11,500\end{array}$ & $\begin{array}{l}1,700 \\
2,300 \\
1,700 \\
1,200 \\
1,300\end{array}$ & $\begin{array}{l}760 \\
800 \\
720 \\
400 \\
510\end{array}$ & $\begin{array}{l}210 \\
340 \\
120 \\
300 \\
170\end{array}$ & $\begin{array}{l}28,980 \\
49,280 \\
31,560 \\
21,960 \\
29,680\end{array}$ \\
\hline 1956 & 420 & 620 & 660 & 550 & 290 & 440 & - & - & - & - & - & - & 34,340 \\
\hline $\begin{array}{l}1964 \\
1965 \\
\end{array}$ & $\begin{array}{l}320 \\
250 \\
\end{array}$ & $\begin{array}{l}450 \\
410 \\
\end{array}$ & $\begin{array}{l}260 \\
400 \\
\end{array}$ & $\begin{array}{l}270 \\
350\end{array}$ & $\begin{array}{l}200 \\
160\end{array}$ & $\begin{array}{l}200 \\
170\end{array}$ & $\begin{array}{r}800 \\
1,300\end{array}$ & $\begin{array}{l}14,000 \\
14,800\end{array}$ & $\begin{array}{l}13,000 \\
18,100\end{array}$ & $\begin{array}{l}2,500 \\
3,500\end{array}$ & $\begin{array}{l}540 \\
790 \\
\end{array}$ & $\begin{array}{l}230 \\
720\end{array}$ & $\begin{array}{l}32,770 \\
40,950\end{array}$ \\
\hline
\end{tabular}

* Based on estimates made for 1950 Compilation. 

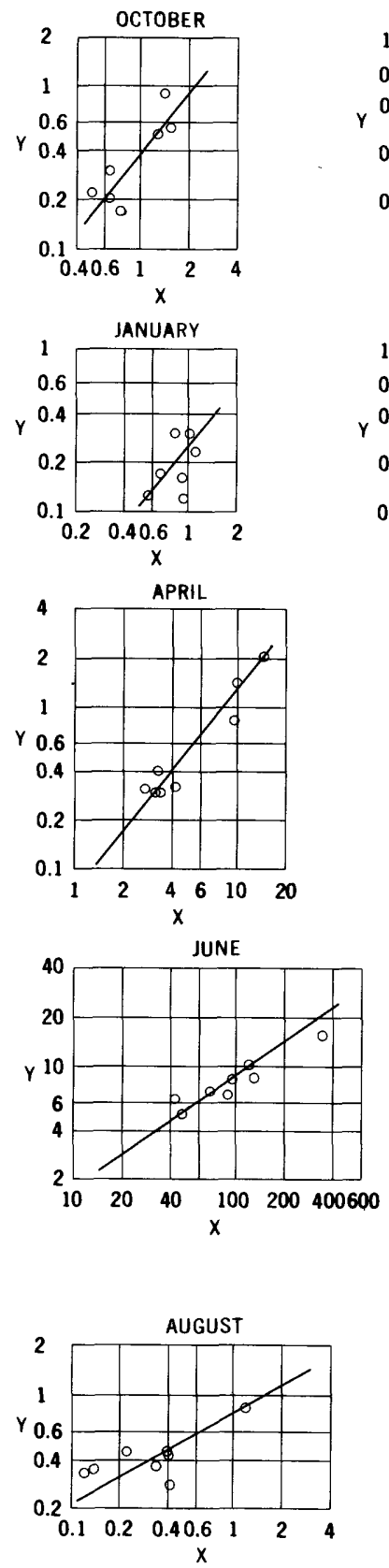
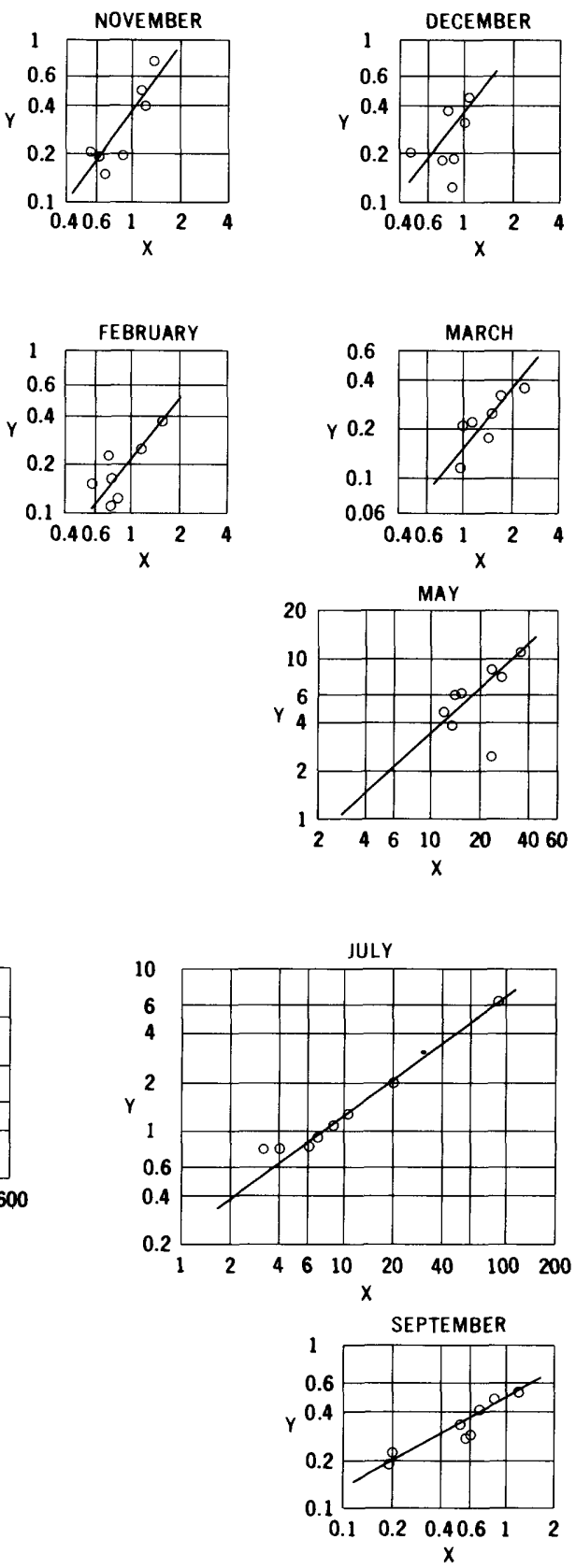

Relationships of monthly mean discharge of Battle Creek near Encampment, Wyo. (Y), to monthly mean discharge of Slater Fork near Slater, Colo. (X). Discharge in thousands of acre-feet. 
2534. Battle Creek near Encampment, wyo.

Location. --Lat $41^{\circ} 08^{\prime} 00^{\prime \prime}$, long $107^{\circ} 03^{\prime} 5^{\prime \prime}$, in NE $\frac{1}{4}$ sec.4, T.13 N., R.86 W., on right bank at sheep bridge, 1 mile downstream from Haskins Creek, 8 miles upstream from Haggerty Creek, and 15 miles west of Encampment. Alt1tude of gage is $8,375 \mathrm{ft}$ (from topographic map).

Drainage area. $--12.8 \mathrm{sq} \mathrm{mi}$.

Records available. --April 1956 to September 1963.

Estimates of streamflow.--August 1931 to March 1956, October 1963 to September 1965, based on relationships of monthly mean discharge with Slater Fork near Slater, Colo. The regression equation used is:

$\log \mathrm{Y}=\mathrm{b} \log \mathrm{X}-\mathrm{c}$

(where $Y$ is discharge of Battle Creek near Encampment, Wyo., and $X$ is discharge of Slater Fork near Slater, Colo., both in acre-feet per month\}.

\begin{tabular}{l|l|l|l|l|l|l|l|l|l|l|l|l}
\multicolumn{10}{c}{ Monthly values of constants 1n above equat1on } \\
\hline & Oct. & Nov. & Dec. & Jan. & Feb. & Mar. & Apr. & May & June & July & Aug. & Sept. \\
\hline b & 1.27 & 1.36 & 1.26 & 1.26 & 1.26 & 1.26 & 1.27 & 0.93 & 0.69 & 0.73 & 0.56 & 0.56 \\
c & 1.22 & 1.52 & 1.23 & 1.33 & 1.48 & 1.64 & 1.97 & .18 & -.49 & -.90 & -1.21 & -1.00 \\
\hline
\end{tabular}

Average discharge. --34 years (1931-65), 22,450 acre-feet per year (31.0 cfs).

Extremes.--1956-63: Maximum discharge, 670 cf s May 29, 1958, from rating curve extended above $320 \mathrm{cf}$ s; minimum daily, $1.7 \mathrm{cf}$ s Nov. 15, 1958, but may have been less during
periods of 1ce effect.

Remarks.--No diversion above station. Estimates of annual flow are within about 15 percent of regression line.

Monthly and annual streamflow, in acre-feet

\begin{tabular}{|c|c|c|c|c|c|c|c|c|c|c|c|c|c|}
\hline $\begin{array}{l}\text { Water } \\
\text { year }\end{array}$ & oct. & Nov. & Dec. & Jan. & Feb. & Mar. & Apr. & May & June & July & Aug. & Sept. & Annual \\
\hline $\begin{array}{l}1956 \\
1957 \\
1958 \\
1959 \\
1960\end{array}$ & $\begin{array}{l}- \\
202 \\
510 \\
174 \\
578\end{array}$ & $\begin{array}{l}- \\
190 \\
385 \\
152 \\
486\end{array}$ & $\begin{array}{l}- \\
184 \\
307 \\
129 \\
369\end{array}$ & $\begin{array}{l}- \\
166 \\
307 \\
123 \\
307\end{array}$ & $\begin{array}{l}- \\
167 \\
250 \\
111 \\
230\end{array}$ & $\begin{array}{l}- \\
215 \\
246 \\
111 \\
358\end{array}$ & $\begin{array}{r}821 \\
298 \\
327 \\
402 \\
1,420\end{array}$ & $\begin{array}{r}8,740 \\
2,580 \\
11,280 \\
3,980 \\
6,010\end{array}$ & $\begin{array}{r}6,920 \\
15,840 \\
10,060 \\
7,040 \\
8,410\end{array}$ & $\begin{array}{r}824 \\
6,230 \\
932 \\
1,260 \\
1,090\end{array}$ & $\begin{array}{l}373 \\
849 \\
368 \\
452 \\
337\end{array}$ & $\begin{array}{l}223 \\
488 \\
282 \\
415 \\
193\end{array}$ & $\begin{array}{r}- \\
27,410 \\
25,250 \\
14,350 \\
19,790\end{array}$ \\
\hline $\begin{array}{l}1961 \\
1962 \\
1963\end{array}$ & $\begin{array}{l}221 \\
902 \\
307\end{array}$ & $\begin{array}{l}196 \\
750 \\
204\end{array}$ & $\begin{array}{l}184 \\
449 \\
206\end{array}$ & $\begin{array}{l}172 \\
235 \\
135\end{array}$ & $\begin{array}{l}150 \\
385 \\
123\end{array}$ & $\begin{array}{l}206 \\
320 \\
177\end{array}$ & $\begin{array}{r}310 \\
2,060 \\
308\end{array}$ & $\begin{array}{l}4,760 \\
7,750 \\
6,060\end{array}$ & $\begin{array}{l}6,340 \\
8,690 \\
5,000\end{array}$ & $\begin{array}{r}807 \\
1,990 \\
778\end{array}$ & $\begin{array}{l}286 \\
463 \\
441\end{array}$ & $\begin{array}{l}538 \\
339 \\
291\end{array}$ & $\begin{array}{l}14,170 \\
24,330 \\
14,030\end{array}$ \\
\hline
\end{tabular}

\begin{tabular}{|c|c|c|c|c|c|c|c|c|c|c|c|c|c|}
\hline $\begin{array}{l}\text { Water } \\
\text { year }\end{array}$ & oct. & Nov. & Dec. & Jan. & Feb. & Mar. & Apr. & May & June & July & Aug. & Sept. & Annual \\
\hline $\begin{array}{l}1931 \\
1932 \\
1933 \\
1934 \\
1935\end{array}$ & $\begin{array}{l}- \\
260 \\
230 \\
140 \\
340\end{array}$ & $\begin{array}{l}- \\
130 \\
200 \\
120 \\
260\end{array}$ & $\begin{array}{l}\overline{130} \\
210 \\
\$ 130 \\
\$ 220\end{array}$ & $\begin{array}{r}\overline{110} \\
150 \\
\neq 110 \\
\neq 150\end{array}$ & $\begin{array}{r}\overrightarrow{120} \\
120 \\
\neq 120 \\
\neq 120\end{array}$ & $\begin{array}{l}\overrightarrow{190} \\
130 \\
210 \\
\neq 180\end{array}$ & $\begin{array}{r}- \\
1,500 \\
120 \\
630 \\
360\end{array}$ & $\begin{array}{r}- \\
12,000 \\
4,800 \\
1,100 \\
7,200\end{array}$ & $\begin{array}{c}- \\
15,200 \\
11,000 \\
2,400 \\
15,200\end{array}$ & $\begin{array}{r}- \\
3,500 \\
510 \\
370 \\
1,800\end{array}$ & $\begin{array}{l}320 \\
730 \\
350 \\
320 \\
450\end{array}$ & $\begin{array}{l}280 \\
340 \\
220 \\
300 \\
260\end{array}$ & $\begin{array}{r}34,210 \\
18,040 \\
5,950 \\
26,540\end{array}$ \\
\hline $\begin{array}{l}1936 \\
1937 \\
1938 \\
1939 \\
1940\end{array}$ & $\begin{array}{l}170 \\
310 \\
440 \\
440 \\
320\end{array}$ & $\begin{array}{l}200 \\
410 \\
450 \\
410 \\
200\end{array}$ & $\begin{array}{r}220 \\
\$ 320 \\
520 \\
440 \\
220\end{array}$ & $\begin{array}{r}\$ 150 \\
\$ 180 \\
310 \\
350 \\
180\end{array}$ & $\begin{array}{r}\neq 120 \\
\neq 120 \\
290 \\
270 \\
180\end{array}$ & $\begin{array}{r}\$ 210 \\
\$ 180 \\
330 \\
420 \\
210\end{array}$ & $\begin{array}{r}920 \\
440 \\
1,400 \\
860 \\
820\end{array}$ & $\begin{array}{l}6,500 \\
8,600 \\
9,500 \\
5,200 \\
5,900\end{array}$ & $\begin{array}{r}5,600 \\
12,200 \\
13,500 \\
5,700 \\
6,300\end{array}$ & $\begin{array}{r}770 \\
3,200 \\
1,900 \\
530 \\
660\end{array}$ & $\begin{array}{l}240 \\
640 \\
560 \\
390 \\
260\end{array}$ & $\begin{array}{l}260 \\
310 \\
450 \\
410 \\
340\end{array}$ & $\begin{array}{l}15,360 \\
26,910 \\
29,650 \\
15,420 \\
15,590\end{array}$ \\
\hline $\begin{array}{l}1941 \\
1942 \\
1943 \\
1944 \\
1945\end{array}$ & $\begin{array}{r}430 \\
1,200 \\
310 \\
390 \\
350\end{array}$ & $\begin{array}{l}360 \\
710 \\
360 \\
260 \\
290\end{array}$ & $\begin{array}{l}280 \\
450 \\
360 \\
280 \\
260\end{array}$ & $\begin{array}{l}190 \\
340 \\
210 \\
200 \\
230\end{array}$ & $\begin{array}{l}180 \\
230 \\
200 \\
200 \\
180\end{array}$ & $\begin{array}{l}260 \\
230 \\
230 \\
180 \\
200\end{array}$ & $\begin{array}{r}400 \\
1,400 \\
1,200 \\
250 \\
280\end{array}$ & $\begin{array}{r}8,200 \\
7,600 \\
4,100 \\
7,000 \\
10,100\end{array}$ & $\begin{array}{r}9,700 \\
12,100 \\
11,700 \\
13,200 \\
17,700\end{array}$ & $\begin{array}{l}1,300 \\
1,600 \\
1,300 \\
2,000 \\
5,600\end{array}$ & $\begin{array}{r}720 \\
360 \\
480 \\
380 \\
1,200\end{array}$ & $\begin{array}{l}420 \\
300 \\
270 \\
340 \\
460\end{array}$ & $\begin{array}{l}22,440 \\
26,520 \\
20,720 \\
24,680 \\
36,850\end{array}$ \\
\hline $\begin{array}{l}1946 \\
1947 \\
1948 \\
1949 \\
1950\end{array}$ & $\begin{array}{l}500 \\
560 \\
530 \\
430 \\
700\end{array}$ & $\begin{array}{l}610 \\
530 \\
460 \\
400 \\
430\end{array}$ & $\begin{array}{l}470 \\
490 \\
570 \\
390 \\
400\end{array}$ & $\begin{array}{l}290 \\
290 \\
360 \\
290 \\
330\end{array}$ & $\begin{array}{l}230 \\
250 \\
230 \\
200 \\
250\end{array}$ & $\begin{array}{l}340 \\
470 \\
250 \\
230 \\
210\end{array}$ & $\begin{array}{r}2,000 \\
640 \\
860 \\
1,100 \\
1,100\end{array}$ & $\begin{array}{r}4,400 \\
9,800 \\
7,800 \\
10,700 \\
8,600\end{array}$ & $\begin{array}{r}7,300 \\
12,800 \\
7,100 \\
15,600 \\
14,800\end{array}$ & $\begin{array}{r}930 \\
3,100 \\
1,000 \\
2,800 \\
2,600\end{array}$ & $\begin{array}{l}560 \\
720 \\
410 \\
520 \\
400\end{array}$ & $\begin{array}{l}390 \\
420 \\
250 \\
370 \\
420\end{array}$ & $\begin{array}{l}18,020 \\
30,070 \\
19,820 \\
33,030 \\
30,240\end{array}$ \\
\hline $\begin{array}{l}1951 \\
1952 \\
1953 \\
1954 \\
1955\end{array}$ & $\begin{array}{l}370 \\
420 \\
300 \\
230 \\
460\end{array}$ & $\begin{array}{l}350 \\
230 \\
270 \\
280 \\
380\end{array}$ & $\begin{array}{l}340 \\
350 \\
330 \\
230 \\
350\end{array}$ & $\begin{array}{l}240 \\
320 \\
260 \\
190 \\
250\end{array}$ & $\begin{array}{l}190 \\
230 \\
210 \\
130 \\
170\end{array}$ & $\begin{array}{l}260 \\
210 \\
240 \\
150 \\
150\end{array}$ & $\begin{array}{r}480 \\
1,300 \\
280 \\
920 \\
600\end{array}$ & $\begin{array}{r}5,500 \\
17,100 \\
4,800 \\
4,300 \\
5,800\end{array}$ & $\begin{array}{r}8,300 \\
16,200 \\
12,400 \\
4,200 \\
8,700\end{array}$ & $\begin{array}{r}1,300 \\
1,900 \\
1,300 \\
950 \\
990\end{array}$ & $\begin{array}{l}700 \\
720 \\
660 \\
380 \\
480\end{array}$ & $\begin{array}{l}290 \\
390 \\
220 \\
350 \\
260\end{array}$ & $\begin{array}{l}18,320 \\
33,360 \\
21,270 \\
12,310 \\
18,590\end{array}$ \\
\hline 1956 & 270 & 390 & 540 & 350 & 200 & 270 & - & - & - & - & - & - & 19,920 \\
\hline $\begin{array}{l}1964 \\
1965\end{array}$ & $\begin{array}{l}210 \\
180\end{array}$ & $\begin{array}{l}280 \\
250\end{array}$ & $\begin{array}{l}210 \\
320\end{array}$ & $\begin{array}{l}190 \\
230\end{array}$ & $\begin{array}{l}140 \\
110\end{array}$ & $\begin{array}{l}120 \\
100\end{array}$ & $\begin{array}{l}290 \\
470\end{array}$ & $\begin{array}{l}7,100 \\
7,700\end{array}$ & $\begin{array}{l}10,100 \\
14,900\end{array}$ & $\begin{array}{l}2,000 \\
3,000\end{array}$ & $\begin{array}{l}500 \\
720\end{array}$ & $\begin{array}{l}310 \\
550\end{array}$ & $\begin{array}{l}21,450 \\
28,530\end{array}$ \\
\hline
\end{tabular}

* Based on estimates made for 1950 Compllation. 

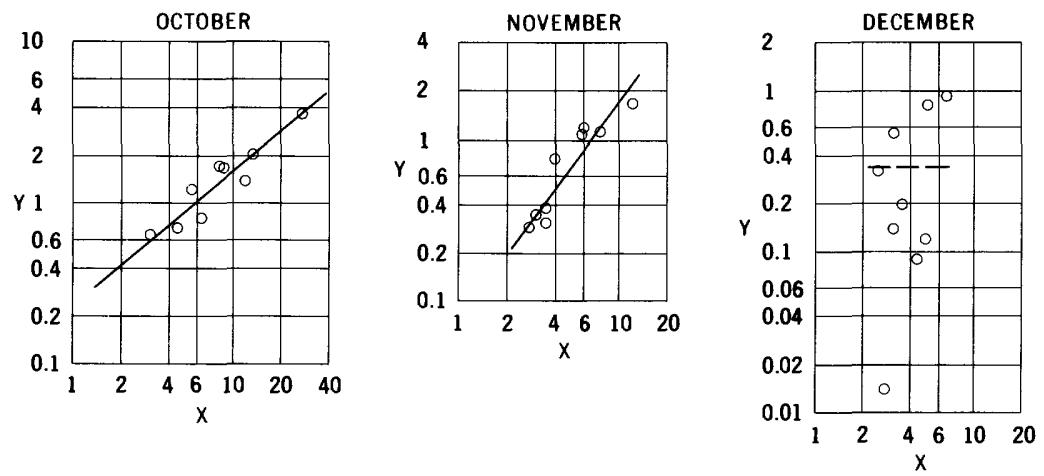

January to March-No flow most years
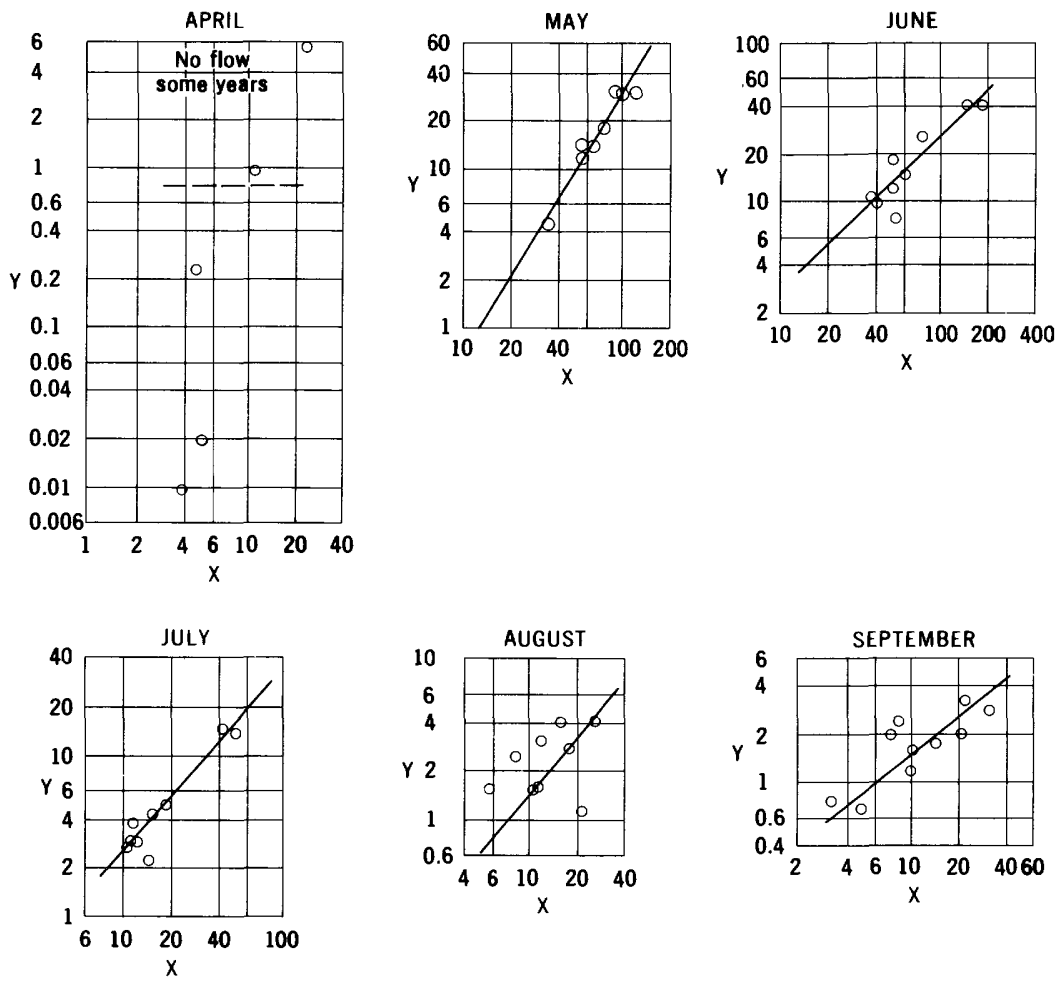

Relationships of monthly mean discharge of East Fork of Dry Fork, near Dry Fork, Utah $(Y)$, to sum of monthly mean discharges of Dry Fork above sinks, near Dry Fork, Utah, and Mosby Canal (X). Discharge in hundreds of acrefeet. 
2690. East Fork of Dry Fork, near Dry Fork, Utah

Location.--Lat 40 39100", long 104.45'40", in sec.14, T.2 S., R.19 E. (unsurveyed), on right bank $3 \frac{1}{2}$ miles upstream from mouth and 8 miles northwest of town of Dry Fork. Altitude of gage is $8,150 \mathrm{ft}$ (by barometer).

Drainage area. $--12 \mathrm{sq} \mathrm{mi}$, approximately.

Records available. --April 1946 to September 1963.

Estimates of streamflow.--May 1939 to September 1942, October 1963 to September 1965 ,

based on relationships of monthly mean discharge with Dry Fork above sinks, near Dry Fork, Utah, adjusted for diversion through Mosby Canal. The regression equation used is :

$$
\log Y=b \log X-c
$$

(where $Y$ is discharge of East Fork of Dry Fork, near Dry Fork, Utah, and $X$ is the sum of discharges of Dry Fork above sinks, near Dry Fork, Utah and Mosby Canal near Lapoint, Utah, all in acre-feet per month).

\begin{tabular}{c|c|c|c|c|c|c|c|c|c|c|c|c}
\hline \multicolumn{10}{c}{ Monthly values of constants 1n above equation } \\
\hline & oct. & Nov. & Dec. & Jan. & Feb. & Mar. & Apr. & May & June & July & Aug. & Sept. \\
\hline b & 0.83 & 1.34 & - & - & - & - & - & 1.63 & 0.97 & 1.13 & 1.18 & 0.78 \\
$\mathrm{c}$ & .29 & 1.79 & - & - & - & - & - & 3.06 & .47 & 1.00 & 1.38 & .17 \\
\hline
\end{tabular}

Average discharge. - -22 years (1939-42, 1946-65), 6,225 acre-feet per year (8.60 cfs).

Extremes.--1946-63: Maximum discharge, 240 cf's June 18, 1949, from rating curve extended above $100 \mathrm{cf}$ 's on basis of slope-area measurement of peak flow; no flow at times.

Remarks.--No diversion above station. Estimates of annual flow are within about 15 percent of regression line.

\begin{tabular}{l|c|r|r|r|r|r|r|r|r|r|r|r|r}
\hline $\begin{array}{l}\text { Water } \\
\text { year }\end{array}$ & Oct. & Nov. & Dec. & Jan. & Feb. & Mar. & Apr. & May & June & July & Aug. & Sept. & Annual \\
\hline 1946 & - & - & - & - & - & - & - & 783 & 483 & 197 & 254 & 391 & \\
1947 & 230 & 176 & 150 & 123 & 117 & 140 & 282 & 5,140 & 3,570 & 1,250 & 412 & 261 & 11,840 \\
1948 & 103 & 52 & 39 & 37 & 29 & 31 & 35 & 2,310 & 1,570 & 261 & 84 & 91 & 4,640 \\
1949 & 89 & 44 & 25 & 18 & 11 & 15 & 69 & 1,800 & 3,630 & 834 & 202 & 131 & 6,870 \\
1950 & 141 & 129 & 47 & 37 & 28 & 31 & 186 & 2,340 & 4,320 & 1,170 & 135 & 70 & 8,680 \\
1951 & 38 & 5.4 & 0 & 0 & 0 & 0 & 0 & 1,280 & 2,190 & 609 & 283 & 203 & 4,610 \\
1952 & 136 & 12 & 0 & 0 & 0 & 0 & 14 & 2,840 & 3,810 & 979 & 554 & 197 & 8,540 \\
1953 & 31 & 9.9 & 0 & 0 & 0 & 0 & 0 & 161 & 2,260 & 374 & 205 & 116 & 3,160 \\
1954 & 111 & 52 & 0 & 0 & 0 & 0 & 3.2 & 2,120 & 010 & 624 & 249 & 156 & 4,130 \\
1955 & 167 & 109 & 8.9 & 0 & 0 & 0 & 1.0 & 1,390 & 1,070 & 285 & 310 & 236 & 3,580 \\
1956 & 122 & 37.9 & 20.0 & 0 & 0 & 0 & 23.4 & 3,000 & 1,850 & 379 & 156 & 74.4 & 5,660 \\
1957 & 64 & 35 & 1.4 & 0 & 0 & 0 & 0 & 111 & 4,090 & 1,350 & 407 & 320 & 6,380 \\
1958 & 202 & 122 & 81 & 24 & 0 & 0 & 2.0 & 2,980 & 2,580 & 493 & 155 & 115 & 6,750 \\
1959 & 70 & 29 & 32 & 0 & 0 & 0 & 0 & 447 & 799 & 431 & 279 & 173 & 2,260 \\
1960 & 138 & 114 & 12 & 0 & 0 & 0 & 97 & 1,420 & 1,210 & 271 & 66 & 67 & 3,400 \\
1961 & 80 & 31 & 14 & 2.0 & 0 & 0 & 0 & 1,160 & 1,480 & 223 & 113 & 275 & 5,380 \\
1962 & 359 & 169 & 92 & 41 & 28 & 30 & 581 & 3,040 & 4,060 & 1,470 & 405 & 195 & 10,470 \\
1963 & 170 & 77 & 55 & 7.0 & 0 & 0 & 0 & 1,010 & 984 & 293 & 160 & 198 & 3,750 \\
\hline
\end{tabular}

Estimated monthly and annual streamflow, in acre-feet

\begin{tabular}{|c|c|c|c|c|c|c|c|c|c|c|c|c|c|}
\hline $\begin{array}{l}\text { Water } \\
\text { year }\end{array}$ & oct. & Nov. & Dec. & Jan. & Feb. & Mar. & Apr. & May & June & $\mathrm{JuIy}$ & Aug. & Sept. & Annual \\
\hline $\begin{array}{l}1939 \\
1940\end{array}$ & 300 & 170 & $\bar{e} 4$ & eit & $\bar{e} \bar{z}$ & ej5 & $\bar{e} \overline{6}$ & $\begin{array}{l}2,300 \\
1,700\end{array}$ & $\begin{array}{l}670 \\
410\end{array}$ & $\begin{array}{l}300 \\
180\end{array}$ & $\begin{array}{r}120 \\
80\end{array}$ & $\begin{array}{l}440 \\
240\end{array}$ & $3, \overline{234}$ \\
\hline $\begin{array}{l}1941 \\
1942\end{array}$ & $\begin{array}{l}420 \\
400\end{array}$ & $\begin{array}{l}370 \\
340\end{array}$ & $\begin{array}{l}\text { e34 } \\
\text { e34 }\end{array}$ & $\begin{array}{l}\text { el7 } \\
\text { e17 }\end{array}$ & $\begin{array}{l}\text { elz } \\
\text { elz }\end{array}$ & $\begin{array}{l}\text { el5 } \\
\text { el5 }\end{array}$ & $\begin{array}{l}\text { e } 76 \\
\text { e } 76\end{array}$ & $\begin{array}{l}5,600 \\
1,800\end{array}$ & $\begin{array}{l}3,700 \\
3,700\end{array}$ & $\begin{array}{l}1,100 \\
1,000\end{array}$ & $\begin{array}{l}380 \\
210\end{array}$ & $\begin{array}{l}250 \\
140\end{array}$ & $\begin{array}{r}11,974 \\
7,744\end{array}$ \\
\hline $\begin{array}{l}1964 \\
1965\end{array}$ & $\begin{array}{r}190 \\
80\end{array}$ & $\begin{array}{l}90 \\
40\end{array}$ & $\begin{array}{l}\text { e34 } \\
\text { e34 }\end{array}$ & $\begin{array}{l}\text { e17 } \\
\text { el7 }\end{array}$ & $\begin{array}{l}e 12 \\
\text { el? }\end{array}$ & $\begin{array}{l}\text { e15 } \\
\text { el5 }\end{array}$ & $\begin{array}{l}\text { e76 } \\
\text { e76 }\end{array}$ & $\begin{array}{r}2,400 \\
500\end{array}$ & $\begin{array}{l}2,300 \\
5,000\end{array}$ & $\begin{array}{r}860 \\
2,800\end{array}$ & $\begin{array}{l}240 \\
640\end{array}$ & $\begin{array}{l}110 \\
330\end{array}$ & $\begin{array}{l}6,344 \\
9,544\end{array}$ \\
\hline
\end{tabular}

e Flat estimate based on average discharge for month. 

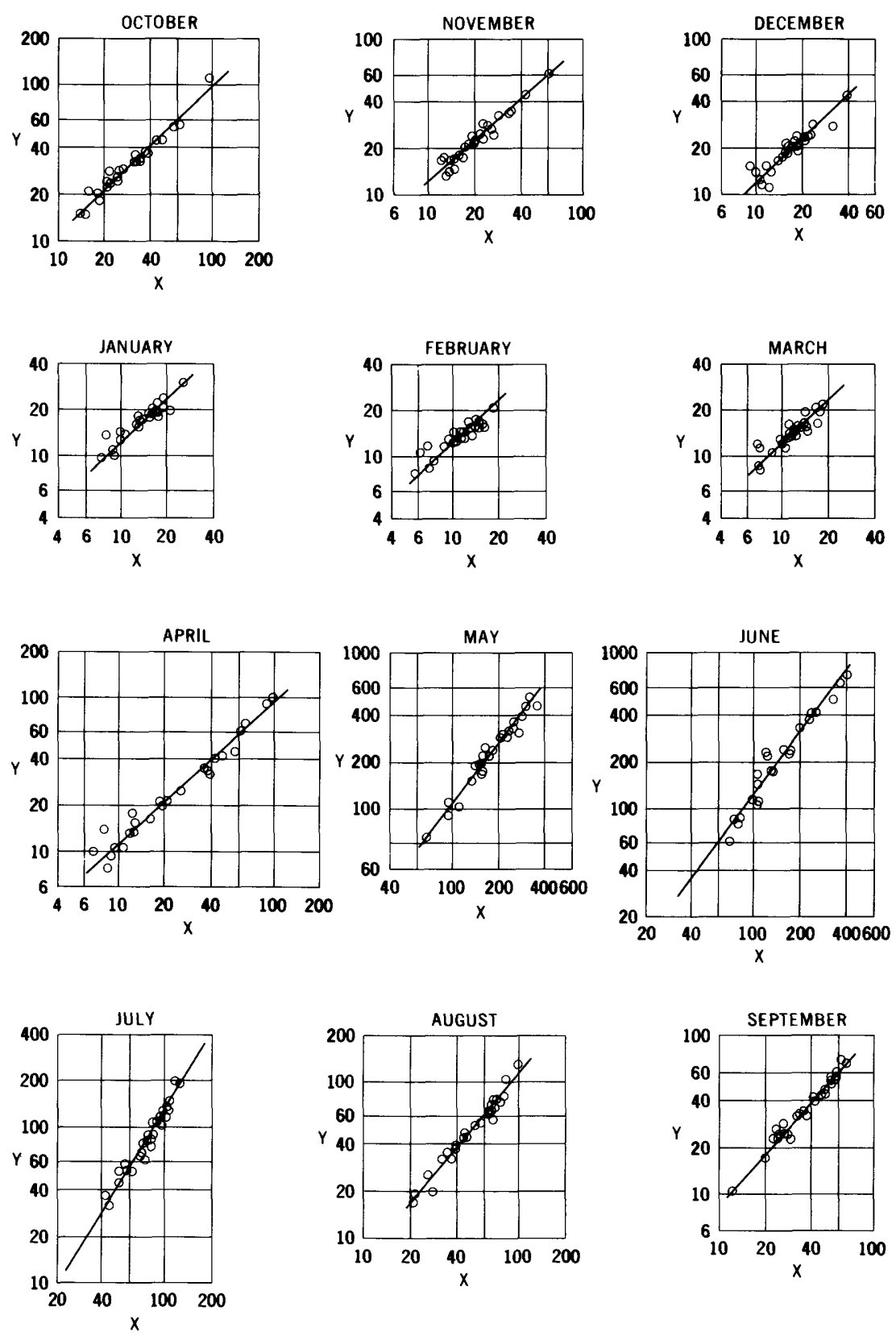

Relationships of monthly mean discharge of Ashley Creek at Sign of the Maine, near Vernal, Utah ( $Y$ ), to monthly mean discharge of Ashley Creek near Vernal, Utah $(X)$. Discharge in hundreds of acre-feet. 
2710. Ashley Creek at Sign of the Maine, near Vernal, Utah

Location.--Lat $40^{\circ} 31_{100^{\prime \prime}}$, long 109.35'40", in NE $\frac{1}{4}$ sec.31, T.3 S., R.21 E., on left bank

three-quarters of a mile downstream from Dry Fork and $4 \frac{3}{4}$ miles northwest of Vernal.

Altitude of gage is $5,750 \mathrm{ft}$ (from topographic map).

Drainage area, $--241 \mathrm{sq} \mathrm{mi}$.

Records available.--March 1900 to December 1904 (published as "near Vernal"), June 1939 to September 1965.

Estimates of streamflow.--October 1930 to June 1939, based on relationships of monthly mean discharge with Ashley Creek near Vernal, Utah. The regression equation used is:

$$
\log Y=b \log X-c
$$

(where $Y$ is discharge of Ashley Creek at Sign of the Maine, near Vernal, Utah, and $X$ is discharge of Ashley Creek near Vernal, Utah, both in acre-feet per month).

\begin{tabular}{c|c|c|c|c|c|c|c|c|c|c|c|c}
\hline \multicolumn{10}{c}{ Monthly values of constants in above equation } \\
\hline & Oct. & Nov. & Dec. & Jan. & Feb. & Mar. & Apr. & May & June & JuIy & Aug. & Sept. \\
\hline $\mathrm{b}$ & 0.97 & 0.87 & 0.95 & 0.95 & 0.95 & 0.95 & 0.92 & 1.30 & 1.34 & 1.64 & 1.20 & 1.11 \\
$\mathrm{c}$ & -.12 & -.48 & -.22 & -.22 & -.25 & -.25 & -.30 & 1.18 & 1.26 & 2.43 & .74 & .40 \\
\hline
\end{tabular}

Average discharge. --35 years $(1930-65), 82,585$ acre-feet per year (115 cfs).

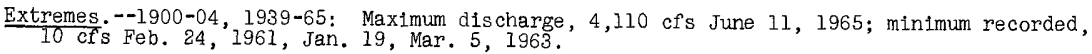

Remarks. - Flow increased since July 1940 by water released from Oaks Park Reservoir (capacity, 6,250 acre-feet) on Big Brush Creek and diverted to Ashley Creek bas in for irrigation. Diversions above station for irrigation and municipal supply. Estimates of annual flow are within about 10 percent of regression line.

\begin{tabular}{|c|c|c|c|c|c|c|c|c|c|c|c|c|c|}
\hline \multicolumn{14}{|c|}{ w, in a } \\
\hline ter & Oct. & Nov. & Dec. & Jan. & Feb. & Mar. & Apr. & May & June & July & Aug. & Sept. & Annua I \\
\hline $\begin{array}{l}939 \\
940\end{array}$ & $5, \overline{4} 20$ & 440 & 370 & $\overline{810}$ & $1, \overline{400}$ & $-\overline{460}$ & $4, \overline{280}$ & $21, \overline{150}$ & 6,120 & & & $\begin{array}{l}5,830 \\
2,370\end{array}$ & $5 \overline{5}, 2$ \\
\hline $\begin{array}{l}.941 \\
942 \\
.943 \\
.944 \\
.945\end{array}$ & $\begin{array}{l}4,470 \\
11,290 \\
3,250 \\
2,360 \\
2,840\end{array}$ & 300 & $\begin{array}{l}2,420 \\
4,370 \\
2,130 \\
1,780 \\
1,840\end{array}$ & $\begin{array}{l}, 030 \\
, 910 \\
810 \\
520 \\
690\end{array}$ & $\begin{array}{l}1,590 \\
2,090 \\
1,470 \\
1,250 \\
1,480\end{array}$ & & $\begin{array}{l}1,650 \\
6,060 \\
9,280 \\
1,790 \\
1,550\end{array}$ & $\begin{array}{l}45,170 \\
29,870 \\
24,740 \\
33,700 \\
19,200\end{array}$ & $\begin{array}{l}40,340 \\
41,060 \\
16,730 \\
65,400 \\
24,090\end{array}$ & & $\begin{array}{l}6,690 \\
5,880 \\
5,610 \\
8,270 \\
7,240\end{array}$ & $\begin{array}{l}7,050 \\
4,610 \\
3,220 \\
4,210 \\
5,250\end{array}$ & $\begin{array}{r}128,300 \\
127,800 \\
80,890 \\
142,300 \\
79,820\end{array}$ \\
\hline $\begin{array}{l}948 \\
949 \\
950\end{array}$ & $\begin{array}{l}3,770 \\
3,210 \\
4,330 \\
2,020 \\
3,590\end{array}$ & 480 & & $\begin{array}{l}1,680 \\
2,360 \\
1,870 \\
1,420 \\
1,980\end{array}$ & $\begin{array}{l}1,550 \\
1,180 \\
1,730\end{array}$ & & $\begin{array}{l}6,830 \\
3,430 \\
2,000 \\
2,490 \\
4,480\end{array}$ & $\begin{array}{l}10,630 \\
52,120 \\
32,510 \\
30,500 \\
36,010\end{array}$ & $\begin{array}{r}7,900 \\
34,930 \\
17,860 \\
41,070 \\
37,710\end{array}$ & $\begin{array}{r}4,440 \\
14,800 \\
6,940 \\
10,840 \\
13,690\end{array}$ & & $\begin{array}{l}3,240 \\
5,510 \\
2,330 \\
3,480 \\
4,330\end{array}$ & $\begin{array}{r}49,060 \\
134,000 \\
81,100 \\
104,600 \\
118,300\end{array}$ \\
\hline $\begin{array}{l}951 \\
952 \\
.953 \\
954 \\
955\end{array}$ & $\begin{array}{l}3,230 \\
3,630 \\
4,420 \\
1,820 \\
2,560\end{array}$ & 230 & & & $\begin{array}{l}1,380 \\
1,530 \\
1,780 \\
1,350 \\
1,260\end{array}$ & & & $\begin{array}{r}21,710 \\
46,570 \\
8,820 \\
23,250 \\
17,460\end{array}$ & & $\begin{array}{r}7,470 \\
11,460 \\
8,370 \\
6,810 \\
5,160\end{array}$ & $\begin{array}{r}6,450 \\
13,500 \\
5,300 \\
3,730 \\
3,980\end{array}$ & $\begin{array}{l}4,340 \\
6,130 \\
2,330 \\
2,480 \\
2,470\end{array}$ & $\begin{array}{r}75,840 \\
132,200 \\
65,120 \\
58,600 \\
52,430\end{array}$ \\
\hline $\begin{array}{l}1956 \\
1957 \\
1958 \\
1959 \\
1960\end{array}$ & $\begin{array}{l}2,400 \\
1,470 \\
3,340 \\
2,070 \\
2,230\end{array}$ & 1,800 & & & $\begin{array}{r}1,600 \\
1,200 \\
928\end{array}$ & & $\begin{array}{l}2,170 \\
1,060 \\
2,110 \\
1,080 \\
3,600\end{array}$ & $\begin{array}{l}31,630 \\
6,440 \\
37,910 \\
10,150 \\
14,810\end{array}$ & & & $\begin{array}{l}3,260 \\
7,560 \\
4,530 \\
3,670 \\
1,910\end{array}$ & $\begin{array}{l}1,720 \\
5,420 \\
2,710 \\
2,410 \\
1,060\end{array}$ & $\begin{array}{l}92,220 \\
42,810 \\
46,690\end{array}$ \\
\hline $\begin{array}{l}7961 \\
1962 \\
1963 \\
1964 \\
1965\end{array}$ & $\begin{array}{l}1,490 \\
6,540 \\
2,900 \\
2,790 \\
2,430\end{array}$ & $\begin{array}{l}1,410 \\
1,690 \\
1,700\end{array}$ & $\begin{array}{l}1,120 \\
2,780 \\
1,150 \\
1,420 \\
1,510\end{array}$ & $\begin{array}{r}988 \\
1,950 \\
966 \\
1,340 \\
1,350\end{array}$ & $\begin{array}{r}855 \\
1,650 \\
795 \\
1,190 \\
1,090\end{array}$ & $\begin{array}{r}829 \\
1,950 \\
871 \\
1,140 \\
1,210\end{array}$ & $\begin{array}{r}946 \\
10,370 \\
776 \\
1,000 \\
1,420\end{array}$ & $\begin{array}{l}16,290 \\
38,940 \\
19,850 \\
28,400 \\
18,400\end{array}$ & $\begin{array}{r}17,680 \\
40,110 \\
8,700 \\
23,540 \\
73,500\end{array}$ & $\begin{array}{r}6,610 \\
12,780 \\
5,160 \\
8,880 \\
19,540\end{array}$ & $\begin{array}{l}4,550 \\
6,800 \\
2,570 \\
4,910 \\
10,510\end{array}$ & $\begin{array}{l}5,630 \\
4,170 \\
2,910 \\
3,290 \\
7,260\end{array}$ & $\begin{array}{r}58,330 \\
132,500 \\
48,060 \\
79,590 \\
139,900\end{array}$ \\
\hline
\end{tabular}

Estimated monthly and annual streamflow, in acre-feet

\begin{tabular}{c|c|c|c|c|c|c|c|c|r|r|r|r|r}
\hline $\begin{array}{l}\text { Water } \\
\text { year }\end{array}$ & oct. & Nov. & Dec. & Jan. & Feb. & Mar. & Apr. & May & June & July & Aug. & Sept. & Annual \\
\hline 1931 & 5,600 & 3,600 & 3,200 & 2,800 & 2,300 & 2,400 & 2,800 & 9,500 & 5,600 & 1,700 & 2,000 & 1,700 & 43,200 \\
1932 & 2,100 & 2,100 & 2,100 & 2,000 & 2,000 & 2,000 & 2,200 & 33,500 & 28,300 & 10,000 & 5,600 & 4,300 & 96,200 \\
1933 & 3,800 & 2,600 & 2,000 & 1,800 & 1,600 & 1,600 & 1,500 & 9,600 & 22,800 & 3,800 & 3,300 & 2,000 & 56,400 \\
1934 & 1,900 & 1,700 & 1,500 & 1,500 & 1,400 & 1,500 & 3,900 & 7,600 & 3,100 & 1,300 & 2,700 & 2,300 & 20,400 \\
1935 & 2,200 & 2,200 & 2,000 & 1,800 & 1,700 & 1,900 & 1,900 & 13,000 & 48,000 & 6,700 & 3,800 & 2,200 & 87,400 \\
& & & & & & & & & & & & & \\
1936 & 2,000 & 1,900 & 1,600 & 1,400 & 1,300 & 1,300 & 2,300 & 12,400 & 7,400 & 3,300 & 4,600 & 4,000 & 43,500 \\
1937 & 3,400 & 2,700 & 1,900 & 1,600 & 1,200 & 1,400 & 2,400 & 46,200 & 23,200 & 10,600 & 5,800 & 3,800 & 104,200 \\
1938 & 3,100 & 2,300 & 1,900 & 1,600 & 1,500 & 1,600 & 2,600 & 26,300 & 42,000 & 11,000 & 5,600 & 4,800 & 104,300 \\
1939 & 6,800 & 4,800 & 3,700 & 2,800 & 2,300 & 2,500 & 7,500 & 22,000 & 8,100 & - & - & - & 71,510 \\
\hline
\end{tabular}



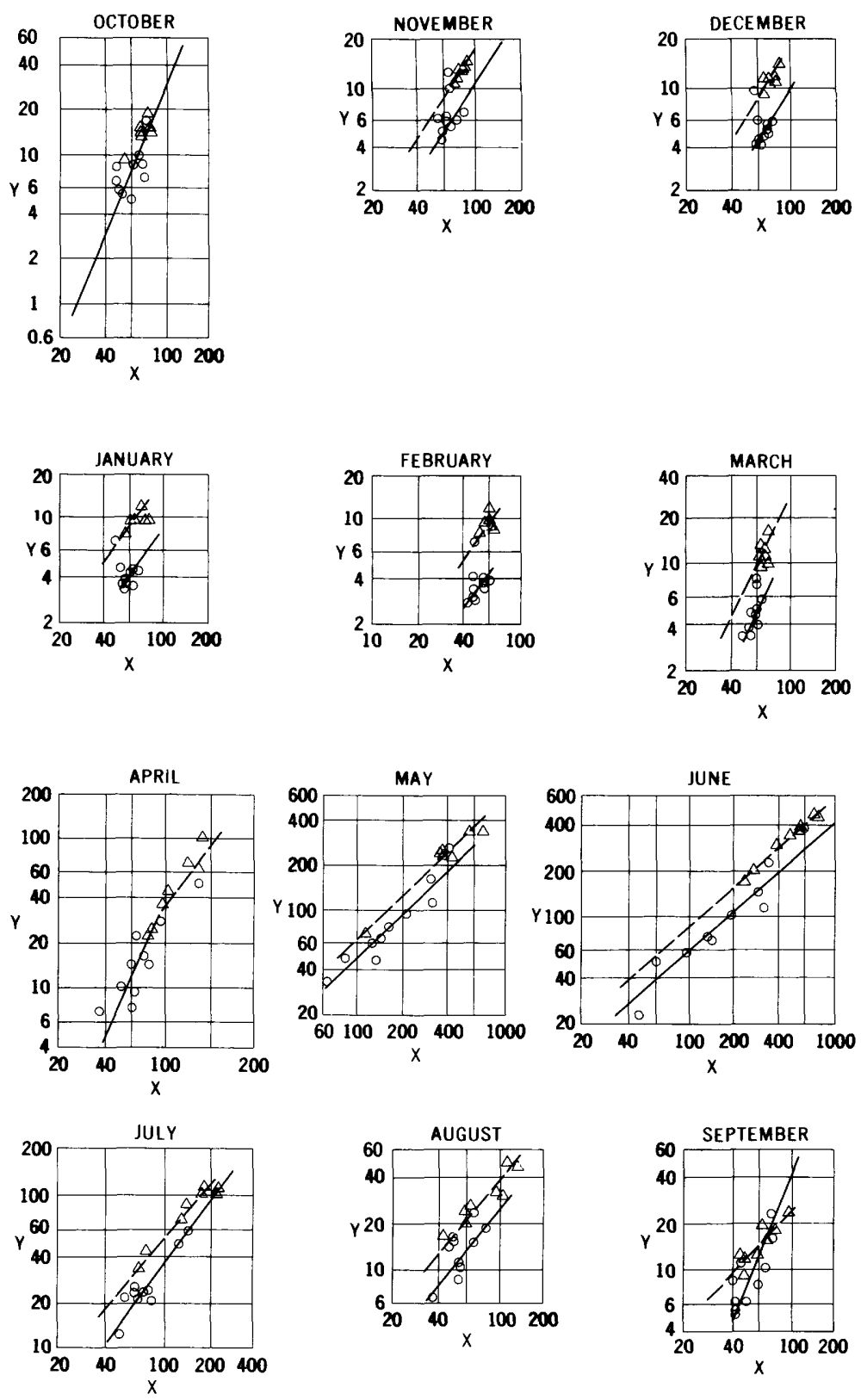

EXPLANATION

$-\longrightarrow-\triangle$ Prior to 1953

o After 1953

Relationships of monthly mean discharge of Duchesne River near Hanna, Utah $(Y)$, to monthly mean discharge of Duchesne River near Tabiona, Utah (X). Discharge in hundreds of acre-feet. 
2740. Duchesne River near Hanna, Utah

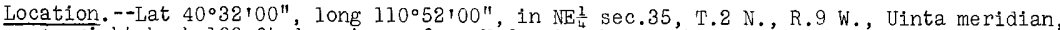
on right bank $100 \mathrm{ft}$ downstream from Hades Creek and il miles northwest of Hanna. Altitude of gage is $7,380 \mathrm{ft}$ (from river-profile map).

Drainage area. $--78 \mathrm{sq} \mathrm{mi}$, approximately.

Records available.--August 1921 to September 1923, June 1929 to September 1930 (fragmentary), March 1946 to September 1963. Prior to 1946 , published as North Fork Duchesne River near Hanna.

Estimates of streamflow.--October 1930 to February 1946, October 1963 to September 1965 , based on relationships of monthly mean discharge with Duchesne River near Tabiona, Utah. The regression equation used is:

$\log Y=b \log X-c$

(where $Y$ is discharge of Duchesne River near Hanna, Utah, and X is discharge of Duchesne River near Tabiona, Utah, both in acre-feet per month).

\begin{tabular}{l|l|l|l|l|l|l|l|l|l|l|l|l|l}
\hline & & Oct. & Nov. & Dec. & Jan. & Feb. & Mar. & Apr. & May & June & July & Aug. & Sept. \\
\hline Oct. 1930 & b & 2.36 & 1.48 & 1.56 & 1.36 & 1.39 & 2.01 & 2.22 & 0.96 & 0.85 & 1.16 & 1.20 & 1.03 \\
Sept. 1953 & c & 6.01 & 2.67 & 2.95 & 2.17 & 2.28 & 4.60 & 5.33 & .04 & -.53 & .93 & 1.21 & .75 \\
\hline Oct. 1953 & b & 2.36 & 1.48 & 1.56 & 1.36 & 1.39 & 2.01 & 2.22 & 0.96 & 0.85 & 1.29 & 1.20 & 2.44 \\
Sept. 1965 & c & 6.01 & 2.91 & 3.26 & 2.49 & 2.61 & 4.90 & 5.33 & .16 & -.37 & 1.60 & 1.42 & 6.10 \\
\hline
\end{tabular}

Average discharge. --23 years $(1931-53), 67,190$ acre-feet per year (92.6 cfs).

Extremes. --1921-23, 1929-30, 1946-63: Maximum discharge, about 17,500 cfs June 16, 1963, caused by failure of Little Deer Creek Dam 8 miles upstream, minimum recorded, 4.6 cf's
Nov. 14, 1956, Mar. 1, 1961 .

Remarks. -Diversions for irrigation of about 60 acres above station. Water is also diverted above station through Duchesne tunnel (capacity, about $600 \mathrm{cfs}$ ) to Provo River for use in The Great Basin; diversion began Oct. 20, 1953. Estimates of annual flow are within about 10 percent of regression line.

\begin{tabular}{c|c|r|r|r|r|r|r|r|r|r|r|r|r|}
\hline $\begin{array}{l}\text { Water } \\
\text { year }\end{array}$ & Oct. & Nov. & Dec. & Jan, & Feb. & Mar. & Apr. & May & June & July & Aug. & Sept. & Annual \\
\hline 1946 & - & - & - & - & - & - & 9,930 & 23,850 & 20,520 & 4,310 & 1,980 & 1,220 & - \\
1947 & 1,460 & 1,240 & 1,110 & 984 & 944 & 1,650 & 3,680 & 32,490 & 28,440 & 8,930 & 3,360 & 1,670 & 85,960 \\
1948 & 1,310 & 1,220 & 1,080 & 984 & 920 & 1,050 & 2,200 & 24,710 & 15,970 & 3,300 & 1,680 & 935 & 56,360 \\
1949 & 932 & 982 & 922 & 827 & 774 & 1,300 & 5,830 & 22,550 & 38,050 & 9,850 & 2,430 & 1,250 & 85,700 \\
1950 & 1,420 & 1,290 & 1,130 & 984 & 833 & 1,110 & 5,670 & 19,530 & 45,600 & 10,570 & 2,520 & 1,850 & 92,510 \\
1951 & 1,370 & 1,460 & 1,410 & 1,250 & 1,170 & 1,290 & 4,450 & 24,150 & 36,240 & 11,110 & 4,890 & 1,920 & 90,710 \\
1952 & 1,860 & 1,230 & 1,110 & 984 & 863 & 922 & 5,655 & 33,320 & 44,180 & 10,720 & 4,650 & 2,390 & 107,900 \\
1953 & 1,450 & 1,070 & 1,050 & 984 & 833 & 994 & 2,430 & 7,080 & 34,410 & 7,010 & 3,280 & 1,270 & 61,860 \\
1954 & 853 & 570 & 515 & 453 & 399 & 451 & 1,660 & 6,590 & 2,280 & 1,220 & 692 & 510 & 16,190 \\
1955 & 499 & 476 & 416 & 382 & 305 & 338 & 738 & 9,580 & 7,010 & 2,120 & 1,130 & 656 & 23,650 \\
1956 & 644 & 504 & 563 & 451 & 403 & 585 & 2,860 & 16,080 & 22,820 & 2,360 & 1,050 & 540 & 48,860 \\
1957 & 542 & 429 & 409 & 372 & 333 & 486 & 1,020 & 4,670 & 38,270 & 5,890 & 1,900 & 1,050 & 55,370 \\
1958 & 845 & 648 & 561 & 469 & 389 & 391 & 1,430 & 25,230 & 14,350 & 2,060 & 885 & 622 & 47,880 \\
1959 & 564 & 585 & 474 & 370 & 293 & 383 & 1,410 & 4,850 & 7,400 & 2,370 & 1,580 & 863 & 21,140 \\
1960 & 980 & 902 & 594 & 484 & 422 & 742 & 2,210 & 6,110 & 5,910 & 2,340 & 1,430 & 1,110 & 23,230 \\
1961 & 811 & 562 & 440 & 369 & 278 & 337 & 722 & 5,320 & 5,040 & 2,170 & 1,670 & 1,640 & 17,360 \\
1962 & 1,610 & 1,200 & 930 & 756 & 702 & 774 & 5,100 & 11,250 & 11,500 & 4,880 & 1,540 & 833 & 41,080 \\
1963 & 694 & 572 & 479 & 394 & 347 & 493 & 968 & 7,710 & 10,140 & 2,540 & 2,360 & 2,380 & 29,080 \\
\hline
\end{tabular}

Estimated monthly and annual streamflow, in acre-feet

\begin{tabular}{l|r|r|r|r|r|r|r|r|r|r|r|r|r|r}
\hline $\begin{array}{l}\text { Water } \\
\text { year }\end{array}$ & Oct. & Nov. & Dec. & Jan. & Feb. & Mar. & Apr. & May & June & July & Aug. & Sept. & Annual \\
\hline 1931 & 3,400 & 1,000 & 800 & 820 & 700 & 760 & 800 & 9,800 & 7,000 & 2,100 & 1,300 & 860 & 29,340 \\
1932 & 260 & 760 & 920 & 960 & 900 & 850 & 2,700 & 17,800 & 31,200 & 9,000 & 2,200 & 1,500 & 69,050 \\
1933 & 760 & 870 & 900 & 1,100 & 740 & 730 & 1,000 & 7,200 & 27,100 & 4,100 & 1,200 & 940 & 46,640 \\
1934 & 400 & 600 & 680 & 730 & 590 & 680 & 1,500 & 4,800 & 5,500 & 1,600 & 1,100 & 670 & 16,850 \\
1935 & 90 & 350 & 520 & 460 & 350 & 310 & 890 & 8,300 & 27,800 & 3,600 & 1,400 & 1,000 & 45,070 \\
1936 & 330 & 630 & 550 & 640 & 580 & 570 & 4,000 & 37,400 & 23,000 & 8,800 & 4,100 & 1,600 & 82,200 \\
1937 & 800 & 850 & 880 & 880 & 730 & 900 & 2,900 & 34,300 & 21,000 & 8,700 & 1,600 & 1,400 & 74,940 \\
1938 & 1,000 & 980 & 880 & 880 & 760 & 1,000 & 4,800 & 24,600 & 30,800 & 5,700 & 2,000 & 2,100 & 75,500 \\
1939 & 1,900 & 1,200 & 1,100 & 880 & 730 & 1,800 & 4,700 & 20,300 & 10,600 & 2,800 & 1,400 & 1,100 & 48,510 \\
1940 & 1,200 & 700 & 700 & 750 & 670 & 800 & 1,400 & 19,500 & 7,800 & 2,100 & 1,200 & 1,500 & 38,320 \\
1941 & 2,100 & 940 & 760 & 760 & 660 & 920 & 1,600 & 25,800 & 27,800 & 6,000 & 2,000 & 1,400 & 70,740 \\
1942 & 2,200 & 1,100 & 1,000 & 900 & 760 & 1,100 & 5,400 & 17,500 & 28,100 & 5,100 & 1,400 & 1,400 & 65,960 \\
1943 & 1,100 & 900 & 860 & 840 & 840 & 1,400 & 8,800 & 27,000 & 31,000 & 9,700 & 3,400 & 1,200 & 87,040 \\
1944 & 1,500 & 1,200 & 1,000 & 1,000 & 880 & 1,300 & 2,800 & 22,000 & 33,800 & 8,600 & 1,800 & 1,300 & 77,180 \\
1945 & 1,300 & 1,000 & 900 & 880 & 740 & 940 & 1,500 & 18,800 & 26,600 & 7,800 & 3,700 & 1,500 & 65,660 \\
1946 & 1,600 & 1,100 & 1,100 & 1,000 & 840 & 1,300 & - & - & - & - & - & - & 68,750 \\
1964 & 680 & 660 & 500 & 430 & 370 & 410 & 1,400 & 11,700 & 16,000 & 4,400 & 1,700 & 920 & 39,170 \\
1965 & 500 & 490 & 540 & 480 & 350 & 390 & 2,800 & 10,400 & 29,000 & 13,300 & 3,000 & 6,000 & 67,250 \\
\hline
\end{tabular}



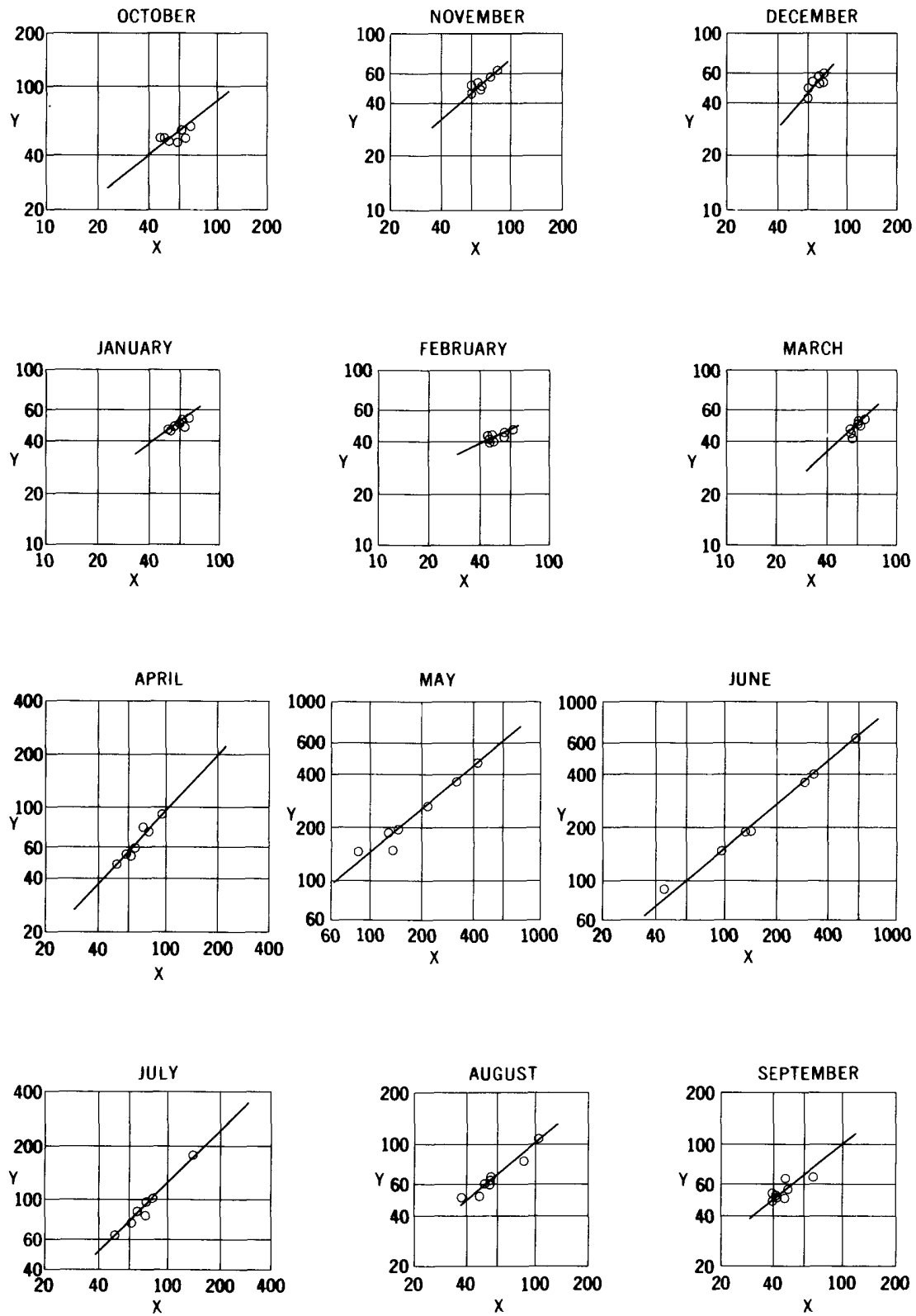

Relationships of monthly mean discharge of Duchesne River at Hanna, Utah $(\mathrm{Y})$, to monthly mean discharge of Duchesne River near Tabiona, Utah (X). Discharge in hundreds of acre-feet. 
2770. Duchesne River at Hanna, Utah

Location.--Lat $40^{\circ} 25^{\prime}$, Iong $110^{\circ} 47^{\prime}$, in SE $\frac{1}{4}$ sec.4, T.1 S., R.8 W., Uinta meredian, at downstream side of 'left abutment' of road bridge, $i$ milé downstream from Sand Creek and 1 mile northwest of Hanna.

Drainage area. $--230 \mathrm{sq} \mathrm{mi}$, approximately.

Records ava1lable. --August 1953 to September 1960.

Estimates of streamflow. - - october 1930 to July 1953, October 1960 to September 1965 , based on relationships of monthly mean discharge with Duchesne River near Tabiona, Utah. The regression equation used is:

$$
\log Y=b \log X-c
$$

(where $\mathrm{Y}$ is discharge of Duchesne River at Hanna, Utah, and X is discharge of Duchesne River near Tabiona, Utah, both in acre-feet per month).

\begin{tabular}{l|l|l|l|l|l|l|l|l|l|l|l|l}
\hline \multicolumn{10}{c}{ Monthly values of constants in above equation } \\
\hline b & oct. & Nov. & Dec. & Jan. & Feb. & Mar. & Apr. & May & June & July & Aug. & Sept. \\
\hline $\mathrm{c}$ & 0.77 & 0.84 & 1.12 & 0.68 & 0.46 & 0.89 & 1.05 & 0.82 & 0.82 & 0.96 & 0.79 & 0.78 \\
\hline
\end{tabular}

Average discharge. --35 years $(1931-65)$, 140,500 acre-feet per year (194 cfs).

Extremes :-1953-60: Maximum discharge, 2,260 cfs June 7, 1957; minimum daily, 52 cfs Mar. $12,15,1956$.

Remarks.--Several diversions above station for irrigation, including a transmountain diversion through Duchesne tunnel. Estimates of annual flow are within about 5 percent of regression line.

Monthly and annual streamflow, in acre-feet

\begin{tabular}{|c|c|c|c|c|c|c|c|c|c|c|c|c|c|}
\hline \begin{tabular}{|l|} 
Water \\
year
\end{tabular} & Oct. & Nov. & Dec. & Jan. & Feb. & Mar. & Apr. & May & June & July & Aug. & Sept. & Annual \\
\hline $\begin{array}{l}19 \\
19 \\
19\end{array}$ & $\begin{array}{c}-\overline{750} \\
4,860\end{array}$ & $\begin{array}{c}5, \overline{6} 20 \\
4,660\end{array}$ & $\begin{array}{l}5, \overline{4} 20 \\
4,830\end{array}$ & $\begin{array}{l}5, \overline{180} \\
4,720\end{array}$ & $\begin{array}{l}600 \\
, 980\end{array}$ & $\begin{array}{l}5, \overline{100} \\
4,340\end{array}$ & $\begin{array}{l}7, \overline{6} 60 \\
5,360\end{array}$ & $\begin{array}{c}\overline{-} \\
29,320 \\
25,740\end{array}$ & $\begin{array}{r}8, \overline{810} \\
18,980\end{array}$ & $\begin{array}{c}- \\
6,290 \\
8,570\end{array}$ & $\begin{array}{l}, 520 \\
, 920 \\
, 930\end{array}$ & & $\begin{array}{l}83^{-}, 230 \\
96,800\end{array}$ \\
\hline $\begin{array}{l}1956 \\
1957 \\
1958 \\
1959 \\
1960\end{array}$ & $\begin{array}{l}5,140 \\
4,900 \\
5,950 \\
5,060 \\
5,090\end{array}$ & $\begin{array}{l}4,900 \\
5,030 \\
6,110 \\
5,210 \\
4,750\end{array}$ & $\begin{array}{l}5,280 \\
5,220 \\
5,910 \\
5,250 \\
4,420\end{array}$ & $\begin{array}{l}4,700 \\
4,570 \\
5,340 \\
4,730 \\
4,530\end{array}$ & $\begin{array}{l}4,400 \\
4,030 \\
4,640 \\
4,250 \\
4,090\end{array}$ & $\begin{array}{l}5,300 \\
4,470 \\
4,850 \\
4,670 \\
4,900\end{array}$ & $\begin{array}{l}9,710 \\
4,820 \\
7,270 \\
5,580 \\
6,990\end{array}$ & $\begin{array}{l}36,080 \\
14,970 \\
47,670 \\
14,360 \\
18,820\end{array}$ & $\begin{array}{l}39,900 \\
63,710 \\
35,810 \\
18,780 \\
14,860\end{array}$ & $\begin{array}{r}9,640 \\
17,690 \\
10,000 \\
7,920 \\
7,170\end{array}$ & $\begin{array}{l}6,520 \\
7,720 \\
6,130 \\
5,820 \\
4,930\end{array}$ & $\begin{array}{l}4,850 \\
6,280 \\
5,350 \\
4,820 \\
4,710\end{array}$ & $\begin{array}{r}135,820 \\
143,400 \\
145,000 \\
86,450 \\
85,260\end{array}$ \\
\hline
\end{tabular}

Estimated monthly and annual streamflow, in acre-feet

\begin{tabular}{|c|c|c|c|c|c|c|c|c|c|c|c|c|c|}
\hline $\begin{array}{l}\text { ter } \\
\text { ar }\end{array}$ & ct. & lov. & ec. & $\operatorname{Jan}$ & Feb. & Mar. & Apr. & May & June & July & Aug. & sept. & Annual \\
\hline 1 & $\begin{array}{l}00 \\
00 \\
00 \\
00 \\
00\end{array}$ & $\begin{array}{l}5,400 \\
4,600 \\
5,000 \\
4,000 \\
3,000\end{array}$ & $\begin{array}{l}, 600 \\
, 000 \\
, 000 \\
, 100 \\
, 300\end{array}$ & & & & & $\begin{array}{l}20,800 \\
34,400 \\
16,100 \\
11,500 \\
18,200\end{array}$ & $\begin{array}{r}12 \\
51 \\
45 \\
6 \\
45\end{array}$ & & & & $\begin{array}{l}, 700 \\
100 \\
, 800 \\
900 \\
, 200\end{array}$ \\
\hline & & $\begin{array}{l}4,100 \\
4,900 \\
5,300 \\
6,000 \\
4,400\end{array}$ & $\begin{array}{l}3,500 \\
4,800 \\
4,900 \\
5,600 \\
4,200\end{array}$ & & & & $\begin{array}{r}10,800 \\
8,800 \\
12,300 \\
12,200 \\
6,200\end{array}$ & & & $\begin{array}{r}18,200 \\
18,000 \\
12,900 \\
7,100 \\
5,700\end{array}$ & $\begin{array}{r}10,600 \\
5,500 \\
6,500 \\
5,200 \\
4,500\end{array}$ & & $\begin{array}{l}174,200 \\
162,600 \\
166,300 \\
121,300 \\
101,800\end{array}$ \\
\hline $\begin{array}{l}1941 \\
1942 \\
1943 \\
1944 \\
1945\end{array}$ & & $\begin{array}{l}5,200 \\
5,600 \\
5,000 \\
6,000 \\
5,400\end{array}$ & $\begin{array}{l}5,400 \\
5,400 \\
4,700 \\
5,600 \\
5,000\end{array}$ & & & & $\begin{array}{r}6,700 \\
13,900 \\
20,300 \\
8,600 \\
6,400\end{array}$ & & & & & $\begin{array}{l}6,200 \\
6,200 \\
5,600 \\
5,800 \\
6,600\end{array}$ & $\begin{array}{l}156,900 \\
151,200 \\
186,500 \\
169,000 \\
150,600\end{array}$ \\
\hline $\begin{array}{l}1946 \\
1947 \\
1948 \\
1949 \\
1950\end{array}$ & $\begin{array}{l}7,000 \\
6,800 \\
6,200 \\
5,000 \\
6,900\end{array}$ & $\begin{array}{l}5,700 \\
6,000 \\
6,000 \\
5,300 \\
6,100\end{array}$ & $\begin{array}{l}5,800 \\
5,700 \\
5,700 \\
5,300 \\
5,600\end{array}$ & $\begin{array}{l}5,300 \\
5,000 \\
5,100 \\
4,800 \\
5,100\end{array}$ & & & $\begin{array}{r}18,800 \\
9,200 \\
7,300 \\
13,800 \\
14,200\end{array}$ & & & & & & $\begin{array}{l}148,900 \\
180,100 \\
131,100 \\
187,300 \\
204,100\end{array}$ \\
\hline $\begin{array}{l}195 \\
1958 \\
1953\end{array}$ & $\begin{array}{l}6,400 \\
6,800 \\
6,200\end{array}$ & $\begin{array}{l}6,400 \\
5,800 \\
5,600\end{array}$ & $\begin{array}{l}6,700 \\
5,800 \\
6,500\end{array}$ & $\begin{array}{l}5,500 \\
5,200 \\
5,800\end{array}$ & & & $\begin{array}{r}10,400 \\
17,500 \\
7,700\end{array}$ & & & & $\begin{array}{l}10,700 \\
12,200\end{array}$ & $\begin{array}{l}6,600 \\
9,400 \\
-\end{array}$ & $\begin{array}{l}187,000 \\
248,700 \\
145,510\end{array}$ \\
\hline $\begin{array}{l}1961 \\
1962 \\
1963 \\
1964 \\
1965\end{array}$ & $\begin{array}{l}600 \\
400 \\
400 \\
200 \\
800\end{array}$ & $\begin{array}{l}4,400 \\
5,000 \\
4,900 \\
5,600 \\
4,900\end{array}$ & $\begin{array}{l}4,800 \\
4,400 \\
5,400 \\
5,200 \\
5,500\end{array}$ & $\begin{array}{l}4,500 \\
4,200 \\
4,700 \\
4,900 \\
5,200\end{array}$ & $\begin{array}{l}4,000 \\
4,200 \\
4,500 \\
4,400 \\
4,400\end{array}$ & $\begin{array}{c}50 \\
00 \\
00 \\
00 \\
00\end{array}$ & $\begin{array}{r}3,400 \\
18,000 \\
6,000 \\
6,300 \\
8,500\end{array}$ & $\begin{array}{r}9,900 \\
38,000 \\
21,600 \\
31,000 \\
28,000\end{array}$ & $\begin{array}{l}10,000 \\
39,000 \\
26,100 \\
38,900 \\
68,200\end{array}$ & $\begin{array}{r}6,600 \\
14,800 \\
7,700 \\
13,700 \\
31,000\end{array}$ & $\begin{array}{r}5,000 \\
7,400 \\
7,500 \\
7,800 \\
11,500\end{array}$ & $\begin{array}{r}7,200 \\
6,400 \\
7,500 \\
5,800 \\
10,500\end{array}$ & $\begin{array}{r}68,500 \\
152,700 \\
107,200 \\
133,300 \\
187,000\end{array}$ \\
\hline
\end{tabular}



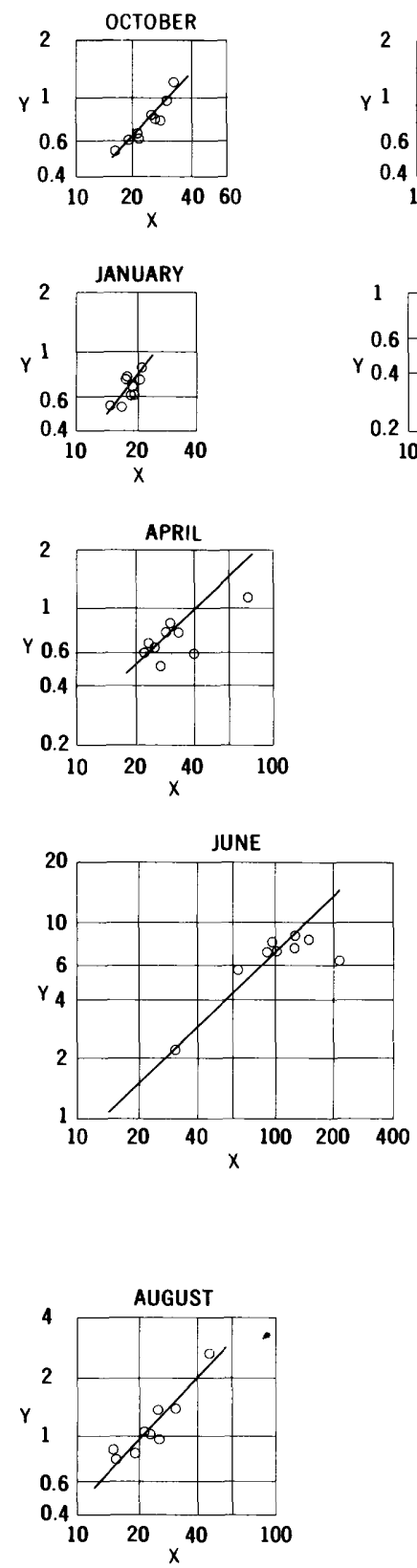
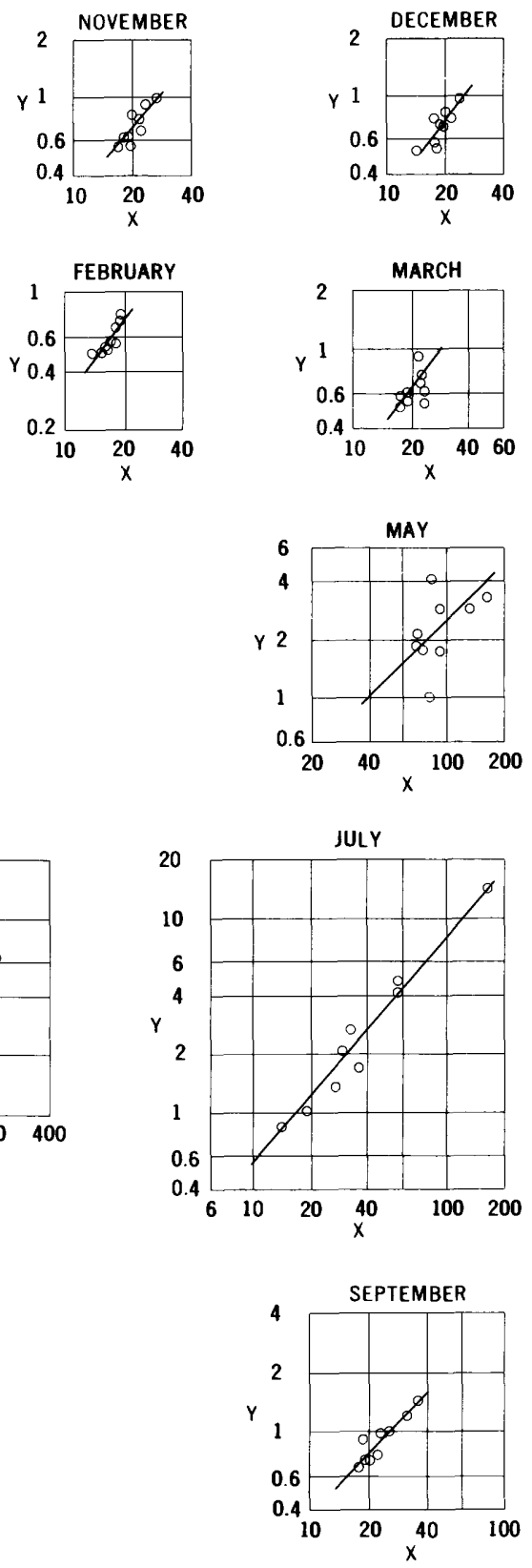

Relationships of monthly mean discharge of White River below Trappers Lake, Colo. $(\mathrm{Y})$, to monthly mean discharge of White River near Meeker, Colo. (X). Discharge in thousands of acre-feet. 
3024. White River below Trappers Lake, Colo.

Location.--Lat 39.59150", long 107.13150", in sec.2, T.1 S., R.88 W., on right bank 200 ft below Trappers Lake, 1 mile upstream from Skinny Fish Creek, and 21 miles east of Bu-

ford. Altitude of gage is $9,600 \mathrm{ft}$ (from topographic map).

Drainage area. $-21.4 \mathrm{sq} \mathrm{mi}$.

Records available.--October 1956 to September 1965.

Estimates of streamflow.--0ctober 1930 to September 1956, based on relationships of monthly mean discharge with white River near Meeker, Colo. The regression equation used is:

$$
\log \mathrm{Y}=b \log \mathrm{X}-\mathrm{c}
$$

(where $\mathrm{Y}$ is discharge of White River below Trappers Lake, Colo., and $\mathrm{X}$ is discharge of White River near Meeker, Colo., both in acre-feet per month).

Monthly values of constants in above equation

\begin{tabular}{l|l|l|l|l|l|l|l|l|l|l|l|l}
\hline & Oct. & Nov. & Dec. & Jan. & Feb. & Mar. & Apr. & May & June & Ju1y & Aug. & Sept. \\
\hline $\mathrm{b}$ & 1.09 & 1.09 & 1.28 & 1.26 & 1.31 & 1.26 & 0.96 & 0.96 & 0.96 & 1.14 & 1.04 & 1.04 \\
$\mathrm{c}$ & 1.88 & 1.87 & 2.63 & 2.54 & 2.76 & 2.62 & 1.41 & 1.38 & .95 & 1.80 & 1.49 & 1.59 \\
\hline
\end{tabular}

Average discharge. --35 years $(1930-65), 19,260$ acre-feet per year $(26.6 \mathrm{cf}$ ).

Extremes.--1956-65: Maximum discharge, $481 \mathrm{cfs}$ July 4, 1957, from rating curve extended above $250 \mathrm{cfs}$; minimum daily, 2.9 cfs Sept. 2, 1964, causéd by temporary storage behind moss jam at lake outlet upstream.

Remarks.--No diversions above station. Natural regulation by Trappers Lake. Estimates of annual flow are within about 15 percent of regression line.

\begin{tabular}{c|c|c|c|c|c|c|c|c|r|r|r|r|r}
\hline $\begin{array}{c}\text { Water } \\
\text { year }\end{array}$ & oct. & Nov. & Dec. & Jan. & Feb. & Mar. & Apr. & May & June & July & Aug. & Sept. & Annual \\
\hline 1957 & 609 & 625 & 584 & 615 & 528 & 612 & 641 & 1,010 & 6,500 & 14,800 & 2,670 & 1,360 & 30,550 \\
1958 & 1,230 & 962 & 984 & 861 & 722 & 738 & 774 & 3,300 & 8,830 & 1,750 & 1,050 & 958 & 22,160 \\
1959 & 837 & 778 & 799 & 738 & 555 & 615 & 869 & 2,160 & 8,310 & 2,160 & 1,360 & 695 & 19,880 \\
1960 & 801 & 811 & 799 & 740 & 575 & 535 & 599 & 1,800 & 7,280 & 1,380 & 775 & 891 & 16,990 \\
& & & & & & & & & & & & & \\
1961 & 624 & 556 & 576 & 543 & 503 & 557 & 601 & 1,880 & 5,970 & 1,050 & 857 & 978 & 14,700 \\
1962 & 990 & 904 & 861 & 797 & 778 & 922 & 1,160 & 2,900 & 7,740 & 4,300 & 950 & 728 & 23,030 \\
1963 & 792 & 674 & 732 & 615 & 666 & 680 & 772 & 4,120 & 2,320 & 849 & 817 & 685 & 13,720 \\
1964 & 541 & 568 & 558 & 553 & 518 & 585 & 678 & 2,910 & 7,290 & 2,640 & 1,020 & 634 & 18,500 \\
1965 & 655 & 608 & 739 & 670 & 532 & 521 & 527 & 1,760 & 8,470 & 4,900 & 1,370 & 1,130 & 21,880 \\
\hline
\end{tabular}

\begin{tabular}{|c|c|c|c|c|c|c|c|c|c|c|c|c|c|}
\hline $\begin{array}{l}\text { Water } \\
\text { year }\end{array}$ & oct. & Nov. & Dec. & Jan. & Feb. & Mar. & Apr. & May & June & July & Aug. & Sept. & Annual \\
\hline $\begin{array}{l}1931 \\
1932 \\
1933 \\
1934 \\
1935\end{array}$ & $\begin{array}{l}930 \\
680 \\
840 \\
710 \\
530\end{array}$ & $\begin{array}{l}900 \\
680 \\
800 \\
720 \\
530\end{array}$ & $\begin{array}{l}920 \\
910 \\
850 \\
780 \\
580\end{array}$ & $\begin{array}{l}780 \\
880 \\
810 \\
550 \\
550\end{array}$ & $\begin{array}{l}680 \\
720 \\
680 \\
490 \\
410\end{array}$ & $\begin{array}{l}720 \\
850 \\
760 \\
740 \\
490\end{array}$ & $\begin{array}{l}850 \\
970 \\
840 \\
830 \\
550\end{array}$ & $\begin{array}{l}2,000 \\
3,100 \\
2,000 \\
1,200 \\
1,400\end{array}$ & $\begin{array}{r}4,400 \\
9,000 \\
10,400 \\
1,200 \\
8,900\end{array}$ & $\begin{array}{r}1,200 \\
4,700 \\
3,100 \\
540 \\
2,500\end{array}$ & $\begin{array}{r}990 \\
1,600 \\
1,200 \\
560 \\
820\end{array}$ & $\begin{array}{r}820 \\
1,000 \\
900 \\
540 \\
660\end{array}$ & $\begin{array}{r}15,190 \\
25,090 \\
23,180 \\
8,860 \\
17,920\end{array}$ \\
\hline $\begin{array}{l}1936 \\
1937 \\
1938 \\
1939 \\
1940\end{array}$ & $\begin{array}{l}510 \\
600 \\
570 \\
730 \\
680\end{array}$ & $\begin{array}{l}600 \\
560 \\
550 \\
640 \\
610\end{array}$ & $\begin{array}{l}560 \\
590 \\
600 \\
740 \\
620\end{array}$ & $\begin{array}{l}700 \\
590 \\
540 \\
710 \\
640\end{array}$ & $\begin{array}{l}580 \\
500 \\
470 \\
470 \\
560\end{array}$ & $\begin{array}{l}540 \\
510 \\
670 \\
640 \\
580\end{array}$ & $\begin{array}{r}970 \\
480 \\
1,000 \\
880 \\
750\end{array}$ & $\begin{array}{l}3,000 \\
2,300 \\
2,800 \\
2,500 \\
2,800\end{array}$ & $\begin{array}{r}6,400 \\
4,300 \\
11,000 \\
4,900 \\
4,500\end{array}$ & $\begin{array}{l}2,100 \\
2,200 \\
3,200 \\
1,400 \\
1,300\end{array}$ & $\begin{array}{r}1,100 \\
720 \\
1,100 \\
910 \\
720\end{array}$ & $\begin{array}{r}690 \\
660 \\
1,000 \\
830 \\
660\end{array}$ & $\begin{array}{l}17,750 \\
14,010 \\
23,500 \\
15,350 \\
14,420\end{array}$ \\
\hline $\begin{array}{l}1941 \\
1942 \\
1943 \\
1944 \\
1945\end{array}$ & $\begin{array}{l}730 \\
930 \\
640 \\
570 \\
630\end{array}$ & $\begin{array}{l}620 \\
740 \\
680 \\
600 \\
600\end{array}$ & $\begin{array}{l}650 \\
730 \\
680 \\
630 \\
590\end{array}$ & $\begin{array}{l}620 \\
740 \\
570 \\
680 \\
610\end{array}$ & $\begin{array}{l}520 \\
580 \\
490 \\
520 \\
560\end{array}$ & $\begin{array}{l}600 \\
620 \\
520 \\
540 \\
600\end{array}$ & $\begin{array}{r}630 \\
1,100 \\
950 \\
480 \\
530\end{array}$ & $\begin{array}{l}3,400 \\
2,800 \\
1,600 \\
2,200 \\
2,600\end{array}$ & $\begin{array}{l}7,300 \\
9,000 \\
6,500 \\
8,000 \\
8,000\end{array}$ & $\begin{array}{l}2,400 \\
2,100 \\
2,200 \\
2,800 \\
4,900\end{array}$ & $\begin{array}{r}1,100 \\
1,000 \\
1,400 \\
960 \\
1,600\end{array}$ & $\begin{array}{l}820 \\
680 \\
690 \\
590 \\
800\end{array}$ & $\begin{array}{l}19,390 \\
21,020 \\
16,920 \\
18,570 \\
22,020\end{array}$ \\
\hline $\begin{array}{l}1946 \\
1947 \\
2948 \\
1949 \\
1950\end{array}$ & $\begin{array}{l}720 \\
770 \\
830 \\
720 \\
810\end{array}$ & $\begin{array}{l}750 \\
630 \\
760 \\
710 \\
740\end{array}$ & $\begin{array}{l}760 \\
660 \\
920 \\
760 \\
780\end{array}$ & $\begin{array}{l}650 \\
640 \\
800 \\
680 \\
780\end{array}$ & $\begin{array}{l}540 \\
560 \\
620 \\
560 \\
660\end{array}$ & $\begin{array}{l}500 \\
760 \\
520 \\
650 \\
620\end{array}$ & $\begin{array}{r}1,000 \\
790 \\
900 \\
890 \\
740\end{array}$ & $\begin{array}{l}1,700 \\
3,900 \\
3,300 \\
2,600 \\
1,700\end{array}$ & $\begin{array}{r}5,700 \\
8,900 \\
6,800 \\
11,400 \\
9,200\end{array}$ & $\begin{array}{l}1,500 \\
4,900 \\
2,000 \\
4,100 \\
2,500\end{array}$ & $\begin{array}{l}1,000 \\
1,700 \\
1,200 \\
1,300 \\
1,000\end{array}$ & $\begin{array}{l}630 \\
900 \\
710 \\
870 \\
800\end{array}$ & $\begin{array}{l}15,450 \\
25,110 \\
19,360 \\
25,240 \\
20,330\end{array}$ \\
\hline $\begin{array}{l}1951 \\
1952 \\
1953 \\
1954 \\
1955\end{array}$ & $\begin{array}{l}720 \\
790 \\
910 \\
700 \\
700\end{array}$ & $\begin{array}{l}660 \\
700 \\
820 \\
680 \\
620\end{array}$ & $\begin{array}{r}760 \\
720 \\
1,100 \\
700 \\
670\end{array}$ & $\begin{array}{l}680 \\
680 \\
940 \\
820 \\
630\end{array}$ & $\begin{array}{l}530 \\
620 \\
660 \\
600 \\
500\end{array}$ & $\begin{array}{l}580 \\
570 \\
700 \\
570 \\
530\end{array}$ & $\begin{array}{l}610 \\
940 \\
700 \\
820 \\
620\end{array}$ & $\begin{array}{l}2,400 \\
3,300 \\
1,900 \\
1,900 \\
2,300\end{array}$ & $\begin{array}{r}8,200 \\
13,600 \\
9,800 \\
2,200 \\
5,500\end{array}$ & $\begin{array}{r}3,500 \\
3,800 \\
2,300 \\
980 \\
1,200\end{array}$ & $\begin{array}{r}1,300 \\
2,000 \\
1,200 \\
700 \\
800\end{array}$ & $\begin{array}{r}760 \\
1,200 \\
650 \\
720 \\
520\end{array}$ & $\begin{array}{l}20,700 \\
28,920 \\
21,680 \\
11,390 \\
14,590\end{array}$ \\
\hline 1956 & 630 & 630 & 720 & 680 & 560 & 620 & 820 & 2,700 & 6,300 & 1,600 & 880 & 510 & 16,650 \\
\hline
\end{tabular}



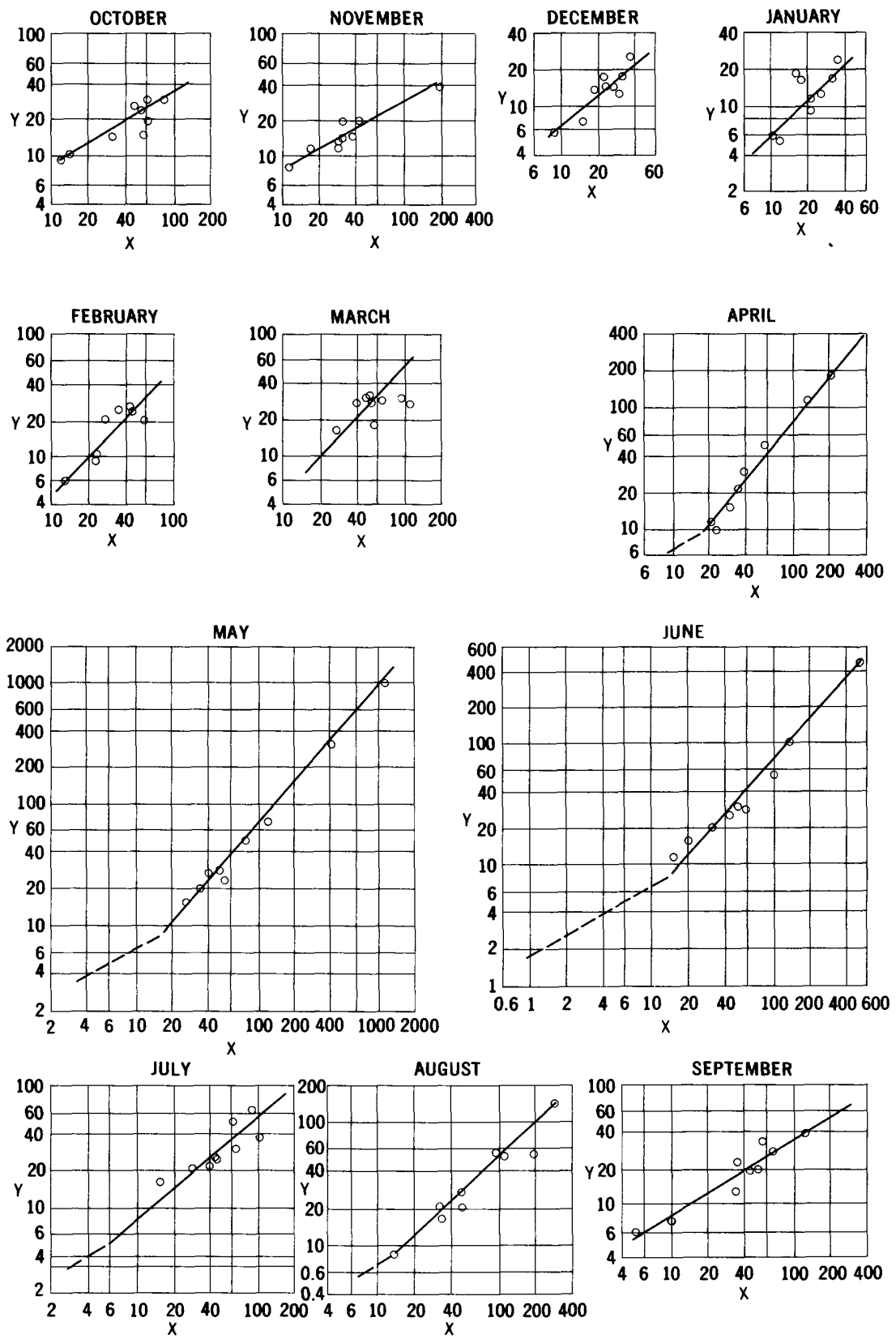

Relationships of monthly mean discharge of Price River near Wellington, Utah (Y), to monthly mean discharge of Price River at Woodside, Utah (X). Discharge in hundreds of acre-feet. 
3140. Price River near Wellington, Utah

Location.--Lat $39^{\circ} 30140^{\prime \prime}$, long $110^{\circ} 40150^{\prime \prime}$, in NW $\frac{1}{4}$ sec.22, T.15 S., R.1l E., on left bank 0.3 mile upstream from Miller Creek and 3.5 milles southeast of 'Weilington. Altitude of gage is $5,300 \mathrm{ft}$ (by barometer).

Drainage area. $--850 \mathrm{sq} \mathrm{mi}$, approximately.

Records available. --0ctober 1949 to September 1958.

Estimates of streamflow:--December 1945 to September 1949, October 1958 to September 1965 , based on relationships of monthly mean discharge with Price River at woodside,
Utah. The regression equation used is:

$$
\log Y=b \log X-c
$$

(where $Y$ is discharge of Price River near Wellington, Utah, and $X$ is discharge of Price River at Woodside, Utah, both in acre-feet per month).

\begin{tabular}{|c|c|c|c|c|c|c|c|c|c|c|c|c|}
\hline & oct. & & Dec & & Feh & & n & & & שרוטר & & \\
\hline b & 0.60 & 0.56 & 0.83 & 0.94 & 7.04 & 1.04 & 131 & 1.14 & 1,11 & 0.83 & 0.83 & 0.62 \\
\hline c & -1.13 & -123 & -.35 & & & .44 & . 98 & & .58 & -.41 & -.355 & -1.08 \\
\hline$x<$ & - & - & - & - & - & - & 1,800 & 1,700 & $|1,400|$ & 600 & $1,300 \mid$ & - \\
\hline
\end{tabular}

Average discharge. --19 years (1946-65), 42,580 acre-feet per year (58.8 cfs).

Extremes.--1949-58: Maximum discharge, 4,190 cfs Aug. 28, 1953, from rating curve extended above $1,800 \mathrm{cfs}$ on basis of slope-area measurements; minimu, $2.4 \mathrm{cfs}$ Nov. 19, 1956.

Remarks. - Many diversions for irrigation above and below station. Flow affected by storage in Scofield Reservoir (capacity, 65,780 acre-ft). Estimates of annual flow are within about 25 percent of regression line.

\begin{tabular}{|c|c|c|c|c|c|c|c|c|c|c|c|c|c|}
\hline $\begin{array}{l}\text { Water } \\
\text { year }\end{array}$ & oct. & Nov. & Dec. & Jan. & Feb. & Mar. & Apr. & May & June & July & Aug. & Sept. & Annual \\
\hline 1950 & 2,890 & 1,380 & 1,740 & 1,970 & 1,960 & 2,850 & 11,250 & 5,020 & 2,600 & 3,590 & 1,660 & 1,980 & 38,890 \\
\hline $\begin{array}{l}1951 \\
1952 \\
1953 \\
1954 \\
1955\end{array}$ & $\begin{array}{l}1,440 \\
1,500 \\
2,600 \\
2,340 \\
2,920\end{array}$ & $\begin{array}{l}1,430 \\
1,940 \\
1,970 \\
1,470 \\
1,160\end{array}$ & $\begin{array}{r}1,270 \\
1,390 \\
2,560 \\
1,490 \\
756\end{array}$ & $\begin{array}{r}1,150 \\
1,680 \\
2,460 \\
1,300 \\
573\end{array}$ & $\begin{array}{r}1,280 \\
2,010 \\
2,430 \\
2,540 \\
613\end{array}$ & $\begin{array}{l}3,180 \\
2,990 \\
2,710 \\
3,010 \\
2,650\end{array}$ & $\begin{array}{r}1,510 \\
36,230 \\
2,960 \\
2,130 \\
4,850\end{array}$ & $\begin{array}{r}2,340 \\
99,510 \\
2,820 \\
2,710 \\
1,980\end{array}$ & $\begin{array}{r}2,870 \\
46,440 \\
3,070 \\
2,040 \\
1,580\end{array}$ & $\begin{array}{l}2,680 \\
6,150 \\
4,890 \\
2,150 \\
1,600\end{array}$ & $\begin{array}{l}5,330 \\
5,490 \\
5,040 \\
2,010 \\
2,630\end{array}$ & $\begin{array}{r}1,350 \\
3,490 \\
2,340 \\
4,070 \\
779\end{array}$ & $\begin{array}{r}25,830 \\
208,800 \\
35,850 \\
27,260 \\
22,090\end{array}$ \\
\hline $\begin{array}{l}1956 \\
1957 \\
1958\end{array}$ & $\begin{array}{r}1,040 \\
932 \\
1,950\end{array}$ & $\begin{array}{r}1,160 \\
801 \\
3,750\end{array}$ & $\begin{array}{r}1,470 \\
615 \\
1,770\end{array}$ & $\begin{array}{r}964 \\
615 \\
1,720\end{array}$ & $\begin{array}{r}914 \\
1,060 \\
2,270\end{array}$ & $\begin{array}{l}2,760 \\
1,640 \\
1,820\end{array}$ & $\begin{array}{r}970 \\
1,120 \\
17,670\end{array}$ & $\begin{array}{r}1,540 \\
7,120 \\
31,300\end{array}$ & $\begin{array}{r}1,150 \\
5,490 \\
10,160\end{array}$ & $\begin{array}{l}2,060 \\
2,920 \\
2,580\end{array}$ & $\begin{array}{r}840 \\
13,440 \\
1,970\end{array}$ & $\begin{array}{r}617 \\
2,050 \\
2,740\end{array}$ & $\begin{array}{l}15,480 \\
37,800 \\
79,700\end{array}$ \\
\hline
\end{tabular}

Estimated monthly and annual streamflow, in acre-feet

\begin{tabular}{|c|c|c|c|c|c|c|c|c|c|c|c|c|c|}
\hline $\begin{array}{c}\text { Water } \\
\text { year }\end{array}$ & oct. & Nov. & Dec. & Jan. & Feb. & Mar. & Apr. & May & June & July & Aug. & Sept. & Annual \\
\hline $\begin{array}{l}1946 \\
1947 \\
1948 \\
1949\end{array}$ & $\begin{array}{c}-, 000 \\
1,700 \\
1,300\end{array}$ & $\begin{array}{l}- \\
1,300 \\
1,200 \\
1,000\end{array}$ & $\begin{array}{r}740 \\
1,300 \\
1,500 \\
1,100\end{array}$ & $\begin{array}{l}600 \\
660 \\
990 \\
980\end{array}$ & $\begin{array}{r}930 \\
1,400 \\
1,400 \\
1,300\end{array}$ & $\begin{array}{l}2,400 \\
2,400 \\
2,200 \\
5,400\end{array}$ & $\begin{array}{r}2,200 \\
3,000 \\
2,700 \\
10,400\end{array}$ & $\begin{array}{l}1,400 \\
4,100 \\
1,700 \\
9,000\end{array}$ & $\begin{array}{r}650 \\
2,100 \\
1,600 \\
11,000\end{array}$ & $\begin{array}{r}940 \\
1,500 \\
1,800 \\
6,700\end{array}$ & $\begin{array}{l}2,700 \\
6,400 \\
3,000 \\
2,500\end{array}$ & $\begin{array}{r}730 \\
1,400 \\
630 \\
2,500\end{array}$ & $\begin{array}{c}- \\
27,560 \\
20,420 \\
53,180\end{array}$ \\
\hline $\begin{array}{l}1959 \\
1960\end{array}$ & $\begin{array}{l}1,600 \\
I, 300\end{array}$ & $\begin{array}{l}1,300 \\
1,100\end{array}$ & $\begin{array}{l}1,700 \\
1,000\end{array}$ & $\begin{array}{r}1,400 \\
920\end{array}$ & $\begin{array}{r}1,200 \\
840\end{array}$ & $\begin{array}{l}1,400 \\
3,300\end{array}$ & $\begin{array}{r}900 \\
1,200\end{array}$ & $\begin{array}{l}880 \\
760\end{array}$ & $\begin{array}{l}790 \\
630\end{array}$ & $\begin{array}{l}520 \\
320\end{array}$ & $\begin{array}{r}3,300 \\
700\end{array}$ & $\begin{array}{l}2,100 \\
1,500\end{array}$ & $\begin{array}{l}17,090 \\
13,570\end{array}$ \\
\hline $\begin{array}{l}1961 \\
1962 \\
1963 \\
1964 \\
1965\end{array}$ & $\begin{array}{r}3,700 \\
920 \\
2,500 \\
1,000 \\
910\end{array}$ & $\begin{array}{r}940 \\
980 \\
1,000 \\
920 \\
980\end{array}$ & $\begin{array}{r}800 \\
860 \\
930 \\
650 \\
1,000\end{array}$ & $\begin{array}{r}400 \\
550 \\
490 \\
420 \\
1,300\end{array}$ & $\begin{array}{r}600 \\
3,800 \\
1,200 \\
490 \\
1,500\end{array}$ & $\begin{array}{r}760 \\
4,400 \\
3,500 \\
940 \\
1,600\end{array}$ & $\begin{array}{r}620 \\
25,900 \\
840 \\
1,900 \\
7,000\end{array}$ & $\begin{array}{r}350 \\
8,800 \\
780 \\
3,600 \\
21,800\end{array}$ & $\begin{array}{r}170 \\
1,800 \\
890 \\
1,800 \\
14,300\end{array}$ & $\begin{array}{r}470 \\
5,300 \\
1,100 \\
1,400 \\
8,200\end{array}$ & $\begin{array}{r}100 \\
540 \\
6,700 \\
4,800 \\
4,000\end{array}$ & $\begin{array}{l}6,700 \\
4,600 \\
4,000 \\
1,300 \\
2,600\end{array}$ & $\begin{array}{l}18,800 \\
58,450 \\
23,830 \\
19,220 \\
65,190\end{array}$ \\
\hline
\end{tabular}

Note--Downward extension of regressions April to August in the direction indicated by each set of monthly data would give estimates at wellington substantially below anything observed, and would suggest inflow per unit area below wellington equal, or nearly equal, to that occurring above. This is not probable because of the difference in basin altitudes, hence a common regression is used below about 1,000 acre-feet. 

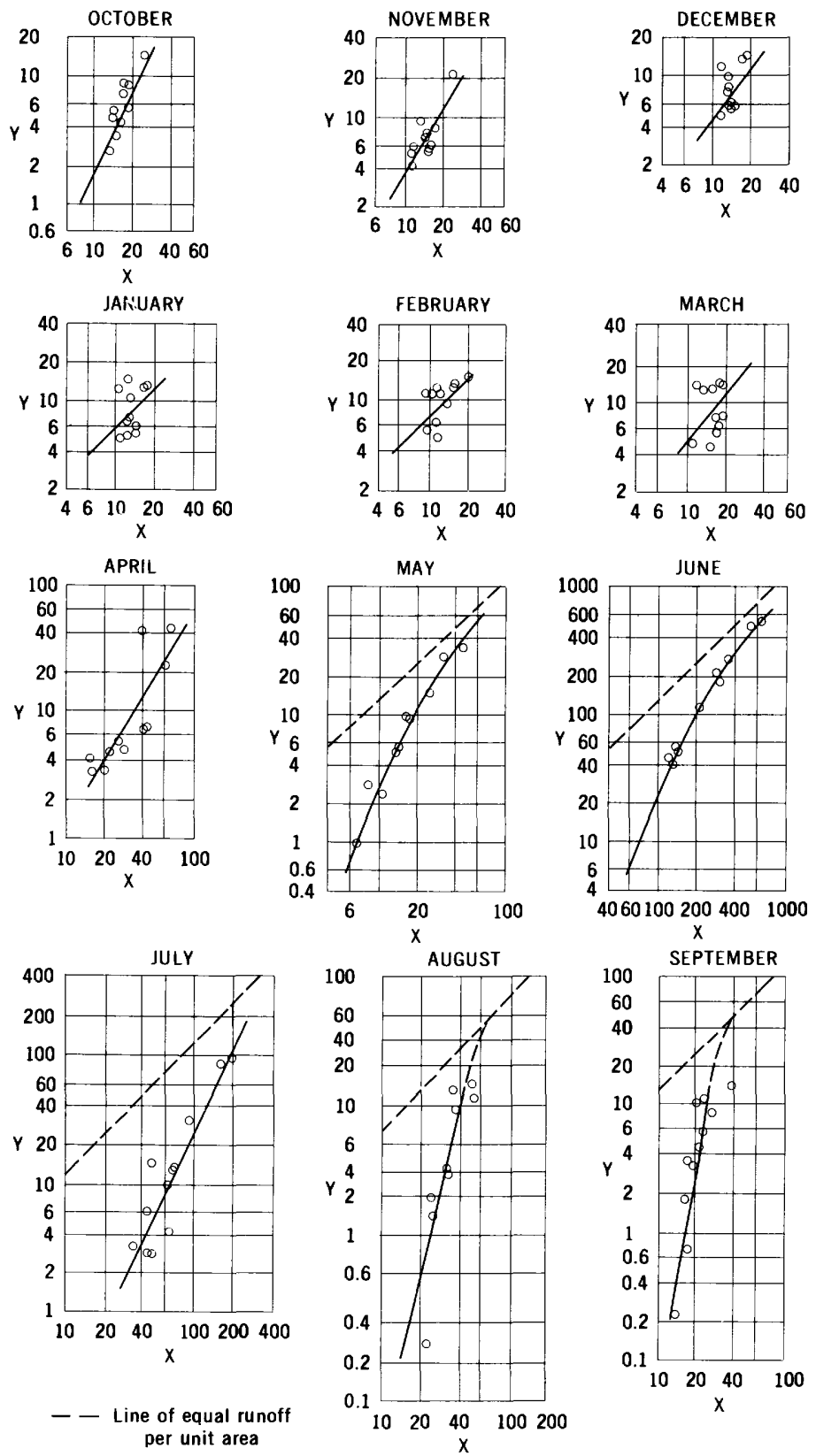

Relationships of monthly mean discharge of Cotton wood Creek near Castle Dale, Utah (Y), to monthly mean discharge of Cottonwood Creek near Orangeville, Utah (X). Discharge in hundreds of acre-feet. 
Location.--Lat $39^{\circ} 10^{\prime}$, long $110^{\circ} 56^{\prime}$, in sec.8, T.19 S., R.9 E., on right bank half a mile upstream from Rock Canyon and 6 miles east of Castle Dale. Altitude of gage is $5,400 \mathrm{ft}$ (from river-profile map).

Drainage area. $--261 \mathrm{sq} \mathrm{mi}$

Records available.--July 1947 to September 1958.

Estimates of streamflow.--May 1932 to June 1947, October 1958 to September 1965, based on relationships of monthly mean discharge with Cottonwood Creek near orangevilie, Utah. The regression equation used is:

$$
\log Y=b \log (X-a)-c
$$

(where $Y$ is discharge of Cottonwood Creek near Castle Dale, Utah and $X$ is discharge of Cottonwood Creek near Orangeville, Utah, both in acre-feet per month).

Monthly values of constants in above equation

\begin{tabular}{l|c|c|c|c|c|c|c|c|c|c|c|c}
\hline & oct. & Nov. & Dec. & Jan. & Feb. & Mar. & Apr. & *May & *June & July & Aug. & Sept. \\
\hline a & 0 & 0 & 0 & 0 & 0 & 0 & 0 & 6,000 & 6,000 & 0 & 0 & 0 \\
b & 2.09 & 1.70 & 1.33 & 1.00 & 1.00 & 1.23 & 1.70 & 1.21 & 1.21 & 2.12 & 4.13 & 5.98 \\
c & 4.01 & 2.52 & 1.34 & .22 & .13 & .99 & 2.99 & 1.00 & 1.00 & 5.07 & 11.70 & 17.26 \\
\hline
\end{tabular}

$\log Y=2.49 \log X-6.58$.

Average discharge, --33 years $(1932-65), 33,776$ acre-feet per year $(46.7$ crs).

Extremes:-1947-58: Maximum discharge, 1,660 cfs about June 3, 1952, from rating curve extended above 820 cfs by logarithmic plotting; no flow for part of Aug. 31, 1954, and for several days during August and September 1956.

Remarks.--Many diversions above station for irri, ation and several transmountain diversions from headwaters to the Great Basin for irrigation in San Pitch River basin. Estimates of annual flow are within about 10 percent of regression line.

Monthly and annual streamflow, in acre-feet

\begin{tabular}{r|r|r|r|r|r|r|r|r|r|r|r|r|r}
\hline $\begin{array}{l}\text { Water } \\
\text { year }\end{array}$ & oct. & Nov. & Dec. & Jan. & Feb. & Mar. & Apr. & May & June & JuIy & Aug. & Sept. & Annual \\
\hline 1947 & - & - & - & - & - & - & - & - & - & 1,250 & 2,580 & 620 & - \\
1948 & 578 & 588 & 799 & 676 & 1,160 & 1,220 & 486 & 9,600 & 5,460 & 602 & 276 & 182 & 21,650 \\
1949 & 447 & 607 & 583 & 523 & 682 & 1,280 & 2,660 & 16,330 & 17,960 & 3,050 & 632 & 337 & 45,090 \\
1950 & 727 & 740 & 559 & 736 & 1,130 & 750 & 734 & 5,660 & 5,020 & 1,410 & 379 & 359 & 18,200 \\
1951 & 552 & 700 & 619 & 553 & 520 & 442 & 346 & 7,640 & 10,960 & 992 & 2,520 & 439 & 26,280 \\
1952 & 870 & 920 & 968 & 1,020 & 1,280 & 1,400 & 4,570 & 34,370 & 52,430 & 8,590 & 2,350 & 1,410 & 110,200 \\
1953 & 881 & 789 & 1,410 & 1,290 & 1,360 & 780 & 585 & 965 & 20,830 & 1,300 & 1,830 & 466 & 32,490 \\
1954 & 830 & 544 & 594 & 633 & 980 & 631 & 777 & 5,350 & 589 & 320 & 137 & 1,070 & 12,460 \\
1955 & 484 & 602 & 1,170 & 1,230 & 1,170 & 1,330 & 430 & 2,370 & 3,940 & 278 & 580 & 72 & 13,660 \\
1956 & 343 & 502 & 756 & 1,470 & 1,240 & 567 & 516 & 9,480 & 4,500 & 281 & 27,6 & 224 & 19,700 \\
1957 & 260 & 414 & 492 & 492 & 595 & 464 & 340 & 2,800 & 47,390 & 9,680 & 2,810 & 884 & 66,620 \\
1958 & 1,460 & 2,110 & 1,300 & 1,230 & 1,530 & 1,360 & 4,390 & 28,680 & 26,100 & 419 & 319 & 1,110 & 70,010 \\
\hline
\end{tabular}

Estimated monthly and annual streamflow, in acre-feet

\begin{tabular}{|c|c|c|c|c|c|c|c|c|c|c|c|c|c|}
\hline $\begin{array}{l}\text { Water } \\
\text { year }\end{array}$ & oct. & Nov. & Dec. & Jan. & Feb. & Mar. & Apr. & May & June & July & Aug. & Sept. & Annual \\
\hline $\begin{array}{l}1932 \\
1933 \\
1934 \\
1935\end{array}$ & $\begin{array}{l}- \\
330 \\
140 \\
100\end{array}$ & $\begin{array}{l}370 \\
230\end{array}$ & $\begin{array}{l}- \\
410 \\
530 \\
340\end{array}$ & $\begin{array}{l}- \\
380 \\
570 \\
500\end{array}$ & $\begin{array}{l}- \\
410 \\
600 \\
580\end{array}$ & $\begin{array}{l}- \\
520 \\
620 \\
560\end{array}$ & $\begin{array}{c}- \\
390 \\
2,300 \\
480\end{array}$ & $\begin{array}{r}9,000 \\
910 \\
910 \\
1,800\end{array}$ & $\begin{array}{r}10,800 \\
22,500 \\
70 \\
39,300\end{array}$ & $\begin{array}{r}860 \\
2,300 \\
30 \\
950\end{array}$ & $\begin{array}{r}310 \\
1,300 \\
20 \\
170\end{array}$ & $\begin{array}{r}70 \\
70 \\
0 \\
20\end{array}$ & $\begin{array}{r}- \\
30,090 \\
\dot{5}, 160 \\
45,030\end{array}$ \\
\hline $\begin{array}{l}1938 \\
1939 \\
1940\end{array}$ & $\begin{array}{l}210 \\
410 \\
470 \\
420 \\
440\end{array}$ & $\begin{array}{l}500 \\
690 \\
450\end{array}$ & $\begin{array}{l}380 \\
610 \\
620 \\
610 \\
470\end{array}$ & $\begin{array}{l}54 \\
61 \\
72 \\
69 \\
57\end{array}$ & $\begin{array}{l}640 \\
740 \\
810 \\
660 \\
710\end{array}$ & $\begin{array}{r}580 \\
730 \\
850 \\
2,000 \\
700\end{array}$ & $\begin{array}{r}2,400 \\
1,100 \\
2,100 \\
3,200 \\
790\end{array}$ & $\begin{array}{l}29,500 \\
19,700 \\
13,800 \\
13,800 \\
22,300\end{array}$ & $\begin{array}{r}11,000 \\
7,500 \\
12,800 \\
3,000 \\
4,200\end{array}$ & $\begin{array}{r}1,200 \\
1,100 \\
440 \\
240 \\
210\end{array}$ & $\begin{array}{r}1,200 \\
430 \\
280 \\
60 \\
20\end{array}$ & $\begin{array}{r}320 \\
1,400 \\
300 \\
180 \\
380\end{array}$ & $\begin{array}{l}48,470 \\
35,090 \\
33,690 \\
25,550 \\
31,240\end{array}$ \\
\hline $\begin{array}{l}1942 \\
1943 \\
1944 \\
1945\end{array}$ & $\begin{array}{r}390 \\
1,600 \\
480 \\
440 \\
510\end{array}$ & $\begin{array}{r}530 \\
1,500 \\
660 \\
570 \\
690\end{array}$ & $\begin{array}{r}610 \\
1,100 \\
780 \\
630 \\
710\end{array}$ & $\begin{array}{r}650 \\
1,000 \\
840 \\
610 \\
750\end{array}$ & $\begin{array}{r}720 \\
1,000 \\
930 \\
780 \\
810\end{array}$ & $\begin{array}{r}810 \\
1,400 \\
930 \\
750 \\
620\end{array}$ & $\begin{array}{r}490 \\
4,300 \\
3,800 \\
530 \\
370\end{array}$ & $\begin{array}{r}20,500 \\
14,700 \\
6,300 \\
15,600 \\
8,200\end{array}$ & $\begin{array}{r}21,300 \\
20,900 \\
4,500 \\
30,700 \\
11,800\end{array}$ & $\begin{array}{l}1,300 \\
1,100 \\
400 \\
2,800 \\
1,400\end{array}$ & $\begin{array}{r}770 \\
280 \\
500 \\
370 \\
1,200\end{array}$ & $\begin{array}{r}500 \\
140 \\
60 \\
160 \\
280\end{array}$ & $\begin{array}{l}48,570 \\
49,020 \\
20,180 \\
53,940 \\
27,340\end{array}$ \\
\hline 1947 & & $\begin{array}{l}900 \\
460\end{array}$ & $\begin{array}{l}750 \\
490\end{array}$ & $\begin{array}{l}800 \\
700\end{array}$ & $\begin{array}{l}820 \\
940\end{array}$ & $\begin{array}{l}1,100 \\
1,200\end{array}$ & $\begin{array}{l}4,300 \\
1,300\end{array}$ & $\begin{array}{r}9,500 \\
16,300\end{array}$ & $\begin{array}{l}3,300 \\
7,700\end{array}$ & $\begin{array}{l}330 \\
-\end{array}$ & $\begin{array}{l}120 \\
-\end{array}$ & -30 & $\begin{array}{l}22,520 \\
33,960\end{array}$ \\
\hline $\begin{array}{l}1959 \\
1960\end{array}$ & $\begin{array}{l}570 \\
340\end{array}$ & $\begin{array}{l}600 \\
410\end{array}$ & $\begin{array}{l}640 \\
350\end{array}$ & $\begin{array}{l}710 \\
450\end{array}$ & $\begin{array}{l}790 \\
600\end{array}$ & $\begin{array}{l}700 \\
850\end{array}$ & $\begin{array}{r}440 \\
1,000\end{array}$ & $\begin{array}{r}680 \\
5,300\end{array}$ & $\begin{array}{l}1,800 \\
5,000\end{array}$ & $\begin{array}{l}200 \\
310\end{array}$ & $\begin{array}{l}50 \\
60\end{array}$ & $\begin{array}{r}20 \\
150\end{array}$ & $\begin{array}{r}7,200 \\
14,820\end{array}$ \\
\hline $\begin{array}{l}1961 \\
1962 \\
1963 \\
1964 \\
1965\end{array}$ & $\begin{array}{r}510 \\
1,000 \\
750 \\
480 \\
270\end{array}$ & $\begin{array}{r}590 \\
1,200 \\
750 \\
540 \\
500\end{array}$ & $\begin{array}{l}640 \\
830 \\
520 \\
430 \\
470\end{array}$ & $\begin{array}{l}650 \\
770 \\
410 \\
550 \\
700\end{array}$ & $\begin{array}{r}660 \\
1,100 \\
780 \\
620 \\
860\end{array}$ & $\begin{array}{r}750 \\
1,300 \\
680 \\
440 \\
760\end{array}$ & $\begin{array}{r}530 \\
3,600 \\
300 \\
270 \\
770\end{array}$ & $\begin{array}{l}2,700 \\
7,800 \\
2,100 \\
5,900 \\
3,400\end{array}$ & $\begin{array}{r}1,300 \\
9,100 \\
2,700 \\
8,500 \\
32,300\end{array}$ & $\begin{array}{r}170 \\
1,500 \\
330 \\
980 \\
19,400\end{array}$ & $\begin{array}{r}400 \\
690 \\
470 \\
420 \\
12,000\end{array}$ & $\begin{array}{r}420 \\
420 \\
820 \\
80 \\
* 5,000\end{array}$ & $\begin{array}{r}9,320 \\
29,310 \\
10,620 \\
19,210 \\
76,930\end{array}$ \\
\hline
\end{tabular}

* Monthly regression not used. Estimated on basis of equal flow per unit area. 

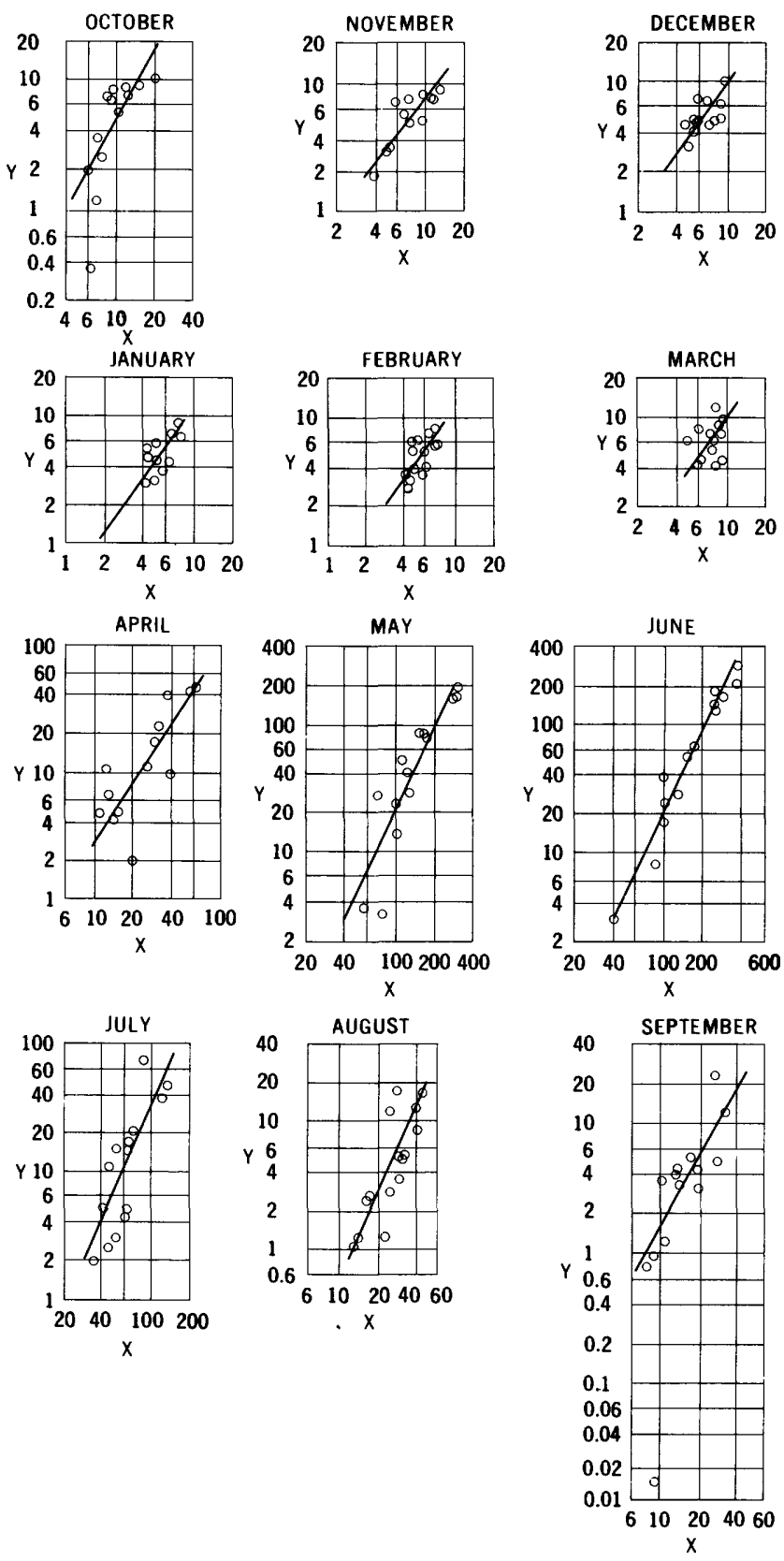

Relationships of monthly mean discharge of Ferron Creek near Castle Dale, Utah $(Y)$, to monthly mean discharge of Ferron Creek (upper station) near Ferron, Utah (X). Discharge in hundreds of acre-feet. 


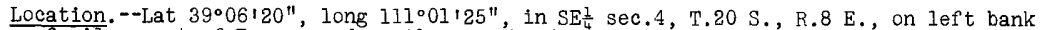
6 miles east of Ferron and 7 miles south of Castle Dale. Altitude of' gage is $5,550 \mathrm{ft}$ (from topographic map).

Drainage area. $--210 \mathrm{sq} \mathrm{mi}$, approximately.

Records avaliable.--June 1911 to September 1914, December 1947 to September 1958.

Estimates of streamflow.--October 1958 to September 1955, based on relationships of monthIy mean discharge with Ferron Creek (upper station) near Ferron, Utah. The regression equation used is:

$\log \mathrm{Y}=\mathrm{b} \log \mathrm{X}-\mathrm{c}$

(where $\mathrm{Y}$ is discharge of Ferron Creek near Castle Dale, Utah, and $\mathrm{X}$ is discharge of Ferron Creek (upper station) near Ferron, Utah, both in acre-feet per month).

Monthly values of constants in above equation

\begin{tabular}{l|l|l|l|l|l|l|l|l|l|l|l|l}
\hline & oct. & Nov. & Dec. & Jan. & Feb. & Mar. & Apr. & May & June & Ju1y & Aug. & Sept. \\
\hline b & 1.69 & 1.30 & 1.40 & 1.40 & 1.40 & 1.40 & 1.55 & 2.13 & 2.14 & 2.33 & 2.25 & 1.76 \\
c & 2.39 & 1.00 & 1.18 & 1.13 & 1.11 & 1.20 & 2.21 & 5.17 & 5.23 & 5.80 & 5.01 & 3.04 \\
\hline
\end{tabular}

Average discharge. --17 years $(1947-65), 19,840$ acre-feet per year $(27.4 \mathrm{cfs})$.

Extremes.--1947-58: Maximum discharge, 1,630 cfs Aug. 3, 1951, from rating curve extended above $650 \mathrm{cf}$ s on basis of slope-area measurements; no flow for several days in September and october 1955.

Remarks.--Flow slightly regulated by small reservoir in mountains (capacity unknown). Many diversions for irrigation above station. Estinates of annual flow are within about 10 percent of regression line.

Monthly and annual streamflow, in acre-feet

\begin{tabular}{c|c|r|r|r|r|r|r|r|r|r|r|r|r}
\hline $\begin{array}{c}\text { Water } \\
\text { year }\end{array}$ & Oct. & Nov. & Dec. & Jan. & Feb. & Mar. & Apr. & May & June & July & Aug. & Sept. & Annual \\
\hline 1948 & - & - & 492 & 430 & 403 & 466 & 1,050 & 4,200 & 3,870 & 509 & 262 & 90 & -5 \\
1949 & 247 & 444 & 399 & 307 & 362 & 1,190 & 4,340 & 7,840 & 13,110 & 1,450 & 862 & 324 & 30,880 \\
1950 & 547 & 494 & 492 & 544 & 550 & 556 & 1,110 & 2,380 & 2,400 & 1,500 & 128 & 119 & 10,820 \\
1951 & 342 & 509 & 462 & 369 & 359 & 408 & 202 & 5,260 & 5,550 & 442 & 1,760 & 424 & 16,090 \\
1952 & 695 & 716 & 732 & 619 & 760 & 865 & 4,600 & 19,780 & 29,600 & 3,800 & 1,710 & 1,210 & 65,090 \\
1953 & 900 & 817 & 676 & 676 & 635 & 735 & 487 & 371 & 6,790 & 1,730 & 1,180 & 307 & 15,300 \\
1954 & 837 & 758 & 714 & 700 & 609 & 640 & 967 & 1,420 & 291 & 202 & 104 & 349 & 7,590 \\
1955 & 196 & 311 & 486 & 430 & 395 & 646 & 473 & 330 & 802 & 15 & 496 & 16 & 4,580 \\
1956 & 34 & 189 & 314 & 292 & 325 & 450 & 134 & 2,890 & 1,740 & 322 & 121 & 76 & 6,890 \\
1957 & 118 & 296 & 461 & 461 & 653 & 425 & 433 & 2,800 & 21,170 & 4,850 & 1,310 & 488 & 33,460 \\
1958 & 1,020 & 889 & 1,020 & 861 & 807 & 958 & 4,010 & 16,490 & 14,340 & 506 & 356 & 443 & 41,700 \\
\hline
\end{tabular}

Estimated monthly and annual streamflow, in acre-feet

\begin{tabular}{c|c|r|r|r|r|r|r|r|r|r|r|r|r}
\hline $\begin{array}{c}\text { Water } \\
\text { year }\end{array}$ & oct. & Nov. & Dec. & Jan. & Feb. & Mar. & Apr. & May & June & July & Aug. & Sept. & Annual \\
\hline $\mathbf{1 9 5 9}$ & 300 & 410 & 350 & 280 & 380 & 430 & 750 & 620 & 560 & 230 & 90 & 90 & 4,490 \\
1960 & 130 & 230 & 160 & 140 & 210 & 870 & 1,200 & 2,400 & 2,300 & 590 & 80 & 150 & 8,460 \\
& & & & & & & & & & & & \\
1961 & 340 & 300 & 240 & 220 & 230 & 410 & 390 & 1,100 & 550 & 290 & 300 & 280 & 4,650 \\
1962 & 510 & 670 & 580 & $\mathbf{5 4 0}$ & 660 & 980 & 5,800 & 7,500 & 11,700 & 1,900 & 840 & 300 & 31,980 \\
1963 & 540 & 290 & 160 & 110 & 230 & 390 & 260 & 2,900 & 2,500 & 420 & 710 & 440 & 8,950 \\
1964 & 250 & 370 & 260 & 280 & 310 & 390 & 260 & 2,300 & 3,600 & 590 & 540 & 180 & 9,330 \\
1965 & 260 & 460 & 520 & 590 & 540 & 400 & 860 & 2,100 & 21,000 & 8,000 & 1,600 & 740 & 37,070 \\
\hline
\end{tabular}



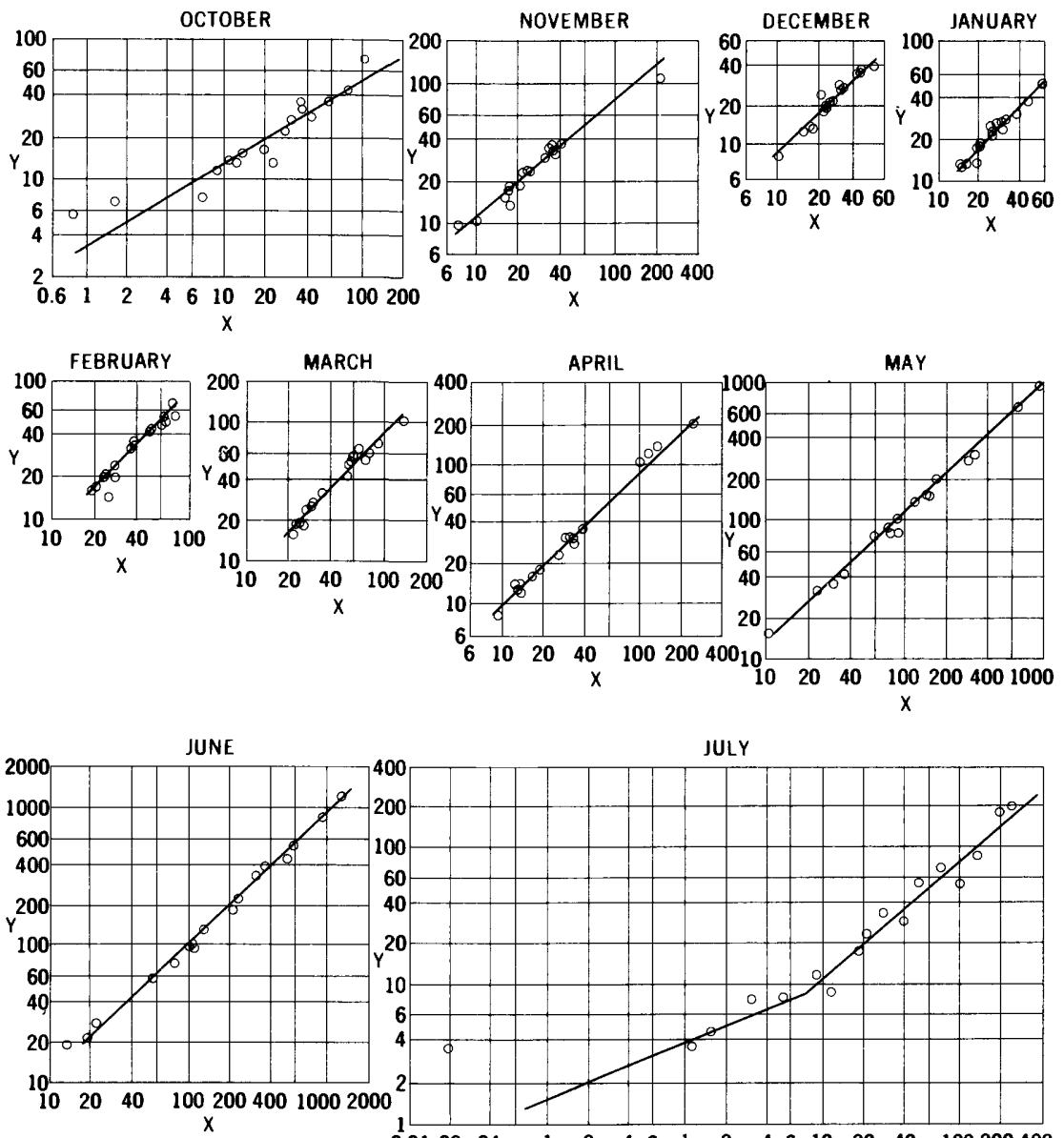

JULY
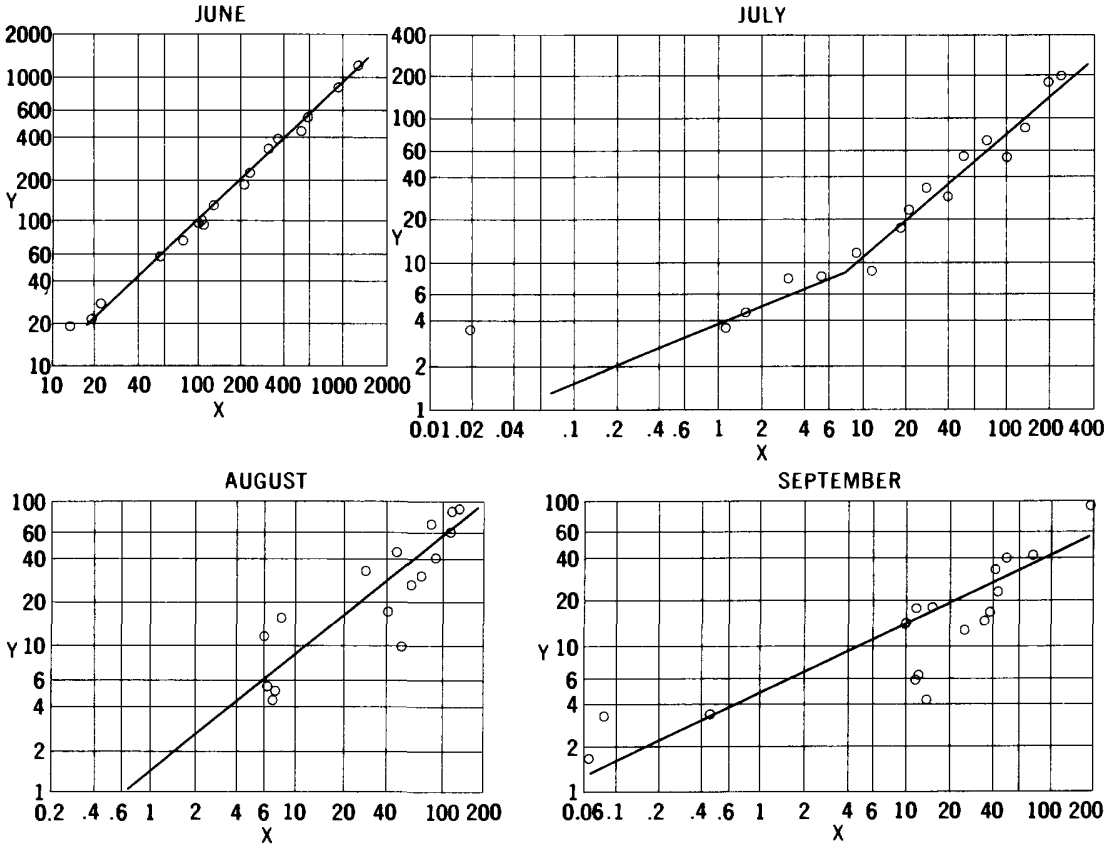

Relationships of monthly mean discharge of San Rafael River near Castle Dale, Utah (Y), to monthly mean discharge of San Rafael River near Green River, Utah (X). Discharge in hundreds of acre-feet. 
3280. San Rafael River near Castle Dale, Utah

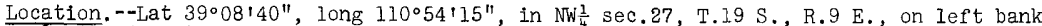
1 mile downstream from Ferron Creek and 8 miles southeast of Cástie Daie. Altitude of gage is 5,320 ft (from river-profile map).

Drainage area. $--927 \mathrm{sq} \mathrm{mi}$.

Records available.--October 1947 to September 1964.

Estimates of streamflow.--october 1945 to September 1947, October 1964 to September 1965, based on relationships of monthly mean discharge with San Rafael River near Green River, Utah. The regression equation used is:

$\log Y=b \log X-c$

(where $Y$ is discharge of San Rafael River near Castle Dale, Utah, and $X$ is discharge of San Rafael River near Green River, Utah, both in acre-f'eet per month).

\begin{tabular}{l|r|r|r|r|r|r|r|r|r|r|r|r}
\hline \multicolumn{10}{c}{ Monthly values of constants in above equation } \\
\hline b & Oct. & Nov. & Dec. & Jan. & Feb. & Mar. & Apr. & May & June & July & Aug. & Sept. \\
\hline $\mathrm{c}$ & -1.59 & 0.83 & 0.94 & 1.02 & 1.02 & 1.05 & 0.94 & 0.94 & 0.95 & 0.86 & 0.80 & 0.48 \\
\hline
\end{tabular}

Average discharge. --20 years $(1945-65), 85,680$ acre-feet per year (118 cfs).

Extremes. --1947-64: Maximum discharge, 4,510 cfs June 3, 1952; minimum recorded, 1.3 cfs Sept. $4,6,1956$.

Remarks.--Diversions for imigation above station, including transmountain diversions to Sevier Lake basin. Estimates of annual flow are within about 10 percent of regression line.

Monthly and annual streamflow, in acre-feet

\begin{tabular}{|c|c|c|c|c|c|c|c|c|c|c|c|c|c|}
\hline $\begin{array}{l}\text { Water } \\
\text { year }\end{array}$ & oet. & Nov. & Dec. & Jan. & Feb. & Mar. & Apr. & May & June & July & Aug. & Sept. & Annual \\
\hline $\begin{array}{l}1948 \\
1949 \\
1950\end{array}$ & $\mid \begin{array}{l}1,640 \\
1,180 \\
2,280\end{array}$ & 1, & $\begin{array}{l}610 \\
890 \\
180\end{array}$ & 2,1 & & $\begin{array}{l}20 \\
30 \\
30\end{array}$ & $\begin{array}{r}3 \\
20 \\
3\end{array}$ & & & & & $\begin{array}{r}327 \\
1,340 \\
1,410\end{array}$ & $\begin{array}{r}62,240 \\
116,700 \\
50,940\end{array}$ \\
\hline $\begin{array}{l}1951 \\
1952 \\
1953 \\
1954 \\
1955\end{array}$ & $\begin{array}{l}1,360 \\
3,770 \\
3,540 \\
3,260 \\
1,320\end{array}$ & & & $\begin{array}{l}2,150 \\
2,490 \\
5,180 \\
3,050 \\
1,840\end{array}$ & & $\begin{array}{r}1,920 \\
10,330 \\
5,130 \\
3,220 \\
6,620\end{array}$ & $\begin{array}{r}1,410 \\
20,980 \\
2,900 \\
3,140 \\
2,360\end{array}$ & $\begin{array}{r}15,510 \\
98,400 \\
3,150 \\
8,590 \\
4,190\end{array}$ & $\begin{array}{r}23,020 \\
125,900 \\
32,020 \\
1,860 \\
5,680\end{array}$ & $\begin{array}{r}3,390 \\
18,660 \\
5,490 \\
884 \\
786\end{array}$ & & $\begin{array}{l}850 \\
200 \\
830 \\
780 \\
334\end{array}$ & $\begin{array}{r}66,660 \\
301,100 \\
78,810 \\
37,360 \\
32,090\end{array}$ \\
\hline $\begin{array}{l}1956 \\
1957 \\
1958 \\
1959 \\
1960\end{array}$ & $\begin{array}{r}711 \\
594 \\
7,350 \\
1,540 \\
752\end{array}$ & $\begin{array}{r}1,030 \\
950 \\
11,270 \\
2,390 \\
1,370\end{array}$ & $\begin{array}{r}2,010 \\
924 \\
3,940 \\
3,390 \\
1,340\end{array}$ & $\begin{array}{l}2,730 \\
1,360 \\
3,810 \\
2,550 \\
1,350\end{array}$ & & $\begin{array}{l}2,410 \\
1,840 \\
5,910 \\
2,550 \\
6,370\end{array}$ & $\begin{array}{r}1,320 \\
1,460 \\
14,490 \\
1,670 \\
3,160\end{array}$ & $\begin{array}{r}13,840 \\
8,460 \\
69,280 \\
1,510 \\
9,020\end{array}$ & $\begin{array}{r}7,520 \\
86,280 \\
55,290 \\
2,710 \\
9,800\end{array}$ & $\begin{array}{r}1,150 \\
19,420 \\
2,350 \\
367 \\
456\end{array}$ & $\begin{array}{l}438 \\
, 080 \\
, 740 \\
506 \\
119\end{array}$ & $\begin{array}{r}171 \\
3,460 \\
2,470 \\
644 \\
615\end{array}$ & $\begin{array}{r}35,840 \\
137,100 \\
185,000 \\
23,460 \\
36,010\end{array}$ \\
\hline $\begin{array}{l}1961 \\
1962 \\
1963 \\
1964\end{array}$ & $\begin{array}{l}4,380 \\
2,640 \\
2,980 \\
1,360\end{array}$ & $\begin{array}{l}1,900 \\
3,350 \\
2,360 \\
1,540\end{array}$ & $\begin{array}{l}1,390 \\
2,490 \\
1,950 \\
1,470\end{array}$ & $\begin{array}{l}1,390 \\
2,450 \\
1,850 \\
1,240\end{array}$ & $\begin{array}{l}1,470 \\
5,620 \\
3,550 \\
2,100\end{array}$ & $\begin{array}{l}1,960 \\
5,010 \\
1,630 \\
2,750\end{array}$ & $\begin{array}{r}1,800 \\
12,610 \\
873 \\
1,220\end{array}$ & $\begin{array}{r}3,530 \\
27,480 \\
7,860 \\
15,530\end{array}$ & $\begin{array}{r}2,110 \\
37,800 \\
9,930 \\
18,610\end{array}$ & $\begin{array}{r}354 \\
7,080 \\
805 \\
2,980\end{array}$ & & $\begin{array}{r}9,970 \\
1,510 \\
4,310 \\
438\end{array}$ & $\begin{array}{r}33,350 \\
109,600 \\
42,290 \\
51,920\end{array}$ \\
\hline
\end{tabular}

Estimated monthly and annual streamflow, in acre-feet

\begin{tabular}{r|r|r|r|r|r|r|r|r|r|r|r|r|r}
\hline $\begin{array}{l}\text { Water } \\
\text { year }\end{array}$ & Oct. & Nov. & Dec. & Jan. & Feb. & Mar. & Apr. & May & June & July & Aug. & Sept. & Annual \\
\hline 1946 & 2,500 & 2,700 & 1,900 & 1,600 & 3,500 & 5,100 & 9,600 & 22,600 & 8,300 & 1,200 & 4,200 & 920 & 64,120 \\
1947 & 2,300 & 4,100 & 3,100 & 2,000 & 4,800 & 3,500 & 3,200 & 36,300 & 25,500 & 4,300 & 9,500 & 2,400 & 101,000 \\
1965 & 410 & 1,300 & 2,700 & 3,100 & 2,800 & 2,700 & 5,100 & 20,500 & 72,000 & 26,300 & 8,000 & 3,100 & 148,000 \\
\hline
\end{tabular}



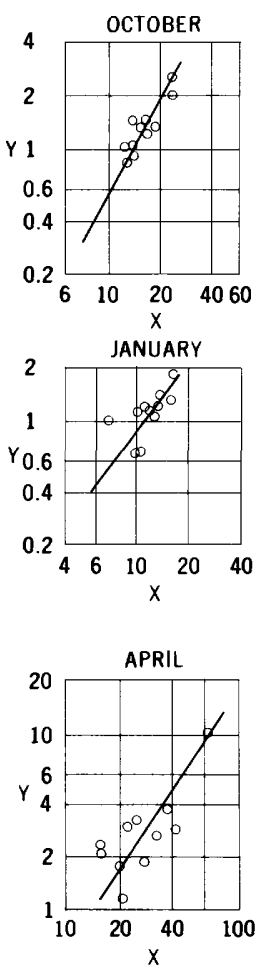
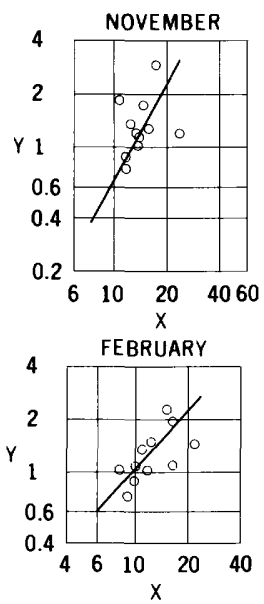
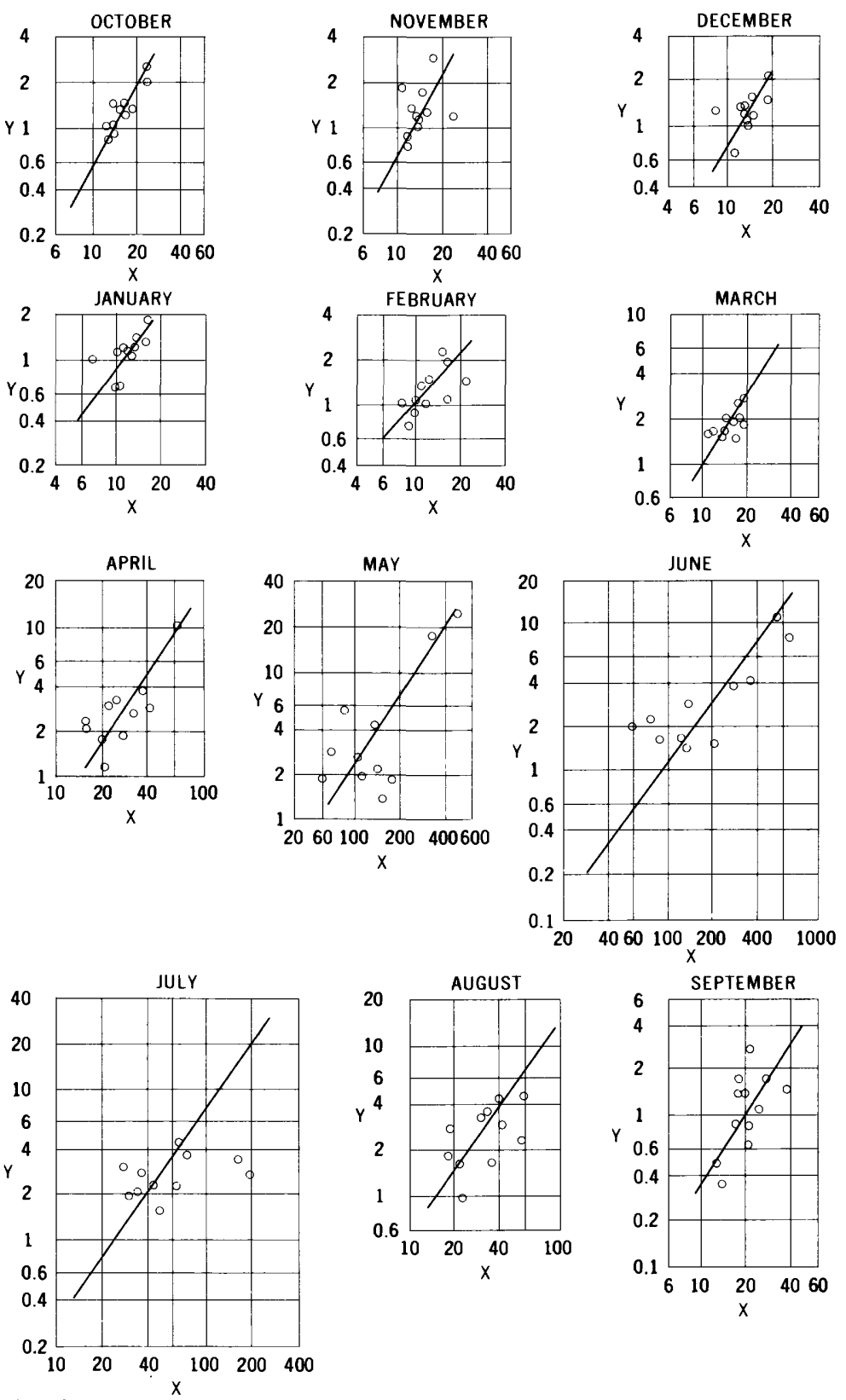

Relationships of monthly mean discharge of Ivie Creek above diversions, near Emery, Utah $(Y)$, to monthly mean discharge of Cottonwood Creek near Orangeville, Utah $(X)$. Discharge in hundreds of acre-feet. 
3315. Ivie Creek above diversions, near Emery, Utah

Location.--Lat $38^{\circ} 45^{1} 30^{\prime \prime}$, long $111^{\circ} 25^{\prime} 15^{\prime \prime}$, in NW $\frac{1}{4} N W_{\frac{1}{4}}$ sec.1, T.24 S., R.4 E., on right bank $l \frac{1}{4}$ miles downstream from Clear Creek and 14 miles southwest of Emery. Altitude of gage is $7,100 \mathrm{ft}$ (by barometer).

Drainage area. - -50 sq mi, approximately.

Records available. - -August 1950 to September 1961.

Estimates of streamflow.--May 1932 to August 1950, october 1961 to September 1965, based on relationships of monthly mean discharge with cottonwood Creek near Orangevilie,

Utah. The regression equation used is:

$$
\log Y=b \log X-c
$$

(where $Y$ is discharge of IVie Creek above diversions, near Emery, Utah, and $X$ is discharge of Cottonwood Creek near Orangeville, Utah, both in acre-feet per month).

\begin{tabular}{l|l|l|l|l|l|l|l|l|l|l|l|l}
\hline \multicolumn{10}{c}{ Monthly values of constants in above equation } \\
\hline & Oct. & Nov. & Dec. & Jan. & Feb. & Mar. & Apr. & May & June & July & Aug. & Sept. \\
\hline b & 1.77 & 1.77 & 1.67 & 1.30 & 1.10 & 1.60 & 1.50 & 1.53 & 1.39 & 1.39 & 1.39 & 1.60 \\
c & 3.59 & 3.48 & 3.12 & 1.98 & 1.26 & 2.79 & 2.73 & 3.73 & 2.50 & 2.68 & 2.39 & 3.17 \\
\hline
\end{tabular}

Average discharge. --33 years $(1932-65), 3,199$ acre-feet per year (4.42 cf's).

Extremes.--1950-61: Maximum discharge, about 700 cfs Aug. 16, 1955, from rating curve extended above $70 \mathrm{cfs}$ by logarithmic plotting; no flow at times.

Remarks. - Flow partly regulated by Sheep Valley Reservoir (capacity, 482 acre-ft). Small diversion for irrigation of 200 acres of meadow above station. Estimates of annual flow are within about 25 percent of regression line.

\begin{tabular}{l|r|r|r|r|r|r|r|r|r|r|r|r|r}
$\begin{array}{l}\text { Water } \\
\text { year }\end{array}$ & Oct. & Nov. & Dec. & Jan. & Feb. & Mar. & Apr. & May & June & July & Aug. & Sept. & Annual \\
\hline 1950 & - & - & - & - & - & - & - & - & - & - & - & 174 & - \\
1951 & 148 & 174 & 152 & 122 & 143 & 201 & 117 & 137 & 158 & 223 & 444 & 153 & 2,170 \\
1952 & 146 & 104 & 99 & 107 & 109 & 204 & 1,000 & 2,440 & 809 & 338 & 468 & 188 & 6,010 \\
1953 & 253 & 286 & 215 & 184 & 194 & 277 & 327 & 284 & 383 & 362 & 304 & 79 & 3,150 \\
1954 & 132 & 128 & 113 & 144 & 222 & 257 & 280 & 220 & 200 & 205 & 167 & 102 & 2,170 \\
1955 & 106 & 138 & 131 & 111 & 109 & 168 & 232 & 193 & 284 & 155 & 169 & 108 & 1,900 \\
1956 & 91 & 89 & 109 & 111 & 105 & 147 & 185 & 189 & 163 & 232 & 97 & 43 & 1,560 \\
1957 & 85 & 77 & 65 & 67 & 88 & 160 & 209 & 555 & 1,120 & 265 & 238 & 216 & 3,140 \\
1958 & 203 & 120 & 145 & 129 & 145 & 185 & 371 & 1,750 & 415 & 442 & 362 & 133 & 4,400 \\
1959 & 124 & 114 & 133 & 117 & 133 & 152 & 173 & 190 & 168 & 198 & 187 & 53 & 1,750 \\
1960 & 103 & 183 & 124 & 98 & 104 & 198 & 265 & 444 & 144 & 275 & 282 & 213 & 2,430 \\
1961 & 130 & 116 & 117 & 66 & 72 & 168 & 293 & 263 & 221 & 300 & 340 & 340 & 2,430 \\
\hline
\end{tabular}

\begin{tabular}{|c|c|c|c|c|c|c|c|c|c|c|c|c|c|}
\hline $\begin{array}{l}\text { Water } \\
\text { year }\end{array}$ & Oct. & Nov. & Dec. & Jan. & $\mathrm{Feb}$. & Mar. & Apr. & May & June & July & Aug. & Sept. & Annual \\
\hline $\begin{array}{l}1932 \\
1933 \\
1934 \\
1935\end{array}$ & $\begin{array}{l}- \\
90 \\
40 \\
30\end{array}$ & $\begin{array}{r}110 \\
70 \\
40\end{array}$ & $\begin{array}{l}70 \\
90 \\
50\end{array}$ & $\begin{array}{l}- \\
40 \\
70 \\
60\end{array}$ & $\begin{array}{l}- \\
60 \\
80 \\
80\end{array}$ & $\begin{array}{l}110 \\
130 \\
120\end{array}$ & $\begin{array}{l}- \\
150 \\
720 \\
180\end{array}$ & $\begin{array}{l}620 \\
130 \\
140 \\
210\end{array}$ & $\begin{array}{r}310 \\
590 \\
20 \\
1,000\end{array}$ & $\begin{array}{r}370 \\
710 \\
40 \\
400\end{array}$ & $\begin{array}{r}250 \\
400 \\
80 \\
200\end{array}$ & $\begin{array}{r}90 \\
90 \\
20 \\
70\end{array}$ & $\begin{array}{l}- \\
2,550 \\
1,500 \\
2,440\end{array}$ \\
\hline $\begin{array}{l}1936 \\
1937 \\
1938 \\
1939 \\
1940\end{array}$ & $\begin{array}{r}60 \\
110 \\
120 \\
110 \\
110\end{array}$ & $\begin{array}{r}100 \\
150 \\
100 \\
130 \\
80\end{array}$ & $\begin{array}{r}60 \\
110 \\
110 \\
110 \\
80\end{array}$ & $\begin{array}{r}70 \\
80 \\
100 \\
100 \\
70\end{array}$ & $\begin{array}{r}90 \\
110 \\
120 \\
100 \\
100\end{array}$ & $\begin{array}{l}120 \\
170 \\
200 \\
620 \\
160\end{array}$ & $\begin{array}{l}750 \\
370 \\
660 \\
980 \\
580\end{array}$ & $\begin{array}{r}2,000 \\
1,300 \\
900 \\
900 \\
1,500\end{array}$ & $\begin{array}{l}310 \\
230 \\
350 \\
130 \\
160\end{array}$ & $\begin{array}{l}480 \\
430 \\
230 \\
150 \\
140\end{array}$ & $\begin{array}{l}390 \\
280 \\
240 \\
140 \\
100\end{array}$ & $\begin{array}{l}130 \\
190 \\
130 \\
110 \\
130\end{array}$ & $\begin{array}{l}4,560 \\
3,530 \\
3,260 \\
3,570 \\
2,910\end{array}$ \\
\hline $\begin{array}{l}1941 \\
1942 \\
1943 \\
1944 \\
1945\end{array}$ & $\begin{array}{l}100 \\
330 \\
120 \\
110 \\
130\end{array}$ & $\begin{array}{l}100 \\
310 \\
130 \\
110 \\
130\end{array}$ & $\begin{array}{l}110 \\
230 \\
150 \\
110 \\
130\end{array}$ & $\begin{array}{r}90 \\
160 \\
120 \\
80 \\
110\end{array}$ & $\begin{array}{l}100 \\
160 \\
140 \\
110 \\
120\end{array}$ & $\begin{array}{l}190 \\
400 \\
230 \\
170 \\
130\end{array}$ & $\begin{array}{r}190 \\
1,300 \\
1,100 \\
200 \\
150\end{array}$ & $\begin{array}{r}I, 300 \\
960 \\
460 \\
I, 000 \\
580\end{array}$ & $\begin{array}{l}540 \\
530 \\
160 \\
800 \\
330\end{array}$ & $\begin{array}{l}500 \\
440 \\
220 \\
840 \\
500\end{array}$ & $\begin{array}{l}330 \\
240 \\
290 \\
260 \\
390\end{array}$ & $\begin{array}{r}150 \\
100 \\
80 \\
110 \\
120\end{array}$ & $\begin{array}{l}3,700 \\
5,160 \\
3,200 \\
3,900 \\
2,820\end{array}$ \\
\hline $\begin{array}{l}1946 \\
1947 \\
1948 \\
1949 \\
1950\end{array}$ & $\begin{array}{l}140 \\
110 \\
160 \\
130 \\
140\end{array}$ & $\begin{array}{r}180 \\
90 \\
160 \\
170 \\
150\end{array}$ & $\begin{array}{r}140 \\
80 \\
130 \\
160 \\
140\end{array}$ & $\begin{array}{l}120 \\
100 \\
110 \\
110 \\
120\end{array}$ & $\begin{array}{l}120 \\
140 \\
120 \\
130 \\
140\end{array}$ & $\begin{array}{l}280 \\
320 \\
160 \\
210 \\
220\end{array}$ & $\begin{array}{r}1,300 \\
450 \\
190 \\
820 \\
440\end{array}$ & $\begin{array}{r}640 \\
I, 100 \\
600 \\
940 \\
440\end{array}$ & $\begin{array}{l}140 \\
230 \\
180 \\
520 \\
190\end{array}$ & $\begin{array}{l}200 \\
470 \\
240 \\
670 \\
270\end{array}$ & $\begin{array}{l}180 \\
390 \\
230 \\
320 \\
220\end{array}$ & $\begin{array}{r}70 \\
160 \\
90 \\
110 \\
-\end{array}$ & $\begin{array}{l}3,510 \\
3,640 \\
2,370 \\
4,290 \\
2,640\end{array}$ \\
\hline $\begin{array}{l}1962 \\
1963 \\
1964 \\
1965\end{array}$ & $\begin{array}{r}230 \\
180 \\
120 \\
70\end{array}$ & $\begin{array}{l}240 \\
150 \\
100 \\
100\end{array}$ & $\begin{array}{r}160 \\
90 \\
70 \\
80\end{array}$ & $\begin{array}{r}110 \\
50 \\
70 \\
100\end{array}$ & $\begin{array}{r}170 \\
110 \\
90 \\
130\end{array}$ & $\begin{array}{r}360 \\
150 \\
80 \\
180\end{array}$ & $\begin{array}{r}1,100 \\
120 \\
110 \\
280\end{array}$ & $\begin{array}{l}540 \\
230 \\
440 \\
310\end{array}$ & $\begin{array}{l}270 \\
120 \\
250 \\
820\end{array}$ & $\begin{array}{r}530 \\
190 \\
400 \\
2,900\end{array}$ & $\begin{array}{r}320 \\
280 \\
270 \\
1,400\end{array}$ & $\begin{array}{r}140 \\
160 \\
90 \\
440\end{array}$ & $\begin{array}{l}4,170 \\
1,830 \\
2,090 \\
6,810\end{array}$ \\
\hline
\end{tabular}



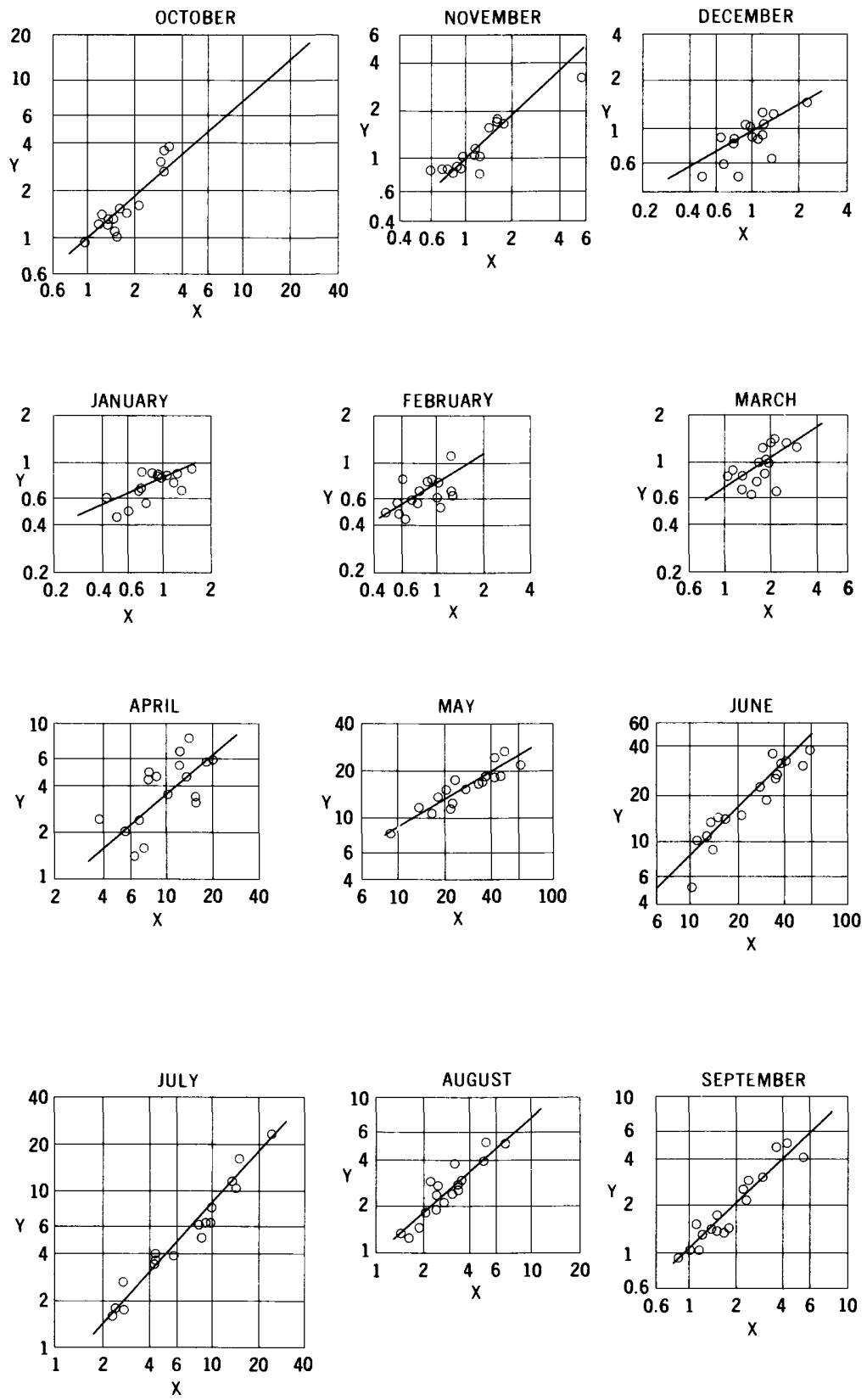

Relationships of monthly mean discharge of West Fork San Juan River above Borns Lake, near Pagosa Springs, Colo. (Y), to monthly mean discharge of East Fork San Juan River near Pagosa Springs, Colo. (X). Discharge in thousands of acre-feet. 
3405. West Fork San Juan River above Borns Lake, near Pagosa Springs, Colo.

Location.--Lat 37\%29100", long 106.55150", in sec.36, T.38 N., R.1 W., on right bank half a mile downstream from Beaver Creek, $1 \frac{1}{2}$ miles upstream from Borns Lake, and 16 miles northwest of Pagosa Springs. Altitude of gage is $8,400 \mathrm{ft}$ (from topographic map).

Drainage area. $--41.2 \mathrm{sq} \mathrm{ml}$.

Records available. --April 1937 to September 1953.

Estimates of streamflow. --May 1935 to March 1937, October 1953 to September 1965, based on relationships of monthly mean discharge with East Fork San Juan River near Pagosa Springs, Colo. The regression equation used is:

$$
\log Y=b \log X-c
$$

(where $Y$ is discharge of West Fork San Juan River above Borns Lake near Pagosa Springs, Colo., and $X$ is discharge of East Fork. San Juan River near Pagosa Springs, Colo., both in acre-feet per month).

Monthly values of constants in above equation

\begin{tabular}{l|l|l|l|r|r|r|r|r|r|r|r|r}
\hline & Oct. & Nov. & Dec. & Jan. & Feb. & Mar. & Apr. & May & June & July & Aug. & Sept. \\
\hline $\mathrm{b}$ & 0.88 & 0.88 & 0.55 & 0.45 & 0.62 & 0.66 & 0.85 & 0.58 & 1.01 & 1.10 & 0.88 & 0.98 \\
$\mathrm{c}$ & -.38 & -.36 & -1.33 & -1.55 & -1.00 & -.86 & -.14 & -1.62 & .14 & .49 & -.35 & -.06 \\
\hline
\end{tabular}

Average discharge. --30 years $(1935-65), 57,480$ acre-feet per year $(79.4$ cfs).

Extremes.--1937-53: Maximum discharge, 1,290 cfs June 18, 1949, from rating curve extended above $750 \mathrm{cfs}$; minimum daily, 6.0 cfs Jan. 30, 31, Feb. 1, 1951 .

Maximum flood known occurred óct. 5 , 1911.

Pemarks. - No regulation or diversion. Estimates of annual flow are within about 25 percent of regression line.

Monthly and annual streamflow, in acre-feet

\begin{tabular}{r|r|r|r|r|r|r|r|r|r|r|r|r|r}
\hline $\begin{array}{l}\text { Water } \\
\text { year }\end{array}$ & oct. & Nov. & Dec. & Jan. & Feb. & Mar. & Apr. & May & June & July & Aug. & Sept. & Annual \\
\hline 1937 & - & - & - & - & - & - & 5,590 & 26,840 & 19,250 & 5,020 & 2,320 & 1,390 & - \\
1938 & 1,540 & 873 & 492 & 553 & 543 & 676 & 5,880 & 16,720 & 34,970 & 7,840 & 2,900 & 5,120 & 78,110 \\
1939 & 3,680 & 1,690 & 1,030 & 669 & 445 & 1,320 & 3,450 & 17,560 & 10,490 & 1,600 & 1,260 & 3,030 & 46,220 \\
1940 & 1,380 & 867 & 492 & 461 & 489 & 1,440 & 4,630 & 11,380 & 5,160 & 1,770 & 1,480 & 2,570 & 32,120 \\
1941 & 3,140 & 1,580 & 1,290 & 861 & 1,110 & 1,360 & 1,420 & 21,600 & 31,800 & 23,060 & 4,950 & 4,140 & 96,310 \\
1942 & 10,330 & 3,160 & 1,480 & 922 & 666 & 861 & 3,330 & 16,980 & 26,030 & 6,330 & 1,910 & 1,350 & 73,350 \\
1943 & 1,030 & 793 & 641 & 676 & 611 & 1,030 & 8,240 & 15,170 & 14,660 & 3,920 & 3,710 & 1,720 & 52,200 \\
1944 & 1,120 & 1,120 & 1,080 & 827 & 678 & 829 & 1,590 & 18,170 & 33,290 & 11,470 & 2,090 & 1,010 & 73,270 \\
1945 & 1,670 & 1,030 & 910 & 752 & 532 & 769 & 2,440 & 18,130 & 23,500 & 6,210 & 2,360 & 1,050 & 59,350 \\
& & & & & & & & & & & & & \\
1946 & 1,350 & 811 & 593 & 499 & 479 & 681 & 4,990 & 7,920 & 11,270 & 2,640 & 2,740 & 2,140 & 36,110 \\
1947 & 2,660 & 1,610 & 1,280 & 801 & 740 & 1,040 & 2,040 & 12,320 & 14,790 & 3,620 & 3,920 & 4,740 & 49,560 \\
1948 & 3,920 & 1,740 & 1,100 & 825 & 601 & 633 & 3,040 & 24,660 & 27,150 & 5,960 & 2,570 & 1,330 & 73,530 \\
1949 & 1,240 & 1,000 & 813 & 696 & 581 & 1,010 & 5,500 & 15,530 & 37,640 & 16,110 & 2,740 & 1,420 & 84,290 \\
1950 & 1,490 & 1,030 & 900 & 837 & 785 & 1,290 & 6,750 & 13,620 & 13,670 & 3,490 & 1,340 & 1,440 & 46,640 \\
1951 & 1,460 & 853 & 869 & 595 & 563 & 908 & 2,470 & 11,980 & 9,190 & 1,720 & 2,900 & 1,540 & 35,050 \\
1952 & 962 & 863 & 857 & 867 & 791 & 821 & 4,570 & 18,560 & 39,750 & 10,030 & 5,160 & 2,930 & 86,160 \\
1953 & 1,230 & 873 & 859 & 851 & 758 & 1,370 & 4,460 & 10,840 & 15,100 & 3,710 & 1,810 & 914 & 42,780 \\
\hline
\end{tabular}

Estimated monthly and annual streamflow, in acre-feet

\begin{tabular}{l|c|r|r|r|r|r|r|r|r|r|r|r|r}
\hline $\begin{array}{l}\text { Water } \\
\text { year }\end{array}$ & Oct. & Nov. & Dec. & Jan. & Feb. & Mar. & Apr. & May & June & July & Aug. & Sept. & Annual \\
\hline 1935 & - & - & - & - & - & - & - & 14,200 & 46,500 & 24,000 & 5,800 & 2,900 & - \\
1936 & 1,900 & 1,100 & 940 & 770 & 670 & 1,200 & 5,900 & 17,100 & 10,100 & 2,700 & 4,800 & 3,800 & 50,980 \\
1937 & 2,000 & 2,000 & 1,100 & 910 & 760 & 1,100 & - & - & - & - & - & - & 68,280 \\
1954 & 1,000 & 1,200 & 890 & 700 & 740 & 750 & 3,500 & 12,300 & 6,800 & 2,600 & 2,400 & 1,600 & 34,480 \\
1955 & 1,700 & 720 & 800 & 730 & 600 & 820 & 1,700 & 11,000 & 12,400 & 3,200 & 4,400 & 1,800 & 39,870 \\
1956 & 1,000 & 800 & 930 & 770 & 750 & 1,600 & 2,800 & 15,600 & 16,500 & 2,100 & 1,600 & 690 & 45,140 \\
1957 & 800 & 970 & 760 & 670 & 690 & 970 & 2,300 & 12,500 & 42,900 & 26,000 & 8,100 & 3,700 & 100,360 \\
1958 & 2,600 & 2,800 & 1,300 & 880 & 790 & 1,000 & 3,700 & 18,400 & 19,500 & 3,300 & 2,400 & 2,800 & 59,470 \\
1959 & 1,400 & 970 & 800 & 630 & 490 & 660 & 1,600 & 9,500 & 8,800 & 1,300 & 2,600 & 1,300 & 30,050 \\
1960 & 4,400 & 3,100 & 1,100 & 840 & 810 & 1,700 & 6,300 & 14,800 & 23,000 & 4,500 & 1,700 & 1,400 & 63,650 \\
1961 & 1,200 & 320 & 740 & 620 & 460 & 740 & 2,600 & 13,500 & 10,700 & 2,000 & 2,500 & 3,300 & 33,880 \\
1962 & 3,000 & 1,300 & 1,100 & 900 & 970 & 1,000 & 6,000 & 15,900 & 19,800 & 5,400 & 1,700 & 1,500 & 59,170 \\
1963 & 1,700 & 1,100 & 920 & 720 & 690 & 1,400 & 3,100 & 12,800 & 5,400 & 1,600 & 1,600 & 1,800 & 32,830 \\
1964 & 1,300 & 910 & 770 & 660 & 510 & 570 & 1,500 & 13,200 & 8,900 & 3,300 & 5,000 & 2,100 & 38,720 \\
1965 & 1,400 & 1,000 & 980 & 830 & 760 & 890 & 3,900 & 17,800 & 36,000 & 20,800 & 5,100 & 7,100 & 96,560 \\
\hline
\end{tabular}



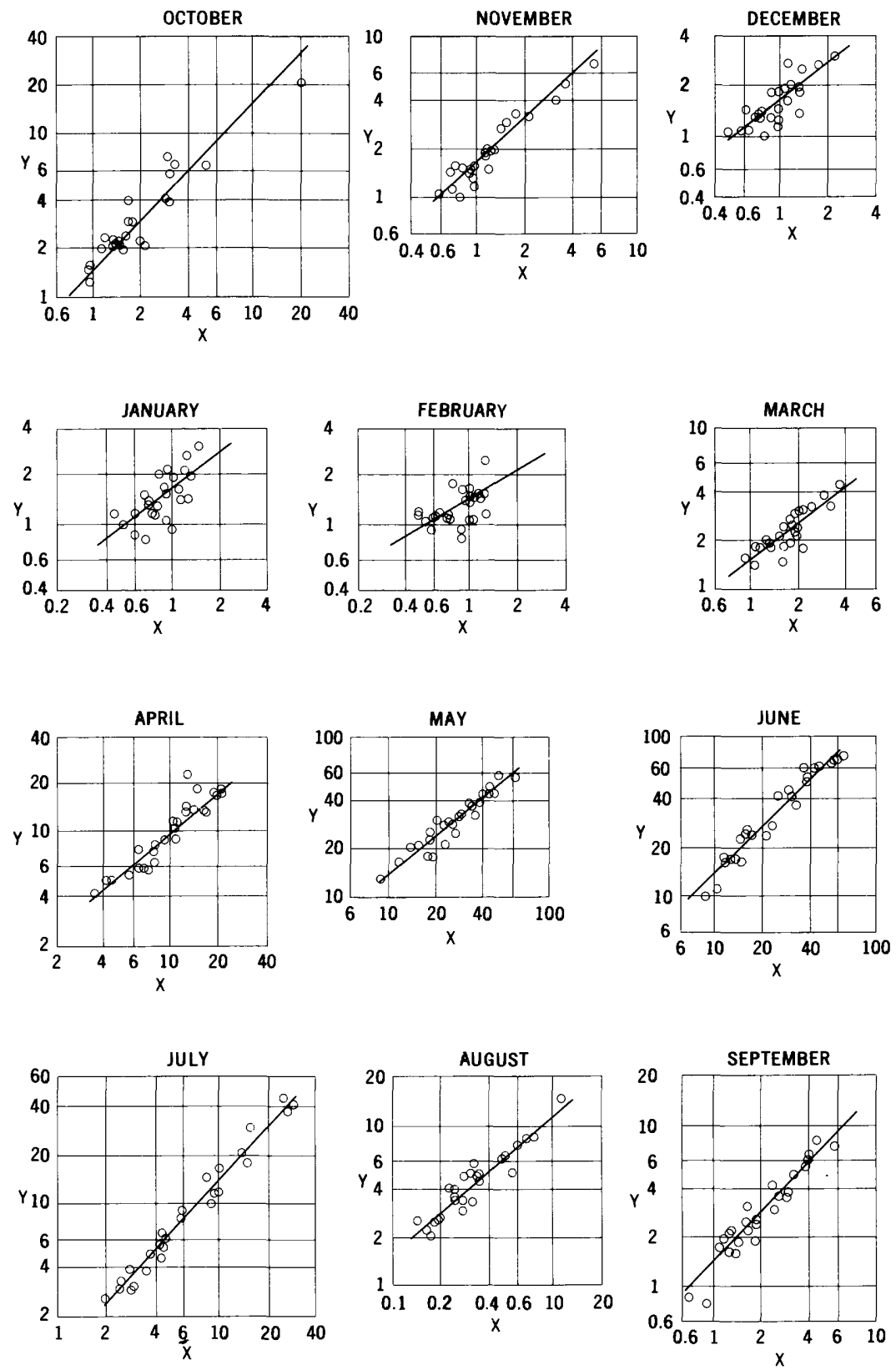

Relationships of monthly mean discharge of West Fork San Juan River near Pagosa Springs, Colo. ( $Y$ ), to monthly mean discharge of East Fork San Juan River near Pagosa Springs, Colo. (X). Discharge in thousands of acre-feet. 
3415. West Fork San Juan River near Pagosa Springs, Colo.

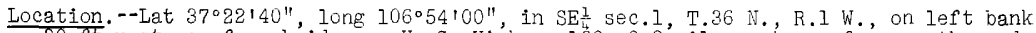
$30 \mathrm{ft}$ upstream from bridge on U. S. Highway lê, 0.9 mile upstream from mouth, and 10 miles northeast of Pagosa Springs. Datum of gage is $7,614.40 \mathrm{ft}$ above mean sea level, datum of 1929 .

Drainage area. $--87.9 \mathrm{sq} \mathrm{mi}$.

Records available. --Apiril 1935 to September 1960.

Estimates of streanflow.--October 1960 to Sertember 1965, based on relationships of monthly mean discharge with East Fork Sar Juan River near Pagosa Springs, Colo. The regression equation used is:

$\log Y=b \log X-c$

(where $Y$ is discharge of West Fork San Juan Fiver near Pagosa Springs, Colo,, and $X$ is discharge of East Fork San Juan River near Pagosa Springs, Colo., both in acre-feet per month).

\begin{tabular}{c|c|c|c|c|c|c|c|c|c|c|c|c}
\hline \multicolumn{10}{c}{ Monthly values of constants in above equation } \\
\hline & Oct. & Nov. & Dec. & Jan. & Feb. & Mar. & Apr. & May & June & July & Aug. & Sept. \\
\hline $\mathrm{b}$ & 1.03 & 0.83 & 0.76 & 0.75 & 0.57 & 0.76 & 0.47 & 0.81 & 1.03 & 1.09 & 0.05 & 1.04 \\
$\mathrm{c}$ & -.07 & -.56 & -.93 & -.98 & -1.47 & -.90 & -.52 & -.89 & -.04 & .01 & -.64 & .95 \\
\hline
\end{tabular}

Average discharge. - -30 years (1935-65), 110,320 acre-feet per year (152 cfs).

Extremes.--1935-60: Maximum discharge, 2,330 cfs June 15, 1952; minimum daily, 5.8 cfs Sept. $21,1956$.

Maximum flood known occurred act. 5, 1911.

Remarks.--Diversions above station for irrigation of about 700 acres above and 100 acres below station. Treasure pass ditch above station exports water to Rio Grande basin. Estimates of annul flow are within about 10 percent of regression line.

Monthly and annual streamflow, in acre-feet

\begin{tabular}{|c|c|c|c|c|c|c|c|c|c|c|c|c|c|}
\hline $\begin{array}{l}\text { water } \\
\text { year }\end{array}$ & Oct. & Nov. & Dec. & Jan. & Feb. & Mar. & Apr. & May & June & July & Aug. & Sept. & Annual \\
\hline 1935 & - & - & - & - & - & - & - & $\therefore 0,750$ & 74,480 & 37,190 & 8,210 & 3,910 & - \\
\hline 1936 & 2,150 & 1,920 & 470 & $92 ?$ & 863 & 3,100 & 17,060 & 0 & 10 & 4,710 &, 430 & 6,520 & 102,000 \\
\hline 1937 & & & & 120 & 1,46 & 0 & 17,100 & 57 & & & & & 148 \\
\hline 1938 & 2,360 & $1,5 \in 0$ & 998 & 1,160 & 1,110 & 1,860 & 17,630 & 37,010 & $F 6,700$ & 16,590 & 4,480 & 8,220 & 159,700 \\
\hline 1939 & & & & 1 & & & 10,770 & & & & & 5,040 & 82,230 \\
\hline 1940 & 2,140 & $1, f 10$ & 1,070 & 982 & 1,260 & 80 & 8,970 & 24,440 & 11 &, 250 & 2,570 & 4,340 & 65,430 \\
\hline 1941 & 7,020 & 710 & 0 & 2,140 & $1,6$. & 340 &, 820 & 55, & 0 & 44 & 40 & 640 & 15,800 \\
\hline 1342 & 20,5 & & & & & & $1 \approx, 600$ & & & 11 , & & 650 & 154,800 \\
\hline 1943 & 1,900 & 20 & 00 & 1,450 & & $\mathrm{CO}$ & 18,640 & 29,010 & & 7,870 & 80 & $\therefore, 570$ & $98,54 \mathrm{C}$ \\
\hline 4 & 2,050 & 1,860 & 1,870 & 1,710 & 1,910 & 1, & 5,780 & 44, & 85 & 20 & 00 & 1,660 & 152,000 \\
\hline & & 2,040 & 610 & 1,430 & 0 & & 5,880 & 39,360 & $4 \varepsilon$ & 11,200 & & 1,780 & 114,100 \\
\hline 1946 & 2,020 & 1,580 & 0 & 881 & 1,140 & 1,890 & 8,510 & 15, & 17 & $z$, & 590 & 100 & 180 \\
\hline 1947 & & & & , & & & & $: 7$ & & & & 990 & 070 \\
\hline 1948 & 6 , & 3 & 070 & 1,940 & & & $1.5,370$ & 48 & & 14 & & 2,260 & 157,900 \\
\hline 1949 & 1,970 & 1,380 &, 280 & $1,=30$ & 1, & & 15,280 & 31, & 65 & 29 & 00 & $=, 410$ & 156,800 \\
\hline 1950 & $=\quad$ & $=, 05 n$ & 1,260 & 1,330 & 1,460 & 2,740 & 14,210 & 27,910 & & 5,500 & 2,570 & 1,920 & 82,640 \\
\hline 1951 & 2, & 1 , & in & 1,15 & 954 & 0,1 & 10 & 120 & & 80 & & 050 & \\
\hline 1 & & & & & & & & & & 17, & & 3,690 & 171,200 \\
\hline 53 & 2,180 & 1,4 & 90 & 2,05 & & & 20 & & & & & 813 & 74,340 \\
\hline 1954 & 1,560 & & & 1,16 & & & 11,770 & 24, & 1 & 50 & 20 & 2,140 & 67,590 \\
\hline 1955 & $z, 850$ & 1,480 & $1,=20$ & 1,300 & 1.190 & $a^{\prime}$ & 4,970 & $=0$ & -5, & 10 & 5,150 & 1,940 & 74,140 \\
\hline $195 \mathrm{~h}$ & & & & 1,050 & 1 & & & & & & & 94 & \\
\hline 1957 & & & & & & & & & & 40,420 & & 5,650 & 160,400 \\
\hline & 3,920 & 4,070 & 3,710 & $\llcorner, 00$ & 1,6 & 1,960 & 11,750 & 44 & & & & 60 & 127,900 \\
\hline 1959 & 2,070 & & & 1,18 & & 1,600 & & 16,800 & & s. & & 2,200 & 58,300 \\
\hline 1960 & 6,650 & 5,020 & 1,910 & 1,670 & 500 & 4,510 & 18,260 & 27,300 & 46,050 & 8,810 & 2,500 & 1,630 & 125,300 \\
\hline
\end{tabular}

Estimated monthly and annual streamflow, in acre-feet

\begin{tabular}{c|c|c|c|r|r|r|r|r|r|r|r|r|r}
\hline $\begin{array}{l}\text { Water } \\
\text { year }\end{array}$ & oct. & Nov. & Dec. & Jan. & Feb. & Mar. & Apr. & May & June & July & Aug. & Sept. & Annual \\
\hline 1961 & 1,800 & 1,600 & 1,200 & 1,100 & 1,000 & 1,600 & 7,400 & 25,500 & 18,500 & 3,600 & 3,800 & 620 & 67,720 \\
1962 & 5,000 & 3,300 & 2,100 & 2,100 & 2,000 & 2,400 & 17,800 & 32,500 & 34,500 & 9,500 & 2,700 & 220 & 114,120 \\
1963 & 2,600 & 1,800 & 1,600 & 1,400 & 1,500 & 5,400 & 9,000 & 23,900 & 3,400 & 2,800 & 2,500 & 280 & 60,180 \\
1964 & 1,800 & 1,500 & 1,200 & 1,200 & 1,100 & 1,200 & 4,300 & 25,000 & 15,800 & 5,600 & 7,600 & 330 & 66,630 \\
1965 & 1,900 & 1,700 & 1,700 & 1,800 & 1,600 & 2,000 & 11,500 & 37,800 & 65,500 & 35,800 & 7,800 & 1,200 & 168,300 \\
\hline
\end{tabular}



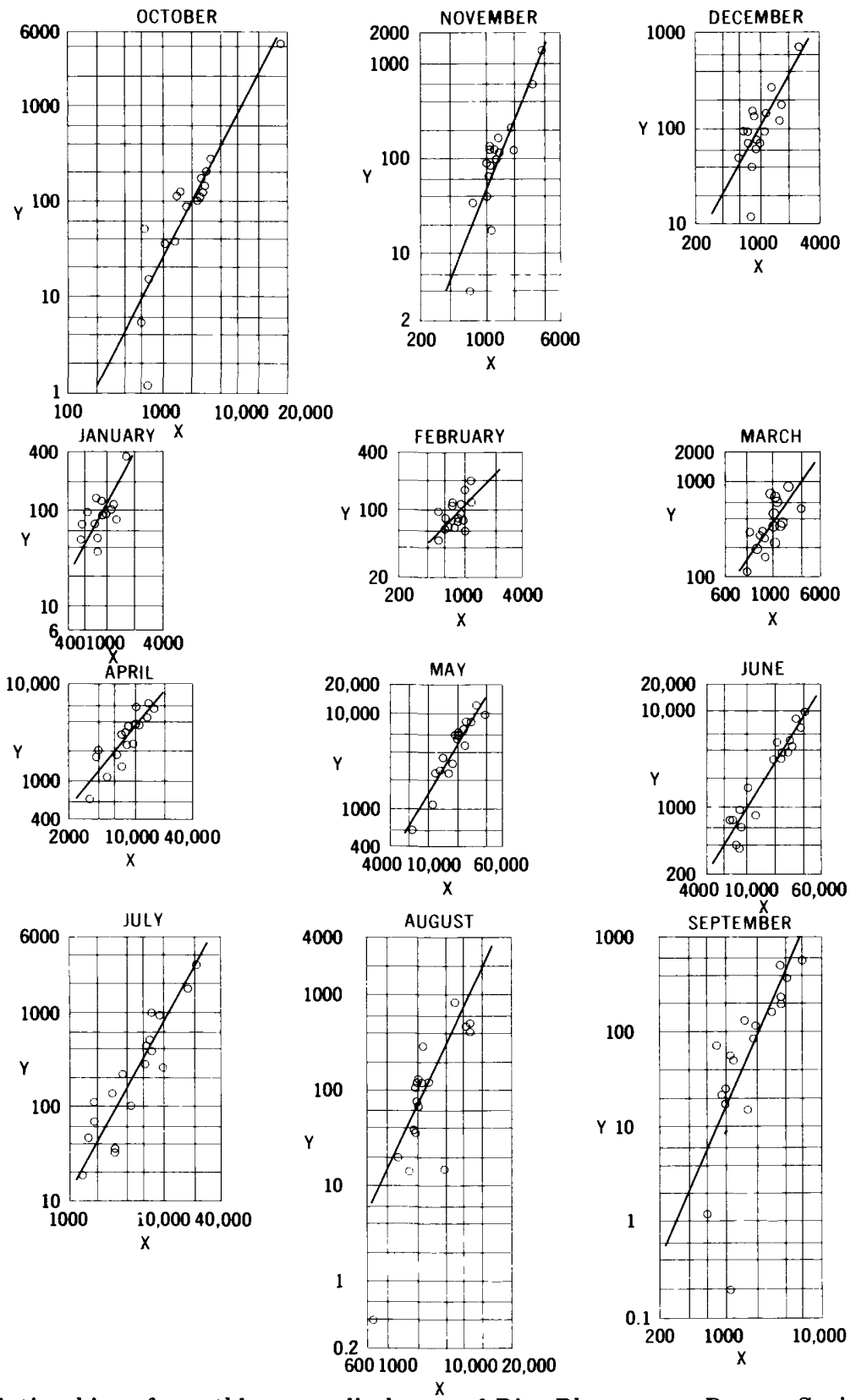

Relationships of monthly mean discharge of Rito Blanco near Pagosa Springs, Colo. (Y), to monthly mean discharge of Rio Blanco near Pagosa Springs, Colo. $(\mathrm{X})$. Discharge in acre-feet. 
3435. Rito Blanco near Pagosa Springs, Colo.

Location.--Lat $37^{\circ} 11^{\prime} 40^{\prime \prime}$, long 106.54'20", in SW $\frac{1}{4}$ sec.12, T.34 N., R.I W., on left bank $130 \mathrm{f} t$ downstream from county highway bridge, $470 \mathrm{ft}$ upstream from sheep Cabin Creek, and $7 \frac{x}{4}$ miles southeast of Pagosa Springs. Altitude of gage is $7,330 \mathrm{ft}$ (from topographic map).

Drainage area. $--23.3 \mathrm{sq}$ mi.

Records available. --May 1935 to September 1952.

Estimates of streamflow. --October 1952 to September 1965, based on relationships of monthly mean discharge with Rio Blanco near Pagosa Springs, Colo. The regression equation used is:

$$
\text { Log } \mathrm{Y}=\mathrm{b} \log \mathrm{X}-\mathrm{c}
$$

(where $\mathrm{Y}$ is discharge of Rito Blanco near Pagosa Springs, Colo., and $\mathrm{X}$ is discharge of Rio Blanco near Fagosa Springs, Colo., both in acre-feet per month).

\begin{tabular}{l|l|l|l|l|l|l|l|l|l|l|l|l}
\hline \multicolumn{10}{c}{ Monthly values of constants in above equation } \\
\hline & Oct. & Nov. & Dec. & Jan. & Feb. & Mar. & Apr. & May & June & July & Aug. & Sept. \\
\hline b & 1.94 & 2.43 & 1.77 & 1.83 & 1.02 & 1.42 & 1.23 & 1.66 & 1.60 & 1.81 & 2.12 & 2.12 \\
c & 4.40 & 5.59 & 3.28 & 3.43 & 1.02 & 2.08 & 1.34 & 3.47 & 3.42 & 4.33 & 5.15 & 5.15 \\
\hline
\end{tabular}

Average discharge. --30 years (1935-65), 12,328 acre-feet per year (17.0 cfs).

Extremes.--1935-52: Maximum discharge, 475 cf's May 13, 1941; no flow at times in many years.

Maximum flood known occurred oct. 5, 1911.

Remarks. --Diversions above station for irrigation of about 150 acres above station and about 600 acres in adjoining drainage basins. Estimates of annual flow are within about 15 percent of regression line.

\begin{tabular}{|c|c|c|c|c|c|c|c|c|c|c|c|c|c|}
\hline $\begin{array}{l}\text { Water } \\
\text { year }\end{array}$ & Oct. & Nov. & Dec. & Jan. & Feb. & Mar. & Apr. & May & June & July & Aug. & Sept. & Annual \\
\hline 1935 & - & - & - & - & - & - & - & 5,520 & 9,690 & 1,770 & 516 & 198 & - \\
\hline $\begin{array}{l}1936 \\
1937 \\
1938 \\
1939 \\
1940\end{array}$ & $\begin{array}{r}147 \\
203 \\
86 \\
275 \\
36\end{array}$ & $\begin{array}{r}106 \\
612 \\
64 \\
131 \\
40\end{array}$ & $\begin{array}{r}155 \\
270 \\
93 \\
97 \\
49\end{array}$ & $\begin{array}{r}135 \\
116 \\
98 \\
91 \\
50\end{array}$ & $\begin{array}{r}111 \\
94 \\
97 \\
65 \\
69\end{array}$ & $\begin{array}{l}872 \\
748 \\
609 \\
697 \\
524\end{array}$ & $\begin{array}{l}5,660 \\
6,420 \\
4,640 \\
2,370 \\
1,880\end{array}$ & $\begin{array}{l}4,510 \\
9,520 \\
6,240 \\
2,350 \\
3,400\end{array}$ & $\begin{array}{r}938 \\
3,270 \\
4,370 \\
722 \\
735\end{array}$ & $\begin{array}{r}136 \\
435 \\
949 \\
46 \\
108\end{array}$ & $\begin{array}{r}418 \\
122 \\
122 \\
20 \\
39\end{array}$ & $\begin{array}{r}365 \\
55 \\
505 \\
161 \\
84\end{array}$ & $\begin{array}{r}13,550 \\
21,860 \\
17,870 \\
7,020 \\
7,010\end{array}$ \\
\hline $\begin{array}{l}1941 \\
1942 \\
1943 \\
1944 \\
1945\end{array}$ & $\begin{array}{r}101 \\
4,500 \\
53 \\
38 \\
113\end{array}$ & $\begin{array}{r}121 \\
1,370 \\
120 \\
85 \\
98\end{array}$ & $\begin{array}{r}122 \\
688 \\
61 \\
72 \\
98\end{array}$ & $\begin{array}{r}81 \\
373 \\
37 \\
73 \\
105\end{array}$ & $\begin{array}{r}121 \\
209 \\
61 \\
65 \\
78\end{array}$ & $\begin{array}{l}349 \\
259 \\
344 \\
198 \\
163\end{array}$ & $\begin{array}{l}2,040 \\
6,060 \\
3,840 \\
1,800 \\
3,070\end{array}$ & $\begin{array}{r}12,030 \\
5,520 \\
2,340 \\
6,670 \\
8,020\end{array}$ & $\begin{array}{l}8,460 \\
4,700 \\
1,590 \\
3,860 \\
3,690\end{array}$ & $\begin{array}{r}3,130 \\
279 \\
218 \\
414 \\
380\end{array}$ & $\begin{array}{r}863 \\
70 \\
147 \\
109 \\
125\end{array}$ & $\begin{array}{r}570 \\
69 \\
50 \\
17 \\
22\end{array}$ & $\begin{array}{r}27,990 \\
24,100 \\
8,860 \\
13,400 \\
15,960\end{array}$ \\
\hline $\begin{array}{l}1946 \\
1947 \\
1948 \\
1949 \\
1950\end{array}$ & $\begin{array}{r}129 \\
125 \\
272 \\
15 \\
119\end{array}$ & $\begin{array}{r}88 \\
208 \\
168 \\
18 \\
124\end{array}$ & $\begin{array}{r}40 \\
184 \\
144 \\
71 \\
138\end{array}$ & $\begin{array}{r}51 \\
92 \\
127 \\
70 \\
111\end{array}$ & $\begin{array}{r}77 \\
167 \\
120 \\
81 \\
112\end{array}$ & $\begin{array}{l}227 \\
442 \\
304 \\
304 \\
363\end{array}$ & $\begin{array}{l}1,380 \\
1,070 \\
3,580 \\
3,180 \\
2,430\end{array}$ & $\begin{array}{r}601 \\
3,050 \\
6,150 \\
6,160 \\
1,130\end{array}$ & $\begin{array}{r}412 \\
836 \\
3,120 \\
5,000 \\
621\end{array}$ & $\begin{array}{r}69 \\
32 \\
100 \\
916 \\
34\end{array}$ & $\begin{array}{r}133 \\
484 \\
74 \\
299 \\
.4\end{array}$ & $\begin{array}{c}116 \\
228 \\
1.2 \\
131 \\
25\end{array}$ & $\begin{array}{r}3,320 \\
6,920 \\
14,160 \\
16,240 \\
5,210\end{array}$ \\
\hline $\begin{array}{l}1951 \\
1952\end{array}$ & $\begin{array}{l}1.2 \\
5.4\end{array}$ & 33.0 & $\begin{array}{l}12 \\
78\end{array}$ & ${ }_{93}^{8.1}$ & $\begin{array}{l}49 \\
84\end{array}$ & $\begin{array}{l}272 \\
117\end{array}$ & $\begin{array}{r}651 \\
3,850\end{array}$ & $\begin{array}{l}2,540 \\
8,250\end{array}$ & $\begin{array}{r}387 \\
6,700\end{array}$ & $\begin{array}{r}19 \\
255\end{array}$ & $\begin{array}{l}37 \\
15\end{array}$ & $15^{.2}$ & $\begin{array}{r}3,980 \\
19,480\end{array}$ \\
\hline
\end{tabular}

Estimated monthly and annual streamflow, in acre-feet

\begin{tabular}{|c|c|c|c|c|c|c|c|c|c|c|c|c|c|}
\hline $\begin{array}{l}\text { Water } \\
\text { year }\end{array}$ & Oct. & Nov. & Dec. & Jan. & Feb. & Mar. & Apr. & May & June & July & Aug. & Sept. & Annual \\
\hline $\begin{array}{l}1953 \\
1954 \\
1955\end{array}$ & $\begin{array}{l}20 \\
30 \\
40\end{array}$ & $\begin{array}{r}120 \\
120 \\
20\end{array}$ & $\begin{array}{l}90 \\
80 \\
60\end{array}$ & $\begin{array}{l}90 \\
60 \\
60\end{array}$ & $\begin{array}{r}100 \\
120 \\
80\end{array}$ & $\begin{array}{l}650 \\
240 \\
210\end{array}$ & $\begin{array}{l}2,000 \\
3,200 \\
1,100\end{array}$ & $\begin{array}{l}1,800 \\
2,200 \\
1,600\end{array}$ & $\begin{array}{r}2,000 \\
370 \\
1,000\end{array}$ & $\begin{array}{r}130 \\
60 \\
80\end{array}$ & $\begin{array}{r}10 \\
150 \\
300\end{array}$ & $\begin{array}{r}2 \\
50 \\
30\end{array}$ & $\begin{array}{l}6,912 \\
6,680 \\
4,580\end{array}$ \\
\hline $\begin{array}{l}1956 \\
1957 \\
1958 \\
1959 \\
1960\end{array}$ & $\begin{array}{r}10 \\
4 \\
230 \\
40 \\
570\end{array}$ & $\begin{array}{r}20 \\
40 \\
820 \\
50 \\
490\end{array}$ & $\begin{array}{r}80 \\
50 \\
380 \\
110 \\
80\end{array}$ & $\begin{array}{r}80 \\
40 \\
250 \\
80 \\
100\end{array}$ & $\begin{array}{r}100 \\
80 \\
120 \\
90 \\
90\end{array}$ & $\begin{array}{r}960 \\
250 \\
230 \\
230 \\
1,300\end{array}$ & $\begin{array}{l}2,000 \\
2,400 \\
3,400 \\
1,500 \\
5,700\end{array}$ & $\begin{array}{r}3,400 \\
3,000 \\
10,400 \\
1,800 \\
3,400\end{array}$ & $\begin{array}{r}1,400 \\
13,000 \\
3,100 \\
540 \\
2,500\end{array}$ & $\begin{array}{r}20 \\
4,700 \\
110 \\
20 \\
120\end{array}$ & $\begin{array}{r}20 \\
2,600 \\
80 \\
70 \\
30\end{array}$ & $\begin{array}{r}1 \\
220 \\
210 \\
6 \\
10\end{array}$ & $\begin{array}{r}8,091 \\
26,384 \\
19,330 \\
4,536 \\
14,390\end{array}$ \\
\hline $\begin{array}{l}1961 \\
1962 \\
1963 \\
1964 \\
1965\end{array}$ & $\begin{array}{r}20 \\
130 \\
90 \\
20 \\
20\end{array}$ & $\begin{array}{r}20 \\
210 \\
100 \\
40 \\
40\end{array}$ & $\begin{array}{r}40 \\
160 \\
130 \\
50 \\
100\end{array}$ & $\begin{array}{r}40 \\
150 \\
80 \\
50 \\
100\end{array}$ & $\begin{array}{r}60 \\
170 \\
110 \\
60 \\
110\end{array}$ & $\begin{array}{l}340 \\
330 \\
770 \\
120 \\
210\end{array}$ & $\begin{array}{l}2,700 \\
4,700 \\
2,400 \\
1,500 \\
3,400\end{array}$ & $\begin{array}{l}3,100 \\
4,300 \\
2,900 \\
3,300 \\
5,600\end{array}$ & $\begin{array}{r}650 \\
1,800 \\
260 \\
530 \\
6,300\end{array}$ & $\begin{array}{r}40 \\
150 \\
40 \\
90 \\
1,500\end{array}$ & $\begin{array}{r}150 \\
20 \\
60 \\
1,000 \\
430\end{array}$ & $\begin{array}{r}340 \\
30 \\
30 \\
40 \\
380\end{array}$ & $\begin{array}{r}7,500 \\
12,150 \\
6,970 \\
6,800 \\
18,190\end{array}$ \\
\hline
\end{tabular}



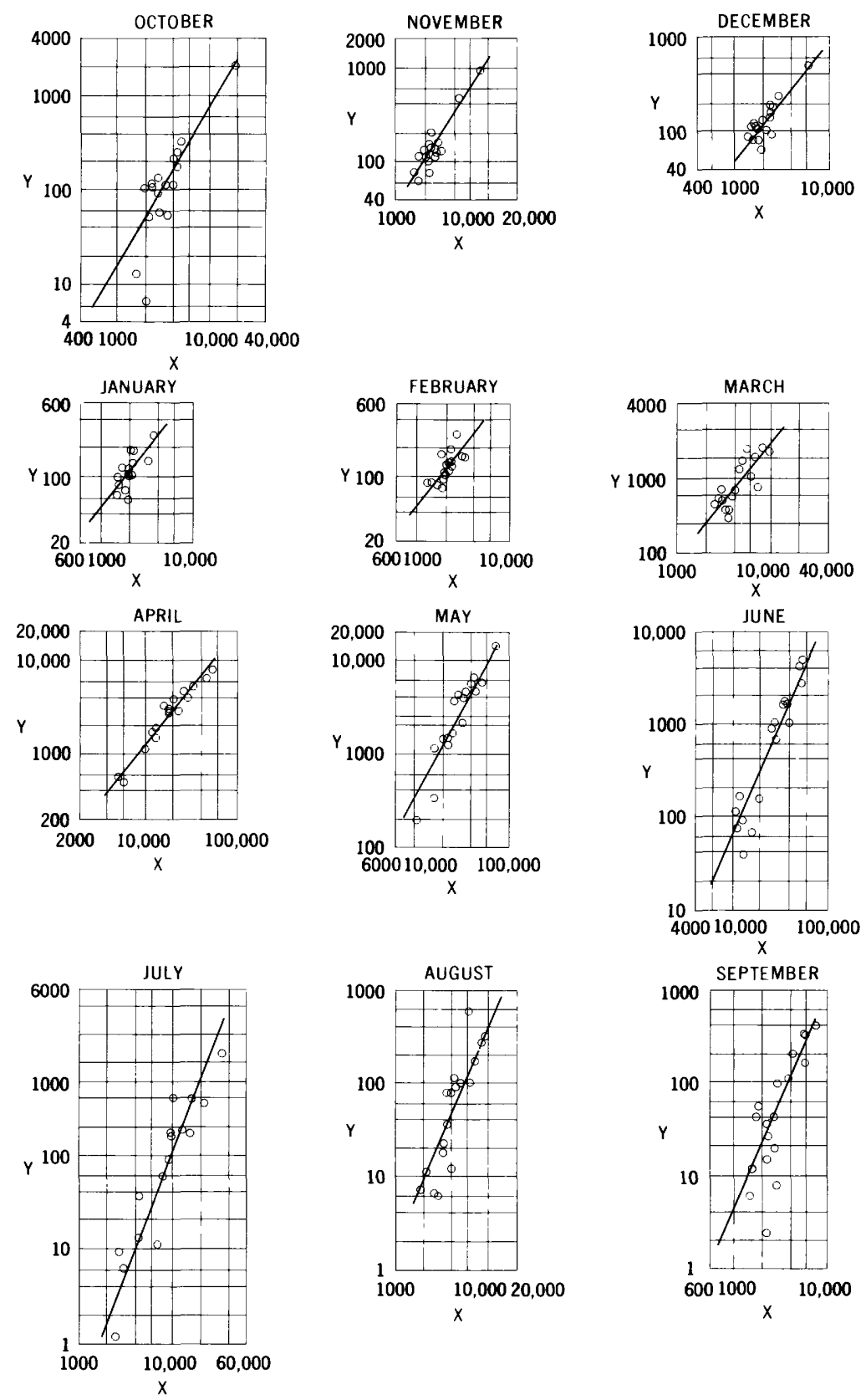

Relationships of monthly mean discharge of Little Navajo River at Chromo, Colo. (Y), to monthly mean discharge of Navajo River at Edith, Colo. (X). Discharge in acre-feet. 
3455. Little Navajo River at Chromo, Colo.

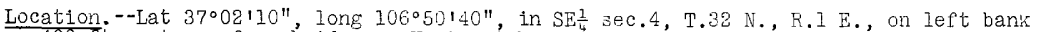
400 f't upstream from bridge on U. S. Highway 84 at Chromo and a quarter of a mile upstream from mouth. Daturn of gage is 7,293.52 ft above mean sea level, datum of 1929.

Drainage area. $--21.9 \mathrm{sq} \mathrm{mi}$.

Records available. --May 1935 to September 1952.

Estimates of streamflow. - - October 1952 to September 1965, based on relationships of monthly mean discharge with Navajo River at Edith, Colo. The regression equation used is:

$$
\log Y=b \log X-c
$$

(where $\mathrm{Y}$ is discharge of Little Navajo River at Chromo, Colo., and $\mathrm{X}$ is discharge of Navajo River at Edith, Colo., woth in acre-feet per month).

\begin{tabular}{l|l|l|l|l|l|l|l|l|l|l|l|l}
\hline \multicolumn{10}{c}{ Monthly values of constants in above equat1on } \\
\hline & oct. & Nov. & Dec. & Jan. & Feb. & Mar. & Apr & May & June & July & Aug. & Sept. \\
\hline $\mathrm{b}$ & 1.70 & 1.55 & 1.25 & 1.25 & 1.25 & 1.25 & 1.25 & 1.81 & 2.36 & 2.64 & 5.36 & 2.28 \\
$\mathrm{c}$ & $\mathbf{5 . 9 2}$ & 3.11 & 2.06 & 2.06 & .06 & 1.84 & 1.91 & 4.70 & 7.65 & 8.50 & 6.85 & 6.21 \\
\hline
\end{tabular}

Average discharge. --35 years $(1930-65), 8,168$ acre-f'eet per year (11.3 cfs).

Extremes.--1935-52: Maximum discharge, 399 cfs May 14, 1941, from rating curve extended above $250 \mathrm{cf}$; no flow at times in 1938-39, 1946-47, 1950-51.

Maximum flood known occurred oct. 5, 1911 .

Remarks.--Diversions above 3 tation for irrigatiun of about 600 acres above station and about 50 acres beluw. Estimates of annual flow are within about 20 percent of regression line.

Monthly and annual streamflow, in acre-feet

\begin{tabular}{|c|c|c|c|c|c|c|c|c|c|c|c|c|c|}
\hline $\begin{array}{l}\text { Water } \\
\text { year }\end{array}$ & Oct. & Nov. & Dec. & Jan. & Feb. & Mar. & Apr. & May & June & July & Aug. & Sept. & Annual \\
\hline 1935 & - & - & - & - & - & - & - & - & 4,090 & 350 & 272 & 323 & - \\
\hline $\begin{array}{l}1936 \\
1937 \\
1938 \\
1939 \\
1940\end{array}$ & $\begin{array}{l}256 \\
324 \\
106 \\
179 \\
107\end{array}$ & $\begin{array}{l}119 \\
474 \\
118 \\
212 \\
118\end{array}$ & $\begin{array}{r}132 \\
240 \\
106 \\
140 \\
87\end{array}$ & $\begin{array}{r}125 \\
153 \\
120 \\
138 \\
87\end{array}$ & $\begin{array}{r}144 \\
157 \\
134 \\
95 \\
76\end{array}$ & $\begin{array}{r}1,500 \\
487 \\
1,010 \\
1,510 \\
631\end{array}$ & $\begin{array}{l}5,550 \\
6,640 \\
4,180 \\
2,850 \\
1,520\end{array}$ & $\begin{array}{l}2,140 \\
5,780 \\
4,550 \\
1,680 \\
1,290\end{array}$ & $\begin{array}{r}116 \\
867 \\
1,630 \\
166 \\
72\end{array}$ & $\begin{array}{r}13 \\
166 \\
405 \\
9.5 \\
6.1\end{array}$ & $\begin{array}{c}173 \\
77 \\
76 \\
11 \\
6.1\end{array}$ & $\begin{array}{c}160 \\
36 \\
316 \\
108 \\
7.7\end{array}$ & $\begin{array}{r}9,850 \\
15,400 \\
12,750 \\
6,900 \\
4,010\end{array}$ \\
\hline $\begin{array}{l}1941 \\
1942 \\
1943 \\
1944 \\
1945\end{array}$ & $\begin{array}{r}106 \\
2,080 \\
133 \\
114 \\
58\end{array}$ & $\begin{array}{r}145 \\
916 \\
168 \\
137 \\
77\end{array}$ & $\begin{array}{r}179 \\
498 \\
187 \\
118 \\
94\end{array}$ & $\begin{array}{l}194 \\
277 \\
197 \\
103 \\
108\end{array}$ & $\begin{array}{r}197 \\
278 \\
178 \\
89 \\
144\end{array}$ & $\begin{array}{r}923 \\
1,230 \\
742 \\
368 \\
357\end{array}$ & $\begin{array}{l}z, 960 \\
8,110 \\
2,890 \\
1,750 \\
3,350\end{array}$ & $\begin{array}{r}13,070 \\
5,520 \\
1,470 \\
3,890 \\
4,740\end{array}$ & $\begin{array}{r}4,670 \\
1,540 \\
157 \\
1,020 \\
686\end{array}$ & $\begin{array}{r}1,590 \\
176 \\
11 \\
189 \\
90\end{array}$ & $\begin{array}{r}314 \\
113 \\
36 \\
12 \\
90\end{array}$ & $\begin{array}{r}401 \\
95 \\
43 \\
15 \\
55\end{array}$ & $\begin{array}{r}25,450 \\
20,830 \\
6,210 \\
7,800 \\
9,850\end{array}$ \\
\hline $\begin{array}{l}1946 \\
1947 \\
1948 \\
1949 \\
1950\end{array}$ & $\begin{array}{r}93 \\
53 \\
210 \\
52 \\
109\end{array}$ & $\begin{array}{l}157 \\
133 \\
131 \\
105 \\
152\end{array}$ & $\begin{array}{r}110 \\
154 \\
106 \\
64 \\
115\end{array}$ & $\begin{array}{r}130 \\
111 \\
109 \\
59 \\
105\end{array}$ & $\begin{array}{r}111 \\
128 \\
115 \\
84 \\
164\end{array}$ & $\begin{array}{l}232 \\
395 \\
277 \\
278 \\
458\end{array}$ & $\begin{array}{r}1,130 \\
512 \\
3,220 \\
3,020 \\
1,890\end{array}$ & $\begin{array}{r}194 \\
1,500 \\
3,660 \\
4,390 \\
340\end{array}$ & $\begin{array}{r}0 \\
66 \\
994 \\
1,650 \\
89\end{array}$ & $\begin{array}{r}0 \\
0 \\
60 \\
402 \\
37\end{array}$ & $\begin{array}{c}18 \\
587 \\
22 \\
100 \\
7.1\end{array}$ & $\begin{array}{c}42 \\
205 \\
11 \\
20 \\
2.4\end{array}$ & $\begin{array}{r}2,220 \\
3,840 \\
8,920 \\
10,120 \\
3,470\end{array}$ \\
\hline $\begin{array}{l}1951 \\
1952\end{array}$ & $13^{6.5}$ & $\begin{array}{l}80 \\
6.3\end{array}$ & $\begin{array}{l}83 \\
81\end{array}$ & $\begin{array}{l}67 \\
74\end{array}$ & $\begin{array}{r}85 \\
104\end{array}$ & $\begin{array}{l}318 \\
453\end{array}$ & $\begin{array}{r}583 \\
4,670\end{array}$ & $\begin{array}{l}1,160 \\
6,460\end{array}$ & $\begin{array}{r}39 \\
2,700\end{array}$ & $174^{1.2}$ & $97^{6.5}$ & $\begin{array}{l}6.0 \\
47\end{array}$ & $\begin{array}{r}2,450 \\
14,940\end{array}$ \\
\hline
\end{tabular}

Estimated monthly and annual streamflow, in acre-feet

\begin{tabular}{|c|c|c|c|c|c|c|c|c|c|c|c|c|c|}
\hline $\begin{array}{l}\text { Water } \\
\text { year }\end{array}$ & oct. & Nov. & Dec. & Jan. & Feb. & Mar. & Apr. & May & June & Ju1y & Aug. & Sept. & Annual \\
\hline $\begin{array}{l}1931 \\
1932 \\
1933 \\
1934 \\
1935\end{array}$ & $\begin{array}{l}- \\
\bar{z} \\
-\end{array}$ & $\begin{array}{l}- \\
- \\
-\end{array}$ & $\begin{array}{l}- \\
\bar{z} \\
\bar{z}\end{array}$ & $\begin{array}{l}- \\
\overline{-} \\
-\end{array}$ & $\begin{array}{l}\overline{-} \\
\overline{-} \\
\end{array}$ & $\begin{array}{l}- \\
\overline{-} \\
-\end{array}$ & $\begin{array}{l}- \\
\overline{-} \\
-\end{array}$ & $\begin{array}{l}- \\
\overline{-} \\
\overline{-}\end{array}$ & $\begin{array}{l}- \\
\bar{z} \\
\overline{-}\end{array}$ & $\begin{array}{l}- \\
\overline{-} \\
\overline{-}\end{array}$ & $\begin{array}{l}\overline{-} \\
\bar{z} \\
\overline{-}\end{array}$ & $\begin{array}{l}\overline{-} \\
\overline{-}\end{array}$ & $\begin{array}{r}* 3,600 \\
* 19,000 \\
* 4,500 \\
* 2,200 \\
* 14,000\end{array}$ \\
\hline $\begin{array}{l}1953 \\
1954 \\
1955\end{array}$ & $\begin{array}{l}60 \\
40 \\
50\end{array}$ & $\begin{array}{r}80 \\
100 \\
70\end{array}$ & $\begin{array}{r}130 \\
90 \\
80\end{array}$ & $\begin{array}{r}130 \\
90 \\
100\end{array}$ & $\begin{array}{r}130 \\
150 \\
90\end{array}$ & $\begin{array}{l}470 \\
340 \\
320\end{array}$ & $\begin{array}{r}1,100 \\
1,600 \\
640\end{array}$ & $\begin{array}{r}640 \\
1,100 \\
720\end{array}$ & $\begin{array}{r}310 \\
50 \\
180\end{array}$ & $\begin{array}{l}20 \\
10 \\
10\end{array}$ & $\begin{array}{l}10 \\
30 \\
50\end{array}$ & $\begin{array}{r}4 \\
20 \\
10\end{array}$ & $\begin{array}{l}3,080 \\
3,6=0 \\
2,220\end{array}$ \\
\hline $\begin{array}{l}1956 \\
1957 \\
1958 \\
1959 \\
1960\end{array}$ & $\begin{array}{r}30 \\
30 \\
180 \\
90 \\
230\end{array}$ & $\begin{array}{r}70 \\
60 \\
380 \\
130 \\
260\end{array}$ & $\begin{array}{r}120 \\
80 \\
220 \\
170 \\
120\end{array}$ & $\begin{array}{r}90 \\
100 \\
200 \\
170 \\
100\end{array}$ & $\begin{array}{l}70 \\
110 \\
190 \\
160 \\
100\end{array}$ & $\begin{array}{r}810 \\
550 \\
650 \\
310 \\
1,500\end{array}$ & $\begin{array}{r}1,500 \\
1,900 \\
3,900 \\
630 \\
4,000\end{array}$ & $\begin{array}{r}1,100 \\
2,300 \\
6,200 \\
490 \\
1,400\end{array}$ & $\begin{array}{r}140 \\
3,900 \\
940 \\
60 \\
580\end{array}$ & $\begin{array}{r}3 \\
2,600 \\
20 \\
30 \\
30\end{array}$ & $\begin{array}{r}10 \\
840 \\
50 \\
40 \\
10\end{array}$ & $\begin{array}{r}5 \\
160 \\
130 \\
10 \\
10\end{array}$ & $\begin{array}{r}3,650 \\
12,630 \\
13,060 \\
2,290 \\
8,350\end{array}$ \\
\hline $\begin{array}{l}1961 \\
1962 \\
1963 \\
1964 \\
1965\end{array}$ & $\begin{array}{r}60 \\
140 \\
110 \\
50 \\
50\end{array}$ & $\begin{array}{r}90 \\
190 \\
160 \\
90 \\
110\end{array}$ & $\begin{array}{r}100 \\
140 \\
140 \\
90 \\
110\end{array}$ & $\begin{array}{r}90 \\
150 \\
120 \\
80 \\
110\end{array}$ & $\begin{array}{r}90 \\
180 \\
190 \\
70 \\
100\end{array}$ & $\begin{array}{l}420 \\
420 \\
860 \\
230 \\
310\end{array}$ & $\begin{array}{r}1,600 \\
3,900 \\
1,500 \\
740 \\
3,100\end{array}$ & $\begin{array}{r}1,600 \\
2,100 \\
990 \\
1,500 \\
2,900\end{array}$ & $\begin{array}{r}170 \\
350 \\
30 \\
60 \\
1,500\end{array}$ & $\begin{array}{r}10 \\
40 \\
3 \\
7 \\
700\end{array}$ & $\begin{array}{r}50 \\
10 \\
30 \\
170 \\
110\end{array}$ & $\begin{array}{r}140 \\
30 \\
50 \\
30 \\
340\end{array}$ & $\begin{array}{l}4,420 \\
7,650 \\
4,180 \\
3,120 \\
9,340\end{array}$ \\
\hline
\end{tabular}

* Annual flgures only; estimated on basis of relationship with San Juan River at Rosa, N. Mex. 

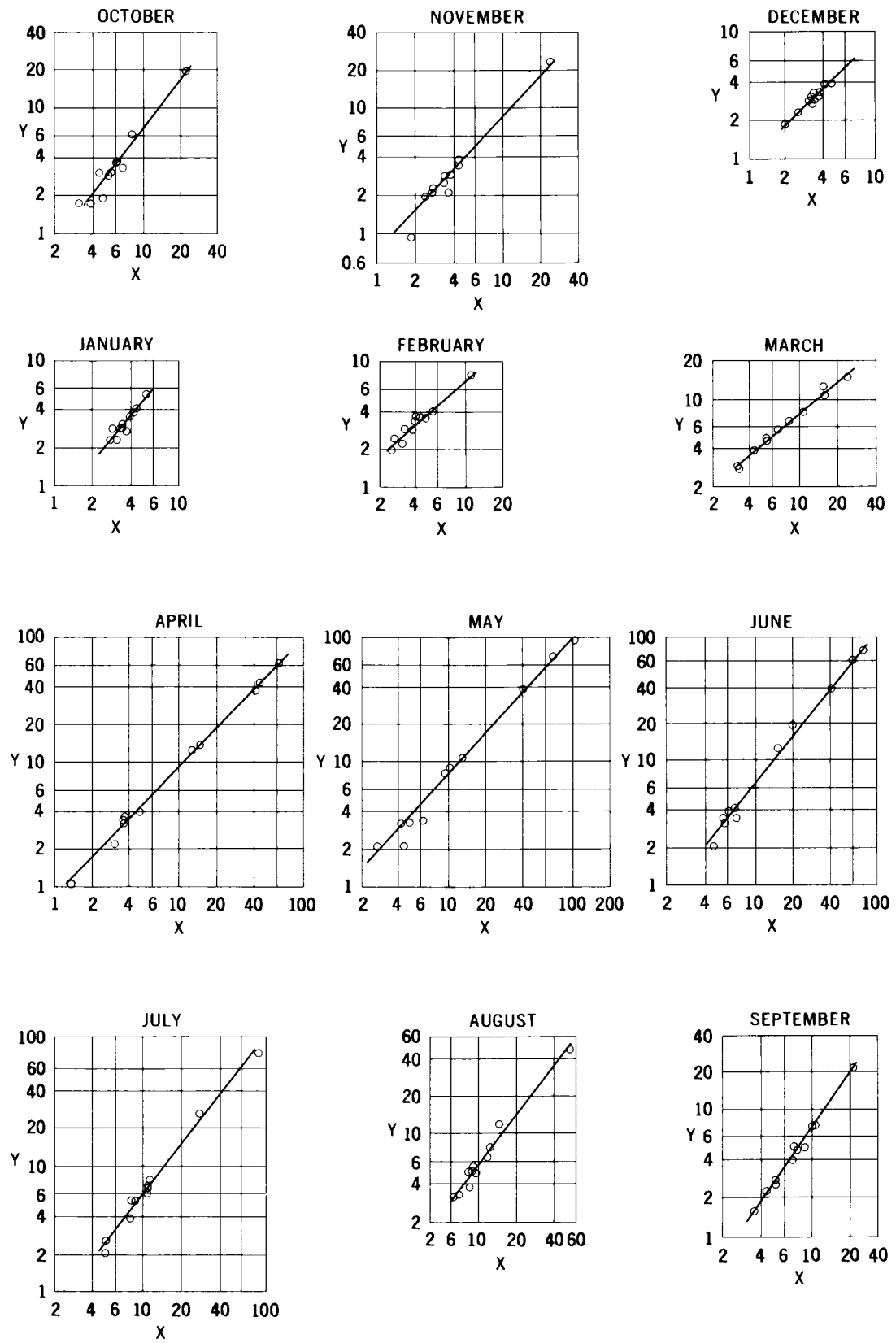

Relationships of monthly mean discharge of Los Pinos River at Ignacio, Colo. (Y), to monthly mean discharge of Los Pinos River at La Boca, Colo. (X). Discharge in thousands of acre-feet. 
3540. Los Pinos River at Ignacio, Colo. (Locally known as Pine RIver)

Location.--Lat $37^{\circ} 07^{145^{\prime \prime}}$, long $107^{\circ} 37^{\prime} 50^{\prime \prime}$, in $\mathrm{S} \frac{1}{2}$ sec.5, T.33 N., R. 7 W. on downstream wing-wall of left abutment of highway bridge, three-quarters of a mile upstream from Ignacio, 2 miles upstream from Rock Creek, and 6 miles south of Bayfield. Datum of gage is $6,468.85 \mathrm{ft}$ above mean sea level, datum of 1929, supplementary adjustment of 1940 .

Drainage area. $--448 \mathrm{sq} \mathrm{m} 1$.

Records available.-April 1899 to May 1900, April to September 1901, April to June 1902 , April to October 1903, September 1910 to September 1961.

Estimates of streamflow.--0ctober 1961 to September 1965 , based on relationships of monthly mean discharge with Los Pinos River at La Boca, Colo. The regression equation used $1 \mathrm{~s}$ :

$$
\log \mathrm{Y}=\mathrm{b} \log \mathrm{X}-\mathrm{c}
$$

(where $Y$ is discharge of Los Pinos River at Ignacio, Colo,, and X is discharge of Los Pinos River at La Boca, Colo., both in acre-feet per monthi).

Monthly values of constants in above equation

\begin{tabular}{l|l|r|r|r|r|r|r|r|r|r|r|l}
\hline & oct. & Nov. & Dec. & Jan. & Feb. & Mar. & Apr. & May & June & July & Aug. & Sept. \\
\hline $\mathrm{b}$ & 1.30 & 1.08 & 0.96 & 1.20 & 0.88 & 0.82 & 1.03 & 1.09 & 1.22 & 1.27 & 1.28 & 1.41 \\
$\mathrm{c}$ & 1.38 & .38 & -.10 & .76 & -.34 & -.60 & .15 & .46 & 1.09 & 1.31 & 1.35 & 1.81 \\
\hline
\end{tabular}

Average discharge. -30 years $(1910-40), 271,060$ acre-feet per year (374 cfs) prior to completion of Vallecito Dam; 25 years $(1940-65), 147,480$ acre-feet per year (204 cfs).

Extremes.--1924-61: Maximum discharge, 7,000 cfs June 29, 1927, from rating curve extended above 3,000 cfs by logarithmic plotting; minimum dáily 1.3 cf's Sept. 8 , 1933 . Maximum flood known occurred 0ct. 5, 1911. A major flood occurred Sept. 5 or 6 ,

Remarks.--Flow regulated by Vallecito Reservolr since April 1941. Diversions for irrigation of about 25,000 acres above station. Estimates of annual flow are within about 5 percent of regression line.

Monthly and annual streamflow, in acre-feet

\begin{tabular}{|c|c|c|c|c|c|c|c|c|c|c|c|c|c|}
\hline $\begin{array}{c}\text { Water } \\
\text { year }\end{array}$ & oct. & Nov. & Dec. & Jan. & Feb, & Mar. & Apr. & May & June & July & Aug. & Sept. & Annual \\
\hline $\begin{array}{l}1931 \\
1932 \\
1933 \\
1934 \\
1935\end{array}$ & $\begin{array}{r}3,730 \\
13,600 \\
3,510 \\
7,680 \\
598\end{array}$ & $\begin{array}{c}50 \\
90 \\
30 \\
30 \\
38\end{array}$ & $\begin{array}{l}6,130 \\
6,350 \\
4,020 \\
5,230 \\
3,000\end{array}$ & $\begin{array}{l}4,140 \\
6,150 \\
4,850 \\
5,340 \\
3,560\end{array}$ & $\begin{array}{l}3,570 \\
9,400 \\
4,010 \\
3,780 \\
4,400\end{array}$ & $\begin{array}{r}4,690 \\
22,600 \\
5,900 \\
5,310 \\
9,230\end{array}$ & $\begin{array}{l}00 \\
00 \\
10 \\
90 \\
20\end{array}$ & $\mid \begin{array}{r}34,050 \\
104,000 \\
20,500 \\
8,910 \\
52,530\end{array}$ & $\begin{array}{r}27,400 \\
81,400 \\
50,600 \\
520 \\
123,500\end{array}$ & $\begin{array}{r}8,140 \\
23,700 \\
6,550 \\
193 \\
25,040\end{array}$ & $\begin{array}{r}1,660 \\
23,700 \\
587 \\
563 \\
8,500\end{array}$ & $\begin{array}{l}5,350 \\
8,050 \\
6,860 \\
2,800 \\
6,370\end{array}$ & $\begin{array}{r}117,000 \\
362,000 \\
118,000 \\
58,800 \\
271,700\end{array}$ \\
\hline $\begin{array}{l}1936 \\
1937 \\
1938 \\
1939 \\
1940\end{array}$ & $\begin{array}{r}4,220 \\
1,760 \\
3,020 \\
15,960 \\
2,040\end{array}$ & $\begin{array}{l}4,680 \\
5,680 \\
3,190 \\
9,030 \\
4,750\end{array}$ & $\begin{array}{l}4,840 \\
5,120 \\
3,240 \\
5,520 \\
3,210\end{array}$ & & $\begin{array}{l}4,110 \\
4,110 \\
4,060 \\
4,000 \\
4,000\end{array}$ & $\begin{array}{r}13,560 \\
12,410 \\
13,260 \\
12,290 \\
8,980\end{array}$ & $\begin{array}{l}49,480 \\
66,180 \\
47,200 \\
25,090 \\
16,610\end{array}$ & $\begin{array}{r}61,820 \\
101,700 \\
68,670 \\
38,710 \\
29,630\end{array}$ & $\begin{array}{r}10,710 \\
27,740 \\
96,810 \\
9,650 \\
2,240\end{array}$ & $\begin{array}{r}916 \\
4,050 \\
17,720 \\
310 \\
270\end{array}$ & $\begin{array}{r}8,000 \\
1,200 \\
779 \\
403 \\
431\end{array}$ & $\mid \begin{array}{r}6,450 \\
1,080 \\
19,270 \\
9,480 \\
7,980\end{array}$ & $\begin{array}{r}173,000 \\
235,200 \\
280,600 \\
135,600 \\
83,720\end{array}$ \\
\hline $\begin{array}{l}1941 \\
1942 \\
1943 \\
1944 \\
1945\end{array}$ & $\begin{array}{r}17,670 \\
52,400 \\
2,160 \\
11,130\end{array}$ & $\begin{array}{r}7,540 \\
18,650 \\
5,790 \\
5,050 \\
1,990\end{array}$ & $\begin{array}{r}7,120 \\
18,070 \\
3,690 \\
6,580 \\
3,200\end{array}$ & $\begin{array}{r}6,360 \\
17,680 \\
3,840 \\
5,080 \\
2,720\end{array}$ & $\begin{array}{r}6, \\
14, \\
3 \\
4 \\
3\end{array}$ & $\begin{array}{r}19,110 \\
9,210 \\
10,460 \\
8,250 \\
6,520\end{array}$ & $\begin{array}{l}30 \\
0 \\
0 \\
0 \\
0\end{array}$ & $\mid \begin{array}{r}125,600 \\
46,290 \\
31,360 \\
66,870 \\
13,780\end{array}$ & $\begin{array}{r}107,700 \\
48,850 \\
11,910 \\
83,050 \\
20,630\end{array}$ & $\begin{array}{r}80,970 \\
10,640 \\
6,360 \\
28,490 \\
7,520\end{array}$ & $\begin{array}{r}10,390 \\
7,590 \\
6,480 \\
13,180 \\
2,930\end{array}$ & $\begin{array}{r}7,900 \\
4,420 \\
4,940 \\
26,820 \\
2,890\end{array}$ & $\begin{array}{r}430,800 \\
295,200 \\
126,500 \\
273,300 \\
90,910\end{array}$ \\
\hline $\begin{array}{l}1946 \\
1947 \\
1948 \\
1949 \\
1950\end{array}$ & $\begin{array}{r}4,580 \\
3,150 \\
18,310 \\
3,050 \\
4,340\end{array}$ & $\begin{array}{r}5,400 \\
2,700 \\
14,940 \\
4,150 \\
1,790\end{array}$ & $\begin{array}{r}3,190 \\
3,730 \\
13,000 \\
4,350 \\
2,530\end{array}$ & $\begin{array}{r}3,280 \\
4,030 \\
12,880 \\
4,670 \\
2,580\end{array}$ & $\begin{array}{r}3,100 \\
3,870 \\
12,520 \\
9,160 \\
3,890\end{array}$ & $\begin{array}{r}3,270 \\
4,200 \\
16,340 \\
31,220 \\
4,410\end{array}$ & $\begin{array}{r}2,380 \\
2,660 \\
76,380 \\
42,090 \\
5,920\end{array}$ & $\begin{array}{r}1,990 \\
22,290 \\
24,200 \\
50,870 \\
4,190\end{array}$ & $\begin{array}{r}2,650 \\
19,480 \\
80,330 \\
65,850 \\
6,000\end{array}$ & $\begin{array}{r}5,000 \\
7,010 \\
11,340 \\
43,730 \\
7,370\end{array}$ & $\begin{array}{r}4,080 \\
12,820 \\
12,780 \\
13,720 \\
4,960\end{array}$ & $\begin{array}{l}3,220 \\
9,600 \\
4,710 \\
9,550 \\
5,100\end{array}$ & $\begin{array}{r}42,140 \\
95,540 \\
297,700 \\
282,400 \\
53,080\end{array}$ \\
\hline $\begin{array}{l}1951 \\
1952 \\
1953 \\
1954 \\
1955\end{array}$ & $\begin{array}{l}1,730 \\
1,740 \\
2,980 \\
3,640 \\
6,060\end{array}$ & $\begin{array}{l}2,160 \\
1,960 \\
2,190 \\
2,260 \\
2,850\end{array}$ & $\begin{array}{l}3,050 \\
3,240 \\
3,910 \\
2,930 \\
2,990\end{array}$ & $\begin{array}{l}2,330 \\
3,550 \\
4,170 \\
2,880 \\
3,090\end{array}$ & $\begin{array}{l}1,990 \\
2,470 \\
3,360 \\
3,650 \\
2,920\end{array}$ & $\begin{array}{r}2,930 \\
10,770 \\
4,950 \\
3,890 \\
4,820\end{array}$ & $\begin{array}{r}1,040 \\
63,650 \\
3,640 \\
3,370 \\
3,330\end{array}$ & $\begin{array}{r}2,080 \\
69,720 \\
3,210 \\
3,240 \\
8,000\end{array}$ & $\begin{array}{r}2,060 \\
60,700 \\
3,310 \\
3,000 \\
3,850\end{array}$ & $\begin{array}{r}2,590 \\
25,330 \\
5,180 \\
6,020 \\
6,970\end{array}$ & $\begin{array}{r}3,170 \\
11,090 \\
5,050 \\
5,050 \\
7,920\end{array}$ & $\begin{array}{l}1,540 \\
4,980 \\
2,750 \\
3,980 \\
5,020\end{array}$ & $\begin{array}{r}26,670 \\
259,200 \\
44,700 \\
43,900 \\
57,800\end{array}$ \\
\hline $\begin{array}{l}1956 \\
1957 \\
1958 \\
1959 \\
1960\end{array}$ & $\begin{array}{r}3,170 \\
1,890 \\
19,110 \\
3,860 \\
2,910\end{array}$ & $\begin{array}{r}3,510 \\
2,590 \\
22,680 \\
3,870 \\
964\end{array}$ & $\begin{array}{r}4,060 \\
2,310 \\
18,610 \\
3,480 \\
2,700\end{array}$ & $\begin{array}{l}3,800 \\
2,900 \\
5,390 \\
2,940 \\
2,290\end{array}$ & $\begin{array}{l}3,550 \\
4,010 \\
7,810 \\
2,860 \\
2,230\end{array}$ & $\begin{array}{r}5,730 \\
6,600 \\
12,910 \\
2,930 \\
15,100\end{array}$ & $\begin{array}{r}4,010 \\
12,100 \\
42,220 \\
2,210 \\
36,550\end{array}$ & $\begin{array}{r}3,390 \\
37,050 \\
95,090 \\
2,130 \\
10,720\end{array}$ & $\begin{array}{r}3,370 \\
74,000 \\
36,000 \\
4,050 \\
18,550\end{array}$ & $\begin{array}{r}3,770 \\
76,470 \\
6,720 \\
2,090 \\
5,280\end{array}$ & $\begin{array}{r}3,850 \\
47,350 \\
6,680 \\
3,320 \\
5,590\end{array}$ & $\begin{array}{r}2,540 \\
21,480 \\
7,340 \\
2,260 \\
4,750\end{array}$ & $\begin{array}{r}44,750 \\
288,800 \\
280,600 \\
36,000 \\
107,700\end{array}$ \\
\hline 1961 & 3,310 & 2,870 & 3,150 & 2,890 & 3,510 & 7,810 & 13,490 & 90 & 12,050 & 7,440 & 5,070 & 7,240 & 77,520 \\
\hline
\end{tabular}

Estimated monthly and annual streamflow, in acre-feet

\begin{tabular}{l|l|l|l|l|l|r|r|r|r|r|r|r|r}
\hline $\begin{array}{l}\text { Water } \\
\text { year }\end{array}$ & oct. & Nov. & Dec. & Jan. & Feb. & Mar. & Apr. & May & June & July & Aug. & Sept. & Annual \\
\hline 1962 & 6,900 & 6,200 & 5,700 & 2,600 & 3,700 & 10,500 & 48,000 & 17,800 & 4,600 & 5,200 & 4,500 & 6,000 & 121,700 \\
1963 & 6,400 & 3,400 & 4,000 & 5,100 & 6,200 & 7,300 & 6,400 & 5,000 & 3,800 & 4,500 & 4,800 & 2,800 & 57,800 \\
1964 & 3,400 & 2,000 & 2,000 & 1,900 & 2,000 & 3,600 & 4,600 & 3,400 & 3,500 & 4,300 & 5,600 & 3,400 & 39,700 \\
1965 & 2,100 & 2,500 & 5,800 & 3,200 & 3,500 & 5,900 & 19,500 & 58,000 & 49,000 & 40,000 & 9,600 & 16,500 & 212,600 \\
\hline
\end{tabular}



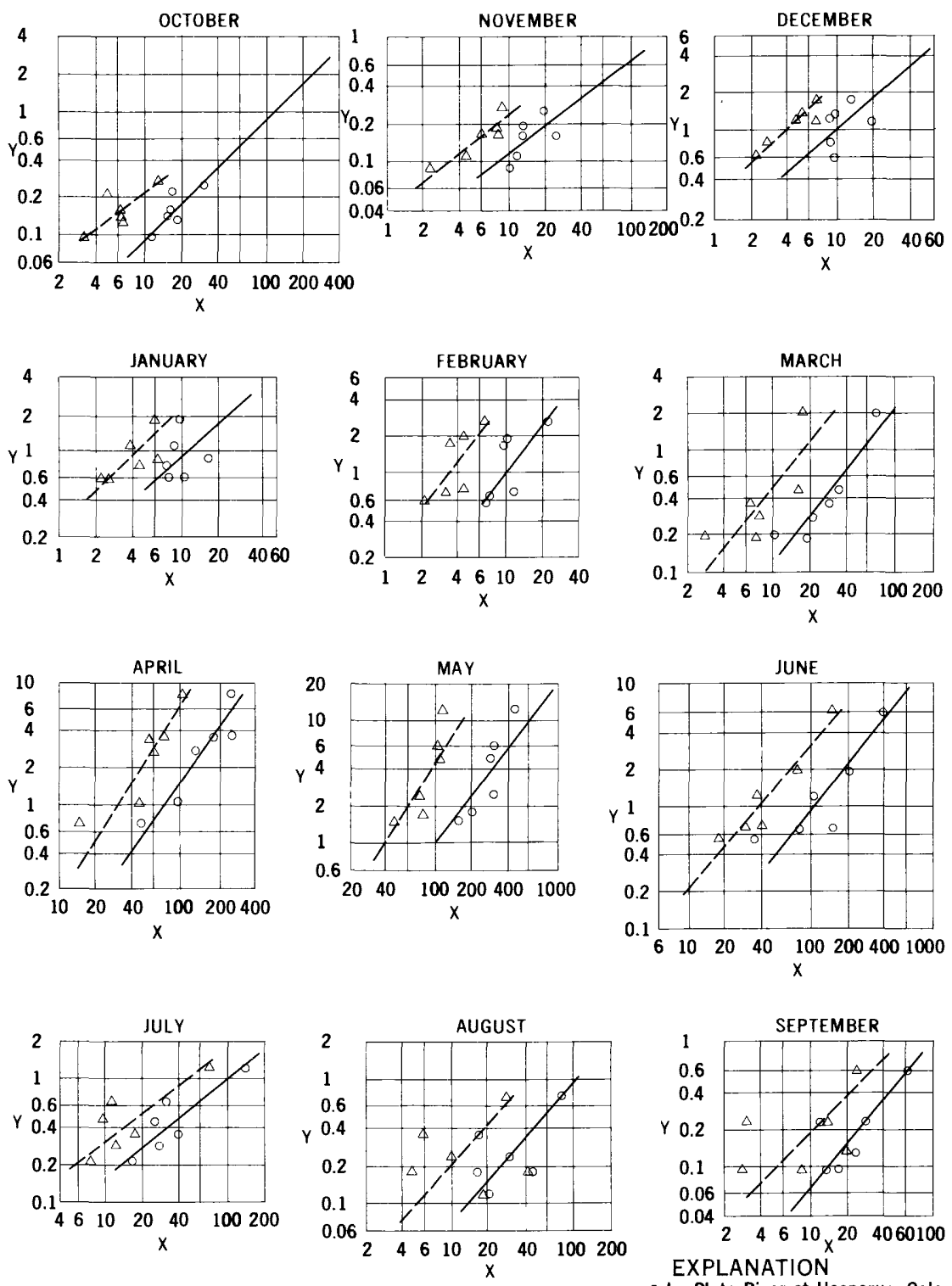

$-\_-\triangle$ Hermosa Creek near Hermosa,

Colo.

Relationships of monthly mean discharge of Falls Creek near Durango, Colo. $(\mathrm{Y})$, to monthly mean discharge of La Plata River at Hesperus, Colo., or Hermosa Creek near Hermosa, Colo. (X). Discharge in hundreds of acre-feet. 
3612. Falls Creek near Durango, Colo.

Location. --Lat 37022100", long 107052100", in SW $\frac{1}{4}$ sec.21, T.36 N., R.9 W., on right bank 1.2 miles upstream from mouth and 6.5 miles north of Durango. 'Altitudé of gage is $7,120 \mathrm{ft}$ (from topographic map).

Drainage area. $--7.18 \mathrm{sq} \mathrm{mi}$.

Records available.--October 1959 to September 1965.

Estimates of streamflow.--October 1930 to September 1940 , based on relationships of monthly mean discharge with La Plata River at Hesperus, Colo.; October 1940 to September

1959 , based on relationshios of monthly mean discharge with Hermosa Creek near Hermosa, colo. The regression equation used is:

$\log \mathrm{Y}=\mathrm{b} \log \mathrm{X}-\mathrm{c}$

(where $Y$ is discharge of Falls Creek near Durango, Colo, and $X$ is discharge of La Plata River at Hesperus, Colo. or Hermosa Creek near Hermosa, Colo., all in acrefeet per month).

\begin{tabular}{|c|c|c|c|c|c|c|c|c|c|c|c|c|c|}
\hline & & Oct. & Nov. & Dec. & Jan. & Feb. & Mar. & Apr. & May & June & July & Aug. & Sept. \\
\hline oct. 1930 & b & & & & 0.98 & & & & & & & & 1.00 \\
\hline Sept. 1940 & c & .91 & .97 & 1.43 & 1.60 & 3.67 & 2.06 & 3.71 & 3.83 & 2.19 & .82 & 1.08 & 1.74 \\
\hline Oct. 1940 & $\mathrm{~b}$ & 0.97 & 0.77 & 0.87 & 0.89 & 1.28 & 1.28 & 1.40 & 1.32 & 1.25 & 0.80 & 1.15 & 1.21 \\
\hline Sept. 1959 & c & 1.96 & 1.24 & 1.59 & 1.70 & 2.84 & 8.77 & 3.42 & 3.28 & 3.04 & 1.20 & 2.62 & 2.82 \\
\hline
\end{tabular}

Average discharge. --35 years $(1930-65), 1,265$ acre-feet per year (1.75 cfs).

Extremes.--1959-65: Maximum discharge, 45 cf's May 19, 1965; minimum daily, 0.1 cfs for many days in most years.

Remarks. --Small diversions above station for Irrigation of meadows. Estimates of annual flow are within about 25 percent of regression line prior to September 1940 , and are within about 15 percent thereafter.

\begin{tabular}{l|c|c|c|c|c|c|c|c|c|c|c|c|c}
\hline $\begin{array}{c}\text { Water } \\
\text { year }\end{array}$ & Oct. & Nov. & Dec. & Jan. & Feb. & Mar. & Apr. & May & June & July & Aug. & Sept. & Annual \\
\hline 1960 & 16 & 25 & 18 & 18 & 17 & 200 & 804 & 626 & 196 & 66 & 36 & 23 & 2,040 \\
& & & & & & & & & & & & & 12 \\
1961 & 22 & 19 & 13 & 7.7 & 6.5 & 28 & 274 & 491 & 123 & 46 & 24 & 23 & 1,080 \\
1962 & 25 & 16 & 12 & 8.7 & 26 & 36 & 363 & 246 & 69 & 36 & 18 & 9.3 & 865 \\
1963 & 13 & 16 & 14 & 11 & 19 & 47 & 106 & 151 & 55 & 22 & 12 & 13 & 479 \\
1964 & 14 & 11 & 8.1 & 6.1 & 5.8 & 20 & 70 & 178 & 67 & 29 & 18 & 9.5 & 436 \\
1965 & 9.5 & 8.7 & 6.1 & 6.1 & 6.9 & 19 & 354 & 1,250 & 603 & 124 & 75 & 60 & 2,520 \\
\hline
\end{tabular}

Estimated monthly and annual streamflow, in acre-feet

\begin{tabular}{|c|c|c|c|c|c|c|c|c|c|c|c|c|c|}
\hline $\begin{array}{l}\text { Water } \\
\text { year }\end{array}$ & Oct. & Nov. & Dec. & Jan. & Feb, & Mar. & Apr. & May & June & July & Aug. & Sept. & Annual \\
\hline $\begin{array}{l}1931 \\
1932 \\
1933 \\
1934 \\
1935\end{array}$ & $\begin{array}{l}10 \\
30 \\
20 \\
30 \\
10\end{array}$ & $\begin{array}{r}10 \\
20 \\
10 \\
9 \\
8\end{array}$ & $\begin{array}{r}10 \\
10 \\
10 \\
8 \\
8\end{array}$ & $\begin{array}{r}6 \\
10 \\
7 \\
7 \\
10\end{array}$ & $\begin{array}{r}6 \\
20 \\
8 \\
20 \\
20\end{array}$ & $\begin{array}{l}10 \\
70 \\
30 \\
50 \\
40\end{array}$ & $\begin{array}{r}30 \\
600 \\
50 \\
140 \\
280\end{array}$ & $\begin{array}{r}170 \\
850 \\
210 \\
80 \\
390\end{array}$ & $\begin{array}{r}70 \\
200 \\
190 \\
20 \\
560\end{array}$ & $\begin{array}{l}40 \\
60 \\
60 \\
20 \\
80\end{array}$ & $\begin{array}{l}20 \\
60 \\
10 \\
10 \\
30\end{array}$ & $\begin{array}{l}20 \\
20 \\
20 \\
10 \\
20\end{array}$ & $\begin{array}{r}402 \\
1,950 \\
625 \\
404 \\
1,456\end{array}$ \\
\hline $\begin{array}{l}1936 \\
1937 \\
1938 \\
1939 \\
1940\end{array}$ & $\begin{array}{l}20 \\
20 \\
10 \\
20 \\
10\end{array}$ & $\begin{array}{r}10 \\
20 \\
6 \\
10 \\
10\end{array}$ & $\begin{array}{r}7 \\
10 \\
5 \\
9 \\
7\end{array}$ & $\begin{array}{r}7 \\
10 \\
4 \\
10 \\
6\end{array}$ & $\begin{array}{r}10 \\
10 \\
6 \\
8 \\
8\end{array}$ & $\begin{array}{l}90 \\
20 \\
10 \\
40 \\
30\end{array}$ & $\begin{array}{l}680 \\
580 \\
760 \\
150 \\
180\end{array}$ & $\begin{array}{l}530 \\
980 \\
710 \\
180 \\
290\end{array}$ & $\begin{array}{r}80 \\
130 \\
280 \\
50 \\
70\end{array}$ & $\begin{array}{l}30 \\
70 \\
60 \\
20 \\
30\end{array}$ & $\begin{array}{r}50 \\
20 \\
20 \\
7 \\
10\end{array}$ & $\begin{array}{r}20 \\
7 \\
30 \\
20 \\
20\end{array}$ & $\begin{array}{r}1,534 \\
1,880 \\
1,900 \\
524 \\
671\end{array}$ \\
\hline $\begin{array}{l}1941 \\
1942 \\
1943 \\
1944 \\
1945\end{array}$ & $\begin{array}{r}20 \\
270 \\
10 \\
20 \\
20\end{array}$ & $\begin{array}{l}20 \\
80 \\
10 \\
20 \\
20\end{array}$ & $\begin{array}{l}10 \\
40 \\
10 \\
20 \\
10\end{array}$ & $\begin{array}{l}10 \\
30 \\
10 \\
10 \\
10\end{array}$ & $\begin{array}{l}20 \\
30 \\
10 \\
10 \\
10\end{array}$ & $\begin{array}{l}60 \\
60 \\
40 \\
40 \\
20\end{array}$ & $\begin{array}{l}190 \\
770 \\
490 \\
270 \\
130\end{array}$ & $\begin{array}{r}1,800 \\
740 \\
370 \\
1,200 \\
510\end{array}$ & $\begin{array}{l}910 \\
300 \\
120 \\
540 \\
200\end{array}$ & $\begin{array}{r}160 \\
60 \\
40 \\
100 \\
50\end{array}$ & $\begin{array}{l}40 \\
20 \\
40 \\
20 \\
20\end{array}$ & $\begin{array}{r}90 \\
10 \\
20 \\
10 \\
9\end{array}$ & $\begin{array}{l}3,330 \\
2,410 \\
1,170 \\
2,260 \\
1,010\end{array}$ \\
\hline $\begin{array}{l}1946 \\
1947 \\
1948 \\
1949 \\
1950\end{array}$ & $\begin{array}{l}10 \\
10 \\
60 \\
10 \\
20\end{array}$ & $\begin{array}{l}10 \\
10 \\
30 \\
20 \\
20\end{array}$ & $\begin{array}{l}10 \\
10 \\
20 \\
10 \\
10\end{array}$ & $\begin{array}{r}7 \\
9 \\
20 \\
10 \\
10\end{array}$ & $\begin{array}{r}6 \\
9 \\
20 \\
10 \\
20\end{array}$ & $\begin{array}{l}20 \\
40 \\
50 \\
40 \\
60\end{array}$ & $\begin{array}{l}110 \\
120 \\
510 \\
510 \\
250\end{array}$ & $\begin{array}{l}120 \\
400 \\
800 \\
830 \\
150\end{array}$ & $\begin{array}{r}80 \\
150 \\
290 \\
550 \\
80\end{array}$ & $\begin{array}{r}30 \\
50 \\
50 \\
110 \\
40\end{array}$ & $\begin{array}{l}20 \\
50 \\
20 \\
30 \\
10\end{array}$ & $\begin{array}{r}10 \\
40 \\
10 \\
10 \\
9\end{array}$ & $\begin{array}{r}433 \\
898 \\
1,880 \\
2,140 \\
679\end{array}$ \\
\hline $\begin{array}{l}1951 \\
195 ? \\
1953 \\
1954 \\
1955\end{array}$ & $\begin{array}{r}10 \\
8 \\
10 \\
10 \\
20\end{array}$ & $\begin{array}{l}10 \\
10 \\
10 \\
20 \\
10\end{array}$ & $\begin{array}{l}10 \\
10 \\
10 \\
10 \\
10\end{array}$ & $\begin{array}{r}8 \\
8 \\
10 \\
8 \\
10\end{array}$ & $\begin{array}{r}7 \\
8 \\
9 \\
10 \\
8\end{array}$ & $\begin{array}{l}20 \\
20 \\
20 \\
20 \\
20\end{array}$ & $\begin{array}{r}30 \\
570 \\
120 \\
180 \\
100\end{array}$ & $\begin{array}{l}140 \\
980 \\
160 \\
200 \\
330\end{array}$ & $\begin{array}{r}70 \\
570 \\
120 \\
30 \\
120\end{array}$ & $\begin{array}{l}30 \\
70 \\
40 \\
40 \\
30\end{array}$ & $\begin{array}{l}10 \\
20 \\
10 \\
10 \\
30\end{array}$ & $\begin{array}{r}6 \\
20 \\
6 \\
20 \\
10\end{array}$ & $\begin{array}{r}351 \\
2,290 \\
525 \\
558 \\
698\end{array}$ \\
\hline $\begin{array}{l}1956 \\
1957 \\
1958 \\
1959 \\
\end{array}$ & $\begin{array}{r}9 \\
7 \\
30 \\
10 \\
\end{array}$ & $\begin{array}{r}10 \\
7 \\
40 \\
10 \\
\end{array}$ & $\begin{array}{r}10 \\
4 \\
40 \\
10 \\
\end{array}$ & $\begin{array}{r}9 \\
5 \\
30 \\
10 \\
\end{array}$ & $\begin{array}{r}10 \\
6 \\
30 \\
7 \\
\end{array}$ & $\begin{array}{l}80 \\
20 \\
60 \\
10 \\
\end{array}$ & $\begin{array}{r}130 \\
190 \\
410 \\
30 \\
\end{array}$ & $\begin{array}{r}290 \\
380 \\
1,200 \\
100 \\
\end{array}$ & $\begin{array}{r}80 \\
830 \\
270 \\
40 \\
\end{array}$ & $\begin{array}{r}30 \\
150 \\
40 \\
20 \\
\end{array}$ & $\begin{array}{r}9 \\
110 \\
20 \\
10 \\
\end{array}$ & $\begin{array}{r}5 \\
50 \\
10 \\
6 \\
\end{array}$ & $\begin{array}{r}672 \\
1,760 \\
2,180 \\
263 \\
\end{array}$ \\
\hline
\end{tabular}



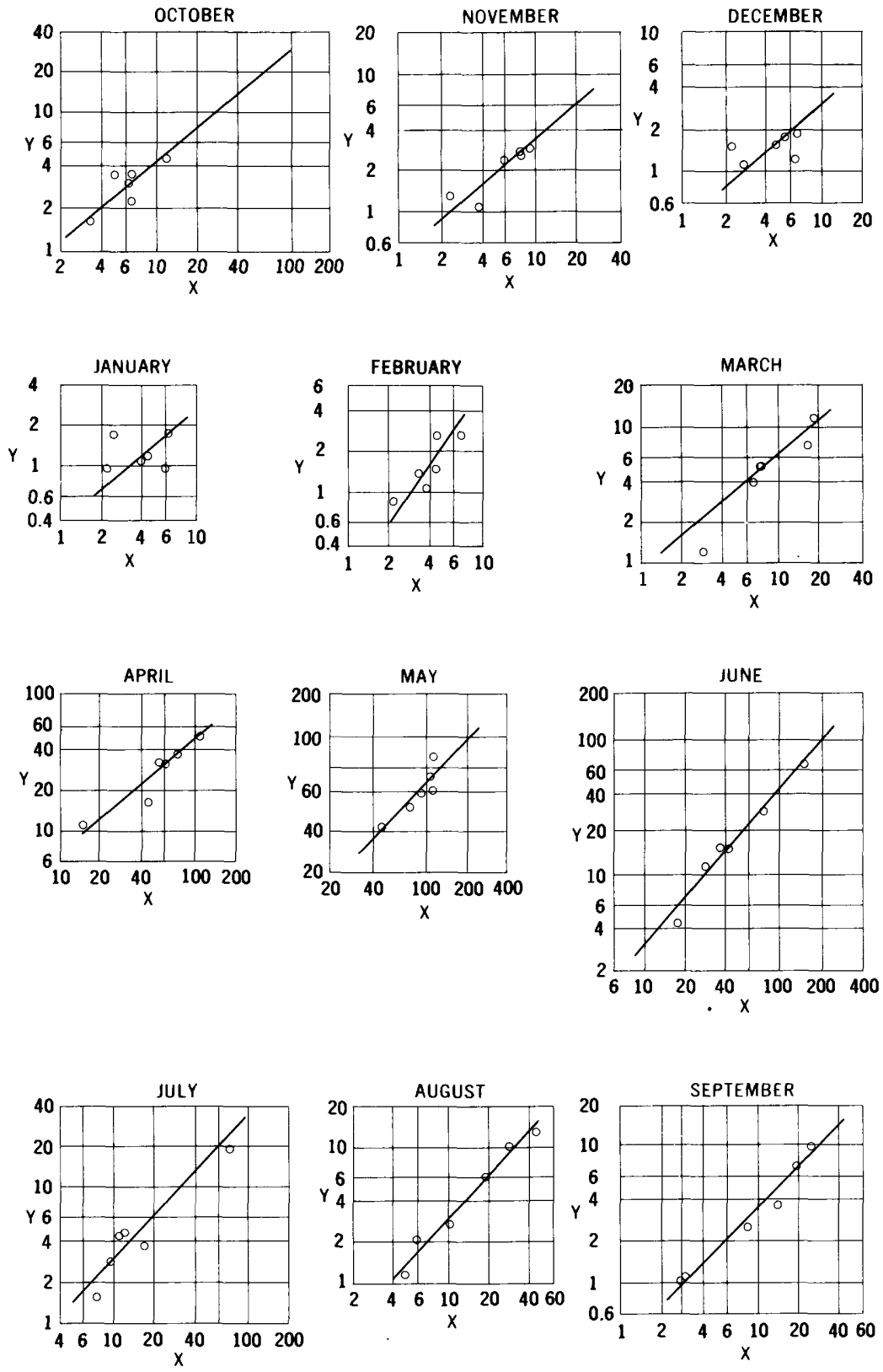

Relationships of monthly mean discharge of Junction Creek near Durango, Colo. (Y), to monthly mean discharge of La Plata River at Hesperus, Colo. $(X)$. Discharge in hundreds of acre-feet. 
3614. Junction Creek near Durango, Colo.

Location. --Lat 37\%20105", long 107054130", in sec.36, T.36 N., R.10 W., on left bank $4 \frac{1}{2}$ miles upstream from mouth and $4 \frac{1}{2}$ miles northwest of Durango. Datum of gage is $7,045.65 \mathrm{ft}^{\mathrm{t}}$ above mean sea level (Bureau of Reclamation bench mark).

Drainage area. $--26.3 \mathrm{sq} \mathrm{mi}$.

Records available. - - october 1959 to September 1965.

Estimates of streamf low.--0ctober 1930 to September 1959, based on relationships of monthly mean discharge with La Plata River at Hesperus, Colo. The regression equation used is:

$\log Y=b \log X-c$

(where $\mathrm{Y}$ is discharge of Junction Creek near Durango, Colo, and $\mathrm{X}$ is discharge of La Plata River at Hesperus, Colo., both in acre-feet per month).

\begin{tabular}{|c|c|c|c|c|c|c|c|c|c|c|c|c|}
\hline \multicolumn{13}{|c|}{ Monthly values of constants in above equation } \\
\hline & oct. & Nov. & Dec. & Jan. & Feb. & Mar. & Apr. & May & June & July & Aug. & Sept. \\
\hline b & 0.84 & 0.84 & 0.84 & 0.84 & 1.46 & 0.84 & 0.84 & 1.06 & 1.15 & 1.07 & 1.07 & 1.01 \\
\hline c & -.13 & -.01 & .06 & .10 & 1.59 & -.28 & -.32 & .57 & .96 & .74 & .74 & .48 \\
\hline
\end{tabular}

Average discharge. --35 years $(1930-65), 13,729$ acre-feet per year (19.0 cfs).

Extremes.--1959-65: Maximum discharge, about 300 cfs May 21, 1965; minimum daily, 0.9 cfs Sept. $3-5,10-13,18,1962$, Dec. 8, 1963 .

Remarks.- No regulation or diversion above station. Estimates of annual f'low are within 10 percent of regression line.

Monthly and annual streamflow, in acre-feet

\begin{tabular}{|c|c|c|c|c|c|c|c|c|c|c|c|c|c|}
\hline $\begin{array}{l}\text { Water } \\
\text { year }\end{array}$ & Oct. & Nov. & Dec. & Jan. & Feb. & Mar. & Apr. & May & June & July & Aug. & Sept. & Annual \\
\hline 1960 & 308 & 289 & 123 & 98 & 105 & 1,190 & 5,080 & 5,110 & 3,000 & 441 & 210 & 108 & 16,060 \\
\hline $\begin{array}{l}1961 \\
1962 \\
1963 \\
1964 \\
1965\end{array}$ & $\begin{array}{l}357 \\
471 \\
359 \\
228 \\
164\end{array}$ & $\begin{array}{l}253 \\
269 \\
234 \\
109 \\
130\end{array}$ & $\begin{array}{l}156 \\
186 \\
176 \\
111 \\
151\end{array}$ & $\begin{array}{r}123 \\
178 \\
113 \\
97 \\
172\end{array}$ & $\begin{array}{r}139 \\
249 \\
150 \\
86 \\
265\end{array}$ & $\begin{array}{l}531 \\
409 \\
729 \\
126 \\
521\end{array}$ & $\begin{array}{l}3,180 \\
3,700 \\
1,680 \\
1,110 \\
3,260\end{array}$ & $\begin{array}{l}4,120 \\
3,120 \\
2,170 \\
3,910 \\
7,310\end{array}$ & $\begin{array}{r}1,610 \\
1,560 \\
451 \\
1,170 \\
6,550\end{array}$ & $\begin{array}{r}284 \\
371 \\
158 \\
458 \\
1,960\end{array}$ & $\begin{array}{r}275 \\
116 \\
607 \\
1,320 \\
1,020\end{array}$ & $\begin{array}{r}379 \\
101 \\
723 \\
258 \\
1,020\end{array}$ & $\begin{array}{r}11,410 \\
10,730 \\
7,550 \\
8,980 \\
22,520\end{array}$ \\
\hline
\end{tabular}

Estimated monthly and annual streamflow, in acre-feet

\begin{tabular}{|c|c|c|c|c|c|c|c|c|c|c|c|c|c|}
\hline $\begin{array}{l}\text { Water } \\
\text { year }\end{array}$ & Oct. & Nov. & Dec. & Jan. & Feb. & Mar. & Apr. & May & June & July & Aug. & Sept. & Annual \\
\hline $\begin{array}{l}1932 \\
1933 \\
1934\end{array}$ & $\begin{array}{l}200 \\
540 \\
320 \\
530 \\
210\end{array}$ & $\begin{array}{l}180 \\
120 \\
100\end{array}$ & $\begin{array}{l}130 \\
140 \\
140 \\
110 \\
110\end{array}$ & $\begin{array}{r}80 \\
160 \\
90 \\
100 \\
130\end{array}$ & $\begin{array}{r}70 \\
240 \\
100 \\
260 \\
220\end{array}$ & $\begin{array}{l}230 \\
770 \\
420 \\
650 \\
560\end{array}$ & $\begin{array}{l}4,300 \\
1,300 \\
3,000\end{array}$ & $\begin{array}{l}2,500 \\
7,100 \\
2,900 \\
7,400 \\
4,300\end{array}$ & $\begin{array}{r}1,000 \\
2,800 \\
2,700 \\
280 \\
7,700\end{array}$ & $\begin{array}{r}420 \\
810 \\
730 \\
190 \\
1,100\end{array}$ & $\begin{array}{l}250 \\
860 \\
190 \\
180 \\
360\end{array}$ & $\begin{array}{l}460 \\
440 \\
410 \\
200 \\
290\end{array}$ & $\begin{array}{r}6,460 \\
18,530 \\
9,480 \\
6,220 \\
18,080\end{array}$ \\
\hline $\begin{array}{l}1936 \\
1937 \\
1938 \\
1939 \\
1940\end{array}$ & $\begin{array}{l}320 \\
360 \\
250 \\
340 \\
210\end{array}$ & $\begin{array}{r}130 \\
210 \\
80 \\
180 \\
130\end{array}$ & $\begin{array}{r}100 \\
180 \\
70 \\
120 \\
90\end{array}$ & $\begin{array}{r}90 \\
160 \\
60 \\
120 \\
90\end{array}$ & $\begin{array}{r}130 \\
170 \\
70 \\
100 \\
90\end{array}$ & $\begin{array}{l}950 \\
350 \\
260 \\
560 \\
490\end{array}$ & & $\begin{array}{l}5,300 \\
7,800 \\
6,300 \\
2,500 \\
3,500\end{array}$ & $\begin{array}{r}1,100 \\
1,900 \\
3,900 \\
770 \\
1,000\end{array}$ & $\begin{array}{l}150 \\
250\end{array}$ & $\begin{array}{l}770 \\
280 \\
270 \\
110 \\
150\end{array}$ & $\begin{array}{l}440 \\
140 \\
600 \\
400 \\
410\end{array}$ & $\begin{array}{r}14,440 \\
16,740 \\
17,780 \\
7,550 \\
8,910\end{array}$ \\
\hline $\begin{array}{l}941 \\
942 \\
943\end{array}$ & $\begin{array}{r}820 \\
2,800 \\
230 \\
250 \\
250\end{array}$ & $\begin{array}{l}160 \\
900 \\
170 \\
230 \\
180\end{array}$ & $\begin{array}{l}160 \\
330 \\
120 \\
150 \\
200\end{array}$ & $\begin{array}{l}130 \\
230 \\
100 \\
140 \\
110\end{array}$ & $\begin{array}{l}150 \\
350 \\
110 \\
210 \\
130\end{array}$ & $\begin{array}{l}460 \\
710 \\
480 \\
370 \\
310\end{array}$ & & $\begin{array}{r}11,500 \\
5,600 \\
5,100 \\
8,400 \\
6,500\end{array}$ & $\begin{array}{r}10,600 \\
3,300 \\
2,200 \\
6,100 \\
2,500\end{array}$ & $\begin{array}{r}2,500 \\
640 \\
490 \\
920 \\
580\end{array}$ & $\begin{array}{l}470 \\
240 \\
340 \\
260 \\
260\end{array}$ & $\begin{array}{r}1,400 \\
180 \\
200 \\
130 \\
120\end{array}$ & $\begin{array}{l}29,950 \\
18,880 \\
14,440 \\
19,060 \\
12,940\end{array}$ \\
\hline $\begin{array}{l}1946 \\
1947 \\
1948 \\
1949 \\
1950\end{array}$ & $\begin{array}{l}240 \\
310 \\
750 \\
210 \\
330\end{array}$ & $\begin{array}{l}170 \\
300 \\
130 \\
270\end{array}$ & $\begin{array}{l}110 \\
160 \\
210 \\
160 \\
150\end{array}$ & $\begin{array}{r}90 \\
140 \\
220 \\
110 \\
110\end{array}$ & $\begin{array}{l}120 \\
190 \\
230 \\
100 \\
150\end{array}$ & $\begin{array}{l}430 \\
640 \\
360 \\
350 \\
370\end{array}$ & $\begin{array}{l}2,200 \\
1,600 \\
3,500 \\
3,000 \\
3,300\end{array}$ & $\begin{array}{l}7,600 \\
4,700 \\
7,300 \\
5,200 \\
2,400\end{array}$ & $\begin{array}{r}1,200 \\
1,600 \\
2,500 \\
10,000 \\
1,400\end{array}$ & $\begin{array}{r}220 \\
510 \\
560 \\
1,700 \\
330\end{array}$ & $\begin{array}{l}270 \\
860 \\
310 \\
520 \\
120\end{array}$ & $\begin{array}{l}260 \\
460 \\
120 \\
180 \\
140\end{array}$ & $\begin{array}{r}6,890 \\
11,340 \\
16,360 \\
21,660 \\
9,070\end{array}$ \\
\hline $\begin{array}{l}1951 \\
1952 \\
1953 \\
1954 \\
1955\end{array}$ & $\begin{array}{l}250 \\
200 \\
260 \\
180 \\
590\end{array}$ & $\begin{array}{l}150 \\
150 \\
140 \\
240 \\
180\end{array}$ & $\begin{array}{l}110 \\
120 \\
160 \\
190 \\
120\end{array}$ & $\begin{array}{r}80 \\
120 \\
140 \\
140 \\
120\end{array}$ & $\begin{array}{r}90 \\
160 \\
160 \\
190 \\
110\end{array}$ & $\begin{array}{l}320 \\
330 \\
430 \\
420 \\
360\end{array}$ & $\begin{array}{l}1,100 \\
4,300 \\
2,600 \\
2,100 \\
1,500\end{array}$ & $\begin{array}{r}3,200 \\
10,000 \\
2,800 \\
2,700 \\
2,800\end{array}$ & $\begin{array}{r}1,700 \\
8,200 \\
2,200 \\
580 \\
1,200\end{array}$ & $\begin{array}{l}380 \\
950 \\
370 \\
660 \\
350\end{array}$ & $\begin{array}{l}250 \\
330 \\
360 \\
310 \\
980\end{array}$ & $\begin{array}{l}170 \\
190 \\
160 \\
370 \\
250\end{array}$ & $\begin{array}{r}7,780 \\
25,050 \\
9,780 \\
8,080 \\
8,560\end{array}$ \\
\hline $\begin{array}{l}1956 \\
1957 \\
1958 \\
1959\end{array}$ & $\begin{array}{l}150 \\
120 \\
360 \\
290\end{array}$ & $\begin{array}{l}110 \\
100 \\
340 \\
190\end{array}$ & $\begin{array}{r}120 \\
80 \\
260 \\
150\end{array}$ & $\begin{array}{r}110 \\
80 \\
190 \\
100\end{array}$ & $\begin{array}{r}150 \\
80 \\
260 \\
100\end{array}$ & $\begin{array}{r}1,300 \\
280 \\
460 \\
290\end{array}$ & $\begin{array}{l}2,100 \\
1,900 \\
3,500 \\
1,100\end{array}$ & $\begin{array}{r}3,300 \\
4,000 \\
10,000 \\
2,100\end{array}$ & $\begin{array}{r}1,200 \\
12,200 \\
3,800 \\
750\end{array}$ & $\begin{array}{r}250 \\
3,300 \\
350 \\
180\end{array}$ & $\begin{array}{r}170 \\
1,500 \\
360 \\
250\end{array}$ & $\begin{array}{r}70 \\
620 \\
410 \\
140\end{array}$ & $\begin{array}{r}9,030 \\
24,260 \\
20,290 \\
5,640\end{array}$ \\
\hline
\end{tabular}



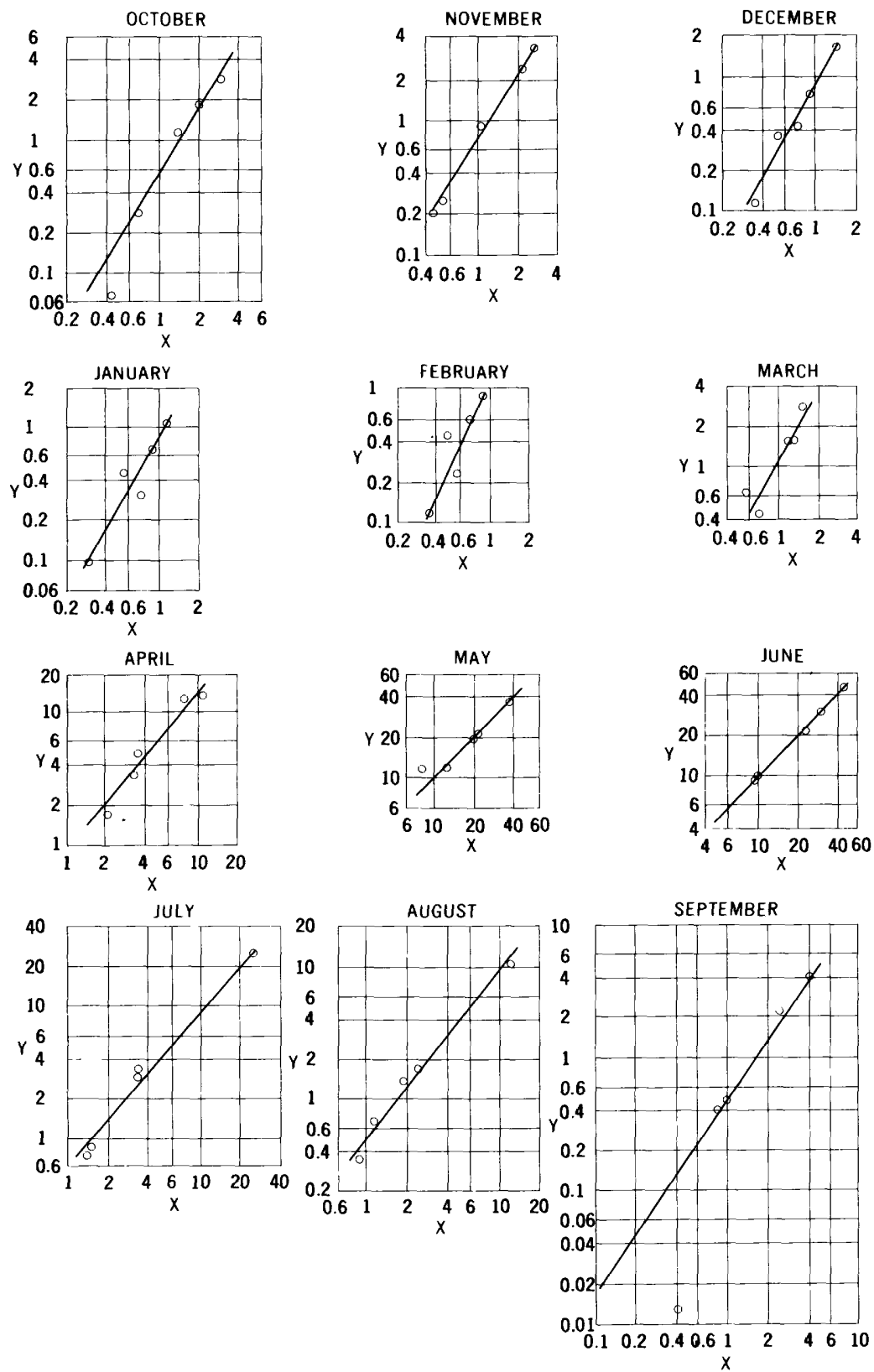

Relationships of monthly mean discharge of Florida River near Durango, Colo. $(Y)$, to monthly mean discharge of Florida River near Hermosa, Colo. (X). Discharge in thousands of acre-feet. 
3630. Florida River near Durango, Colo.

Location. --Lat 37019140", long 107\%44140", in sec.4, T.35 N., R. 8 W., on left bank just downstream from Red Creek, 9 miles northeast of Durango. 'Datum of gage is 7,301.88 $\mathrm{ft}^{\mathrm{t}}$ above mean sea level, datum of 1929. Prior to Mar. 26, 1928, staff gage or water-stage recorder at several sites within $4 \frac{1}{2}$ miles downstream at various datums. Mar. 26, 1928 to Sept. 30, 1934, water-stage recorder at site a quarter of a mile downstream at different ratum.

Drainage area. --96 sq mi, approximately.

Records available.--May to July 1899, April to June 1901, April to October 1902, April to October 1903, September 1910 to October 1911, January to December 1912, June 1917 to September 1924, October 1926 to September 1960. Monthly discharge only for some periods.

Estimates of streamf low.--October 1960 to September 1963, based on relationships of monthly mean discharge with Florida River near Hermosa, Colo. The regression equation used is:

$$
\log Y=b \log X-c
$$

(where $\mathrm{Y}$ is discharge of Florida River near Durango, Colo, and $\mathrm{X}$ is discharge of Florida hiver near Hermosa, Colo., both in acre-feet per month).

Monthly values of constants in above equation

\begin{tabular}{c|c|c|c|c|r|r|r|r|r|r|r|l}
\hline & oct. & Nov. & Dec. & Jan. & Feb. & Mar. & Apr. & May & June & July & Aug. & Sept. \\
\hline b & 1.62 & 1.59 & 1.72 & 1.78 & 2.15 & 1.77 & 1.23 & 1.01 & 1.02 & 1.15 & 1.28 & 1.45 \\
c & 2.11 & 1.89 & 2.50 & 2.38 & 3.40 & 2.26 & .76 & .04 & .10 & .65 & 1.14 & 1.66 \\
\hline
\end{tabular}

Average discharge. -33 years $(1931-63), 67,949$ acre-feet per year (93.9 cf's).

Extremes. - $-1899,1901-3,1910-12,1917-24,1926-60$ : Maximum discharge not determined, occurred Oct. 5, 1911; maximum determined, 3,200 cfs June 28, 1927 from rating curve extended above $900 \mathrm{cfs}$; minimum daily, no flow Sept. 13-18, sept. 22 to oct. 16, 1956 .

Maximum flood known occurred oct. 5, 1911.

Remarks.--Diversions for irrigation of about 160 acres above station. Regulated by Lemon Reservoir (capacity 40,300 acre-feet) since November 1963. Estimates of annual flow are within about 7 percent of regression line.

\begin{tabular}{|c|c|c|c|c|c|c|c|c|c|c|c|c|c|}
\hline $\begin{array}{l}\text { Water } \\
\text { year }\end{array}$ & oct. & Nov. & Dec. & Jan. & Feb. & Mar. & Apr. & May & June & July & Aug. & Sept. & Annual \\
\hline $\begin{array}{l}1931 \\
1932 \\
1933 \\
1934 \\
1935\end{array}$ & $\begin{array}{l}1,160 \\
4,030 \\
1,630 \\
2,750 \\
1,300\end{array}$ & $\begin{array}{r}670 \\
1,240 \\
893 \\
851 \\
645\end{array}$ & $\begin{array}{l}492 \\
922 \\
615 \\
492 \\
553\end{array}$ & $\begin{array}{l}307 \\
492 \\
338 \\
369 \\
430\end{array}$ & $\begin{array}{l}278 \\
863 \\
389 \\
333 \\
389\end{array}$ & $\begin{array}{r}553 \\
2,990 \\
676 \\
1,170 \\
1,580\end{array}$ & $\begin{array}{r}2,210 \\
13,810 \\
1,820 \\
8,330 \\
8,540\end{array}$ & $\begin{array}{r}10,620 \\
32,660 \\
8,960 \\
9,650 \\
15,290\end{array}$ & $\begin{array}{r}14,860 \\
31,930 \\
25,100 \\
1,270 \\
45,260\end{array}$ & $\begin{array}{r}4,060 \\
9,500 \\
6,810 \\
682 \\
12,760\end{array}$ & $\begin{array}{r}2,620 \\
9,620 \\
1,940 \\
904 \\
8,370\end{array}$ & $\begin{array}{l}, 430 \\
, 030 \\
, 440 \\
, 170 \\
, 480\end{array}$ & $\begin{array}{r}41,080 \\
111,100 \\
51,610 \\
27,970 \\
99,600\end{array}$ \\
\hline $\begin{array}{l}1936 \\
1937 \\
1938 \\
1939 \\
1940\end{array}$ & $\begin{array}{l}2,820 \\
2,070 \\
1,560 \\
4,350 \\
1,590\end{array}$ & $\begin{array}{r}1,080 \\
1,840 \\
833 \\
1,780 \\
959\end{array}$ & $\begin{array}{l}615 \\
922 \\
553 \\
909 \\
453\end{array}$ & $\begin{array}{l}430 \\
615 \\
430 \\
653 \\
355\end{array}$ & $\begin{array}{l}414 \\
444 \\
500 \\
321 \\
327\end{array}$ & $\begin{array}{l}2,080 \\
1,500 \\
1,760 \\
1,700 \\
1,360\end{array}$ & $\begin{array}{r}11,040 \\
15,950 \\
13,730 \\
5,750 \\
5,620\end{array}$ & $\begin{array}{l}28,320 \\
31,550 \\
24,530 \\
17,050 \\
17,440\end{array}$ & $\begin{array}{r}10,960 \\
16,430 \\
35,410 \\
5,670 \\
4,970\end{array}$ & $\begin{array}{r}3,160 \\
5,190 \\
8,290 \\
902 \\
1,820\end{array}$ & $\begin{array}{l}6,950 \\
2,110 \\
2,240 \\
1,000 \\
1,010\end{array}$ & $\begin{array}{l}4,280 \\
1,820 \\
8,500 \\
5,440 \\
5,980\end{array}$ & $\begin{array}{l}72,150 \\
80,440 \\
98,340 \\
45,520 \\
39,880\end{array}$ \\
\hline $\begin{array}{l}1941 \\
1942 \\
1943 \\
1944 \\
1945\end{array}$ & $\begin{array}{r}5,480 \\
15,080 \\
851 \\
1,360 \\
1,520\end{array}$ & $\begin{array}{r}1,570 \\
6,050 \\
838 \\
1,100 \\
1,300\end{array}$ & $\begin{array}{r}1,050 \\
2,740 \\
536 \\
742 \\
615\end{array}$ & $\begin{array}{r}553 \\
1,480 \\
510 \\
488 \\
369\end{array}$ & $\begin{array}{l}889 \\
778 \\
528 \\
424 \\
305\end{array}$ & $\begin{array}{r}2,140 \\
1,580 \\
1,320 \\
805 \\
672\end{array}$ & $\begin{array}{r}6,490 \\
13,250 \\
11,540 \\
4,630 \\
5,310\end{array}$ & $\begin{array}{l}39,850 \\
23,800 \\
21,590 \\
28,490 \\
25,280\end{array}$ & $\begin{array}{l}45,380 \\
32,310 \\
14,810 \\
36,980 \\
23,420\end{array}$ & $\begin{array}{r}25,760 \\
5,460 \\
3,300 \\
9,930 \\
5,820\end{array}$ & $\begin{array}{l}5,440 \\
1,510 \\
4,320 \\
1,430 \\
2,540\end{array}$ & $\begin{array}{r}7,870 \\
1,270 \\
1,580 \\
764 \\
699\end{array}$ & $\begin{array}{r}142,500 \\
105,300 \\
61,720 \\
87,140 \\
67,850\end{array}$ \\
\hline $\begin{array}{l}1946 \\
1947 \\
1948 \\
1949 \\
1950\end{array}$ & $\begin{array}{l}2,220 \\
2,280 \\
3,740 \\
1,450 \\
1,310\end{array}$ & $\begin{array}{r}1,110 \\
1,430 \\
2,000 \\
763 \\
1,360\end{array}$ & $\begin{array}{r}417 \\
932 \\
1,080 \\
450 \\
875\end{array}$ & $\begin{array}{l}234 \\
449 \\
726 \\
556 \\
742\end{array}$ & $\begin{array}{l}218 \\
548 \\
514 \\
353 \\
520\end{array}$ & $\begin{array}{r}690 \\
966 \\
1,120 \\
1,150 \\
1,240\end{array}$ & $\begin{array}{r}3,710 \\
2,730 \\
2,740 \\
9,160 \\
8,240\end{array}$ & $\begin{array}{r}8,730 \\
20,620 \\
35,530 \\
25,330 \\
15,820\end{array}$ & $\begin{array}{r}16,890 \\
19,230 \\
34,850 \\
47,410 \\
9,360\end{array}$ & $\begin{array}{r}1,990 \\
4,550 \\
6,960 \\
14,370 \\
2,070\end{array}$ & $\begin{array}{r}1,780 \\
8,460 \\
2,350 \\
2,270 \\
328\end{array}$ & $\begin{array}{r}1,100 \\
4,620 \\
740 \\
808 \\
700\end{array}$ & $\begin{array}{r}39,090 \\
66,820 \\
102,400 \\
104,100 \\
42,560\end{array}$ \\
\hline $\begin{array}{l}1951 \\
1952 \\
1953 \\
1954 \\
1955\end{array}$ & $\begin{array}{r}1,150 \\
523 \\
973 \\
448 \\
3,500\end{array}$ & $\begin{array}{r}387 \\
438 \\
618 \\
1,110 \\
739\end{array}$ & $\begin{array}{l}290 \\
359 \\
393 \\
512 \\
462\end{array}$ & $\begin{array}{l}246 \\
316 \\
337 \\
430 \\
328\end{array}$ & $\begin{array}{l}228 \\
311 \\
327 \\
382 \\
268\end{array}$ & $\begin{array}{l}544 \\
609 \\
773 \\
565 \\
438\end{array}$ & $\begin{array}{r}1,120 \\
1,020 \\
3,460 \\
5,370 \\
2,200\end{array}$ & $\begin{array}{l}10,400 \\
26,620 \\
11,370 \\
16,030 \\
12,370\end{array}$ & $\begin{array}{r}11,810 \\
44,580 \\
14,560 \\
7,700 \\
14,210\end{array}$ & $\begin{array}{l}1,270 \\
9,740 \\
2,660 \\
6,000 \\
2,060\end{array}$ & $\begin{array}{l}1,790 \\
2,790 \\
1,580 \\
2,650 \\
4,790\end{array}$ & $\begin{array}{r}825 \\
1,640 \\
237 \\
2,590 \\
1,020\end{array}$ & $\begin{array}{l}30,060 \\
98,940 \\
37,290 \\
43,790 \\
42,380\end{array}$ \\
\hline $\begin{array}{l}1956 \\
1957 \\
1958 \\
1959 \\
1960\end{array}$ & $\begin{array}{r}291 \\
70 \\
1,800 \\
1,150 \\
2,880\end{array}$ & $\begin{array}{r}207 \\
247 \\
3,480 \\
877 \\
2,390\end{array}$ & $\begin{array}{r}364 \\
115 \\
1,660 \\
430 \\
738\end{array}$ & $\begin{array}{r}460 \\
96 \\
1,050 \\
307 \\
676\end{array}$ & $\begin{array}{l}434 \\
111 \\
889 \\
222 \\
575\end{array}$ & $\begin{array}{r}1,540 \\
618 \\
1,500 \\
430 \\
2,790\end{array}$ & $\begin{array}{r}3,440 \\
4,870 \\
12,500 \\
1,690 \\
13,400\end{array}$ & $\begin{array}{l}19,140 \\
11,470 \\
37,320 \\
11,910 \\
20,240\end{array}$ & $\begin{array}{r}8,910 \\
44,370 \\
29,750 \\
9,590 \\
21,570\end{array}$ & $\begin{array}{r}737 \\
25,610 \\
2,880 \\
862 \\
3,310\end{array}$ & $\begin{array}{r}350 \\
10,940 \\
1,370 \\
1,670 \\
699\end{array}$ & $\begin{array}{r}13 \\
4,100 \\
2,120 \\
465 \\
391\end{array}$ & $\begin{array}{r}35,890 \\
102,600 \\
96,320 \\
29,600 \\
69,660\end{array}$ \\
\hline
\end{tabular}

Estimated monthly and annual streamflow, in acre-feet

\begin{tabular}{l|r|r|r|r|r|r|r|r|r|r|r|r|r}
\hline $\begin{array}{l}\text { Water } \\
\text { year }\end{array}$ & Oct. & Nov. & Dec. & Jan. & Feb. & Mar. & Apr. & May & June & July & Aug. & Sept. & Annual \\
\hline 1961 & 860 & 960 & 450 & 300 & 210 & 640 & 4,500 & 25,000 & 12,000 & 1,300 & 2,800 & 6,000 & 55,020 \\
1962 & 4,200 & 1,600 & 760 & 450 & 500 & 810 & 14,000 & 22,000 & 18,000 & 3,100 & 600 & 4,500 & 70,520 \\
1963 & 1,500 & 1,500 & 740 & 420 & 450 & 2,100 & 6,700 & 19,000 & 4,300 & 960 & 1,900 & 3,500 & 43,070 \\
\hline
\end{tabular}



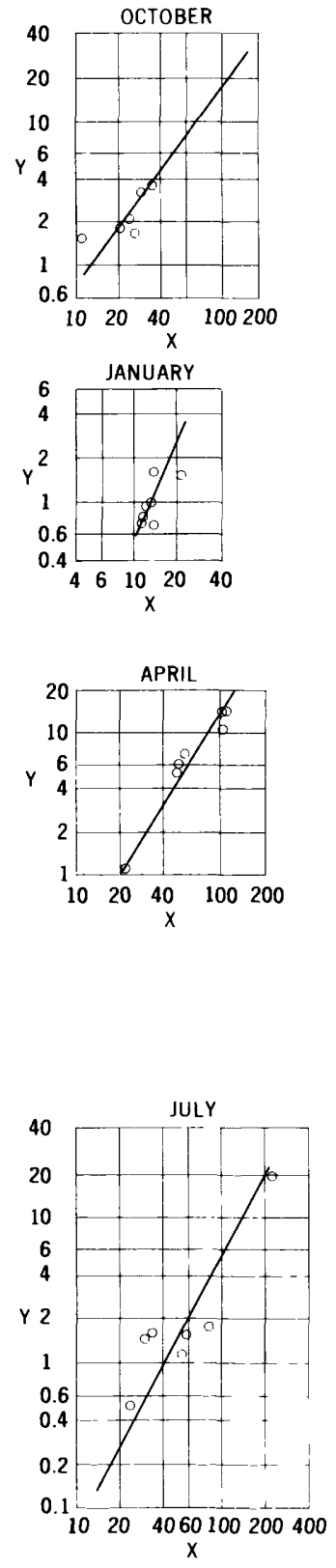
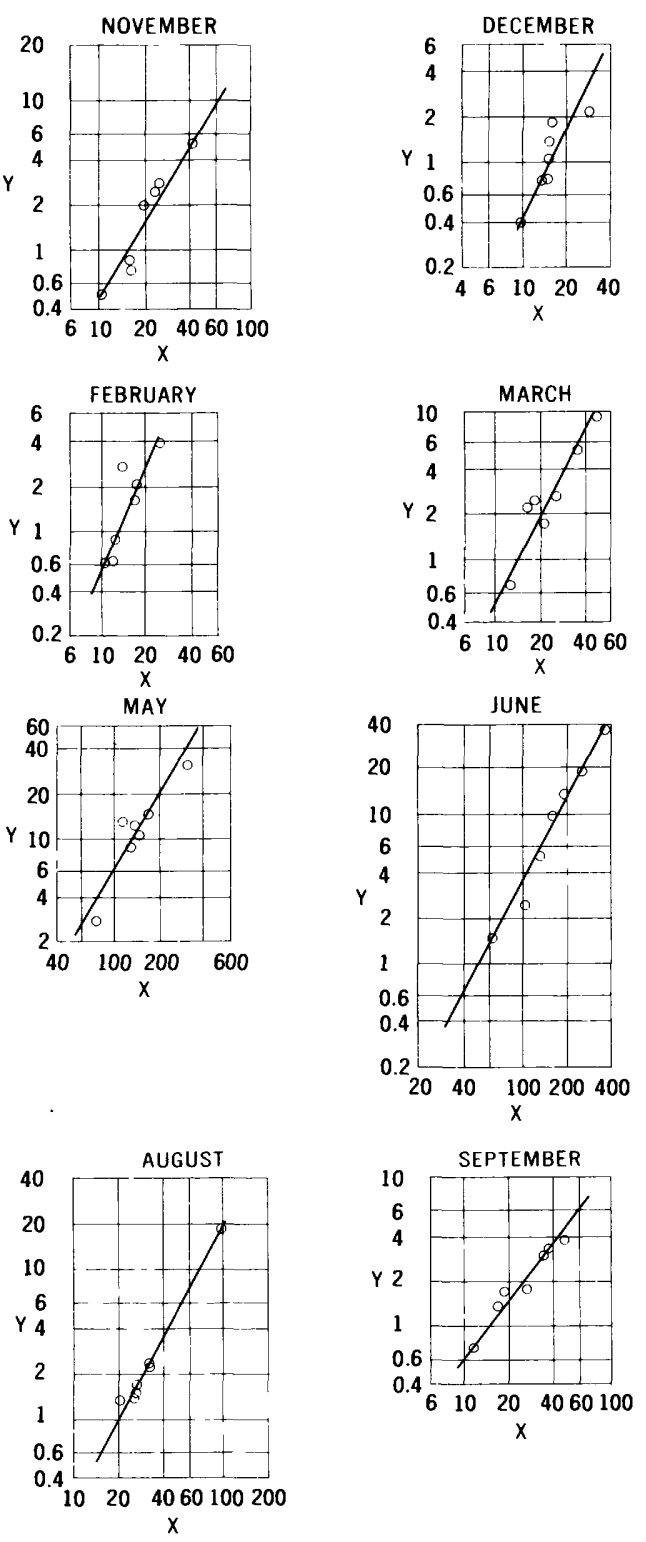

Relationships of monthly mean discharge of Florida River at Bondad, Colo. ( $Y$ ), to monthly mean discharge of Animas River near Cedar Hill, New Mexico (X). Discharge in thousands of acre-feet. 
3632. Florida River at Bondad, Colo.

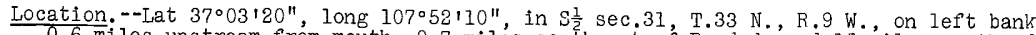
0.6 miles upstream from mouth, $0.7 \mathrm{miles}$ southeast of Bondad, and 15 miles south of Durango. Altitude of gage is $6,000 \mathrm{ft}$ (from topographic map).

Drainage area. - -221 sq mi.

Records available.--October 1956 to september 1963.

Estimates of streamflow. --October 1933 to September 1956, October 1963 to September 1965, based on relationships of monthly mean discharge with Animas River near cedar Hill,

N. Mex. The regression equation used is:

$$
\log Y=b \log X-c
$$

(where $Y$ is discharge of Florida River at Bondad, Colo., and $X$ is discharge of Animas River near Cedar Hill, N. Mex, both in acre-feet per month).

\begin{tabular}{l|c|c|c|c|c|c|c|c|c|c|c|c}
\hline \multicolumn{10}{c}{ Monthly values of constants in above equat1on } \\
\hline & oct. & Nov. & Dec. & Jan. & Feb. & Mar. & Apr. & May & June & July & Aug. & Sept. \\
\hline b & 1.40 & 1.70 & 2.00 & 2.31 & 2.31 & 1.91 & 1.63 & 1.70 & 1.84 & 1.84 & 1.85 & 1.29 \\
c & 2.77 & 4.10 & 5.37 & 6.51 & 6.51 & 4.93 & 4.01 & 4.72 & 5.66 & 5.46 & 4.99 & 2.37 \\
\hline
\end{tabular}

Average discharge. --32 years $(1933-65), 55,362$ acre-feet per year (76.5 cf's).

Extremes. --1956-63: Maximum discharge determined, 1,430 cf's June 7, 1958, but may have been higher on July 26, 1957; minimum daily, 4.6 cf's July 24, 1959.

Remarks.--Diversions for irrigation of about 14,000 acres above station. Estimates of annual flow are within about 20 percent of regression line.

Monthly and annual streamflow, in acre-feet

\begin{tabular}{r|r|r|r|r|r|r|r|r|r|r|r|r|r}
\hline $\begin{array}{l}\text { Water } \\
\text { year }\end{array}$ & Oct. & Nov. & Dec. & Jan. & Feb. & Mar. & Apr. & May & June & July & Aug. & Sept. & Annual \\
\hline 1957 & 1,540 & 505 & 398 & 750 & 2,690 & 2,250 & 5,240 & 12,880 & 37,230 & 19,650 & 18,990 & 3,890 & 106,100 \\
1958 & 3,180 & 5,160 & 2,110 & 1,570 & 3,840 & 5,410 & 13,800 & 31,190 & 19,530 & 1,150 & 1,640 & 1,780 & 90,360 \\
1959 & 1,730 & 855 & 754 & 772 & 625 & 687 & 1,110 & 2,750 & 2,430 & 513 & 1,350 & 723 & 14,300 \\
1960 & 1,670 & 2,870 & 1,020 & 940 & 885 & 9,160 & 13,980 & 12,390 & 13,380 & 1,570 & 1,320 & 1,370 & 60,560 \\
& & & & & & & & & & & & & \\
1961 & 1,750 & 758 & 768 & 718 & 633 & 2,500 & 7,010 & 14,850 & 5,230 & 1,630 & 2,300 & 3,280 & 41,430 \\
1962 & 3,630 & 2,490 & 1,850 & 1,630 & 1,990 & 1,760 & 10,340 & 10,280 & 9,560 & 1,780 & 1,440 & 1,720 & 48,470 \\
1963 & 2,070 & 2,070 & 1,320 & 1,010 & 1,660 & 2,650 & 6,070 & 8,700 & 1,470 & 1,460 & 2,190 & 3,080 & 33,750 \\
\hline
\end{tabular}

Estimated monthly and annual streamflow, in acre-feet

\begin{tabular}{|c|c|c|c|c|c|c|c|c|c|c|c|c|c|}
\hline $\begin{array}{l}\text { Water } \\
\text { year }\end{array}$ & Oct. & Nov. & Dec. & Jan. & Feb. & Mar. & Apr. & May & June & July & Aug. & Sept. & Annual \\
\hline $\begin{array}{l}1934 \\
1935\end{array}$ & $\begin{array}{r}\neq 2,400 \\
1,100\end{array}$ & $\neq 1,000$ & $\begin{array}{l}870 \\
520\end{array}$ & $\begin{array}{r}.000 \\
770\end{array}$ & $\begin{array}{l}680 \\
760\end{array}$ & $\begin{array}{l}1,300 \\
3,100\end{array}$ & $\begin{array}{l}4,400 \\
7,000\end{array}$ & 300 & $\begin{array}{r}320 \\
26,900\end{array}$ & $\begin{array}{r}140 \\
5,600\end{array}$ & $\begin{array}{r}500 \\
5,400\end{array}$ & $\begin{array}{l}1,400 \\
2,800\end{array}$ & $\begin{array}{r}\$ 18,510 \\
62,280\end{array}$ \\
\hline $\begin{array}{l}1936 \\
1937 \\
1938 \\
1939 \\
1940\end{array}$ & $\begin{array}{l}2,500 \\
1,700 \\
1,800 \\
4,800 \\
1,600\end{array}$ & $\begin{array}{r}1,200 \\
1,500 \\
950 \\
1,900 \\
1,000\end{array}$ & $\begin{array}{r}650 \\
760 \\
770 \\
1,000 \\
610\end{array}$ & $\begin{array}{r}650 \\
880 \\
950 \\
1,100 \\
1,100\end{array}$ & $\begin{array}{l}830 \\
840 \\
980 \\
680 \\
860\end{array}$ & $\begin{array}{l}5,600 \\
5,700 \\
5,200 \\
3,700 \\
2,000\end{array}$ & $\begin{array}{r}15,000 \\
18,200 \\
17,000 \\
5,600 \\
4,700\end{array}$ & $\begin{array}{r}19,000 \\
27,200 \\
15,200 \\
9,200 \\
9,800\end{array}$ & $\begin{array}{r}3,800 \\
4,900 \\
23,300 \\
2,700 \\
2,000\end{array}$ & $\begin{array}{r}820 \\
1,800 \\
6,600 \\
510 \\
360\end{array}$ & $\begin{array}{r}5,700 \\
1,200 \\
2,200 \\
650 \\
590\end{array}$ & & $\begin{array}{l}58,450 \\
66,080 \\
79,950 \\
35,540 \\
27,120\end{array}$ \\
\hline $\begin{array}{l}1941 \\
1942 \\
1943 \\
1944 \\
1945\end{array}$ & $\begin{array}{r}4,900 \\
28,100 \\
1,600 \\
2,200 \\
1,900\end{array}$ & $\begin{array}{r}1,400 \\
11,600 \\
1,100 \\
1,700 \\
1,300\end{array}$ & $\begin{array}{r}1,200 \\
4,800 \\
870 \\
1,100 \\
840\end{array}$ & $\begin{array}{r}1,600 \\
3,900 \\
1,300 \\
1,000 \\
950\end{array}$ & $\begin{array}{r}2,400 \\
2,400 \\
1,200 \\
940 \\
850\end{array}$ & $\begin{array}{l}6,000 \\
3,700 \\
3,200 \\
1,800 \\
1,500\end{array}$ & $\begin{array}{r}7,400 \\
16,500 \\
15,700 \\
4,100 \\
3,400\end{array}$ & $\begin{array}{l}50,500 \\
15,300 \\
10,600 \\
26,200 \\
14,900\end{array}$ & $\begin{array}{r}33,300 \\
17,200 \\
5,600 \\
25,500 \\
8,600\end{array}$ & $\begin{array}{r}20,700 \\
4,600 \\
1,800 \\
8,400 \\
2,600\end{array}$ & $\begin{array}{l}6,700 \\
2,500 \\
5,800 \\
2,300 \\
2,400\end{array}$ & & $\begin{array}{r}143,500 \\
112,400 \\
51,570 \\
76,340 \\
40,540\end{array}$ \\
\hline $\begin{array}{l}1946 \\
1947 \\
1948 \\
1949 \\
1950\end{array}$ & $\begin{array}{l}2,000 \\
2,000 \\
6,400 \\
1,800 \\
2,000\end{array}$ & $\begin{array}{l}1,200 \\
1,400 \\
2,600 \\
1,000 \\
1,300\end{array}$ & $\begin{array}{r}650 \\
1,000 \\
1,400 \\
720 \\
860\end{array}$ & $\begin{array}{r}900 \\
840 \\
1,500 \\
1,600 \\
1,700\end{array}$ & $\begin{array}{r}880 \\
940 \\
1,400 \\
1,000 \\
1,400\end{array}$ & $\begin{array}{l}1,100 \\
1,300 \\
1,900 \\
3,000 \\
1,800\end{array}$ & $\begin{array}{r}4,000 \\
2,300 \\
13,500 \\
11,100 \\
7,500\end{array}$ & $\begin{array}{r}3,000 \\
14,300 \\
27,200 \\
20,400 \\
5,300\end{array}$ & $\begin{array}{r}6,600 \\
8,800 \\
17,000 \\
32,900 \\
4,600\end{array}$ & $\begin{array}{r}1,100 \\
4,400 \\
4,200 \\
11,400 \\
1,300\end{array}$ & $\begin{array}{r}2,000 \\
8,400 \\
3,000 \\
3,500 \\
710\end{array}$ & $\begin{array}{l}1,400 \\
5,000 \\
1,200 \\
1,500 \\
1,500\end{array}$ & $\begin{array}{l}24,830 \\
50,680 \\
81,300 \\
89,920 \\
29,970\end{array}$ \\
\hline $\begin{array}{l}1951 \\
1952 \\
1953 \\
1954 \\
1955\end{array}$ & $\begin{array}{l}1,400 \\
1,000 \\
1,600 \\
1,200 \\
4,200\end{array}$ & $\begin{array}{r}760 \\
660 \\
920 \\
1,200 \\
1,100\end{array}$ & $\begin{array}{l}720 \\
810 \\
540 \\
730 \\
760\end{array}$ & $\begin{array}{r}800 \\
2,100 \\
880 \\
580 \\
800\end{array}$ & $\begin{array}{r}500 \\
1,400 \\
760 \\
570 \\
570\end{array}$ & $\begin{array}{r}560 \\
2,900 \\
1,400 \\
730 \\
1,200\end{array}$ & $\begin{array}{r}880 \\
16,500 \\
3,600 \\
4,600 \\
2,100\end{array}$ & $\begin{array}{r}4,500 \\
27,900 \\
4,400 \\
7,000 \\
6,500\end{array}$ & $\begin{array}{r}4,800 \\
33,900 \\
7,400 \\
2,100 \\
5,700\end{array}$ & $\begin{array}{r}820 \\
7,200 \\
1,000 \\
2,100 \\
1,000\end{array}$ & $\begin{array}{l}1,300 \\
3,900 \\
1,400 \\
1,700 \\
2,800\end{array}$ & $\begin{array}{r}1,000 \\
2,200 \\
900 \\
2,000 \\
1,200\end{array}$ & $\begin{array}{r}18,040 \\
100,470 \\
24,800 \\
24,510 \\
27,930\end{array}$ \\
\hline 1956 & 880 & 680 & 800 & 1,000 & 880 & 2,900 & 3,600 & 10,100 & 4,400 & 460 & 560 & 560 & 26,820 \\
\hline $\begin{array}{l}1964 \\
1965\end{array}$ & $\begin{array}{l}1,300 \\
1,200\end{array}$ & $\begin{array}{l}780 \\
890\end{array}$ & $\begin{array}{l}470 \\
950\end{array}$ & $\begin{array}{r}590 \\
1,200\end{array}$ & $\begin{array}{l}390 \\
920\end{array}$ & $\begin{array}{r}460 \\
1,700\end{array}$ & $\begin{array}{l}1,200 \\
9,200\end{array}$ & $\begin{array}{r}8,000 \\
14,000\end{array}$ & $\begin{array}{r}2,700 \\
19,500\end{array}$ & $\begin{array}{r}620 \\
17,900\end{array}$ & $\begin{array}{r}3,100 \\
10,500\end{array}$ & $\begin{array}{l}1,500 \\
6,000\end{array}$ & $\begin{array}{l}21,110 \\
83,960\end{array}$ \\
\hline
\end{tabular}

\# Based on estimates for 1950 Compilation. 

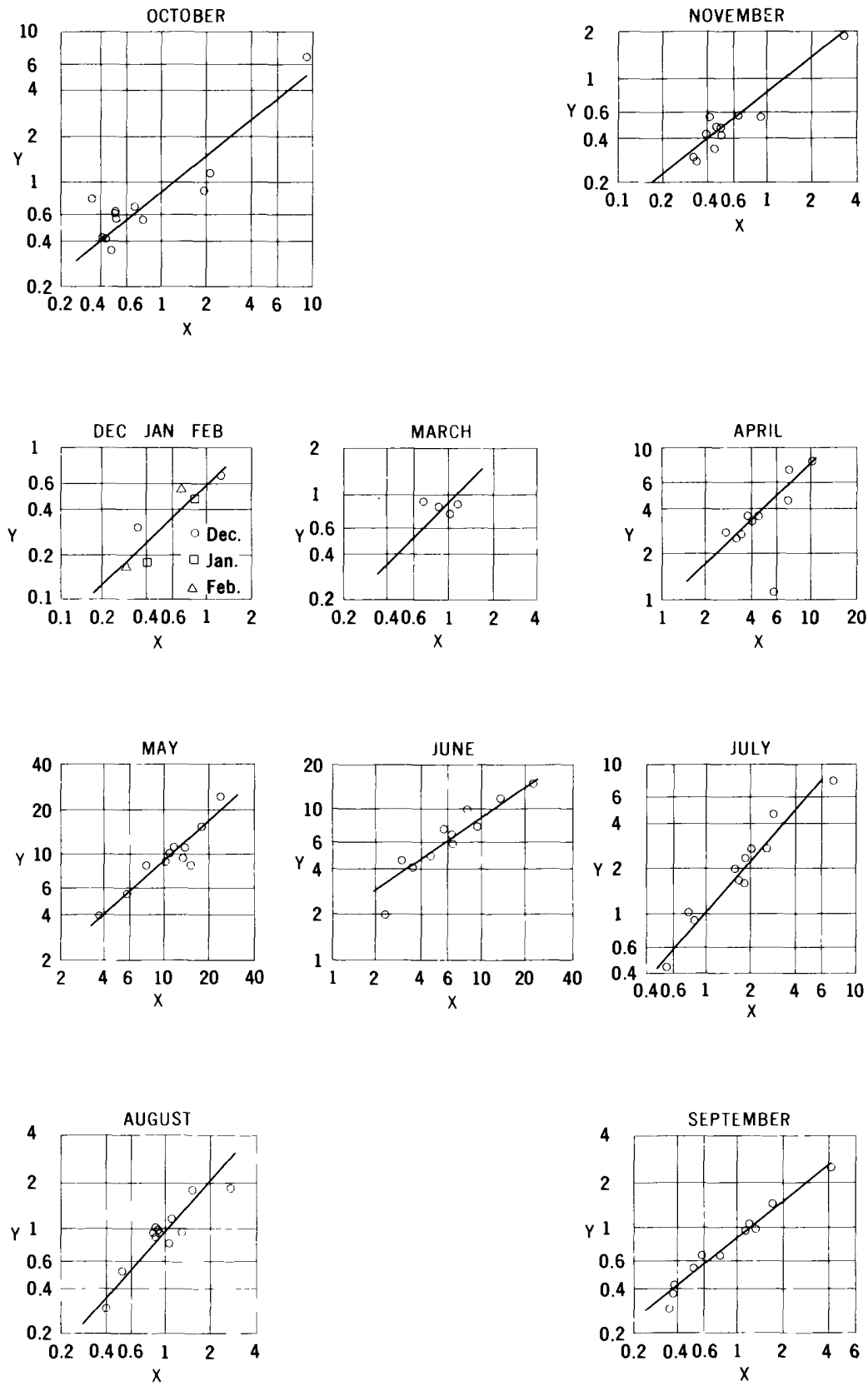

Relationships of monthly mean discharge of West Mancos River near Mancos, Colo. (Y), to monthly mean discharge of La Plata River at Hesperus, Colo. $(X)$. Discharge in thousands of acre-feet. 
3685. West Mancos River near Mancos, Colo.

Location,--Lat $37^{\circ} 22^{1} 30^{\prime \prime}$, long 108 15120", in sec.14, T. $36 \mathrm{~N}, \mathrm{R}, 13 \mathrm{~W}$, on right bank $1 \frac{1}{2}$ miles upstream from confluence with'East Mancos River and $3 \frac{1}{2}$ miles northeast of Mancos. Altitude of gage is $7,450 \mathrm{ft}$ (from nearby Bureau of Reclamation level line).

Drainage area. $--42.1 \mathrm{sq} \mathrm{mi}$.

Records available. --September 1910 to September 1911 (no winter records), October to December 1911 (gage heights only), May 1938 to September 1953. Monthly discharge only for some periods.

Estimates of streamflow.--October 1930 to April 1938, based on relationships of monthly mean discharge with LaPlata River at Hesperus, Colo. The regression equation used is:

$\log Y=b \log X-c$

(where $\mathrm{Y}$ is discharge of West Mancos River near Mancos, Colo., and $\mathrm{X}$ is discharge of LaPlata River at Hesperus, Colo., both in acre-feet per month\}.

Monthly values of constants in above equation

\begin{tabular}{l|r|r|r|r|r|r|r|r|r|r|r|r}
\hline & Oct. & Nov. & Dec. & Jan. & Feb. & Mar. & Apr. & May & June & July & Aug. & Sept. \\
\hline $\mathrm{b}$ & 0.80 & 0.78 & 0.98 & 0.98 & 0.98 & 0.98 & 0.98 & 0.90 & 0.77 & 1.12 & 1.10 & 0.80 \\
$\mathrm{c}$ & $\mathbf{- . 5 2}$ & $\mathbf{- . 5 7}$ & .14 & .14 & .14 & -.02 & -.02 & -.39 & $\mathbf{- . 8 8}$ & .36 & .33 & $\mathbf{- . 5 2}$ \\
\hline
\end{tabular}

Average discharge. - -23 years $(1930-53), 27,527$ acre-feet per year (38.0 cfs).

Extremes.--1938-53: Maximum discharge, 1,080 cfs May 13, 1941, f'rom rating curve extended above 260 cf's; minimum daily recorded, 0.1 cfs Apr. 11-13, 1951.

Remarks.--Diversion above station for irrigation of about 2,500 acres below since March 1949. Regulation for irrigation by Jackson Gulch Reservoir (capacity, 10,000 acre-feet) since March 1949. Estimates of annual flow are within about 10 percent of regression line.

Monthly and annual streamflow, in acre-feet

\begin{tabular}{|c|c|c|c|c|c|c|c|c|c|c|c|c|c|}
\hline $\begin{array}{c}\text { Water } \\
\text { year }\end{array}$ & oct. & Nov. & Dec. & Jan. & $\mathrm{Feb}$. & Mar. & Apr. & May & June & July & Aug. & Sept. & Annual \\
\hline $\begin{array}{l}1938 \\
1939 \\
1940\end{array}$ & $\begin{array}{l}- \\
564 \\
428\end{array}$ & $\begin{array}{l}- \\
417 \\
279\end{array}$ & $\begin{array}{c}\overline{3} \\
\pm 243\end{array}$ & $\begin{array}{r}\overline{184} \\
\neq 215\end{array}$ & $\begin{array}{r}\overline{167} \\
+230\end{array}$ & $\begin{array}{r}789 \\
+677\end{array}$ & $\begin{array}{l}3,440 \\
3,670\end{array}$ & $\begin{array}{l}9,560 \\
5,540 \\
8,460\end{array}$ & $\begin{array}{l}7,690 \\
2,010 \\
4,630\end{array}$ & $\begin{array}{r}2,660 \\
438 \\
882\end{array}$ & $\begin{array}{l}924 \\
293 \\
508\end{array}$ & $\begin{array}{r}1,470 \\
986 \\
1,080\end{array}$ & $\begin{array}{c}- \\
15,140 \\
\neq 21,300\end{array}$ \\
\hline $\begin{array}{l}1941 \\
1942 \\
1943 \\
1944 \\
1945\end{array}$ & $\begin{array}{r}1,170 \\
6,980 \\
351 \\
637 \\
574\end{array}$ & $\begin{array}{r}547 \\
1,910 \\
340 \\
571 \\
464\end{array}$ & $\begin{array}{l}\neq 553 \\
700 \\
\neq 277 \\
\neq 430 \\
\neq 553\end{array}$ & $\begin{array}{r}\$ 492 \\
492 \\
\neq 246 \\
\neq 430 \\
\$ 369\end{array}$ & $\begin{array}{r}\neq 444 \\
555 \\
\neq 250 \\
\neq 431 \\
\$ 333\end{array}$ & $\begin{array}{r}856 \\
819 \\
\neq 827 \\
\neq 508 \\
\neq 400\end{array}$ & $\begin{array}{l}2,360 \\
7,460 \\
8,520 \\
2,680 \\
2,600\end{array}$ & $\begin{array}{l}24,460 \\
11,350 \\
10,120 \\
15,550 \\
11,260\end{array}$ & $\begin{array}{r}15,120 \\
10,100 \\
7,430 \\
11,990 \\
6,740\end{array}$ & $\begin{array}{l}7,470 \\
2,660 \\
1,950 \\
4,490 \\
2,310\end{array}$ & $\begin{array}{r}1,780 \\
927 \\
1,120 \\
1,010 \\
896\end{array}$ & $\begin{array}{r}2,580 \\
543 \\
665 \\
427 \\
374\end{array}$ & $\begin{array}{r}+57,830 \\
44,500 \\
\$ 32,100 \\
\$ 39,150 \\
\neq 26,870\end{array}$ \\
\hline $\begin{array}{l}1946 \\
1947 \\
1948 \\
1949 \\
1950\end{array}$ & $\begin{array}{l}622 \\
683 \\
884 \\
423 \\
643\end{array}$ & $\begin{array}{l}417 \\
475 \\
543 \\
292 \\
546\end{array}$ & $\begin{array}{l}\ddagger 307 \\
\ddagger 523 \\
\ddagger 400 \\
\ddagger 430 \\
\ddagger 369\end{array}$ & $\begin{array}{l}\$ 307 \\
\$ 492 \\
\$ 430 \\
\$ 338 \\
\$ 277\end{array}$ & $\begin{array}{l}\ddagger 333 \\
\ddagger 523 \\
\ddagger 345 \\
\ddagger 250 \\
\ddagger 413\end{array}$ & $\begin{array}{r}\neq 615 \\
712 \\
\neq 448 \\
\neq 354 \\
\neq 31\end{array}$ & $\begin{array}{r}3,680 \\
2,810 \\
4,610 \\
1,150 \\
676\end{array}$ & $\begin{array}{l}3,940 \\
8,910 \\
8,590 \\
5,490 \\
3,970\end{array}$ & $\begin{array}{r}4,190 \\
4,960 \\
5,960 \\
13,500 \\
4,800\end{array}$ & $\begin{array}{r}988 \\
1,660 \\
1,570 \\
3,630 \\
2,670\end{array}$ & $\begin{array}{r}976 \\
1,810 \\
792 \\
3,190 \\
2,030\end{array}$ & $\begin{array}{r}670 \\
987 \\
294 \\
1,600 \\
1,180\end{array}$ & $\begin{array}{l}\neq 17,050 \\
¥ 24,550 \\
¥ 24,870 \\
¥ 30,630 \\
\neq 17,600\end{array}$ \\
\hline $\begin{array}{l}1951 \\
1952 \\
1953\end{array}$ & $\begin{array}{r}608 \\
570 \\
1,520\end{array}$ & $\begin{array}{r}+126 \\
229 \\
457\end{array}$ & $\begin{array}{r}+215 \\
246 \\
221\end{array}$ & $\begin{array}{r}+184 \\
277 \\
178\end{array}$ & $\begin{array}{r}+222 \\
316 \\
167\end{array}$ & $\begin{array}{r}\neq 184 \\
430 \\
208\end{array}$ & $\begin{array}{r}157 \\
2,290 \\
304\end{array}$ & $\begin{array}{l}1,160 \\
9,450 \\
1,210\end{array}$ & $\begin{array}{r}4,450 \\
13,970 \\
4,480\end{array}$ & $\begin{array}{l}2,580 \\
3,240 \\
2,650\end{array}$ & $\begin{array}{l}1,550 \\
2,500 \\
2,210\end{array}$ & $\begin{array}{r}783 \\
1,730 \\
1,180\end{array}$ & $\begin{array}{r}\neq 12,220 \\
35,250 \\
14,780\end{array}$ \\
\hline
\end{tabular}

* Estimated for 1950 and 1960 compilations.

Estimated monthly and annual streamflow, in acre-feet

\begin{tabular}{l|r|r|r|r|r|r|r|r|r|r|r|r|r|}
\hline $\begin{array}{l}\text { Water } \\
\text { year }\end{array}$ & Oct. & Nov. & Dec. & Jan. & Feb. & Mar. & Apr. & May & June & July & Aug. & Sept. & Annual \\
\hline $\mathbf{1 9 3 1}$ & 420 & 370 & 230 & 160 & 140 & 280 & 1,300 & 5,500 & 3,500 & 1,400 & 800 & 1,100 & 15,200 \\
1932 & 1,100 & 510 & 270 & 340 & 320 & 1,100 & 7,800 & 13,400 & 6,800 & 2,900 & 2,700 & 1,000 & 38,240 \\
1933 & 650 & 460 & 270 & 180 & 190 & 550 & 1,800 & 6,200 & 6,700 & 2,600 & 580 & 1,000 & 21,180 \\
1934 & 1,100 & 310 & 200 & 200 & 540 & 910 & 3,300 & 3,400 & $* 930$ & 620 & 550 & 560 & 12,420 \\
1935 & 430 & 270 & 200 & 270 & 310 & 760 & 4,900 & 8,700 & 13,300 & 4,100 & 1,200 & 750 & 35,190 \\
& & & & & & & & & & & & & \\
1936 & 640 & 330 & 170 & 180 & 220 & 1,400 & 8,400 & 10,400 & 3,700 & 1,000 & 2,400 & 1,100 & 29,940 \\
1937 & 730 & 510 & 340 & 330 & 260 & 440 & 7,500 & 14,600 & 5,200 & 3,300 & 870 & 440 & 34,420 \\
$\mathbf{1 9 3 8}$ & 500 & 210 & 120 & 110 & 140 & 310 & 9,000 & - & - & - & - & - & 32,690 \\
\hline
\end{tabular}

* Used July relation. 